Supporting Information

\title{
Enantioselective Synthesis of Axially Chiral Biaryls by Diels-Alder/Retro-Diels-Alder Reaction of 2-Pyrones with Alkynes
}

Meng-Meng Xu, ${ }^{\dagger}$ Xin-Yu You, ${ }^{\dagger}$ Yu-Zhen Zhang, ${ }^{\dagger}$ Yang Lu, ${ }^{\dagger}$ Kui Tan,${ }^{\dagger}$ Limin Yang,, and Quan Cai*,ं

'Department of Chemistry, Fudan University, 220 Handan Rd., Shanghai 200433, China

College of Materials, Chemistry and Chemical Engineering, Hangzhou Normal University, Hangzhou 311121, China 


\section{Table of Contents}

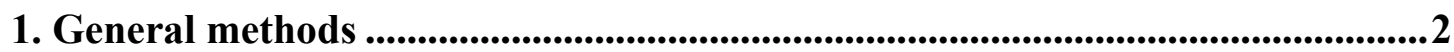

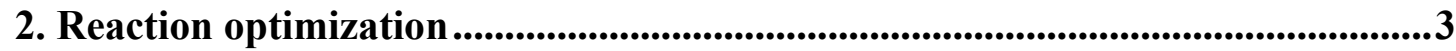

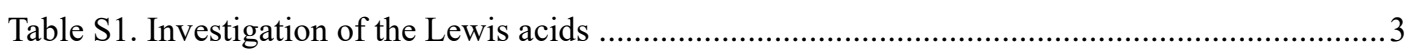

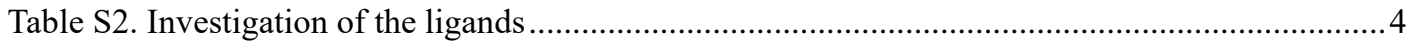

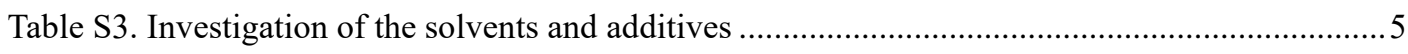

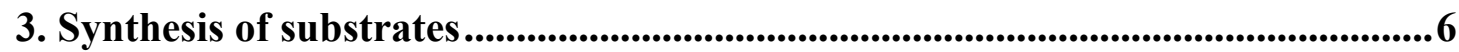

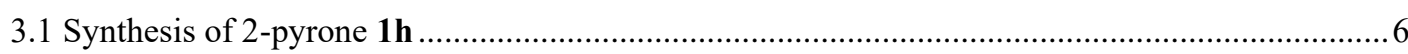

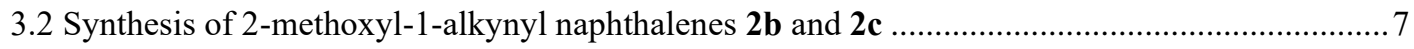

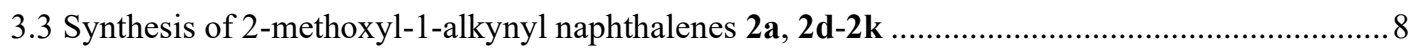

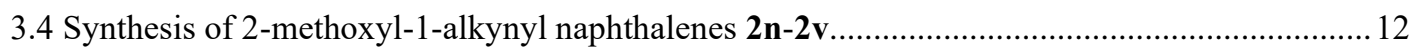

3.5 Synthesis of 2-alkoxyl-1-alkynyl naphthalenes $\mathbf{2 w - 2 y}$ and 1-alkynyl naphthalene $\mathbf{2 z}$................17

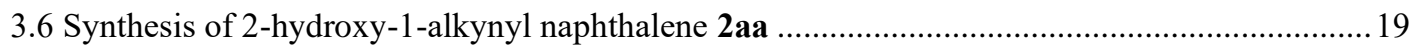

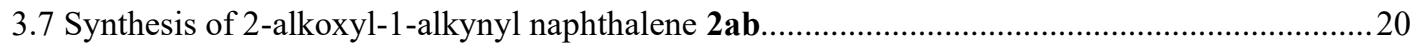

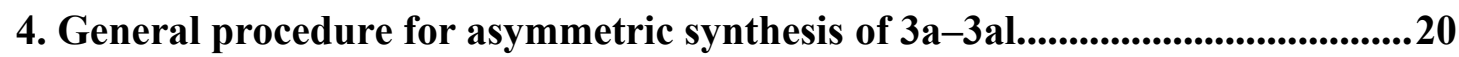

5. Procedure for asymmetric synthesis of 3am .............................................................65

6. Density Functional Theory (DFT) calculations ..................................................68

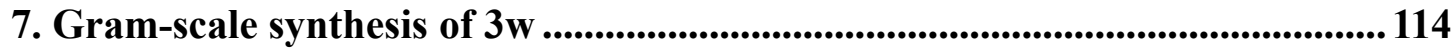

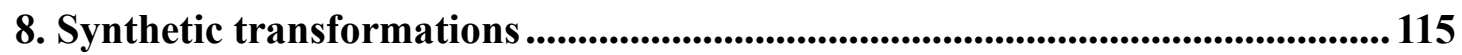

9. X-ray Crystallographic Data of 3w (CCDC 2054016) ..................................127

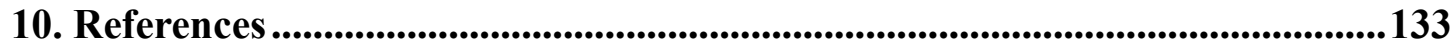

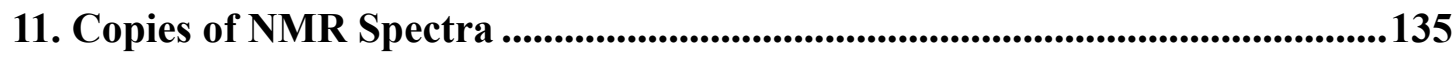




\section{General methods}

Unless stated otherwise, all solvents and reagents were purified and dried according to standard methods prior to use. All reactions were carried out in flame-dried glassware under an atmosphere of argon with magnetic stirring. Reactions were monitored by thin-layer chromatography (TLC) carried out on Huanghai silica gel HSGF254 precoated plates $(0.2 \mathrm{~mm} \pm 0.03 \mathrm{~mm})$ using UV light as visualizing agent and an ethanolic solution of phosphomolybdic acid, an aqueous solution of cerium sulfate or a basic aqueous solution of potassium permanganate as developing agents. Silica gel (300-400 mesh) purchased from Tansoole was used for flash chromatography. ${ }^{1} \mathrm{H}$ and ${ }^{13} \mathrm{C}$ NMR spectra were recorded on a Bruker AVANCE III HD (400 MHz and $101 \mathrm{MHz}$, respectively) and internally referenced to tetramethylsilane signal or residual protio solvent signals $\left(\mathrm{CDCl}_{3}, \delta_{\mathrm{H}}=7.26 \mathrm{ppm}, \delta_{\mathrm{C}}=77.16 \mathrm{ppm}\right)$. The following abbreviations were used to designate multiplicities: $\mathrm{s}=$ singlet, $\mathrm{d}=$ doublet, $\mathrm{t}=$ triplet, $\mathrm{q}=$ quartet, $\mathrm{m}$ $=$ multiplet, $\mathrm{br}=$ broad. High-resolution mass spectra (HRMS) were recorded on an Agilent Technologies 6224 TOF LC/MS using ESI (electrospray ionization) or an Agilent GCQTOF mass spectrometer using EI (electronic ionization). Optical Rotations were measured on a Rudolph Autopol III S2 polarimeter. 2-Pyrones 1a-1g and 1i-1I were prepared according to the literature. ${ }^{1} \mathbf{2} \mathbf{l}$ and $\mathbf{2} \mathbf{m}$ were prepared according to the literatures. ${ }^{2,3}$ 


\section{Reaction optimization}

Table S1. Investigation of the Lewis acids. ${ }^{a}$<smiles>COC(=O)c1cccoc1=O</smiles>

1a<smiles>COc1ccc2ccccc2c1C#CC(C)C</smiles>

2a
Lewis acid (10 mol\%) $(S, S)$-L1 $(12 \mathrm{~mol} \%)$ $\mathrm{DCM}, 25^{\circ} \mathrm{C}, t \mathrm{~h}$<smiles>COc1cccc(C(C)C)c1-c1c(C(C)C)ccc(OC)c1-c1ccccc1</smiles>

3a

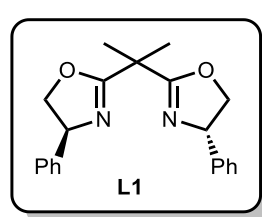

\begin{tabular}{ccccc}
\hline entry & Lewis acid & $t(\mathrm{~h})$ & ${\text { yield }(\%)^{b}}^{b}$ & ee $(\%)^{c}$ \\
\hline 1 & $\mathrm{Cu}\left(\mathrm{ClO}_{4}\right)_{2} \bullet 6 \mathrm{H}_{2} \mathrm{O}$ & 72 & 61 & 64 \\
2 & $\mathrm{Cu}\left(\mathrm{BF}_{4}\right)_{2} \bullet \mathrm{XH}_{2} \mathrm{O}$ & 72 & 9 & 60 \\
3 & $\mathrm{Cu}(\mathrm{NTf})_{2}$ & 72 & 0 & -- \\
4 & $\mathrm{Cu}(\mathrm{OTf})_{2}$ & 72 & 37 & 66 \\
5 & $\mathrm{Cu}\left(\mathrm{SbF}_{6}\right)_{2}$ & 72 & 0 & -- \\
6 & $\mathrm{Cu}\left(\mathrm{PF}_{6}\right)_{2}$ & 72 & 0 & -- \\
7 & $\mathrm{In}\left(\mathrm{OTf}_{3}\right.$ & 72 & 0 & -- \\
8 & $\mathrm{Ni}\left(\mathrm{ClO}_{4}\right)_{2} \bullet 6 \mathrm{H}_{2} \mathrm{O}$ & 72 & 0 & -- \\
9 & $\mathrm{Co}\left(\mathrm{ClO}_{4}\right)_{2} \bullet 6 \mathrm{H}_{2} \mathrm{O}$ & 72 & 0 & -- \\
\hline
\end{tabular}

${ }^{a}$ Reaction conditions: 1a $(0.1 \mathrm{mmol}), \mathbf{2 a}(0.3 \mathrm{mmol})$, Lewis acid $(10 \mathrm{~mol} \%)$ and $(S, S)-$ L1 (12 mol\%) in DCM (1.0 mL) at $25^{\circ} \mathrm{C} .{ }^{b}$ Isolated yield. ${ }^{c}$ Determined by HPLC analysis. 
Table S2. Investigation of the ligands. ${ }^{a, b, c}$

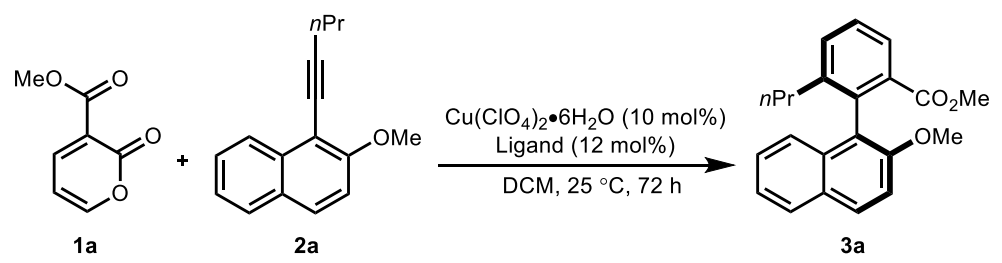

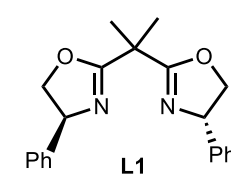

$61 \%, 64 \%$ ee

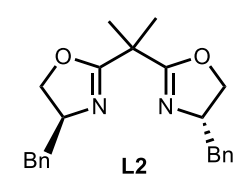

$47 \%,-34 \%$ ee

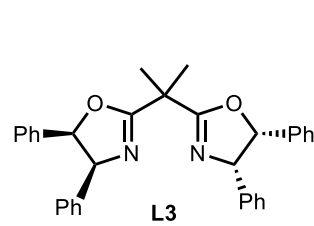

$47 \%, 79 \%$ ee

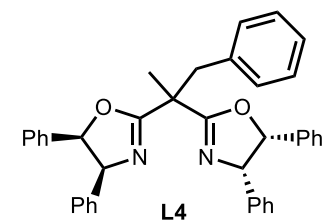

\% $74 \%$ ee<smiles>CC(Cc1ccc(C(C)(C)C)cc1)(C1=N[C@H](c2ccccc2)[C@H](c2ccccc2)O1)C1=N[C@H](c2ccccc2)[C@H](c2ccccc2)O1</smiles><smiles>CC(Cc1cc(C(C)(C)C)cc(C(C)(C)C)c1)(C1=N[C@H](c2ccccc2)[C@H](c2ccccc2)O1)C1=N[C@H](c2ccccc2)[C@H](c2ccccc2)O1</smiles><smiles>COc1cc(CC(C)(C2=N[C@H](c3ccccc3)[C@H](c3ccccc3)O2)C2=N[C@H](c3ccccc3)[C@H](c3ccccc3)O2)cc(OC)c1</smiles>

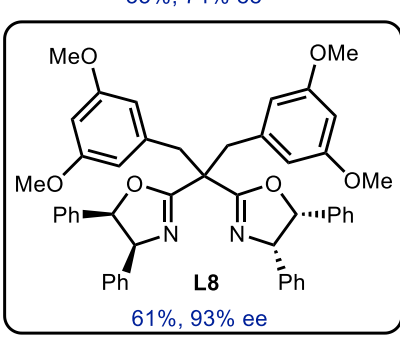<smiles>CC(CC1CCCCC1)(C1=N[C@H](c2ccccc2)[C@H](P)O1)C1=N[C@H](c2ccccc2)[C@H](P)O1</smiles>

$34 \%, 73 \%$ ee

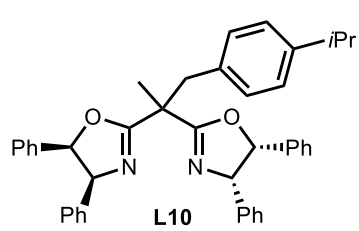

$41 \%, 75 \%$ ee<smiles>CC(Cc1ccc([N+](=O)[O-])cc1)(C1=N[C@H](c2ccccc2)[C@H](P)O1)C1=N[C@H](c2ccccc2)[C@H](c2ccccc2)O1</smiles>

$34 \%, 71 \%$ ee

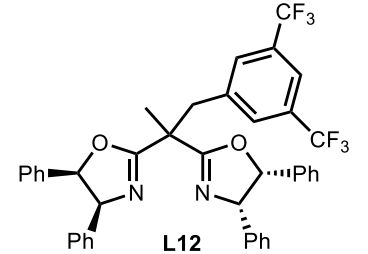

$59 \%, 84 \%$ ee

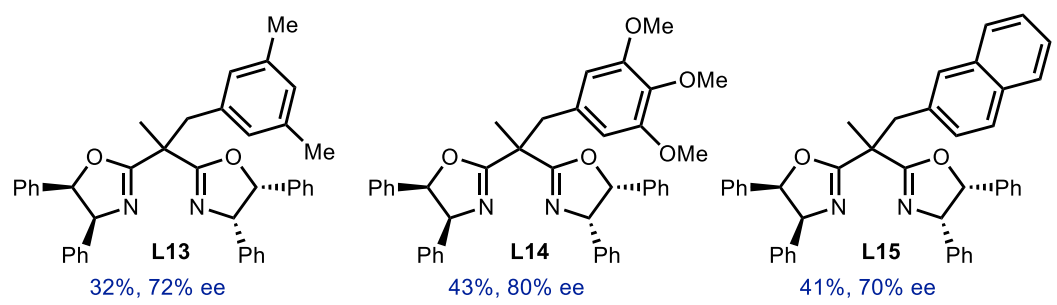

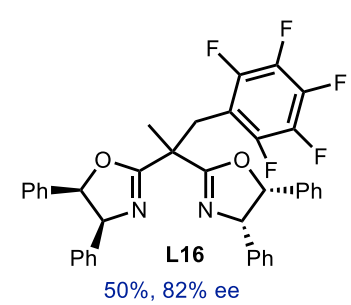

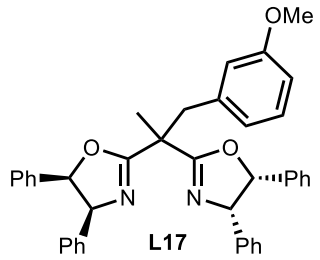

$42 \%, 82 \%$ ee

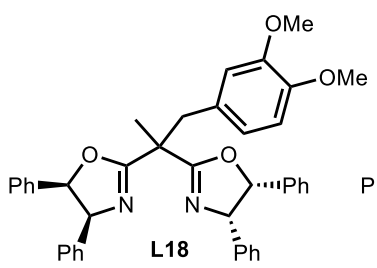

$48 \%, 73 \%$ ee

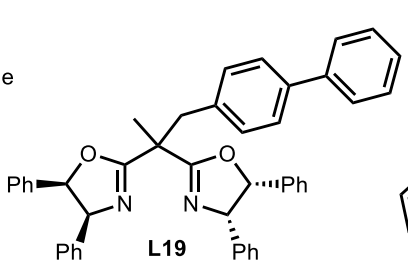

$35 \%, 74 \%$ ee

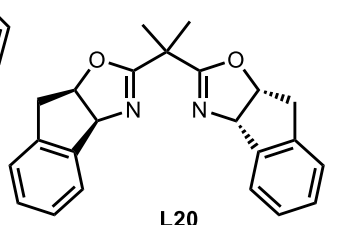

$9 \%, 7 \%$ ee

${ }^{a}$ Reaction conditions: $\mathbf{1 a}(0.1 \mathrm{mmol}), \mathbf{2 a}(0.3 \mathrm{mmol}), \mathrm{Cu}\left(\mathrm{ClO}_{4}\right)_{2} \bullet 6 \mathrm{H}_{2} \mathrm{O}(10 \mathrm{~mol} \%)$ and ligand $(12 \mathrm{mmol} \%)$ in DCM $(1.0 \mathrm{~mL})$ at $25^{\circ} \mathrm{C} .{ }^{b}$ Isolated yield. ${ }^{c}$ Determined by HPLC analysis. 
Table S3. Investigation of the solvents and additives. ${ }^{a}$

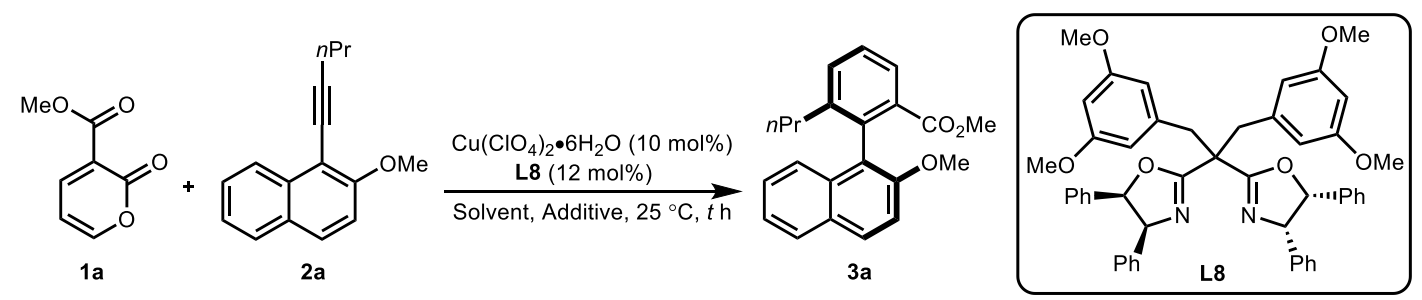

\begin{tabular}{cccccc}
\hline entry & solvent & additive & $t(\mathrm{~h})$ & ${\text { yield }(\%)^{b}}^{\text {ee }(\%)^{c}}$ \\
\hline 1 & DCM & - & 72 & 61 & 93 \\
2 & DCE & - & 36 & 88 & 95 \\
3 & DCE & $3 \AA$ A.S. & 36 & 90 & 96 \\
4 & DCE & $4 \AA$ M.S. & 36 & 89 & 96 \\
5 & DCE & $5 \AA$ M.S. & 36 & 91 & 96 \\
$6^{d}$ & DCE & $5 \AA$ M.S. & 72 & 97 & 96
\end{tabular}

${ }^{a}$ Reaction conditions: 1a $(0.1 \mathrm{mmol}), \mathbf{2 a}(0.3 \mathrm{mmol}), \mathrm{Cu}\left(\mathrm{ClO}_{4}\right)_{2} \bullet 6 \mathrm{H}_{2} \mathrm{O}(10 \mathrm{~mol} \%)$ and L8 (12 mol\%) in DCM (1.0 mL) at $25{ }^{\circ} \mathrm{C} .{ }^{b}$ Isolated yield. ${ }^{c}$ Determined by HPLC analysis. ${ }^{d} \mathbf{1 a}(0.2 \mathrm{mmol}), \mathbf{2 a}(0.3 \mathrm{mmol}), \mathrm{Cu}\left(\mathrm{ClO}_{4}\right)_{2} \bullet 6 \mathrm{H}_{2} \mathrm{O}(5 \mathrm{~mol} \%), \mathbf{L 8}(6 \mathrm{~mol} \%)$ and $5 \AA$ M.S. $(50 \mathrm{mg})$ in DCE $(1.0 \mathrm{~mL})$ at $25^{\circ} \mathrm{C}$. 


\section{Synthesis of substrates}

\subsection{Synthesis of 2-pyrone $1 \mathrm{~h}$}

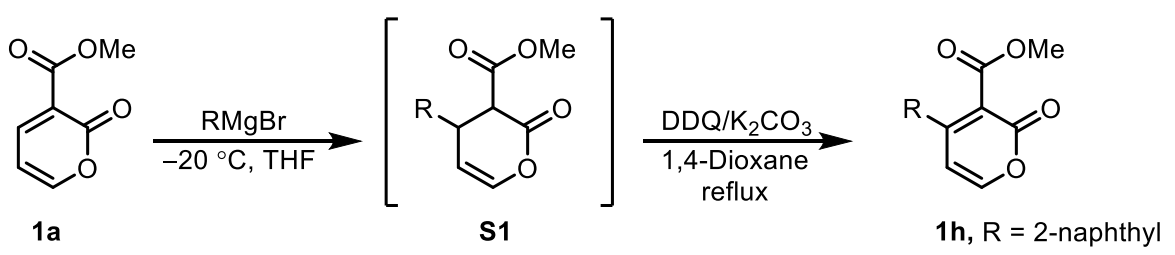

To a solution of methyl 2-oxo-2H-pyron-3-carboxylate $1 \mathrm{a}(1.54 \mathrm{~g}, 10 \mathrm{mmol})$ in THF (100 mL) was added fresh prepared 2-naphthyl magnesium bromide (1.0 M in THF, 12 $\mathrm{mL}$ ) dropwise at $-20{ }^{\circ} \mathrm{C}$. The reaction was stirred at this temperature for $15 \mathrm{~min}$. The reaction mixture was quenched with $\mathrm{NH}_{4} \mathrm{Cl}(5 \%$ aq., $15 \mathrm{~mL})$. The aqueous layer was extracted with dichloromethane $(150 \mathrm{~mL} \times 3)$. The combined extracts were washed with brine $(200 \mathrm{~mL})$, dried over $\mathrm{Na}_{2} \mathrm{SO}_{4}$ and concentrated under reduced pressure. The residue was purified by flash column chromatography (silica gel, ethyl acetate/petroleum ether 1:10) to afford $\mathbf{S} 1$ as a viscous oil.

To a solution of $\mathbf{S 1}(360 \mathrm{mg}, 1.28 \mathrm{mmol})$ in 1,4-dioxane $(13.0 \mathrm{~mL})$ was added DDQ (583 mg, $2.56 \mathrm{~mol})$ and $\mathrm{K}_{2} \mathrm{CO}_{3}(265 \mathrm{mg}, 1.92 \mathrm{mmol})$. The reaction mixture was stirred at reflux for $2 \mathrm{~h}$. The reaction mixture was quenched with $\mathrm{Na}_{2} \mathrm{~S}_{2} \mathrm{O}_{3}(5 \%$ aq., $15 \mathrm{~mL})$. The aqueous layer was extracted with dichloromethane $(150 \mathrm{~mL} \times 3)$. The combined extracts were dried over $\mathrm{Na}_{2} \mathrm{SO}_{4}$ and concentrated under reduced pressure. The residue was purified by flash column chromatography (silica gel, ethyl acetate/petroleum ether $1: 10)$ to afford $\mathbf{1 h}$ as a yellow solid.

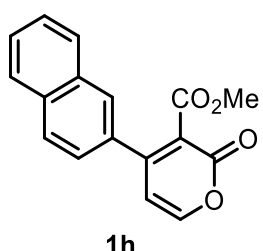

Brown solid (31\% yield over 2 steps); m.p. $96.6-97.0{ }^{\circ} \mathrm{C} ;{ }^{1} \mathrm{H}$ NMR $\left(400 \mathrm{MHz}, \mathrm{CHCl}_{3}\right) \delta 7.93-7.87(\mathrm{~m}, 4 \mathrm{H}), 7.61-7.56(\mathrm{~m}, 3 \mathrm{H}), 7.47$ $(\mathrm{dd}, J=8.8,2.0 \mathrm{~Hz}, 1 \mathrm{H}), 6.49(\mathrm{~d}, J=5.2 \mathrm{~Hz}, 1 \mathrm{H}), 3.68(\mathrm{~s}, 3 \mathrm{H}) ;{ }^{13} \mathrm{C}$ NMR (101 MHz, $\left.\mathrm{CHCl}_{3}\right) \delta 165.3,159.2,154.0,151.6,133.9,132.9$, 132.7, 129.0, 128.7, 127.88, 127.86, 127.6, 127.2, 124.0, 119.2, 108.7, 52.8; FT-IR 
(neat): $v_{\max }=1711,1625,1537,1238,1108,1026,964,794,762 \mathrm{~cm}^{-1}$; HRMS (EI): exact mass calculated for: $\mathrm{C}_{17} \mathrm{H}_{12} \mathrm{O}_{4}[\mathrm{M}]^{+}: 280.0730$, found 280.0732 .

\subsection{Synthesis of 2-methoxyl-1-alkynyl naphthalenes $2 \mathrm{~b}$ and $2 \mathrm{c}$}

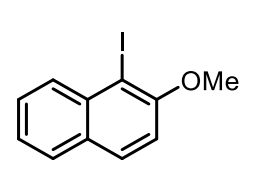

S2

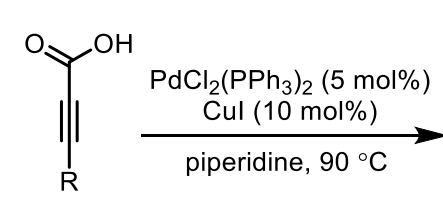

S3

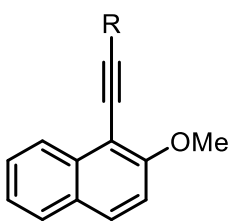

2b, $\mathrm{R}=\mathrm{Me}$ 2c, $R=E t$

A mixture of S2 (1.00 g, $3.52 \mathrm{mmol})$, alkynyl carboxylic acid $\mathbf{S 3}$ (17.6 mmol), $\mathrm{PdCl}_{2}\left(\mathrm{PPh}_{3}\right)_{2}(124 \mathrm{mg}, 0.18 \mathrm{mmol})$ and $\mathrm{CuI}(67 \mathrm{mg}, 0.35 \mathrm{mmol})$ in piperidine $(11 \mathrm{~mL})$ was stirred overnight at $90{ }^{\circ} \mathrm{C}$. Then, the reaction mixture was filtered through a pad of celite and washed with ethyl acetate. The mixture was concentrated under reduced pressure. The residue was purified by flash column chromatography (silica gel, ethyl acetate/petroleum ether 1:99) to afford the corresponding coupling product.<smiles>CC#Cc1c(OC)ccc2ccccc12</smiles>

2b

Yellow solid (89\% yield); m.p. 69.6-72.0 ${ }^{\circ} \mathrm{C}$; ${ }^{1} \mathrm{H}$ NMR $(400 \mathrm{MHz}$, $\left.\mathrm{CHCl}_{3}\right) \delta 8.27(\mathrm{~d}, J=8.4 \mathrm{~Hz}, 1 \mathrm{H}), 7.79-7.76(\mathrm{~m}, 2 \mathrm{H}), 7.52(\mathrm{ddd}, J=$ 8.4, 7.2, $1.6 \mathrm{~Hz}, 1 \mathrm{H}), 7.36$ (ddd, $J=8.4,6.8,1.2 \mathrm{~Hz}, 1 \mathrm{H}), 7.24$ (d, $J=$ $9.2 \mathrm{~Hz}, 1 \mathrm{H}), 4.03(\mathrm{~s}, 3 \mathrm{H}), 2.29(\mathrm{~s}, 3 \mathrm{H}) ;{ }^{13} \mathrm{C} \mathrm{NMR}\left(101 \mathrm{MHz}, \mathrm{CHCl}_{3}\right) \delta$ 158.6, 135.0, 129.3, 128.7, 128.1, 127.2, 125.5, 124.2, 112.7, 107.1, 95.7, 73.9, 56.7, 5.2; FT-IR (neat): $v_{\max }=3055,3001,2935,2913,2840,2233,1647,1589,1507,1469$, $1386,1330,1273,1258,1178,1147,1107,1063,1019,901,805,779,747,661,561$ $\mathrm{cm}^{-1}$; HRMS (EI): exact mass calculated for: $\mathrm{C}_{14} \mathrm{H}_{12} \mathrm{O}[\mathrm{M}]^{+}:$196.0883, found 196.0883.<smiles>CCC#Cc1c(OC)ccc2ccccc12</smiles>

2c

Brown oil (96\% yield); ${ }^{1} \mathrm{H}$ NMR (400 MHz, $\left.\mathrm{CHCl}_{3}\right) \delta 8.28(\mathrm{~d}, J=8.4$ $\mathrm{Hz}, 1 \mathrm{H}), 7.78-7.76(\mathrm{~m}, 2 \mathrm{H}), 7.53(\mathrm{ddd}, J=8.4,6.8,1.2 \mathrm{~Hz}, 1 \mathrm{H}), 7.37$ (ddd, $J=8.0,6.8,1.2 \mathrm{~Hz}, 1 \mathrm{H}), 7.24$ (d, $J=9.2 \mathrm{~Hz}, 1 \mathrm{H}), 4.02(\mathrm{~s}, 3 \mathrm{H})$, $2.67(\mathrm{q}, J=7.6 \mathrm{~Hz}, 2 \mathrm{H}), 1.39$ (t, $J=7.6 \mathrm{~Hz}, 3 \mathrm{H}) ;{ }^{13} \mathrm{C} \mathrm{NMR}(101 \mathrm{MHz}$, 
$\left.\mathrm{CHCl}_{3}\right) \delta 158.6,135.0,129.3,128.7,128.1,127.2,125.5,124.2,112.9,107.2,101.8$ 74.1, 56.8, 14.4, 14.0; HRMS (EI): exact mass calculated for: $\mathrm{C}_{15} \mathrm{H}_{14} \mathrm{O}[\mathrm{M}]^{+}$: 210.1039, found 210.1038 .

\subsection{Synthesis of 2-methoxyl-1-alkynyl naphthalenes $2 \mathrm{a}, 2 \mathrm{~d}-2 \mathrm{k}$}

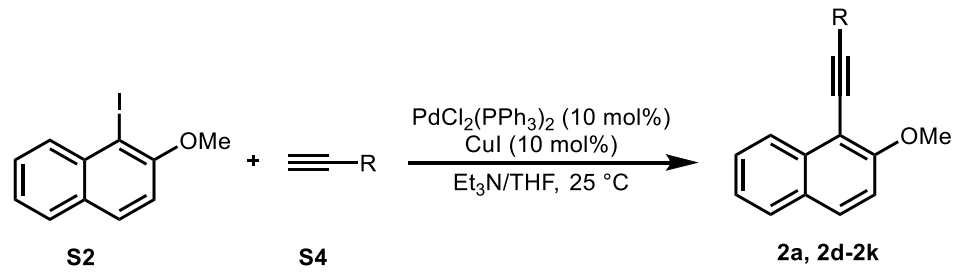

To a stirred solution of $\mathbf{S 2}(1.00 \mathrm{~g}, 3.52 \mathrm{mmol}), \mathrm{PdCl}_{2}\left(\mathrm{PPh}_{3}\right)_{2}(247 \mathrm{mg}, 0.35 \mathrm{mmol})$, $\mathrm{CuI}(67 \mathrm{mg}, 0.35 \mathrm{mmol})$ in triethylamine $(11 \mathrm{~mL})$ and THF $(11 \mathrm{~mL})$ was added terminal alkyne S4 $(5.28 \mathrm{mmol})$ at $25^{\circ} \mathrm{C}$. The reaction mixture was stirred at $25{ }^{\circ} \mathrm{C}$. After the reaction was complete (monitored by TLC), the reaction mixture was filtered through a pad of celite and washed with ethyl acetate. The mixture was concentrated under reduced pressure. The residue was purified by flash column chromatography (silica gel, ethyl acetate/petroleum ether 1:99) to afford the corresponding coupling product.<smiles>COc1ccc2ccccc2c1C#CC(C)C</smiles>

Brown oil (93\% yield); ${ }^{1} \mathrm{H}$ NMR (400 MHz, $\left.\mathrm{CHCl}_{3}\right) \delta 8.27(\mathrm{~d}, J=8.4$ $\mathrm{Hz}, 1 \mathrm{H}), 7.78-7.76(\mathrm{~m}, 2 \mathrm{H}), 7.52(\mathrm{ddd}, J=8.0,6.8,1.2 \mathrm{~Hz}, 1 \mathrm{H}), 7.36$ (ddd, $J=8.0,6.8,1.2 \mathrm{~Hz}, 1 \mathrm{H}), 7.24(\mathrm{~d}, J=8.8 \mathrm{~Hz}, 1 \mathrm{H}), 4.02(\mathrm{~s}, 3 \mathrm{H})$, $2.63(\mathrm{t}, J=7.2 \mathrm{~Hz}, 2 \mathrm{H}), 1.77(\mathrm{q}, J=7.2 \mathrm{~Hz}, 2 \mathrm{H}), 1.15(\mathrm{t}, J=7.2 \mathrm{~Hz}$, $3 \mathrm{H}),{ }^{13} \mathrm{C}$ NMR $\left(101 \mathrm{MHz}, \mathrm{CHCl}_{3}\right) \delta 158.7,135.0,129.3,128.7,128.1,127.2,125.5$, 124.2, 112.9, 107.3, 100.4, 74.9, 56.8, 22.7, 22.3, 13.9; FT-IR (neat): $v_{\max }=2960,2930$, $2871,2838,1621,1590,1507,1462,1384,1330,1272,1256,1147,1112,1063,1020$, 903, 805, $747 \mathrm{~cm}^{-1}$; HRMS (EI): exact mass calculated for: $\mathrm{C}_{16} \mathrm{H}_{16} \mathrm{O}[\mathrm{M}]^{+}: 224.1196$, found 224.1195 . 


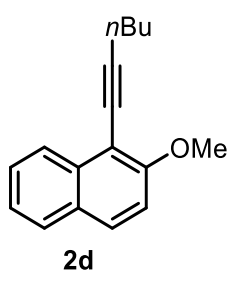

Brown solid (92\% yield); m.p. $44.3-45.0{ }^{\circ} \mathrm{C} ;{ }^{1} \mathrm{H}$ NMR $(400 \mathrm{MHz}$, $\left.\mathrm{CHCl}_{3}\right) \delta 8.28(\mathrm{dd}, J=8.8,5.6 \mathrm{~Hz}, 1 \mathrm{H}), 7.77(\mathrm{~d}, J=8.8 \mathrm{~Hz}, 2 \mathrm{H}), 7.56$ $-7.51(\mathrm{~m}, 1 \mathrm{H}), 7.39-7.35(\mathrm{~m}, 1 \mathrm{H}), 7.24(\mathrm{~d}, J=9.2 \mathrm{~Hz}, 1 \mathrm{H}), 4.02(\mathrm{~s}$, $3 \mathrm{H}), 2.66(\mathrm{td}, J=7.2,3.2 \mathrm{~Hz}, 2 \mathrm{H}), 1.78-1.71(\mathrm{~m}, 2 \mathrm{H}), 1.65-1.56(\mathrm{~m}$, 2H), $1.01(\mathrm{td}, J=7.6,3.2 \mathrm{~Hz}, 3 \mathrm{H}) ;{ }^{13} \mathrm{C} \mathrm{NMR}\left(101 \mathrm{MHz}, \mathrm{CHCl}_{3}\right) \delta 158.7,135.0,129.3$, 128.8, 128.1, 127.2 125.5, 124.2, 112.9, 107.3, 100.5, 74.7, 56.8, 31.3, 22.3, 20.0, 13.9; FT-IR (neat): $v_{\max }=3058,2999,2956,2931,2860,2838,1621,1590,1507,1463,1442$, $1431,1384,1330,1272,1257,1178,1147,1112,1065,1020,903,805,778,746,709$ $\mathrm{cm}^{-1}$; HRMS (EI): exact mass calculated for: $\mathrm{C}_{17} \mathrm{H}_{18} \mathrm{O}[\mathrm{M}]^{+}: 238.1352$, found 238.1355 .

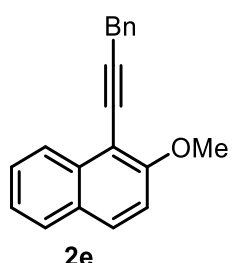

Brown solid (87\% yield); m.p. 47.5-48.7 ${ }^{\circ} \mathrm{C}$; ${ }^{1} \mathrm{H}$ NMR (400 MHz, $\left.\mathrm{CHCl}_{3}\right) \delta 8.27(\mathrm{~d}, J=8.4 \mathrm{~Hz}, 1 \mathrm{H}), 7.78(\mathrm{t}, J=8.8 \mathrm{~Hz}, 2 \mathrm{H}), 7.55(\mathrm{~d}, J=$ $7.2 \mathrm{~Hz}, 2 \mathrm{H}), 7.50$ (ddd, $J=8.8,6.8,1.2 \mathrm{~Hz}, 1 \mathrm{H}), 7.38-7.34(\mathrm{~m}, 3 \mathrm{H})$, $7.28-7.23(\mathrm{~m}, 2 \mathrm{H}), 4.08(\mathrm{~s}, 2 \mathrm{H}), 4.02(\mathrm{~s}, 3 \mathrm{H}) ;{ }^{13} \mathrm{C}$ NMR $(101 \mathrm{MHz}$, $\left.\mathrm{CHCl}_{3}\right) \delta 159.0,137.2,135.0,129.7,128.70,128.67,128.20,128.15,127.3,126.7$, 125.5, 124.2, 112.9, 106.9, 97.3, 56.8, 26.6; FT-IR (neat): $v_{\max }=3060,3027,2934$, 2839, 1621, 1590, 1508, 1494, 1453, 1330, 1273, 1256, 1178, 1147, 1107, 1061, 1029, 1020, 903, 806, 729, 695, 670, 600, $559 \mathrm{~cm}^{-1}$, HRMS (EI): exact mass calculated for: $\mathrm{C}_{20} \mathrm{H}_{16} \mathrm{O}[\mathrm{M}]^{+}:$272.1196, found 272.1197.

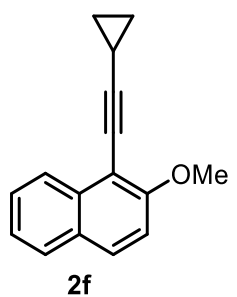

Yellow solid (96\% yield); m.p. $39.8-40.3{ }^{\circ} \mathrm{C} ;{ }^{1} \mathrm{H}$ NMR $(400 \mathrm{MHz}$, $\left.\mathrm{CHCl}_{3}\right) \delta 8.22(\mathrm{~d}, J=8.4 \mathrm{~Hz}, 1 \mathrm{H}), 7.77-7.75(\mathrm{~m}, 2 \mathrm{H}), 7.52(\mathrm{t}, J=7.6$ $\mathrm{Hz}, 1 \mathrm{H}), 7.36(\mathrm{t}, J=7.6 \mathrm{~Hz}, 1 \mathrm{H}), 7.22(\mathrm{~d}, J=9.2 \mathrm{~Hz}, 1 \mathrm{H}), 4.01(\mathrm{~s}, 3 \mathrm{H})$, $1.72-1.66(\mathrm{~m}, 1 \mathrm{H}), 0.98-0.96(\mathrm{~m}, 4 \mathrm{H}) ;{ }^{13} \mathrm{C} \mathrm{NMR}\left(101 \mathrm{MHz}, \mathrm{CHCl}_{3}\right)$ $\delta 158.9,135.1,129.2,128.7,128.1,127.1,125.4,124.1,112.8,107.1,103.5,69.9,56.7$, 9.3, 1.0; FT-IR (neat): $v_{\max }=3057,3008,2936,2839,2219,1621,1589,1506,1462$, $1431,1396,1330,1272,1257,1215,1177,1147,1125,1080,1048,1019,945,904$, $857,805,747,708,653,561 \mathrm{~cm}^{-1}$; HRMS (EI): exact mass calculated for: $\mathrm{C}_{16} \mathrm{H}_{14} \mathrm{O}$ $[\mathrm{M}]^{+}:$222.1039, found 222.1037. 


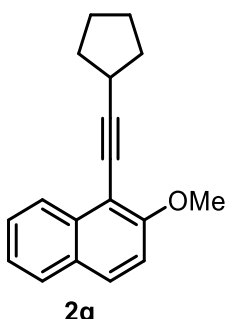

Brown solid (80\% yield); m.p. 50.6-51.3 ${ }^{\circ} \mathrm{C} ;{ }^{1} \mathrm{H}$ NMR $(400 \mathrm{MHz}$, $\left.\mathrm{CHCl}_{3}\right) \delta 8.24(\mathrm{~d}, J=8.4 \mathrm{~Hz}, 1 \mathrm{H}), 7.76(\mathrm{~d}, J=8.8 \mathrm{~Hz}, 2 \mathrm{H}), 7.51$ (ddd, $J=8.0,6.4,1.2 \mathrm{~Hz}, 1 \mathrm{H}), 7.36(\mathrm{ddd}, J=8.0,6.8,1.2 \mathrm{~Hz}, 1 \mathrm{H}), 7.23(\mathrm{~d}$, $J=9.2 \mathrm{~Hz}, 1 \mathrm{H}), 4.01(\mathrm{~s}, 3 \mathrm{H}), 3.10-3.03(\mathrm{~m}, 1 \mathrm{H}), 2.15-2.06(\mathrm{~m}, 2 \mathrm{H})$, $1.92-1.83(\mathrm{~m}, 4 \mathrm{H}), 1.71-1.61(\mathrm{~m}, 2 \mathrm{H}) ;{ }^{13} \mathrm{C} \mathrm{NMR}\left(101 \mathrm{MHz}, \mathrm{CHCl}_{3}\right)$ $\delta 158.5,135.0,129.2,128.8,128.1,127.1,125.5,124.1,113.0,107.4,104.8,74.2,56.9$, 34.4, 31.7, 25.3; FT-IR (neat): $v_{\max }=2940,2840,1589,1507,1328,1272,1257,1149$, 1113, 1066, 1018, 904, 806, 778, 753, $561 \mathrm{~cm}^{-1}$; HRMS (EI): exact mass calculated for: $\mathrm{C}_{18} \mathrm{H}_{18} \mathrm{O}[\mathrm{M}]^{+}:$250.1352, found 250.1353 .

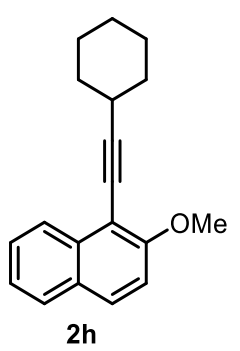

Brown solid (80\% yield); m.p. $60.7-61.5{ }^{\circ} \mathrm{C}$; ${ }^{1} \mathrm{H}$ NMR $(400 \mathrm{MHz}$, $\left.\mathrm{CHCl}_{3}\right) \delta 8.27(\mathrm{~d}, J=8.8 \mathrm{~Hz}, 1 \mathrm{H}), 7.75(\mathrm{~d}, J=8.8 \mathrm{~Hz}, 2 \mathrm{H}), 7.51$ (ddd, $J=8.0,6.8,1.2 \mathrm{~Hz}, 1 \mathrm{H}), 7.35(\mathrm{ddd}, J=8.0,6.8,1.2 \mathrm{~Hz}, 1 \mathrm{H}), 7.22(\mathrm{~d}$, $J=9.2 \mathrm{~Hz}, 1 \mathrm{H}), 4.00(\mathrm{~s}, 3 \mathrm{H}), 2.86-2.79(\mathrm{~m}, 1 \mathrm{H}), 2.05-1.99(\mathrm{~m}, 2 \mathrm{H})$, $1.88-1.81(\mathrm{~m}, 2 \mathrm{H}), 1.74-1.65(\mathrm{~m}, 2 \mathrm{H}), 1.62-1.58(\mathrm{~m}, 1 \mathrm{H}), 1.47-$ $1.39(\mathrm{~m}, 3 \mathrm{H}) ;{ }^{13} \mathrm{C} \mathrm{NMR}\left(101 \mathrm{MHz}, \mathrm{CHCl}_{3}\right) \delta 158.6,135.0,129.2,128.8,128.1,127.1$, 125.6, 124.1, 113.1, 107.4, 104.6, 74.7, 56.9, 33.1, 30.5, 26.2, 25.1; FT-IR (neat): $v_{\max }$ $=3057,3001,2927,2851,2218,1621,1590,1507,1463,1447,1385,1330,1271,1147$, 1093, 1019, 904, 805, 746, $562 \mathrm{~cm}^{-1}$; HRMS (EI): exact mass calculated for: $\mathrm{C}_{19} \mathrm{H}_{20} \mathrm{O}$ $[\mathrm{M}]^{+}:$264.1509, found 264.1510.

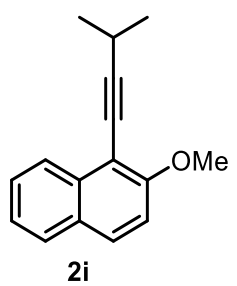

Yellow solid (82\% yield); m.p. $74.8-75.5{ }^{\circ} \mathrm{C} ;{ }^{1} \mathrm{H}$ NMR $(400 \mathrm{MHz}$, $\left.\mathrm{CHCl}_{3}\right) \delta 8.25(\mathrm{~d}, J=8.4 \mathrm{~Hz}, 1 \mathrm{H}), 7.75(\mathrm{~d}, J=8.8 \mathrm{~Hz}, 2 \mathrm{H}), 7.52(\mathrm{ddd}$, $J=8.4,6.8,1.2 \mathrm{~Hz}, 1 \mathrm{H}), 7.35(\mathrm{ddd}, J=8.0,6.8,1.2 \mathrm{~Hz}, 1 \mathrm{H}), 7.22(\mathrm{~d}$, $J=8.8 \mathrm{~Hz}, 1 \mathrm{H}), 4.01(\mathrm{~s}, 3 \mathrm{H}), 3.01$ (hept, $J=6.8 \mathrm{~Hz}, 1 \mathrm{H}), 1.40(\mathrm{~d}, J=$ $7.2 \mathrm{~Hz}, 6 \mathrm{H}) ;{ }^{13} \mathrm{C} \mathrm{NMR}\left(101 \mathrm{MHz}, \mathrm{CHCl}_{3}\right) \delta 158.5,135.0,129.3,128.8,128.1,127.2$, $125.5,124.1,113.1,107.2,106.0,73.9,56.9,23.5,22.0$; FT-IR (neat): $v_{\max }=3057$, $2967,2933,2869,2839,1621,1590,1506,1462,1382,1331,1316,1272,1256,1178$, 1147, 1114, 1082, 903, 805, 747, $656 \mathrm{~cm}^{-1}$; HRMS (EI): exact mass calculated for: $\mathrm{C}_{16} \mathrm{H}_{16} \mathrm{O}[\mathrm{M}]^{+}:$224.1196, found 224.1197. 


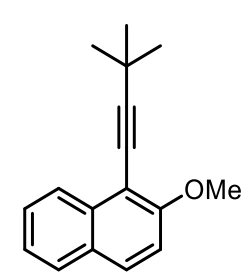

2j

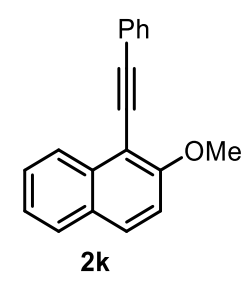

Yellow solid (94\% yield); ${ }^{1} \mathrm{H}$ NMR (400 MHz, $\left.\mathrm{CHCl}_{3}\right) \delta 8.24$ (d, $J=$ $8.4 \mathrm{~Hz}, 1 \mathrm{H}), 7.75$ (d, $J=8.8 \mathrm{~Hz}, 2 \mathrm{H}), 7.51$ (t, $J=7.2 \mathrm{~Hz}, 1 \mathrm{H}), 7.36$ (t, $J=7.2 \mathrm{~Hz}, 1 \mathrm{H}), 7.22(\mathrm{~d}, J=8.8 \mathrm{~Hz}, 1 \mathrm{H}), 4.01$ (s, 3H), $1.46(\mathrm{~s}, 9 \mathrm{H})$. The spectroscopic data was consistent with those reported in the literature. ${ }^{4}$ Yellow solid (71\% yield); ${ }^{1} \mathrm{H}$ NMR $\left(400 \mathrm{MHz}, \mathrm{CHCl}_{3}\right) \delta 8.36(\mathrm{~d}, J=$ $8.4 \mathrm{~Hz}, 1 \mathrm{H}), 7.84$ (d, $J=9.2 \mathrm{~Hz}, 1 \mathrm{H}), 7.80(\mathrm{~d}, J=8.0 \mathrm{~Hz}, 1 \mathrm{H}), 7.69-$ $7.66(\mathrm{~m}, 2 \mathrm{H}), 7.58-7.54(\mathrm{~m}, 1 \mathrm{H}), 7.41-7.35$ (m, 4H), 7.28 (d, $J=8.8$ $\mathrm{Hz}, 1 \mathrm{H}), 4.07$ (s, 3H). The spectroscopic data was consistent with those reported in the literature. ${ }^{5}$ 


\subsection{Synthesis of 2-methoxyl-1-alkynyl naphthalenes 2n-2v}

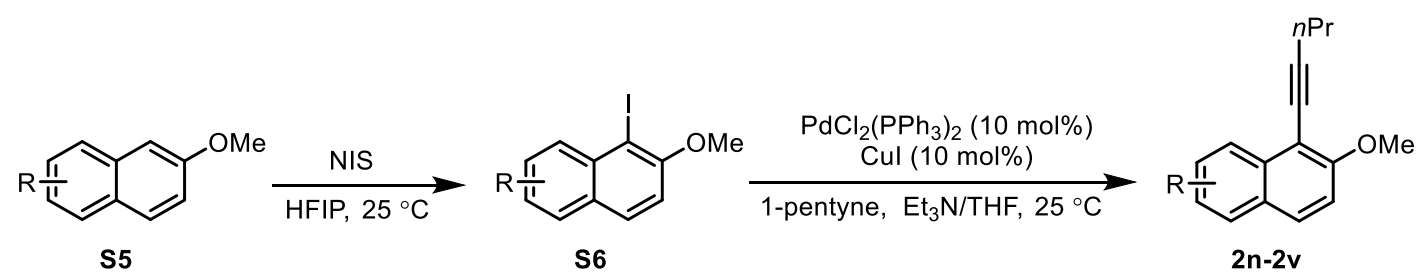

To a stirred solution of $\mathbf{S 5}$ in HFIP was added NIS at $25^{\circ} \mathrm{C}$. The reaction mixture was stirred at $25^{\circ} \mathrm{C}$. After the reaction was complete (monitored by TLC), the solvent was removed under reduced pressure. The residue was purified by recrystallization from ethyl acetate/petroleum ether to afford the corresponding iodide intermediate $\mathbf{S 6}$.

To a stirred solution of the above iodide intermediate $\mathbf{S 6}(3.52 \mathrm{mmol}), \mathrm{PdCl}_{2}\left(\mathrm{PPh}_{3}\right)_{2}$ (247 mg, $0.35 \mathrm{mmol}), \mathrm{CuI}(67 \mathrm{mg}, 0.35 \mathrm{mmol})$ in triethylamine $(11 \mathrm{~mL})$ and THF (11 $\mathrm{mL})$ was added 1 -pentyne $(5.28 \mathrm{mmol})$ at $25^{\circ} \mathrm{C}$. The reaction mixture was stirred at 25 ${ }^{\circ} \mathrm{C}$. After the reaction was complete (monitored by TLC), the reaction mixture was filtered through a pad of celite and washed with ethyl acetate. The mixture was concentrated under reduced pressure. The residue was purified by flash column chromatography (silica gel, ethyl acetate/petroleum ether 1:99) to afford the corresponding coupling product.

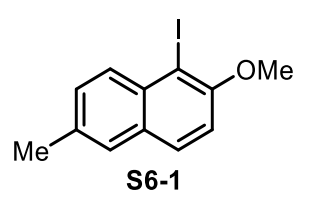

Yellow solid (87\% yield); m.p. $79.8-80.5{ }^{\circ} \mathrm{C} ;{ }^{1} \mathrm{H}$ NMR $(400 \mathrm{MHz}$, $\left.\mathrm{CHCl}_{3}\right) \delta 8.03(\mathrm{~d}, J=8.8 \mathrm{~Hz}, 1 \mathrm{H}), 7.73(\mathrm{~d}, J=8.8 \mathrm{~Hz}, 1 \mathrm{H}), 7.51(\mathrm{~s}$, 1H), $7.37(\mathrm{~d}, J=8.8 \mathrm{~Hz}, 1 \mathrm{H}), 7.16(\mathrm{dd}, J=8.8,3.2 \mathrm{~Hz}, 1 \mathrm{H}), 4.00$ (s, 3H), $2.50(\mathrm{~s}, 3 \mathrm{H}) ;{ }^{13} \mathrm{C} \mathrm{NMR}\left(101 \mathrm{MHz}, \mathrm{CHCl}_{3}\right) \delta 156.2,134.1,131.1,130.5,130.2$, 129.7, 127.2, 113.2, 87.7, 57.4, 21.3; FT-IR (neat): $v_{\max }=2907,2839,1593,1477,1462$, 1344, 1330, 1260, 1245, 1224, 1182, 1154, 1139, 1066, 1055, 976, 857, $798 \mathrm{~cm}^{-1}$; HRMS (EI): exact mass calculated for: $\mathrm{C}_{12} \mathrm{H}_{11} \mathrm{IO}[\mathrm{M}]^{+}:$297.9849, found 297.9852. 


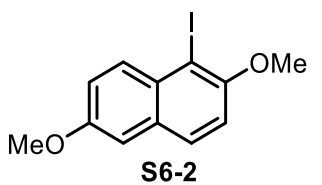

Yellow solid (27\% yield); m.p. 85.6-86.4 ${ }^{\circ} \mathrm{C} ;{ }^{1} \mathrm{H}$ NMR $(400 \mathrm{MHz}$, $\left.\mathrm{CHCl}_{3}\right) \delta 8.06(\mathrm{~d}, J=9.2 \mathrm{~Hz}, 1 \mathrm{H}), 7.71(\mathrm{~d}, J=8.8 \mathrm{~Hz}, 1 \mathrm{H}), 7.20$ $(\mathrm{dd}, J=9.2,2.4 \mathrm{~Hz}, 1 \mathrm{H}), 7.16(\mathrm{~d}, J=8.8 \mathrm{~Hz}, 1 \mathrm{H}), 7.07(\mathrm{~d}, J=2.8$ $\mathrm{Hz}, 1 \mathrm{H}), 3.99$ (s, 3H), 3.92 (s, 3H); ${ }^{13} \mathrm{C} \mathrm{NMR}\left(101 \mathrm{MHz}, \mathrm{CHCl}_{3}\right) \delta$ 156.7, 155.4, 133.0, 131.2, 130.8, 129.0, 120.9, 113.7, 106.1, 88.1, 57.6, 55.6; FT-IR (neat): $v_{\max }=2954$, 2922, 2868, 2850, 1597, 1495, 1456, 1370, 1348, 1254, 1229, 1198, 1168, 1125, 1058, 1023, 849, 820, $793 \mathrm{~cm}^{-1}$ HRMS (EI): exact mass calculated for: $\mathrm{C}_{12} \mathrm{H}_{11} \mathrm{IO}_{2}[\mathrm{M}]^{+}$: 313.9798, found 313.9802 .

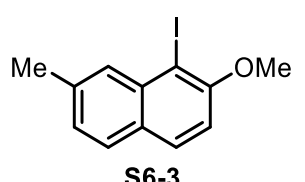

Yellow solid (74\% yield); m.p. 89.3-90.1 ${ }^{\circ} \mathrm{C} ;{ }^{1} \mathrm{H}$ NMR (400 MHz, $\left.\mathrm{CHCl}_{3}\right) \delta 7.92(\mathrm{~s}, 1 \mathrm{H}), 7.77(\mathrm{~d}, J=8.8 \mathrm{~Hz}, 1 \mathrm{H}), 7.64(\mathrm{~d}, J=8.4 \mathrm{~Hz}$, 1H), $7.21(\mathrm{dd}, J=8.4,1.2 \mathrm{~Hz}, 1 \mathrm{H}), 7.14(\mathrm{dd}, J=8.8,1.2 \mathrm{~Hz}, 1 \mathrm{H})$, $4.01(\mathrm{~s}, 3 \mathrm{H}), 2.57$ (s, 3H); ${ }^{13} \mathrm{C}$ NMR (101 MHz, $\left.\mathrm{CHCl}_{3}\right) \delta 156.8,138.3,135.9,130.3$, 130.2, 128.3, 128.2, 126.8, 112.2, 87.2, 57.3, 22.2; FT-IR (neat): $v_{\max }=2921,2854$, $1626,1595,1505,1461,1435,1350,1312,1263,1244,1173,1153,1066,801,758 \mathrm{~cm}^{-1}$; HRMS (EI): exact mass calculated for: $\mathrm{C}_{12} \mathrm{H}_{11} \mathrm{IO}[\mathrm{M}]^{+}: 297.9849$, found 297.9850 .

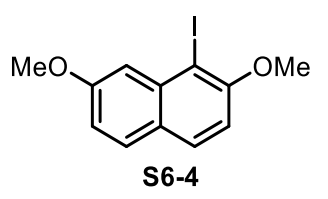

Yellow solid (84\% yield); m.p. 96.7-97.1 ${ }^{\circ} \mathrm{C} ;{ }^{1} \mathrm{H}$ NMR (400 MHz, $\left.\mathrm{CHCl}_{3}\right) \delta 7.74(\mathrm{~d}, J=8.8 \mathrm{~Hz}, 1 \mathrm{H}), 7.64(\mathrm{~d}, J=8.8 \mathrm{~Hz}, 1 \mathrm{H}), 7.47$ $(\mathrm{d}, J=2.4 \mathrm{~Hz}, 1 \mathrm{H}), 7.05(\mathrm{~d}, J=8.8 \mathrm{~Hz}, 1 \mathrm{H}), 7.02(\mathrm{dd}, J=9.2,2.4$ $\mathrm{Hz}, 1 \mathrm{H}), 4.01$ (s, 3H), 3.98 (s, 3H); ${ }^{13} \mathrm{C} \mathrm{NMR}\left(101 \mathrm{MHz}, \mathrm{CHCl}_{3}\right) \delta$ 159.9, 157.3, 137.3, 130.14, 130.09, 125.4, 117.3, 110.4, 110.1, 86.7, 57.2, 55.5; FT-IR (neat): $v_{\max }=1619$, 1504, 1459, 1377, 1261, 1220, 1153, 1063, 1022, 958, 829, $767 \mathrm{~cm}^{-1}$; HRMS (EI): exact mass calculated for: $\mathrm{C}_{12} \mathrm{H}_{11} \mathrm{IO}_{2}[\mathrm{M}]^{+}: 313.9798$, found 313.9796 .

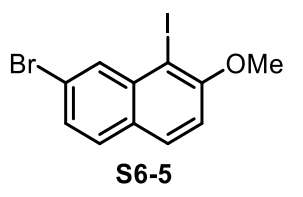

White solid (80\% yield); m.p. $113.8-114.2{ }^{\circ} \mathrm{C} ;{ }^{1} \mathrm{H}$ NMR $(400 \mathrm{MHz}$, $\left.\mathrm{CHCl}_{3}\right) \delta 8.34(\mathrm{~d}, J=1.6 \mathrm{~Hz}, 1 \mathrm{H}), 7.78(\mathrm{~d}, J=8.8 \mathrm{~Hz}, 1 \mathrm{H}), 7.60(\mathrm{~d}$, $J=8.8 \mathrm{~Hz}, 1 \mathrm{H}), 7.44(\mathrm{dd}, J=8.8,2.0 \mathrm{~Hz}, 1 \mathrm{H}), 7.20(\mathrm{~d}, J=8.8 \mathrm{~Hz}$, 1H), $4.02(\mathrm{~s}, 3 \mathrm{H}) ;{ }^{13} \mathrm{C} \mathrm{NMR}\left(101 \mathrm{MHz}, \mathrm{CHCl}_{3}\right) \delta 157.5,137.2,133.5,130.5,130.0$, 128.4, 128.0, 123.1, 113.2, 86.2, 57.3; FT-IR (neat): $v_{\max }=1606,1490,1337,1250$, 
1154, 1062, 908, $820 \mathrm{~cm}^{-1}$; HRMS (EI): exact mass calculated for: $\mathrm{C}_{11} \mathrm{H}_{8} \mathrm{BrIO}[\mathrm{M}]^{+}$: 361.8798 , found 361.8800 .

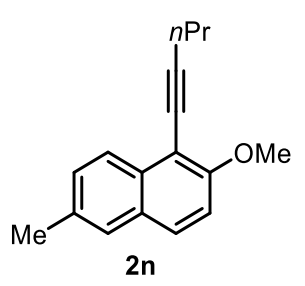

Yellow oil (24\% yield); ${ }^{1} \mathrm{H}$ NMR $\left(400 \mathrm{MHz}, \mathrm{CHCl}_{3}\right) \delta 8.16(\mathrm{~d}, J=$ $8.4 \mathrm{~Hz}, 1 \mathrm{H}), 7.68(\mathrm{~d}, J=9.2 \mathrm{~Hz}, 1 \mathrm{H}), 7.53(\mathrm{~s}, 1 \mathrm{H}), 7.35(\mathrm{dd}, J=8.4$, $1.6 \mathrm{~Hz}, 1 \mathrm{H}), 7.20(\mathrm{~d}, J=9.2 \mathrm{~Hz}, 1 \mathrm{H}), 4.00(\mathrm{~s}, 3 \mathrm{H}), 2.62$ (t, $J=7.2$ Hz, 2H), 2.48 (s, 3H), 1.77 (sext, $J=7.2 \mathrm{~Hz}, 2 \mathrm{H}), 1.15$ (t, $J=7.2$ $\mathrm{Hz}, 3 \mathrm{H}) ;{ }^{13} \mathrm{C} \mathrm{NMR}\left(101 \mathrm{MHz}, \mathrm{CHCl}_{3}\right) \delta 158.1,133.6,133.2,129.5,128.9,128.6,127.1$, 125.4, 113.0, 107.2, 100.1, 75.0, 56.8, 22.7, 22.3, 21.5, 13.9; FT-IR (neat): $v_{\max }=2961$, 2933, 2870, 2838, 1592, 1503, 1481, 1462, 1334, 1273, 1257, 1116, 1061, 921, 877, 818, 800, 672, $642 \mathrm{~cm}^{-1}$; HRMS (EI): exact mass calculated for: $\mathrm{C}_{17} \mathrm{H}_{18} \mathrm{O}[\mathrm{M}]^{+}$: 238.1352 , found 238.1350 .

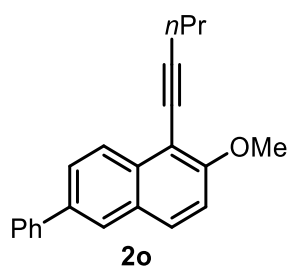

White solid (85\% yield); m.p. 93.1-93.5 ${ }^{\circ} \mathrm{C} ;{ }^{1} \mathrm{H}$ NMR (400 MHz, $\left.\mathrm{CHCl}_{3}\right) \delta 8.33(\mathrm{~d}, J=8.4 \mathrm{~Hz}, 1 \mathrm{H}), 7.96(\mathrm{~d}, J=1.2 \mathrm{~Hz}, 1 \mathrm{H}), 7.83-$ $7.78(\mathrm{~m}, 2 \mathrm{H}), 7.71(\mathrm{~d}, J=7.2 \mathrm{~Hz}, 2 \mathrm{H}), 7.48(\mathrm{~d}, J=7.6 \mathrm{~Hz}, 2 \mathrm{H}), 7.36$ $(\mathrm{t}, J=7.6 \mathrm{~Hz}, 1 \mathrm{H}), 7.26(\mathrm{~d}, J=8.0 \mathrm{~Hz}, 1 \mathrm{H}), 4.03(\mathrm{~s}, 3 \mathrm{H}), 2.64(\mathrm{t}, J$ $=7.2 \mathrm{~Hz}, 2 \mathrm{H}), 1.79(\mathrm{sext}, J=7.2 \mathrm{~Hz}, 2 \mathrm{H}), 1.16(\mathrm{t}, J=7.2 \mathrm{~Hz}, 3 \mathrm{H}) ;{ }^{13} \mathrm{C} \mathrm{NMR}(101 \mathrm{MHz}$, $\left.\mathrm{CHCl}_{3}\right) \delta 158.7,141.1,136.9,134.2,129.6,129.0,128.9,127.4,127.3,126.8,126.1$, 126.0, 113.3, 107.2, 100.4, 74.9, 56.8, 22.7, 22.3, 13.9; FT-IR (neat): $v_{\max }=2959,2925$, $2865,1588,1493,1460,1438,1345,1334,1282,1258,1175,1122,1067,1030,891$, 801, 759, $698 \mathrm{~cm}^{-1}$; HRMS (EI): exact mass calculated for: $\mathrm{C}_{22} \mathrm{H}_{20} \mathrm{O}[\mathrm{M}]^{+}: 300.1509$, found 300.1508 .

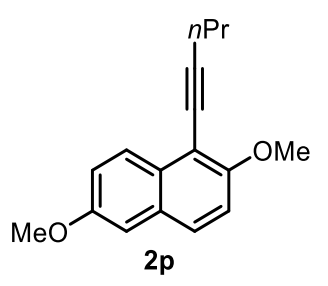

Yellow solid (87\% yield); m.p. $41.9-42.8{ }^{\circ} \mathrm{C} ;{ }^{1} \mathrm{H}$ NMR $(400 \mathrm{MHz}$, $\left.\mathrm{CHCl}_{3}\right) \delta 8.17(\mathrm{~d}, J=9.2 \mathrm{~Hz}, 1 \mathrm{H}), 7.65(\mathrm{~d}, J=9.2 \mathrm{~Hz}, 1 \mathrm{H}), 7.19$ (dd, $J=9.2,2.4 \mathrm{~Hz}, 2 \mathrm{H}), 7.07$ (d, $J=2.4 \mathrm{~Hz}, 1 \mathrm{H}), 3.98(\mathrm{~s}, 3 \mathrm{H})$, $3.89(\mathrm{~s}, 3 \mathrm{H}), 2.61(\mathrm{t}, J=7.2 \mathrm{~Hz}, 2 \mathrm{H}), 1.76(\mathrm{sext}, J=7.2 \mathrm{~Hz}, 2 \mathrm{H})$, $1.14(\mathrm{t}, J=7.2 \mathrm{~Hz}, 3 \mathrm{H}) ;{ }^{13} \mathrm{C}$ NMR $\left(101 \mathrm{MHz}, \mathrm{CHCl}_{3}\right) \delta 157.3,156.5,130.4,129.6$, $127.8,127.1,119.9,113.6,107.7,106.1,100.1,75.0,57.0,55.4,22.7,22.3,13.9$; FT- 
IR (neat): $v_{\max }=2960,2933,2871,2837,1590,1505,1462,1336,1271,1255,1244$, 1166, 1106, 1066, 1026, 845, 822, $795 \mathrm{~cm}^{-1}$; HRMS (EI): exact mass calculated for: $\mathrm{C}_{17} \mathrm{H}_{18} \mathrm{O}_{2}[\mathrm{M}]^{+}:$254.1301, found 254.1301.

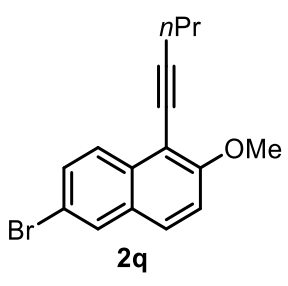

Yellow oil (53\% yield); ${ }^{1} \mathrm{H}$ NMR (400 MHz, $\left.\mathrm{CHCl}_{3}\right) \delta 8.13(\mathrm{~d}, J=$ $9.2 \mathrm{~Hz}, 1 \mathrm{H}), 7.90(\mathrm{~d}, J=2.0 \mathrm{~Hz}, 1 \mathrm{H}), 7.65(\mathrm{~d}, J=8.8 \mathrm{~Hz}, 1 \mathrm{H}), 7.56$ $(\mathrm{dd}, J=9.2,2.0 \mathrm{~Hz}, 1 \mathrm{H}), 7.23(\mathrm{~d}, J=9.2 \mathrm{~Hz}, 1 \mathrm{H}), 4.00(\mathrm{~s}, 3 \mathrm{H}), 2.61$ (t, $J=7.2 \mathrm{~Hz}, 2 \mathrm{H}), 1.76$ (sext, $J=7.2 \mathrm{~Hz}, 2 \mathrm{H}), 1.14$ (t, $J=7.6 \mathrm{~Hz}$, $3 \mathrm{H}) ;{ }^{13} \mathrm{C} \mathrm{NMR}\left(101 \mathrm{MHz}, \mathrm{CHCl}_{3}\right) \delta 158.8,133.5,130.4,130.0,129.7,128.2,127.5$, 117.9, 113.9, 107.6, 100.9, 74.4, 56.8, 22.6, 22.2, 13.8; FT-IR (neat): $v_{\max }=3011,2964$, 2934, 2870, 2838, 1583, 1495, 1458, 1326, 1270, 1256, 1175, 1119, 1064, 901, 871, 818, $802 \mathrm{~cm}^{-1}$; HRMS (EI): exact mass calculated for: $\mathrm{C}_{16} \mathrm{H}_{15} \mathrm{BrO}_{2}[\mathrm{M}]^{+}$: 302.0301 , found 302.0299 .

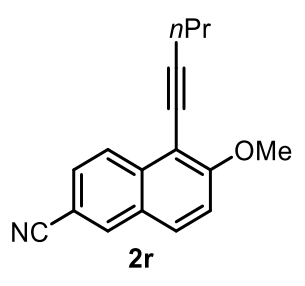

Yellow solid (85\% yield); m.p. $61.8-62.4{ }^{\circ} \mathrm{C} ;{ }^{1} \mathrm{H}$ NMR (400 MHz, $\left.\mathrm{CHCl}_{3}\right) \delta 8.31(\mathrm{~d}, J=8.8 \mathrm{~Hz}, 1 \mathrm{H}), 8.13(\mathrm{~d}, J=1.2 \mathrm{~Hz}, 1 \mathrm{H}), 7.80(\mathrm{~d}$, $J=9.2 \mathrm{~Hz}, 1 \mathrm{H}), 7.61(\mathrm{dd}, J=8.8,1.2 \mathrm{~Hz}, 1 \mathrm{H}), 7.33(\mathrm{~d}, J=9.2 \mathrm{~Hz}$, 1H), 4.04 (s, 3H), 2.62 (t, $J=7.2 \mathrm{~Hz}, 2 \mathrm{H}), 1.77$ (sext, $J=7.2 \mathrm{~Hz}$, 2H), $1.14(\mathrm{t}, J=7.2 \mathrm{~Hz}, 3 \mathrm{H}) ;{ }^{13} \mathrm{C} \mathrm{NMR}\left(101 \mathrm{MHz}, \mathrm{CHCl}_{3}\right) \delta 160.7,136.5,134.3,129.7$, $127.5,127.4,126.9,119.6,114.2,107.9,107.4,101.6,73.9,56.7,22.5,22.2$, 13.8; FTIR (neat): $v_{\max }=2933,2838,2219,1618,1586,1475,1453,1337,1275,1256,1114$, 1058, 889, 832, $781 \mathrm{~cm}^{-1}$; HRMS (EI): exact mass calculated for: $\mathrm{C}_{17} \mathrm{H}_{15} \mathrm{NO}[\mathrm{M}]^{+}$: 249.1148 , found 249.1149 .

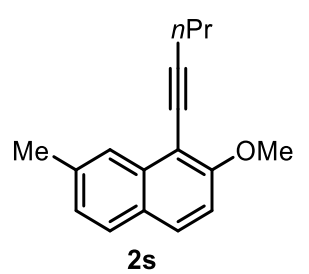

Yellow solid (74\% yield); m.p. $39.1-39.5{ }^{\circ} \mathrm{C} ;{ }^{1} \mathrm{H}$ NMR (400 MHz, $\left.\mathrm{CHCl}_{3}\right) \delta 8.04(\mathrm{~s}, 1 \mathrm{H}), 7.69(\mathrm{~d}, J=9.2 \mathrm{~Hz}, 1 \mathrm{H}), 7.64(\mathrm{~d}, J=8.0 \mathrm{~Hz}$, 1H), $7.18(\mathrm{dd}, J=8.4,1.2 \mathrm{~Hz}, 1 \mathrm{H}), 7.13(\mathrm{~d}, J=9.2 \mathrm{~Hz}, 1 \mathrm{H}), 3.98$ (s, 3H), 2.63 (t, $J=7.2 \mathrm{~Hz}, 2 \mathrm{H}), 2.52$ (s, 3H), 1.77 (sext, $J=7.2 \mathrm{~Hz}$, 2H), $1.16(\mathrm{t}, J=7.6 \mathrm{~Hz}, 3 \mathrm{H}) ;{ }^{13} \mathrm{C} \mathrm{NMR}\left(101 \mathrm{MHz}, \mathrm{CHCl}_{3}\right) \delta 158.8,137.1,135.2,129.0$, $127.9,127.0,126.4,124.5,111.9,106.6,100.1,75.1,56.7,22.7,22.3,22.2,13.8$; FT- 
IR (neat): $v_{\max }=2961,2931,2839,1626,1594,1511,1462,1431,1317,1269,1174$, 1152, 1118, 1064, 831, $773 \mathrm{~cm}^{-1}$; HRMS (EI): exact mass calculated for: $\mathrm{C}_{17} \mathrm{H}_{18} \mathrm{O}[\mathrm{M}]^{+}$: 238.1352 , found 238.1352 .

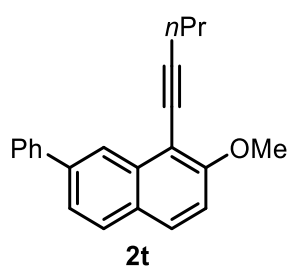

Yellow solid (95\% yield); m.p. 87.3-87.8 ${ }^{\circ} \mathrm{C} ;{ }^{1} \mathrm{H}$ NMR $(400 \mathrm{MHz}$, $\left.\mathrm{CHCl}_{3}\right) \delta 8.52(\mathrm{~s}, 1 \mathrm{H}), 7.77(\mathrm{~d}, J=8.4 \mathrm{~Hz}, 1 \mathrm{H}), 7.75-7.73(\mathrm{~m}, 2 \mathrm{H})$, $7.70(\mathrm{~d}, J=9.2 \mathrm{~Hz}, 1 \mathrm{H}), 7.59(\mathrm{dd}, J=8.4,2.0 \mathrm{~Hz}, 1 \mathrm{H}), 7.46(\mathrm{t}, J=$ $7.6 \mathrm{~Hz}, 2 \mathrm{H}), 7.35$ (t, $J=7.2 \mathrm{~Hz}, 1 \mathrm{H}), 7.14(\mathrm{~d}, J=9.2 \mathrm{~Hz}, 1 \mathrm{H}), 3.94$ (s, 3H), 2.62 (t, $J=7.2 \mathrm{~Hz}, 2 \mathrm{H}), 1.75$ (sext, $J=7.2 \mathrm{~Hz}, 2 \mathrm{H}), 1.16$ (t, $J=7.6 \mathrm{~Hz}, 3 \mathrm{H})$; ${ }^{13} \mathrm{C} \mathrm{NMR}\left(101 \mathrm{MHz}, \mathrm{CHCl}_{3}\right) \delta 158.9,141.4,139.7,135.2,128.9,128.6,127.8,127.5$, 127.4, 123.8, 123.5, 112.8, 107.5, 100.6, 75.0, 56.6, 22.6, 22.3, 13.8; FT-IR (neat): $v_{\max }$ $=2928,2841,1592,1492,1454,1369,1250,1164,1114,1062,837,760 \mathrm{~cm}^{-1}$; HRMS (EI): exact mass calculated for: $\mathrm{C}_{22} \mathrm{H}_{20} \mathrm{O}[\mathrm{M}]^{+}: 300.1509$, found 300.1506 .

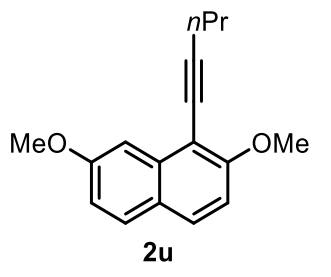

Yellow solid (48\% yield); m.p. $36.5-37.0{ }^{\circ} \mathrm{C} ;{ }^{1} \mathrm{H}$ NMR $(400 \mathrm{MHz}$, $\left.\mathrm{CHCl}_{3}\right) \delta 7.69(\mathrm{~d}, J=9.2 \mathrm{~Hz}, 1 \mathrm{H}), 7.65(\mathrm{~d}, J=8.8 \mathrm{~Hz}, 1 \mathrm{H}), 7.59$ $(\mathrm{d}, J=2.0 \mathrm{~Hz}, 1 \mathrm{H}), 7.07(\mathrm{~d}, J=9.2 \mathrm{~Hz}, 1 \mathrm{H}), 7.02(\mathrm{dd}, J=8.8,2.4$ Hz, 1H), 4.00 (s, 3H), 3.95 (s, 3H), 2.64 (t, $J=7.2 \mathrm{~Hz}, 2 \mathrm{H}), 1.77$ (sext, $J=7.2 \mathrm{~Hz}, 2 \mathrm{H}), 1.18(\mathrm{t}, J=7.6 \mathrm{~Hz}, 3 \mathrm{H}) ;{ }^{13} \mathrm{C} \mathrm{NMR}\left(101 \mathrm{MHz}, \mathrm{CHCl}_{3}\right) \delta 159.2$, 159.1, 136.6, 129.7, 129.0, 124.2, 117.0, 110.1, 106.2, 103.8, 100.1, 75.3, 56.7, 55.3, 22.7, 22.3, 13.8; FT-IR (neat): $v_{\max }=2929,2838,1618,1504,1457,1319,1258,1217$, 1060, 1027, 829, 775 $\mathrm{cm}^{-1}$; HRMS (EI): exact mass calculated for: $\mathrm{C}_{17} \mathrm{H}_{18} \mathrm{O}_{2}[\mathrm{M}]^{+}$: 254.1301, found 254.1297.

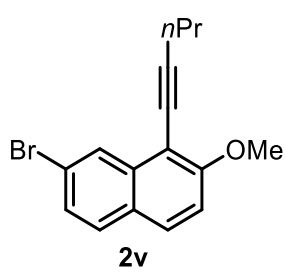

Colorless oil (40\% yield); ${ }^{1} \mathrm{H}$ NMR (400 MHz, $\left.\mathrm{CHCl}_{3}\right) \delta 8.42(\mathrm{~d}, J$ $=1.6 \mathrm{~Hz}, 1 \mathrm{H}), 7.69(\mathrm{~d}, J=8.8 \mathrm{~Hz}, 1 \mathrm{H}), 7.59(\mathrm{~d}, J=8.8 \mathrm{~Hz}, 1 \mathrm{H})$, $7.41(\mathrm{dd}, J=8.4,1.6 \mathrm{~Hz}, 1 \mathrm{H}), 7.20(\mathrm{~d}, J=9.2 \mathrm{~Hz}, 1 \mathrm{H}), 3.99(\mathrm{~s}, 3 \mathrm{H})$, $2.62(\mathrm{t}, J=6.8 \mathrm{~Hz}, 2 \mathrm{H}), 1.77(\mathrm{sext}, J=7.2 \mathrm{~Hz}, 2 \mathrm{H}), 1.15(\mathrm{t}, J=7.6$ $\mathrm{Hz}, 3 \mathrm{H}) ;{ }^{13} \mathrm{C} \mathrm{NMR}\left(101 \mathrm{MHz}, \mathrm{CHCl}_{3}\right) \delta 159.2,136.2,129.7,129.1,127.8,127.5,127.0$, $121.9,113.1,106.6,100.9,74.3,56.7,22.6,22.3,13.8$; FT-IR (neat): $v_{\max }=2961,2932$, 
2838, 1610, 1498, 1457, 1360, 1316, 1256, 1149, 1117, 1062, 824, $723 \mathrm{~cm}^{-1}$; HRMS (EI): exact mass calculated for: $\mathrm{C}_{16} \mathrm{H}_{15} \mathrm{BrO}[\mathrm{M}]^{+}: 302.0301$, found 302.0302 .

\subsection{Synthesis of 2-alkoxyl-1-alkynyl naphthalenes 2w-2y and 1-alkynyl} naphthalene $2 z$

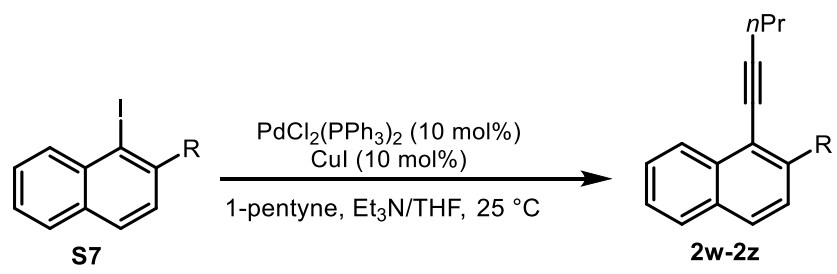

To a stirred solution of $\mathbf{S} 7$ (3.52 mmol), $\mathrm{PdCl}_{2}\left(\mathrm{PPh}_{3}\right)_{2}$ (247 mg, $\left.0.35 \mathrm{mmol}\right), \mathrm{CuI}(67$ $\mathrm{mg}, 0.35 \mathrm{mmol})$ in triethylamine $(11 \mathrm{~mL})$ and THF $(11 \mathrm{~mL})$ was added 1-pentyne $(5.28$ mmol) at $25{ }^{\circ} \mathrm{C}$. The reaction mixture was stirred at $25^{\circ} \mathrm{C}$. After the reaction was complete (monitored by TLC), the reaction mixture was filtered through a pad of celite and washed with ethyl acetate. The mixture was concentrated under reduced pressure. The residue was purified by flash column chromatography (silica gel, ethyl acetate/petroleum ether 1:99) to afford the corresponding coupling product.

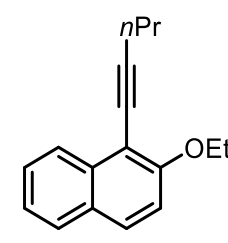

2w

Brown oil (85\% yield); ${ }^{1} \mathrm{H}$ NMR (400 $\left.\mathrm{MHz}, \mathrm{CHCl}_{3}\right) \delta 8.28(\mathrm{~d}, J=8.4$ $\mathrm{Hz}, 1 \mathrm{H}), 7.73(\mathrm{~d}, J=8.4 \mathrm{~Hz}, 1 \mathrm{H}), 7.70(\mathrm{~d}, J=8.8 \mathrm{~Hz}, 1 \mathrm{H}), 7.52-7.48$ (m, 1H), $7.36-7.32(\mathrm{~m}, 1 \mathrm{H}), 7.17(\mathrm{~d}, J=9.2 \mathrm{~Hz}, 1 \mathrm{H}), 4.21(\mathrm{q}, J=6.8$ $\mathrm{Hz}, 2 \mathrm{H}), 2.60(\mathrm{t}, J=6.8 \mathrm{~Hz}, 2 \mathrm{H}), 1.74(\mathrm{sext}, J=7.2 \mathrm{~Hz}, 2 \mathrm{H}), 1.47$ (t, $J$ $=7.2 \mathrm{~Hz}, 3 \mathrm{H}), 1.15(\mathrm{t}, J=7.2 \mathrm{~Hz}, 3 \mathrm{H}) ;{ }^{13} \mathrm{C} \mathrm{NMR}\left(101 \mathrm{MHz}, \mathrm{CHCl}_{3}\right) \delta$ 158.3, 135.0, $129.1,128.8,128.0,127.0,125.6,124.1,114.8,108.2$, 100.0, 75.1, 65.5, 22.6, 22.2, 15.2, 13.7; FT-IR (neat): $v_{\max }=2962,2931,1588,1506,1464,1384,1328,1271,1253$, 1113, 1058, 1022, 803, $747 \mathrm{~cm}^{-1}$; HRMS (EI): exact mass calculated for: $\mathrm{C}_{17} \mathrm{H}_{18} \mathrm{O}[\mathrm{M}]^{+}$: 238.1352 , found 238.1352 . 


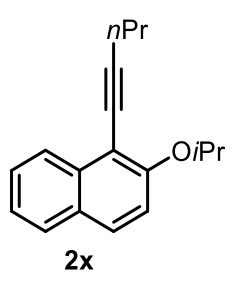

Brown oil (65\% yield); ${ }^{1} \mathrm{H} \mathrm{NMR}\left(400 \mathrm{MHz}, \mathrm{CHCl}_{3}\right) \delta 8.27(\mathrm{~d}, J=8.4$ $\mathrm{Hz}, 1 \mathrm{H}), 7.75(\mathrm{~d}, J=8.4 \mathrm{~Hz}, 1 \mathrm{H}), 7.70(\mathrm{~d}, J=8.8 \mathrm{~Hz}, 1 \mathrm{H}), 7.52-7.48$ (m, 1H), $7.38-7.34(\mathrm{~m}, 1 \mathrm{H}), 7.19(\mathrm{~d}, J=9.2 \mathrm{~Hz}, 1 \mathrm{H}), 4.71$ (sept, $J=$ $6.0 \mathrm{~Hz}, 1 \mathrm{H}), 2.60$ (t, $J=6.8 \mathrm{~Hz}, 2 \mathrm{H}), 1.75$ (sext, $J=7.2 \mathrm{~Hz}, 2 \mathrm{H}), 1.41$ (s, 3H), 1.39 (s, 3H), 1.15 (t, $J=7.2 \mathrm{~Hz}, 3 \mathrm{H}) ;{ }^{13} \mathrm{C} \mathrm{NMR}\left(101 \mathrm{MHz}, \mathrm{CHCl}_{3}\right) \delta$ 157.7, $135.0,129.2,128.9,128.0,126.9,125.8,124.4,118.2,110.5,99.9,75.3,73.5,22.7$, 22.6, 22.2, 13.8; FT-IR (neat): $v_{\max }=2969,2931,1588,1504,1464,1381,1325,1271$, 1250, 1110, 1003, 806,748 $\mathrm{cm}^{-1}$; HRMS (EI): exact mass calculated for: $\mathrm{C}_{18} \mathrm{H}_{20} \mathrm{O}[\mathrm{M}]^{+}$: 252.1509 , found 252.1511 .

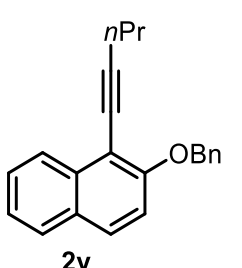

Yellow solid (40\% yield); m.p. 43.0-43.5 ${ }^{\circ} \mathrm{C}$; ${ }^{1} \mathrm{H}$ NMR $(400 \mathrm{MHz}$, $\left.\mathrm{CHCl}_{3}\right) \delta 8.30(\mathrm{~d}, J=8.4 \mathrm{~Hz}, 1 \mathrm{H}), 7.76(\mathrm{~d}, J=8.0 \mathrm{~Hz}, 1 \mathrm{H}), 7.71(\mathrm{~d}, J$ $=8.8 \mathrm{~Hz}, 1 \mathrm{H}), 7.55-7.51(\mathrm{~m}, 3 \mathrm{H}), 7.40-7.35(\mathrm{~m}, 3 \mathrm{H}), 7.33-7.29(\mathrm{~m}$, $1 \mathrm{H}), 7.23(\mathrm{~d}, J=9.2 \mathrm{~Hz}, 1 \mathrm{H}), 5.32(\mathrm{~s}, 2 \mathrm{H}), 2.63(\mathrm{t}, J=6.8 \mathrm{~Hz}, 2 \mathrm{H}), 1.76$ (sext, $J=7.2 \mathrm{~Hz}, 2 \mathrm{H}), 1.13(\mathrm{t}, J=7.2 \mathrm{~Hz}, 3 \mathrm{H}) ;{ }^{13} \mathrm{C} \mathrm{NMR}\left(101 \mathrm{MHz}, \mathrm{CHCl}_{3}\right) \delta 158.0$, 137.4, 135.0, 129.1, 128.6, 128.1, 127.9, 127.3, 127.1, 125.7, 124.4, 115.5, 108.9, 100.4, 75.0, 71.7, 22.6, 22.3, 13.8; FT-IR (neat): $v_{\max }=1590,1510,1274,1257,1057,1017$, 801, 711, $681 \mathrm{~cm}^{-1}$; HRMS (EI): exact mass calculated for: $\mathrm{C}_{17} \mathrm{H}_{15} \mathrm{NO}[\mathrm{M}]^{+}: 300.1509$, found 300.1511 .

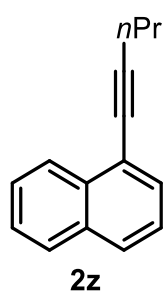

Brown oil (97\% yield); ${ }^{1} \mathrm{H}$ NMR $\left(400 \mathrm{MHz}, \mathrm{CHCl}_{3}\right) \delta 8.38(\mathrm{~d}, J=8.4 \mathrm{~Hz}$, 1H), $7.85(\mathrm{~d}, J=8.0 \mathrm{~Hz}, 1 \mathrm{H}), 7.79(\mathrm{~d}, J=8.0 \mathrm{~Hz}, 1 \mathrm{H}), 7.65$ (d, $J=7.2 \mathrm{~Hz}$, $1 \mathrm{H}), 7.57$ (ddd, $J=8.4,7.2,1.2 \mathrm{~Hz}, 1 \mathrm{H}), 7.51(\mathrm{ddd}, J=8.0,6.8,1.2 \mathrm{~Hz}, 1 \mathrm{H})$, $7.41(\mathrm{dd}, J=8.0,7.2 \mathrm{~Hz}, 1 \mathrm{H}), 2.57$ (t, $J=7.2 \mathrm{~Hz}, 2 \mathrm{H}), 1.76$ (sext, $J=7.2 \mathrm{~Hz}$, $2 \mathrm{H}), 1.16(\mathrm{t}, J=7.2 \mathrm{~Hz}, 3 \mathrm{H})$. The spectroscopic data was consistent with those reported in the literature. ${ }^{6}$ 


\subsection{Synthesis of 2-hydroxy-1-alkynyl naphthalene 2aa}<smiles>Oc1ccc2ccccc2c1I</smiles>

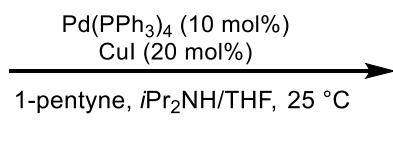

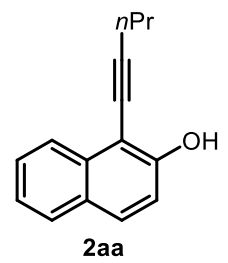

To a stirred solution of $\mathbf{S 8}(1.1 \mathrm{~g}, 4.1 \mathrm{mmol}), \mathrm{Pd}\left(\mathrm{PPh}_{3}\right)_{4}$ (474 mg, $\left.0.41 \mathrm{mmol}\right), \mathrm{CuI}$ (156 mg, $0.82 \mathrm{mmol})$ in $i \mathrm{Pr}_{2} \mathrm{NH}(1.7 \mathrm{~mL})$ and THF $(17 \mathrm{~mL})$ was added 1-pentyne (559 $\mathrm{mg}, 8.2 \mathrm{mmol}$ ) at $25^{\circ} \mathrm{C}$. After the reaction mixture was stirred at this temperature for 2 h. Then, the mixture was quenched with $\mathrm{H}_{2} \mathrm{O}(20 \mathrm{~mL})$. The aqueous layer was extracted with dichloromethane $(30 \mathrm{~mL} \times 3)$. The combined organic layers were washed with brine $(30 \mathrm{~mL})$, dried over $\mathrm{Na}_{2} \mathrm{SO}_{4}$, and concentrated under reduced pressure. The residue was purified by flash column chromatography (silica gel, dichloromethane/petroleum ether 1:10) to afford $2 \mathbf{a a}$ as a colorless oil (373 $\mathrm{mg}, 44 \%$ yield).<smiles>CC(C)C#Cc1c(O)ccc2ccccc12</smiles>

2aa ${ }^{1} \mathrm{H}$ NMR $\left(400 \mathrm{MHz}, \mathrm{CHCl}_{3}\right) \delta 8.09(\mathrm{~d}, \mathrm{~J}=8.4 \mathrm{~Hz}, 1 \mathrm{H}), 7.75(\mathrm{~d}, \mathrm{~J}=8.0$ $\mathrm{Hz}, 1 \mathrm{H}), 7.70(\mathrm{~d}, \mathrm{~J}=8.8 \mathrm{~Hz}, 1 \mathrm{H}), 7.51(\mathrm{t}, \mathrm{J}=7.6 \mathrm{~Hz}, 1 \mathrm{H}), 7.34$ (t, J = $7.2 \mathrm{~Hz}, 1 \mathrm{H}), 7.18(\mathrm{~d}, \mathrm{~J}=9.2 \mathrm{~Hz}, 1 \mathrm{H}), 6.14(\mathrm{~s}, 1 \mathrm{H}), 2.61$ (t, J = 7.2 Hz, 2H), 1.75 (sext, J = 7.2 Hz, 2H), 1.13 (t, J = 7.2 Hz, 3H); ${ }^{13} \mathrm{C}$ NMR $\left(101 \mathrm{MHz}, \mathrm{CHCl}_{3}\right) \delta 155.9,133.9,129.8,128.6,128.3,127.3,125.0,124.0,116.3$, 103.6, 102.8, 73.2, 22.6, 22.1, 13.8; FT-IR (neat): $v_{\max }=3483,1633,1591,1464,1389$, 1273, 1210, 1171, 836, $747 \mathrm{~cm}^{-1}$; HRMS (EI): exact mass calculated for: $\mathrm{C}_{15} \mathrm{H}_{14} \mathrm{O}[\mathrm{M}]^{+}$: 210.1039 , found 210.1040 . 
3.7 Synthesis of 2-alkoxyl-1-alkynyl naphthalene 2ab

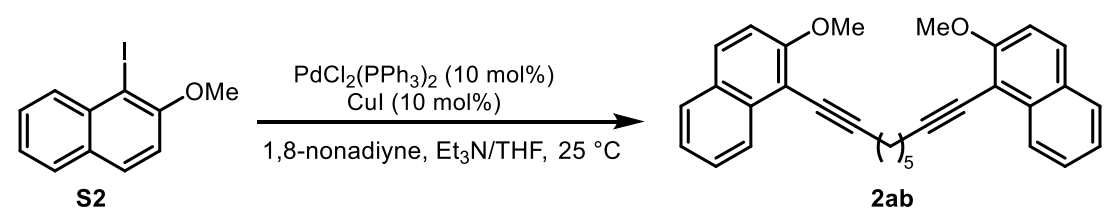

To a stirred solution of $\mathbf{S 2}(3.52 \mathrm{mmol}), \mathrm{PdCl}_{2}\left(\mathrm{PPh}_{3}\right)_{2}$ (247 mg, $\left.0.35 \mathrm{mmol}\right), \mathrm{CuI}$ (67 $\mathrm{mg}, 0.35 \mathrm{mmol})$ in triethylamine $(11 \mathrm{~mL})$ and THF $(11 \mathrm{~mL})$ was added 1,8-nonadiyne (170 mg, $1.41 \mathrm{mmol}$ ) at $25^{\circ} \mathrm{C}$. After the reaction mixture was stirred at this temperature for $36 \mathrm{~h}$, the crude mixture was filtered through a pad of celite and washed with ethyl acetate. The mixture was concentrated under reduced pressure. The residue was purified by flash column chromatography (silica gel, ethyl acetate/petroleum ether 1:9) to afford 2ab as a brown oil (300 mg, 49\% yield).

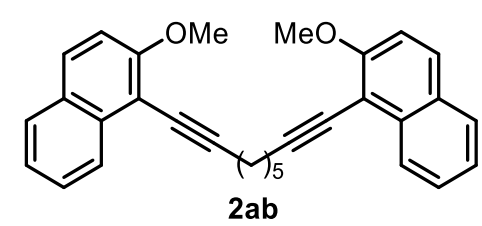

${ }^{1} \mathrm{H}$ NMR $\left(400 \mathrm{MHz}, \mathrm{CHCl}_{3}\right) \delta 8.26(\mathrm{~d}, J=8.4 \mathrm{~Hz}, 1 \mathrm{H})$, $7.76(\mathrm{~d}, J=8.8 \mathrm{~Hz}, 1 \mathrm{H}), 7.75(\mathrm{~d}, J=8.0 \mathrm{~Hz}, 1 \mathrm{H}), 7.48$ (ddd, $J=8.0,6.8,1.2 \mathrm{~Hz}, 1 \mathrm{H}), 7.33$ (ddd, $J=8.0,6.8,0.8$ Hz, 1H), 7.22 (d, J = 9.2 Hz, 1H), 4.00 (s, 3H), $2.72-$ $2.69(\mathrm{~m}, 2 \mathrm{H}), 1.88-1.85(\mathrm{~m}, 3 \mathrm{H}) ;{ }^{13} \mathrm{C} \mathrm{NMR}\left(101 \mathrm{MHz}, \mathrm{CHCl}_{3}\right) \delta 158.7,135.0,129.3$, $128.7,128.1,127.2,125.5,124.2,112.9,107.3,100.2,75.0,56.8,28.9,28.7,20.3$; FTIR (neat): $v_{\max }=3055,3000,2933,2838,1620,1589,1506,1461,1432,1384,1329$, 1272, 1256, 1146, 1062, 804, $746 \mathrm{~cm}^{-1}$; HRMS (ESI): exact mass calculated for: $\mathrm{C}_{31} \mathrm{H}_{28} \mathrm{O}_{2} \mathrm{Na}[\mathrm{M}+\mathrm{Na}]^{+}:$455.1982, found 155.1977 .

\section{General procedure for asymmetric synthesis of 3a-3al}

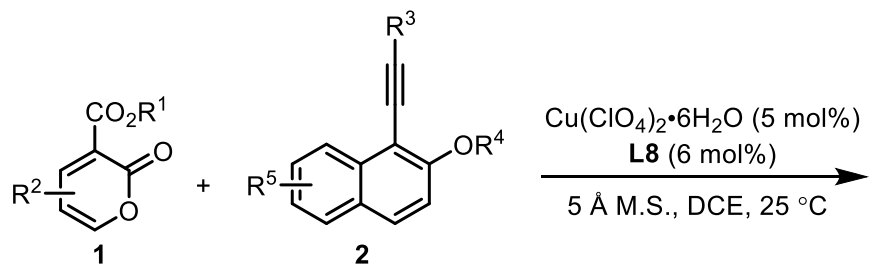<smiles>[R]COc1ccc2ccccc2c1-c1c([R])cccc1OC(=O)O</smiles> 
A mixture of $\mathrm{Cu}\left(\mathrm{ClO}_{4}\right)_{2} \bullet 6 \mathrm{H}_{2} \mathrm{O}(3.7 \mathrm{mg}, 0.01 \mathrm{mmol}), 5 \AA$ M.S. (50.0 mg) and $\mathbf{L 8}(9.1$ $\mathrm{mg}, 0.012 \mathrm{mmol})$ in DCE $(1.0 \mathrm{~mL})$ was stirred at $25{ }^{\circ} \mathrm{C}$ for $2 \mathrm{~h}$. Then, 2-pyrone $1(0.2$ mmol) was added. After stirred at $25{ }^{\circ} \mathrm{C}$ for $20 \mathrm{~min}$, the corresponding alkyne $2(0.3$ mmol) was added in one portion. After 2-pyrone 1 was consumed completely (monitored by TLC), the crude mixture was filtrated through celite and washed with dichloromethane. The filtrate was concentrated under reduced pressure. The residue was purified by flash column chromatography (silica gel, ethyl acetate/petroleum ether $1: 20)$ to afford 3.

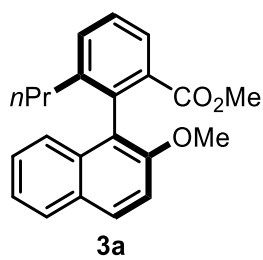

Colorless oil (64.7 mg, 97\% yield, 96\% ee, $48 \mathrm{~h}$ ); the enantiomeric excess was determined by CHIRALPAK IG column, hexanes/ $i$-PrOH, $97: 3 \mathrm{v} / \mathrm{v}, \mathrm{v}=0.3 \mathrm{~mL} / \mathrm{min}, \lambda=250 \mathrm{~nm}, 25^{\circ} \mathrm{C}, \mathrm{t}_{\mathrm{R}}($ major $)=18.8 \mathrm{~min}$, $\mathrm{t}_{\mathrm{R}}($ minor $)=20.4 \mathrm{~min} ;[\alpha]_{\mathrm{D}}{ }^{24}=+15.28\left(c=1.0, \mathrm{CHCl}_{3}\right)$.

3a: ${ }^{1} \mathrm{H}$ NMR $\left(400 \mathrm{MHz}, \mathrm{CHCl}_{3}\right) \delta 7.89(\mathrm{~d}, J=9.2 \mathrm{~Hz}, 1 \mathrm{H}), 7.86(\mathrm{dd}, J=8.0,1.6 \mathrm{~Hz}$, 1H), $7.80(\mathrm{dd}, J=7.2,1.6 \mathrm{~Hz}, 1 \mathrm{H}), 7.54(\mathrm{dd}, J=7.6,1.2 \mathrm{~Hz}, 1 \mathrm{H}), 7.44(\mathrm{t}, J=7.6 \mathrm{~Hz}$, 1H), $7.35(\mathrm{~d}, J=9.2 \mathrm{~Hz}, 1 \mathrm{H}), 7.31-7.23(\mathrm{~m}, 2 \mathrm{H}), 7.09-7.07$ (m, 1H), $3.81(\mathrm{~s}, 3 \mathrm{H})$, $3.36(\mathrm{~s}, 3 \mathrm{H}), 2.23-2.18(\mathrm{~m}, 2 \mathrm{H}), 1.39-1.28(\mathrm{~m}, 2 \mathrm{H}), 0.65(\mathrm{t}, J=7.6 \mathrm{~Hz}, 3 \mathrm{H}) ;{ }^{13} \mathrm{C}$ NMR $\left(101 \mathrm{MHz}, \mathrm{CHCl}_{3}\right) \delta 168.1,153.7,143.3,136.5,133.7,132.7,132.3,129.1,128.9$, $128.0,127.8,127.5,126.4,124.6,123.4,122.9,113.3,56.5,51.7,35.4,23.4$, 14.1; FTIR (neat): $v_{\max }=3059,2996,2957,2934,2870,2837,1728,1621,1593,1508,1456$, 1431, 1378, 1333, 1254, 1189, 1138, 1121, 1099, 1067, 1020, 908, 807, 747, 701, 681, $640 \mathrm{~cm}^{-1}$; HRMS (ESI): exact mass calculated for: $\mathrm{C}_{22} \mathrm{H}_{22} \mathrm{O}_{3}[\mathrm{M}]^{+}: 334.1563$, found 334.1565 . 


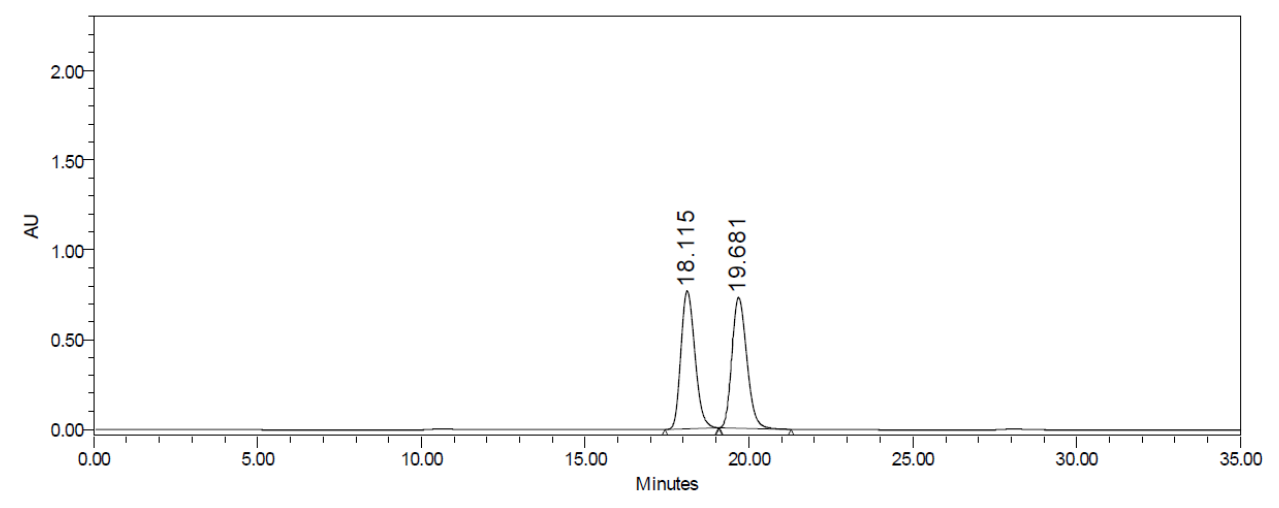

\begin{tabular}{|c|c|c|r|c|}
\hline & RT & Area & $\%$ Area & Height \\
\hline 1 & 18.115 & 22989552 & 49.83 & 767684 \\
\hline 2 & 19.681 & 23149105 & 50.17 & 728467 \\
\hline
\end{tabular}

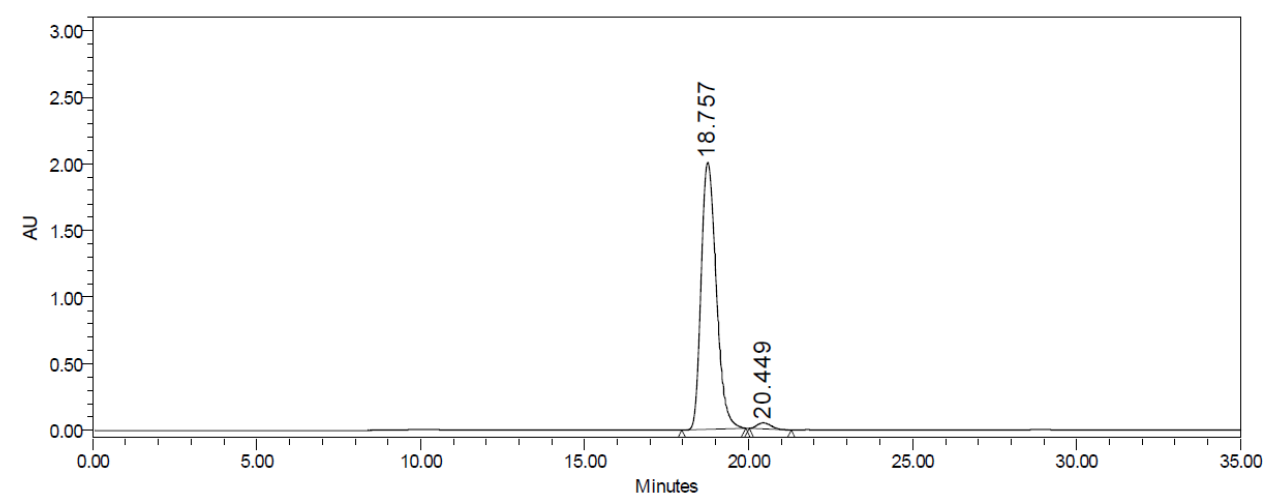

\begin{tabular}{|l|c|c|r|r|}
\hline & RT & Area & $\%$ Area & Height \\
\hline 1 & 18.757 & 64425649 & 97.91 & 2005916 \\
\hline 2 & 20.449 & 1375363 & 2.09 & 45822 \\
\hline
\end{tabular}

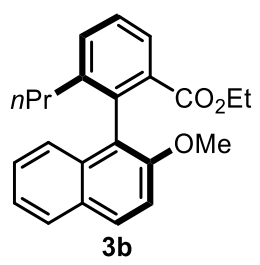

Colorless oil (58.6 mg, 84\% yield, 95\% ee, $108 \mathrm{~h}$ ); the enantiomeric excess was determined by CHIRALPAK IG column, hexanes/i-PrOH, $97: 3 \mathrm{v} / \mathrm{v}, \mathrm{v}=0.3 \mathrm{~mL} / \mathrm{min}, \lambda=254 \mathrm{~nm}, 25{ }^{\circ} \mathrm{C}, \mathrm{t}_{\mathrm{R}}($ major $)=20.4 \mathrm{~min}$, $\mathrm{t}_{\mathrm{R}}($ minor $)=23.0 \mathrm{~min} ;[\alpha]_{\mathrm{D}}{ }^{24}=-3.46\left(c=1.0, \mathrm{CHCl}_{3}\right)$.

3b: ${ }^{1} \mathrm{H}$ NMR $\left(400 \mathrm{MHz}, \mathrm{CHCl}_{3}\right) \delta 7.88(\mathrm{~d}, J=8.8 \mathrm{~Hz}, 1 \mathrm{H}), 7.85(\mathrm{dd}, J=7.6,1.2 \mathrm{~Hz}$, 1H), $7.80-7.78(\mathrm{~m}, 1 \mathrm{H}), 7.52(\mathrm{dd}, J=7.6,1.2 \mathrm{~Hz}, 1 \mathrm{H}), 7.44$ (t, $J=7.6 \mathrm{~Hz}, 1 \mathrm{H}), 7.33$ $(\mathrm{d}, J=9.2 \mathrm{~Hz}, 1 \mathrm{H}), 7.31-7.23(\mathrm{~m}, 2 \mathrm{H}), 7.12-7.10(\mathrm{~m}, 1 \mathrm{H}), 3.81(\mathrm{~s}, 3 \mathrm{H}), 3.80-3.71$ (m, 2H), $2.24-2.20(\mathrm{~m}, 2 \mathrm{H}), 1.41-1.26(\mathrm{~m}, 2 \mathrm{H}), 0.65(\mathrm{t}, J=7.2 \mathrm{~Hz}, 3 \mathrm{H}), 0.60(\mathrm{t}, J=$ $7.2 \mathrm{~Hz}, 3 \mathrm{H}) ;{ }^{13} \mathrm{C} \mathrm{NMR}\left(101 \mathrm{MHz}, \mathrm{CHCl}_{3}\right) \delta 168.2,153.8,143.2,136.0,133.8,133.1$, $132.5,129.1,128.9,127.9,127.8,127.5,126.4,124.8,123.4,123.1,113.2,60.3,56.5$, 
35.4, 23.4, 14.0, 13.4; FT-IR (neat): $v_{\max }=3059,2959,2932,2870,2838,1725,1707$, 1593, 1509, 1474, 1463, 1366, 1333, 1270, 1254, 1176, 1141, 1120, 1068, 1020, 807, 761, $747 \mathrm{~cm}^{-1}$; HRMS (ESI): exact mass calculated for: $\mathrm{C}_{23} \mathrm{H}_{24} \mathrm{O}_{3}[\mathrm{M}]^{+}: 348.1720$, found 348.1717 .
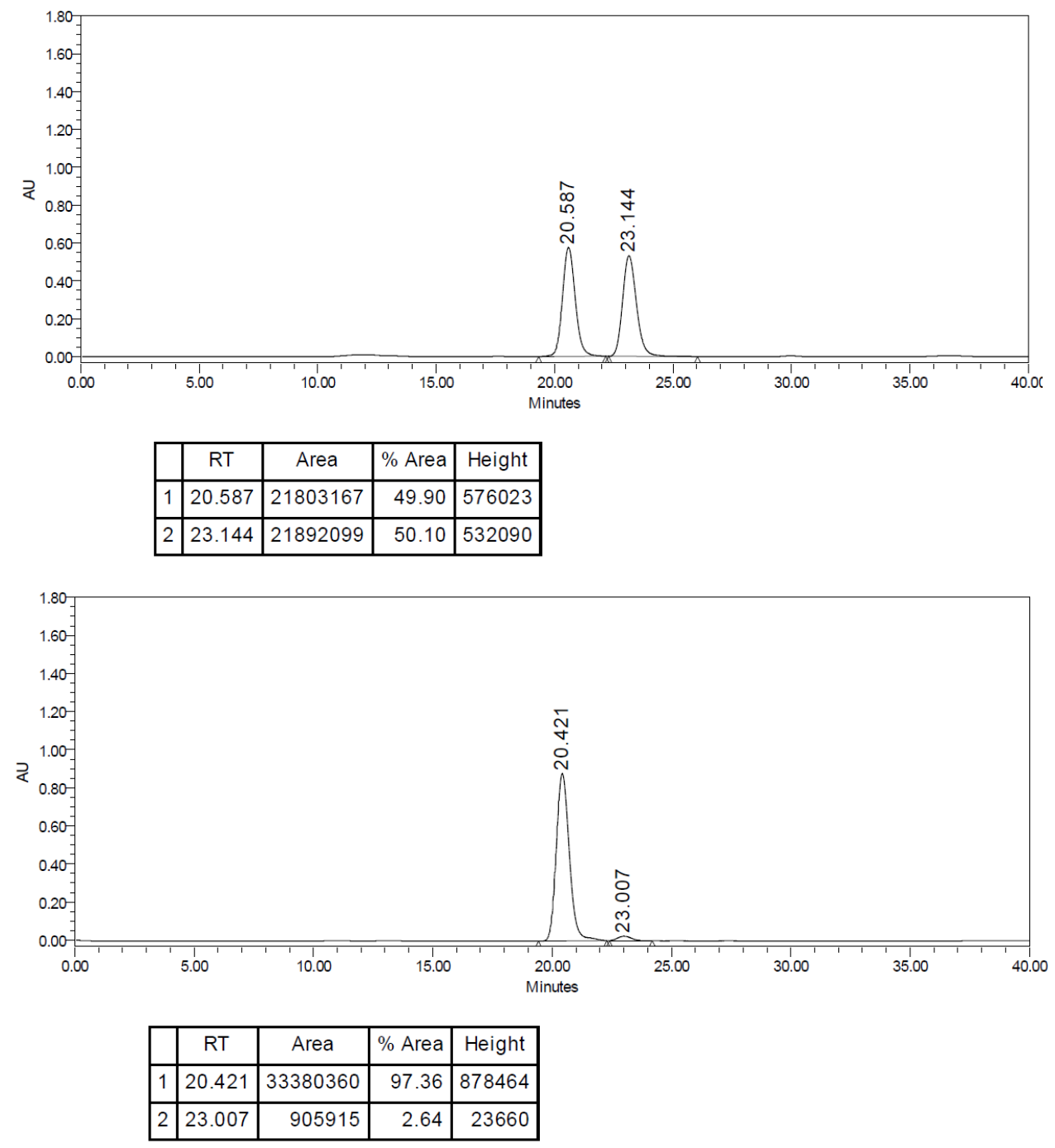

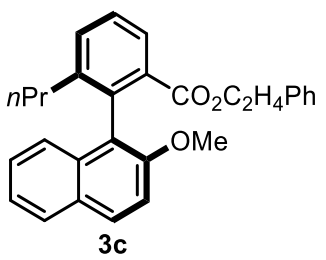

Colorless oil (62.0 $\mathrm{mg}, 73 \%$ yield, $95 \%$ ee, $36 \mathrm{~h})$; the enantiomeric excess was determined by CHIRALPAK IC column, hexanes $/ i-\mathrm{PrOH}, 97: 3 \mathrm{v} / \mathrm{v}, v=0.3 \mathrm{~mL} / \mathrm{min}, \lambda=254 \mathrm{~nm}, 25^{\circ} \mathrm{C}$, $\mathrm{t}_{\mathrm{R}}($ major $)=27.5 \mathrm{~min}, \mathrm{t}_{\mathrm{R}}($ minor $)=24.3 \mathrm{~min} ;[\alpha]_{\mathrm{D}}{ }^{24}=+8.46(c=$

$\left.1.0, \mathrm{CHCl}_{3}\right)$. 
3c: ${ }^{1} \mathrm{H}$ NMR $\left(400 \mathrm{MHz}, \mathrm{CHCl}_{3}\right) \delta 7.89(\mathrm{~d}, J=8.8 \mathrm{~Hz}, 1 \mathrm{H}), 7.81(\mathrm{dd}, J=8.0,1.2 \mathrm{~Hz}$, 2H), $7.53(\mathrm{dd}, J=7.6,1.2 \mathrm{~Hz}, 1 \mathrm{H}), 7.43(\mathrm{t}, J=7.6 \mathrm{~Hz}, 1 \mathrm{H}), 7.33$ (d, $J=8.8 \mathrm{~Hz}, 1 \mathrm{H})$, $7.30-7.24(\mathrm{~m}, 2 \mathrm{H}), 7.21-7.11(\mathrm{~m}, 4 \mathrm{H}), 6.93-6.91(\mathrm{~m}, 2 \mathrm{H}), 3.94-3.90(\mathrm{~m}, 2 \mathrm{H})$, $3.78(\mathrm{~s}, 3 \mathrm{H}), 2.31-2.18(\mathrm{~m}, 4 \mathrm{H}), 1.39-1.28(\mathrm{~m}, 2 \mathrm{H}), 0.65(\mathrm{t}, J=7.2 \mathrm{~Hz}, 3 \mathrm{H}) ;{ }^{13} \mathrm{C}$ $\operatorname{NMR}\left(101 \mathrm{MHz}, \mathrm{CHCl}_{3}\right) \delta 168.0,153.7,143.2,137.9,136.1,133.8,132.8,132.6,129.1$, $128.9,128.8,128.4,128.0,127.9,127.5,126.5,126.4,124.8,123.5,123.1,113.3,65.0$, 56.4, 35.5, 34.4, 23.4, 14.1; FT-IR (neat): $v \max =3060,2041,2959,2933,2870,2837$, $1725,1593,1509,1455,1270,1254,1174,1139,1121,1098,1068,1020,807,746$, $699 \mathrm{~cm}^{-1}$; HRMS (ESI): exact mass calculated for: $\mathrm{C}_{29} \mathrm{H}_{28} \mathrm{O}_{3}[\mathrm{M}]^{+}:$424.2033, found 424.2033.
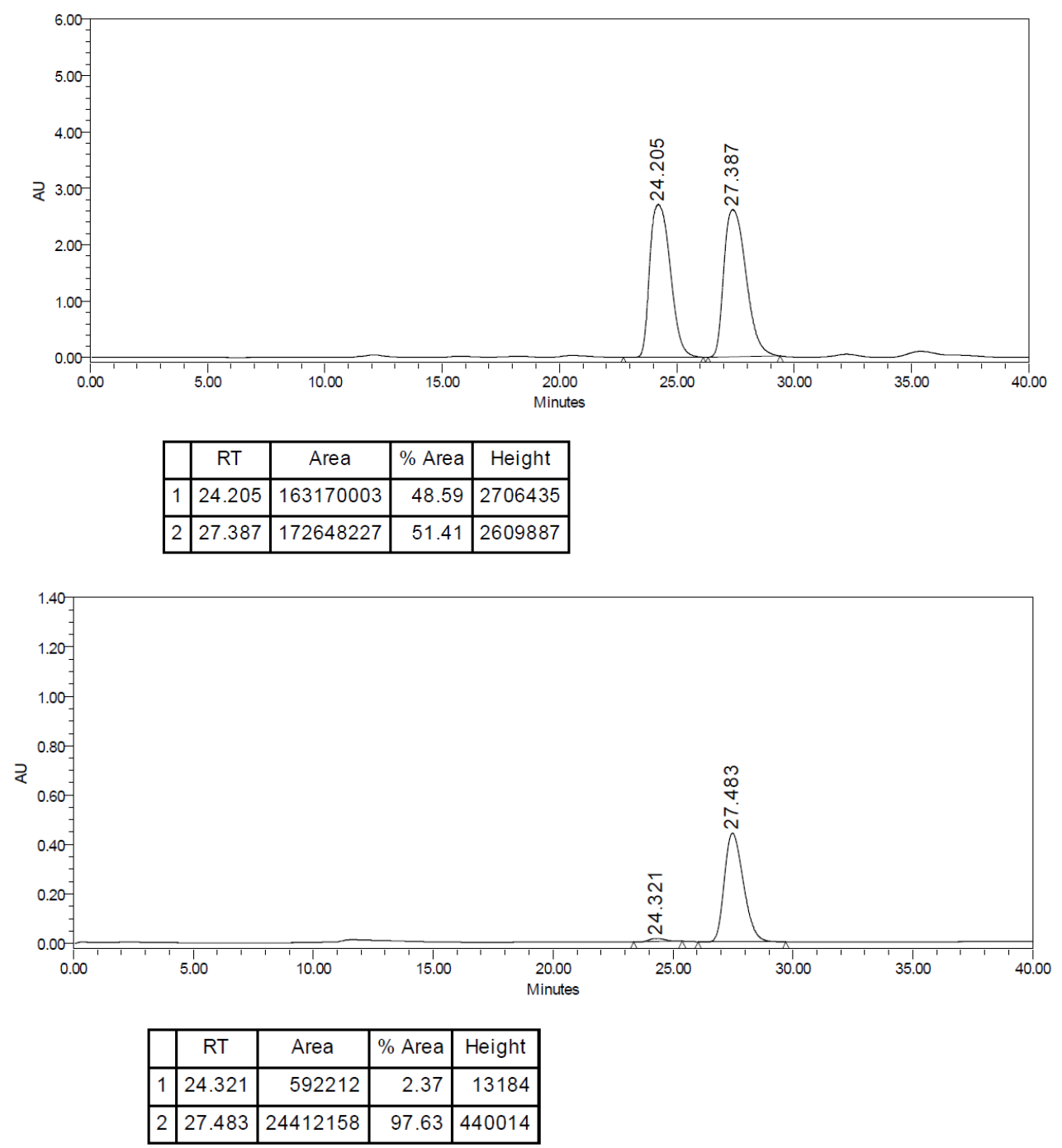


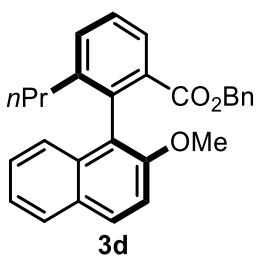

Colorless oil (60.1 mg, 73\% yield, 94\% ee, $168 \mathrm{~h}$ ); the enantiomeric excess was determined by CHIRALPAK IE column, hexanes $/ i$-PrOH, $97: 3 \mathrm{v} / \mathrm{v}, v=0.3 \mathrm{~mL} / \mathrm{min}, \lambda=254 \mathrm{~nm}, 25{ }^{\circ} \mathrm{C}, \mathrm{t}_{\mathrm{R}}$ (major) $=29.8 \mathrm{~min}$, $\mathrm{t}_{\mathrm{R}}($ minor $)=32.4 \mathrm{~min} ;[\alpha]_{\mathrm{D}}^{23}=+9.42\left(c=1.0, \mathrm{CHCl}_{3}\right)$.

3d: ${ }^{1} \mathrm{H}$ NMR $\left(400 \mathrm{MHz}, \mathrm{CHCl}_{3}\right) \delta 7.88(\mathrm{dd}, J=7.6,1.2 \mathrm{~Hz}, 1 \mathrm{H}), 7.80-7.77(\mathrm{~m}, 2 \mathrm{H})$, $7.52(\mathrm{dd}, J=8.0,1.2 \mathrm{~Hz}, 1 \mathrm{H}), 7.43(\mathrm{t}, J=7.6 \mathrm{~Hz}, 1 \mathrm{H}), 7.31-7.24(\mathrm{~m}, 2 \mathrm{H}), 7.22-7.13$ (m, 4H), $7.09(\mathrm{~d}, J=8.4 \mathrm{~Hz}, 1 \mathrm{H}), 6.81(\mathrm{~d}, J=6.8 \mathrm{~Hz}, 2 \mathrm{H}), 4.79\left(\mathrm{AB}, J_{A B}=12.4 \mathrm{~Hz}\right.$, $\left.J_{B A}=22.0 \mathrm{~Hz}, 2 \mathrm{H}\right), 3.69(\mathrm{~s}, 3 \mathrm{H}), 2.20-2.15(\mathrm{~m}, 2 \mathrm{H}), 1.40-1.22(\mathrm{~m}, 2 \mathrm{H}), 0.63(\mathrm{t}, J=$ 7.2 Hz, 2H); ${ }^{13} \mathrm{C}$ NMR (101 MHz, $\left.\mathrm{CHCl}_{3}\right) \delta 167.9,153.7,143.3,136.2,135.7,133.7$, $132.7,132.6,129.1,128.9,128.3,128.2,128.03,127.99,127.9,127.5,126.4,124.7$, 123.4, 122.7, 113.2, 66.5, 56.3, 35.4, 23.4, 14.0; FT-IR (neat): $v_{\max }=3060,2958,2933$, $2870,1726,1593,1578,1509,1455,1375,1333,1271,1254,1174,1135,1120,1097$, 1068, 969, 806, 746, 715, $696 \mathrm{~cm}^{-1}$; HRMS (ESI): exact mass calculated for: $\mathrm{C}_{28} \mathrm{H}_{26} \mathrm{O}_{3}$ $[\mathrm{M}]^{+}:$410.1876, found 410.1875 .

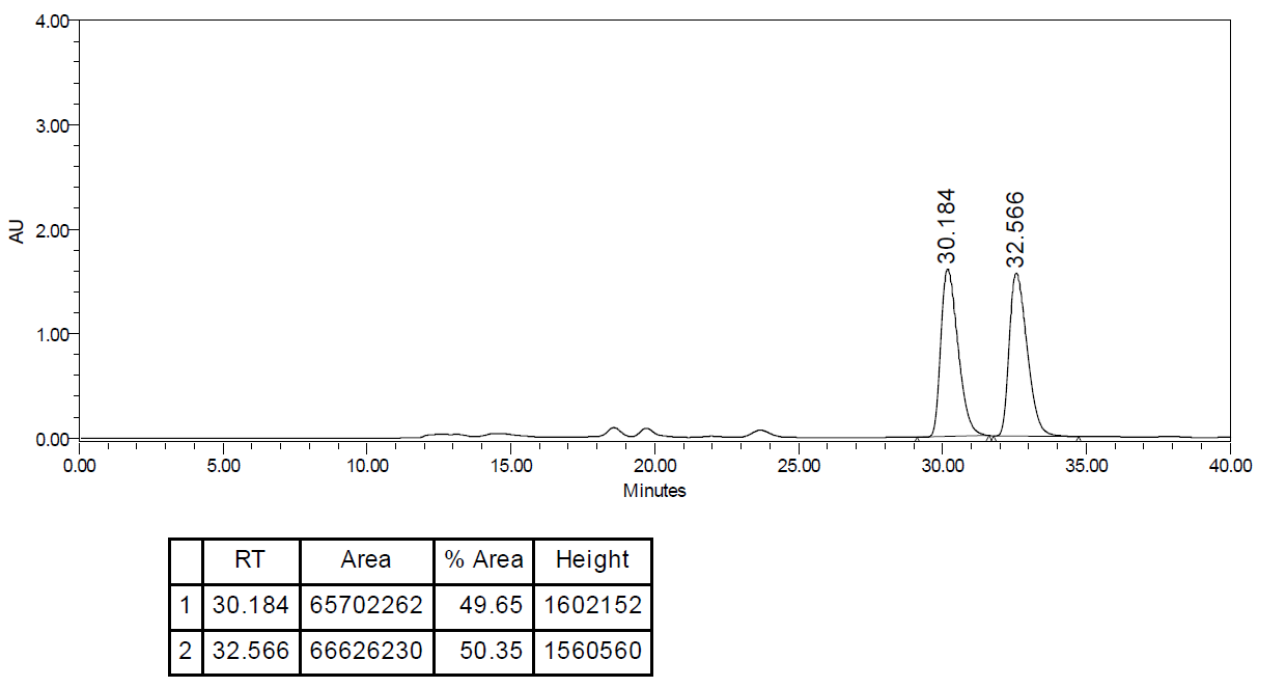



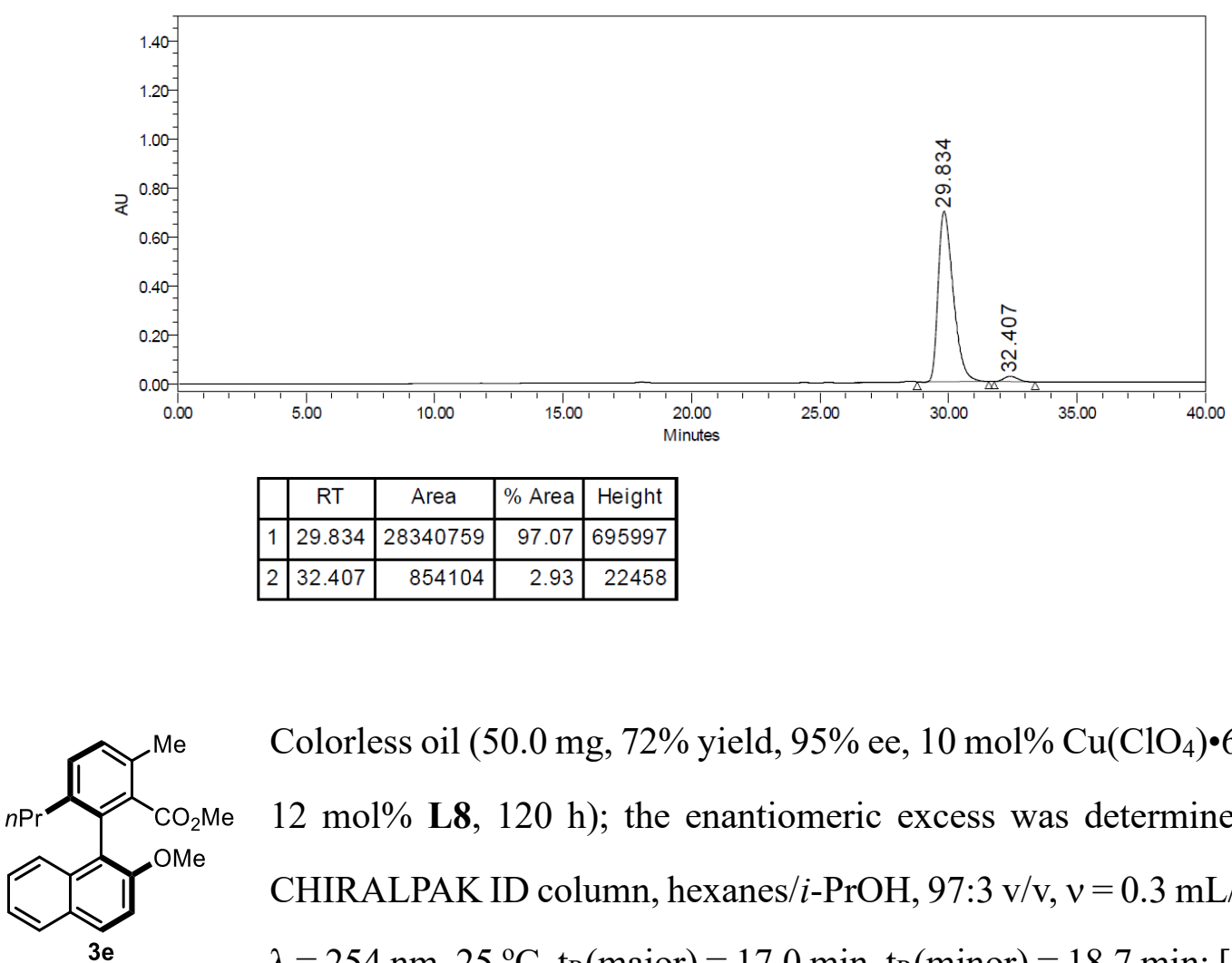

Colorless oil (50.0 mg, $72 \%$ yield, $95 \%$ ee, $10 \mathrm{~mol} \% \mathrm{Cu}\left(\mathrm{ClO}_{4}\right) \cdot 6 \mathrm{H}_{2} \mathrm{O}$, $12 \mathrm{~mol} \% \mathbf{L 8}, 120 \mathrm{~h}$ ); the enantiomeric excess was determined by CHIRALPAK ID column, hexanes $/ i$-PrOH, $97: 3 \mathrm{v} / \mathrm{v}, v=0.3 \mathrm{~mL} / \mathrm{min}$, $\lambda=254 \mathrm{~nm}, 25^{\circ} \mathrm{C}, \mathrm{t}_{\mathrm{R}}($ major $)=17.0 \mathrm{~min}, \mathrm{t}_{\mathrm{R}}($ minor $)=18.7 \mathrm{~min} ;[\alpha]_{\mathrm{D}}^{23}$ $=-39.76\left(c=1.0, \mathrm{CHCl}_{3}\right)$.

3e: ${ }^{1} \mathrm{H}$ NMR (400 MHz, $\left.\mathrm{CHCl}_{3}\right) \delta 7.86(\mathrm{~d}, J=9.2 \mathrm{~Hz}, 1 \mathrm{H}), 7.80-7.75(\mathrm{~m}, 1 \mathrm{H}), 7.33-$ $7.27(\mathrm{~m}, 4 \mathrm{H}), 7.25-7.18(\mathrm{~m}, 2 \mathrm{H}), 3.84(\mathrm{~s}, 3 \mathrm{H}), 3.13(\mathrm{~s}, 3 \mathrm{H}), 2.36(\mathrm{~s}, 3 \mathrm{H}), 2.17(\mathrm{td}, J=$ 8.0, $3.2 \mathrm{~Hz}, 2 \mathrm{H}), 1.40-1.30(\mathrm{~m}, 2 \mathrm{H}), 0.66(\mathrm{t}, J=7.2 \mathrm{~Hz}, 3 \mathrm{H}) ;{ }^{13} \mathrm{C} \mathrm{NMR}(101 \mathrm{MHz}$, $\left.\mathrm{CHCl}_{3}\right) \delta 167.0,154.4,139.9,135.5,133.8,133.6,132.4,130.1,129.60,129.57,128.8$, 127.7, 126.3, 125.4, 123.5, 121.9, 113.2, 56.6, 51.2, 35.2, 23.3, 19.7, 14.1; FT-IR (neat): $v_{\max }=3056,3004,2958,2932,2870,2839,1728,1593,1508,1464,1433,1333,1257$, 1236, 1146, 1122, 1074, 1020, 808, 747, $702 \mathrm{~cm}^{-1}$; HRMS (ESI): exact mass calculated for: $\mathrm{C}_{23} \mathrm{H}_{24} \mathrm{O}_{3}[\mathrm{M}]^{+}: 348.1720$, found 348.1722 

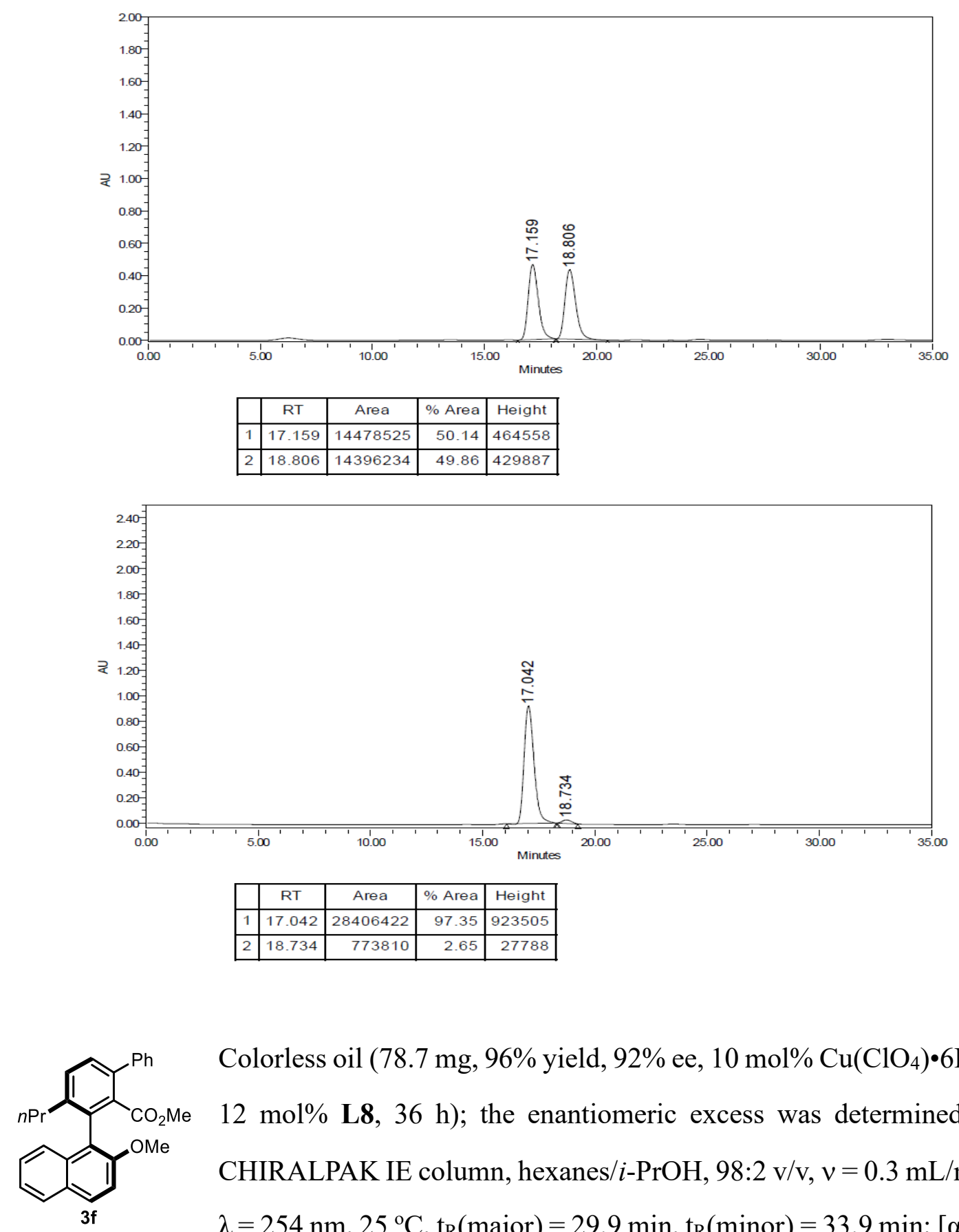

Colorless oil (78.7 mg, $96 \%$ yield, $92 \%$ ee, $10 \mathrm{~mol} \% \mathrm{Cu}\left(\mathrm{ClO}_{4}\right) \cdot 6 \mathrm{H}_{2} \mathrm{O}$, $12 \mathrm{~mol} \% \mathbf{L 8}, 36 \mathrm{~h}$ ); the enantiomeric excess was determined by CHIRALPAK IE column, hexanes $/ i-\mathrm{PrOH}, 98: 2 \mathrm{v} / \mathrm{v}, v=0.3 \mathrm{~mL} / \mathrm{min}$, $\lambda=254 \mathrm{~nm}, 25^{\circ} \mathrm{C}, \mathrm{t}_{\mathrm{R}}($ major $)=29.9 \mathrm{~min}, \mathrm{t}_{\mathrm{R}}($ minor $)=33.9 \mathrm{~min} ;[\alpha]_{\mathrm{D}}{ }^{24}$ $=-84.72\left(c=1.0, \mathrm{CHCl}_{3}\right)$.

3f: ${ }^{1} \mathrm{H}$ NMR $\left(400 \mathrm{MHz}, \mathrm{CHCl}_{3}\right) \delta 7.87(\mathrm{~d}, J=9.2 \mathrm{~Hz}, 1 \mathrm{H}), 7.79-7.76(\mathrm{~m}, 1 \mathrm{H}), 7.50-$ $7.42(\mathrm{~m}, 4 \mathrm{H}), 7.38-7.24(\mathrm{~m}, 7 \mathrm{H}), 3.85(\mathrm{~s}, 3 \mathrm{H}), 2.92(\mathrm{~s}, 3 \mathrm{H}), 2.24(\mathrm{td}, J=8.4,1.6 \mathrm{~Hz}$, 2H), $1.45-1.38(\mathrm{~m}, 2 \mathrm{H}), 0.70(\mathrm{t}, J=7.2 \mathrm{~Hz}, 3 \mathrm{H}) ;{ }^{13} \mathrm{C} \mathrm{NMR}\left(101 \mathrm{MHz}, \mathrm{CHCl}_{3}\right) \delta 169.7$, 154.6, 141.7, 140.9, 137.6, 134.8, 134.3, 133.8, 130.1, 129.8, 129.1, 128.8, 128.6, 128.3, $127.7,127.3,126.4,125.4,123.5,121.6,113.4,56.7,51.2,35.3,23.3,14.2$; FT-IR 
(neat): $v_{\max }=3058,2958,2933,2870,2838,1728,1593,1509,1463,1432,1263,1234$, 1146, 1124, 1096, 1062, 1020, 809, 734, $699 \mathrm{~cm}^{-1}$; HRMS (EI): exact mass calculated for: $\mathrm{C}_{28} \mathrm{H}_{26} \mathrm{O}_{3}[\mathrm{M}]^{+}: 410.1876$, found 410.1878
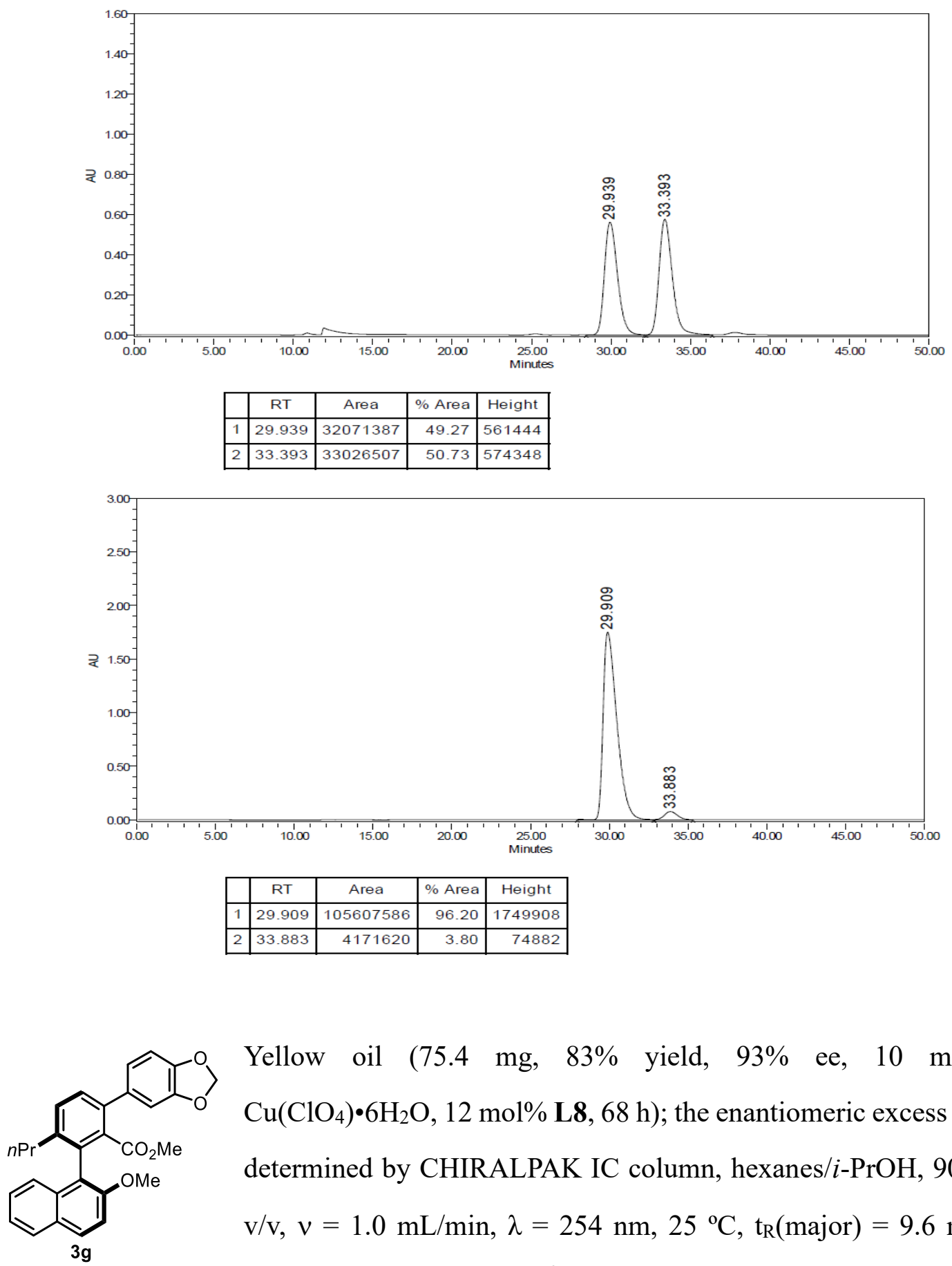

Yellow oil $(75.4 \mathrm{mg}, \quad 83 \%$ yield, $93 \%$ ee, $10 \quad \mathrm{~mol} \%$ $\left.\mathrm{Cu}\left(\mathrm{ClO}_{4}\right) \cdot 6 \mathrm{H}_{2} \mathrm{O}, 12 \mathrm{~mol} \% \mathbf{L 8}, 68 \mathrm{~h}\right)$; the enantiomeric excess was determined by CHIRALPAK IC column, hexanes/i-PrOH, 90:10 $\mathrm{v} / \mathrm{v}, v=1.0 \mathrm{~mL} / \mathrm{min}, \lambda=254 \mathrm{~nm}, 25{ }^{\circ} \mathrm{C}, \mathrm{t}_{\mathrm{R}}$ (major) $=9.6 \mathrm{~min}$, $\mathrm{t}_{\mathrm{R}}($ minor $)=10.7 \mathrm{~min} ;[\alpha]_{\mathrm{D}}^{25}=-73.62\left(c=1.0, \mathrm{CHCl}_{3}\right)$. 
3g: ${ }^{1} \mathrm{H}$ NMR $\left(400 \mathrm{MHz}, \mathrm{CHCl}_{3}\right) \delta 7.86(\mathrm{~d}, J=8.8 \mathrm{~Hz}, 1 \mathrm{H}), 7.78-7.76(\mathrm{~m}, 1 \mathrm{H}), 7.45$ $(\mathrm{d}, J=8.0 \mathrm{~Hz}, 1 \mathrm{H}), 7.38(\mathrm{~d}, J=8.0 \mathrm{~Hz}, 1 \mathrm{H}), 7.34-7.23(\mathrm{~m}, 4 \mathrm{H}), 6.96(\mathrm{~d}, J=1.2 \mathrm{~Hz}$, 1H), $6.91(\mathrm{dd}, J=8.0,1.6 \mathrm{~Hz}, 1 \mathrm{H}), 6.79$ (d, $J=8.0 \mathrm{~Hz}, 1 \mathrm{H}), 5.94(\mathrm{~s}, 2 \mathrm{H}), 3.84(\mathrm{~s}, 3 \mathrm{H})$, $2.96(\mathrm{~s}, 3 \mathrm{H}), 2.22(\mathrm{t}, J=7.2 \mathrm{~Hz}, 2 \mathrm{H}), 1.45-1.33(\mathrm{~m}, 2 \mathrm{H}), 0.69(\mathrm{t}, J=7.6 \mathrm{~Hz}, 3 \mathrm{H}) ;{ }^{13} \mathrm{C}$ NMR (101 MHz, $\left.\mathrm{CHCl}_{3}\right) \delta 169.7,154.5,147.7,147.0,141.5,137.1,134.9,134.8,134.2$, 133.8, 130.0, 129.7, 129.0, 128.7, 127.7, 126.4, 125.4, 123.5, 122.2, 121.5, 113.4, 109.2, 108.2, 101.2, 56.6, 51.2, 35.3, 23.3, 14.1; FT-IR (neat): $v_{\max }=2960,2915,2879,1727$, 1594, 1509, 1464, 1433, 1332, 1256, 1226, 1145, 1122, 1107, 1074, 1036, 935, 806, 728, 703, $613 \mathrm{~cm}^{-1}$; HRMS (EI): exact mass calculated for: $\mathrm{C}_{29} \mathrm{H}_{26} \mathrm{O}_{5}[\mathrm{M}]^{+}: 454.1775$, found 454.1775 .
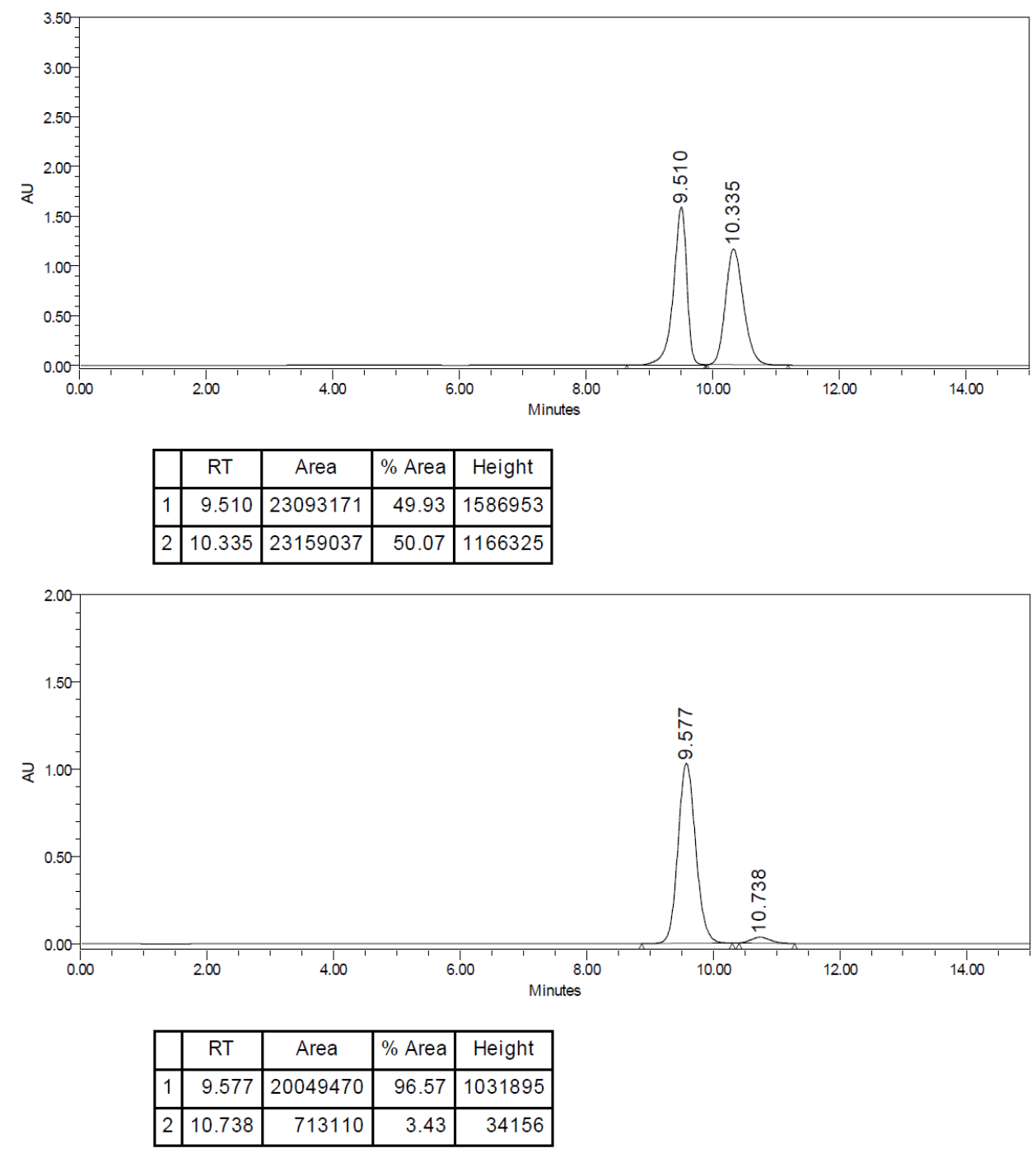


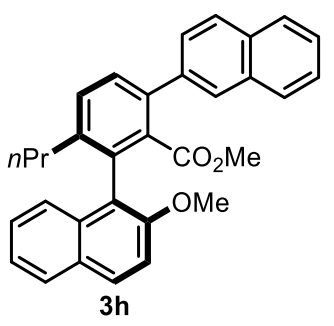

Yellow oil $(86.0 \mathrm{mg}, 93 \%$ yield, $93 \%$ ee, $10 \mathrm{~mol} \%$ $\left.\mathrm{Cu}\left(\mathrm{ClO}_{4}\right) \cdot 6 \mathrm{H}_{2} \mathrm{O}, 12 \mathrm{~mol} \% \mathbf{L 8}, 48 \mathrm{~h}\right)$; the enantiomeric excess was determined by CHIRALPAK IC column, hexanes/i-PrOH, 90:10 $\mathrm{v} / \mathrm{v}, \mathrm{v}=1.0 \mathrm{~mL} / \mathrm{min}, \lambda=254 \mathrm{~nm}, 25{ }^{\circ} \mathrm{C}, \mathrm{t}_{\mathrm{R}}$ (major) $=5.9 \mathrm{~min}$, $\mathrm{t}_{\mathrm{R}}($ minor $)=7.0 \mathrm{~min} ;[\alpha]_{\mathrm{D}}{ }^{26}=-90.52\left(c=1.0, \mathrm{CHCl}_{3}\right)$.

3h: ${ }^{1} \mathrm{H}$ NMR (400 MHz, $\left.\mathrm{CHCl}_{3}\right) \delta 7.94(\mathrm{~d}, J=1.2 \mathrm{~Hz}, 1 \mathrm{H}), 7.88(\mathrm{~d}, J=9.2 \mathrm{~Hz}, 1 \mathrm{H})$, $7.84-7.77(\mathrm{~m}, 4 \mathrm{H}), 7.60(\mathrm{dd}, J=8.4,1.6 \mathrm{~Hz}, 1 \mathrm{H}), 7.53(\mathrm{dd}, J=10.0,8.0 \mathrm{~Hz}, 1 \mathrm{H}), 7.48$ - 7.43 (m, 2H), $7.36-7.28$ (m, 4H), 3.87 (s, 3H), 2.90 (s, 3H), 2.27 (td, J= 8.4, 2.0 Hz, 2H), $1.48-1.38(\mathrm{~m}, 2 \mathrm{H}), 0.72(\mathrm{t}, J=7.2 \mathrm{~Hz}, 3 \mathrm{H}),{ }^{13} \mathrm{C} \mathrm{NMR}\left(101 \mathrm{MHz}, \mathrm{CHCl}_{3}\right) \delta 169.7$, 154.6, 141.8, 138.4, 137.5, 135.0, 134.4, 133.8, 133.5, 132.6, 130.2, 129.8, 129.4, 128.8, $128.3,127.9,127.8,127.7,127.4,127.0,126.5,126.3,126.0,125.4,123.6,121.5,113.4$ 56.7, 51.3, 35.4, 23.3, 14.2; FT-IR (neat): $v_{\max }=3053,2966,2932,2346,1728,1593$, 1507, 1463, 1431, 1255, 1235, 1146, 1121, 1092, 1020, 809, 745, $635 \mathrm{~cm}^{-1}$; HRMS (EI): exact mass calculated for: $\mathrm{C}_{32} \mathrm{H}_{28} \mathrm{O}_{3}[\mathrm{M}]^{+}:$460.2033, found 460.2035.

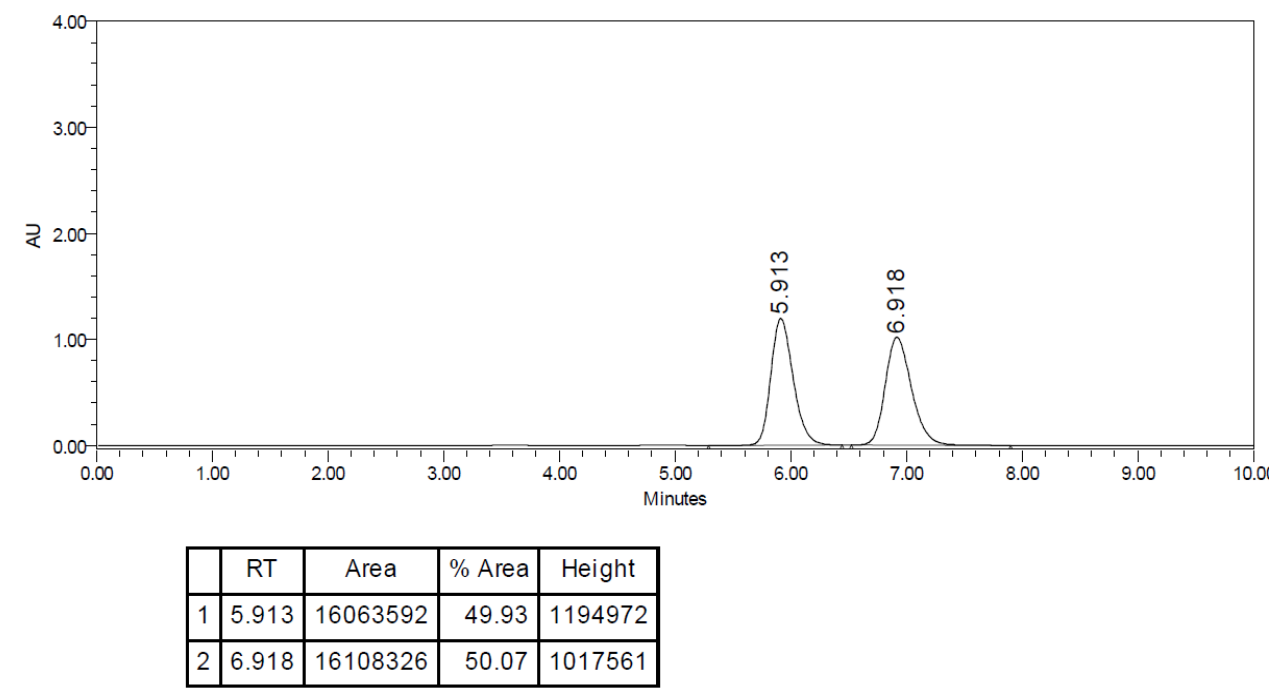



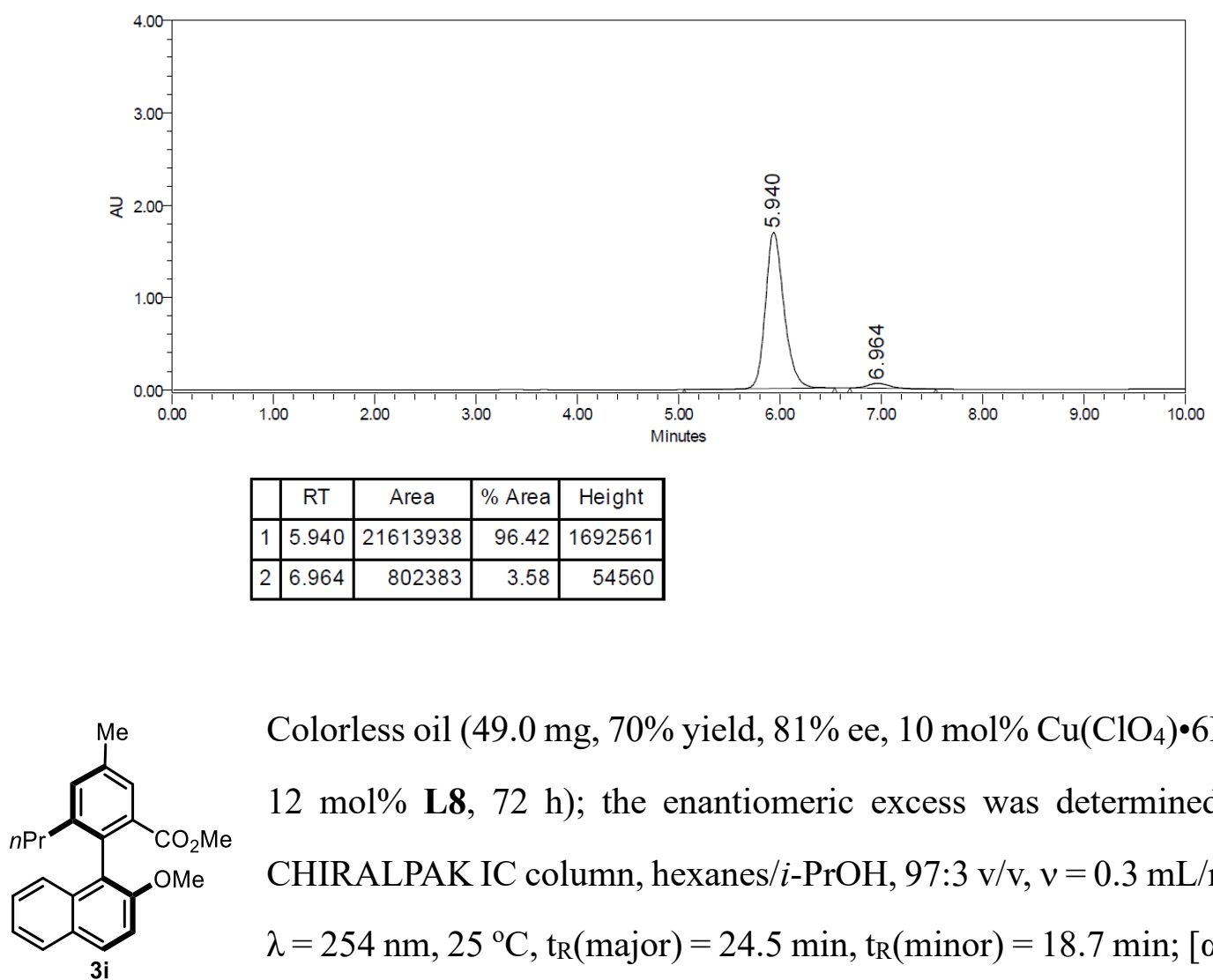

Colorless oil (49.0 mg, $70 \%$ yield, $81 \%$ ee, $10 \mathrm{~mol} \% \mathrm{Cu}\left(\mathrm{ClO}_{4}\right) \cdot 6 \mathrm{H}_{2} \mathrm{O}$, $12 \mathrm{~mol} \% \mathbf{L 8}, 72 \mathrm{~h}$ ); the enantiomeric excess was determined by CHIRALPAK IC column, hexanes $/ i-\mathrm{PrOH}, 97: 3 \mathrm{v} / \mathrm{v}, v=0.3 \mathrm{~mL} / \mathrm{min}$, $\lambda=254 \mathrm{~nm}, 25^{\circ} \mathrm{C}, \mathrm{t}_{\mathrm{R}}($ major $)=24.5 \mathrm{~min}, \mathrm{t}_{\mathrm{R}}($ minor $)=18.7 \mathrm{~min} ;[\alpha]_{\mathrm{D}}{ }^{23}$ $=+13.26\left(c=1.0, \mathrm{CHCl}_{3}\right)$.

3i: ${ }^{1} \mathrm{H}$ NMR (400 MHz, $\left.\mathrm{CHCl}_{3}\right) \delta 7.87(\mathrm{~d}, J=8.8 \mathrm{~Hz}, 1 \mathrm{H}), 7.80-7.78(\mathrm{~m}, 1 \mathrm{H}), 7.68$ (s, 1H), $7.35(\mathrm{~s}, 1 \mathrm{H}), 7.34(\mathrm{~d}, J=8.8 \mathrm{~Hz}, 1 \mathrm{H}), 7.30-7.22(\mathrm{~m}, 2 \mathrm{H}), 7.10(\mathrm{~d}, J=8.0 \mathrm{~Hz}$, 1H), $3.81(\mathrm{~s}, 3 \mathrm{H}), 3.35(\mathrm{~s}, 3 \mathrm{H}), 2.46(\mathrm{~s}, 3 \mathrm{H}), 2.19-2.14(\mathrm{~m}, 2 \mathrm{H}), 1.38-1.27(\mathrm{~m}, 2 \mathrm{H})$, $0.64(\mathrm{t}, J=7.2 \mathrm{~Hz}, 3 \mathrm{H}) ;{ }^{13} \mathrm{C}$ NMR $\left(101 \mathrm{MHz}, \mathrm{CHCl}_{3}\right) \delta 168.3,153.9,143.1,137.1$, $133.8,133.6,133.5,132.3,129.0,128.9,128.5,128.0,126.3,124.7,123.4,123.0,113.3$, 56.5, 51.7, 35.4, 23.5, 21.4, 14.1; FT-IR (neat): $v_{\max }=2955,2931,2870,1725,1593$, $1510,1463,1433,1305,1255,1241,1194,1138,1068,1018,807,792,750 \mathrm{~cm}^{-1}$; HRMS (EI): exact mass calculated for: $\mathrm{C}_{23} \mathrm{H}_{24} \mathrm{O}_{3}[\mathrm{M}]^{+}: 348.1720$, found 348.1721. 

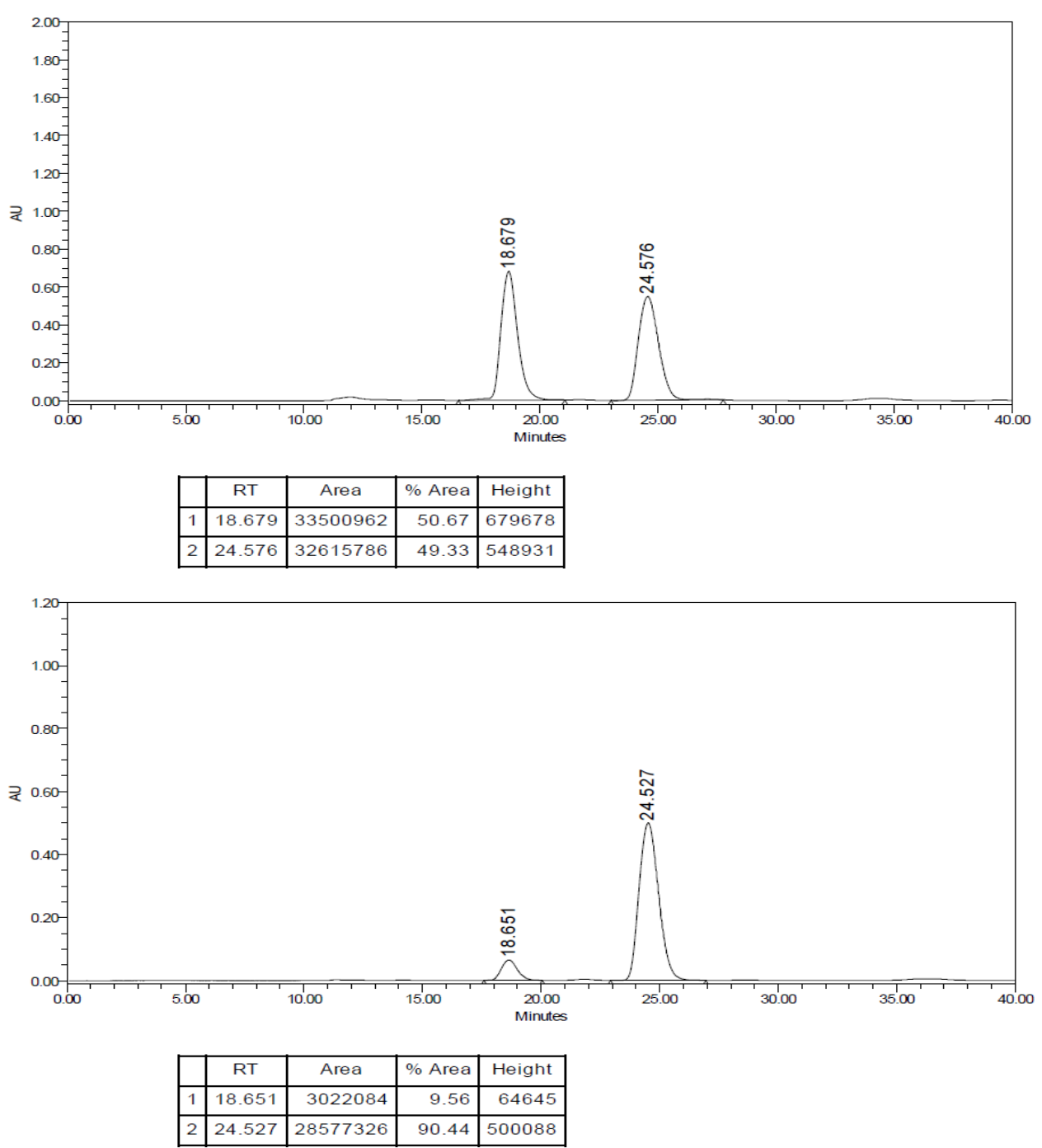

Colorless oil (67.4 mg, $82 \%$ yield, $82 \%$ ee, $10 \mathrm{~mol} \% \mathrm{Cu}\left(\mathrm{ClO}_{4}\right) \cdot 6 \mathrm{H}_{2} \mathrm{O}$, $12 \mathrm{~mol} \% \mathbf{L 8}, 72 \mathrm{~h}$ ); the enantiomeric excess was determined by CHIRALPAK IC column, hexanes $/ i-\mathrm{PrOH}, 97: 3 \mathrm{v} / \mathrm{v}, v=0.3 \mathrm{~mL} / \mathrm{min}$, $\lambda=254 \mathrm{~nm}, 25^{\circ} \mathrm{C}, \mathrm{t}_{\mathrm{R}}($ major $)=28.1 \mathrm{~min}, \mathrm{t}_{\mathrm{R}}($ minor $)=23.7 \mathrm{~min} ;[\alpha]_{\mathrm{D}}{ }^{24}$ $=+13.54\left(c=1.0, \mathrm{CHCl}_{3}\right)$.

3j: ${ }^{1} \mathrm{H}$ NMR (400 MHz, $\left.\mathrm{CHCl}_{3}\right) \delta 8.10(\mathrm{~d}, J=2.0 \mathrm{~Hz}, 1 \mathrm{H}), 7.90(\mathrm{~d}, J=9.2 \mathrm{~Hz}, 1 \mathrm{H})$, $7.82(\mathrm{dd}, J=6.4,2.4 \mathrm{~Hz}, 1 \mathrm{H}), 7.76(\mathrm{~d}, J=1.6 \mathrm{~Hz}, 1 \mathrm{H}), 7.74-7.71(\mathrm{~m}, 2 \mathrm{H}), 7.49(\mathrm{t}, J$ $=7.6 \mathrm{~Hz}, 2 \mathrm{H}), 7.41-7.36(\mathrm{~m}, 2 \mathrm{H}), 7.32-7.24(\mathrm{~m}, 2 \mathrm{H}), 7.18-7.16(\mathrm{~m}, 1 \mathrm{H}), 3.84(\mathrm{~s}$, $3 \mathrm{H}), 3.39(\mathrm{~s}, 3 \mathrm{H}), 2.30-2.25(\mathrm{~m}, 2 \mathrm{H}), 1.46-1.32(\mathrm{~m}, 2 \mathrm{H}), 0.68(\mathrm{t}, J=7.2 \mathrm{~Hz}, 3 \mathrm{H})$; ${ }^{13} \mathrm{C}$ NMR $\left(101 \mathrm{MHz}, \mathrm{CHCl}_{3}\right) \delta 168.1,153.8,143.8,140.5,140.3,135.5,133.7,132.8$, 
$131.3,129.2,129.0,128.9,128.1,127.7,127.3,126.6,126.5,124.7,123.5,122.6,113.3$, 56.5, 51.8, 35.6, 23.5, 14.1; FT-IR (neat): $v_{\max }=3058,2958,2933,2870,2838,1727$, $1593,1510,1461,1452,1433,1329,1254,1230,1144,1069,1020,807,735,697 \mathrm{~cm}^{-1}$; HRMS (EI): exact mass calculated for: $\mathrm{C}_{28} \mathrm{H}_{26} \mathrm{O}_{3}[\mathrm{M}]^{+}: 410.1876$, found 410.1880 .
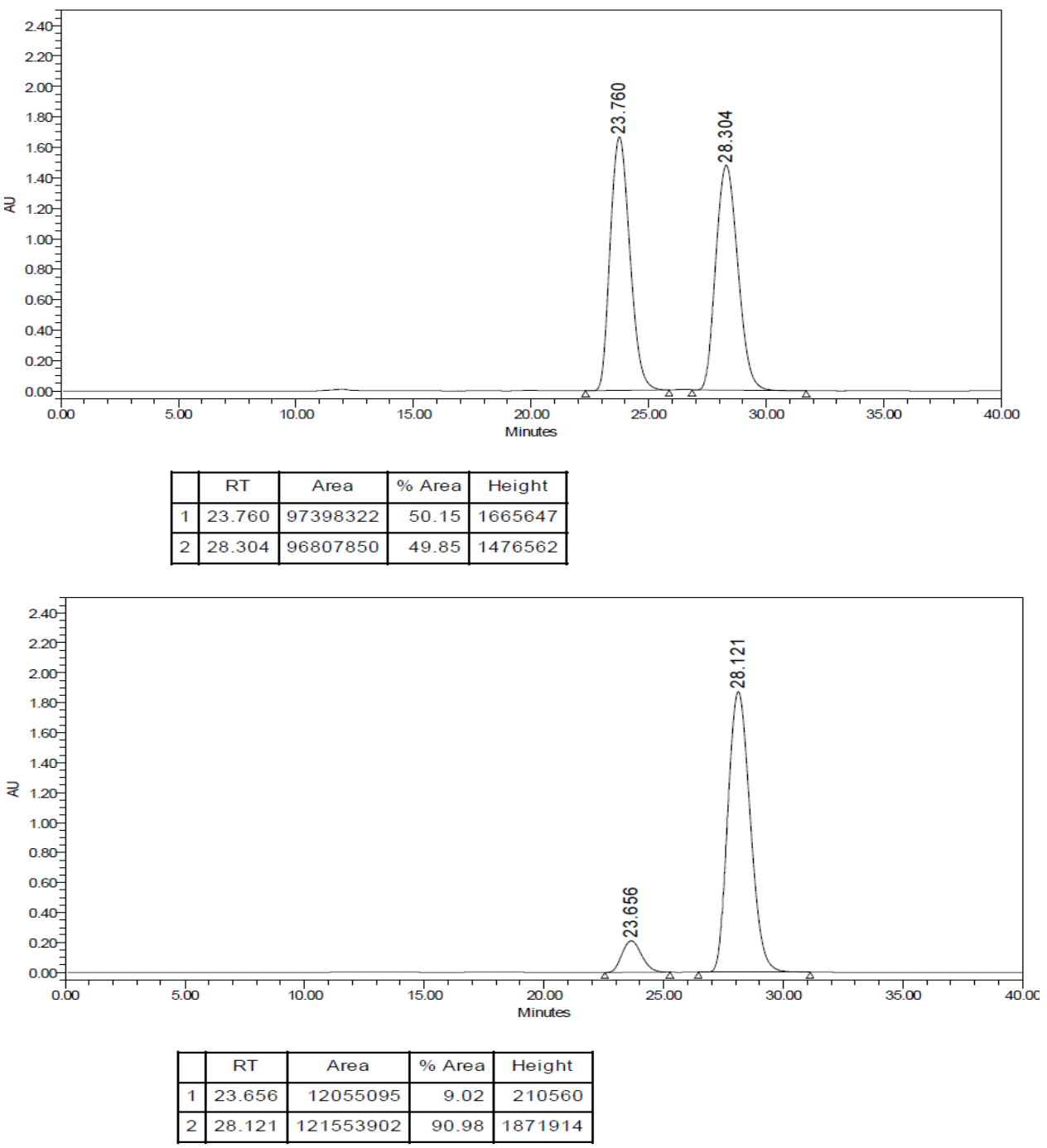

Colorless oil (54.7 mg, $66 \%$ yield, $72 \%$ ee, $10 \mathrm{~mol} \% \mathrm{Cu}\left(\mathrm{ClO}_{4}\right) \cdot 6 \mathrm{H}_{2} \mathrm{O}$, $12 \mathrm{~mol} \% \mathbf{L 8}, 36 \mathrm{~h}$ ); the enantiomeric excess was determined by CHIRALPAK IE column, hexanes $/ i-\mathrm{PrOH}, 97: 3 \mathrm{v} / \mathrm{v}, v=0.3 \mathrm{~mL} / \mathrm{min}$, $\lambda=250 \mathrm{~nm}, 25^{\circ} \mathrm{C}, \mathrm{t}_{\mathrm{R}}($ major $)=21.0 \mathrm{~min}, \mathrm{t}_{\mathrm{R}}($ minor $)=22.8 \mathrm{~min} ;[\alpha]_{\mathrm{D}}{ }^{24}$ $=+13.34\left(c=1.0, \mathrm{CHCl}_{3}\right)$. 
3k: ${ }^{1} \mathrm{H}$ NMR $\left(400 \mathrm{MHz}, \mathrm{CHCl}_{3}\right) \delta 8.00(\mathrm{~d}, J=2.0 \mathrm{~Hz}, 1 \mathrm{H}), 7.89(\mathrm{~d}, J=9.2 \mathrm{~Hz}, 1 \mathrm{H})$, $7.82-7.79(\mathrm{~m}, 1 \mathrm{H}), 7.67(\mathrm{~d}, J=2.4 \mathrm{~Hz}, 1 \mathrm{H}), 7.34(\mathrm{~d}, J=9.2 \mathrm{~Hz}, 1 \mathrm{H}), 7.30-7.25(\mathrm{~m}$, 2H), $7.08-7.06(\mathrm{~m}, 1 \mathrm{H}), 3.81(\mathrm{~s}, 3 \mathrm{H}), 3.38(\mathrm{~s}, 3 \mathrm{H}), 2.19-2.15(\mathrm{~m}, 2 \mathrm{H}), 1.40-1.26$ $(\mathrm{m}, 2 \mathrm{H}), 0.65$ (t, $J=7.6 \mathrm{~Hz}, 3 \mathrm{H}) ;{ }^{13} \mathrm{C} \mathrm{NMR}\left(101 \mathrm{MHz}, \mathrm{CHCl}_{3}\right) \delta 166.6,153.7,145.8$, 135.7, 135.4, 134.0, 133.4, 130.8, 129.5, 128.9, 128.1, 126.6, 124.3, 123.6, 121.6, 121.4, 113.1, 56.4, 52.0, 35.2, 23.2, 14.0; FT-IR (neat): $v_{\max }=2956,2931,2870,2836,1730$, $1593,1570,1509,1453,1433,1270,1254,1226,1145,1067,807,749,736 \mathrm{~cm}^{-1}$; HRMS (ESI): exact mass calculated for: $\mathrm{C}_{22} \mathrm{H}_{21} \mathrm{BrO}_{3}[\mathrm{M}]^{+}: 412.0669$, found 412.0664 .
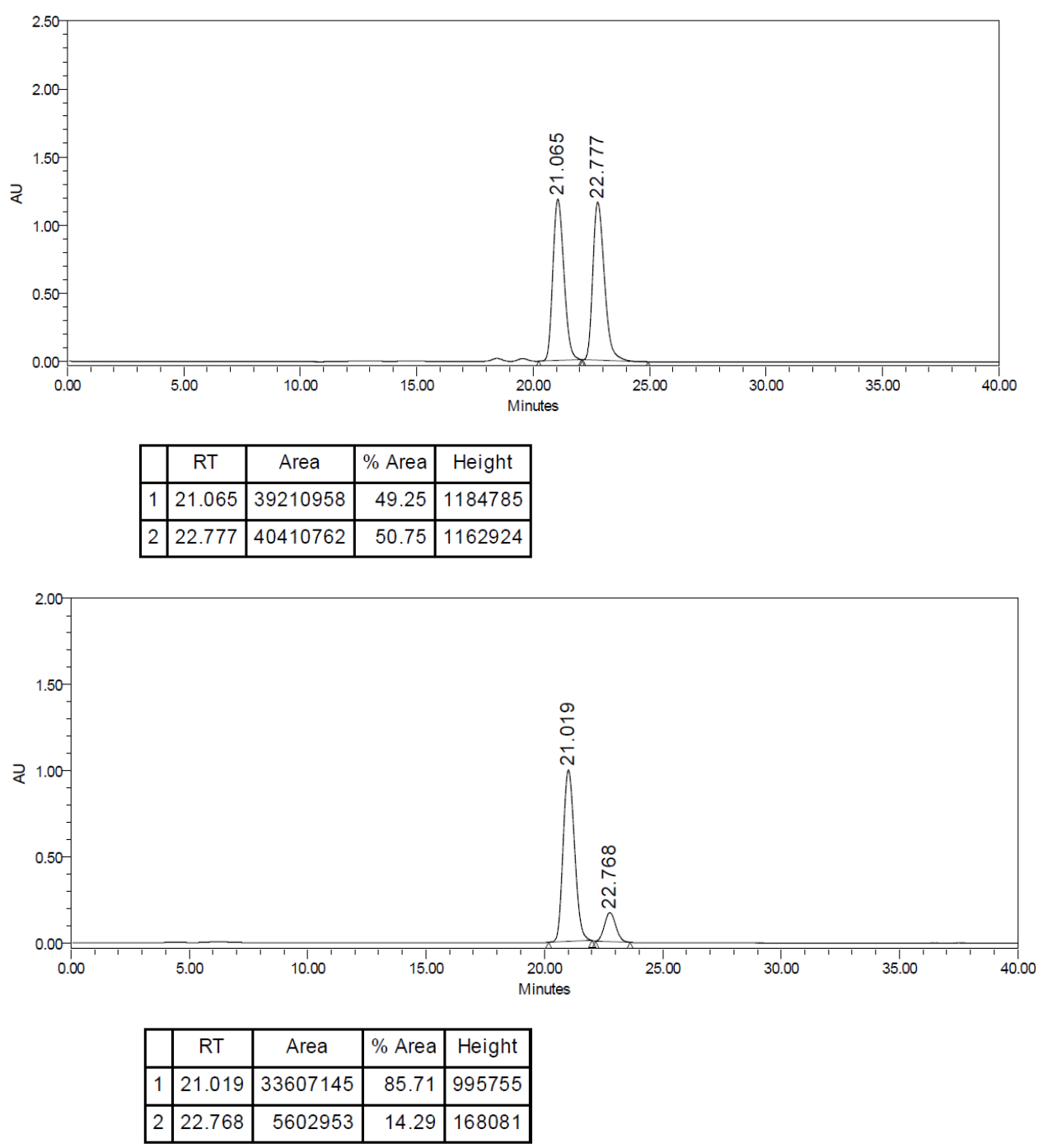


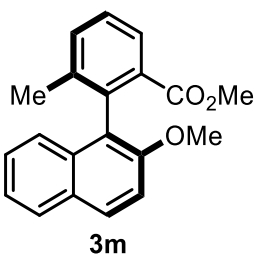

Colorless oil (51.3 mg, 84\% yield, 95\% ee, $72 \mathrm{~h}$ ); the enantiomeric excess was determined by CHIRALPAK IC column, hexanes/ $i-\mathrm{PrOH}$, $97: 3 \mathrm{v} / \mathrm{v}, v=0.3 \mathrm{~mL} / \mathrm{min}, \lambda=250 \mathrm{~nm}, 25^{\circ} \mathrm{C}, \mathrm{t}_{\mathrm{R}}($ major $)=29.9 \mathrm{~min}$, $\mathrm{t}_{\mathrm{R}}($ minor $)=25.3 \mathrm{~min} ;[\alpha]_{\mathrm{D}}^{24}=-2.18\left(c=1.0, \mathrm{CHCl}_{3}\right)$.

3m: ${ }^{1} \mathrm{H}$ NMR (400 MHz, $\left.\mathrm{CHCl}_{3}\right) \delta 7.88(\mathrm{~d}, J=8.8 \mathrm{~Hz}, 2 \mathrm{H}), 7.81(\mathrm{dd}, J=7.2,1.6 \mathrm{~Hz}$, $1 \mathrm{H}), 7.51(\mathrm{~d}, J=7.2 \mathrm{~Hz}, 1 \mathrm{H}), 7.40(\mathrm{t}, J=7.6 \mathrm{~Hz}, 1 \mathrm{H}), 7.36(\mathrm{~d}, J=8.8 \mathrm{~Hz}, 1 \mathrm{H}), 7.32-$ $7.24(\mathrm{~m}, 2 \mathrm{H}), 7.10-7.07(\mathrm{~m}, 1 \mathrm{H}), 3.82(\mathrm{~s}, 3 \mathrm{H}), 3.38(\mathrm{~s}, 3 \mathrm{H}), 1.93(\mathrm{~s}, 3 \mathrm{H}) ;{ }^{13} \mathrm{C} \mathrm{NMR}$ $\left(101 \mathrm{MHz}, \mathrm{CHCl}_{3}\right) \delta 168.0,153.4,139.0,137.1,133.7,133.1,132.1,129.10,129.06$, 128.1, 127.9, 127.5, 126.5, 124.3, 123.5, 123.3, 113.6, 56.7, 51.7, 20.2; FT-IR (neat): $v_{\max }=3057,2997,2948,2838,1621,1593,1508,1458,1432,1377,1333,1291,1211$, 1190, 1174, 1138, 1121, 1089, 1065, 1019, 906, 877, 807, 748, 101, $638 \mathrm{~cm}^{-1}$; HRMS (ESI): exact mass calculated for: $\mathrm{C}_{20} \mathrm{H}_{18} \mathrm{O}_{3}[\mathrm{M}]^{+}: 306.1250$, found 306.1252 .

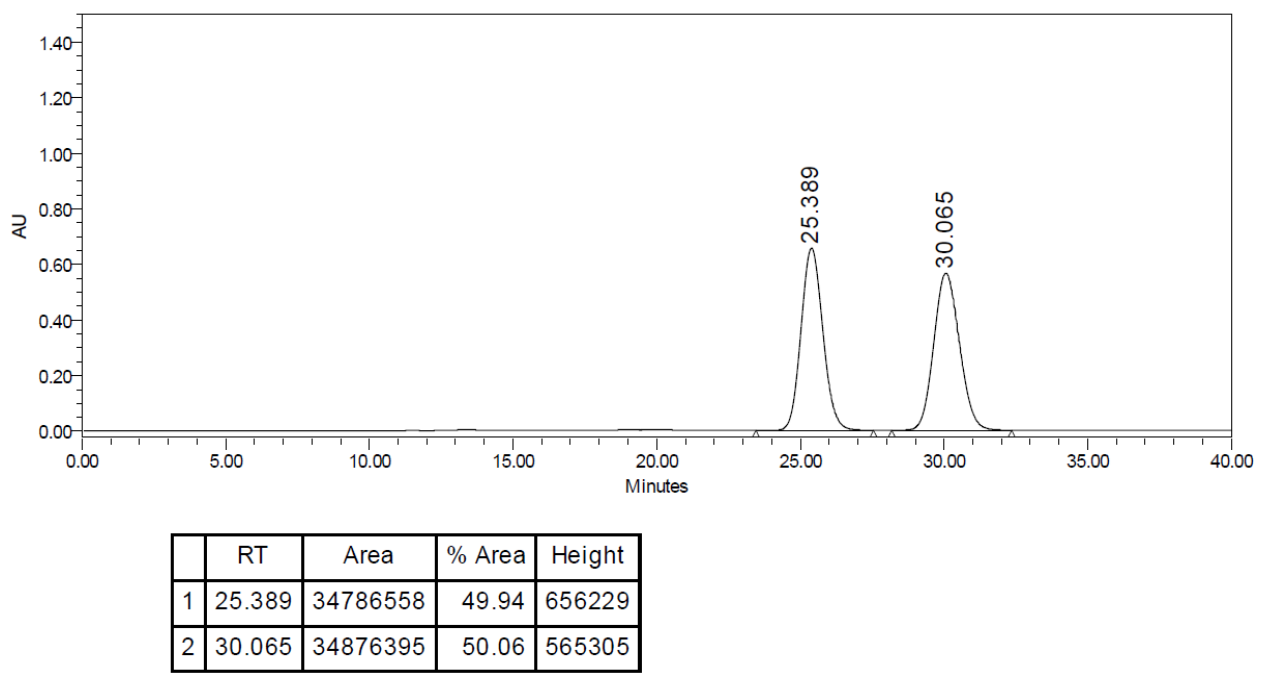



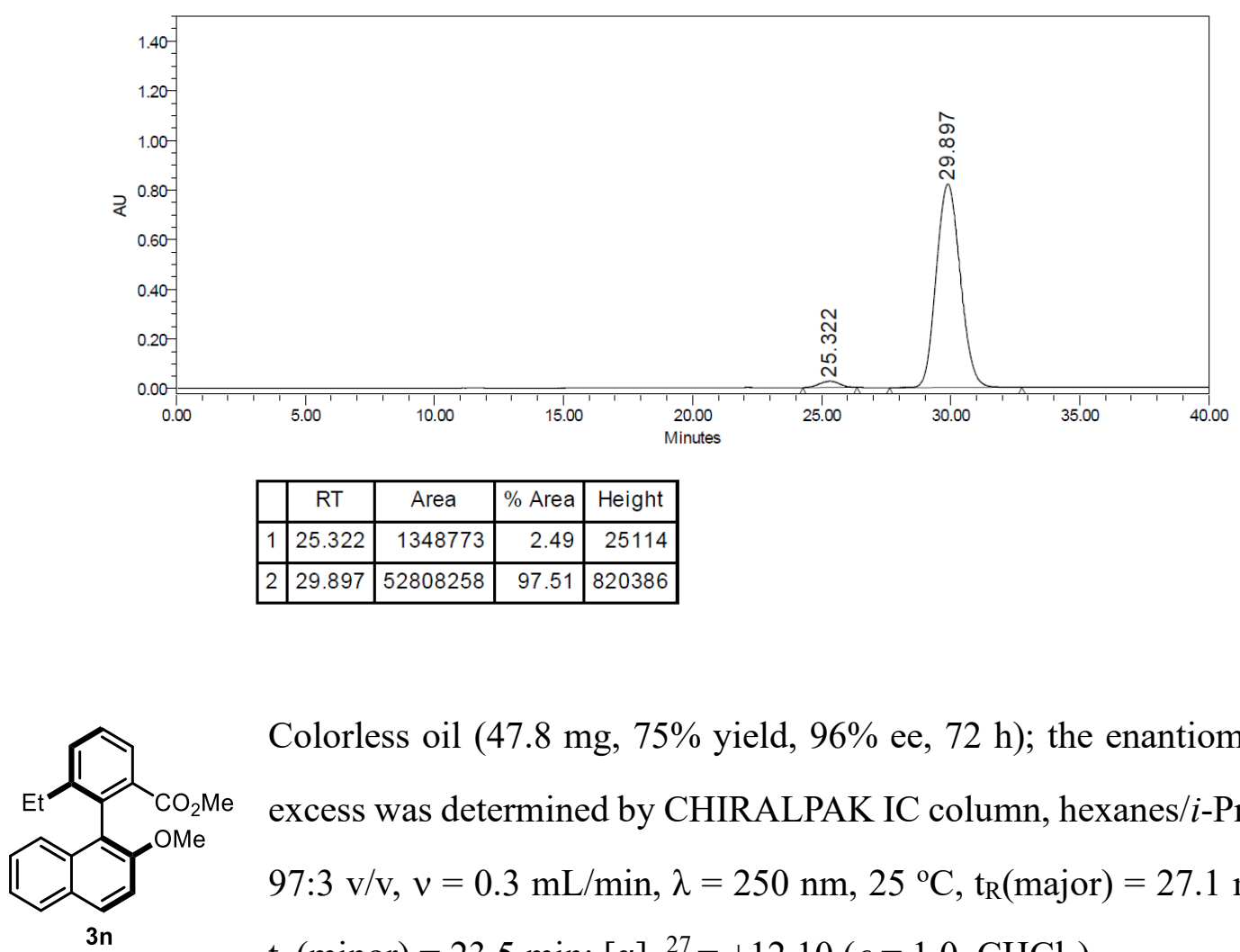

Colorless oil (47.8 mg, $75 \%$ yield, $96 \%$ ee, $72 \mathrm{~h}$ ); the enantiomeric excess was determined by CHIRALPAK IC column, hexanes $/ i$-PrOH, $97: 3 \mathrm{v} / \mathrm{v}, v=0.3 \mathrm{~mL} / \mathrm{min}, \lambda=250 \mathrm{~nm}, 25^{\circ} \mathrm{C}, \mathrm{t}_{\mathrm{R}}$ (major) $=27.1 \mathrm{~min}$, $\mathrm{t}_{\mathrm{R}}($ minor $)=23.5 \mathrm{~min} ;[\alpha]_{\mathrm{D}}^{27}=+12.10\left(c=1.0, \mathrm{CHCl}_{3}\right)$.

3n: ${ }^{1} \mathrm{H}$ NMR (400 MHz, $\left.\mathrm{CHCl}_{3}\right) \delta 7.90-7.86(\mathrm{~m}, 2 \mathrm{H}), 7.80(\mathrm{dd}, J=7.2,1.6 \mathrm{~Hz}, 1 \mathrm{H})$, $7.56(\mathrm{dd}, J=7.6,1.2 \mathrm{~Hz}, 1 \mathrm{H}), 7.46$ (t, $J=7.6 \mathrm{~Hz}, 1 \mathrm{H}), 7.35$ (d, $J=9.2 \mathrm{~Hz}, 1 \mathrm{H}), 7.31-$ $7.24(\mathrm{~m}, 2 \mathrm{H}), 7.09-7.07(\mathrm{~m}, 1 \mathrm{H}), 3.82(\mathrm{~s}, 3 \mathrm{H}), 3.37$ (s, 3H), $2.24(\mathrm{q}, J=7.6 \mathrm{~Hz}, 2 \mathrm{H})$, $0.93(\mathrm{t}, J=7.6 \mathrm{~Hz}, 3 \mathrm{H}) ;{ }^{13} \mathrm{C} \mathrm{NMR}\left(101 \mathrm{MHz}, \mathrm{CHCl}_{3}\right) \delta 168.0,153.7,144.7,136.3$, $133.6,132.2,131.9,129.1,128.9,128.0,127.8,127.7,126.4,124.6,123.5,122.9,113.4$, 56.6, 51.7, 26.4, 14.8; FT-IR (neat): $v_{\max }=3060,3002,2948,2837,1728,1621,1593$, $1509,1464,1431,1290,1270,1248,1199,1132,1094,1065,1020,993,908,807,762$, 747, 735, 700, 681, $638 \mathrm{~cm}^{-1}$; HRMS (ESI): exact mass calculated for: $\mathrm{C}_{21} \mathrm{H}_{20} \mathrm{O}_{3}[\mathrm{M}]^{+}$: 320.1407 , found 320.1407 . 


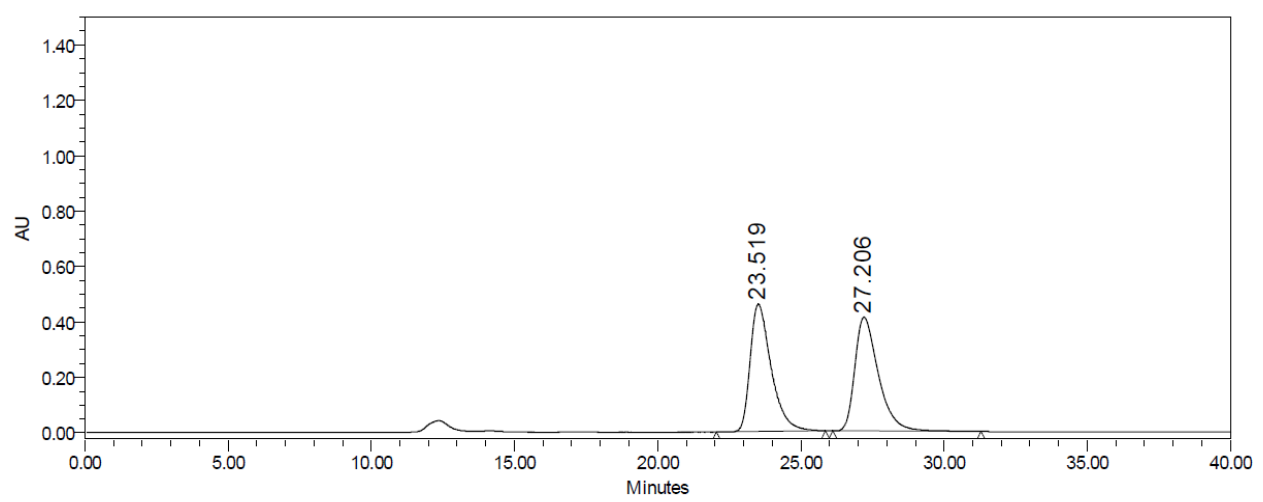

\begin{tabular}{|c|c|c|c|c|}
\hline & RT & Area & $\%$ Area & Height \\
\hline 1 & 23.519 & 23543470 & 49.76 & 460033 \\
\hline 2 & 27.206 & 23773098 & 50.24 & 411368 \\
\hline
\end{tabular}

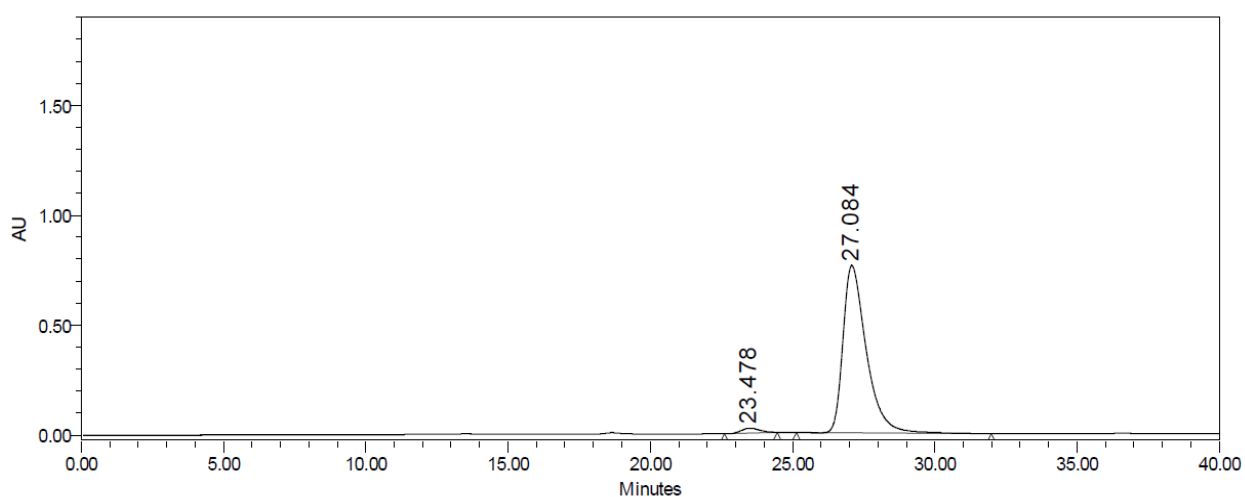

\begin{tabular}{|c|c|c|r|r|}
\hline & RT & Area & \% Area & Height \\
\hline 1 & 23.478 & 1006059 & 2.25 & 22549 \\
\hline 2 & 27.084 & 43732589 & 97.75 & 761099 \\
\hline
\end{tabular}

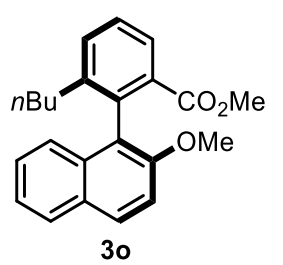

Colorless oil (63.8 mg, 92\% yield, $96 \%$ ee, $42 \mathrm{~h}$ ); the enantiomeric excess was determined by CHIRALPAK IG column, hexanes/ $i$-PrOH, $97: 3 \mathrm{v} / \mathrm{v}, v=0.3 \mathrm{~mL} / \mathrm{min}, \lambda=250 \mathrm{~nm}, 25{ }^{\circ} \mathrm{C}, \mathrm{t}_{\mathrm{R}}$ (major) $=17.7 \mathrm{~min}$, $\mathrm{t}_{\mathrm{R}}($ minor $)=19.7 \mathrm{~min} ;[\alpha]_{\mathrm{D}}^{25}=+24.62\left(c=1.0, \mathrm{CHCl}_{3}\right)$.

3o: ${ }^{1} \mathrm{H}$ NMR $\left(400 \mathrm{MHz}, \mathrm{CHCl}_{3}\right) \delta 7.89-7.85(\mathrm{~m}, 2 \mathrm{H}), 7.81-7.79(\mathrm{~m}, 1 \mathrm{H}), 7.53(\mathrm{dd}$, $J=8.0,1.2 \mathrm{~Hz}, 1 \mathrm{H}), 7.43(\mathrm{t}, J=8.0 \mathrm{~Hz}, 1 \mathrm{H}), 7.34(\mathrm{~d}, J=9.2 \mathrm{~Hz}, 1 \mathrm{H}), 7.31-7.23(\mathrm{~m}$, 2H), $7.09-7.07(\mathrm{~m}, 1 \mathrm{H}), 3.81(\mathrm{~s}, 3 \mathrm{H}), 3.37(\mathrm{~s}, 3 \mathrm{H}), 2.28-2.16(\mathrm{~m}, 2 \mathrm{H}), 1.33-1.24$ $(\mathrm{m}, 2 \mathrm{H}), 1.07-0.98(\mathrm{~m}, 2 \mathrm{H}), 0.60(\mathrm{t}, J=7.2 \mathrm{~Hz}, 3 \mathrm{H}) ;{ }^{13} \mathrm{C} \mathrm{NMR}\left(101 \mathrm{MHz}, \mathrm{CHCl}_{3}\right) \delta$ $168.1,153.7,143.5,136.5,133.7,132.7,132.3,129.1,128.9,128.0,127.8,127.5,126.4$, 124.6, 123.4, 122.9, 113.2, 56.5, 51.7, 33.0, 32.5, 22.4, 13.8; FT-IR (neat): $v_{\max }=3058$, 
2998, 2953, 2870, 2859, 1729, 1621, 1593, 1509, 1457, 1431, 1377, 1333, 1268, 1254 , 1190, 1138, 1121, 1102, 1063, 1020, 908, 806, 760, 747, 701, 681, $641 \mathrm{~cm}^{-1}$; HRMS (ESI): exact mass calculated for: $\mathrm{C}_{23} \mathrm{H}_{24} \mathrm{O}_{3}[\mathrm{M}]^{+}: 348.1720$, found 348.1723.
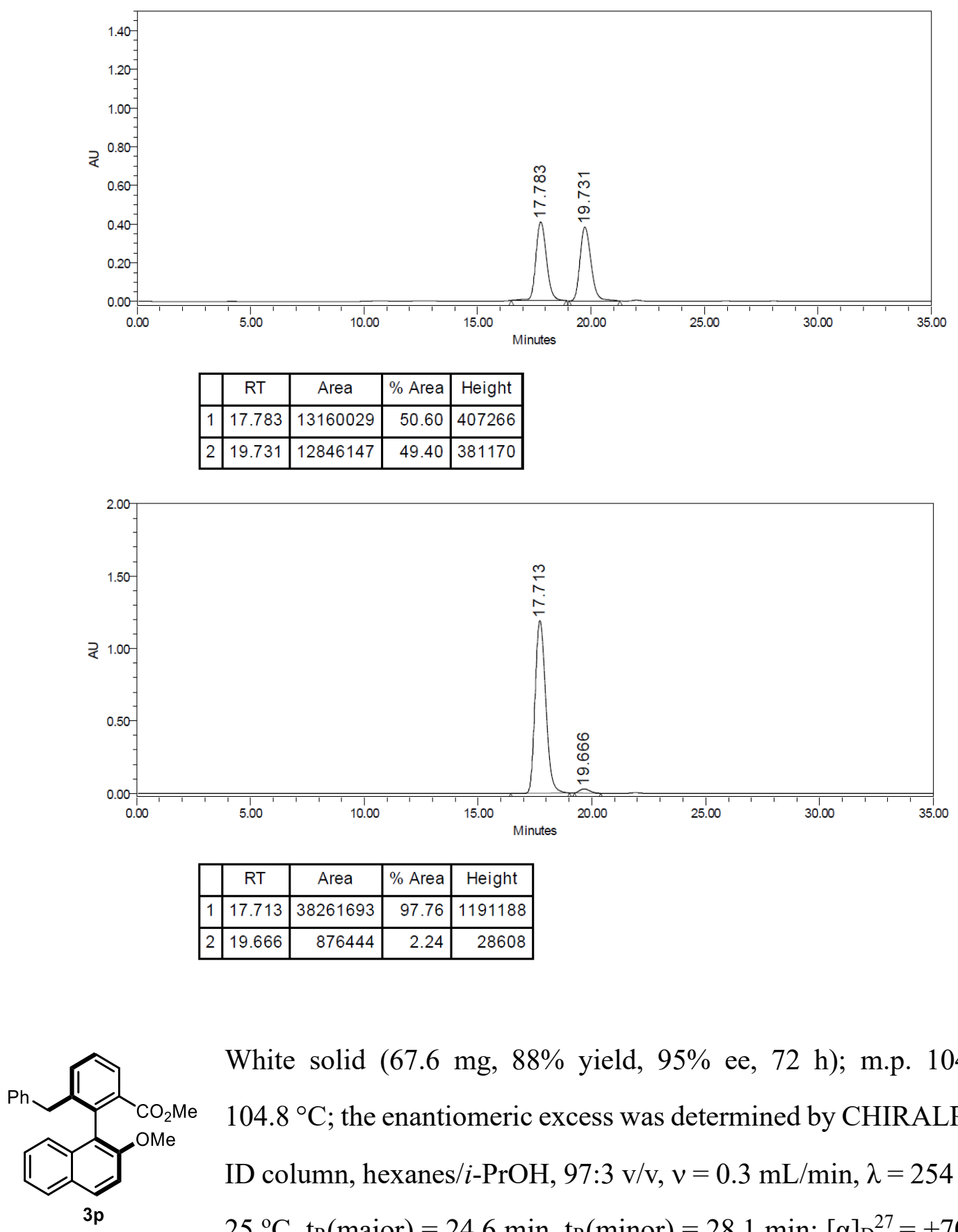

White solid (67.6 mg, 88\% yield, 95\% ee, 72 h); m.p. 104.2$104.8^{\circ} \mathrm{C}$; the enantiomeric excess was determined by CHIRALPAK ID column, hexanes $/ i$-PrOH, 97:3 v/v, $v=0.3 \mathrm{~mL} / \mathrm{min}, \lambda=254 \mathrm{~nm}$, $25^{\circ} \mathrm{C}, \mathrm{t}_{\mathrm{R}}($ major $)=24.6 \mathrm{~min}, \mathrm{t}_{\mathrm{R}}($ minor $)=28.1 \mathrm{~min} ;[\alpha]_{\mathrm{D}}^{27}=+70.92$ $\left(c=1.0, \mathrm{CHCl}_{3}\right)$. 
3p: ${ }^{1} \mathrm{H}$ NMR $\left(400 \mathrm{MHz}, \mathrm{CHCl}_{3}\right) \delta 7.92-7.86(\mathrm{~m}, 2 \mathrm{H}), 7.81-7.79(\mathrm{~m}, 1 \mathrm{H}), 7.42(\mathrm{~d}, J$ $=4.8 \mathrm{~Hz}, 2 \mathrm{H}), 7.31-7.24(\mathrm{~m}, 3 \mathrm{H}), 7.10-7.05(\mathrm{~m}, 4 \mathrm{H}), 6.80-6.78(\mathrm{~m}, 2 \mathrm{H}), 3.65(\mathrm{~s}$, 3H), $3.56(\mathrm{~s}, 2 \mathrm{H}), 3.39$ (s, 3H); ${ }^{13} \mathrm{C} \mathrm{NMR}\left(101 \mathrm{MHz}, \mathrm{CHCl}_{3}\right) \delta$ 167.9, 153.7, 142.1, 140.6, 137.0, 133.7, 133.4, 132.5, 129.3, 129.2, 128.9, 128.4, 128.1, 127.6, 126.6, 125.8, 124.4, 123.4, 122.5, 113.1, 56.2, 51.8, 39.4; FT-IR (neat): $v_{\max }=3057,3024,2941$, $2841,2357,2337,1725,1593,1508,1432,1375,1335,1267,1253,1201,1180,1133$, 1086, 1062, 1020, 1008, 908, 809, 771, 732, 694, 646, $586 \mathrm{~cm}^{-1}$; HRMS (ESI): exact mass calculated for: $\mathrm{C}_{26} \mathrm{H}_{22} \mathrm{O}_{3}[\mathrm{M}]^{+}: 382.1563$, found 382.1566 .
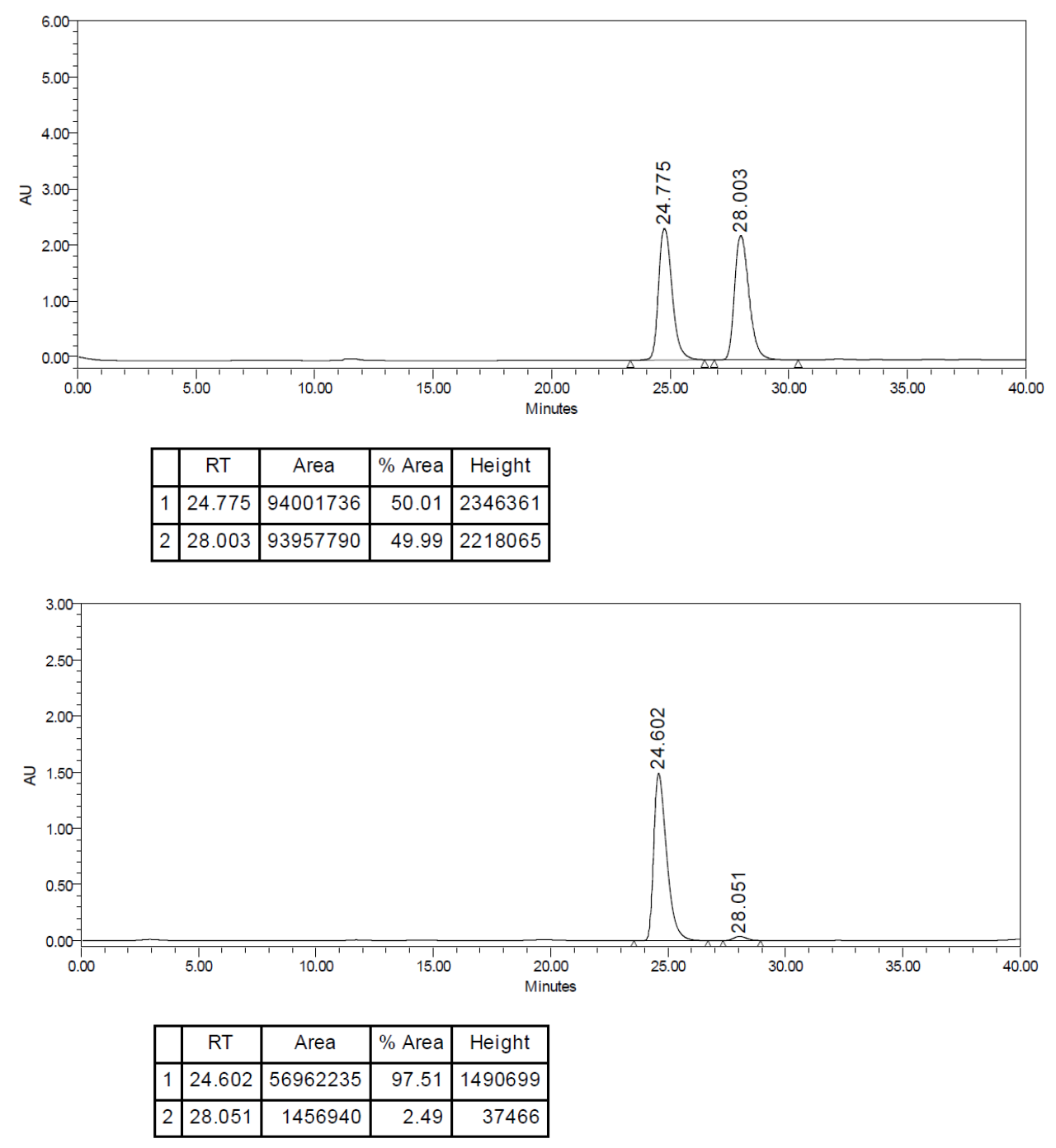


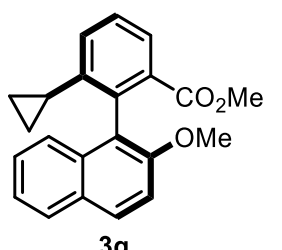

$3 q$

Colorless oil (57.3 mg, 87\% yield, $96 \%$ ee, $10 \mathrm{~mol} \% \mathrm{Cu}\left(\mathrm{ClO}_{4}\right) \cdot 6 \mathrm{H}_{2} \mathrm{O}$, $12 \mathrm{~mol} \% \mathbf{L 8}, 48 \mathrm{~h}$ ); the enantiomeric excess was determined by CHIRALPAK IC column, hexanes $/ i-\mathrm{PrOH}, 97: 3 \mathrm{v} / \mathrm{v}, v=0.3 \mathrm{~mL} / \mathrm{min}$, $\lambda=254 \mathrm{~nm}, 25^{\circ} \mathrm{C}, \mathrm{t}_{\mathrm{R}}($ major $)=33.0 \mathrm{~min}, \mathrm{t}_{\mathrm{R}}($ minor $)=27.0 \mathrm{~min} ;[\alpha]_{\mathrm{D}}{ }^{28}$ $=+51.04\left(c=1.0, \mathrm{CHCl}_{3}\right)$.

3q: 1H NMR (400 MHz, $\left.\mathrm{CHCl}_{3}\right) \delta 7.88(\mathrm{~d}, J=8.8 \mathrm{~Hz}, 1 \mathrm{H}), 7.84-7.80(\mathrm{~m}, 2 \mathrm{H}), 7.41$ $(\mathrm{t}, J=7.6 \mathrm{~Hz}, 1 \mathrm{H}), 7.36(\mathrm{~d}, J=9.2 \mathrm{~Hz}, 1 \mathrm{H}), 7.32-7.25(\mathrm{~m}, 2 \mathrm{H}), 7.19-7.14(\mathrm{~m}, 2 \mathrm{H})$, $3.82(\mathrm{~s}, 3 \mathrm{H}), 3.39(\mathrm{~s}, 3 \mathrm{H}), 1.38(\mathrm{tt}, J=8.4,5.6 \mathrm{~Hz}, 1 \mathrm{H}), 0.60-0.42(\mathrm{~m}, 4 \mathrm{H}) ;{ }^{13} \mathrm{C} \mathrm{NMR}$ $\left(101 \mathrm{MHz}, \mathrm{CHCl}_{3}\right) \delta 168.0,153.8,143.9,137.5,133.6,132.1,129.1,129.0,128.0$, $127.7,127.5,126.4,124.8,123.5,123.3,113.6,56.7,51.8,13.4,8.34,8.27$; FT-IR (neat): $v_{\max }=3059,3002,2948,2837,1728,1621,1593,1508,1464,1432,1290,1270$, 1248, 1198, 1132, 1094, 1065, 1020, 993, 909, 787, 747, 736, 700, $638 \mathrm{~cm}^{-1}$; HRMS (ESI): exact mass calculated for: $\mathrm{C}_{22} \mathrm{H}_{20} \mathrm{O}_{3}[\mathrm{M}]^{+}: 332.1407$, found 332.1409.

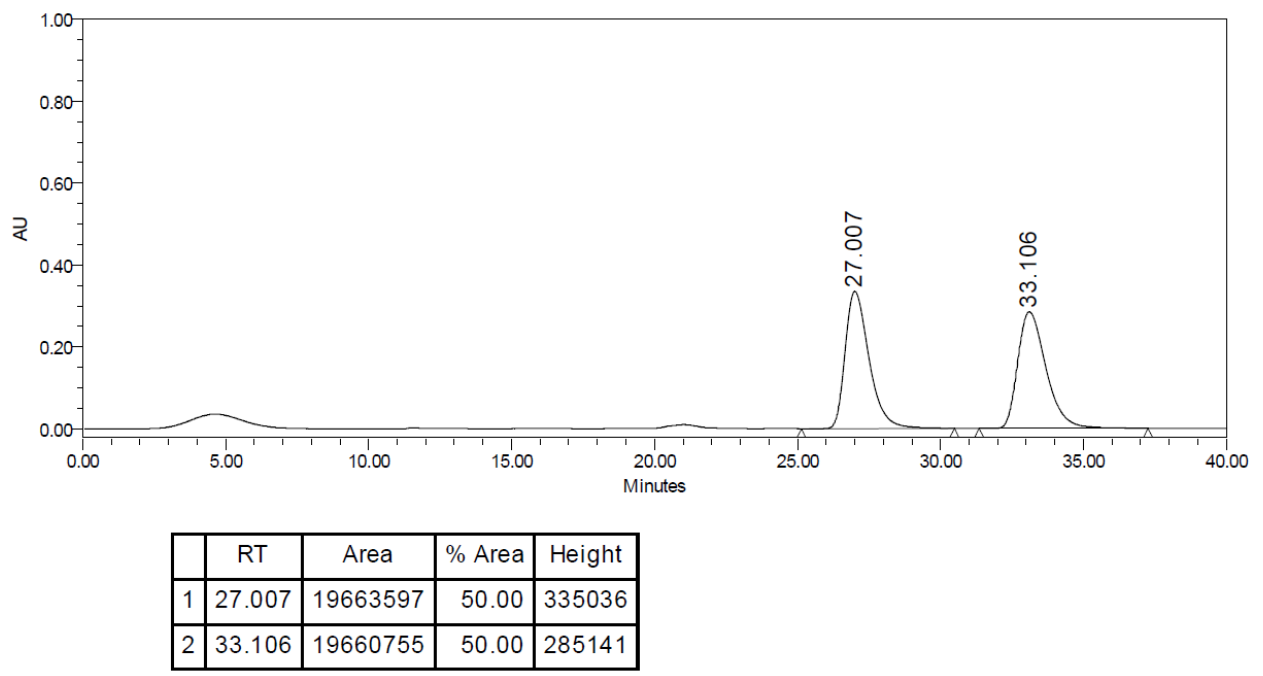



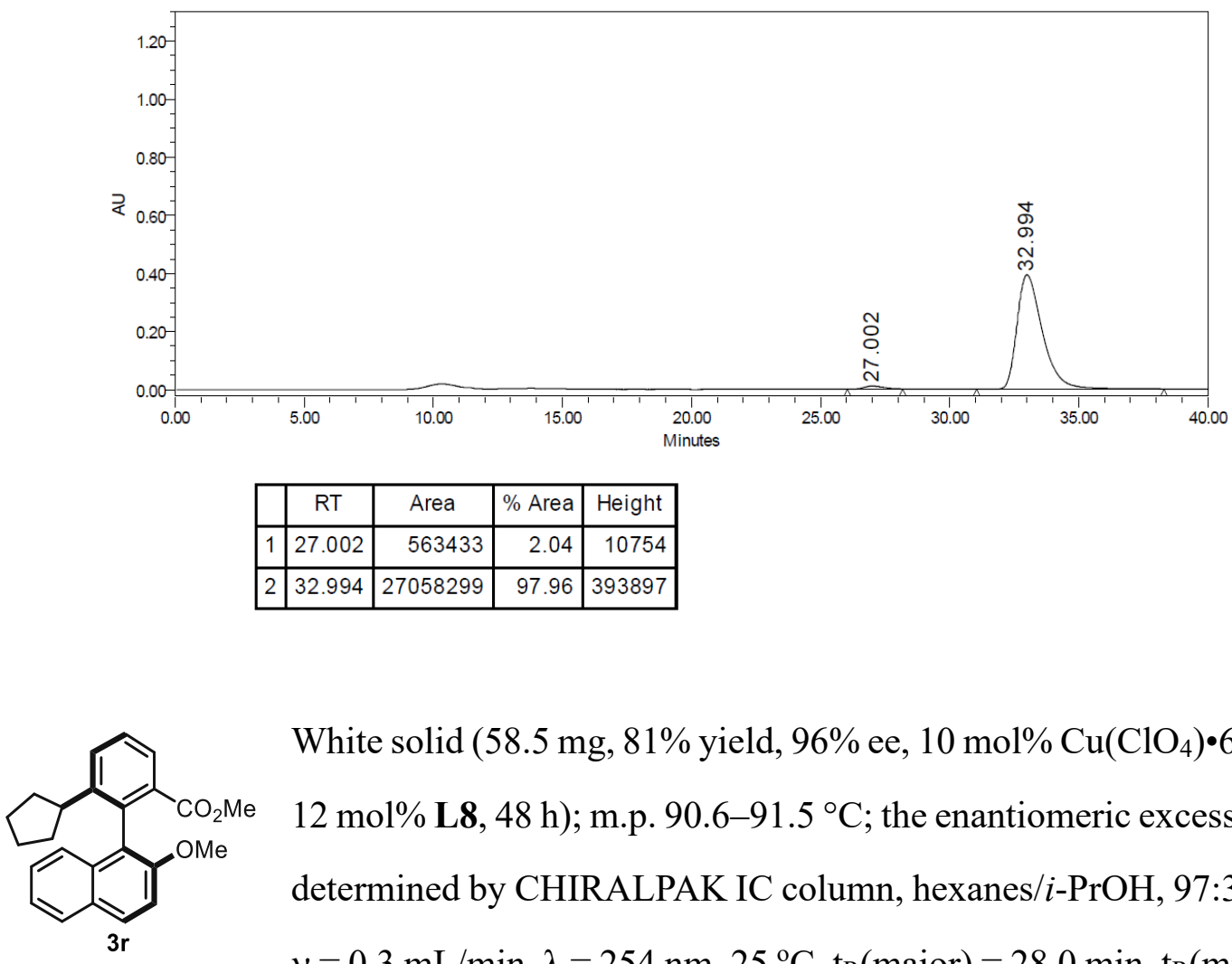

White solid (58.5 mg, $81 \%$ yield, $96 \%$ ee, $10 \mathrm{~mol} \% \mathrm{Cu}\left(\mathrm{ClO}_{4}\right) \cdot 6 \mathrm{H}_{2} \mathrm{O}$, 12 mol\% L8, 48 h); m.p. $90.6-91.5^{\circ} \mathrm{C}$; the enantiomeric excess was determined by CHIRALPAK IC column, hexanes/i-PrOH, 97:3 v/v, $v=0.3 \mathrm{~mL} / \mathrm{min}, \lambda=254 \mathrm{~nm}, 25^{\circ} \mathrm{C}, \mathrm{t}_{\mathrm{R}}($ major $)=28.0 \mathrm{~min}, \mathrm{t}_{\mathrm{R}}($ minor $)$ $=24.1 \mathrm{~min} ;[\alpha]_{\mathrm{D}}^{23}=+55.72\left(c=1.0, \mathrm{CHCl}_{3}\right)$.

3r: ${ }^{1} \mathrm{H}$ NMR (400 MHz, $\left.\mathrm{CHCl}_{3}\right) \delta 7.88(\mathrm{~d}, J=9.2 \mathrm{~Hz}, 1 \mathrm{H}), 7.84-7.79(\mathrm{~m}, 2 \mathrm{H}), 7.63$ $(\mathrm{dd}, J=8.0,1.2 \mathrm{~Hz}, 1 \mathrm{H}), 7.47(\mathrm{t}, J=8.0 \mathrm{~Hz}, 1 \mathrm{H}), 7.34(\mathrm{~d}, J=9.2 \mathrm{~Hz}, 1 \mathrm{H}), 7.31-7.23$ (m, 2H), $7.09(\mathrm{~d}, J=8.0 \mathrm{~Hz}, 1 \mathrm{H}), 3.81(\mathrm{~s}, 3 \mathrm{H}), 3.34(\mathrm{~s}, 3 \mathrm{H}), 2.63-2.55(\mathrm{~m}, 1 \mathrm{H}), 1.83$ $-1.75(\mathrm{~m}, 1 \mathrm{H}), 1.70-1.61(\mathrm{~m}, 2 \mathrm{H}), 1.57-1.40(\mathrm{~m}, 3 \mathrm{H}), 1.39-1.25(\mathrm{~m}, 2 \mathrm{H}) ;{ }^{13} \mathrm{C}$ NMR (101 MHz, $\left.\mathrm{CHCl}_{3}\right) \delta 168.2,154.0,148.0,136.1,134.0,132.1,129.9,129.1,128.8$, $127.9,127.8,127.6,126.3,124.9,123.4,123.1,113.3,56.6,51.7,42.0,35.6,35.3$, 26.12, 26.08; FT-IR (neat): $v_{\max }=2949,2864,1728,1620,1592,1508,1454,1428$, 1270, 1256, 1243, 1184, 1131, 1085, 1064, 1017, 1004, 809, $752 \mathrm{~cm}^{-1}$; HRMS (ESI): exact mass calculated for: $\mathrm{C}_{24} \mathrm{H}_{24} \mathrm{O}_{3}[\mathrm{M}]^{+}: 360.1720$, found 360.1720 . 


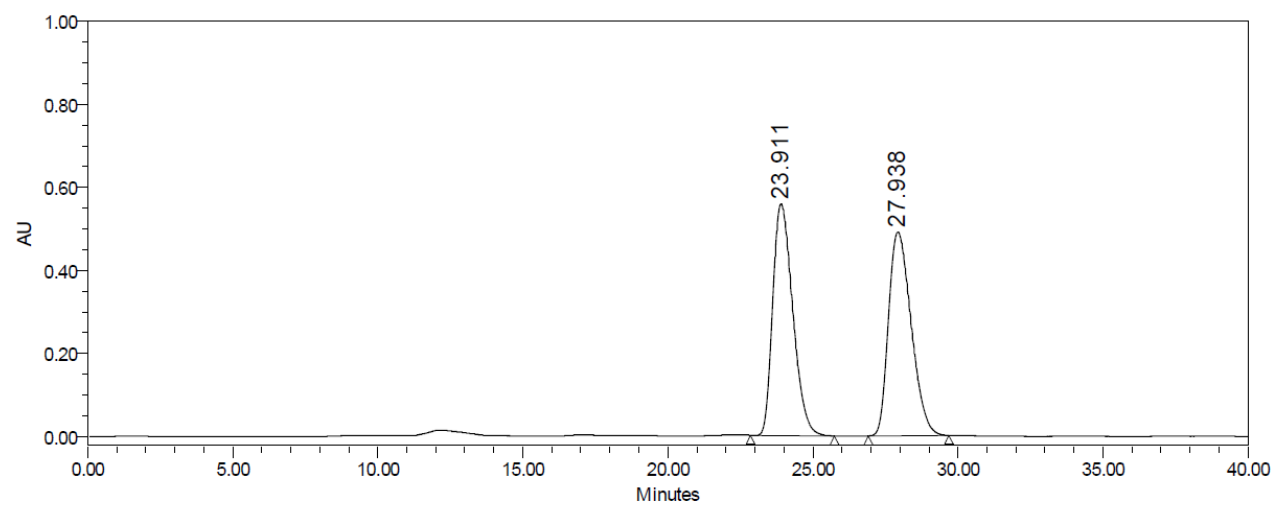

\begin{tabular}{|c|c|c|r|c|}
\hline & RT & Area & $\%$ Area & Height \\
\hline 1 & 23.911 & 26611175 & 50.01 & 557750 \\
\hline 2 & 27.938 & 26595611 & 49.99 & 490803 \\
\hline
\end{tabular}

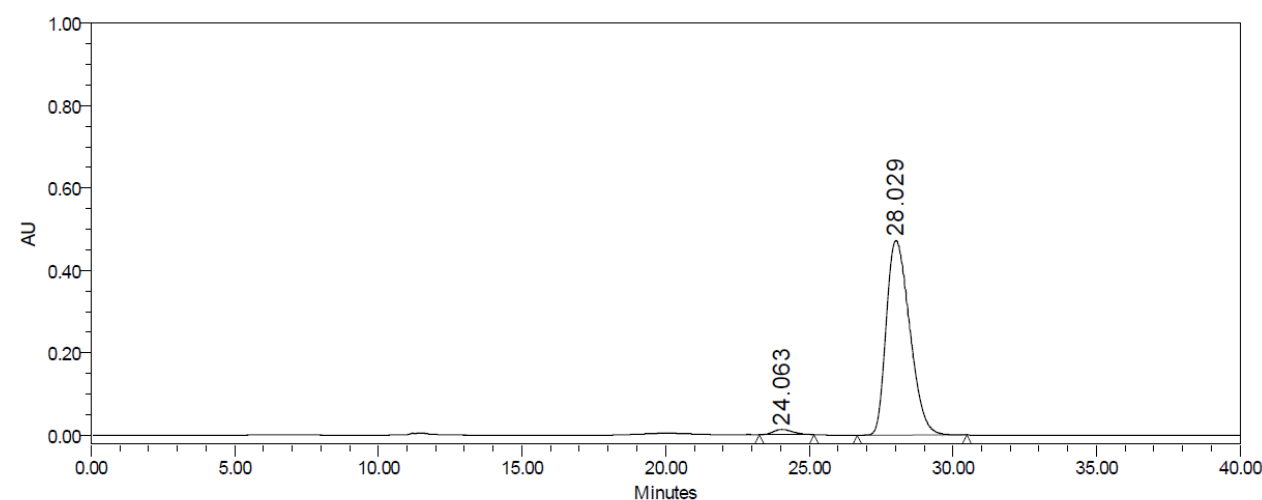

\begin{tabular}{|l|c|r|r|r|}
\hline & RT & Area & $\%$ Area & Height \\
\hline 1 & 24.063 & 604516 & 2.21 & 12759 \\
\hline 2 & 28.029 & 26730331 & 97.79 & 472519 \\
\hline
\end{tabular}

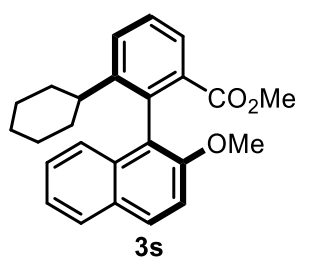

Colorless oil $(70.9 \mathrm{mg}, 95 \%$ yield, $95 \%$ ee, $10 \mathrm{~mol} \%$ $\left.\mathrm{Cu}\left(\mathrm{ClO}_{4}\right) \cdot 6 \mathrm{H}_{2} \mathrm{O}, 12 \mathrm{~mol} \% \mathbf{L 8}, 36 \mathrm{~h}\right)$; the enantiomeric excess was determined by CHIRALPAK IC column, hexanes/ $i-\mathrm{PrOH}, 97: 3 \mathrm{v} / \mathrm{v}$, $v=0.3 \mathrm{~mL} / \mathrm{min}, \lambda=254 \mathrm{~nm}, 25^{\circ} \mathrm{C}, \mathrm{t}_{\mathrm{R}}$ (major) $=27.9 \mathrm{~min}, \mathrm{t}_{\mathrm{R}}($ minor $)$ $=23.0 \mathrm{~min} ;[\alpha]_{\mathrm{D}}^{24}=+28.04\left(c=1.0, \mathrm{CHCl}_{3}\right)$.

3s: ${ }^{1} \mathrm{H}$ NMR $\left(400 \mathrm{MHz}, \mathrm{CHCl}_{3}\right) \delta 7.88(\mathrm{~d}, J=9.2 \mathrm{~Hz}, 1 \mathrm{H}), 7.83(\mathrm{dd}, J=7.6,1.2 \mathrm{~Hz}$, 1H), $7.81-7.78(\mathrm{~m}, 1 \mathrm{H}), 7.60(\mathrm{dd}, J=8.0,1.2 \mathrm{~Hz}, 1 \mathrm{H}), 7.47$ (t, $J=7.6 \mathrm{~Hz}, 1 \mathrm{H}), 7.35$ $(\mathrm{d}, J=8.8 \mathrm{~Hz}, 1 \mathrm{H}), 7.30-7.21(\mathrm{~m}, 2 \mathrm{H}), 7.07(\mathrm{~d}, J=8.4 \mathrm{~Hz}, 1 \mathrm{H}), 3.81(\mathrm{~s}, 3 \mathrm{H}), 3.33(\mathrm{~s}$, $3 \mathrm{H}), 2.12(\mathrm{tt}, J=12.0,3.2 \mathrm{~Hz}, 1 \mathrm{H}), 1.74(\mathrm{~d}, J=12.8 \mathrm{~Hz}, 1 \mathrm{H}), 1.65(\mathrm{~d}, J=12.8 \mathrm{~Hz}, 1 \mathrm{H})$, $1.50(\mathrm{~d}, J=12.0 \mathrm{~Hz}, 2 \mathrm{H}), 1.45-1.42(\mathrm{~m}, 1 \mathrm{H}), 1.37-1.27(\mathrm{~m}, 1 \mathrm{H}), 1.17-1.06(\mathrm{~m}$, 
1H), $0.99-0.88(\mathrm{~m}, 1 \mathrm{H}), 0.79-0.68(\mathrm{~m}, 1 \mathrm{H}) ;{ }^{13} \mathrm{C} \mathrm{NMR}\left(101 \mathrm{MHz}, \mathrm{CHCl}_{3}\right) \delta 168.2$, $153.9,148.5,135.6,134.0,132.2,130.1,129.1,128.9,127.9,127.73,127.65,126.3$, 124.7, 123.4, 122.8, 113.1, 56.5, 51.7, 41.0, 34.5, 34.2, 26.9, 26.7, 26.2; FT-IR (neat): $v_{\max }=3060,2999,2926,2859,1728,1622,1509,1448,1432,1286,1269,1253,1227$, 1144, 1122, 1084, 1064, 984, 807, 759, 747, 735, 701, $666 \mathrm{~cm}^{-1}$; HRMS (ESI): exact mass calculated for: $\mathrm{C}_{25} \mathrm{H}_{26} \mathrm{O}_{3}[\mathrm{M}]^{+}: 374.1876$, found 374.1876 .

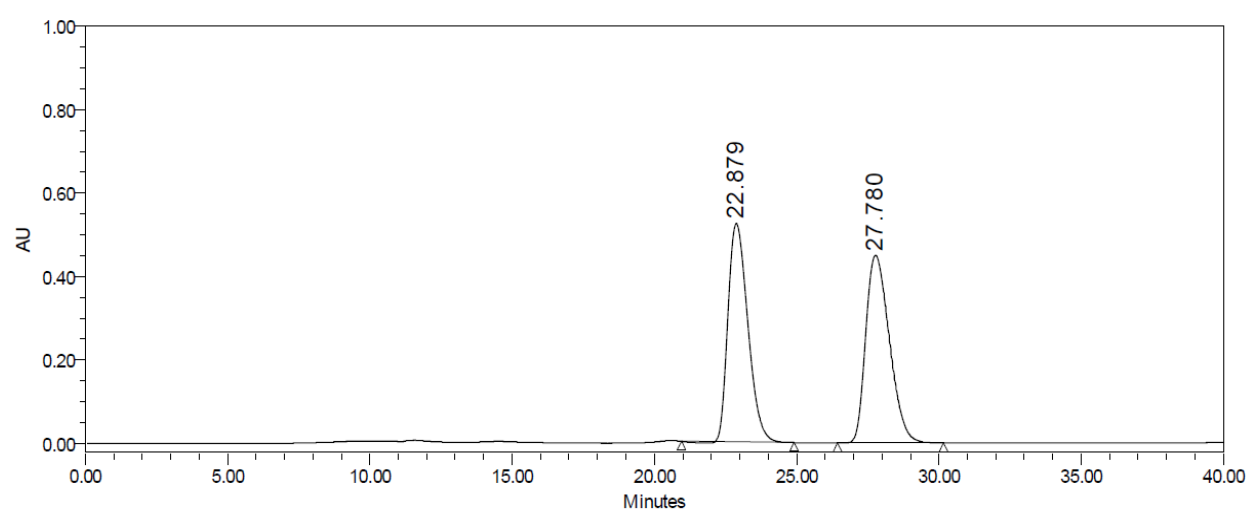

\begin{tabular}{|c|c|c|r|c|}
\hline & RT & Area & $\%$ Area & Height \\
\hline 1 & 22.879 & 25836842 & 49.75 & 523780 \\
\hline 2 & 27.780 & 26093421 & 50.25 & 449866 \\
\hline
\end{tabular}

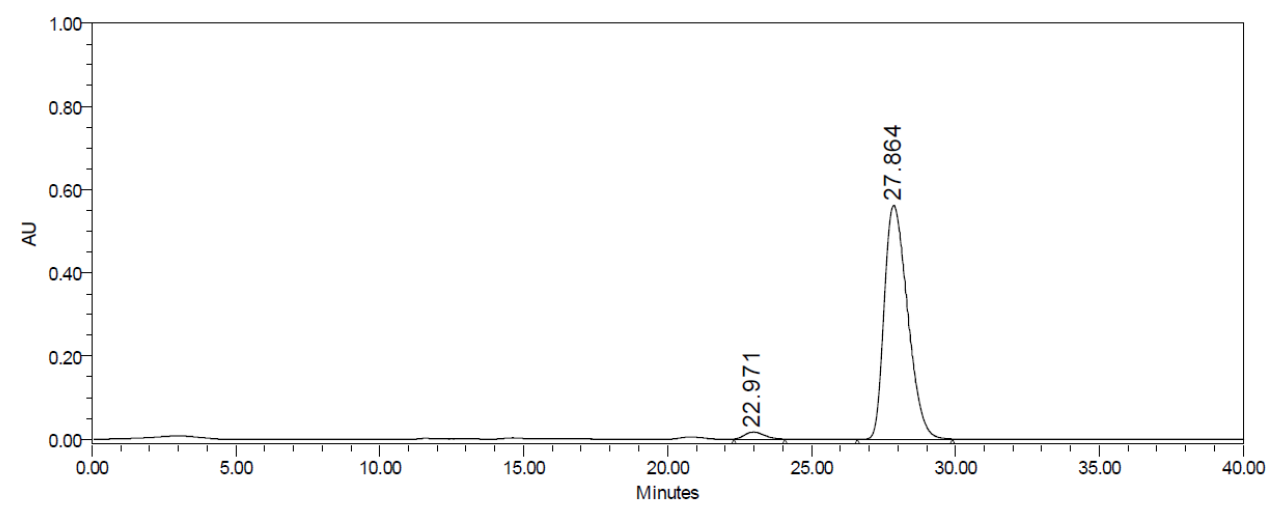

\begin{tabular}{|c|c|r|r|r|}
\hline & RT & \multicolumn{1}{|c|}{ Area } & \% Area & Height \\
\hline 1 & 22.971 & 766837 & 2.37 & 16716 \\
\hline 2 & 27.864 & 31617421 & 97.63 & 561943 \\
\hline
\end{tabular}

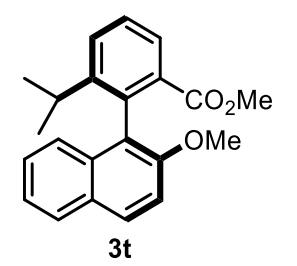

Colorless oil (63.8 mg, $95 \%$ yield, $96 \%$ ee, $10 \mathrm{~mol} \% \mathrm{Cu}\left(\mathrm{ClO}_{4}\right) \cdot 6 \mathrm{H}_{2} \mathrm{O}$, $12 \mathrm{~mol} \% \mathbf{L 8}, 36 \mathrm{~h}$ ); the enantiomeric excess was determined by CHIRALPAK IC column, hexanes $/ i-\mathrm{PrOH}, 97: 3 \mathrm{v} / \mathrm{v}, v=0.3 \mathrm{~mL} / \mathrm{min}$, $\lambda=254 \mathrm{~nm}, 25^{\circ} \mathrm{C}, \mathrm{t}_{\mathrm{R}}($ major $)=24.6 \mathrm{~min}, \mathrm{t}_{\mathrm{R}}($ minor $)=21.0 \mathrm{~min} ;[\alpha]_{\mathrm{D}}^{23}$ $=+29.10\left(c=1.0, \mathrm{CHCl}_{3}\right)$. 
3t: ${ }^{1} \mathrm{H}$ NMR $\left(400 \mathrm{MHz}, \mathrm{CHCl}_{3}\right) \delta 7.89(\mathrm{~d}, J=9.2 \mathrm{~Hz}, 1 \mathrm{H}), 7.84(\mathrm{dd}, J=7.6,1.2 \mathrm{~Hz}$, 1H), $7.80(\mathrm{dd}, J=7.6,1.2 \mathrm{~Hz}, 1 \mathrm{H}), 7.63(\mathrm{dd}, J=8.0,1.2 \mathrm{~Hz}, 1 \mathrm{H}), 7.49$ (t, $J=7.6 \mathrm{~Hz}$, 1H), 7.35 (d, J = 9.2 Hz, 1H), $7.31-7.23(\mathrm{~m}, 2 \mathrm{H}), 7.10-7.07(\mathrm{~m}, 1 \mathrm{H}), 3.82(\mathrm{~s}, 3 \mathrm{H})$, $3.35(\mathrm{~s}, 3 \mathrm{H}), 2.53(\mathrm{sept}, J=6.8 \mathrm{~Hz}, 1 \mathrm{H}), 1.06(\mathrm{~d}, J=6.8 \mathrm{~Hz}, 3 \mathrm{H}), 0.94(\mathrm{~d}, J=6.8 \mathrm{~Hz}$, $3 \mathrm{H}) ;{ }^{13} \mathrm{C} \mathrm{NMR}\left(101 \mathrm{MHz}, \mathrm{CHCl}_{3}\right) \delta 168.1,153.9,149.6,135.4,133.9,132.1,129.3$, 129.1, 128.9, 128.0, 127.9, 127.7, 126.4, 124.7, 123.5, 122.8, 113.2, 56.4, 51.7, 30.2, 24.2, 24.0; FT-IR (neat): $v_{\max }=3059,2961,2868,2838,1728,1622,1508,1463,1432$, 1334, 1270,1252, 1190, 1147, 1124, 1096, 1066, 807, 747, 735, 682, $640 \mathrm{~cm}^{-1}$; HRMS (ESI): exact mass calculated for: $\mathrm{C}_{22} \mathrm{H}_{22} \mathrm{O}_{3}[\mathrm{M}]^{+}:$334.1563, found 334.1566.
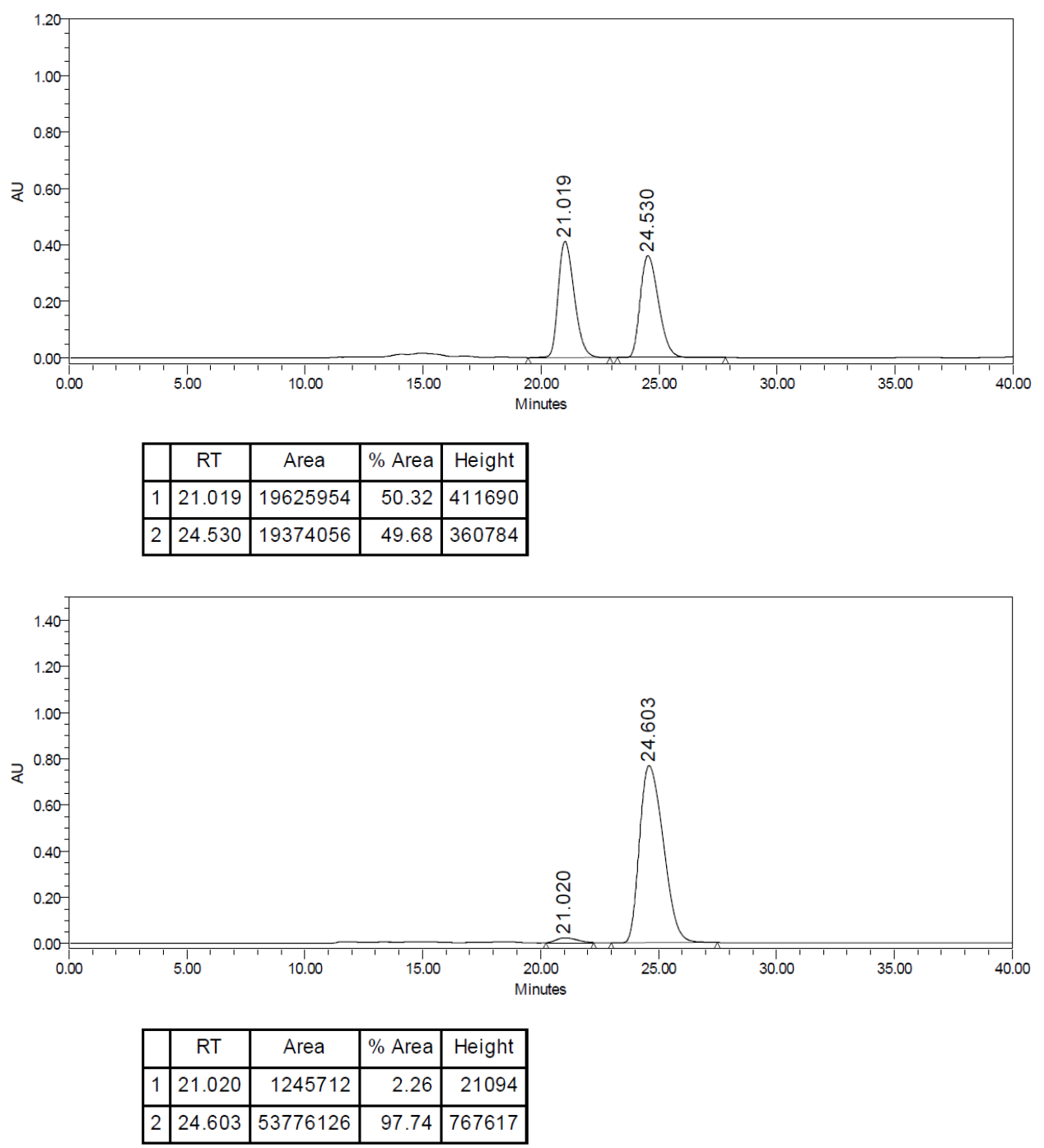


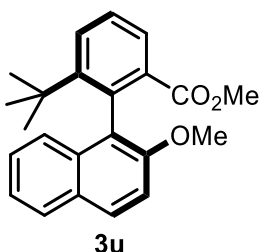

Colorless oil (25.7 mg, $37 \%$ yield, $94 \%$ ee, $10 \mathrm{~mol} \% \mathrm{Cu}\left(\mathrm{ClO}_{4}\right) \cdot 6 \mathrm{H}_{2} \mathrm{O}$, $12 \mathrm{~mol} \% \mathbf{L 8}, 72 \mathrm{~h}$ ); the enantiomeric excess was determined by CHIRALPAK ID column, hexanes $/ i-\mathrm{PrOH}, 97: 3 \mathrm{v} / \mathrm{v}, v=0.3 \mathrm{~mL} / \mathrm{min}$, $\lambda=254 \mathrm{~nm}, 25^{\circ} \mathrm{C}, \mathrm{t}_{\mathrm{R}}($ major $)=19.3 \mathrm{~min}, \mathrm{t}_{\mathrm{R}}($ minor $)=21.6 \mathrm{~min} ;[\alpha]_{\mathrm{D}}{ }^{27}$ $=-28.06\left(c=1.0, \mathrm{CHCl}_{3}\right)$.

3u: ${ }^{1} \mathrm{H}$ NMR $\left(400 \mathrm{MHz}, \mathrm{CHCl}_{3}\right) \delta 7.88(\mathrm{~d}, J=9.2 \mathrm{~Hz}, 1 \mathrm{H}), 7.79(\mathrm{dd}, J=8.0,1.2 \mathrm{~Hz}$, 1H), $7.77-7.75(\mathrm{~m}, 1 \mathrm{H}), 7.59$ (dd, $J=7.6,1.2 \mathrm{~Hz}, 1 \mathrm{H}), 7.43$ (t, $J=8.0 \mathrm{~Hz}, 1 \mathrm{H}), 7.30$ - $7.24(\mathrm{~m}, 3 \mathrm{H}), 7.10-7.06(\mathrm{~m}, 1 \mathrm{H}), 3.85(\mathrm{~s}, 3 \mathrm{H}), 3.18(\mathrm{~s}, 3 \mathrm{H}), 1.04(\mathrm{~s}, 9 \mathrm{H}) ;{ }^{13} \mathrm{C}$ NMR $\left(101 \mathrm{MHz}, \mathrm{CHCl}_{3}\right) \delta 169.3,154.6,150.3,135.2,135.0,134.2,130.7,129.6,128.5$, $127.7,127.5,126.6,126.1,126.0,124.2,123.3,112.6,56.1,51.6,37.3,32.2$; FT-IR (neat): $v_{\max }=3002,2952,2905,2871,2837,1731,1622,1593,1508,1463,1431,1375$, $1363,1332,1290,1270,1251,1203,1147,1129,1107,1065,1020,976,907,907,807$, 749, 701, $635 \mathrm{~cm}^{-1}$; HRMS (ESI): exact mass calculated for: $\mathrm{C}_{23} \mathrm{H}_{24} \mathrm{O}_{3}[\mathrm{M}]^{+}: 348.1720$, found 348.1724 .

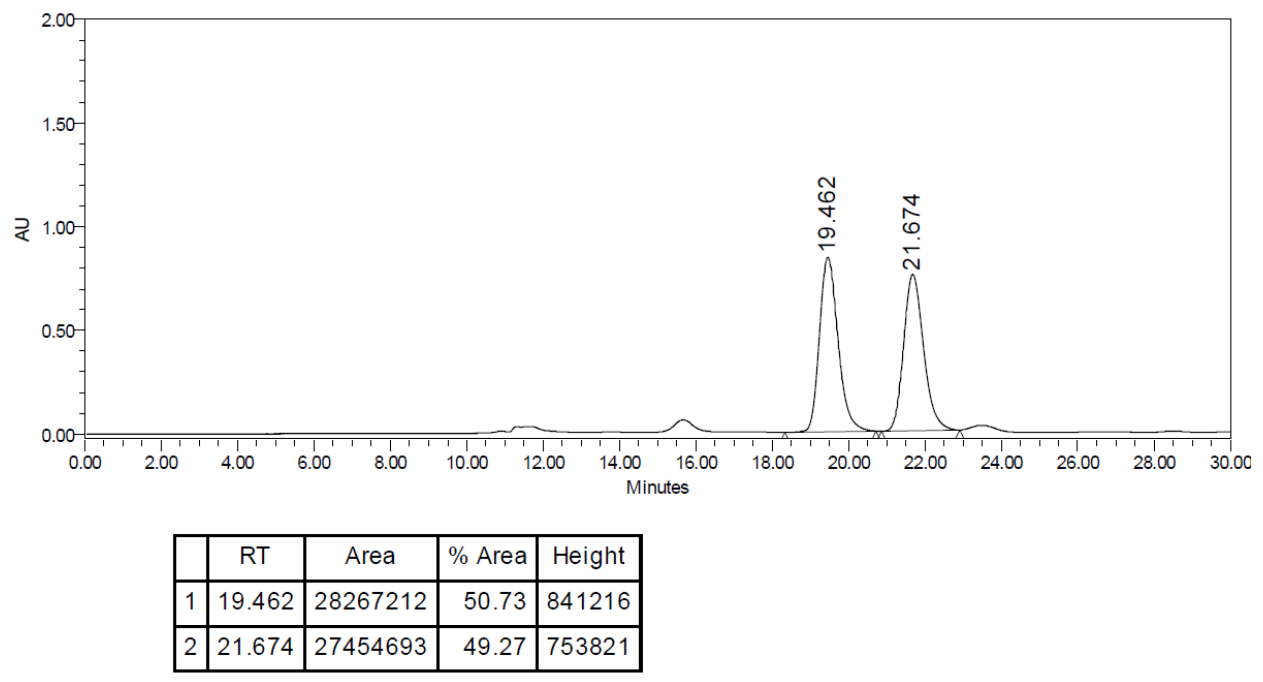



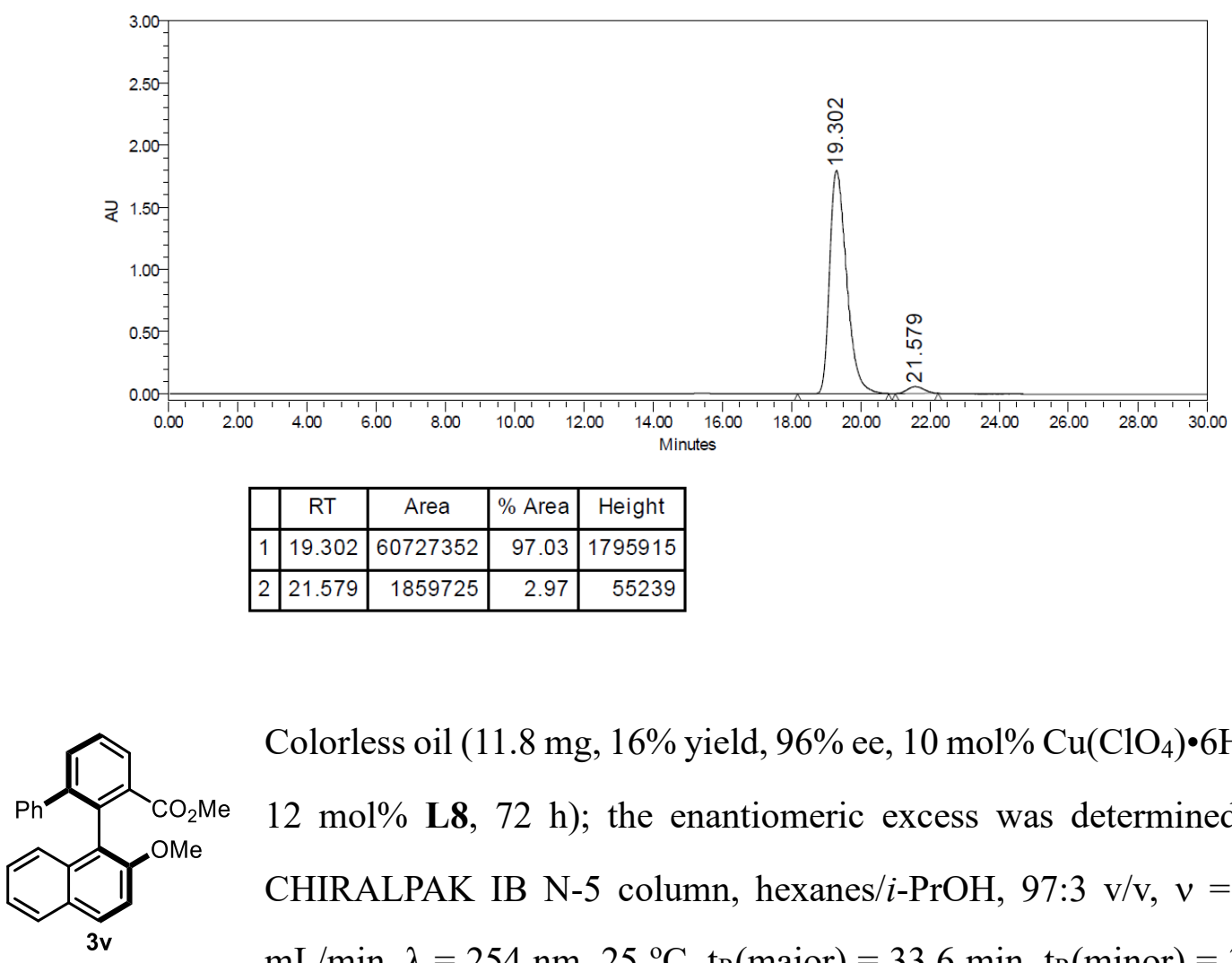

Colorless oil (11.8 mg, 16\% yield, $96 \%$ ee, $10 \mathrm{~mol} \% \mathrm{Cu}\left(\mathrm{ClO}_{4}\right) \cdot 6 \mathrm{H}_{2} \mathrm{O}$, $12 \mathrm{~mol} \% \mathbf{L 8}, 72 \mathrm{~h}$ ); the enantiomeric excess was determined by CHIRALPAK IB N-5 column, hexanes $/ i-\mathrm{PrOH}, 97: 3 \mathrm{v} / \mathrm{v}, v=0.3$ $\mathrm{mL} / \mathrm{min}, \lambda=254 \mathrm{~nm}, 25^{\circ} \mathrm{C}, \mathrm{t}_{\mathrm{R}}($ major $)=33.6 \mathrm{~min}, \mathrm{t}_{\mathrm{R}}($ minor $)=30.1$ $\min ; \alpha]_{\mathrm{D}}{ }^{24}=+23.60\left(c=0.5, \mathrm{CHCl}_{3}\right)$.

3v: ${ }^{1} \mathrm{H}$ NMR $\left(400 \mathrm{MHz}, \mathrm{CHCl}_{3}\right) \delta 7.99(\mathrm{dd}, J=7.6,1.2 \mathrm{~Hz}, 1 \mathrm{H}), 7.73-7.68(\mathrm{~m}, 2 \mathrm{H})$, $7.62(\mathrm{dd}, J=7.6,1.6 \mathrm{~Hz}, 1 \mathrm{H}), 7.55(\mathrm{t}, J=7.6 \mathrm{~Hz}, 1 \mathrm{H}), 7.29-7.21(\mathrm{~m}, 3 \mathrm{H}), 7.08(\mathrm{~d}, J$ $=9.2 \mathrm{~Hz}, 1 \mathrm{H}), 7.00-6.93(\mathrm{~m}, 5 \mathrm{H}), 3.59(\mathrm{~s}, 3 \mathrm{H}), 3.34(\mathrm{~s}, 3 \mathrm{H}) ;{ }^{13} \mathrm{C}$ NMR $(101 \mathrm{MHz}$, $\left.\mathrm{CHCl}_{3}\right) \delta 168.2,153.6,144.3,141.2,135.6,133.8,133.6,133.0,129.22,129.15,128.7$, 128.6, 128.0, 127.7, 127.2, 126.6, 126.4, 124.5, 123.2, 122.8, 112.8, 56.2, 51.9; FT-IR (neat): $v_{\max }=3057,2934,2917,2850,1731,1594,1509,1431,1304,1271,1254,1195$, 1183, 1142, 1064, 1021, 908, 808, 764, 746, 701, $645 \mathrm{~cm}^{-1}$; HRMS (ESI): exact mass calculated for: $\mathrm{C}_{25} \mathrm{H}_{20} \mathrm{O}_{3}[\mathrm{M}]^{+}: 368.1407$, found 368.1409 . 


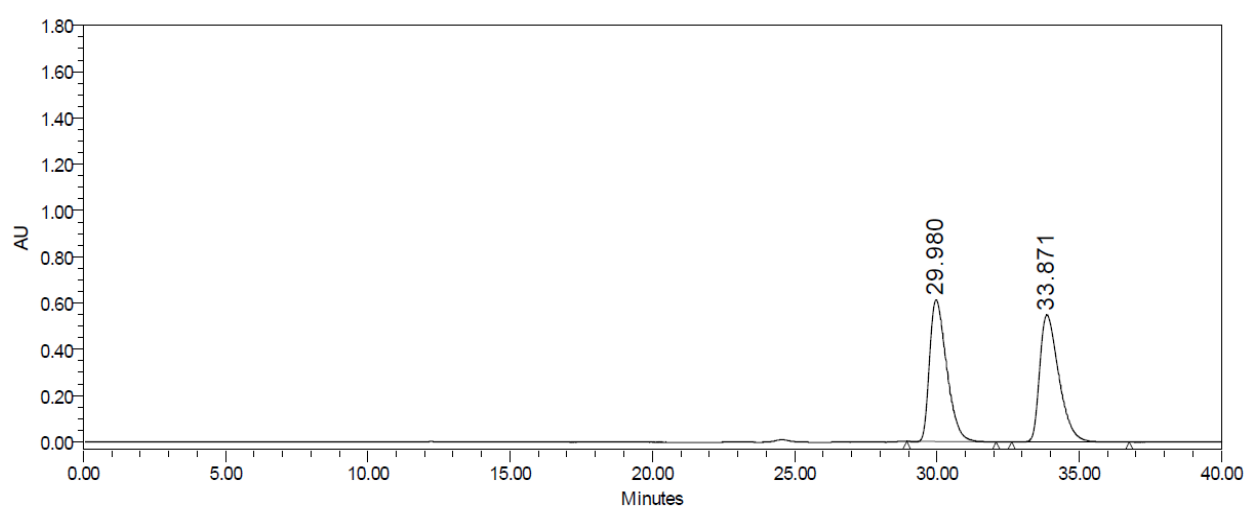

\begin{tabular}{|c|c|c|c|c|}
\hline & RT & Area & \% Area & Height \\
\hline 1 & 29.980 & 25623470 & 49.89 & 612863 \\
\hline 2 & 33.871 & 25736062 & 50.11 & 548912 \\
\hline
\end{tabular}

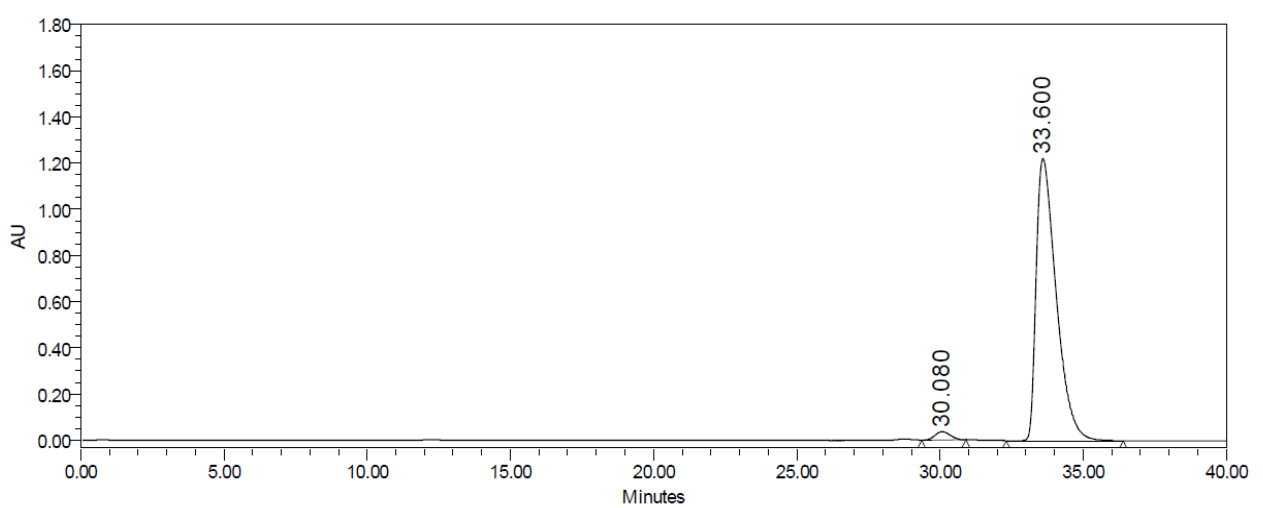

\begin{tabular}{|r|c|r|r|r|}
\hline & RT & Area & $\%$ Area & Height \\
\hline 1 & 30.080 & 1348841 & 2.22 & 36160 \\
\hline 2 & 33.600 & 59487831 & 97.78 & 1220647 \\
\hline
\end{tabular}

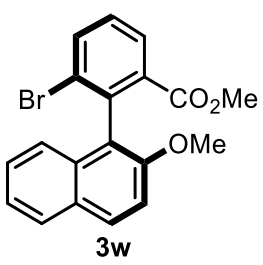

Yellow solid (62.2 mg, 84\% yield, 94\% ee, 72 h); m.p. $90.1-90.9^{\circ} \mathrm{C}$; the enantiomeric excess was determined by CHIRALPAK IC column, hexanes/i-PrOH, 97:3 v/v, $v=0.3 \mathrm{~mL} / \mathrm{min}, \lambda=254 \mathrm{~nm}, 25^{\circ} \mathrm{C}$, $\mathrm{t}_{\mathrm{R}}($ major $)=28.2 \mathrm{~min}, \mathrm{t}_{\mathrm{R}}($ minor $)=23.6 \mathrm{~min} ;[\alpha]_{\mathrm{D}}^{32}=+6.54(c=1.0$,

$\left.\mathrm{CHCl}_{3}\right)$.

3w: ${ }^{1} \mathrm{H}$ NMR (400 MHz, $\left.\mathrm{CHCl}_{3}\right) \delta 7.99(\mathrm{dd}, J=8.0,1.2 \mathrm{~Hz}, 1 \mathrm{H}), 7.93-7.90(\mathrm{~m}, 2 \mathrm{H})$, $7.83-7.81(\mathrm{~m}, 1 \mathrm{H}), 7.37$ (t, $J=7.6 \mathrm{~Hz}, 2 \mathrm{H}), 7.33-7.29(\mathrm{~m}, 2 \mathrm{H}), 7.10-7.08(\mathrm{~m}, 1 \mathrm{H})$, 3.85 (s, 3H), 3.39 (s, 3H); ${ }^{13} \mathrm{C}$ NMR (101 MHz, $\left.\mathrm{CHCl}_{3}\right) \delta 166.7,153.6,138.4,136.4$, $134.1,132.8,129.9,129.4,128.91,128.87,128.2,127.3,126.8,124.0,123.6,123.5$, 113.5, 56.9, 52.1; FT-IR (neat): $v_{\max }=2926,2844,1722,1590,1507,1434,1257,1199$, 
1142, 1069, 1019, 804, 752, $704 \mathrm{~cm}^{-1}$; HRMS (EI): exact mass calculated for: $\mathrm{C}_{19} \mathrm{H}_{15} \mathrm{BrO}_{3}[\mathrm{M}]^{+}: 370.0199$, found 370.0201 .
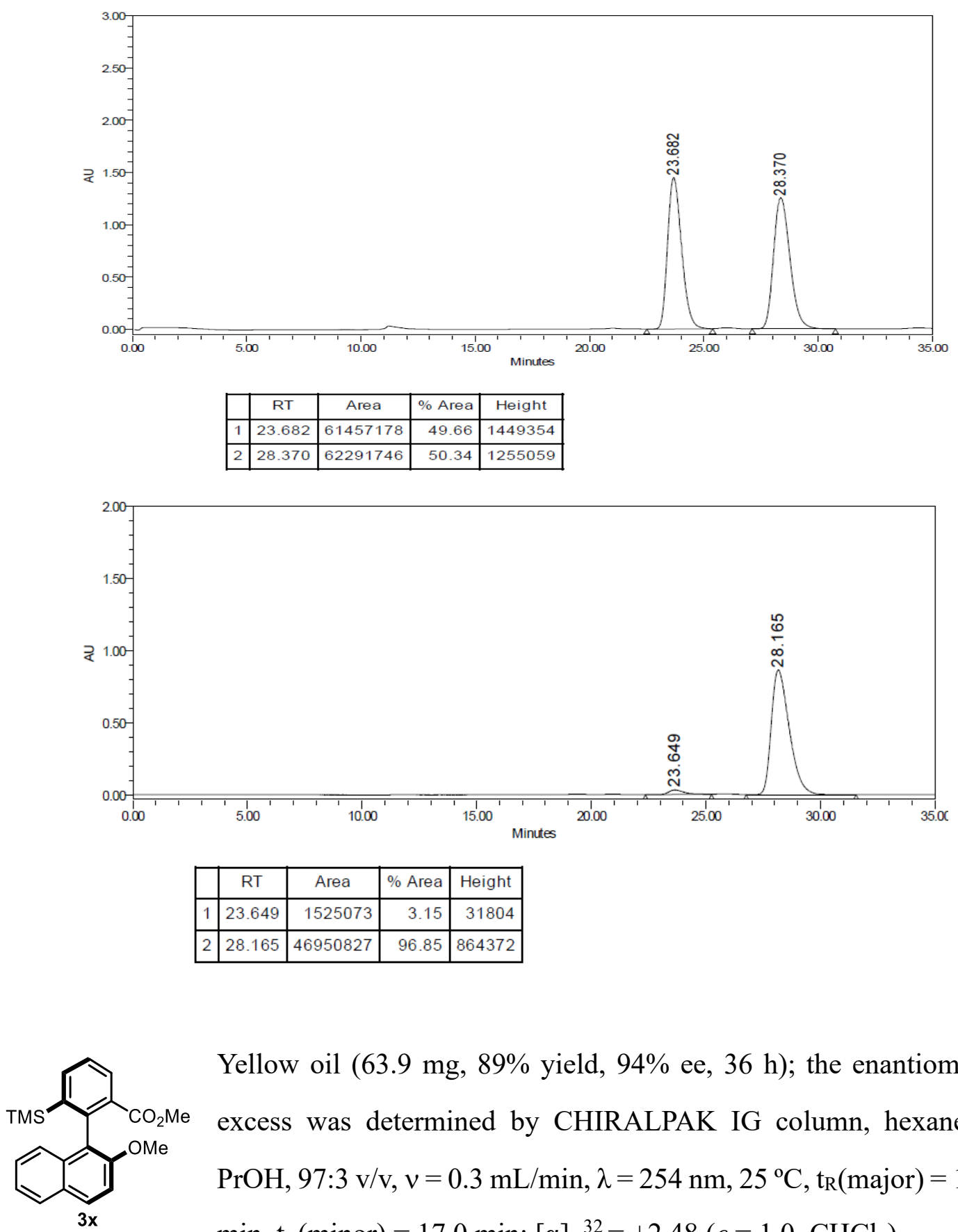

Yellow oil (63.9 mg, 89\% yield, 94\% ee, $36 \mathrm{~h}$ ); the enantiomeric excess was determined by CHIRALPAK IG column, hexanes $/ i$ PrOH, $97: 3 \mathrm{v} / \mathrm{v}, v=0.3 \mathrm{~mL} / \mathrm{min}, \lambda=254 \mathrm{~nm}, 25^{\circ} \mathrm{C}, \mathrm{t}_{\mathrm{R}}$ (major) $=15.4$ $\min , t_{\mathrm{R}}(\operatorname{minor})=17.0 \mathrm{~min} ;[\alpha]_{\mathrm{D}}{ }^{32}=+2.48\left(c=1.0, \mathrm{CHCl}_{3}\right)$.

3x: ${ }^{1} \mathrm{H}$ NMR $\left(400 \mathrm{MHz}, \mathrm{CHCl}_{3}\right) \delta 7.99(\mathrm{dd}, J=8.0,1.2 \mathrm{~Hz}, 1 \mathrm{H}), 7.90-7.85(\mathrm{~m}, 2 \mathrm{H})$, $7.78(\mathrm{dd}, J=7.2,1.6 \mathrm{~Hz}, 1 \mathrm{H}), 7.49$ (t, $J=7.6 \mathrm{~Hz}, 1 \mathrm{H}), 7.30(\mathrm{~d}, J=9.2 \mathrm{~Hz}, 1 \mathrm{H}), 7.27-$ $7.21(\mathrm{~m}, 2 \mathrm{H}), 7.01(\mathrm{~d}, J=8.4 \mathrm{~Hz}, 1 \mathrm{H}), 3.81(\mathrm{~s}, 3 \mathrm{H}), 3.37$ (s, 3H), $-0.31(\mathrm{~s}, 9 \mathrm{H}) ;{ }^{13} \mathrm{C}$ 
$\operatorname{NMR}\left(101 \mathrm{MHz}, \mathrm{CHCl}_{3}\right) \delta 168.1,154.0,143.1,142.6,138.4,134.7,132.0,130.7,129.4$, 128.6, 127.8, 126.8, 126.2, 125.4, 124.6, 123.3, 112.6, 56.0, 51.7, -0.05; FT-IR (neat): $v_{\max }=2950,2897,2838,1732,1593,1509,1433,1371,1246,1106,1083,1064,863$, 836, 806, $764 \mathrm{~cm}^{-1}$; HRMS (EI): exact mass calculated for: $\mathrm{C}_{22} \mathrm{H}_{24} \mathrm{O}_{3} \mathrm{Si}[\mathrm{M}]^{+}: 364.1489$, found 364.1495 .
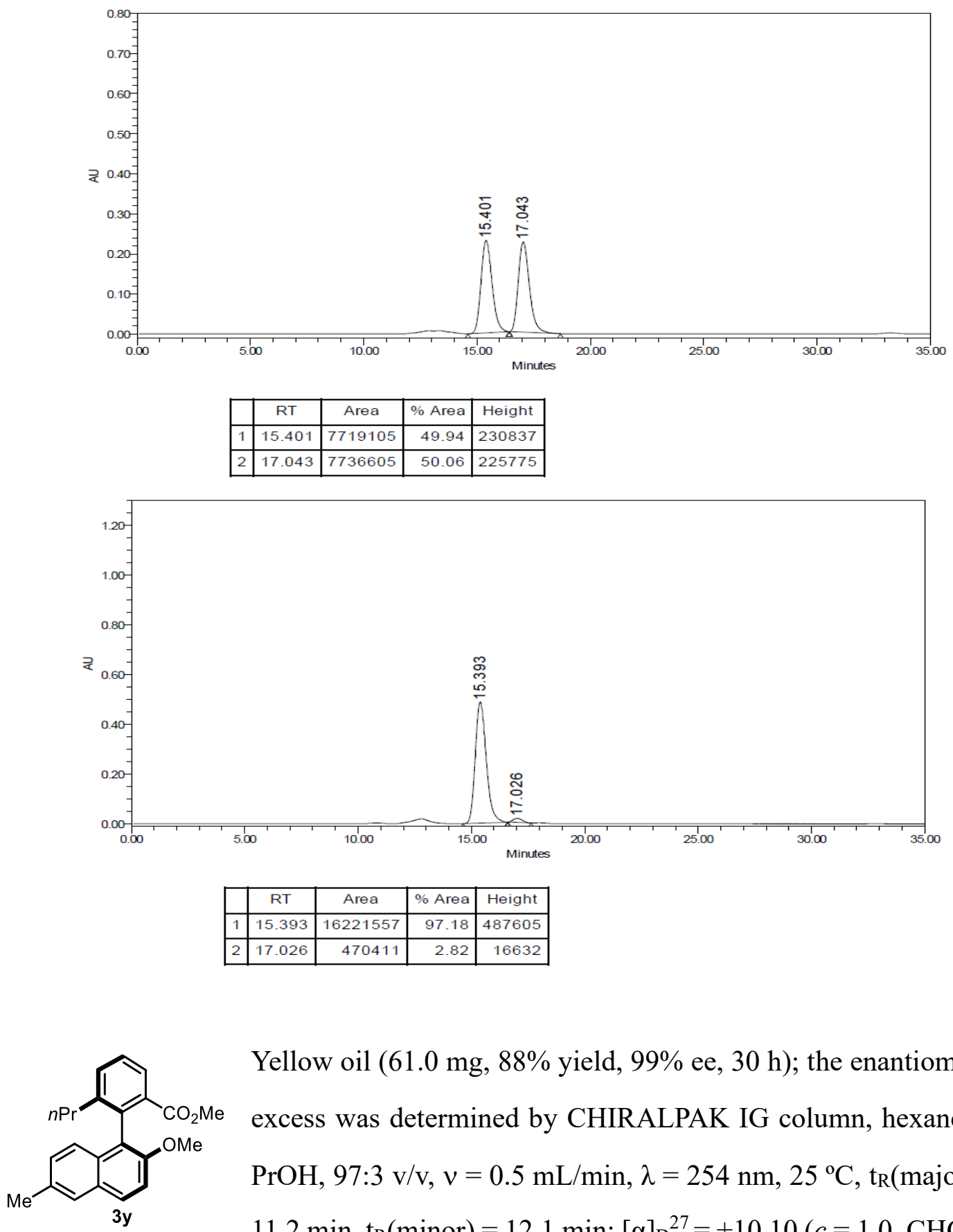

Yellow oil (61.0 mg, 88\% yield, 99\% ee, $30 \mathrm{~h}$ ); the enantiomeric excess was determined by CHIRALPAK IG column, hexanes $/ i$ PrOH, 97:3 v/v, $v=0.5 \mathrm{~mL} / \mathrm{min}, \lambda=254 \mathrm{~nm}, 25^{\circ} \mathrm{C}$, $\mathrm{t}_{\mathrm{R}}$ (major) $=$ $11.2 \mathrm{~min}, \mathrm{t}_{\mathrm{R}}(\operatorname{minor})=12.1 \mathrm{~min} ;[\alpha]_{\mathrm{D}}^{27}=+10.10\left(c=1.0, \mathrm{CHCl}_{3}\right)$. 
3y: ${ }^{1} \mathrm{H}$ NMR (400 MHz, $\left.\mathrm{CHCl}_{3}\right) \delta 7.84(\mathrm{dd}, J=7.6,1.2 \mathrm{~Hz}, 1 \mathrm{H}), 7.79(\mathrm{~d}, J=8.8 \mathrm{~Hz}$, 1H), $7.57(\mathrm{~s}, 1 \mathrm{H}), 7.52(\mathrm{dd}, J=8.0,1.2 \mathrm{~Hz}, 2 \mathrm{H}), 7.43(\mathrm{t}, J=8.0 \mathrm{~Hz}, 1 \mathrm{H}), 7.31(\mathrm{~d}, J=$ $9.2 \mathrm{~Hz}, 1 \mathrm{H}), 7.09$ (dd, $J=8.8,1.6 \mathrm{~Hz}, 1 \mathrm{H}), 6.98$ (d, $J=8.4 \mathrm{~Hz}, 1 \mathrm{H}), 3.79$ (s, 3H), 3.36 (s, 3H), 2.44 (s, 3H), $2.27-2.15(\mathrm{~m}, 2 \mathrm{H}), 1.41-1.26(\mathrm{~m}, 2 \mathrm{H}), 0.65$ (t, J=7.2 Hz, 3H); ${ }^{13} \mathrm{C} \mathrm{NMR}\left(101 \mathrm{MHz}, \mathrm{CHCl}_{3}\right) \delta 168.2,153.2,143.3,136.6,132.8,132.6,132.4,131.9$, $129.1,128.8,128.4,127.8,127.4,127.0,124.5,122.8,113.4,56.5,51.7,35.4,23.4$, 21.5, 14.1; FT-IR (neat): $v_{\max }=2956,2929,2869,2837,1728,1597,1458,1254,1190$, $1138,1126,1067,817,799,757 \mathrm{~cm}^{-1}$; HRMS (EI): exact mass calculated for: $\mathrm{C}_{23} \mathrm{H}_{24} \mathrm{O}_{3}$ $[\mathrm{M}]^{+}: 348.1720$, found 348.1720 .
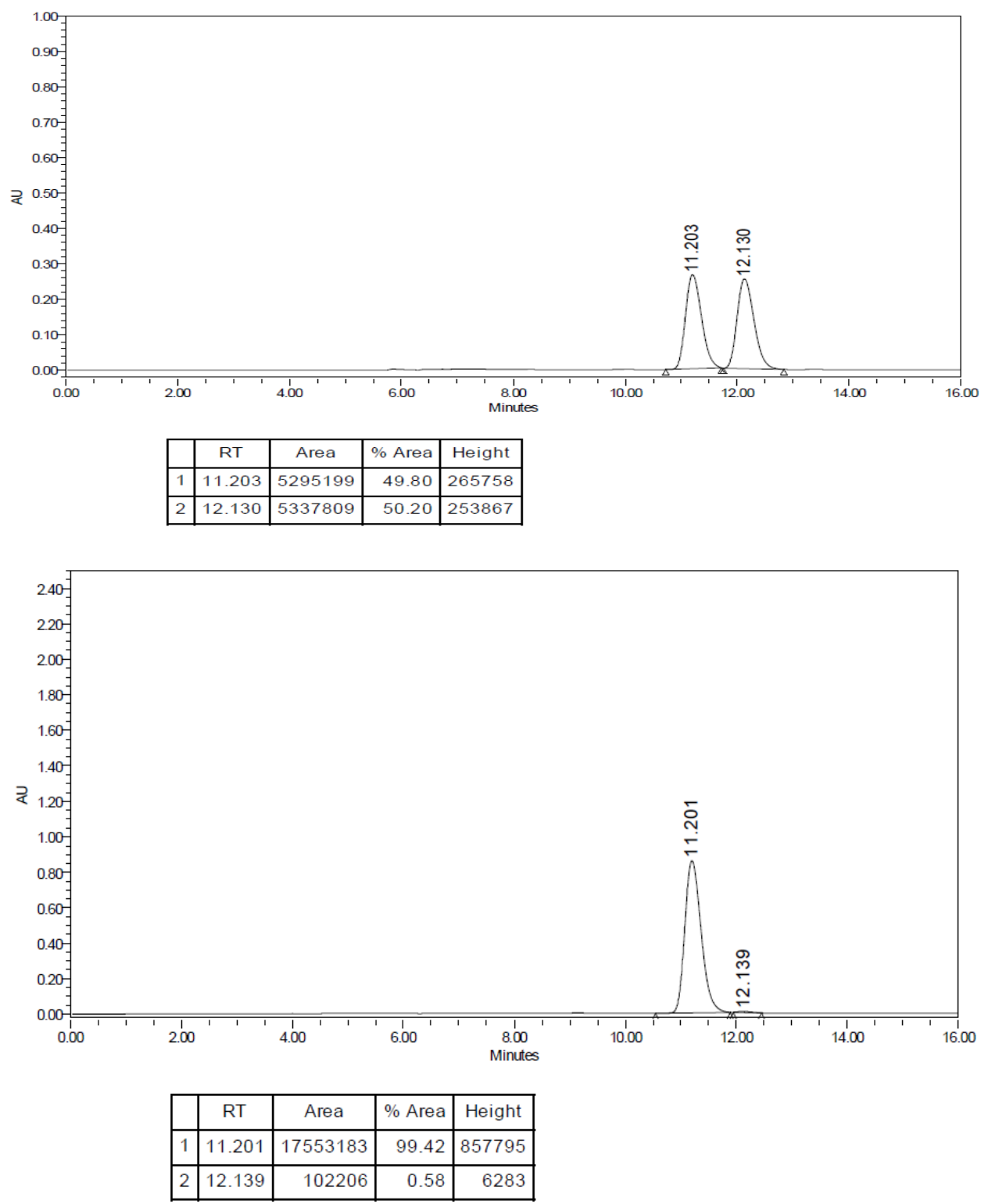


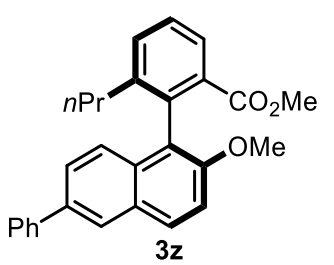

Yellow oil (76.1 mg, 93\% yield, 98\% ee, $42 \mathrm{~h}$ ); the enantiomeric excess was determined by CHIRALPAK IC column, hexanes $/ i$ PrOH, 97:3 v/v, v $=0.3 \mathrm{~mL} / \mathrm{min}, \lambda=254 \mathrm{~nm}, 25^{\circ} \mathrm{C}, \mathrm{t}_{\mathrm{R}}$ (major) $=$ $27.4 \min , \mathrm{t}_{\mathrm{R}}($ minor $)=29.9 \mathrm{~min} ;[\alpha]_{\mathrm{D}}{ }^{27}=+11.76\left(c=1.0, \mathrm{CHCl}_{3}\right)$.

3z: ${ }^{1} \mathrm{H}$ NMR (400 MHz, $\left.\mathrm{CHCl}_{3}\right) \delta 8.01(\mathrm{~d}, J=1.6 \mathrm{~Hz}, 1 \mathrm{H}), 7.94(\mathrm{~d}, J=9.2 \mathrm{~Hz}, 1 \mathrm{H})$, $7.88(\mathrm{dd}, J=7.6,0.8 \mathrm{~Hz}, 1 \mathrm{H}), 7.68(\mathrm{~d}, J=7.6 \mathrm{~Hz}, 2 \mathrm{H}), 7.56-7.52(\mathrm{~m}, 2 \mathrm{H}), 7.47-7.42$ (m, 3H), 7.37 (d, $J=8.8 \mathrm{~Hz}, 1 \mathrm{H}), 7.32$ (t, $J=7.2 \mathrm{~Hz}, 1 \mathrm{H}), 7.16(\mathrm{~d}, J=8.8 \mathrm{~Hz}, 1 \mathrm{H})$, $3.82(\mathrm{~s}, 3 \mathrm{H}), 3.40(\mathrm{~s}, 3 \mathrm{H}), 2.28-2.20(\mathrm{~m}, 2 \mathrm{H}), 1.41-1.31(\mathrm{~m}, 2 \mathrm{H}), 0.66(\mathrm{t}, J=7.2 \mathrm{~Hz}$, $3 \mathrm{H}),{ }^{13} \mathrm{C} \mathrm{NMR}\left(101 \mathrm{MHz}, \mathrm{CHCl}_{3}\right) \delta 168.1,153.9,143.3,141.2,136.4,136.0,132.9$, $132.7,132.3,129.5,129.1,128.9,127.9,127.5,127.3,127.1,126.1,125.9,125.2,122.8$, 113.6, 56.5, 51.8, 35.4, 23.4, 14.1; FT-IR (neat): $v_{\max }=2956,2931,2869,2838,1728$, 1594, 1492, 1254, 1190, 1174, 1129, 1068, 801, 754, $696 \mathrm{~cm}^{-1}$; HRMS (EI): exact mass calculated for: $\mathrm{C}_{28} \mathrm{H}_{26} \mathrm{O}_{3}[\mathrm{M}]^{+}: 410.1876$, found 410.1880 .

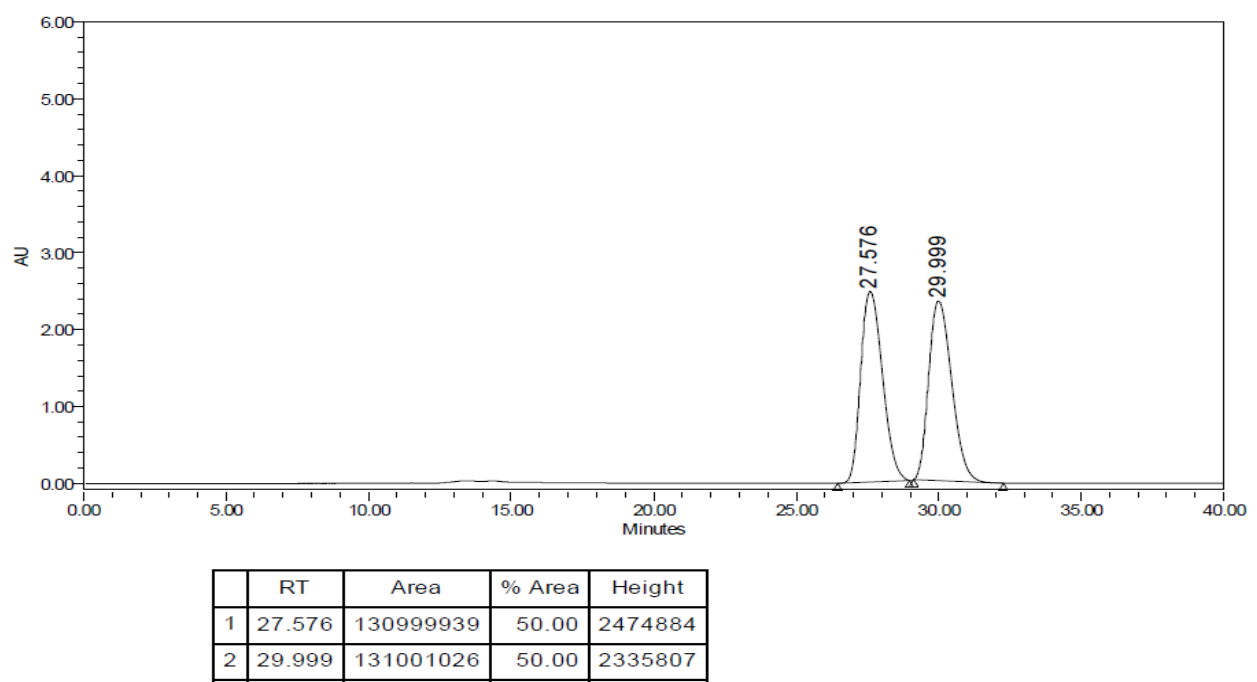



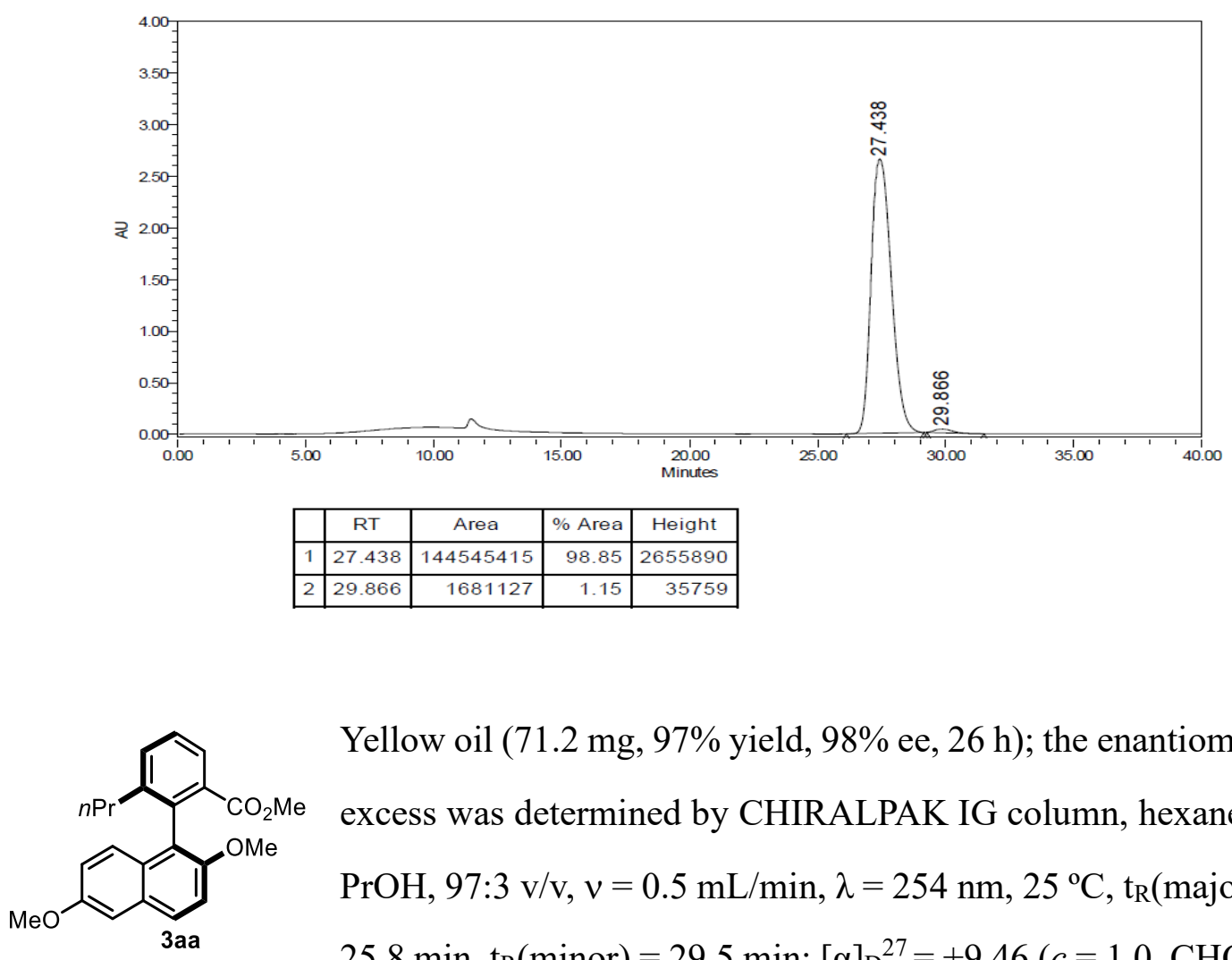

Yellow oil (71.2 mg, 97\% yield, 98\% ee, $26 \mathrm{~h}$ ); the enantiomeric excess was determined by CHIRALPAK IG column, hexanes $/ i$ PrOH, 97:3 v/v, $v=0.5 \mathrm{~mL} / \mathrm{min}, \lambda=254 \mathrm{~nm}, 25^{\circ} \mathrm{C}, \mathrm{t}_{\mathrm{R}}$ (major) $=$ $25.8 \mathrm{~min}, \mathrm{t}_{\mathrm{R}}($ minor $)=29.5 \mathrm{~min} ;[\alpha]_{\mathrm{D}}{ }^{27}=+9.46\left(c=1.0, \mathrm{CHCl}_{3}\right)$.

3aa: ${ }^{1} \mathrm{H}$ NMR $\left(400 \mathrm{MHz}, \mathrm{CHCl}_{3}\right) \delta 7.84(\mathrm{dd}, J=7.6,1.2 \mathrm{~Hz}, 1 \mathrm{H}), 7.77(\mathrm{~d}, J=8.8 \mathrm{~Hz}$, 1H), $7.52(\mathrm{dd}, J=8.0,1.2 \mathrm{~Hz}, 1 \mathrm{H}), 7.42(\mathrm{t}, J=8.0 \mathrm{~Hz}, 1 \mathrm{H}), 7.31(\mathrm{~d}, J=8.8 \mathrm{~Hz}, 1 \mathrm{H})$, $7.12(\mathrm{~d}, J=2.8 \mathrm{~Hz}, 1 \mathrm{H}), 7.00(\mathrm{~d}, J=9.6 \mathrm{~Hz}, 1 \mathrm{H}), 6.95(\mathrm{dd}, J=9.2,2.4 \mathrm{~Hz}, 1 \mathrm{H}), 3.88$ $(\mathrm{s}, 3 \mathrm{H}), 3.77(\mathrm{~s}, 3 \mathrm{H}), 3.38(\mathrm{~s}, 3 \mathrm{H}), 2.25-2.17(\mathrm{~m}, 2 \mathrm{H}), 1.39-1.24(\mathrm{~m}, 2 \mathrm{H}), 0.65(\mathrm{t}, J$ $=7.2 \mathrm{~Hz}, 3 \mathrm{H}) ;{ }^{13} \mathrm{C} \mathrm{NMR}\left(101 \mathrm{MHz}, \mathrm{CHCl}_{3}\right) \delta 168.1,155.9,152.3,143.2,136.5,132.6$, $132.3,129.8,129.1,127.73,127.66,127.4,126.2,123.3,119.2,114.0,105.9,56.6,55.3$, 51.7, 35.4, 23.4, 14.0; FT-IR (neat): $v_{\max }=2952,2928,2827,2838,1702,1595,1508$, $1459,1298,1276,1259,1246,1195,1168,1142,1117,1099,1074,1028,876,823,806$, $758 \mathrm{~cm}^{-1}$; HRMS (EI): exact mass calculated for: $\mathrm{C}_{23} \mathrm{H}_{24} \mathrm{O}_{4}[\mathrm{M}]^{+}: 364.1669$, found 364.1670. 

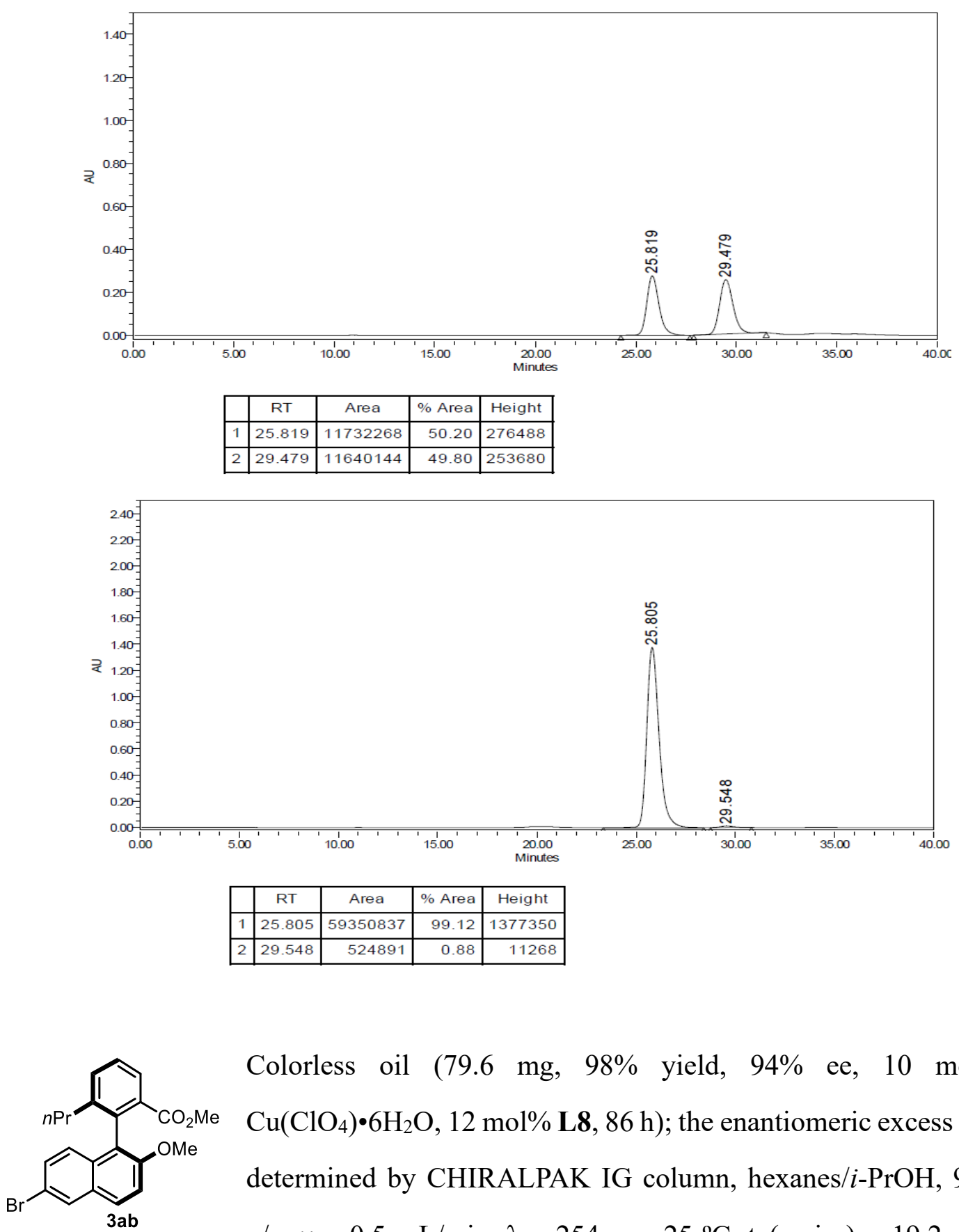

Colorless oil (79.6 mg, $98 \%$ yield, $94 \%$ ee, $10 \mathrm{~mol} \%$ $\left.\mathrm{Cu}\left(\mathrm{ClO}_{4}\right) \cdot 6 \mathrm{H}_{2} \mathrm{O}, 12 \mathrm{~mol} \% \mathbf{L 8}, 86 \mathrm{~h}\right)$; the enantiomeric excess was determined by CHIRALPAK IG column, hexanes/i-PrOH, 97:3 $\mathrm{v} / \mathrm{v}, v=0.5 \mathrm{~mL} / \mathrm{min}, \lambda=254 \mathrm{~nm}, 25^{\circ} \mathrm{C}, \mathrm{t}_{\mathrm{R}}($ major $)=19.2 \mathrm{~min}$, $\mathrm{t}_{\mathrm{R}}($ minor $)=21.1 \mathrm{~min} ;[\alpha]_{\mathrm{D}}^{25}=+16.92\left(c=1.0, \mathrm{CHCl}_{3}\right)$.

3ab: ${ }^{1} \mathrm{H}$ NMR (400 MHz, $\left.\mathrm{CHCl}_{3}\right) \delta 7.96(\mathrm{~d}, J=2.0 \mathrm{~Hz}, 1 \mathrm{H}), 7.87(\mathrm{dd}, J=7.6,0.8 \mathrm{~Hz}$, 1H), $7.79(\mathrm{~d}, J=8.8 \mathrm{~Hz}, 1 \mathrm{H}), 7.53(\mathrm{~d}, J=6.8 \mathrm{~Hz}, 1 \mathrm{H}), 7.45(\mathrm{t}, J=8.0 \mathrm{~Hz}, 1 \mathrm{H}), 7.36(\mathrm{~d}$, $J=9.2 \mathrm{~Hz}, 1 \mathrm{H}), 7.31(\mathrm{dd}, J=8.8,2.0 \mathrm{~Hz}, 1 \mathrm{H}), 6.96(\mathrm{~d}, J=9.2 \mathrm{~Hz}, 1 \mathrm{H}), 3.80(\mathrm{~s}, 3 \mathrm{H})$, $3.40(\mathrm{~s}, 3 \mathrm{H}), 2.23-2.11(\mathrm{~m}, 2 \mathrm{H}), 1.39-1.24(\mathrm{~m}, 2 \mathrm{H}), 0.65(\mathrm{t}, J=7.2 \mathrm{~Hz}, 3 \mathrm{H}) ;{ }^{13} \mathrm{C}$ $\mathrm{NMR}\left(101 \mathrm{MHz}, \mathrm{CHCl}_{3}\right) \delta 167.8,154.0,143.2,135.9,132.9,132.2,132.1,130.0,129.9$, 
$129.7,128.3,128.0,127.7,126.5,123.2,117.2,114.2,56.5,51.8,35.4,23.4,14.0$; FTIR (neat): $v_{\max }=2945,2928,2869,2853,1722,1585,1495,1459,1255,1191,1139$, 1128, 1069, 1055, 906, 812, 799, $757 \mathrm{~cm}^{-1}$; HRMS (EI): exact mass calculated for: $\mathrm{C}_{22} \mathrm{H}_{21} \mathrm{BrO}_{3}[\mathrm{M}]^{+}:$412.0669, found 412.0670.
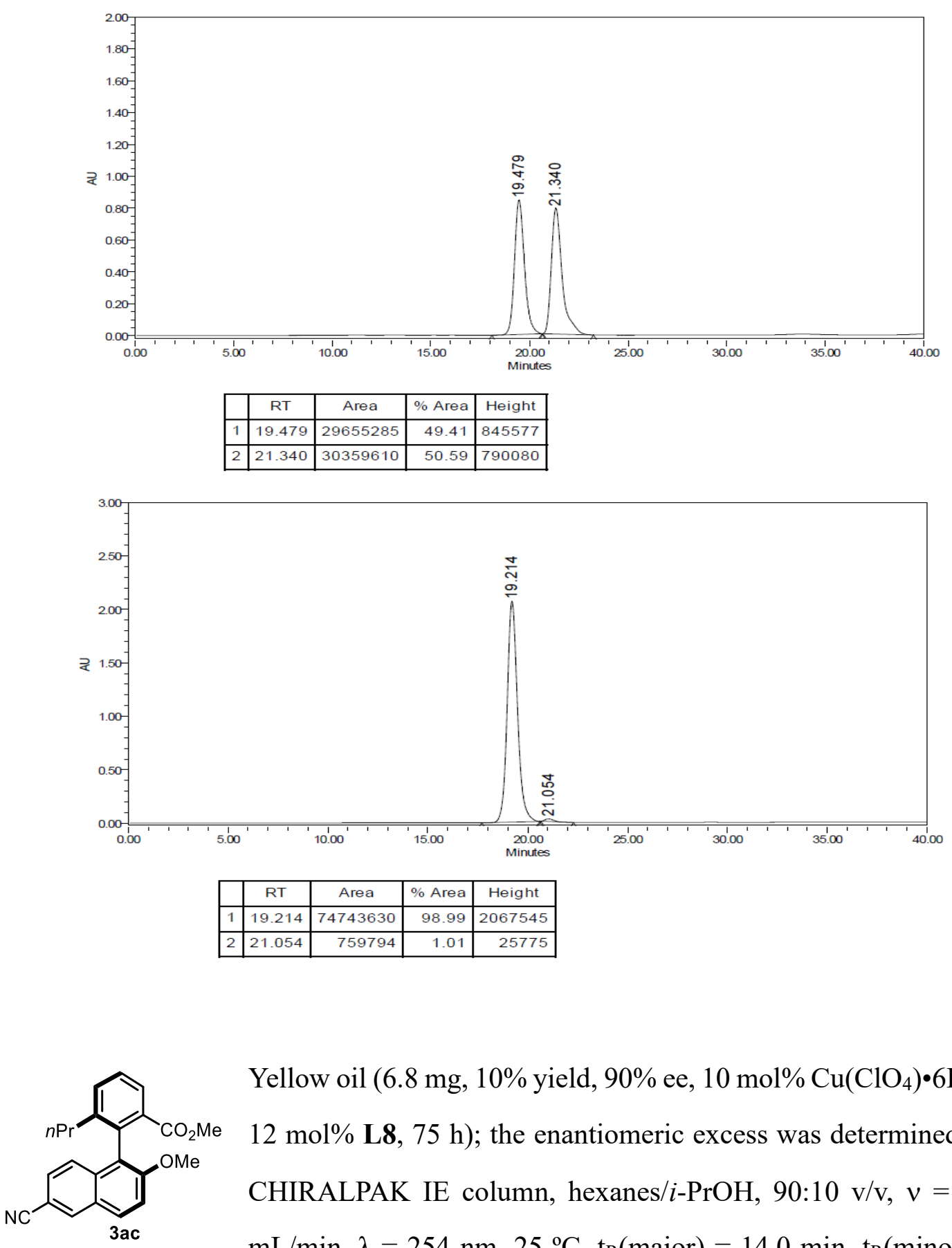

Yellow oil (6.8 mg, 10\% yield, $90 \%$ ee, $10 \mathrm{~mol} \% \mathrm{Cu}\left(\mathrm{ClO}_{4}\right) \cdot 6 \mathrm{H}_{2} \mathrm{O}$, $12 \mathrm{~mol} \% \mathbf{L 8}, 75 \mathrm{~h}$ ); the enantiomeric excess was determined by CHIRALPAK IE column, hexanes/i-PrOH, 90:10 v/v, $v=1.0$ $\mathrm{mL} / \mathrm{min}, \lambda=254 \mathrm{~nm}, 25^{\circ} \mathrm{C}, \mathrm{t}_{\mathrm{R}}($ major $)=14.0 \mathrm{~min}, \mathrm{t}_{\mathrm{R}}($ minor $)=$ $15.3 \mathrm{~min} ;[\alpha]_{\mathrm{D}}^{21}=+20.10\left(c=0.2, \mathrm{CHCl}_{3}\right)$. 
3ac: ${ }^{1} \mathrm{H}$ NMR $\left(400 \mathrm{MHz}, \mathrm{CHCl}_{3}\right) \delta 8.21(\mathrm{~d}, J=1.2 \mathrm{~Hz}, 1 \mathrm{H}), 7.96(\mathrm{~d}, J=8.8 \mathrm{~Hz}, 1 \mathrm{H})$, $7.91(\mathrm{dd}, J=8.0,1.2 \mathrm{~Hz}, 1 \mathrm{H}), 7.56(\mathrm{dd}, J=8.0,1.2 \mathrm{~Hz}, 1 \mathrm{H}), 7.49-7.45(\mathrm{~m}, 2 \mathrm{H}), 7.37$ $(\mathrm{dd}, J=8.8,1.2 \mathrm{~Hz}, 1 \mathrm{H}), 7.16(\mathrm{~d}, J=8.8 \mathrm{~Hz}, 1 \mathrm{H}), 3.85$ (s, 3H), $3.43(\mathrm{~s}, 3 \mathrm{H}), 2.21-$ $2.08(\mathrm{~m}, 2 \mathrm{H}), 1.35-1.27(\mathrm{~m}, 2 \mathrm{H}), 0.65$ (t, J=7.2 Hz, 3H); ${ }^{13} \mathrm{C} \mathrm{NMR}\left(101 \mathrm{MHz}, \mathrm{CHCl}_{3}\right)$ $\delta 167.6,156.2,143.2,135.3,135.1,134.6,133.1,131.8,123.0,128.2,128.0,127.5$, $126.8,125.8,123.5,119.8,114.5,106.7,56.4,51.9,35.4,23.5,14.0$; IR (neat): $v_{\max }=$ 2957, 2925, 2852, 2223, 1725, 1619, 1480, 1458, 1274, 1258, 1139, 1100, 1055, 801, $759 \mathrm{~cm}^{-1}$; HRMS (EI): exact mass calculated for: $\mathrm{C}_{23} \mathrm{H}_{21} \mathrm{NO}_{3}[\mathrm{M}]^{+}: 359.1516$, found 359.1518 .
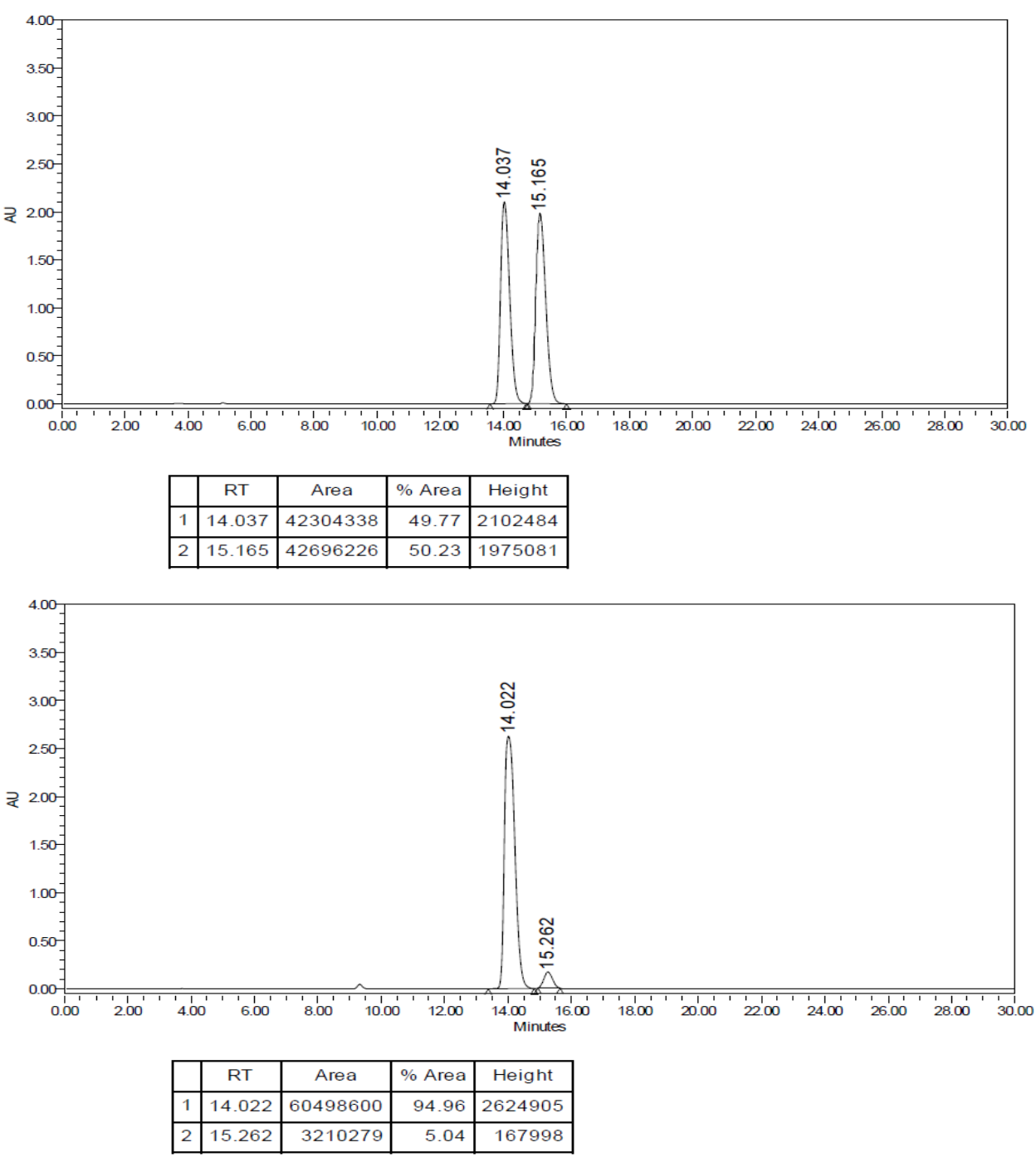


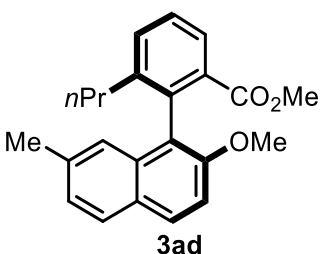

$\left.1.0, \mathrm{CHCl}_{3}\right)$.

Yellow oil (64.9 mg, 93\% yield, 93\% ee, $36 \mathrm{~h}$ ); the enantiomeric excess was determined by CHIRALCEL OD-H column, hexanes $/ i-\mathrm{PrOH}, 98: 2 \mathrm{v} / \mathrm{v}, v=0.3 \mathrm{~mL} / \mathrm{min}, \lambda=254 \mathrm{~nm}, 25^{\circ} \mathrm{C}$, $\mathrm{t}_{\mathrm{R}}($ major $)=22.9 \mathrm{~min}, \mathrm{t}_{\mathrm{R}}($ minor $)=20.5 \mathrm{~min} ;[\alpha]_{\mathrm{D}}^{27}=+27.86(c=$

3ad: ${ }^{1} \mathrm{H}$ NMR $\left(400 \mathrm{MHz}, \mathrm{CHCl}_{3}\right) \delta 7.86(\mathrm{dd}, J=7.6,0.8 \mathrm{~Hz}, 1 \mathrm{H}), 7.82(\mathrm{~d}, J=9.2 \mathrm{~Hz}$, 1H), $7.69(\mathrm{~d}, J=8.4 \mathrm{~Hz}, 1 \mathrm{H}), 7.54(\mathrm{dd}, J=8.0,1.2 \mathrm{~Hz}, 1 \mathrm{H}), 7.44(\mathrm{t}, J=8.0 \mathrm{~Hz}, 1 \mathrm{H})$, $7.26(\mathrm{~d}, J=9.2 \mathrm{~Hz}, 1 \mathrm{H}), 7.12(\mathrm{dd}, J=8.4,1.2 \mathrm{~Hz}, 1 \mathrm{H}), 6.83(\mathrm{~s}, 1 \mathrm{H}), 3.79$ (s, 3H), 3.37 $(\mathrm{s}, 3 \mathrm{H}), 2.30(\mathrm{~s}, 3 \mathrm{H}), 2.24-2.16(\mathrm{~m}, 2 \mathrm{H}), 1.42-1.28(\mathrm{~m}, 2 \mathrm{H}), 0.65(\mathrm{t}, J=7.2 \mathrm{~Hz}, 3 \mathrm{H})$; ${ }^{13} \mathrm{C}$ NMR $\left(101 \mathrm{MHz}, \mathrm{CHCl}_{3}\right) \delta 168.2,153.8,143.3,136.7,136.0,133.8,132.7,132.3$, $128.8,127.9,127.8,127.4,127.2,125.8,123.5,122.3,112.3,56.4,51.7,35.4,23.4$, 22.1, 14.0; FT-IR (neat): $v_{\max }=2950,1726,1509,1449,1371,1249,1184,1136,1063$, 825, $754 \mathrm{~cm}^{-1}$; HRMS (EI): exact mass calculated for: $\mathrm{C}_{23} \mathrm{H}_{24} \mathrm{O}_{3}[\mathrm{M}]^{+}: 348.1720$, found 348.1719.

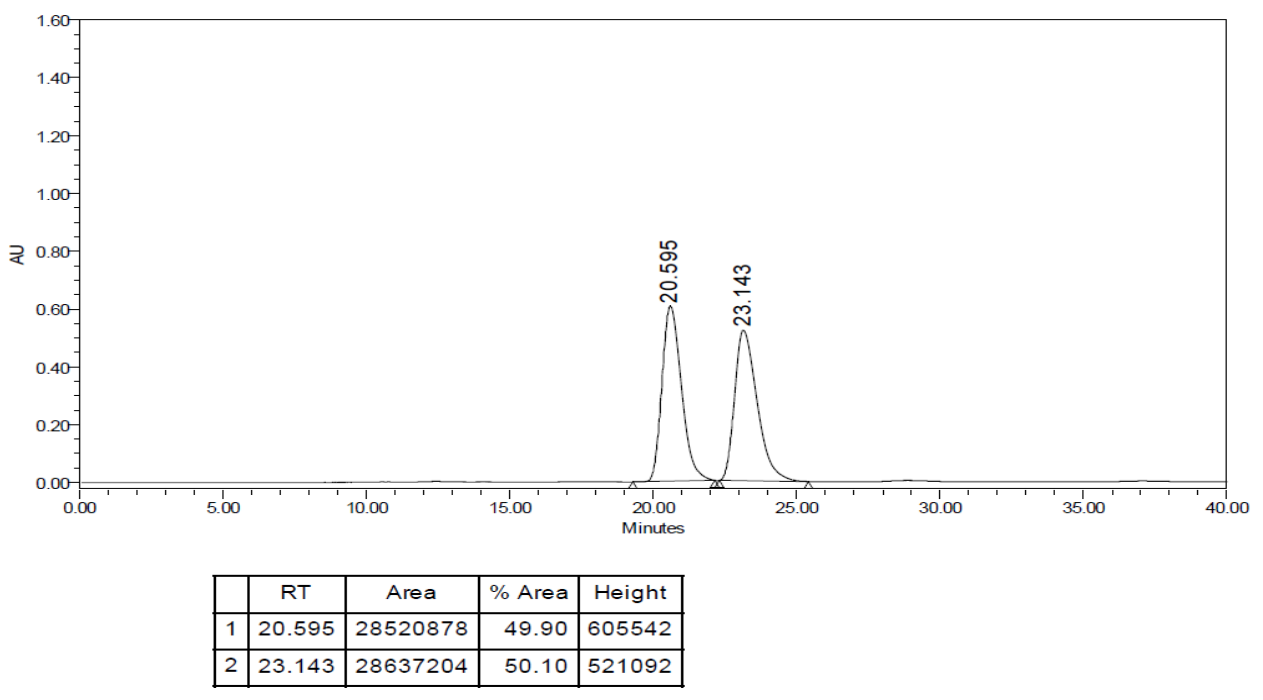



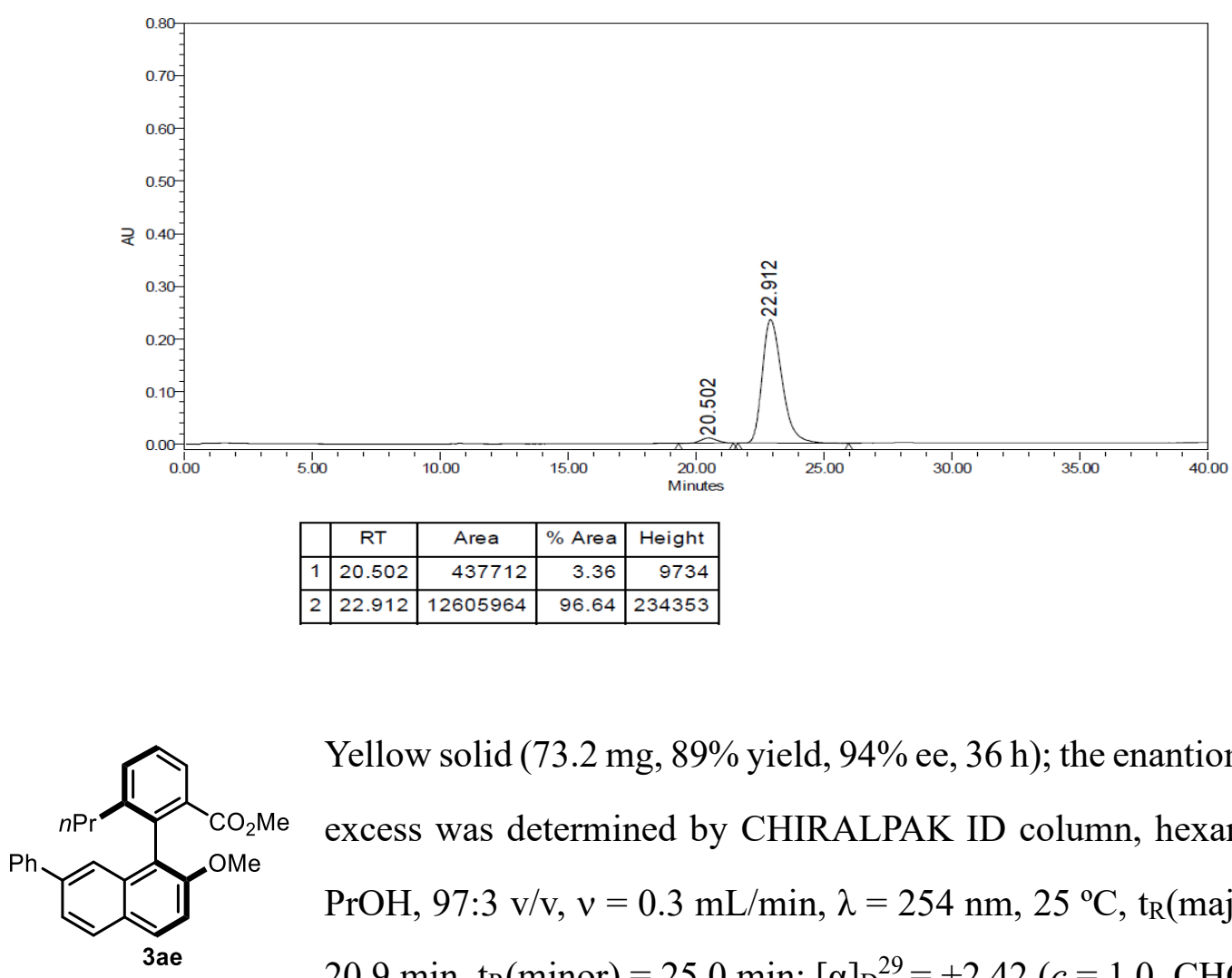

Yellow solid (73.2 mg, 89\% yield, 94\% ee, $36 \mathrm{~h}$ ); the enantiomeric excess was determined by CHIRALPAK ID column, hexanes $/ i$ $\mathrm{PrOH}, 97: 3 \mathrm{v} / \mathrm{v}, \mathrm{v}=0.3 \mathrm{~mL} / \mathrm{min}, \lambda=254 \mathrm{~nm}, 25^{\circ} \mathrm{C}, \mathrm{t}_{\mathrm{R}}$ (major) $=$ $20.9 \min , \mathrm{t}_{\mathrm{R}}($ minor $)=25.0 \mathrm{~min} ;[\alpha]_{\mathrm{D}}^{29}=+2.42\left(c=1.0, \mathrm{CHCl}_{3}\right)$.

3ae: ${ }^{1} \mathrm{H}$ NMR (400 MHz, $\left.\mathrm{CHCl}_{3}\right) \delta 7.92(\mathrm{~d}, J=9.2 \mathrm{~Hz}, 1 \mathrm{H}), 7.89(\mathrm{~d}, J=8.4 \mathrm{~Hz}, 2 \mathrm{H})$, $7.59-7.55(\mathrm{~m}, 2 \mathrm{H}), 7.51-7.49(\mathrm{~m}, 2 \mathrm{H}), 7.46(\mathrm{t}, J=8.0 \mathrm{~Hz}, 1 \mathrm{H}), 7.40-7.36(\mathrm{~m}, 3 \mathrm{H})$, $7.32-7.28(\mathrm{~m}, 2 \mathrm{H}), 3.84(\mathrm{~s}, 3 \mathrm{H}), 3.40(\mathrm{~s}, 3 \mathrm{H}), 2.29-2.25(\mathrm{~m}, 2 \mathrm{H}), 1.44-1.32(\mathrm{~m}$, 2H), $0.68(\mathrm{t}, J=7.6 \mathrm{~Hz}, 3 \mathrm{H}) ;{ }^{13} \mathrm{C} \mathrm{NMR}\left(101 \mathrm{MHz}, \mathrm{CHCl}_{3}\right) \delta 168.1,154.1,143.3,141.7$, 139.0, 136.3, 133.9, 132.8, 132.4, 128.9, 128.8, 128.6, 128.1, 127.9, 127.57, 127.55, 127.2, 123.33, 123.26, 122.6, 113.3, 56.5, 51.7, 35.5, 23.5, 14.1; FT-IR (neat): $v_{\max }=$ 2957, 2932, 1731, 1622, 1491, 1446, 1285, 1243, 1139, 1070, 1042, 834, 760, $698 \mathrm{~cm}^{-1}$; HRMS (EI): exact mass calculated for: $\mathrm{C}_{28} \mathrm{H}_{26} \mathrm{O}_{3}[\mathrm{M}]^{+}: 410.1876$, found 410.1877 . 

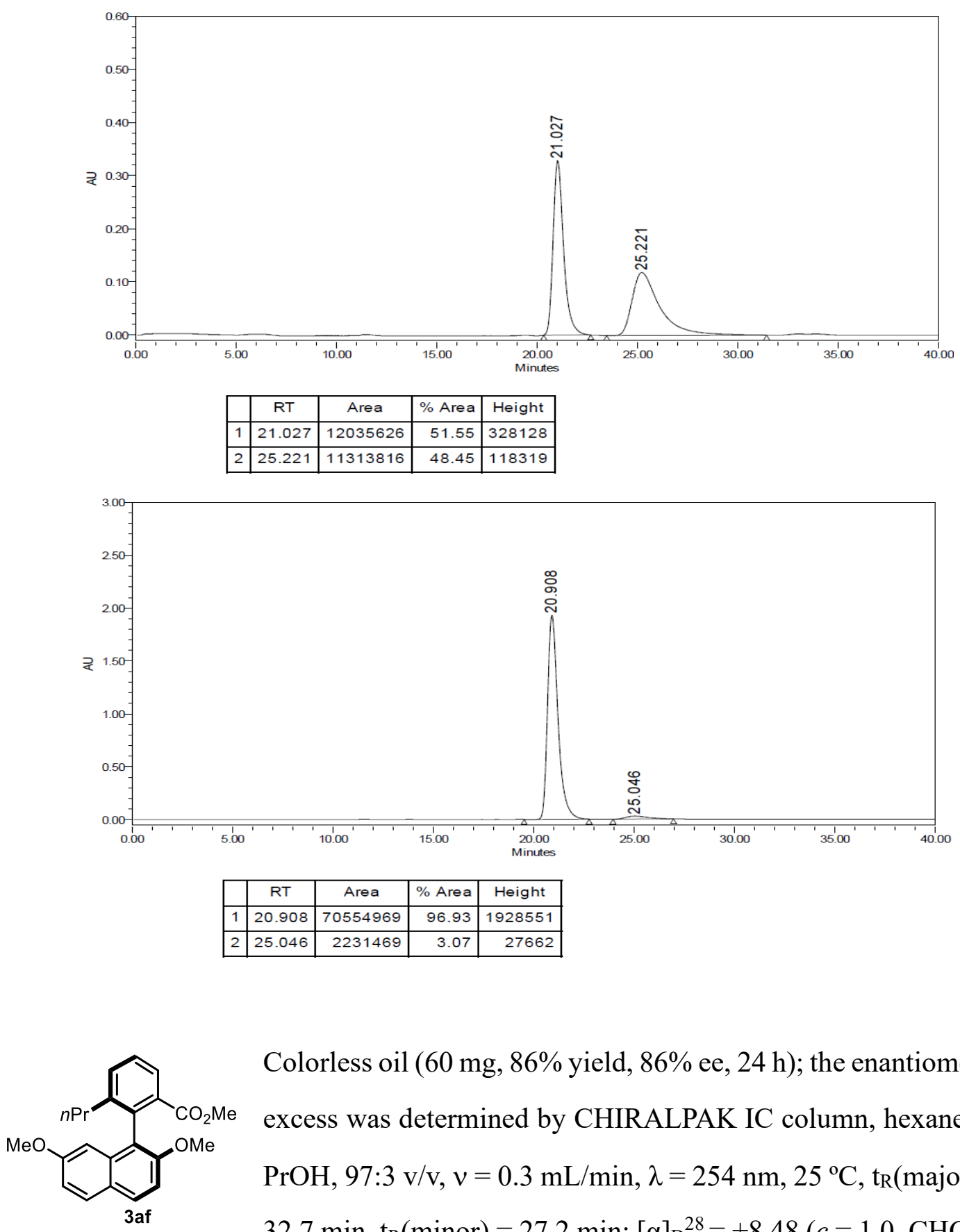

Colorless oil ( $60 \mathrm{mg}, 86 \%$ yield, $86 \%$ ee, $24 \mathrm{~h}$ ); the enantiomeric excess was determined by CHIRALPAK IC column, hexanes/ $i$ $\mathrm{PrOH}, 97: 3 \mathrm{v} / \mathrm{v}, v=0.3 \mathrm{~mL} / \mathrm{min}, \lambda=254 \mathrm{~nm}, 25^{\circ} \mathrm{C}$, $\mathrm{t}_{\mathrm{R}}$ (major) $=$ $32.7 \mathrm{~min}, \mathrm{t}_{\mathrm{R}}($ minor $)=27.2 \mathrm{~min} ;[\alpha]_{\mathrm{D}}{ }^{28}=+8.48\left(c=1.0, \mathrm{CHCl}_{3}\right)$.

3af: ${ }^{1} \mathrm{H}$ NMR $\left(400 \mathrm{MHz}, \mathrm{CHCl}_{3}\right) \delta 7.86(\mathrm{dd}, J=7.6,1.2 \mathrm{~Hz}, 1 \mathrm{H}), 7.80(\mathrm{~d}, J=8.8 \mathrm{~Hz}$, 1H), $7.70(\mathrm{~d}, J=8.8 \mathrm{~Hz}, 1 \mathrm{H}), 7.54(\mathrm{dd}, J=7.6,0.8 \mathrm{~Hz}, 1 \mathrm{H}), 7.44(\mathrm{t}, J=7.6 \mathrm{~Hz}, 1 \mathrm{H})$, $7.19(\mathrm{~d}, J=9.2 \mathrm{~Hz}, 1 \mathrm{H}), 6.97(\mathrm{dd}, J=9.2,2.8 \mathrm{~Hz}, 1 \mathrm{H}), 6.36(\mathrm{~d}, J=2.4 \mathrm{~Hz}, 1 \mathrm{H}), 3.80$ (s, 3H), $3.62(\mathrm{~s}, 3 \mathrm{H}), 3.39(\mathrm{~s}, 3 \mathrm{H}), 2.29-2.21(\mathrm{~m}, 2 \mathrm{H}), 1.41-1.31(\mathrm{~m}, 2 \mathrm{H}), 0.67(\mathrm{t}, J$ $=7.6 \mathrm{~Hz}, 3 \mathrm{H}) ;{ }^{13} \mathrm{C} \mathrm{NMR}\left(101 \mathrm{MHz}, \mathrm{CHCl}_{3}\right) \delta 168.2,158.1,154.4,143.2,136.6,134.9$, $132.7,132.4,129.6,128.8,127.9,127.4,124.5,121.9,116.0,110.7,103.1,56.4,55.1$, 
51.7, 35.4, 23.4, 14.0; FT-IR (neat): $v_{\max }=2956,1730,1623,1510,1462,1254,1224$, 1182, 1137, 1068, 1032, 826, $756 \mathrm{~cm}^{-1}$; HRMS (EI): exact mass calculated for: $\mathrm{C}_{23} \mathrm{H}_{24} \mathrm{O}_{4}[\mathrm{M}]^{+}: 364.1669$, found 364.1672.
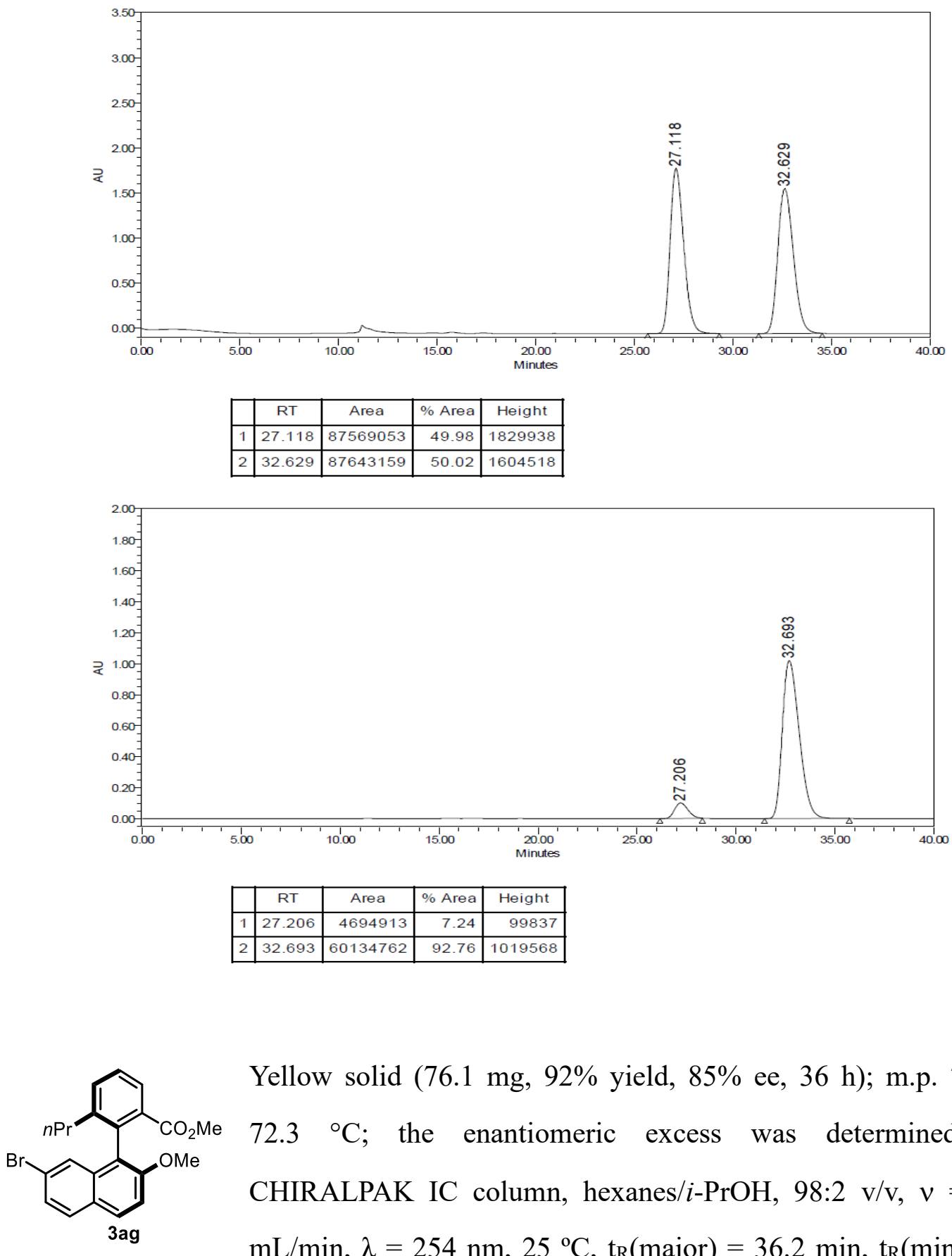

Yellow solid (76.1 mg, 92\% yield, 85\% ee, 36 h); m.p. 71.5$72.3{ }^{\circ} \mathrm{C}$; the enantiomeric excess was determined by CHIRALPAK IC column, hexanes $/ i-\mathrm{PrOH}, 98: 2 \mathrm{v} / \mathrm{v}, v=0.3$ $\mathrm{mL} / \min , \lambda=254 \mathrm{~nm}, 25^{\circ} \mathrm{C}, \mathrm{t}_{\mathrm{R}}($ major $)=36.2 \mathrm{~min}, \mathrm{t}_{\mathrm{R}}($ minor $)=$ $33.4 \mathrm{~min} ;[\alpha]_{\mathrm{D}}^{25}=+13.84\left(c=1.0, \mathrm{CHCl}_{3}\right)$. 
3ag: ${ }^{1} \mathrm{H}$ NMR $\left(400 \mathrm{MHz}, \mathrm{CHCl}_{3}\right) \delta 7.90(\mathrm{dd}, J=8.0,1.2 \mathrm{~Hz}, 1 \mathrm{H}), 7.85(\mathrm{~d}, J=9.2 \mathrm{~Hz}$, 1H), $7.67(\mathrm{~d}, J=8.8 \mathrm{~Hz}, 1 \mathrm{H}), 7.55(\mathrm{~d}, J=7.2 \mathrm{~Hz}, 1 \mathrm{H}), 7.46(\mathrm{t}, J=8.0 \mathrm{~Hz}, 1 \mathrm{H}), 7.37-$ $7.33(\mathrm{~m}, 2 \mathrm{H}), 7.22(\mathrm{~d}, J=1.6 \mathrm{~Hz}, 1 \mathrm{H}), 3.80(\mathrm{~s}, 3 \mathrm{H}), 3.43(\mathrm{~s}, 3 \mathrm{H}), 2.23-2.10(\mathrm{~m}, 2 \mathrm{H})$, $1.40-1.30(\mathrm{~m}, 2 \mathrm{H}), 0.66(\mathrm{t}, J=7.6 \mathrm{~Hz}, 3 \mathrm{H}) ;{ }^{13} \mathrm{C} \mathrm{NMR}\left(101 \mathrm{MHz}, \mathrm{CHCl}_{3}\right) \delta 167.7$, $154.5,143.2,135.8,134.9,133.0,131.9,129.8,129.1$, 128.2, 127.8, 127.2 , 126.9, 126.6, 122.4, 121.0, 113.5, 56.4, 51.8, 35.4, 23.4, 14.0; FT-IR (neat): $v_{\max }=2927,2852,1723$, 1614, 1497, 1458, 1251, 1136, 1065, 924, 823, $754 \mathrm{~cm}^{-1}$; HRMS (EI): exact mass calculated for: $\mathrm{C}_{22} \mathrm{H}_{21} \mathrm{BrO}_{3}[\mathrm{M}]^{+}: 412.0669$, found 412.0666.
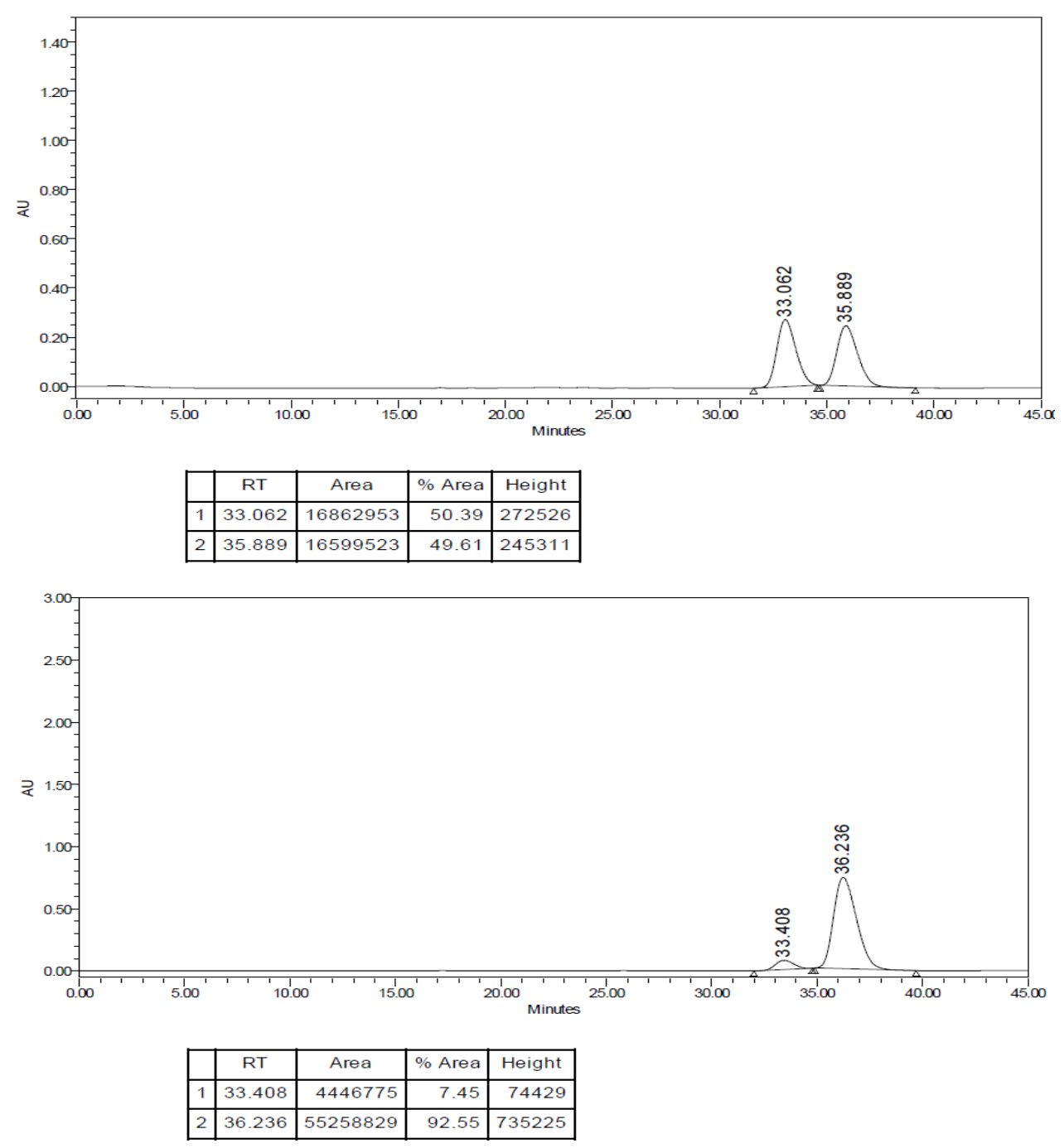


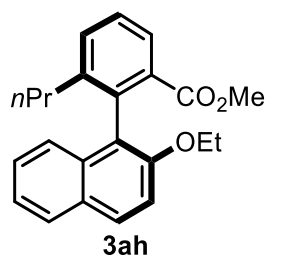

Yellow oil (64 mg, 93\% yield, 97\% ee, $24 \mathrm{~h}$ ); the enantiomeric excess was determined by CHIRALPAK IE column, hexanes/i-PrOH, 98:2 $\mathrm{v} / \mathrm{v}, v=0.2 \mathrm{~mL} / \mathrm{min}, \lambda=254 \mathrm{~nm}, 25^{\circ} \mathrm{C}, \mathrm{t}_{\mathrm{R}}$ (major) $=34.0 \mathrm{~min}$, $\mathrm{t}_{\mathrm{R}}(\operatorname{minor})=31.2 \mathrm{~min} ;[\alpha]_{\mathrm{D}}^{25}=+22.98\left(c=1.0, \mathrm{CHCl}_{3}\right)$.

3ah: ${ }^{1} \mathrm{H}$ NMR $\left(400 \mathrm{MHz}, \mathrm{CHCl}_{3}\right) \delta 7.85(\mathrm{~d}, J=8.4 \mathrm{~Hz}, 2 \mathrm{H}), 7.79(\mathrm{dd}, J=6.8,1.6 \mathrm{~Hz}$, $1 \mathrm{H}), 7.52(\mathrm{~d}, J=6.8 \mathrm{~Hz}, 1 \mathrm{H}), 7.42(\mathrm{t}, J=7.6 \mathrm{~Hz}, 1 \mathrm{H}), 7.31(\mathrm{~d}, J=8.8 \mathrm{~Hz}, 1 \mathrm{H}), 7.29-$ $7.23(\mathrm{~m}, 2 \mathrm{H}), 7.10(\mathrm{~d}, J=8.0 \mathrm{~Hz}, 1 \mathrm{H}), 4.07$ (q, $J=6.8 \mathrm{~Hz}, 2 \mathrm{H}), 3.35(\mathrm{~s}, 3 \mathrm{H}), 2.26-$ $2.16(\mathrm{~m}, 2 \mathrm{H}), 1.41-1.26(\mathrm{~m}, 2 \mathrm{H}), 1.18(\mathrm{t}, J=6.8 \mathrm{~Hz}, 3 \mathrm{H}), 0.64(\mathrm{t}, J=7.2 \mathrm{~Hz}, 3 \mathrm{H}) ;{ }^{13} \mathrm{C}$ NMR $\left(101 \mathrm{MHz}, \mathrm{CHCl}_{3}\right) \delta 168.2,153.0,143.3,136.7,133.8,132.6,132.4,129.0,128.9$, $128.0,127.7,127.3,126.3,124.7,123.42,123.35,114.5,64.6,51.7,35.4,23.4,15.1$, 14.0; FT-IR (neat): $v_{\max }=2958,2929,2871,1730,1593,1507,1431,1251,1138,1120$, 1058, 805, $747 \mathrm{~cm}^{-1}$; HRMS (EI): exact mass calculated for: $\mathrm{C}_{23} \mathrm{H}_{24} \mathrm{O}_{3}[\mathrm{M}]^{+}: 348.1720$, found 348.1721 .

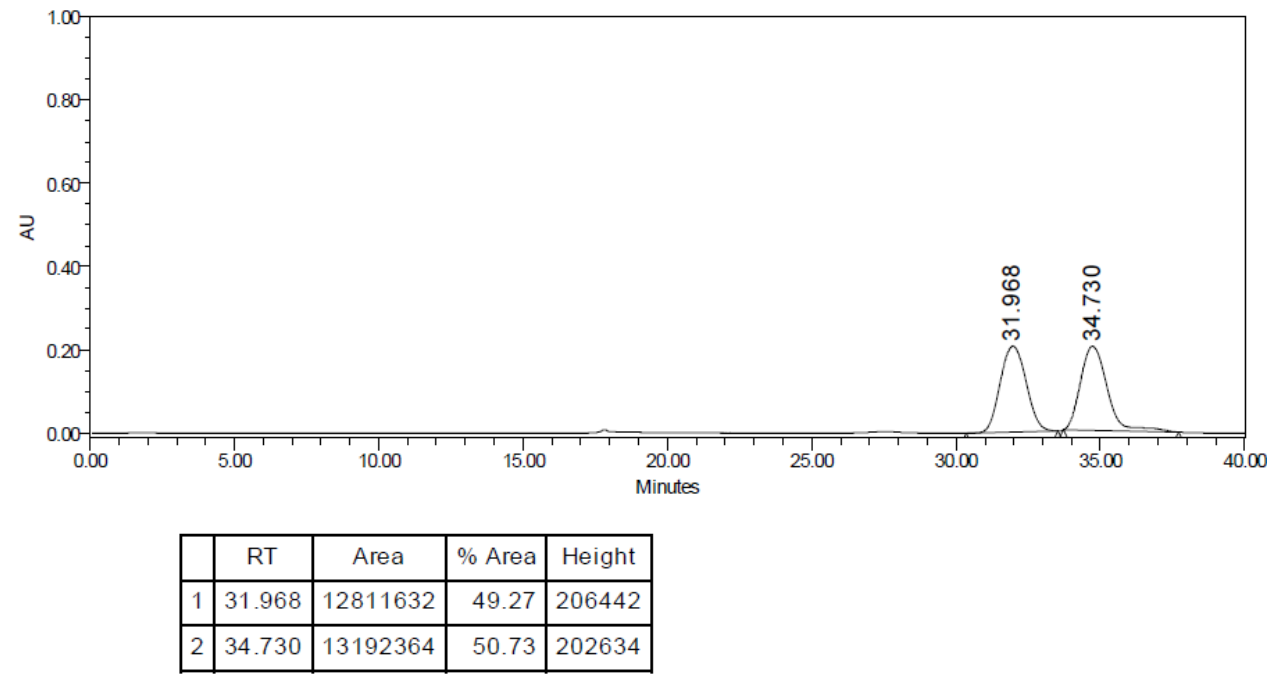



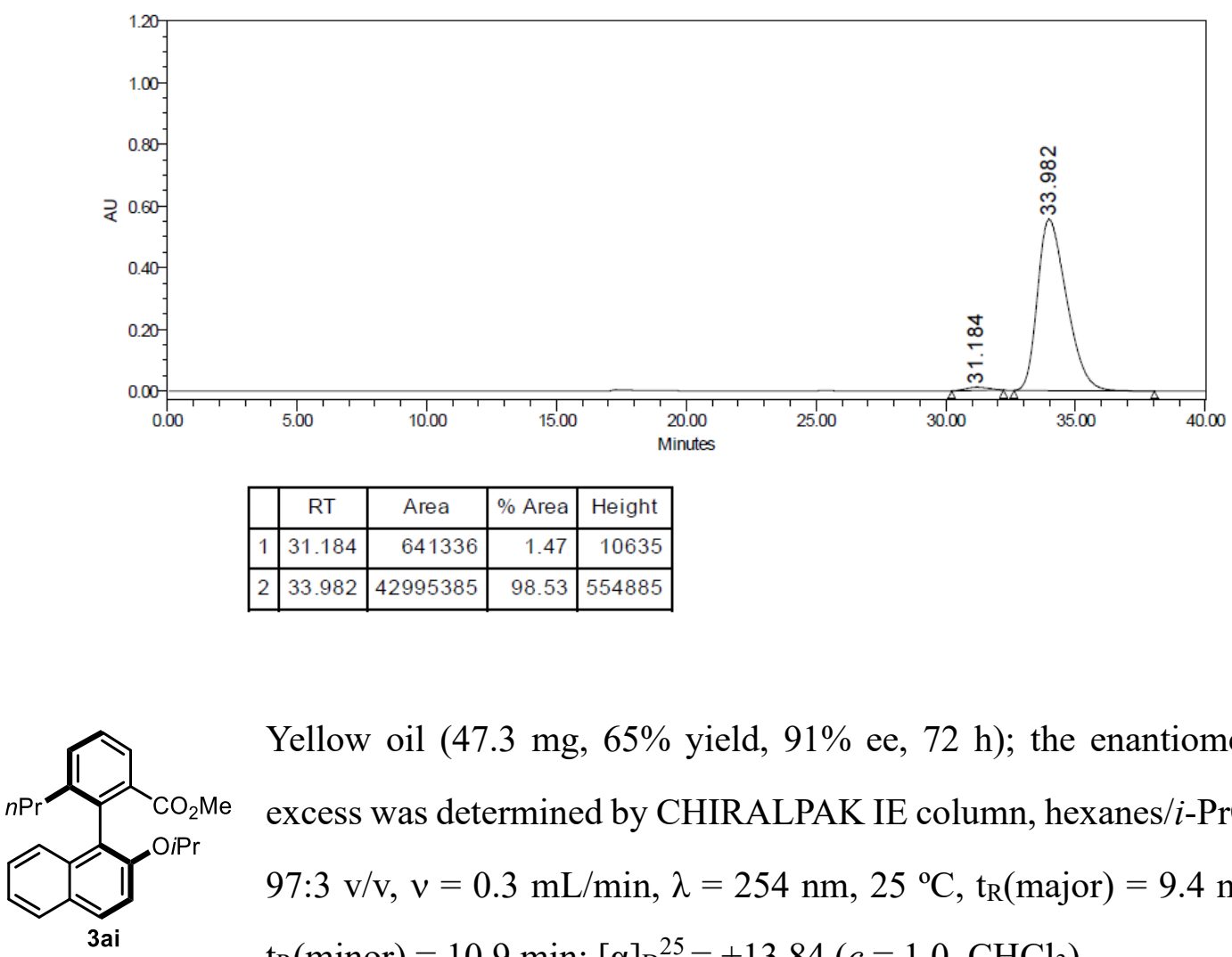

Yellow oil (47.3 mg, 65\% yield, 91\% ee, $72 \mathrm{~h}$ ); the enantiomeric excess was determined by CHIRALPAK IE column, hexanes $/ i$-PrOH, $97: 3 \mathrm{v} / \mathrm{v}, v=0.3 \mathrm{~mL} / \mathrm{min}, \lambda=254 \mathrm{~nm}, 25{ }^{\circ} \mathrm{C}, \mathrm{t}_{\mathrm{R}}($ major $)=9.4 \mathrm{~min}$, $\mathrm{t}_{\mathrm{R}}($ minor $)=10.9 \mathrm{~min} ;[\alpha]_{\mathrm{D}}{ }^{25}=+13.84\left(c=1.0, \mathrm{CHCl}_{3}\right)$.

3ai: ${ }^{1} \mathrm{H}$ NMR (400 MHz, $\left.\mathrm{CHCl}_{3}\right) \delta 7.85-7.77(\mathrm{~m}, 3 \mathrm{H}), 7.51(\mathrm{dd}, J=8.0,1.2 \mathrm{~Hz}, 1 \mathrm{H})$, $7.41(\mathrm{t}, J=8.0 \mathrm{~Hz}, 1 \mathrm{H}), 7.29-7.22(\mathrm{~m}, 3 \mathrm{H}), 7.13-7.11(\mathrm{~m}, 1 \mathrm{H}), 4.50$ (sept, $J=6.0$ $\mathrm{Hz}, 1 \mathrm{H}), 3.34(\mathrm{~s}, 3 \mathrm{H}), 2.24(\mathrm{t}, J=7.2 \mathrm{~Hz}, 2 \mathrm{H}), 1.40-1.29(\mathrm{~m}, 2 \mathrm{H}), 1.17(\mathrm{~d}, J=6.0 \mathrm{~Hz}$, $2 \mathrm{H}), 1.09(\mathrm{~d}, J=6.0 \mathrm{~Hz}, 2 \mathrm{H}), 0.63(\mathrm{t}, J=7.6 \mathrm{~Hz}, 3 \mathrm{H}) ;{ }^{13} \mathrm{C} \mathrm{NMR}\left(101 \mathrm{MHz}, \mathrm{CHCl}_{3}\right) \delta$ $168.3,152.0,143.3,136.8,134.1,132.5,132.4,128.9,128.7,127.9,127.7,127.2,126.2$, $124.8,124.3,123.3,116.0,70.7,51.6,35.4,23.3,22.6,22.3,14.0$; FT-IR (neat): $v_{\max }=$ 2961, 2931, 2870, 1731, 1592, 1507, 1453, 1431, 1377, 1250, 1137, 1112, 1005, 805, $748 \mathrm{~cm}^{-1}$; HRMS (EI): exact mass calculated for: $\mathrm{C}_{24} \mathrm{H}_{26} \mathrm{O}_{3}[\mathrm{M}]^{+}: 362.1876$, found 362.1877 . 

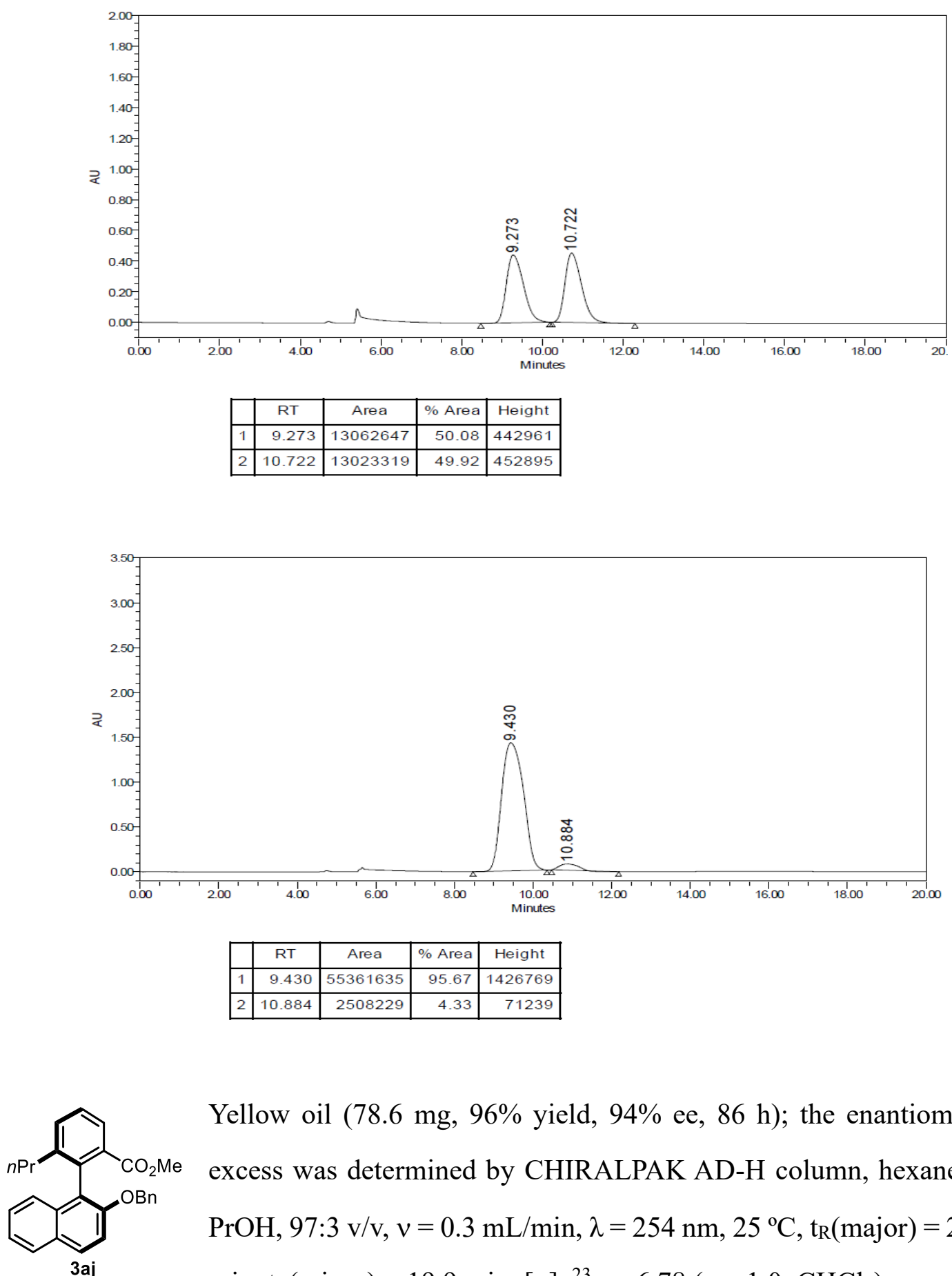

Yellow oil (78.6 mg, 96\% yield, 94\% ee, $86 \mathrm{~h}$ ); the enantiomeric excess was determined by CHIRALPAK AD-H column, hexanes $/ i$ $\operatorname{PrOH}, 97: 3 \mathrm{v} / \mathrm{v}, \mathrm{v}=0.3 \mathrm{~mL} / \mathrm{min}, \lambda=254 \mathrm{~nm}, 25^{\circ} \mathrm{C}, \mathrm{t}_{\mathrm{R}}($ major $)=24.4$ $\min , \mathrm{t}_{\mathrm{R}}($ minor $)=19.9 \mathrm{~min} ;[\alpha]_{\mathrm{D}}^{23}=-6.78\left(c=1.0, \mathrm{CHCl}_{3}\right)$.

3aj: ${ }^{1} \mathrm{H}$ NMR $\left(400 \mathrm{MHz}, \mathrm{CHCl}_{3}\right) \delta 7.89(\mathrm{dd}, J=8.0,1.2 \mathrm{~Hz}, 1 \mathrm{H}), 7.83(\mathrm{~d}, J=8.8 \mathrm{~Hz}$, 1H), $7.79(\mathrm{dd}, J=7.2,2.0 \mathrm{~Hz}, 1 \mathrm{H}), 7.54(\mathrm{~d}, J=6.8 \mathrm{~Hz}, 1 \mathrm{H}), 7.45(\mathrm{t}, J=7.6 \mathrm{~Hz}, 1 \mathrm{H})$, $7.32(\mathrm{~d}, J=8.8 \mathrm{~Hz}, 1 \mathrm{H}), 7.30-7.26(\mathrm{~m}, 2 \mathrm{H}), 7.25-7.21(\mathrm{~m}, 3 \mathrm{H}), 7.16-7.13(\mathrm{~m}, 3 \mathrm{H})$, $5.10(\mathrm{~s}, 2 \mathrm{H}), 3.34(\mathrm{~s}, 3 \mathrm{H}), 2.28-2.20(\mathrm{~m}, 2 \mathrm{H}), 1.41-1.26(\mathrm{~m}, 2 \mathrm{H}), 0.62(\mathrm{t}, J=7.2 \mathrm{~Hz}$, $3 \mathrm{H}) ;{ }^{13} \mathrm{C} \mathrm{NMR}\left(101 \mathrm{MHz}, \mathrm{CHCl}_{3}\right) \delta 168.1,152.8,143.4,137.9,136.6,133.8,132.7$, 
132.3, 129.2, 129.0, 128.4, 128.0, 127.9, 127.49, 127.45, 126.6, 126.4, 124.8, 124.0, 123.6, 115.1, 71.0, 51.7, 35.5, 23.4, 14.0; FT-IR (neat): $v_{\max }=3058,2961,2924,2869$, $1728,1592,1508,1453,1431,1256,1219,1190,1138,1121,1099,1082,1057,1018$, 803, 713, $695 \mathrm{~cm}^{-1}$; HRMS (EI): exact mass calculated for: $\mathrm{C}_{28} \mathrm{H}_{26} \mathrm{O}_{3}[\mathrm{M}]^{+}: 410.1876$, found 410.1878 .
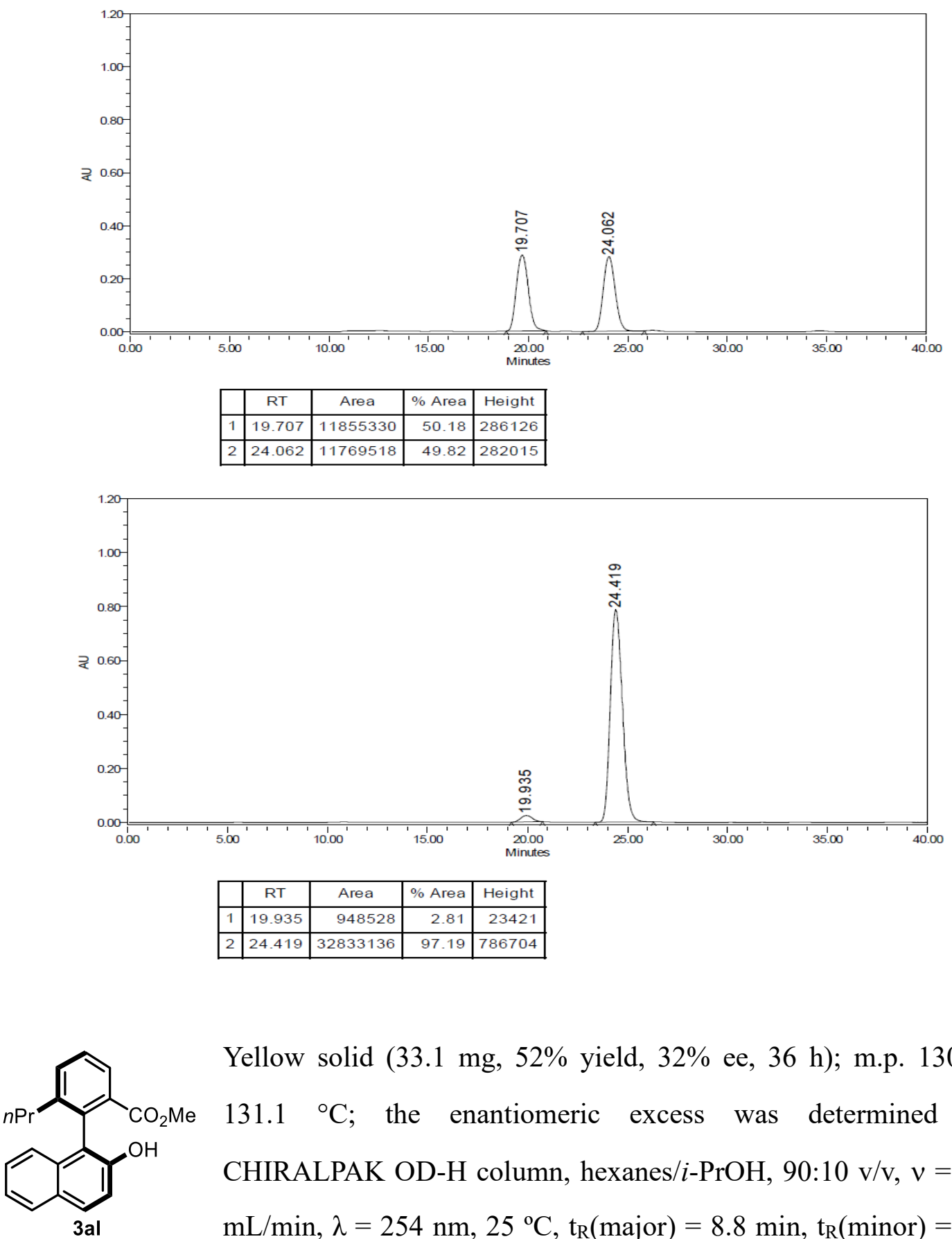

Yellow solid (33.1 mg, 52\% yield, 32\% ee, 36 h); m.p. $130.5-$ $131.1{ }^{\circ} \mathrm{C}$; the enantiomeric excess was determined by CHIRALPAK OD-H column, hexanes/ $i-\mathrm{PrOH}, 90: 10 \mathrm{v} / \mathrm{v}, v=1.0$ $\mathrm{mL} / \mathrm{min}, \lambda=254 \mathrm{~nm}, 25{ }^{\circ} \mathrm{C}, \mathrm{t}_{\mathrm{R}}($ major $)=8.8 \mathrm{~min}, \mathrm{t}_{\mathrm{R}}($ minor $)=6.8$ $\min ;[\alpha]_{\mathrm{D}}^{19}=+0.22\left(c=1.0, \mathrm{CHCl}_{3}\right)$. 
3al: ${ }^{1} \mathrm{H}$ NMR (400 MHz, $\left.\mathrm{CHCl}_{3}\right) \delta 7.86-7.79(\mathrm{~m}, 3 \mathrm{H}), 7.59$ (d, J = 7.6 Hz, 1H), 7.52 (t, J = 7.6 Hz, 1H), $7.31-7.24(\mathrm{~m}, 3 \mathrm{H}), 6.98(\mathrm{~d}, \mathrm{~J}=7.6 \mathrm{~Hz}, 1 \mathrm{H}), 4.96(\mathrm{~s}, 1 \mathrm{H}), 3.42(\mathrm{~s}$, $3 \mathrm{H}), 2.28-2.15(\mathrm{~m}, 2 \mathrm{H}), 1.41-1.31(\mathrm{~m}, 2 \mathrm{H}), 0.66(\mathrm{t}, \mathrm{J}=6.8 \mathrm{~Hz}, 3 \mathrm{H}) ;{ }^{13} \mathrm{C} \mathrm{NMR}(101$ $\left.\mathrm{MHz}, \mathrm{CHCl}_{3}\right) \delta 168.2,150.5,144.9,133.9,133.6,133.3,133.1,129.6,129.0,128.8$, $128.2,128.1,126.6,124.2,123.3,119.0,117.6,52.2,35.4,23.6,14.1$; FT-IR (neat): $v_{\max }=1434,1261,1144,985,798,754,684 \mathrm{~cm}^{-1} ;$ HRMS (EI): exact mass calculated for: $\mathrm{C}_{21} \mathrm{H}_{20} \mathrm{O}_{3}[\mathrm{M}]^{+}:$320.1407, found 320.1410.
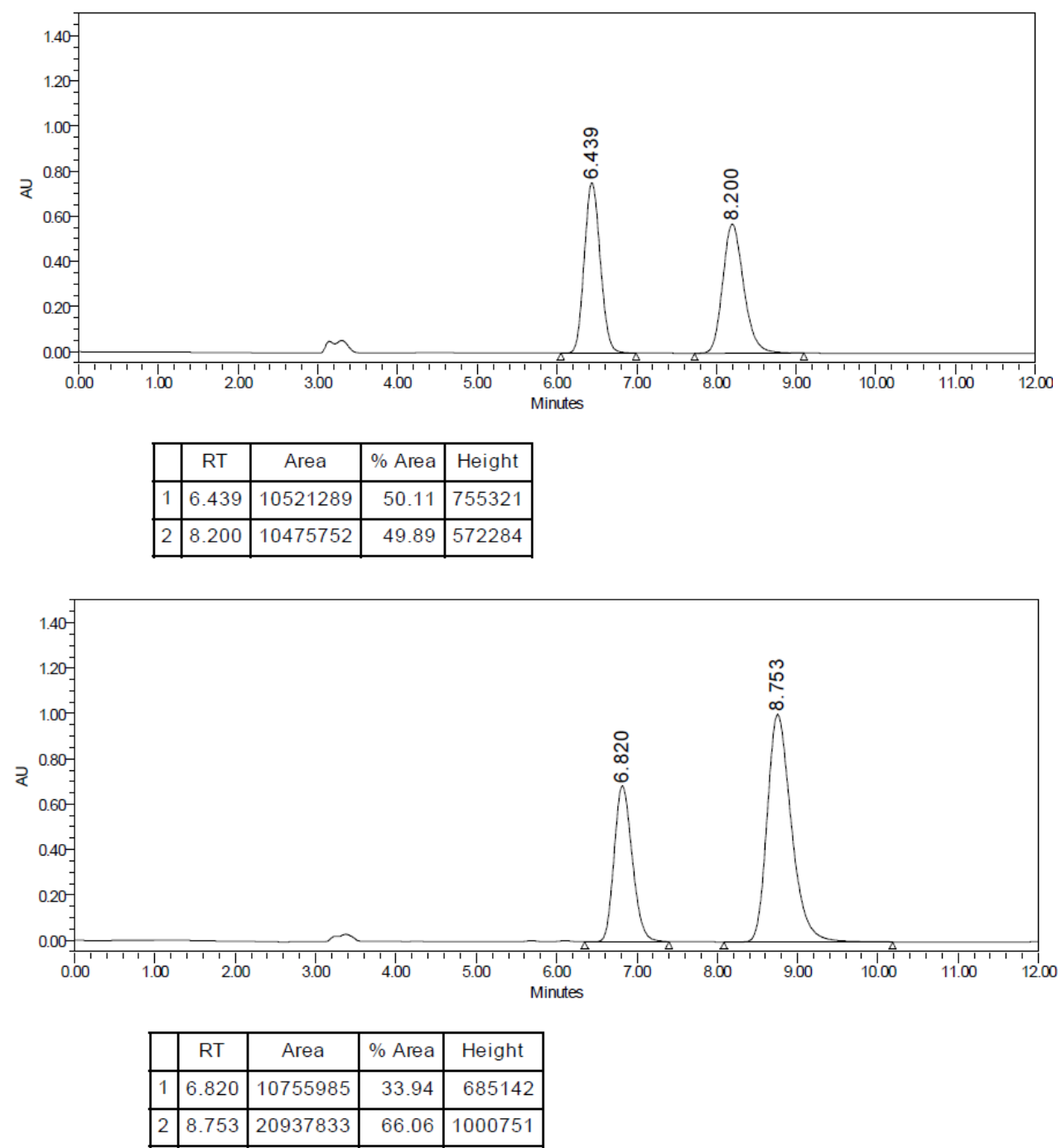

\section{Procedure for asymmetric synthesis of 3am}




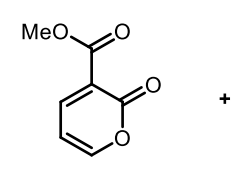

$1 \mathrm{a}$

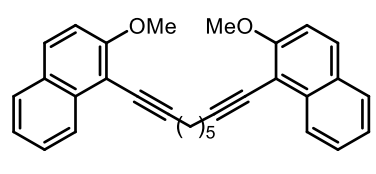

$2 \mathrm{ab}$

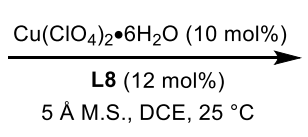

5 A M.S., DCE, $25^{\circ} \mathrm{C}$

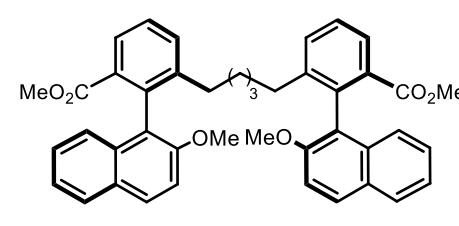

3am

A mixture of $\mathrm{Cu}\left(\mathrm{ClO}_{4}\right)_{2} \bullet 6 \mathrm{H}_{2} \mathrm{O}(7.4 \mathrm{mg}, 0.02 \mathrm{mmol}), 5 \AA$ M.S. $(50.0 \mathrm{mg})$ and $\mathbf{L 8}(18.2$ $\mathrm{mg}, 0.024 \mathrm{mmol})$ in DCE $(0.5 \mathrm{~mL})$ was stirred at $25^{\circ} \mathrm{C}$ for $2 \mathrm{~h}$. Then, 2-pyrone $1 \mathrm{a}(0.2$ mmol) was added. After stirred at $25{ }^{\circ} \mathrm{C}$ for $20 \mathrm{~min}$, the solution of alkyne $2 \mathbf{a b}(0.1$ $\mathrm{mmol})$ in DCE $(0.5 \mathrm{~mL})$ was added in one portion. After the reaction mixture was stirred at this temperature for $72 \mathrm{~h}$, the crude mixture was filtrated through celite and washed with dichloromethane. The filtrate was concentrated under reduced pressure. The residue was purified by flash column chromatography (silica gel, ethyl acetate/petroleum ether 1:10) to afford 3am.

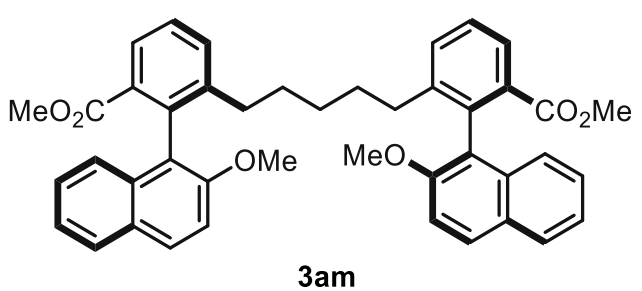

Yellow oil (33.4 mg, 51\% yield, 99.9\% ee, 19:1 dr $72 \mathrm{~h})$; the enantiomeric excess was determined by CHIRALPAK IC column, hexanes $/ i-\mathrm{PrOH}, 90: 10 \mathrm{v} / \mathrm{v}, v=1.0 \mathrm{~mL} / \mathrm{min}, \lambda$

$=254 \mathrm{~nm}, 25{ }^{\circ} \mathrm{C}, \mathrm{t}_{\mathrm{R}}($ major $)=23.7 \mathrm{~min}, \mathrm{t}_{\mathrm{R}}($ minor $)=17.0 \mathrm{~min} ;[\alpha]_{\mathrm{D}}{ }^{24}=+9.26(c=1.0$, $\left.\mathrm{CHCl}_{3}\right)$.

3am: ${ }^{1} \mathrm{H}$ NMR (400 MHz, $\left.\mathrm{CHCl}_{3}\right) \delta 7.85-7.81(\mathrm{~m}, 4 \mathrm{H}), 7.78(\mathrm{~d}, J=8.0 \mathrm{~Hz}, 2 \mathrm{H}), 7.38$ (t, $J=7.6 \mathrm{~Hz}, 2 \mathrm{H}), 7.32(\mathrm{dd}, J=7.6,1.2 \mathrm{~Hz}, 2 \mathrm{H}), 7.28-7.23(\mathrm{~m}, 4 \mathrm{H}), 7.20-7.16(\mathrm{~m}$, 2H), $6.99(\mathrm{~d}, J=8.4 \mathrm{~Hz}, 2 \mathrm{H}), 3.67(\mathrm{~s}, 6 \mathrm{H}), 3.34(\mathrm{~s}, 6 \mathrm{H}), 2.04-1.95(\mathrm{~m}, 4 \mathrm{H}), 1.10-$ $0.96(\mathrm{~m}, 4 \mathrm{H}), 0.74-0.67(\mathrm{~m}, 2 \mathrm{H}) ;{ }^{13} \mathrm{C} \mathrm{NMR}\left(101 \mathrm{MHz}, \mathrm{CHCl}_{3}\right) \delta$ 168.0, 153.6, 143.3, $136.3,133.6,132.6,132.3,129.1,128.8,128.0,127.8,127.4,126.4,124.6,123.4,122.8$, 113.1, 56.4, 51.7, 33.0, 29.9, 29.0; FT-IR (neat): $v_{\max }=3058,2943,2933,2857,1728$, 1593, 1508, 1456, 1431, 1255, 1190, 1137, 1120, 1065, 1020, 806, 747, 734, $700 \mathrm{~cm}^{-1}$; HRMS (EI): exact mass calculated for: $\mathrm{C}_{23} \mathrm{H}_{25} \mathrm{NO}_{2}[\mathrm{M}]^{+}: 652.2819$, found 652.2825. 


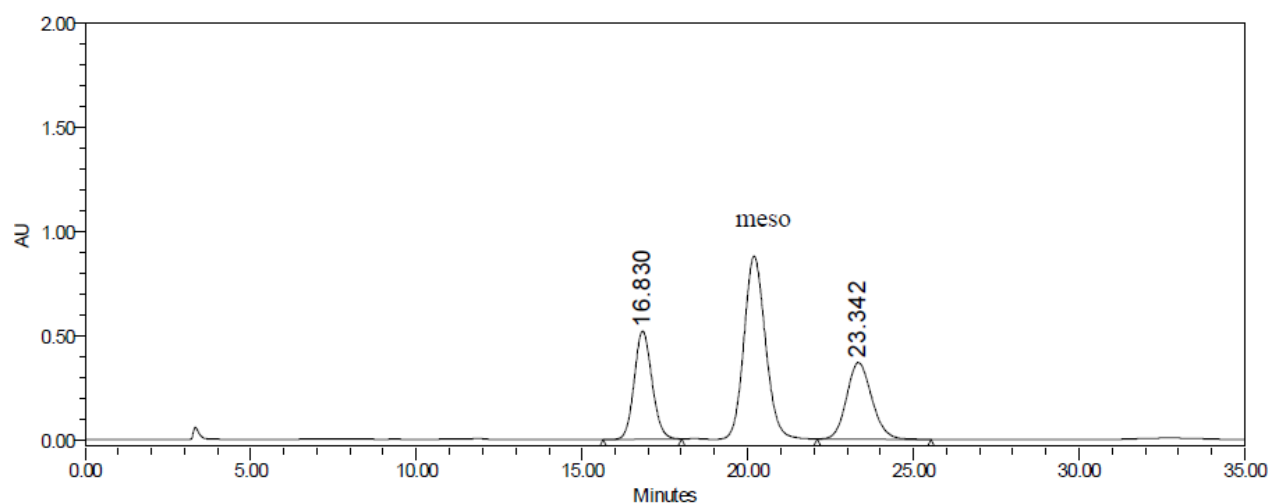

\begin{tabular}{|c|c|c|r|c|}
\hline & RT & Area & $\%$ Area & Height \\
\hline 1 & 16.830 & 19435973 & 50.00 & 520315 \\
\hline 2 & 23.342 & 19432445 & 50.00 & 367827 \\
\hline
\end{tabular}

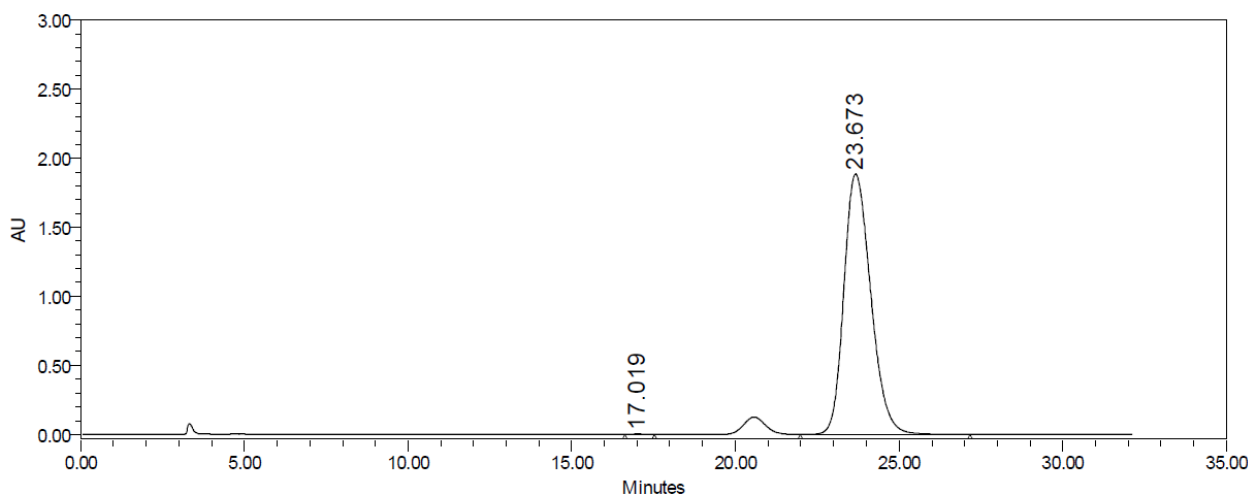

\begin{tabular}{|l|c|r|r|r|}
\hline & RT & Area & \% Area & Height \\
\hline 1 & 17.019 & 74725 & 0.07 & 2343 \\
\hline 2 & 23.673 & 108068176 & 99.93 & 1885670 \\
\hline
\end{tabular}

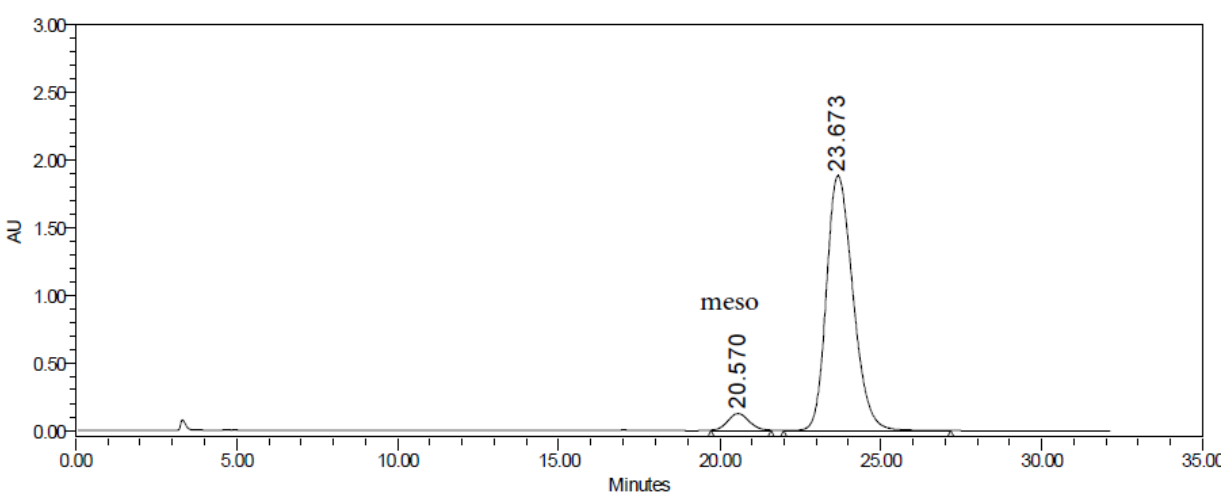

\begin{tabular}{|c|c|c|r|c|}
\hline & RT & Area & $\%$ Area & Height \\
\hline 1 & 20.570 & 5589564 & 4.92 & 123783 \\
\hline 2 & 23.673 & 108068176 & 95.08 & 1885670 \\
\hline
\end{tabular}




\section{Density Functional Theory (DFT) calculations}

Density-functional theory (DFT) calculations were performed with Gaussian $16^{7}$ Geometry optimizations were performed with the $\omega$ B97X-D functional. ${ }^{8}$ The LANL2DZ basis set with effective core potential (ECP) ${ }^{9}$ was used for $\mathrm{Cu}$, and the 6$31 \mathrm{G}(\mathrm{d})$ basis set was used for other atoms. Frequency calculations were performed at the same level of theory as for geometry optimization to characterize the stationary points as either minima (no imaginary frequencies) or first-order saddle points (one imaginary frequency) on the potential energy surface, as well as to obtain thermal Gibbs free energy corrections. Intrinsic reaction coordinate calculations were performed to ensure that the first-order saddle points found were true transition states (TS) connecting the reactants and the products. Single-point energies were calculated with orca package ${ }^{10}$ with the $\omega \mathrm{B} 97 \mathrm{M}-\mathrm{V}$ functional, ${ }^{11}$ def2-TZVP basis set. ${ }^{12}$ Solvation effects were incorporated with single-point energy calculations with the $\mathrm{SMD}^{13}$ solvation model. Molecular structure visualizations were obtained using CYLview. ${ }^{14}$ 


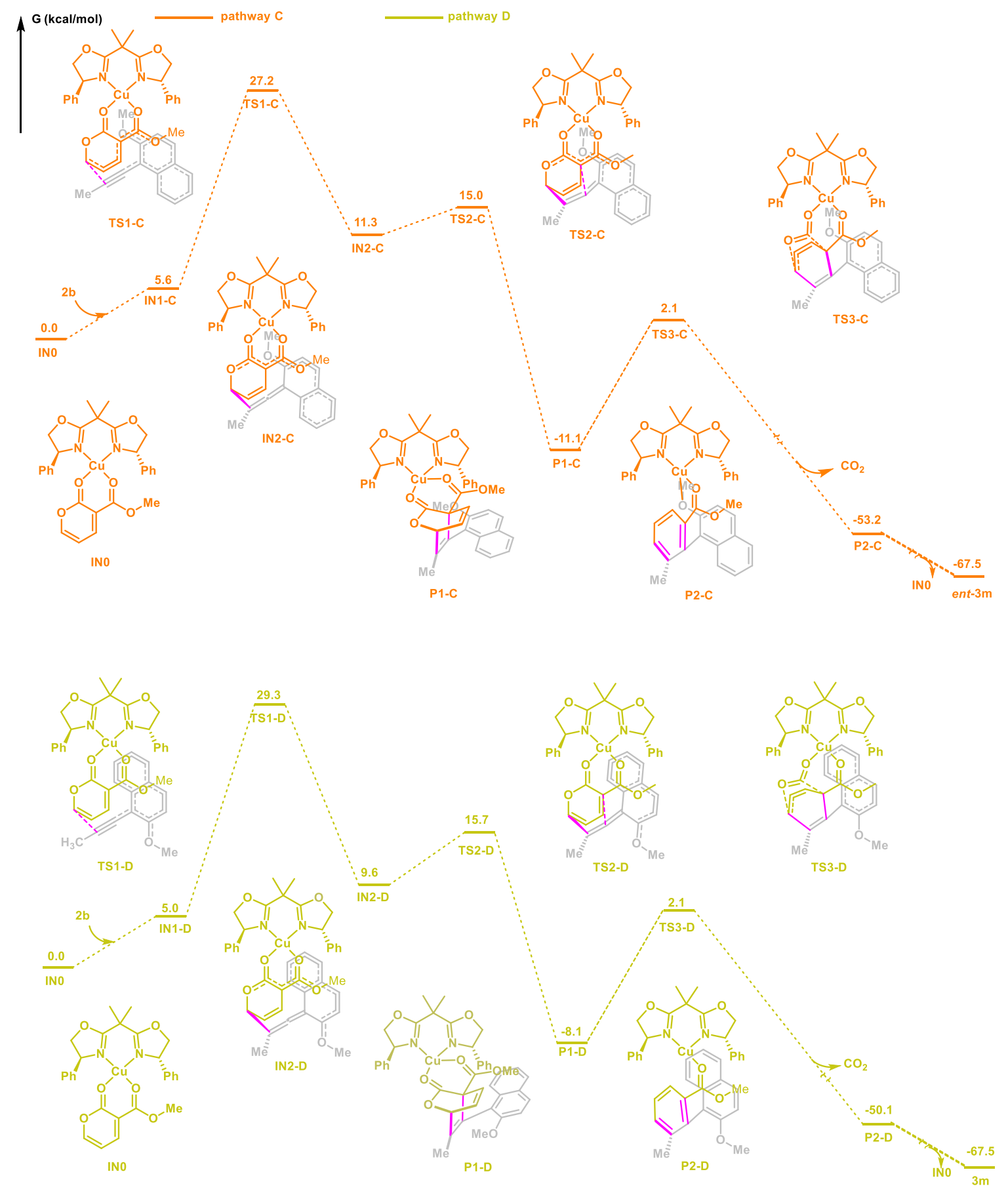

Figure S1. Calculated free energy profile for pathway C and D. 


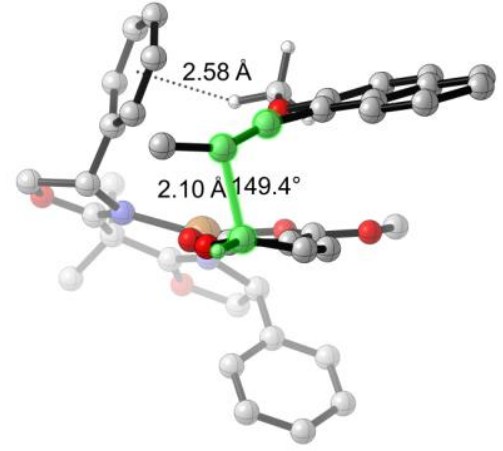

TS1-A

$\Delta \Delta \mathrm{G}^{\ddagger}=0 \mathrm{kcal} / \mathrm{mo}$

$\Delta \Delta \mathrm{G}^{\ddagger}=0 \mathrm{kcal} / \mathrm{mo}$

$\Delta \Delta \mathrm{G}^{\ddagger}=0 \mathrm{kcal} / \mathrm{mol}$

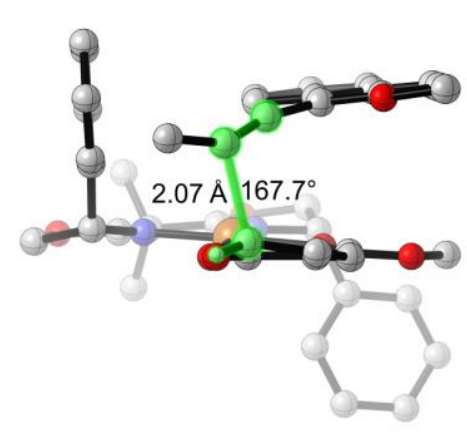

TS1-B

$\Delta \Delta G^{\ddagger}=1.0 \mathrm{kcal} / \mathrm{mol}$ $\Delta \Delta \mathrm{G}^{\ddagger}=1.7 \mathrm{kcal} / \mathrm{mol}$

$\Delta \Delta \mathrm{G}^{\ddagger}=1.5 \mathrm{kcal} / \mathrm{mol}$

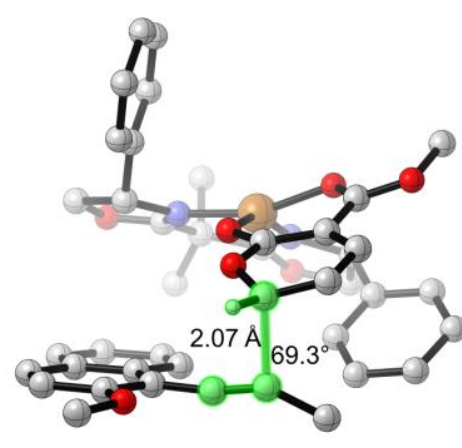

TS1-C

$\Delta \Delta \mathrm{G}^{\ddagger}=4.1 \mathrm{kcal} / \mathrm{mol}$ $\Delta \Delta \mathrm{G}^{\ddagger}=3.9 \mathrm{kcal} / \mathrm{mol}$ $\Delta \Delta \mathrm{G}^{\ddagger}=3.0 \mathrm{kcal} / \mathrm{mol}$

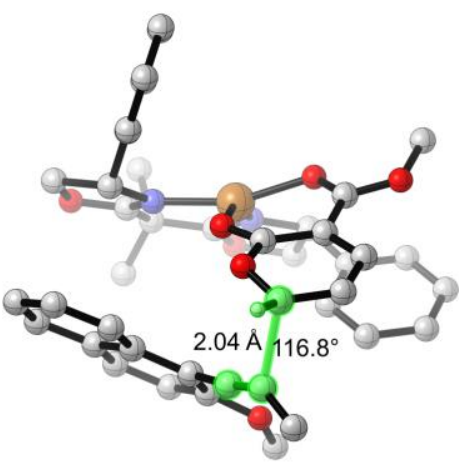

TS1-D

$\Delta \Delta G^{\ddagger}=6.2 \mathrm{kcal} / \mathrm{mol}$

$\Delta \Delta \mathrm{G}^{\ddagger}=6.1 \mathrm{kcal} / \mathrm{mol}$

$\Delta \Delta \mathrm{G}^{\ddagger}=5.0 \mathrm{kcal} / \mathrm{mol}$

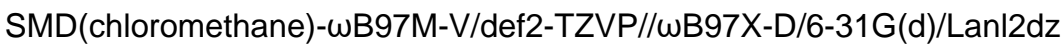

SMD (chloromethane)- $\omega$ B97X-V/def2-TZVP//wB97X-D/6-31G(d)/Lanl2dz

SMD (chloromethane)-TPSSh-D3BJ/def2-TZVP//WB97X-D/6-31G(d)/Lanl2dz

Figure S2. Relative energies of TS-1-A and TS-1-B in $\mathrm{kcal} \mathrm{mol}^{-1}$. The relative energies are consistent in terms of predicted enantioselectivity calculated by three commonly used density functional methods. 


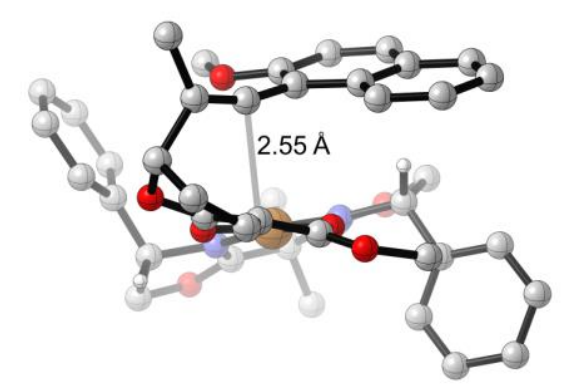

TS2-A

$\Delta \mathrm{G} \ddagger=17.5 \mathrm{kcal} / \mathrm{mol}$

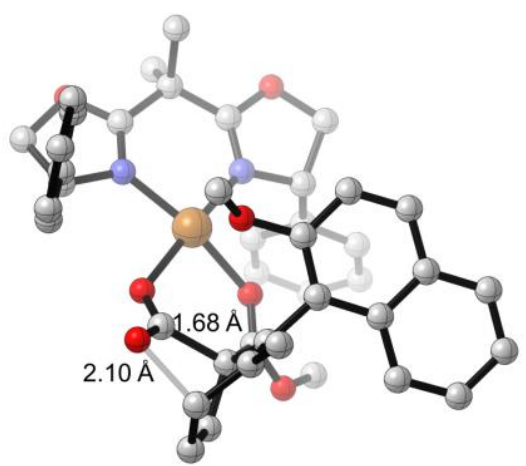

TS3-A

$\Delta \mathrm{G}^{\ddagger}=13.9 \mathrm{kcal} / \mathrm{mol}$

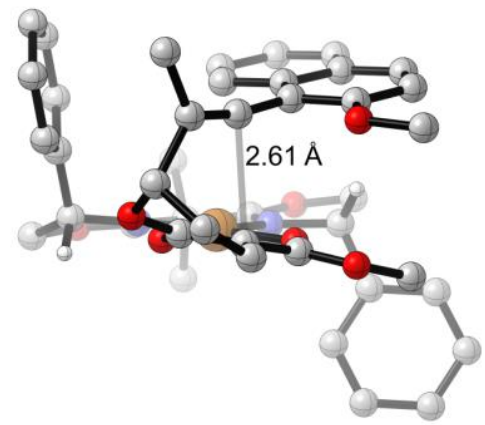

TS2-B

$\Delta \mathrm{G}^{\ddagger}=14.5 \mathrm{kcal} / \mathrm{mol}$

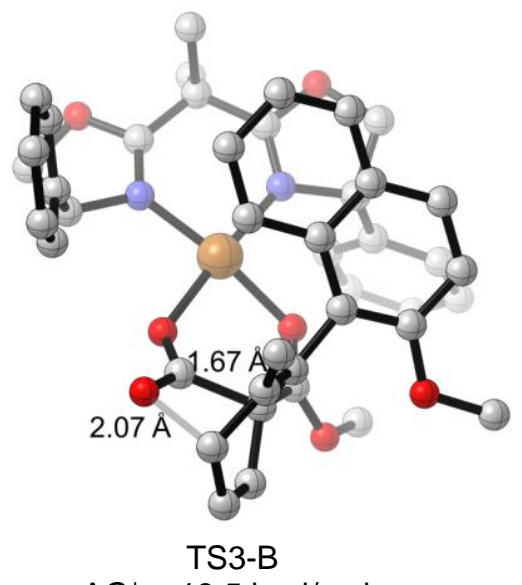

$\Delta \mathrm{G}^{\ddagger}=13.5 \mathrm{kcal} / \mathrm{mol}$

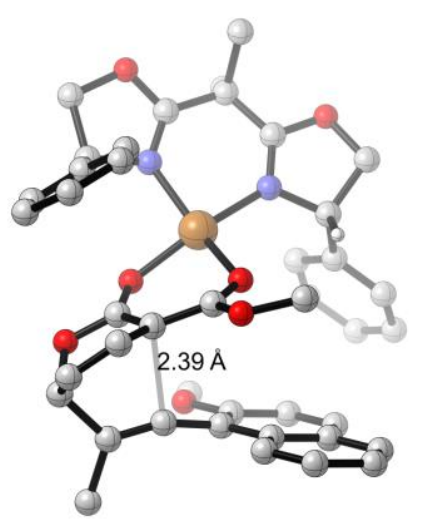

TS2-C

$\Delta \Delta \mathrm{G}^{\ddagger}=15.0 \mathrm{kcal} / \mathrm{mol}$

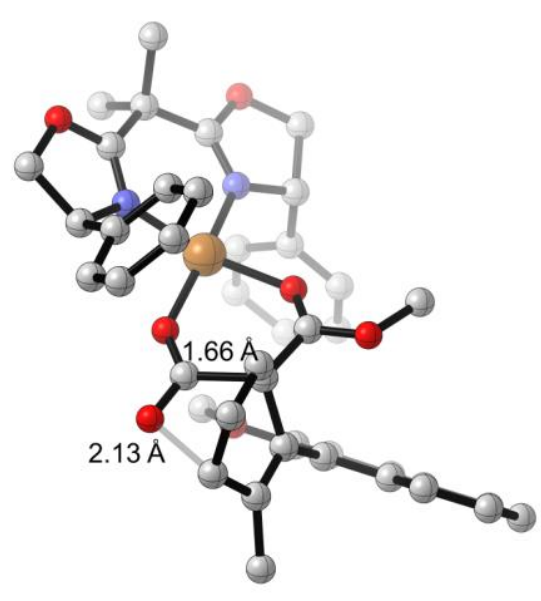

TS3-C

$\Delta \mathrm{G}^{\ddagger}=13.2 \mathrm{kcal} / \mathrm{mol}$

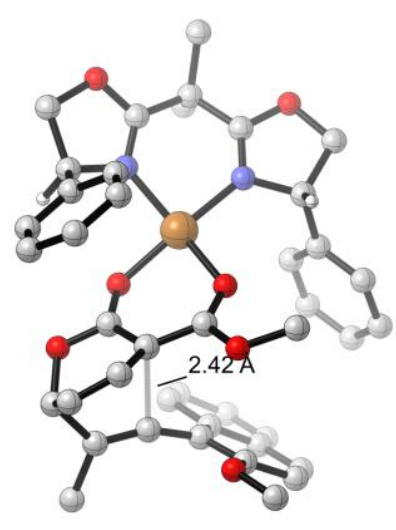

TS2-D

$\Delta \Delta \mathrm{G}^{\ddagger}=15.7 \mathrm{kcal} / \mathrm{mol}$

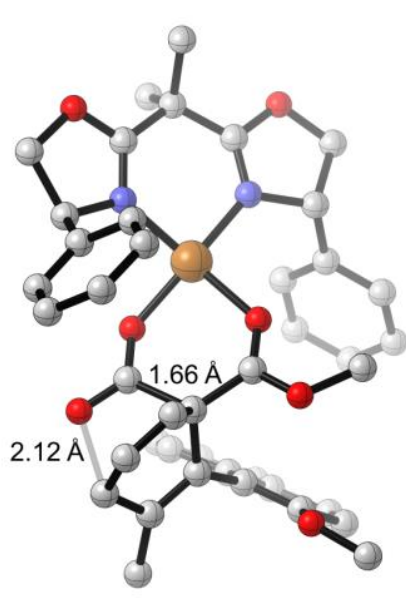

TS3-D

$\Delta \mathrm{G}^{\ddagger}=10.2 \mathrm{kcal} / \mathrm{mol}$

Figure S3. DFT-optimized transition structures and relative free energies in $\mathrm{kcal} \mathrm{mol}^{-1}$. 


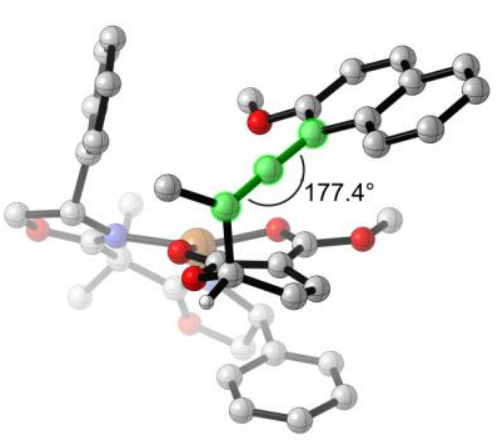

$\Delta \mathrm{G}^{\ddagger}=10.0 \mathrm{kcal} / \mathrm{mol}$

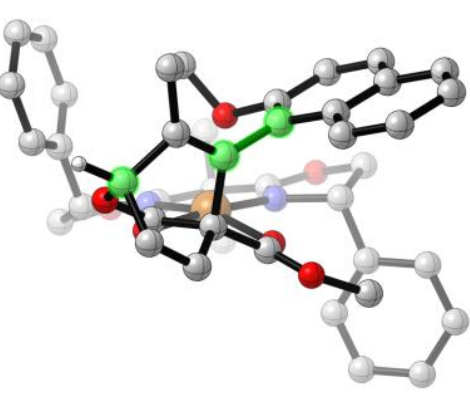

P1-A

$\Delta G^{\ddagger}=-13.2 \mathrm{kcal} / \mathrm{mol}$

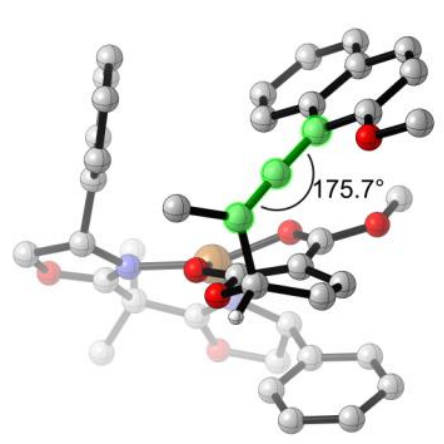

IN2-B

$\Delta \mathrm{G}^{\ddagger}=9.5 \mathrm{kcal} / \mathrm{mol}$

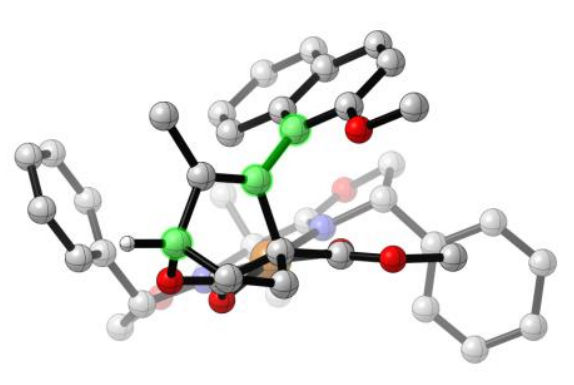

$\Delta G^{\ddagger}=-10.4 \mathrm{kcal} / \mathrm{mol}$

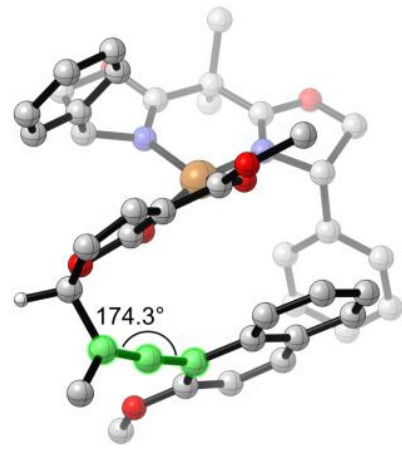

IN2-C

$\Delta G^{\ddagger}=11.3 \mathrm{kcal} / \mathrm{mol}$

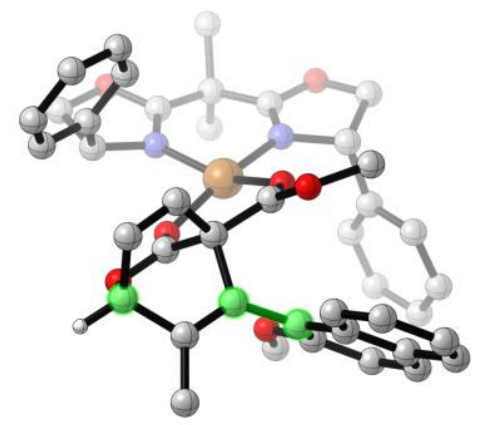

$\Delta \mathrm{G}^{\ddagger}=-11.1 \mathrm{kcal} / \mathrm{mol}$

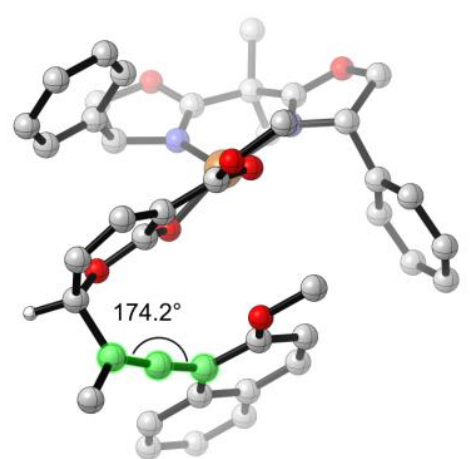

IN2-D

$\Delta \mathrm{G}^{\ddagger}=9.6 \mathrm{kcal} / \mathrm{mol}$

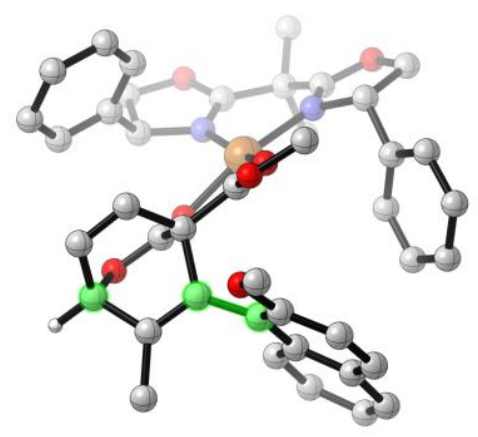

P1-D

$\Delta G^{\ddagger}=-8.1 \mathrm{kcal} / \mathrm{mol}$ 


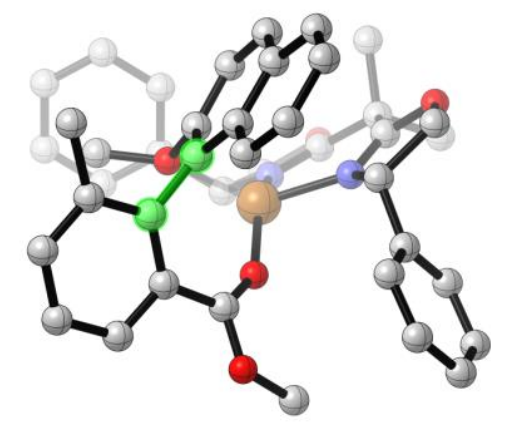

P2-A

$\Delta \mathrm{G}^{\ddagger}=-48.0 \mathrm{kcal} / \mathrm{mol}$

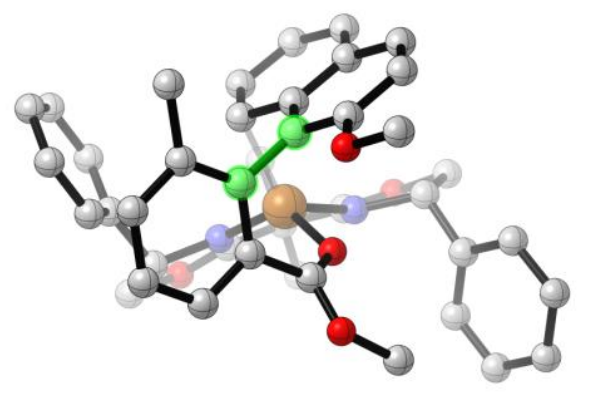

P2-B

$\Delta \mathrm{G}^{\ddagger}=-48.3 \mathrm{kcal} / \mathrm{mol}$

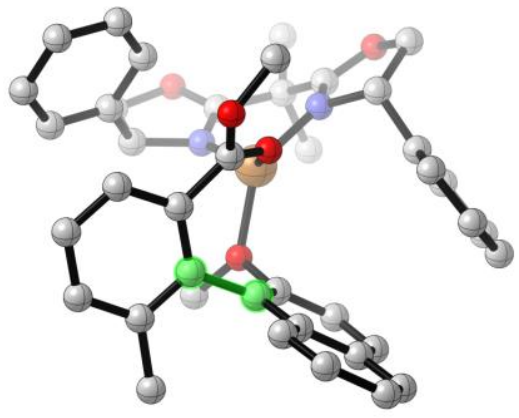

P2-C

$\Delta \mathrm{G}^{\ddagger}=-53.2 \mathrm{kcal} / \mathrm{mol}$

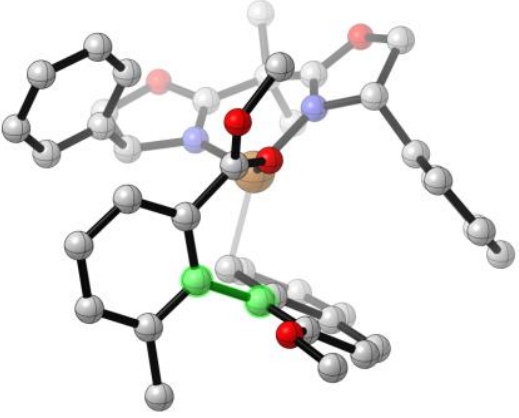

P2-D

$\Delta \mathrm{G}^{\ddagger}=-50.1 \mathrm{kcal} / \mathrm{mol}$

Figure S4. DFT-optimized intermediates and relative free energies in $\mathrm{kcal} \mathrm{mol}^{-1}$. 


\begin{tabular}{|c|c|c|c|c|c|c|}
\hline Structure & E_SPC & $\mathrm{E}$ & $\mathrm{ZPE}$ & H_SPC & T.S & $\mathrm{G}(\mathrm{T}) \_\mathrm{SPC}$ \\
\hline 1a & -571.308919 & -571.051377 & 0.125124 & -571.173108 & 0.047729 & -571.220837 \\
\hline $2 b$ & -615.886375 & -615.670149 & 0.220646 & -615.651433 & 0.055328 & -615.706760 \\
\hline ent $-3 \mathrm{~m}$ & -998.688544 & -998.333716 & 0.335780 & -998.331450 & 0.071897 & -998.403347 \\
\hline $3 \mathrm{~m}$ & -998.688787 & -998.334118 & 0.335999 & -998.331506 & 0.071981 & -998.403487 \\
\hline $\mathrm{CO}_{2}$ & -188.622260 & -188.520000 & 0.011825 & -188.606859 & 0.024935 & -188.631795 \\
\hline INO & -3284.965620 & -1839.879042 & 0.528978 & -3284.402278 & 0.103332 & -3284.505610 \\
\hline IN1-A & -3900.869005 & -2455.586773 & 0.752026 & -3900.068148 & 0.134010 & -3900.202158 \\
\hline IN1-B & -3900.879046 & -2455.600850 & 0.752758 & -3900.077992 & 0.129355 & -3900.207348 \\
\hline IN1-C & -3900.873135 & -2455.593571 & 0.752802 & -3900.071802 & 0.131584 & -3900.203385 \\
\hline IN1-D & -3900.873451 & -2455.601901 & 0.752156 & -3900.072556 & 0.131911 & -3900.204467 \\
\hline IN2-A & -3900.866499 & -2455.593797 & 0.753867 & -3900.064982 & 0.131442 & -3900.196424 \\
\hline IN2-B & -3900.867283 & -2455.597442 & 0.753853 & -3900.065720 & 0.131573 & -3900.197293 \\
\hline IN2-C & -3900.866090 & -2455.595291 & 0.753715 & -3900.064822 & 0.129536 & -3900.194358 \\
\hline IN2-D & -3900.867540 & -2455.595672 & 0.753797 & -3900.066106 & 0.130998 & -3900.197104 \\
\hline P1-A & -3900.908152 & -2455.632213 & 0.754991 & -3900.106358 & 0.127005 & -3900.233363 \\
\hline P1-B & -3900.905720 & -2455.632537 & 0.755803 & -3900.103504 & 0.125470 & -3900.228974 \\
\hline P1-C & -3900.903197 & -2455.623662 & 0.755047 & -3900.101185 & 0.128805 & -3900.229990 \\
\hline P1-D & -3900.896945 & -2455.618873 & 0.754901 & -3900.094981 & 0.130224 & -3900.225205 \\
\hline P2-A & -3712.317441 & -2267.147357 & 0.739576 & -3711.532839 & 0.124207 & -3711.657046 \\
\hline P2-B & -3712.316793 & -3711.356897 & 0.739346 & -3711.532349 & 0.125160 & -3711.657509 \\
\hline $\mathrm{P} 2-\mathrm{C}$ & -3712.326762 & -3711.363703 & 0.739896 & -3711.542106 & 0.123181 & -3711.665288 \\
\hline P2-D & -3712.322552 & -3711.360975 & 0.739868 & -3711.537925 & 0.123110 & -3711.661034 \\
\hline TS1-A & -3900.843192 & -2455.570296 & 0.751767 & -3900.043723 & 0.131801 & -3900.175525 \\
\hline TS1-B & -3900.846647 & -2455.576406 & 0.751855 & -3900.048257 & 0.125661 & -3900.173918 \\
\hline
\end{tabular}




$\begin{array}{lllllll}\text { TS1-C } & -3900.838032 & -2455.569158 & 0.751694 & -3900.038563 & 0.130479 & -3900.169042 \\ \text { TS1-D } & -3900.835887 & -2455.565029 & 0.752025 & -3900.036253 & 0.129395 & -3900.165648 \\ \text { TS2-A } & -3900.859437 & -2455.585448 & 0.753832 & -3900.059303 & 0.125233 & -3900.184536 \\ \text { TS2-B } & -3900.862652 & -2455.591469 & 0.753481 & -3900.062594 & 0.126682 & -3900.189276 \\ \text { TS2-C } & -3900.859679 & -2455.587190 & 0.752958 & -3900.059855 & 0.128632 & -3900.188487 \\ \text { TS2-D } & -3900.856724 & -2455.583139 & 0.752878 & -3900.056784 & 0.130561 & -3900.187345 \\ \text { TS3-A } & -3900.882984 & -2455.608152 & 0.752212 & -3900.083748 & 0.127487 & -3900.211235 \\ \text { TS3-B } & -3900.882351 & -2455.610574 & 0.753332 & -3900.082527 & 0.124961 & -3900.207488 \\ \text { TS3-C } & -3900.878711 & -2455.600262 & 0.752207 & -3900.079232 & 0.129866 & -3900.209097 \\ \text { TS3-D } & -3900.872199 & -2455.595071 & 0.752101 & -3900.072849 & 0.129926 & -3900.202775\end{array}$




\begin{tabular}{|c|c|c|c|c|c|c|c|}
\hline $1 a$ & & & & C & -3.010628 & 3.356701 & -0.000016 \\
\hline C & 2.801867 & -0.707000 & 0.012582 & $\mathrm{H}$ & -4.055670 & 3.029693 & -0.001130 \\
\hline C & 1.911338 & -1.720463 & 0.035484 & $\mathrm{H}$ & -2.853738 & 3.981294 & 0.886127 \\
\hline C & 0.118303 & -0.077785 & 0.009353 & $\mathrm{H}$ & -2.852205 & 3.982453 & -0.885065 \\
\hline C & 0.529910 & -1.377014 & 0.034655 & $\mathrm{O}$ & -2.373826 & -1.267393 & -0.000013 \\
\hline $\mathrm{H}$ & 2.245337 & -2.749150 & 0.059896 & $C$ & -2.960079 & -2.548880 & 0.000088 \\
\hline $\mathrm{H}$ & -0.218846 & -2.161079 & 0.059720 & $\mathrm{H}$ & -2.685561 & -3.120952 & 0.895569 \\
\hline $\mathrm{H}$ & 3.879618 & -0.827812 & 0.018568 & $\mathrm{H}$ & -4.037654 & -2.381114 & 0.000212 \\
\hline $\mathrm{O}$ & 2.444863 & 0.574765 & -0.019631 & $\mathrm{H}$ & -2.685814 & -3.120999 & -0.895447 \\
\hline C & 1.099541 & 1.008532 & -0.036947 & & & & \\
\hline $\mathrm{O}$ & 0.923583 & 2.189344 & -0.095022 & \multicolumn{2}{|c|}{$e n t-3 m$} & & \\
\hline C & -1.330088 & 0.273974 & 0.040456 & C & 0.500352 & -0.062111 & 0.444821 \\
\hline $\mathrm{O}$ & -1.787128 & 1.379524 & 0.181776 & C & 1.673751 & 0.239296 & -0.297915 \\
\hline O & -2.096515 & -0.828635 & -0.100958 & C & 0.477861 & -1.172585 & 1.266015 \\
\hline C & -3.501931 & -0.591451 & -0.052367 & C & 2.817155 & -0.599013 & -0.179068 \\
\hline $\mathrm{H}$ & -3.804622 & 0.084640 & -0.855528 & C & 1.748153 & 1.361347 & -1.169286 \\
\hline $\mathrm{H}$ & -3.968350 & -1.568260 & -0.178088 & C & 1.611955 & -2.010456 & 1.382808 \\
\hline \multirow[t]{2}{*}{$\mathrm{H}$} & -3.785194 & -0.151085 & 0.906818 & C & 3.989680 & -0.288555 & -0.917203 \\
\hline & & & & C & 2.750009 & -1.723194 & 0.675624 \\
\hline $2 b$ & & & & C & 2.894823 & 1.635670 & -1.866797 \\
\hline C & -2.110148 & 2.207672 & 0.000007 & $\mathrm{H}$ & 0.876546 & 1.999138 & -1.280069 \\
\hline C & -1.380598 & 1.244441 & -0.000011 & $\mathrm{H}$ & 1.589539 & -2.880540 & 2.028558 \\
\hline C & -0.499077 & 0.120068 & -0.000022 & $\mathrm{C}$ & 4.033198 & 0.803947 & -1.741151 \\
\hline C & 0.918439 & 0.305810 & -0.000016 & $\mathrm{H}$ & 4.855262 & -0.938815 & -0.815883 \\
\hline C & -1.023885 & -1.167826 & -0.000041 & $\mathrm{H}$ & 3.620230 & -2.367726 & 0.768713 \\
\hline C & 1.774634 & -0.829321 & -0.000018 & $\mathrm{H}$ & 2.930798 & 2.496595 & -2.528059 \\
\hline C & 1.506722 & 1.598278 & 0.000010 & $\mathrm{H}$ & 4.934436 & 1.033387 & -2.301593 \\
\hline C & -0.164308 & -2.291784 & -0.000051 & $\mathrm{O}$ & -0.683731 & -1.394229 & 1.940333 \\
\hline C & 3.181841 & -0.640539 & 0.000008 & C & -0.788330 & -2.543438 & 2.746932 \\
\hline C & 1.195603 & -2.119866 & -0.000038 & $\mathrm{H}$ & -0.067058 & -2.527880 & 3.574560 \\
\hline C & 2.868933 & 1.748164 & 0.000034 & $\mathrm{H}$ & -1.799369 & -2.528484 & 3.156190 \\
\hline $\mathrm{H}$ & 0.854398 & 2.465437 & 0.000015 & $\mathrm{H}$ & -0.649642 & -3.462456 & 2.163140 \\
\hline $\mathrm{H}$ & -0.575057 & -3.294529 & -0.000057 & C & -0.694497 & 0.831303 & 0.383667 \\
\hline C & 3.720929 & 0.618170 & 0.000033 & C & -1.815368 & 0.533310 & -0.408738 \\
\hline $\mathrm{H}$ & 3.824558 & -1.517463 & 0.000008 & C & -0.698112 & 1.996537 & 1.171119 \\
\hline $\mathrm{H}$ & 1.847659 & -2.989421 & -0.000040 & C & -2.918232 & 1.391406 & -0.422791 \\
\hline $\mathrm{H}$ & 3.301225 & 2.744548 & 0.000055 & C & -1.804705 & 2.843896 & 1.128890 \\
\hline $\mathrm{H}$ & 4.798192 & 0.753686 & 0.000053 & C & -2.908792 & 2.550264 & 0.337302 \\
\hline
\end{tabular}




$\begin{array}{llll}\mathrm{H} & -3.775922 & 1.143046 & -1.036515 \\ \mathrm{H} & -1.801043 & 3.747424 & 1.733485 \\ \mathrm{H} & -3.761643 & 3.221850 & 0.318187 \\ \mathrm{C} & 0.476420 & 2.328054 & 2.057470 \\ \mathrm{H} & 0.694792 & 1.503610 & 2.743962 \\ \mathrm{H} & 1.383397 & 2.503330 & 1.468661 \\ \mathrm{H} & 0.276300 & 3.225006 & 2.650454 \\ \mathrm{C} & -1.841209 & -0.696275 & -1.258173 \\ \mathrm{O} & -0.878216 & -1.316189 & -1.643846 \\ \mathrm{O} & -3.102841 & -1.051027 & -1.576224 \\ \mathrm{C} & -3.210567 & -2.193257 & -2.419736 \\ \mathrm{H} & -2.774040 & -3.070906 & -1.936317 \\ \mathrm{H} & -4.278435 & -2.338608 & -2.582692 \\ \mathrm{H} & -2.697108 & -2.023281 & -3.369632\end{array}$

$\begin{array}{llll}\text { C } & -1.866663 & 0.401506 & -0.059473 \\ \text { C } & -1.290495 & -1.329714 & -1.656825 \\ \text { C } & -3.141301 & 0.500723 & -0.623747 \\ \text { C } & -2.573212 & -1.225256 & -2.192786 \\ \text { C } & -3.496159 & -0.317769 & -1.684640 \\ \text { H } & -3.846737 & 1.217824 & -0.220068 \\ \text { H } & -2.850357 & -1.863626 & -3.028010 \\ \text { H } & -4.489049 & -0.248161 & -2.118384 \\ \mathrm{C} & -0.300967 & -2.313274 & -2.227611 \\ \text { H } & 0.629332 & -1.815996 & -2.521381 \\ \text { H } & -0.042118 & -3.073861 & -1.483179 \\ \text { H } & -0.712688 & -2.819379 & -3.105487 \\ \mathrm{C} & -1.525576 & 1.260359 & 1.115624 \\ \mathrm{O} & -0.744407 & 0.983299 & 1.994987 \\ \mathrm{O} & -2.222572 & 2.413743 & 1.100065 \\ \mathrm{C} & -1.981934 & 3.275998 & 2.208575 \\ \mathrm{H} & -0.928987 & 3.565895 & 2.246706 \\ \mathrm{H} & -2.613372 & 4.148988 & 2.044090 \\ \mathrm{H} & -2.246507 & 2.782475 & 3.147390\end{array}$

$\begin{array}{llll}\text { C } & 0.796680 & -1.558386 & 0.875841\end{array}$

$\begin{array}{llll}C & 2.775935 & 0.245087 & 0.024767\end{array}$

$\begin{array}{llll}\text { C } & 1.169611 & 1.342982 & -1.454263\end{array}$

$\mathrm{CO}_{2}$

$\begin{array}{llll}\text { C } & 2.110927 & -1.646196 & 1.390671\end{array}$

$\begin{array}{llll}\text { C } & -0.000000 & 0.000000 & 0.000001\end{array}$

$\begin{array}{llll}\text { C } & 3.761539 & 1.163925 & -0.422509\end{array}$

$\begin{array}{llll}\text { O } & -0.000000 & 0.000000 & 1.164616\end{array}$

$\begin{array}{llll}\text { C } & 3.069903 & -0.760162 & 0.973860\end{array}$

$\begin{array}{llll}\text { C } & 2.144067 & 2.213338 & -1.866477\end{array}$

$\begin{array}{llll}H & 0.166331 & 1.417945 & -1.861786\end{array}$

$\begin{array}{llll}\text { O } & 0.000000 & -0.000000 & -1.164616\end{array}$

$\begin{array}{llll}H & 2.366457 & -2.406833 & 2.119181\end{array}$

$\begin{array}{llll}\text { C } & 3.457489 & 2.127981 & -1.345900\end{array}$

$\begin{array}{llll}H & 4.766951 & 1.086277 & -0.015722\end{array}$

$\begin{array}{llll}H & 4.078010 & -0.828222 & 1.374460\end{array}$

$\begin{array}{llll}H & 1.907793 & 2.977448 & -2.601417\end{array}$

$\begin{array}{llll}H & 4.218329 & 2.825979 & -1.681900\end{array}$

$\begin{array}{llll}\text { O } & -0.199333 & -2.411875 & 1.231070\end{array}$

$\begin{array}{llll}\text { C } & 0.029165 & -3.313707 & 2.288836\end{array}$

$\begin{array}{llll}H & 0.316419 & -2.789411 & 3.208579\end{array}$

$\begin{array}{llll}H & -0.918570 & -3.828965 & 2.450360\end{array}$

$\begin{array}{llll}H & 0.799263 & -4.054208 & 2.035350\end{array}$

$\begin{array}{llll}\text { C } & -0.931387 & -0.510316 & -0.573396\end{array}$

INO

$\begin{array}{llll}\text { C } & 4.520122 & 0.188439 & -1.923765 \\ \text { C } & 5.041020 & 0.804867 & -0.834800 \\ \text { C } & 2.789042 & 0.993842 & 0.044752 \\ \text { C } & 4.141836 & 1.229163 & 0.165914 \\ \text { H } & 6.106650 & 0.978222 & -0.760121 \\ \text { H } & 4.513447 & 1.753217 & 1.040381 \\ \text { H } & 5.075164 & -0.167805 & -2.782734 \\ \text { O } & 3.208682 & -0.042698 & -2.050926 \\ \text { C } & 2.304338 & 0.303666 & -1.112571 \\ \text { O } & 1.137292 & -0.013890 & -1.381815 \\ \text { C } & 1.830931 & 1.463157 & 1.066355 \\ \text { O } & 0.616910 & 1.223681 & 1.058952\end{array}$




\begin{tabular}{|c|c|c|c|c|c|c|c|}
\hline O & 2.383967 & 2.180765 & 2.001985 & C & -0.501801 & 4.563247 & -1.721791 \\
\hline C & 1.533481 & 2.713210 & 3.043489 & $\mathrm{H}$ & -0.239082 & 5.749956 & 0.055273 \\
\hline $\mathrm{H}$ & 0.776000 & 3.356483 & 2.594228 & $\mathrm{H}$ & -0.961766 & 3.197814 & -3.322511 \\
\hline $\mathrm{H}$ & 2.200202 & 3.282474 & 3.686247 & $\mathrm{H}$ & 0.017564 & 5.266993 & -2.364033 \\
\hline $\mathrm{H}$ & 1.066981 & 1.893115 & 3.590959 & C & 1.332936 & -2.705416 & 0.017904 \\
\hline $\mathrm{Cu}$ & -0.391464 & 0.002356 & -0.172427 & C & 2.603371 & -2.895631 & -0.527648 \\
\hline N & -2.177440 & 0.387343 & 0.495866 & C & 1.214576 & -2.331510 & 1.359903 \\
\hline N & -0.916780 & -1.853191 & -0.565566 & C & 3.740990 & -2.709647 & 0.254877 \\
\hline C & -3.245119 & -0.320465 & 0.336602 & $\mathrm{H}$ & 2.705261 & -3.192459 & -1.568447 \\
\hline O & -4.386563 & 0.309787 & 0.501384 & C & 2.352449 & -2.133262 & 2.139617 \\
\hline C & -2.038469 & -2.427532 & -0.293282 & $\mathrm{H}$ & 0.231401 & -2.209986 & 1.810846 \\
\hline O & -2.043984 & -3.741722 & -0.331136 & C & 3.617947 & -2.320048 & 1.587170 \\
\hline C & -4.101041 & 1.721077 & 0.701399 & $\mathrm{H}$ & 4.723803 & -2.884276 & -0.172227 \\
\hline $\mathrm{H}$ & -4.634995 & 2.039687 & 1.595083 & $\mathrm{H}$ & 2.249435 & -1.858897 & 3.184961 \\
\hline $\mathrm{H}$ & -4.475945 & 2.254904 & -0.173933 & $\mathrm{H}$ & 4.503885 & -2.186539 & 2.199874 \\
\hline C & -2.560768 & 1.775897 & 0.835574 & & & & \\
\hline $\mathrm{H}$ & -2.264591 & 1.959137 & 1.872850 & IN1-A & & & \\
\hline C & 0.103474 & -2.885681 & -0.848672 & C & -2.581009 & 0.516523 & 3.003543 \\
\hline $\mathrm{H}$ & 0.388876 & -2.793740 & -1.900396 & C & -3.264231 & 1.320877 & 2.154449 \\
\hline C & -0.689962 & -4.193469 & -0.600512 & C & -1.349405 & 1.239805 & 0.670888 \\
\hline $\mathrm{H}$ & -0.731465 & -4.853129 & -1.466040 & C & -2.632471 & 1.675135 & 0.947588 \\
\hline $\mathrm{H}$ & -0.351382 & -4.744533 & 0.278440 & $\mathrm{H}$ & -4.275061 & 1.631071 & 2.383298 \\
\hline C & -3.369990 & -1.788224 & 0.011706 & $\mathrm{H}$ & -3.151100 & 2.297395 & 0.226539 \\
\hline C & -4.264383 & -1.954342 & -1.244719 & $\mathrm{H}$ & -2.934356 & 0.155594 & 3.960636 \\
\hline $\mathrm{H}$ & -4.373151 & -3.016458 & -1.474715 & O & -1.338326 & 0.087048 & 2.742331 \\
\hline $\mathrm{H}$ & -3.832109 & -1.446901 & -2.112611 & C & -0.702732 & 0.358356 & 1.585897 \\
\hline $\mathrm{H}$ & -5.253821 & -1.538001 & -1.046939 & O & 0.404289 & -0.195668 & 1.468889 \\
\hline C & -4.018346 & -2.491699 & 1.229727 & C & -0.692414 & 1.587124 & -0.592547 \\
\hline $\mathrm{H}$ & -4.161232 & -3.550425 & 1.007271 & $\mathrm{O}$ & 0.447058 & 1.228795 & -0.936577 \\
\hline $\mathrm{H}$ & -4.992669 & -2.043149 & 1.432998 & O & -1.417929 & 2.349857 & -1.361204 \\
\hline $\mathrm{H}$ & -3.393727 & -2.400912 & 2.123555 & C & -0.880617 & 2.771632 & -2.627886 \\
\hline C & -1.860612 & 2.765463 & -0.068453 & $\mathrm{H}$ & -0.603119 & 1.904088 & -3.227384 \\
\hline C & -1.315651 & 3.934300 & 0.462015 & $\mathrm{H}$ & -1.684816 & 3.328966 & -3.102133 \\
\hline C & -1.732317 & 2.507268 & -1.437380 & $\mathrm{H}$ & -0.012074 & 3.408500 & -2.458625 \\
\hline C & -0.644542 & 4.833680 & -0.362002 & $\mathrm{Cu}$ & 1.731779 & 0.140527 & 0.064451 \\
\hline $\mathrm{H}$ & -1.429175 & 4.151087 & 1.521759 & $N$ & 3.308098 & 1.094817 & -0.640151 \\
\hline C & -1.048686 & 3.400687 & -2.259907 & $N$ & 2.853106 & -1.362082 & 0.594520 \\
\hline $\mathrm{H}$ & -2.190937 & 1.623123 & -1.878304 & C & 4.544041 & 0.734971 & -0.564953 \\
\hline
\end{tabular}




\begin{tabular}{|c|c|c|c|c|c|c|c|}
\hline 0 & 5.439838 & 1.671170 & -0.792459 & C & 1.568240 & 3.572876 & 2.318023 \\
\hline C & 4.111683 & -1.544602 & 0.386355 & $\mathrm{H}$ & 3.030395 & 2.103535 & 1.761409 \\
\hline O & 4.604247 & -2.698642 & 0.787063 & C & 0.767264 & 4.630310 & 1.893288 \\
\hline c & 4.742351 & 2.929194 & -0.982759 & $\mathrm{H}$ & 0.199636 & 5.872617 & 0.228262 \\
\hline $\mathrm{H}$ & 5.033863 & 3.588949 & -0.163961 & $\mathrm{H}$ & 1.570292 & 3.274879 & 3.361903 \\
\hline $\mathrm{H}$ & 5.068907 & 3.344231 & -1.935387 & $\mathrm{H}$ & 0.136258 & 5.154980 & 2.603353 \\
\hline C & 3.243791 & 2.538756 & -0.949739 & C & -3.122899 & -2.343594 & 1.734299 \\
\hline c & 2.273109 & -2.611175 & 1.152681 & C & -3.434020 & -1.655856 & 0.786751 \\
\hline C & 3.549331 & -3.413388 & 1.484865 & C & -3.890868 & -0.843337 & -0.294752 \\
\hline $\mathrm{H}$ & 3.538920 & -4.436079 & 1.110695 & C & -5.219135 & -0.299168 & -0.275416 \\
\hline $\mathrm{H}$ & 3.804009 & -3.401412 & 2.545872 & C & -3.057390 & -0.581720 & -1.375467 \\
\hline C & 5.104119 & -0.641466 & -0.304186 & C & -5.667553 & 0.470702 & -1.383926 \\
\hline C & 5.432262 & -1.276243 & -1.684565 & C & -6.098153 & -0.494077 & 0.819676 \\
\hline $\mathrm{H}$ & 5.880534 & -2.260833 & -1.533807 & C & -3.522812 & 0.155223 & -2.487940 \\
\hline $\mathrm{H}$ & 4.531522 & -1.381286 & -2.297119 & C & -6.975234 & 1.022425 & -1.365407 \\
\hline $\mathrm{H}$ & 6.151526 & -0.645909 & -2.213093 & C & -4.797444 & 0.660412 & -2.486681 \\
\hline C & 6.383449 & -0.535832 & 0.554534 & C & -7.355789 & 0.057248 & 0.810437 \\
\hline $\mathrm{H}$ & 6.797727 & -1.532134 & 0.715198 & $\mathrm{H}$ & -5.769418 & -1.099400 & 1.658392 \\
\hline $\mathrm{H}$ & 7.126339 & 0.072929 & 0.037446 & $\mathrm{H}$ & -2.880173 & 0.315543 & -3.346469 \\
\hline $\mathrm{H}$ & 6.176364 & -0.081295 & 1.527912 & C & -7.802038 & 0.824357 & -0.290324 \\
\hline $\mathrm{H}$ & 1.718045 & -2.356742 & 2.057030 & $\mathrm{H}$ & -7.312047 & 1.601218 & -2.220882 \\
\hline $\mathrm{H}$ & 2.790916 & 2.648814 & -1.939542 & $\mathrm{H}$ & -5.155072 & 1.220926 & -3.345917 \\
\hline c & 1.348944 & -3.266652 & 0.149213 & $\mathrm{H}$ & -8.021949 & -0.106217 & 1.651802 \\
\hline C & -0.019229 & -3.338442 & 0.402429 & $\mathrm{H}$ & -8.801606 & 1.245853 & -0.283318 \\
\hline C & 1.859201 & -3.826697 & -1.028610 & C & -2.748452 & -3.215045 & 2.846485 \\
\hline c & -0.868591 & -3.992451 & -0.487574 & $\mathrm{H}$ & -1.966218 & -2.759764 & 3.465325 \\
\hline $\mathrm{H}$ & -0.426243 & -2.879822 & 1.298384 & $\mathrm{H}$ & -3.611372 & -3.422759 & 3.486849 \\
\hline C & 1.013200 & -4.479458 & -1.917762 & $\mathrm{H}$ & -2.369175 & -4.174349 & 2.478022 \\
\hline $\mathrm{H}$ & 2.925654 & -3.781522 & -1.243068 & $\mathrm{O}$ & -1.767843 & -1.007032 & -1.267071 \\
\hline C & -0.351766 & -4.572605 & -1.641074 & C & -1.031361 & -1.256212 & -2.453196 \\
\hline $\mathrm{H}$ & -1.932653 & -4.033205 & -0.279469 & $\mathrm{H}$ & -0.765084 & -0.326407 & -2.969662 \\
\hline $\mathrm{H}$ & 1.418256 & -4.929491 & -2.818456 & $\mathrm{H}$ & -0.119021 & -1.764332 & -2.137391 \\
\hline $\mathrm{H}$ & -1.008643 & -5.096262 & -2.328321 & $\mathrm{H}$ & -1.589374 & -1.906392 & -3.133891 \\
\hline C & 2.405044 & 3.289235 & 0.063607 & & & & \\
\hline C & 1.615369 & 4.362337 & -0.349917 & \multicolumn{2}{|c|}{ IN1-B } & & \\
\hline C & 2.389868 & 2.909698 & 1.408498 & C & -4.346226 & -1.533468 & -2.492051 \\
\hline C & 0.801616 & 5.032462 & 0.559486 & C & -2.116746 & -1.533711 & -1.551376 \\
\hline $\mathrm{H}$ & 1.645099 & 4.687000 & -1.387722 & C & -3.279367 & -2.204065 & -1.854744 \\
\hline
\end{tabular}




\begin{tabular}{|c|c|c|c|c|c|c|c|}
\hline H & -5.271696 & -2.035063 & -2.741866 & C & -0.608934 & 3.765436 & -0.608840 \\
\hline $\mathrm{H}$ & -3.364381 & -3.257327 & -1.615014 & C & -1.987214 & 3.635923 & -0.767371 \\
\hline $\mathrm{O}$ & -3.036673 & 0.428685 & -2.519985 & C & -0.111555 & 4.470552 & 0.492314 \\
\hline C & -2.013971 & -0.140070 & -1.850500 & C & -2.856052 & 4.199344 & 0.165430 \\
\hline O & -1.066084 & 0.621554 & -1.611146 & $\mathrm{H}$ & -2.382582 & 3.097417 & -1.624048 \\
\hline C & -0.977407 & -2.208008 & -0.911273 & C & -0.977198 & 5.020775 & 1.429901 \\
\hline O & 0.047951 & -1.635222 & -0.517001 & $\mathrm{H}$ & 0.961242 & 4.606031 & 0.618371 \\
\hline O & -1.122787 & -3.500208 & -0.827239 & C & -2.354917 & 4.882656 & 1.268257 \\
\hline c & -0.020536 & -4.278168 & -0.309035 & $\mathrm{H}$ & -3.928351 & 4.117086 & 0.025085 \\
\hline $\mathrm{H}$ & 0.269599 & -3.893900 & 0.669002 & $\mathrm{H}$ & -0.580730 & 5.569334 & 2.278300 \\
\hline $\mathrm{H}$ & -0.405500 & -5.292586 & -0.234839 & $\mathrm{H}$ & -3.033338 & 5.321103 & 1.992577 \\
\hline $\mathrm{H}$ & 0.814787 & -4.226730 & -1.008236 & C & 3.004201 & -2.517418 & -0.266095 \\
\hline $\mathrm{Cu}$ & 0.586697 & 0.287109 & -0.589901 & C & 3.306457 & -3.808178 & 0.177988 \\
\hline$N$ & 2.332718 & -0.154470 & 0.206030 & C & 3.010061 & -2.250665 & -1.632767 \\
\hline$N$ & 1.194205 & 2.120943 & -1.005122 & C & 3.601882 & -4.818257 & -0.730914 \\
\hline C & 3.313145 & 0.653892 & 0.428515 & $\mathrm{H}$ & 3.309181 & -4.029970 & 1.243719 \\
\hline $\mathrm{O}$ & 4.296054 & 0.190227 & 1.171927 & C & 3.296659 & -3.264390 & -2.546661 \\
\hline C & 2.376706 & 2.607408 & -0.845198 & $\mathrm{H}$ & 2.796759 & -1.249286 & -1.996254 \\
\hline O & 2.595178 & 3.799755 & -1.366656 & C & 3.590350 & -4.549071 & -2.099381 \\
\hline C & 3.893127 & -1.107395 & 1.668578 & $\mathrm{H}$ & 3.843046 & -5.814113 & -0.373143 \\
\hline $\mathrm{H}$ & 4.740548 & -1.782216 & 1.559392 & $\mathrm{H}$ & 3.302912 & -3.045234 & -3.609821 \\
\hline $\mathrm{H}$ & 3.633499 & -0.979693 & 2.721313 & $\mathrm{H}$ & 3.822617 & -5.334531 & -2.810988 \\
\hline C & 2.683781 & -1.469715 & 0.783328 & C & -4.223053 & 0.811347 & 0.379147 \\
\hline C & 0.343840 & 3.177939 & -1.627653 & C & -3.378238 & 0.113734 & 0.891818 \\
\hline C & 1.415921 & 4.162497 & -2.129744 & C & -2.432436 & -0.733606 & 1.546028 \\
\hline $\mathrm{H}$ & 1.191485 & 5.206579 & -1.918005 & C & -1.221240 & -0.205426 & 2.094791 \\
\hline $\mathrm{H}$ & 1.663323 & 4.032643 & -3.185501 & C & -2.726033 & -2.087499 & 1.700477 \\
\hline C & 3.546890 & 2.049247 & -0.082766 & C & -0.369027 & -1.054928 & 2.854279 \\
\hline C & 3.808134 & 2.991177 & 1.120314 & C & -0.841603 & 1.153734 & 1.916187 \\
\hline $\mathrm{H}$ & 4.020722 & 3.998663 & 0.757681 & C & -1.857609 & -2.930728 & 2.438717 \\
\hline $\mathrm{H}$ & 2.940702 & 3.027652 & 1.786443 & C & 0.798113 & -0.515833 & 3.455337 \\
\hline $\mathrm{H}$ & 4.671234 & 2.633099 & 1.684160 & C & -0.722383 & -2.419049 & 3.010187 \\
\hline C & 4.787875 & 2.004576 & -1.011108 & C & 0.309611 & 1.636923 & 2.494686 \\
\hline $\mathrm{H}$ & 5.001017 & 3.008669 & -1.381921 & $\mathrm{H}$ & -1.494217 & 1.819509 & 1.357815 \\
\hline $\mathrm{H}$ & 5.653002 & 1.646363 & -0.450206 & $\mathrm{H}$ & -2.102241 & -3.976689 & 2.581380 \\
\hline $\mathrm{H}$ & 4.621429 & 1.340107 & -1.864638 & C & 1.136497 & 0.802383 & 3.280239 \\
\hline $\mathrm{H}$ & -0.213125 & 2.741666 & -2.456029 & $\mathrm{H}$ & 1.409205 & -1.165073 & 4.078252 \\
\hline $\mathrm{H}$ & 1.846492 & -1.809932 & 1.396257 & $\mathrm{H}$ & -0.084955 & -3.065073 & 3.608210 \\
\hline
\end{tabular}




\begin{tabular}{|c|c|c|c|c|c|c|c|}
\hline $\mathrm{H}$ & 0.559010 & 2.685235 & 2.368610 & O & -1.141655 & -4.116239 & -0.510750 \\
\hline $\mathrm{H}$ & 2.017704 & 1.212562 & 3.764776 & $C$ & -5.062950 & 0.151559 & 0.701722 \\
\hline C & -5.291830 & 1.626906 & -0.190175 & $\mathrm{H}$ & -5.558917 & 0.075829 & 1.670874 \\
\hline $\mathrm{H}$ & -4.948676 & 2.187610 & -1.067360 & $\mathrm{H}$ & -5.730216 & 0.602225 & -0.031888 \\
\hline $\mathrm{H}$ & -6.138575 & 0.999945 & -0.488625 & C & -3.670948 & 0.825578 & 0.769394 \\
\hline $\mathrm{H}$ & -5.660316 & 2.348331 & 0.546258 & $\mathrm{H}$ & -3.500588 & 1.262418 & 1.755714 \\
\hline O & -3.849493 & -2.530751 & 1.100948 & C & 0.707516 & -2.757534 & 0.074477 \\
\hline C & -4.318376 & -3.840631 & 1.393569 & $\mathrm{H}$ & 1.265148 & -2.199168 & -0.679676 \\
\hline$H$ & -4.458276 & -3.980036 & 2.470003 & C & 0.307015 & -4.153231 & -0.460028 \\
\hline $\mathrm{H}$ & -5.283001 & -3.926266 & 0.894036 & $\mathrm{H}$ & 0.662309 & -4.343983 & -1.471978 \\
\hline$H$ & -3.635730 & -4.605052 & 1.003241 & $\mathrm{H}$ & 0.587853 & -4.968244 & 0.207751 \\
\hline C & -4.165425 & -0.232046 & -2.814434 & $\mathrm{C}$ & -3.031610 & -2.774457 & -0.018697 \\
\hline \multirow[t]{2}{*}{$H$} & -4.874909 & 0.400606 & -3.332388 & C & -3.666311 & -3.206757 & -1.361657 \\
\hline & & & & $\mathrm{H}$ & -3.384450 & -4.237580 & -1.581292 \\
\hline \multicolumn{2}{|c|}{ IN1-C } & & & $\mathrm{H}$ & -3.330296 & -2.569015 & -2.183831 \\
\hline C & 4.045197 & 1.649052 & 1.441534 & $\mathrm{H}$ & -4.753468 & -3.146618 & -1.292292 \\
\hline C & 3.697667 & 2.791291 & 2.086015 & C & -3.534839 & -3.694026 & 1.128165 \\
\hline C & 1.389248 & 2.089333 & 1.915082 & $\mathrm{H}$ & -3.253958 & -4.727151 & 0.913959 \\
\hline C & 2.328582 & 3.009437 & 2.330771 & $\mathrm{H}$ & -4.623054 & -3.634315 & 1.194565 \\
\hline $\mathrm{H}$ & 4.455477 & 3.500500 & 2.391100 & $\mathrm{H}$ & -3.103188 & -3.401022 & 2.090491 \\
\hline $\mathrm{H}$ & 2.004255 & 3.910517 & 2.840556 & C & -3.465129 & 1.882855 & -0.295143 \\
\hline $\mathrm{H}$ & 5.052554 & 1.342011 & 1.195079 & C & -3.896818 & 3.188743 & -0.046342 \\
\hline O & 3.144935 & 0.737377 & 1.065917 & C & -2.944584 & 1.566506 & -1.548455 \\
\hline C & 1.820138 & 0.905788 & 1.238565 & C & -3.797984 & 4.164106 & -1.034758 \\
\hline O & 1.122943 & -0.011077 & 0.791048 & $\mathrm{H}$ & -4.340662 & 3.440011 & 0.915188 \\
\hline C & -0.050279 & 2.309695 & 2.098577 & C & -2.828202 & 2.545298 & -2.533740 \\
\hline O & -0.940679 & 1.535697 & 1.712900 & $\mathrm{H}$ & -2.638972 & 0.547228 & -1.765655 \\
\hline O & -0.333534 & 3.422114 & 2.716579 & C & -3.256109 & 3.844885 & -2.279100 \\
\hline C & -1.721438 & 3.758849 & 2.922123 & $\mathrm{H}$ & -4.149121 & 5.171640 & -0.836090 \\
\hline $\mathrm{H}$ & -2.224235 & 3.823300 & 1.955879 & $\mathrm{H}$ & -2.415245 & 2.286381 & -3.503705 \\
\hline $\mathrm{H}$ & -1.705336 & 4.724769 & 3.421061 & $\mathrm{H}$ & -3.178961 & 4.604537 & -3.049945 \\
\hline $\mathrm{H}$ & -2.191802 & 3.004657 & 3.555407 & C & 1.498350 & -2.806803 & 1.360852 \\
\hline $\mathrm{Cu}$ & -0.807495 & -0.219454 & 0.762734 & C & 2.872972 & -2.573885 & 1.327766 \\
\hline N & -2.762749 & -0.332155 & 0.606661 & C & 0.883205 & -3.123457 & 2.572866 \\
\hline N & -0.611757 & -2.101764 & 0.240510 & C & 3.625797 & -2.658430 & 2.495098 \\
\hline C & -3.469038 & -1.368172 & 0.295827 & $\mathrm{H}$ & 3.351279 & -2.315568 & 0.385741 \\
\hline O & -4.773075 & -1.196486 & 0.262900 & C & 1.633943 & -3.196991 & 3.743167 \\
\hline C & -1.536038 & -2.931961 & -0.095637 & $\mathrm{H}$ & -0.186277 & -3.320881 & 2.60900 \\
\hline
\end{tabular}




\begin{tabular}{|c|c|c|c|c|c|c|c|}
\hline C & 3.007239 & -2.965575 & 3.705214 & $\mathrm{H}$ & 5.417807 & 1.884346 & -0.289992 \\
\hline$H$ & 4.697966 & -2.490899 & 2.460853 & $\mathrm{H}$ & 5.029125 & -0.866349 & -3.591012 \\
\hline $\mathrm{H}$ & 1.150455 & -3.448635 & 4.681813 & $\mathrm{O}$ & 3.375284 & -0.390244 & -2.552636 \\
\hline$H$ & 3.594803 & -3.035966 & 4.614918 & $C$ & 2.710874 & 0.259193 & -1.568275 \\
\hline C & 0.752199 & 3.000434 & -1.119354 & O & 1.501776 & 0.006440 & -1.524015 \\
\hline C & 1.432462 & 2.088471 & -1.533998 & $C$ & 2.794743 & 1.926050 & 0.317522 \\
\hline C & -0.051644 & 4.131999 & -0.666065 & $\mathrm{O}$ & 1.579867 & 1.945450 & 0.500266 \\
\hline$H$ & -1.044460 & 3.799649 & -0.341243 & $\mathrm{O}$ & 3.629115 & 2.634399 & 1.041133 \\
\hline $\mathrm{H}$ & 0.435910 & 4.659302 & 0.162123 & C & 3.074249 & 3.440011 & 2.097084 \\
\hline $\mathrm{H}$ & -0.196731 & 4.853250 & -1.476534 & $\mathrm{H}$ & 2.398364 & 4.187035 & 1.677855 \\
\hline C & 3.542298 & 0.922706 & -1.974235 & $\mathrm{H}$ & 3.929037 & 3.917408 & 2.570376 \\
\hline C & 2.154146 & 0.954854 & -2.015705 & $\mathrm{H}$ & 2.542074 & 2.803248 & 2.806173 \\
\hline C & 3.567405 & -1.298399 & -2.928999 & $\mathrm{Cu}$ & 0.105576 & 0.523762 & -0.192909 \\
\hline C & 1.436923 & -0.170690 & -2.530691 & $N$ & -1.179497 & 1.166472 & 1.136088 \\
\hline C & 4.248041 & -0.214655 & -2.440501 & $\mathrm{~N}$ & 0.295774 & -1.230062 & 0.831904 \\
\hline C & 2.149801 & -1.310928 & -2.988136 & $C$ & -1.736061 & 0.496204 & 2.085310 \\
\hline C & 0.021788 & -0.173952 & -2.615560 & O & -2.603467 & 1.164278 & 2.832330 \\
\hline$H$ & 5.331489 & -0.231062 & -2.419209 & $C$ & -0.452304 & -1.660362 & 1.781888 \\
\hline $\mathrm{H}$ & 1.982295 & -3.283626 & -3.861702 & $\mathrm{O}$ & -0.254155 & -2.906988 & 2.177201 \\
\hline$H$ & 4.119364 & -2.161521 & -3.290137 & $C$ & -2.766139 & 2.472159 & 2.234973 \\
\hline C & -0.649559 & -1.261464 & -3.117217 & $\mathrm{H}$ & -2.812954 & 3.206753 & 3.036650 \\
\hline $\mathrm{H}$ & -0.511593 & 0.722978 & -2.317622 & $\mathrm{H}$ & -3.702790 & 2.466156 & 1.672399 \\
\hline$H$ & -1.731689 & -1.228493 & -3.217901 & $C$ & -1.534595 & 2.601948 & 1.333477 \\
\hline C & 0.055161 & -2.406330 & -3.555554 & $\mathrm{H}$ & -0.697261 & 3.024871 & 1.901517 \\
\hline $\mathrm{H}$ & -0.485975 & -3.252015 & -3.968210 & C & 1.284043 & -2.271335 & 0.509668 \\
\hline C & 1.427625 & -2.422746 & -3.497279 & $\mathrm{H}$ & 1.248188 & -2.472643 & -0.562015 \\
\hline $\mathrm{O}$ & 4.161322 & 1.999164 & -1.434964 & $C$ & 0.734141 & -3.486987 & 1.290536 \\
\hline C & 5.531948 & 2.222121 & -1.744362 & $\mathrm{H}$ & 0.213751 & -4.195722 & 0.641911 \\
\hline $\mathrm{H}$ & 6.185000 & 1.501345 & -1.236897 & $\mathrm{H}$ & 1.474249 & -3.997169 & 1.905979 \\
\hline $\mathrm{H}$ & 5.756132 & 3.227143 & -1.386974 & $C$ & -1.462805 & -0.899135 & 2.598835 \\
\hline \multirow[t]{2}{*}{$\mathrm{H}$} & 5.704657 & 2.174576 & -2.823831 & C & -2.769751 & -1.704717 & 2.728430 \\
\hline & & & & $\mathrm{H}$ & -2.562526 & -2.656957 & 3.220260 \\
\hline \multicolumn{2}{|c|}{ IN1-D } & & & $\mathrm{H}$ & -3.209309 & -1.909114 & 1.750851 \\
\hline C & 4.695075 & -0.270627 & -2.750943 & $\mathrm{H}$ & -3.485969 & -1.147471 & 3.335475 \\
\hline C & 5.470755 & 0.514409 & -1.969852 & $C$ & -0.828017 & -0.713406 & 4.009818 \\
\hline C & 3.476841 & 1.140533 & -0.735269 & $\mathrm{H}$ & -0.574023 & -1.691403 & 4.423283 \\
\hline C & 4.834241 & 1.234896 & -0.933204 & $\mathrm{H}$ & -1.544560 & -0.226218 & 4.673412 \\
\hline $\mathrm{H}$ & 6.535061 & 0.588822 & -2.149964 & $\mathrm{H}$ & 0.079304 & -0.102574 & 3.960388 \\
\hline
\end{tabular}




\begin{tabular}{|c|c|c|c|c|c|c|c|}
\hline C & -1.737412 & 3.448140 & 0.102665 & C & -0.188957 & -3.890889 & -2.454046 \\
\hline C & -0.886508 & 4.532895 & -0.118878 & $\mathrm{H}$ & -0.121586 & -1.757418 & -2.569737 \\
\hline C & -2.799168 & 3.224136 & -0.777912 & $\mathrm{H}$ & 0.757767 & -4.015788 & -2.971767 \\
\hline C & -1.096270 & 5.390912 & -1.196602 & C & -0.904946 & -5.033631 & -2.032146 \\
\hline $\mathrm{H}$ & -0.065212 & 4.722427 & 0.567870 & $\mathrm{H}$ & -0.503960 & -6.024294 & -2.218489 \\
\hline C & -3.009893 & 4.079587 & -1.853535 & $\mathrm{C}$ & -2.122852 & -4.882019 & -1.417617 \\
\hline $\mathrm{H}$ & -3.439898 & 2.359208 & -0.645422 & $\mathrm{O}$ & -4.161207 & 0.233061 & -0.457812 \\
\hline C & -2.163325 & 5.167573 & -2.062189 & C & -5.482931 & 0.420397 & 0.039044 \\
\hline $\mathrm{H}$ & -0.437794 & 6.239879 & -1.349526 & $\mathrm{H}$ & -5.592501 & -0.009095 & 1.040666 \\
\hline $\mathrm{H}$ & -3.839659 & 3.899400 & -2.529784 & $\mathrm{H}$ & -6.225734 & -0.008517 & -0.639885 \\
\hline $\mathrm{H}$ & -2.338289 & 5.841425 & -2.894704 & $\mathrm{H}$ & -5.627406 & 1.499969 & 0.088525 \\
\hline C & 2.685471 & -1.834312 & 0.889133 & & & & \\
\hline C & 3.772699 & -2.330463 & 0.164643 & \multicolumn{2}{|c|}{ IN2-A } & & \\
\hline C & 2.923037 & -0.971131 & 1.961383 & C & -2.442808 & 0.328162 & 2.919668 \\
\hline C & 5.074574 & -1.978453 & 0.511619 & $\mathrm{C}$ & -2.830123 & 1.656762 & 2.352512 \\
\hline $\mathrm{H}$ & 3.599804 & -3.010340 & -0.666800 & C & -1.085345 & 1.309400 & 0.709370 \\
\hline C & 4.224130 & -0.609441 & 2.302596 & C & -2.195179 & 2.080069 & 1.257591 \\
\hline $\mathrm{H}$ & 2.088909 & -0.576948 & 2.534555 & $\mathrm{H}$ & -3.622143 & 2.216944 & 2.835095 \\
\hline C & 5.303107 & -1.112592 & 1.579340 & $\mathrm{H}$ & -2.452096 & 3.019832 & 0.781621 \\
\hline $\mathrm{H}$ & 5.911457 & -2.387552 & -0.045943 & $\mathrm{H}$ & -2.555161 & 0.300356 & 4.005897 \\
\hline $\mathrm{H}$ & 4.397576 & 0.052119 & 3.145822 & $\mathrm{O}$ & -1.055327 & 0.021753 & 2.703486 \\
\hline $\mathrm{H}$ & 6.317824 & -0.847042 & 1.858567 & C & -0.497383 & 0.322453 & 1.522056 \\
\hline C & -1.106908 & 0.975576 & -2.133778 & $\mathrm{O}$ & 0.558034 & -0.335252 & 1.295455 \\
\hline C & -1.763554 & 0.018018 & -1.744069 & C & -0.593119 & 1.540205 & -0.594327 \\
\hline C & -0.497249 & 2.052175 & -2.925147 & $\mathrm{O}$ & 0.446003 & 1.036911 & -1.115404 \\
\hline $\mathrm{H}$ & 0.412772 & 1.684597 & -3.407850 & O & -1.343915 & 2.341409 & -1.340629 \\
\hline $\mathrm{H}$ & -1.197564 & 2.374115 & -3.700824 & C & -0.892896 & 2.643726 & -2.662119 \\
\hline $\mathrm{H}$ & -0.252060 & 2.920750 & -2.307860 & $\mathrm{H}$ & -0.894043 & 1.745591 & -3.284681 \\
\hline C & -3.727337 & -1.013068 & -0.719879 & $\mathrm{H}$ & -1.603686 & 3.372086 & -3.048424 \\
\hline C & -2.474969 & -1.137550 & -1.326105 & $\mathrm{H}$ & 0.112448 & 3.067295 & -2.640116 \\
\hline C & -3.944443 & -3.414222 & -0.611992 & $\mathrm{Cu}$ & 1.806908 & 0.123299 & -0.093098 \\
\hline C & -1.926044 & -2.441318 & -1.585481 & $\mathrm{~N}$ & 3.315273 & 1.149642 & -0.875107 \\
\hline C & -4.474286 & -2.170285 & -0.389642 & $\mathrm{~N}$ & 3.067687 & -1.288748 & 0.471379 \\
\hline C & -2.662522 & -3.589882 & -1.192698 & C & 4.566887 & 0.853024 & -0.847320 \\
\hline C & -0.681237 & -2.625372 & -2.235485 & $\mathrm{O}$ & 5.413041 & 1.843205 & -1.049009 \\
\hline $\mathrm{H}$ & -5.459392 & -2.081885 & 0.051290 & C & 4.317370 & -1.411104 & 0.188745 \\
\hline $\mathrm{H}$ & -2.698424 & -5.751096 & -1.111956 & $\mathrm{O}$ & 4.923610 & -2.484048 & 0.659376 \\
\hline $\mathrm{H}$ & -4.522530 & -4.293773 & -0.342918 & $\mathrm{C}$ & 4.645487 & 3.070645 & -1.157178 \\
\hline
\end{tabular}




\begin{tabular}{|c|c|c|c|c|c|c|c|}
\hline $\mathrm{H}$ & 4.931469 & 3.707035 & -0.318144 & $\mathrm{H}$ & 1.712364 & 3.020765 & 3.332809 \\
\hline $\mathrm{H}$ & 4.918196 & 3.545454 & -2.098959 & $\mathrm{H}$ & 0.065353 & 4.781777 & 2.744856 \\
\hline C & 3.169827 & 2.602905 & -1.095005 & $C$ & -3.233242 & -0.879070 & 2.352155 \\
\hline C & 2.630568 & -2.471694 & 1.247110 & $C$ & -3.727305 & -0.789105 & 1.160709 \\
\hline C & 3.970842 & -3.211144 & 1.477015 & $C$ & -4.200395 & -0.651139 & -0.082837 \\
\hline $\mathrm{H}$ & 3.966437 & -4.246316 & 1.136529 & $C$ & -5.561947 & -0.180589 & -0.358582 \\
\hline $\mathrm{H}$ & 4.324715 & -3.156970 & 2.507343 & $C$ & -3.309691 & -0.960895 & -1.184996 \\
\hline C & 5.181189 & -0.517057 & -0.673446 & $C$ & -5.963755 & -0.049201 & -1.709889 \\
\hline C & 5.263839 & -1.170841 & -2.080550 & $C$ & -6.467853 & 0.141351 & 0.654751 \\
\hline $\mathrm{H}$ & 5.726536 & -2.157405 & -1.997360 & $C$ & -3.752655 & -0.810117 & -2.508086 \\
\hline $\mathrm{H}$ & 4.270653 & -1.273177 & -2.528039 & $C$ & -7.267118 & 0.397897 & -2.018925 \\
\hline $\mathrm{H}$ & 5.885857 & -0.552887 & -2.733373 & $C$ & -5.038646 & -0.373110 & -2.739208 \\
\hline C & 6.589119 & -0.399451 & -0.057080 & $C$ & -7.742636 & 0.579085 & 0.330812 \\
\hline $\mathrm{H}$ & 7.034800 & -1.390972 & 0.031722 & $\mathrm{H}$ & -6.174007 & 0.046302 & 1.695101 \\
\hline $\mathrm{H}$ & 7.222887 & 0.213452 & -0.699409 & $\mathrm{H}$ & -3.104164 & -1.043859 & -3.342660 \\
\hline $\mathrm{H}$ & 6.556472 & 0.058777 & 0.935353 & $C$ & -8.148753 & 0.708692 & -1.006362 \\
\hline $\mathrm{H}$ & 2.202747 & -2.119686 & 2.187271 & $\mathrm{H}$ & -7.565035 & 0.491928 & -3.059053 \\
\hline $\mathrm{H}$ & 2.673196 & 2.748247 & -2.058788 & $\mathrm{H}$ & -5.369764 & -0.270153 & -3.769972 \\
\hline C & 1.593296 & -3.278808 & 0.494819 & $\mathrm{H}$ & -8.438880 & 0.823697 & 1.126541 \\
\hline C & 0.336754 & -3.503448 & 1.057275 & $\mathrm{H}$ & -9.151370 & 1.050392 & -1.237936 \\
\hline C & 1.897904 & -3.843549 & -0.747915 & $C$ & -3.276541 & -2.132177 & 3.190359 \\
\hline C & -0.593084 & -4.307842 & 0.402279 & $\mathrm{H}$ & -2.253034 & -2.415846 & 3.455865 \\
\hline $\mathrm{H}$ & 0.089643 & -3.047572 & 2.011463 & $\mathrm{H}$ & -3.822798 & -1.935774 & 4.118880 \\
\hline C & 0.968893 & -4.644899 & -1.404818 & $\mathrm{H}$ & -3.756137 & -2.962753 & 2.669960 \\
\hline $\mathrm{H}$ & 2.875993 & -3.681560 & -1.197477 & $\mathrm{O}$ & -2.113697 & -1.372623 & -0.848186 \\
\hline C & -0.275814 & -4.886232 & -0.824096 & C & -1.142290 & -1.699771 & -1.849634 \\
\hline $\mathrm{H}$ & -1.560849 & -4.493806 & 0.857524 & $\mathrm{H}$ & -0.926777 & -0.823624 & -2.465975 \\
\hline $\mathrm{H}$ & 1.222754 & -5.097163 & -2.358314 & $\mathrm{H}$ & -0.255522 & -1.994207 & -1.292405 \\
\hline $\mathrm{H}$ & -0.991494 & -5.531425 & -1.324191 & $\mathrm{H}$ & -1.492011 & -2.538201 & -2.456492 \\
\hline C & 2.331989 & 3.240698 & -0.005551 & & & & \\
\hline C & 1.417833 & 4.244398 & -0.324285 & \multicolumn{2}{|c|}{ IN2-B } & & \\
\hline C & 2.439632 & 2.813920 & 1.321494 & C & -2.967484 & -1.944043 & -2.166238 \\
\hline C & 0.607619 & 4.800938 & 0.661917 & C & -1.207751 & -1.362526 & -0.607439 \\
\hline $\mathrm{H}$ & 1.340301 & 4.600680 & -1.348710 & C & -2.309283 & -2.214691 & -1.036659 \\
\hline C & 1.622165 & 3.362272 & 2.306619 & $\mathrm{H}$ & -3.752146 & -2.574997 & -2.566088 \\
\hline $\mathrm{H}$ & 3.168998 & 2.054886 & 1.598354 & $\mathrm{H}$ & -2.537105 & -3.093690 & -0.443757 \\
\hline C & 0.700674 & 4.352925 & 1.976966 & $\mathrm{O}$ & -1.228007 & -0.339947 & -2.749101 \\
\hline $\mathrm{H}$ & -0.095739 & 5.585398 & 0.401455 & $\mathrm{C}$ & -0.647587 & -0.479264 & -1.545497 \\
\hline
\end{tabular}




\begin{tabular}{|c|c|c|c|c|c|c|c|}
\hline O & 0.389265 & 0.234235 & -1.414002 & $\mathrm{H}$ & -0.105353 & 3.107088 & -2.116340 \\
\hline c & -0.694252 & -1.419255 & 0.711785 & C & 0.964196 & 4.543393 & 1.317267 \\
\hline $\mathrm{O}$ & 0.361219 & -0.868800 & 1.136022 & $\mathrm{H}$ & 2.844020 & 3.564917 & 0.974925 \\
\hline O & -1.436624 & -2.104668 & 1.568571 & C & -0.305707 & 4.820571 & 0.812822 \\
\hline C & -0.980235 & -2.189914 & 2.920993 & $\mathrm{H}$ & -1.675967 & 4.521672 & -0.818581 \\
\hline $\mathrm{H}$ & -0.945763 & -1.195804 & 3.371139 & $\mathrm{H}$ & 1.271815 & 4.953060 & 2.274199 \\
\hline $\mathrm{H}$ & -1.710642 & -2.816198 & 3.430390 & $\mathrm{H}$ & -0.989966 & 5.445059 & 1.378115 \\
\hline $\mathrm{H}$ & 0.009130 & -2.649113 & 2.965111 & C & 2.153209 & -3.220292 & 0.249068 \\
\hline $\mathrm{Cu}$ & 1.680709 & -0.072766 & -0.014321 & C & 1.240651 & -4.158118 & 0.731128 \\
\hline$N$ & 3.211454 & -1.055471 & 0.791445 & C & 2.221934 & -2.981135 & -1.126443 \\
\hline$N$ & 2.935942 & 1.261105 & -0.739653 & C & 0.397384 & -4.837233 & -0.144230 \\
\hline C & 4.467036 & -0.809895 & 0.658359 & $\mathrm{H}$ & 1.189641 & -4.365346 & 1.797391 \\
\hline O & 5.295854 & -1.792310 & 0.955383 & C & 1.371263 & -3.651596 & -2.001562 \\
\hline C & 4.200050 & 1.381935 & -0.535336 & $\mathrm{H}$ & 2.944886 & -2.272516 & -1.525935 \\
\hline O & 4.778631 & 2.449201 & -1.054431 & C & 0.454765 & -4.578164 & -1.511097 \\
\hline C & 4.503387 & -2.957881 & 1.298114 & $\mathrm{H}$ & -0.303758 & -5.569509 & 0.243345 \\
\hline $\mathrm{H}$ & 4.736246 & -3.735773 & 0.569473 & $\mathrm{H}$ & 1.431320 & -3.455213 & -3.067253 \\
\hline $\mathrm{H}$ & 4.803639 & -3.275842 & 2.296159 & $\mathrm{H}$ & -0.205620 & -5.103063 & -2.193475 \\
\hline C & 3.038456 & -2.459503 & 1.215105 & C & -3.411951 & 0.570470 & -2.460755 \\
\hline C & 2.452671 & 2.441312 & -1.488855 & C & -3.925040 & 0.589350 & -1.274614 \\
\hline C & 3.776419 & 3.176791 & -1.809373 & C & -4.384652 & 0.542042 & -0.018490 \\
\hline $\mathrm{H}$ & 3.796321 & 4.212627 & -1.471211 & C & -3.534349 & 0.907919 & 1.119322 \\
\hline $\mathrm{H}$ & 4.060393 & 3.119310 & -2.860934 & C & -5.726745 & 0.048124 & 0.216521 \\
\hline C & 5.125057 & 0.489241 & 0.258295 & C & -4.040652 & 0.711797 & 2.424397 \\
\hline C & 5.501464 & 1.239517 & 1.564675 & C & -2.247753 & 1.430456 & 0.959354 \\
\hline $\mathrm{H}$ & 6.011864 & 2.172800 & 1.316143 & C & -6.192572 & -0.114377 & 1.535496 \\
\hline $\mathrm{H}$ & 4.612153 & 1.462916 & 2.162174 & C & -3.243491 & 1.028224 & 3.544731 \\
\hline $\mathrm{H}$ & 6.179864 & 0.620630 & 2.157167 & C & -5.364055 & 0.205684 & 2.583519 \\
\hline C & 6.391542 & 0.203238 & -0.577547 & C & -1.477429 & 1.731640 & 2.070373 \\
\hline $\mathrm{H}$ & 6.878580 & 1.144206 & -0.837375 & $\mathrm{H}$ & -1.847692 & 1.612647 & -0.032030 \\
\hline $\mathrm{H}$ & 7.087487 & -0.403885 & 0.002904 & $\mathrm{H}$ & -7.190501 & -0.485542 & 1.730671 \\
\hline $\mathrm{H}$ & 6.149078 & -0.332421 & -1.500147 & C & -1.970480 & 1.530155 & 3.368570 \\
\hline $\mathrm{H}$ & 1.961207 & 2.088026 & -2.397177 & $\mathrm{H}$ & -3.645792 & 0.879586 & 4.542686 \\
\hline $\mathrm{H}$ & 2.569388 & -2.456876 & 2.203043 & $\mathrm{H}$ & -5.739576 & 0.072671 & 3.595398 \\
\hline C & 1.470845 & 3.244385 & -0.662381 & $\mathrm{H}$ & -0.483472 & 2.142616 & 1.925086 \\
\hline C & 0.193446 & 3.512193 & -1.153432 & $\mathrm{H}$ & -1.358582 & 1.781493 & 4.228278 \\
\hline C & 1.847015 & 3.757340 & 0.582773 & C & -3.451803 & 1.729226 & -3.424501 \\
\hline C & -0.689905 & 4.301923 & -0.420877 & $\mathrm{H}$ & -2.429020 & 1.970916 & -3.730700 \\
\hline
\end{tabular}




$\begin{array}{llll}\text { H } & -4.012594 & 1.440779 & -4.319726 \\ \text { H } & -3.917304 & 2.615174 & -2.989755 \\ \text { O } & -6.425782 & -0.225572 & -0.850583 \\ \text { C } & -7.768843 & -0.728862 & -0.743168 \\ \text { H } & -8.404371 & 0.000508 & -0.235308 \\ \text { H } & -8.102440 & -0.863516 & -1.769498 \\ \text { H } & -7.771516 & -1.686838 & -0.217527 \\ \text { C } & -2.613211 & -0.688217 & -2.897027 \\ \text { H } & -2.749979 & -0.790673 & -3.975965\end{array}$

\section{IN2-C}

C

(

\section{c}

r

$\begin{array}{llll}\mathrm{H} & 4.984965 & -3.000188 & 0.749244 \\ \mathrm{H} & 3.204504 & -2.451988 & 2.411030 \\ \mathrm{H} & 4.481789 & -2.416094 & -1.627789 \\ \mathrm{O} & 2.654531 & -1.662635 & -1.283209 \\ \mathrm{C} & 1.742375 & -1.226337 & -0.406432\end{array}$

$\begin{array}{llll}\text { C } & 1.742375 & -1.226337 & -0.406432 \\ \text { O } & 0.690143 & -0.791993 & -0.964107\end{array}$

$\begin{array}{llll}\text { O } & 0.690143 & -0.791993 & -0.964107 \\ \mathrm{C} & 1.102664 & -0.745661 & 1.909021\end{array}$

$\begin{array}{llll}0 & 0.027823 & -0.132980 & 1.644043\end{array}$

○ $\quad 1.468436 \quad-0.862976 \quad 3.174664$

$\begin{array}{llll}\text { C } & 0.581862 & -0.353319 & 4.175781\end{array}$

$\begin{array}{llll}H & -0.388936 & -0.849900 & 4.114481\end{array}$

$\begin{array}{llll}H & 0.456341 & 0.724975 & 4.055970\end{array}$

$\begin{array}{llll}H & 1.064073 & -0.579210 & 5.125128\end{array}$

Cu $\quad-0.929392 \quad-0.461815 \quad-0.018565$

$\begin{array}{llll}\mathrm{N} & -2.008478 & -1.719763 & -1.116949\end{array}$

$\begin{array}{llll}N & -2.583184 & 0.439579 & 0.565785\end{array}$

C $\quad-3.276189 \quad-1.931263 \quad-1.058044$

$\begin{array}{llll}\mathrm{O} & -3.714543 & -3.044653 & -1.614658\end{array}$

$\begin{array}{llll}\text { C } & -3.803956 & 0.065779 & 0.382527\end{array}$

$\begin{array}{llll}\text { O } & -4.729632 & 0.804265 & 0.967466\end{array}$

$\begin{array}{llll}\text { C } & -2.576561 & -3.715559 & -2.214744\end{array}$

$\begin{array}{llll}H & -2.701630 & -3.658603 & -3.297342\end{array}$

$\begin{array}{llll}H & -2.594901 & -4.753549 & -1.884747\end{array}$

$\begin{array}{llll}\text { C } & -1.358751 & -2.915014 & -1.695587 \\ \text { C } & -2.603844 & 1.731960 & 1.274673 \\ \text { C } & -4.044781 & 1.749173 & 1.824244 \\ \text { H } & -4.120719 & 1.371788 & 2.847057 \\ \text { H } & -4.549174 & 2.709312 & 1.731645 \\ \text { C } & -4.351743 & -1.084198 & -0.425462\end{array}$

$\begin{array}{llll}\text { C } & -5.206587 & -1.978719 & 0.505459\end{array}$

$\begin{array}{llll}H & -6.005686 & -1.383625 & 0.950845\end{array}$

$\begin{array}{llll}H & -4.601040 & -2.411864 & 1.307987\end{array}$

H $\quad-5.652712 \quad-2.790065 \quad-0.071519$

$\begin{array}{llll}\text { C } & -5.232743 & -0.493922 & -1.558002\end{array}$

$\begin{array}{llll}H & -6.052965 & 0.081977 & -1.125148\end{array}$

$\begin{array}{llll}H & -5.652280 & -1.309580 & -2.150761\end{array}$

$\begin{array}{llll}H & -4.650166 & 0.158600 & -2.215622\end{array}$

$\begin{array}{llll}\text { C } & 4.722076 & -0.414094 & -0.903538\end{array}$

$\begin{array}{llll}\text { C } & 3.985225 & 0.644879 & -0.851120\end{array}$

$\begin{array}{llll}\text { C } & 3.122002 & 1.666322 & -0.749000\end{array}$

$\begin{array}{llll}\text { C } & 2.673452 & 2.155700 & 0.556770\end{array}$

C $\quad 2.556460 \quad 2.226090 \quad-1.957026$

$\begin{array}{llll}\text { C } & 1.654201 & 3.131962 & 0.594327\end{array}$

$\begin{array}{llll}\text { C } & 3.211099 & 1.683637 & 1.758832\end{array}$

$\begin{array}{llll}\text { C } & 1.533197 & 3.189918 & -1.869235\end{array}$

$\begin{array}{llll}\text { C } & 1.194072 & 3.624441 & 1.832924\end{array}$

$\begin{array}{llll}\text { C } & 1.111382 & 3.612245 & -0.632657\end{array}$

$\begin{array}{llll}\text { C } & 2.750029 & 2.182383 & 2.966036\end{array}$

$\begin{array}{llll}H & 3.992574 & 0.930860 & 1.744330\end{array}$

$\begin{array}{llll}\text { H } & 1.081351 & 3.606189 & -2.760495\end{array}$

$\begin{array}{llll}\text { C } & 1.739872 & 3.155947 & 3.009617\end{array}$

$\begin{array}{llll}H & 0.419329 & 4.384754 & 1.841457\end{array}$

$\begin{array}{llll}H & 0.323497 & 4.358969 & -0.582733\end{array}$

$\begin{array}{llll}H & 3.184670 & 1.817147 & 3.891144\end{array}$

H $\quad 1.405704 \quad 3.548206 \quad 3.964446$

$\begin{array}{llll}\text { C } & 6.228526 & -0.423316 & -0.953879\end{array}$

$\begin{array}{llll}\text { H } & 6.562830 & -0.921891 & -1.869608\end{array}$

H $\quad 6.620203 \quad-0.982069 \quad-0.097670$

$\begin{array}{llll}H & 6.642243 & 0.585596 & -0.931506\end{array}$

$\begin{array}{llll}\text { O } & 3.046148 & 1.769542 & -3.078130\end{array}$

$\begin{array}{llll}\text { C } & 2.552973 & 2.241020 & -4.340186\end{array}$ 


\begin{tabular}{|c|c|c|c|c|c|c|c|}
\hline $\mathrm{H}$ & 1.492407 & 1.998294 & -4.445302 & $\mathrm{O}$ & -0.667451 & 0.807363 & -0.626718 \\
\hline $\mathrm{H}$ & 3.134211 & 1.707439 & -5.088803 & $C$ & -0.475629 & 1.025521 & 2.250842 \\
\hline $\mathrm{H}$ & 2.720430 & 3.316904 & -4.434038 & $\mathrm{O}$ & 0.454035 & 0.267859 & 1.838179 \\
\hline C & -0.527254 & -3.630335 & -0.647658 & $\mathrm{O}$ & -0.585690 & 1.225217 & 3.556896 \\
\hline C & 0.683588 & -4.219562 & -1.012583 & $C$ & 0.439259 & 0.702108 & 4.405721 \\
\hline C & -0.956653 & -3.720646 & 0.679320 & $\mathrm{H}$ & 1.413345 & 1.109351 & 4.125003 \\
\hline C & 1.454030 & -4.889042 & -0.066996 & $\mathrm{H}$ & 0.464944 & -0.389128 & 4.351026 \\
\hline $\mathrm{H}$ & 1.032540 & -4.144952 & -2.038931 & $\mathrm{H}$ & 0.170408 & 1.022844 & 5.410407 \\
\hline C & -0.180900 & -4.382594 & 1.627888 & $\mathrm{Cu}$ & 1.074716 & 0.325553 & -0.006255 \\
\hline $\mathrm{H}$ & -1.905649 & -3.283826 & 0.983016 & $\mathrm{~N}$ & 1.959757 & 1.192446 & -1.555833 \\
\hline C & 1.025812 & -4.968303 & 1.255525 & $\mathrm{~N}$ & 2.753348 & -0.636140 & 0.406256 \\
\hline $\mathrm{H}$ & 2.393215 & -5.345092 & -0.362631 & $C$ & 3.181456 & 1.088300 & -1.941165 \\
\hline $\mathrm{H}$ & -0.527240 & -4.453873 & 2.654269 & $\mathrm{O}$ & 3.627726 & 2.024172 & -2.754274 \\
\hline $\mathrm{H}$ & 1.627156 & -5.491117 & 1.992184 & $C$ & 3.856310 & -0.641472 & -0.262517 \\
\hline $\mathrm{H}$ & -0.711757 & -2.600208 & -2.516625 & $\mathrm{O}$ & 4.849946 & -1.337956 & 0.253786 \\
\hline $\mathrm{H}$ & -1.864450 & 1.709620 & 2.079172 & $C$ & 2.542611 & 2.960667 & -2.991224 \\
\hline C & -2.333220 & 2.900276 & 0.337167 & $\mathrm{H}$ & 2.303838 & 2.916179 & -4.054229 \\
\hline C & -2.292607 & 2.760453 & -1.049515 & $\mathrm{H}$ & 2.906083 & 3.953713 & -2.726987 \\
\hline C & -2.228881 & 4.180228 & 0.893216 & $C$ & 1.396997 & 2.453966 & -2.078801 \\
\hline C & -2.167899 & 3.883409 & -1.869069 & $C$ & 2.909923 & -1.512229 & 1.584466 \\
\hline $\mathrm{H}$ & -2.382828 & 1.778149 & -1.504312 & $C$ & 4.426721 & -1.821465 & 1.551663 \\
\hline C & -2.109861 & 5.300822 & 0.078046 & $\mathrm{H}$ & 4.998927 & -1.273056 & 2.302001 \\
\hline $\mathrm{H}$ & -2.267874 & 4.305109 & 1.973629 & $\mathrm{H}$ & 4.659838 & -2.884345 & 1.601804 \\
\hline C & -2.084485 & 5.154673 & -1.310489 & $C$ & 4.155434 & -0.016410 & -1.607658 \\
\hline $\mathrm{H}$ & -2.160955 & 3.761939 & -2.948031 & $C$ & 5.599996 & 0.522173 & -1.625189 \\
\hline $\mathrm{H}$ & -2.052600 & 6.289297 & 0.522862 & $\mathrm{H}$ & 6.298806 & -0.290725 & -1.424092 \\
\hline \multirow[t]{2}{*}{$\mathrm{H}$} & -2.012206 & 6.029689 & -1.948347 & $\mathrm{H}$ & 5.746762 & 1.303114 & -0.873766 \\
\hline & & & & $\mathrm{H}$ & 5.823584 & 0.942143 & -2.606661 \\
\hline \multicolumn{2}{|c|}{ IN2-D } & & & C & 3.984985 & -1.128260 & -2.679710 \\
\hline C & -3.713072 & 2.347827 & 0.005456 & $\mathrm{H}$ & 4.682157 & -1.944894 & -2.475704 \\
\hline C & -3.472791 & 3.003889 & 1.328462 & $\mathrm{H}$ & 4.214429 & -0.717611 & -3.666390 \\
\hline C & -1.456088 & 1.674175 & 1.462981 & $\mathrm{H}$ & 2.961651 & -1.515867 & -2.687518 \\
\hline C & -2.404749 & 2.615802 & 2.033639 & C & -4.605858 & 1.093486 & 0.176474 \\
\hline $\mathrm{H}$ & -4.178474 & 3.744995 & 1.684109 & C & -4.078085 & -0.053427 & -0.098209 \\
\hline $\mathrm{H}$ & -2.198737 & 3.026403 & 3.015672 & $C$ & -3.417474 & -1.176327 & -0.413477 \\
\hline $\mathrm{H}$ & -4.220316 & 3.010914 & -0.700082 & C & -3.412679 & -1.723420 & -1.773327 \\
\hline O & -2.494077 & 1.985860 & -0.648827 & C & -2.619853 & -1.811307 & 0.614044 \\
\hline$C$ & -1.501333 & 1.451544 & 0.073699 & $C$ & -2.594533 & -2.846471 & -2.041927 \\
\hline
\end{tabular}




\begin{tabular}{|c|c|c|c|c|c|c|c|}
\hline C & -4.180618 & -1.178421 & -2.806660 & $\mathrm{H}$ & 2.436578 & -3.116381 & -0.597933 \\
\hline C & -1.803507 & -2.909533 & 0.296742 & C & 0.774067 & -4.480250 & 2.622254 \\
\hline C & -2.566160 & -3.404485 & -3.338549 & $\mathrm{H}$ & 1.527729 & -2.709690 & 3.583878 \\
\hline C & -1.811051 & -3.395583 & -0.988984 & $C$ & 0.702067 & -5.210176 & 1.436943 \\
\hline C & -4.139291 & -1.740532 & -4.071866 & $\mathrm{H}$ & 1.261990 & -5.282839 & -0.641911 \\
\hline $\mathrm{H}$ & -4.814632 & -0.319312 & -2.613494 & $\mathrm{H}$ & 0.327611 & -4.872182 & 3.530926 \\
\hline $\mathrm{H}$ & -1.182402 & -3.382468 & 1.047316 & $\mathrm{H}$ & 0.200803 & -6.172749 & 1.420113 \\
\hline C & -3.332792 & -2.857155 & -4.344054 & & & & \\
\hline$H$ & -1.940790 & -4.271595 & -3.530766 & \multicolumn{2}{|c|}{ P1-A } & & \\
\hline $\mathrm{H}$ & -1.183952 & -4.255330 & -1.212784 & $C$ & 1.799817 & -3.764722 & -1.760983 \\
\hline$H$ & -4.745902 & -1.313475 & -4.863950 & $C$ & 2.599479 & -3.148018 & -2.882511 \\
\hline $\mathrm{H}$ & -3.320173 & -3.286854 & -5.339581 & $C$ & 1.754979 & -1.282392 & -1.665803 \\
\hline C & -6.008783 & 1.307647 & 0.682880 & $C$ & 2.557058 & -1.822222 & -2.848185 \\
\hline $\mathrm{H}$ & -6.530966 & 2.025154 & 0.041162 & $\mathrm{H}$ & 3.105489 & -3.750685 & -3.625692 \\
\hline $\mathrm{H}$ & -5.969969 & 1.719413 & 1.696212 & $\mathrm{H}$ & 2.999180 & -1.144814 & -3.566818 \\
\hline $\mathrm{H}$ & -6.577137 & 0.376947 & 0.705042 & $\mathrm{H}$ & 1.672944 & -4.842536 & -1.816379 \\
\hline O & -2.712505 & -1.288061 & 1.809965 & O & 0.420653 & -3.222886 & -1.883686 \\
\hline C & -1.967341 & -1.843288 & 2.900100 & $C$ & 0.376539 & -1.926389 & -1.772100 \\
\hline $\mathrm{H}$ & -2.287790 & -2.870821 & 3.091529 & $\mathrm{O}$ & -0.708217 & -1.339677 & -1.735595 \\
\hline $\mathrm{H}$ & -2.204711 & -1.209425 & 3.752402 & $C$ & 1.738752 & 0.220264 & -1.549570 \\
\hline$H$ & -0.897024 & -1.807405 & 2.679402 & $\mathrm{O}$ & 0.801872 & 0.878378 & -1.092746 \\
\hline C & 1.017422 & 3.382389 & -0.941728 & $\mathrm{O}$ & 2.856849 & 0.757174 & -1.921473 \\
\hline C & -0.140435 & 4.154683 & -1.034673 & $C$ & 3.027621 & 2.183841 & -1.727554 \\
\hline C & 1.805285 & 3.469234 & 0.210038 & $\mathrm{H}$ & 2.226357 & 2.727256 & -2.229783 \\
\hline C & -0.510419 & 4.997333 & 0.009210 & $\mathrm{H}$ & 3.017470 & 2.393291 & -0.657773 \\
\hline $\mathrm{H}$ & -0.765285 & 4.085582 & -1.920840 & $\mathrm{H}$ & 3.998005 & 2.408968 & -2.162130 \\
\hline C & 1.429651 & 4.305343 & 1.258901 & $\mathrm{Cu}$ & -1.027068 & 0.247563 & -0.547920 \\
\hline $\mathrm{H}$ & 2.723870 & 2.891573 & 0.293183 & $\mathrm{~N}$ & -2.964677 & -0.148965 & -0.521301 \\
\hline C & 0.269517 & 5.069079 & 1.160394 & $\mathrm{~N}$ & -1.315565 & 1.901346 & 0.486189 \\
\hline $\mathrm{H}$ & -1.413156 & 5.593619 & -0.073767 & $C$ & -3.910546 & 0.618945 & -0.097872 \\
\hline$H$ & 2.052155 & 4.372087 & 2.145824 & $\mathrm{O}$ & -5.142386 & 0.238789 & -0.375041 \\
\hline $\mathrm{H}$ & -0.020252 & 5.725702 & 1.974332 & $C$ & -2.423361 & 2.329296 & 0.990476 \\
\hline$H$ & 0.503360 & 2.216841 & -2.660244 & $\mathrm{O}$ & -2.330979 & 3.355143 & 1.812533 \\
\hline $\mathrm{H}$ & 2.637837 & -0.943922 & 2.476032 & $C$ & -5.058259 & -0.921285 & -1.242284 \\
\hline C & 2.051797 & -2.757519 & 1.496079 & $\mathrm{H}$ & -5.782421 & -1.653220 & -0.888175 \\
\hline C & 1.961117 & -3.485904 & 0.307357 & $\mathrm{H}$ & -5.315144 & -0.589324 & -2.250231 \\
\hline C & 1.446647 & -3.259827 & 2.649065 & $C$ & -3.592663 & -1.373057 & -1.102316 \\
\hline C & 1.294710 & -4.707922 & 0.278928 & C & -0.239464 & 2.833132 & 0.891761 \\
\hline
\end{tabular}




\begin{tabular}{|c|c|c|c|c|c|c|c|}
\hline C & -0.922370 & 3.597023 & 2.043726 & C & 2.507833 & -1.054929 & 0.833141 \\
\hline $\mathrm{H}$ & -0.760281 & 4.673131 & 2.019978 & C & 3.825883 & -0.630859 & 1.186834 \\
\hline $\mathrm{H}$ & -0.685525 & 3.194910 & 3.032121 & C & 1.429636 & -0.592035 & 1.553232 \\
\hline C & -3.833037 & 1.867193 & 0.740310 & C & 3.990081 & 0.233366 & 2.308148 \\
\hline C & -4.578874 & 3.017642 & 0.016033 & C & 4.972359 & -1.032871 & 0.454552 \\
\hline $\mathrm{H}$ & -4.566393 & 3.913022 & 0.640333 & C & 1.586875 & 0.254593 & 2.669498 \\
\hline $\mathrm{H}$ & -4.111281 & 3.251685 & -0.945361 & C & 5.296083 & 0.655421 & 2.669376 \\
\hline $\mathrm{H}$ & -5.616061 & 2.725756 & -0.157951 & C & 2.846430 & 0.653780 & 3.036613 \\
\hline C & -4.512343 & 1.555002 & 2.098466 & C & 6.220491 & -0.602175 & 0.827562 \\
\hline $\mathrm{H}$ & -4.495171 & 2.445403 & 2.729241 & $\mathrm{H}$ & 4.854054 & -1.687100 & -0.404054 \\
\hline $\mathrm{H}$ & -5.551364 & 1.266043 & 1.929742 & $\mathrm{H}$ & 0.706950 & 0.564495 & 3.225733 \\
\hline $\mathrm{H}$ & -3.997412 & 0.741708 & 2.620006 & C & 6.386804 & 0.246586 & 1.946699 \\
\hline $\mathrm{H}$ & 0.611386 & 2.253882 & 1.259661 & $\mathrm{H}$ & 5.418437 & 1.304552 & 3.531662 \\
\hline $\mathrm{H}$ & -3.145825 & -1.572128 & -2.075998 & $\mathrm{H}$ & 2.983637 & 1.299511 & 3.899377 \\
\hline C & 0.211846 & 3.745241 & -0.234422 & $\mathrm{H}$ & 7.092037 & -0.917632 & 0.263058 \\
\hline C & 1.319737 & 4.564755 & -0.001073 & $\mathrm{H}$ & 7.382374 & 0.570068 & 2.231461 \\
\hline C & -0.443625 & 3.819452 & -1.460860 & C & 2.791241 & -4.215272 & 0.624036 \\
\hline C & 1.768772 & 5.442356 & -0.982244 & $\mathrm{H}$ & 1.995523 & -4.920960 & 0.890031 \\
\hline $\mathrm{H}$ & 1.838026 & 4.518294 & 0.955112 & $\mathrm{H}$ & 3.635882 & -4.806799 & 0.252026 \\
\hline C & 0.009799 & 4.692819 & -2.448800 & $\mathrm{H}$ & 3.114746 & -3.689572 & 1.525187 \\
\hline $\mathrm{H}$ & -1.309859 & 3.194147 & -1.657813 & $\mathrm{O}$ & 0.145828 & -0.937405 & 1.150977 \\
\hline C & 1.115615 & 5.504617 & -2.212792 & C & -0.395157 & -2.066022 & 1.858757 \\
\hline $\mathrm{H}$ & 2.624904 & 6.080023 & -0.786441 & $\mathrm{H}$ & -0.355521 & -1.887358 & 2.937085 \\
\hline $\mathrm{H}$ & -0.509390 & 4.745605 & -3.400583 & $\mathrm{H}$ & -1.427798 & -2.174513 & 1.533499 \\
\hline $\mathrm{H}$ & 1.461395 & 6.190882 & -2.978830 & $\mathrm{H}$ & 0.172178 & -2.967762 & 1.610101 \\
\hline C & -3.388039 & -2.558131 & -0.183666 & & & & \\
\hline C & -2.639739 & -3.655760 & -0.605443 & & & & \\
\hline C & -3.952481 & -2.565039 & 1.097196 & C & 2.546133 & -3.507513 & -1.483525 \\
\hline C & -2.442550 & -4.740819 & 0.246215 & C & 3.549804 & -2.801713 & -2.363248 \\
\hline $\mathrm{H}$ & -2.211571 & -3.666769 & -1.602475 & C & 2.288819 & -1.037507 & -1.386489 \\
\hline C & -3.757622 & -3.648169 & 1.947017 & C & 3.395377 & -1.484245 & -2.334497 \\
\hline $\mathrm{H}$ & -4.559245 & -1.727581 & 1.436321 & $\mathrm{H}$ & 4.271961 & -3.345402 & -2.958282 \\
\hline C & -2.995670 & -4.737243 & 1.523320 & $\mathrm{H}$ & 3.949816 & -0.757519 & -2.912786 \\
\hline $\mathrm{H}$ & -1.869426 & -5.596824 & -0.096025 & $\mathrm{H}$ & 2.521894 & -4.589800 & -1.577875 \\
\hline $\mathrm{H}$ & -4.207864 & -3.649799 & 2.934448 & $\mathrm{O}$ & 1.206278 & -3.065092 & -1.949854 \\
\hline $\mathrm{H}$ & -2.851658 & -5.587119 & 2.182665 & C & 1.028259 & -1.787281 & -1.802336 \\
\hline C & 2.323920 & -3.256656 & -0.420527 & $\mathrm{O}$ & -0.097692 & -1.295600 & -1.949340 \\
\hline C & 2.264137 & -1.926634 & -0.337396 & C & 2.079056 & 0.450473 & -1.27680 \\
\hline
\end{tabular}




\begin{tabular}{|c|c|c|c|c|c|c|c|}
\hline O & 1.012932 & 0.975574 & -0.936269 & $\mathrm{H}$ & -1.303222 & 3.049906 & -1.935693 \\
\hline $\mathrm{O}$ & 3.142392 & 1.143522 & -1.526270 & C & 0.895287 & 5.628775 & -2.153962 \\
\hline C & 3.077279 & 2.579842 & -1.344559 & $\mathrm{H}$ & 2.088849 & 6.377004 & -0.523425 \\
\hline $\mathrm{H}$ & 2.270457 & 2.997335 & -1.948131 & $\mathrm{H}$ & -0.430537 & 4.687286 & -3.562394 \\
\hline $\mathrm{H}$ & 2.907573 & 2.790327 & -0.288129 & $\mathrm{H}$ & 1.269352 & 6.354512 & -2.868593 \\
\hline $\mathrm{H}$ & 4.047812 & 2.945642 & -1.669915 & C & -2.536727 & -3.005576 & -0.566920 \\
\hline $\mathrm{Cu}$ & -0.807695 & 0.181930 & -0.801430 & C & -1.591578 & -3.949404 & -0.962209 \\
\hline$N$ & -2.621755 & -0.563461 & -0.933909 & C & -3.112529 & -3.107949 & 0.704002 \\
\hline$N$ & -1.463155 & 1.743173 & 0.194314 & C & -1.197058 & -4.960850 & -0.087938 \\
\hline C & -3.723139 & -0.008549 & -0.556246 & $\mathrm{H}$ & -1.165682 & -3.900597 & -1.958457 \\
\hline $\mathrm{O}$ & -4.834511 & -0.641993 & -0.873616 & C & -2.721706 & -4.116735 & 1.576952 \\
\hline C & -2.663770 & 1.947573 & 0.617417 & $\mathrm{H}$ & -3.884094 & -2.405439 & 1.015877 \\
\hline O & -2.825317 & 2.947049 & 1.458138 & C & -1.753617 & -5.040929 & 1.184697 \\
\hline C & -4.473542 & -1.768008 & -1.715191 & $\mathrm{H}$ & -0.471268 & -5.700189 & -0.412907 \\
\hline $\mathrm{H}$ & -5.041081 & -2.632017 & -1.373283 & $\mathrm{H}$ & -3.178917 & -4.191496 & 2.558388 \\
\hline $\mathrm{H}$ & -4.754047 & -1.508504 & -2.737929 & $\mathrm{H}$ & -1.455243 & -5.833693 & 1.863044 \\
\hline C & -2.952198 & -1.899330 & -1.513326 & C & 2.671190 & -2.997504 & -0.048984 \\
\hline C & -0.587172 & 2.799422 & 0.743907 & C & 2.513004 & -1.676463 & 0.029175 \\
\hline C & -1.514571 & 3.466861 & 1.784120 & C & 2.510874 & -0.776299 & 1.199844 \\
\hline $\mathrm{H}$ & -1.558190 & 4.551853 & 1.702122 & C & 1.319655 & -0.502041 & 1.928945 \\
\hline $\mathrm{H}$ & -1.297105 & 3.163573 & 2.809672 & C & 3.685372 & -0.092591 & 1.489701 \\
\hline C & -3.942054 & 1.241424 & 0.253391 & C & 1.351421 & 0.485919 & 2.954926 \\
\hline C & -4.807781 & 2.231878 & -0.568315 & C & 0.083527 & -1.162511 & 1.667229 \\
\hline $\mathrm{H}$ & -5.007706 & 3.124618 & 0.027540 & C & 3.716298 & 0.872683 & 2.528593 \\
\hline $\mathrm{H}$ & -4.302095 & 2.529630 & -1.492018 & C & 0.165291 & 0.789952 & 3.673073 \\
\hline $\mathrm{H}$ & -5.758839 & 1.759970 & -0.822259 & C & 2.574179 & 1.148790 & 3.232107 \\
\hline C & -4.679387 & 0.832766 & 1.553719 & C & -1.047195 & -0.836517 & 2.379744 \\
\hline $\mathrm{H}$ & -4.883494 & 1.721447 & 2.152977 & $\mathrm{H}$ & 0.045124 & -1.987813 & 0.957619 \\
\hline $\mathrm{H}$ & -5.627154 & 0.353081 & 1.303588 & $\mathrm{H}$ & 4.636482 & 1.389351 & 2.773662 \\
\hline $\mathrm{H}$ & -4.075880 & 0.138885 & 2.147640 & C & -1.016833 & 0.155098 & 3.385551 \\
\hline $\mathrm{H}$ & 0.260249 & 2.319722 & 1.240627 & $\mathrm{H}$ & 0.214608 & 1.526314 & 4.471555 \\
\hline $\mathrm{H}$ & -2.434901 & -2.008902 & -2.466251 & $\mathrm{H}$ & 2.602297 & 1.887439 & 4.028408 \\
\hline C & -0.083130 & 3.766065 & -0.309860 & $\mathrm{H}$ & -1.965179 & -1.378624 & 2.181202 \\
\hline C & 0.870757 & 4.711613 & 0.076724 & $\mathrm{H}$ & -1.913814 & 0.380701 & 3.954016 \\
\hline C & -0.550169 & 3.767535 & -1.621918 & C & 2.928530 & -3.947791 & 1.071720 \\
\hline C & 1.355693 & 5.640928 & -0.837582 & $\mathrm{H}$ & 2.105524 & -4.666877 & 1.159991 \\
\hline $\mathrm{H}$ & 1.238858 & 4.723582 & 1.101163 & $\mathrm{H}$ & 3.846622 & -4.518754 & 0.892783 \\
\hline C & -0.058150 & 4.692059 & -2.542818 & $\mathrm{H}$ & 3.031846 & -3.415551 & 2.019715 \\
\hline
\end{tabular}




\begin{tabular}{|c|c|c|c|c|c|c|c|}
\hline O & 4.742843 & -0.391635 & 0.714877 & $\mathrm{H}$ & -2.653847 & 4.593298 & 1.793288 \\
\hline C & 5.988916 & 0.250581 & 0.953733 & C & -4.740382 & 1.150020 & 0.190885 \\
\hline $\mathrm{H}$ & 6.360594 & 0.029864 & 1.959396 & C & -5.858649 & 1.019278 & 1.249866 \\
\hline $\mathrm{H}$ & 6.676492 & -0.161500 & 0.216604 & $\mathrm{H}$ & -6.153121 & 2.010821 & 1.596127 \\
\hline \multirow[t]{2}{*}{$\mathrm{H}$} & 5.909650 & 1.334490 & 0.812807 & $\mathrm{H}$ & -5.527938 & 0.430496 & 2.110797 \\
\hline & & & & $\mathrm{H}$ & -6.727549 & 0.530264 & 0.807644 \\
\hline P1-C & & & & C & -5.240178 & 1.991039 & -1.016962 \\
\hline c & 2.052742 & -3.523687 & -1.448847 & $\mathrm{H}$ & -5.538137 & 2.983933 & -0.672635 \\
\hline C & 1.854393 & -4.032336 & -0.040578 & $\mathrm{H}$ & -6.110313 & 1.502758 & -1.461990 \\
\hline c & 1.425151 & -1.697129 & 0.111360 & $\mathrm{H}$ & -4.462204 & 2.092010 & -1.780151 \\
\hline C & 1.501876 & -3.064756 & 0.795746 & C & 3.104013 & -2.423663 & -1.451412 \\
\hline $\mathrm{H}$ & 1.973827 & -5.077596 & 0.213244 & C & 2.762583 & -1.414555 & -0.650877 \\
\hline $\mathrm{H}$ & 1.246216 & -3.176857 & 1.841325 & C & 3.392610 & -0.083173 & -0.537095 \\
\hline $\mathrm{H}$ & 2.197644 & -4.289675 & -2.205721 & C & 4.566133 & 0.133720 & 0.241785 \\
\hline O & 0.774151 & -2.864024 & -1.833443 & C & 2.787570 & 0.969368 & -1.201790 \\
\hline C & 0.445103 & -1.896120 & -1.033843 & C & 5.124034 & 1.442448 & 0.288948 \\
\hline O & -0.589625 & -1.255179 & -1.253840 & C & 5.190468 & -0.902676 & 0.984853 \\
\hline C & 1.130827 & -0.566271 & 1.062432 & C & 3.352356 & 2.264911 & -1.168428 \\
\hline O & 0.213044 & 0.258838 & 0.969639 & C & 6.291817 & 1.672635 & 1.062999 \\
\hline 0 & 2.002064 & -0.531788 & 2.020236 & C & 4.494548 & 2.481938 & -0.440663 \\
\hline C & 2.060804 & 0.630673 & 2.872902 & C & 6.314517 & -0.645766 & 1.728258 \\
\hline $\mathrm{H}$ & 1.126758 & 0.737096 & 3.426407 & $\mathrm{H}$ & 4.769024 & -1.903196 & 0.958662 \\
\hline $\mathrm{H}$ & 2.255898 & 1.506076 & 2.250518 & $\mathrm{H}$ & 2.895225 & 3.078854 & -1.716938 \\
\hline $\mathrm{H}$ & 2.896234 & 0.442488 & 3.542362 & C & 6.875608 & 0.652736 & 1.767850 \\
\hline $\mathrm{Cu}$ & -1.447765 & 0.061452 & -0.115465 & $\mathrm{H}$ & 6.718253 & 2.671670 & 1.083951 \\
\hline N & -3.204949 & -0.686156 & -0.592792 & $\mathrm{H}$ & 4.936438 & 3.474543 & -0.421473 \\
\hline $\mathrm{N}$ & -2.301931 & 1.606709 & 0.723077 & $\mathrm{H}$ & 6.784806 & -1.446995 & 2.289719 \\
\hline C & -4.375136 & -0.216727 & -0.326331 & $\mathrm{H}$ & 7.769961 & 0.834505 & 2.354346 \\
\hline O & -5.392837 & -1.014767 & -0.561103 & C & 4.303933 & -2.521503 & -2.333209 \\
\hline C & -3.563424 & 1.877915 & 0.793412 & $\mathrm{H}$ & 4.004648 & -2.602960 & -3.384445 \\
\hline $\mathrm{O}$ & -3.884298 & 2.978583 & 1.438942 & $\mathrm{H}$ & 4.891057 & -3.414571 & -2.089705 \\
\hline C & -4.875567 & -2.253754 & -1.114049 & $\mathrm{H}$ & 4.946321 & -1.646309 & -2.217395 \\
\hline $\mathrm{H}$ & -5.242243 & -2.327465 & -2.138424 & $\mathrm{O}$ & 1.634984 & 0.663062 & -1.857664 \\
\hline $\mathrm{H}$ & -5.269943 & -3.070490 & -0.510160 & C & 1.024087 & 1.650826 & -2.671542 \\
\hline C & -3.337162 & -2.095292 & -1.019804 & $\mathrm{H}$ & 0.690723 & 2.506910 & -2.076669 \\
\hline C & -1.543791 & 2.727221 & 1.331134 & $\mathrm{H}$ & 0.162958 & 1.161481 & -3.129353 \\
\hline C & -2.674135 & 3.527887 & 2.017415 & $\mathrm{H}$ & 1.703922 & 1.989149 & -3.460768 \\
\hline $\mathrm{H}$ & -2.732219 & 3.366301 & 3.095381 & C & -2.667590 & -3.039410 & -0.040530 \\
\hline
\end{tabular}




$\begin{array}{lrrr}\text { C } & -2.110601 & -4.229435 & -0.512468 \\ \text { C } & -2.632064 & -2.764361 & 1.329232 \\ \text { C } & -1.543400 & -5.140084 & 0.373889 \\ \text { H } & -2.125290 & -4.449017 & -1.576851 \\ \text { C } & -2.050166 & -3.670226 & 2.214901 \\ \text { H } & -3.081419 & -1.853614 & 1.720017 \\ \text { C } & -1.510944 & -4.862489 & 1.738909 \\ \text { H } & -1.132234 & -6.071514 & -0.002357 \\ \text { H } & -2.043730 & -3.455947 & 3.279231 \\ \text { H } & -1.078897 & -5.578633 & 2.430679 \\ \text { H } & -2.872330 & -2.204978 & -2.001564 \\ \text { H } & -0.847472 & 2.319759 & 2.065946 \\ \text { C } & -0.787289 & 3.530069 & 0.295604 \\ \text { C } & -1.426779 & 3.982191 & -0.863611 \\ \text { C } & 0.523818 & 3.928173 & 0.551420 \\ \text { C } & -0.766777 & 4.831869 & -1.744905 \\ \text { H } & -2.453858 & 3.692229 & -1.074177 \\ \text { C } & 1.181251 & 4.790029 & -0.323703 \\ \text { H } & 1.027462 & 3.589165 & 1.452955 \\ \text { C } & 0.537634 & 5.243757 & -1.471481 \\ \text { H } & -1.273693 & 5.187043 & -2.636294 \\ \text { H } & 2.194331 & 5.110201 & -0.102695 \\ \text { H } & 1.045129 & 5.923256 & -2.148531\end{array}$

$\begin{array}{lllr}\mathrm{C} & 1.620980 & -0.354840 & -3.264701 \\ \mathrm{H} & 0.611273 & -0.410626 & -3.674581 \\ \mathrm{H} & 1.885119 & -1.287976 & -2.764722 \\ \mathrm{H} & 2.341509 & -0.100356 & -4.037994 \\ \mathrm{Cu} & -1.519503 & -0.149832 & 0.068024 \\ \mathrm{~N} & -3.242810 & 0.376633 & 0.839896 \\ \mathrm{~N} & -2.333262 & -1.785245 & -0.666648\end{array}$

$\begin{array}{llll}\text { C } & -4.347728 & -0.283709 & 0.860685\end{array}$

$\begin{array}{llll}\mathrm{O} & -5.424992 & 0.388119 & 1.197005\end{array}$

$\begin{array}{llll}\text { C } & -3.482416 & -2.287635 & -0.351755\end{array}$

$\begin{array}{llll}0 & -3.780616 & -3.448255 & -0.888617\end{array}$

$\begin{array}{llll}\text { C } & -5.045320 & 1.777432 & 1.396583\end{array}$

$\begin{array}{llll}H & -5.347976 & 2.055178 & 2.405491\end{array}$

$\begin{array}{llll}H & -5.591426 & 2.369853 & 0.661309\end{array}$

$\begin{array}{llll}\text { C } & -3.510124 & 1.789449 & 1.180323\end{array}$

$\begin{array}{llll}\text { C } & -1.648885 & -2.708504 & -1.599626\end{array}$

$\begin{array}{llll}\text { C } & -2.658623 & -3.879963 & -1.697526\end{array}$

$\begin{array}{llll}H & -3.033392 & -4.061127 & -2.704431\end{array}$

$\begin{array}{llll}H & -2.279334 & -4.805561 & -1.262738\end{array}$

$\begin{array}{llll}\text { C } & -4.527806 & -1.757532 & 0.603879\end{array}$

$\begin{array}{llll}\text { C } & -5.938398 & -2.036125 & 0.046242\end{array}$

$\begin{array}{llll}\mathrm{H} & -6.072331 & -3.108960 & -0.096273\end{array}$

$\begin{array}{llll}H & -6.100341 & -1.532606 & -0.910870\end{array}$

$\begin{array}{llll}H & -6.688196 & -1.681534 & 0.754747\end{array}$

\section{P1-D}

$\begin{array}{llll}\text { C } & 1.813087 & 3.676755 & 1.257629 \\ \text { C } & 1.582580 & 4.175522 & -0.148575 \\ \text { C } & 1.266064 & 1.817457 & -0.310787 \\ \text { C } & 1.272325 & 3.193407 & -0.983878 \\ \text { H } & 1.655977 & 5.224885 & -0.402986 \\ \text { H } & 1.014487 & 3.295322 & -2.029750 \\ \text { H } & 1.930639 & 4.448025 & 2.013965 \\ \text { O } & 0.575336 & 2.955830 & 1.651220 \\ \text { C } & 0.308592 & 1.956622 & 0.865975 \\ \text { O } & -0.638373 & 1.213201 & 1.150678 \\ \text { C } & 0.934545 & 0.702784 & -1.275240 \\ \text { O } & 0.038701 & -0.141348 & -1.147203 \\ \text { O } & 1.706885 & 0.724890 & -2.311638\end{array}$

$\begin{array}{llll}\text { C } & -4.331788 & -2.493576 & 1.959821\end{array}$

$\begin{array}{llll}H & -4.471563 & -3.567380 & 1.814482\end{array}$

$\begin{array}{llll}H & -5.080534 & -2.141465 & 2.673972\end{array}$

$\begin{array}{llll}H & -3.334753 & -2.312095 & 2.372291\end{array}$

$\begin{array}{llll}\text { C } & 2.911493 & 2.625727 & 1.247491\end{array}$

$\begin{array}{llll}\text { C } & 2.629445 & 1.598597 & 0.443368\end{array}$

$\begin{array}{llll}\text { C } & 3.419807 & 0.355566 & 0.311914\end{array}$

$\begin{array}{llll}\text { C } & 3.366975 & -0.652548 & 1.323893\end{array}$

$\begin{array}{llll}\text { C } & 4.331257 & 0.251769 & -0.730666\end{array}$

$\begin{array}{llll}\text { C } & 4.252738 & -1.762890 & 1.237416\end{array}$

$\begin{array}{llll}\text { C } & 2.497654 & -0.576685 & 2.446160\end{array}$

$\begin{array}{llll}\text { C } & 5.172797 & -0.883716 & -0.837168\end{array}$

$\begin{array}{llll}\text { C } & 4.262733 & -2.736439 & 2.270091\end{array}$

$\begin{array}{llll}\text { C } & 5.129544 & -1.853769 & 0.128503\end{array}$ 


\begin{tabular}{|c|c|c|c|c|c|c|c|}
\hline C & 2.530655 & -1.533031 & 3.430478 & $\mathrm{H}$ & 1.540311 & -3.683398 & 1.690263 \\
\hline $\mathrm{H}$ & 1.826109 & 0.270028 & 2.550427 & $\mathrm{H}$ & 2.623837 & -4.353621 & -2.407502 \\
\hline $\mathrm{H}$ & 5.876681 & -0.973877 & -1.655673 & $\mathrm{H}$ & 3.196741 & -4.417995 & 0.008832 \\
\hline C & 3.425461 & -2.625359 & 3.350117 & & & & \\
\hline $\mathrm{H}$ & 4.962723 & -3.564931 & 2.199456 & \multicolumn{2}{|c|}{$P 2-A$} & & \\
\hline $\mathrm{H}$ & 5.799686 & -2.706361 & 0.058863 & $C$ & -2.802270 & -4.638109 & 0.829580 \\
\hline$H$ & 1.875892 & -1.441284 & 4.291898 & $C$ & -2.976020 & -4.234612 & 2.146223 \\
\hline $\mathrm{H}$ & 3.453600 & -3.362223 & 4.146028 & $C$ & -2.091732 & -2.058673 & 1.570826 \\
\hline C & 4.114897 & 2.793783 & 2.113701 & C & -2.629156 & -2.945510 & 2.515014 \\
\hline $\mathrm{H}$ & 3.828971 & 2.810594 & 3.172012 & $\mathrm{H}$ & -3.378964 & -4.921632 & 2.882250 \\
\hline$H$ & 4.617053 & 3.743066 & 1.894744 & $\mathrm{H}$ & -2.759410 & -2.621078 & 3.540102 \\
\hline $\mathrm{H}$ & 4.826460 & 1.980836 & 1.958364 & $\mathrm{H}$ & -3.072235 & -5.649436 & 0.539960 \\
\hline O & 4.373186 & 1.290296 & -1.591590 & $\mathrm{C}$ & -1.703003 & -0.730403 & 2.088397 \\
\hline C & 5.351033 & 1.298841 & -2.619410 & $\mathrm{O}$ & -0.778696 & -0.008976 & 1.673319 \\
\hline $\mathrm{H}$ & 6.363992 & 1.250607 & -2.206013 & $\mathrm{O}$ & -2.405600 & -0.346111 & 3.117607 \\
\hline $\mathrm{H}$ & 5.220432 & 2.245649 & -3.142539 & $C$ & -2.005529 & 0.844736 & 3.826462 \\
\hline $\mathrm{H}$ & 5.201153 & 0.471503 & -3.323371 & $\mathrm{H}$ & -2.821911 & 1.048232 & 4.514914 \\
\hline C & -3.022940 & 2.719367 & 0.088941 & $\mathrm{H}$ & -1.083434 & 0.637874 & 4.374462 \\
\hline C & -2.444631 & 3.943134 & 0.427375 & $\mathrm{H}$ & -1.864593 & 1.670845 & 3.131851 \\
\hline C & -3.146787 & 2.369063 & -1.259236 & $\mathrm{Cu}$ & 0.842685 & 0.257384 & 0.660880 \\
\hline C & -2.002983 & 4.809841 & -0.568375 & $\mathrm{~N}$ & 2.698471 & 0.812492 & 0.624945 \\
\hline $\mathrm{H}$ & -2.337453 & 4.220431 & 1.472663 & $\mathrm{~N}$ & 0.242381 & 1.913747 & -0.229311 \\
\hline C & -2.691527 & 3.231278 & -2.255015 & $C$ & 3.168312 & 1.881574 & 0.069976 \\
\hline $\mathrm{H}$ & -3.620992 & 1.431002 & -1.542023 & O & 4.436182 & 2.140273 & 0.293341 \\
\hline C & -2.121263 & 4.454486 & -1.910220 & $C$ & 0.999649 & 2.690483 & -0.922907 \\
\hline $\mathrm{H}$ & -1.569805 & 5.766699 & -0.295009 & $\mathrm{O}$ & 0.398962 & 3.477578 & -1.789333 \\
\hline $\mathrm{H}$ & -2.806097 & 2.958474 & -3.299518 & $C$ & 4.948166 & 1.151535 & 1.221427 \\
\hline $\mathrm{H}$ & -1.785330 & 5.135572 & -2.685691 & $\mathrm{H}$ & 5.861816 & 0.740173 & 0.794247 \\
\hline $\mathrm{H}$ & -2.984759 & 2.019305 & 2.110462 & $\mathrm{H}$ & 5.164100 & 1.668915 & 2.157136 \\
\hline $\mathrm{H}$ & -1.551219 & -2.196947 & -2.562168 & $C$ & 3.801475 & 0.115773 & 1.334596 \\
\hline C & -0.283210 & -3.145079 & -1.124677 & $C$ & -1.171380 & 2.150248 & -0.611484 \\
\hline C & 0.035419 & -3.206906 & 0.231546 & C & -1.018348 & 3.165891 & -1.769947 \\
\hline C & 0.655082 & -3.558289 & -2.070990 & $\mathrm{H}$ & -1.556740 & 4.098313 & -1.603447 \\
\hline C & 1.289605 & -3.654144 & 0.635867 & $\mathrm{H}$ & -1.273590 & 2.746594 & -2.744452 \\
\hline $\mathrm{H}$ & -0.695176 & -2.916723 & 0.984061 & $C$ & 2.496871 & 2.848199 & -0.873506 \\
\hline C & 1.904026 & -4.021116 & -1.666148 & $C$ & 2.811893 & 4.299989 & -0.436240 \\
\hline $\mathrm{H}$ & 0.407417 & -3.527800 & -3.130192 & $\mathrm{H}$ & 2.345673 & 4.998525 & -1.133382 \\
\hline C & 2.223581 & -4.062131 & -0.311435 & $\mathrm{H}$ & 2.435849 & 4.501809 & 0.571363 \\
\hline
\end{tabular}




\begin{tabular}{|c|c|c|c|c|c|c|c|}
\hline $\mathrm{H}$ & 3.890743 & 4.462831 & -0.446867 & $C$ & -0.439945 & -0.290604 & -3.193550 \\
\hline C & 3.069981 & 2.575272 & -2.289271 & $C$ & -4.611197 & -0.573019 & -2.575785 \\
\hline $\mathrm{H}$ & 2.652053 & 3.294636 & -2.995878 & $\mathrm{H}$ & -4.151589 & -1.632179 & -0.780780 \\
\hline $\mathrm{H}$ & 4.155884 & 2.689840 & -2.275445 & $\mathrm{H}$ & 1.504588 & -0.705037 & -2.367087 \\
\hline $\mathrm{H}$ & 2.821538 & 1.564275 & -2.628299 & C & -4.109626 & 0.076292 & -3.727916 \\
\hline $\mathrm{H}$ & -1.580109 & 1.211108 & -0.985525 & $\mathrm{H}$ & -2.366916 & 0.645658 & -4.827480 \\
\hline $\mathrm{H}$ & 3.513459 & -0.020492 & 2.380766 & $\mathrm{H}$ & -0.062549 & 0.174923 & -4.099713 \\
\hline C & -2.034739 & 2.650112 & 0.520487 & $\mathrm{H}$ & -5.683590 & -0.638184 & -2.423432 \\
\hline C & -3.302033 & 2.095931 & 0.701806 & $\mathrm{H}$ & -4.800674 & 0.499773 & -4.449082 \\
\hline C & -1.626795 & 3.716771 & 1.324561 & $C$ & -2.154365 & -4.260980 & -1.563350 \\
\hline C & -4.160130 & 2.609294 & 1.672263 & $\mathrm{H}$ & -2.253940 & -5.347748 & -1.607161 \\
\hline $\mathrm{H}$ & -3.627502 & 1.272214 & 0.069145 & $\mathrm{H}$ & -2.929395 & -3.831234 & -2.207453 \\
\hline C & -2.482045 & 4.227300 & 2.296758 & $\mathrm{H}$ & -1.187159 & -3.991526 & -1.999447 \\
\hline $\mathrm{H}$ & -0.643346 & 4.161448 & 1.187824 & $\mathrm{O}$ & 0.827031 & -1.706151 & -0.084182 \\
\hline C & -3.752068 & 3.676651 & 2.468134 & $C$ & 1.302660 & -3.066321 & -0.015002 \\
\hline $\mathrm{H}$ & -5.148954 & 2.180924 & 1.800566 & $\mathrm{H}$ & 1.756727 & -3.334675 & -0.970871 \\
\hline $\mathrm{H}$ & -2.163723 & 5.061829 & 2.913244 & $\mathrm{H}$ & 2.053540 & -3.090268 & 0.771316 \\
\hline $\mathrm{H}$ & -4.422968 & 4.084169 & 3.217433 & $\mathrm{H}$ & 0.468562 & -3.728660 & 0.225391 \\
\hline C & 4.132540 & -1.223405 & 0.716845 & & & & \\
\hline C & 4.448764 & -2.301931 & 1.543492 & \multicolumn{2}{|c|}{ P2-B } & & \\
\hline C & 4.215085 & -1.371760 & -0.670195 & $C$ & 4.765158 & -0.061947 & -1.531092 \\
\hline C & 4.841058 & -3.519721 & 0.989753 & $C$ & 4.115265 & 0.077786 & -2.753228 \\
\hline $\mathrm{H}$ & 4.402061 & -2.192503 & 2.624490 & $C$ & 2.219839 & 1.004191 & -1.603172 \\
\hline C & 4.602680 & -2.587769 & -1.223045 & $\mathrm{C}$ & 2.839597 & 0.622758 & -2.796355 \\
\hline $\mathrm{H}$ & 3.988857 & -0.533129 & -1.325303 & $\mathrm{H}$ & 4.609534 & -0.223170 & -3.670803 \\
\hline C & 4.915344 & -3.664102 & -0.392930 & $\mathrm{H}$ & 2.328330 & 0.759797 & -3.743399 \\
\hline $\mathrm{H}$ & 5.094516 & -4.351006 & 1.639458 & $\mathrm{H}$ & 5.770740 & -0.472929 & -1.507878 \\
\hline $\mathrm{H}$ & 4.674624 & -2.694531 & -2.300903 & $C$ & 0.852885 & 1.566567 & -1.670036 \\
\hline $\mathrm{H}$ & 5.227860 & -4.609380 & -0.824422 & $\mathrm{O}$ & -0.089998 & 1.250720 & -0.916585 \\
\hline C & -2.292633 & -3.771475 & -0.141777 & $\mathrm{O}$ & 0.680719 & 2.428534 & -2.626892 \\
\hline C & -1.927641 & -2.461852 & 0.229234 & C & -0.640288 & 2.981650 & -2.822180 \\
\hline C & -1.434375 & -1.583995 & -0.876503 & $\mathrm{H}$ & -1.352317 & 2.180401 & -3.023030 \\
\hline C & -2.349091 & -1.051122 & -1.839948 & $\mathrm{H}$ & -0.944548 & 3.529754 & -1.930797 \\
\hline C & -0.087914 & -1.395619 & -1.090731 & $\mathrm{H}$ & -0.542002 & 3.644473 & -3.678290 \\
\hline C & -1.843989 & -0.392322 & -3.001368 & $\mathrm{Cu}$ & -0.506623 & -0.436661 & -0.021498 \\
\hline C & -3.755891 & -1.128475 & -1.657255 & $\mathrm{~N}$ & -0.930010 & -2.167387 & -0.727584 \\
\hline C & 0.426809 & -0.772211 & -2.249809 & $N$ & -2.347911 & -0.060379 & 0.444666 \\
\hline C & -2.757041 & 0.160787 & -3.936724 & $C$ & -2.066388 & -2.770169 & -0.642119 \\
\hline
\end{tabular}




\begin{tabular}{|c|c|c|c|c|c|c|c|}
\hline O & -2.187117 & -3.892135 & -1.318017 & C & 2.243215 & -4.680723 & 0.935739 \\
\hline C & -3.251065 & -0.957666 & 0.639479 & $\mathrm{H}$ & 0.144475 & -4.731787 & 0.492098 \\
\hline $\mathrm{O}$ & -4.319439 & -0.585037 & 1.313560 & C & 3.488239 & -4.156364 & 0.589528 \\
\hline C & -0.959808 & -4.110160 & -2.060281 & $\mathrm{H}$ & 4.564604 & -2.872199 & -0.760953 \\
\hline $\mathrm{H}$ & -0.595059 & -5.106106 & -1.810257 & $\mathrm{H}$ & 2.158817 & -5.384468 & 1.757429 \\
\hline $\mathrm{H}$ & -1.210915 & -4.060639 & -3.119943 & $\mathrm{H}$ & 4.372984 & -4.451619 & 1.143821 \\
\hline C & -0.022978 & -2.970454 & -1.594880 & C & 4.171943 & 0.340955 & -0.331609 \\
\hline C & -2.825270 & 1.222081 & 1.012018 & C & 2.881708 & 0.887781 & -0.368973 \\
\hline C & -4.102966 & 0.775252 & 1.766902 & C & 2.252999 & 1.428271 & 0.871666 \\
\hline $\mathrm{H}$ & -4.987098 & 1.355063 & 1.505879 & C & 1.418990 & 0.625367 & 1.673119 \\
\hline $\mathrm{H}$ & -3.979346 & 0.737397 & 2.850587 & C & 2.476650 & 2.755306 & 1.229975 \\
\hline C & -3.262234 & -2.403034 & 0.202730 & C & 0.774443 & 1.180290 & 2.818381 \\
\hline C & -4.562893 & -2.665278 & -0.590958 & C & 1.162132 & -0.749756 & 1.342688 \\
\hline $\mathrm{H}$ & -5.425693 & -2.425233 & 0.032046 & C & 1.846884 & 3.308967 & 2.377020 \\
\hline $\mathrm{H}$ & -4.604742 & -2.057618 & -1.499741 & C & -0.058095 & 0.359368 & 3.612611 \\
\hline $\mathrm{H}$ & -4.616700 & -3.717447 & -0.873716 & C & 1.014409 & 2.539619 & 3.142577 \\
\hline C & -3.216431 & -3.296036 & 1.470974 & C & 0.377647 & -1.529289 & 2.196462 \\
\hline $\mathrm{H}$ & -4.088990 & -3.090580 & 2.094268 & $\mathrm{H}$ & 1.815472 & -1.251531 & 0.629566 \\
\hline $\mathrm{H}$ & -3.238811 & -4.348282 & 1.178318 & $\mathrm{H}$ & 2.025303 & 4.341339 & 2.652447 \\
\hline $\mathrm{H}$ & -2.310435 & -3.107038 & 2.055386 & C & -0.265572 & -0.972363 & 3.311611 \\
\hline $\mathrm{H}$ & -2.071342 & 1.589226 & 1.714171 & $\mathrm{H}$ & -0.525529 & 0.793149 & 4.493258 \\
\hline $\mathrm{H}$ & 0.271973 & -2.344796 & -2.442507 & $\mathrm{H}$ & 0.539523 & 2.967275 & 4.020829 \\
\hline C & -3.090322 & 2.274784 & -0.040007 & $\mathrm{H}$ & 0.277826 & -2.588561 & 1.982215 \\
\hline C & -2.755357 & 3.602219 & 0.224986 & $\mathrm{H}$ & -0.877832 & -1.592823 & 3.956965 \\
\hline C & -3.765029 & 1.958887 & -1.220238 & C & 4.910371 & 0.213380 & 0.974418 \\
\hline C & -3.104313 & 4.608253 & -0.673148 & $\mathrm{H}$ & 4.338613 & -0.375763 & 1.699788 \\
\hline $\mathrm{H}$ & -2.231175 & 3.856171 & 1.143312 & $\mathrm{H}$ & 5.879842 & -0.269909 & 0.833968 \\
\hline C & -4.104803 & 2.961470 & -2.123401 & $\mathrm{H}$ & 5.088164 & 1.198264 & 1.418511 \\
\hline $\mathrm{H}$ & -4.038675 & 0.928784 & -1.436927 & $\mathrm{O}$ & 3.293722 & 3.448155 & 0.430542 \\
\hline C & -3.779384 & 4.288858 & -1.848640 & C & 3.623944 & 4.798175 & 0.747543 \\
\hline $\mathrm{H}$ & -2.854937 & 5.641035 & -0.451759 & $\mathrm{H}$ & 4.115600 & 4.863049 & 1.723198 \\
\hline $\mathrm{H}$ & -4.638286 & 2.710429 & -3.034712 & $\mathrm{H}$ & 4.316030 & 5.116201 & -0.030178 \\
\hline $\mathrm{H}$ & -4.060477 & 5.072420 & -2.544763 & $\mathrm{H}$ & 2.734153 & 5.435816 & 0.727589 \\
\hline C & 1.212444 & -3.411168 & -0.846451 & & & & \\
\hline C & 2.460884 & -2.897705 & -1.194517 & \multicolumn{2}{|c|}{$\mathrm{P} 2-\mathrm{C}$} & & \\
\hline C & 1.111246 & -4.311178 & 0.218739 & C & -2.110116 & 4.354630 & -0.924157 \\
\hline C & 3.596199 & -3.270696 & -0.477579 & C & -1.375393 & 4.651904 & 0.216576 \\
\hline $\mathrm{H}$ & 2.553990 & -2.201391 & -2.024813 & C & -1.251757 & 2.302085 & 0.7164 \\
\hline
\end{tabular}




\begin{tabular}{|c|c|c|c|c|c|c|c|}
\hline C & -0.959565 & 3.626724 & 1.051450 & C & -3.780115 & 0.171192 & -0.378194 \\
\hline $\mathrm{H}$ & -1.139472 & 5.682029 & 0.461084 & C & -1.665100 & -0.262474 & -1.424835 \\
\hline $\mathrm{H}$ & -0.391529 & 3.841709 & 1.948359 & C & -4.220448 & -1.116464 & -0.800729 \\
\hline $\mathrm{H}$ & -2.447935 & 5.162582 & -1.566899 & C & -4.664697 & 0.983449 & 0.378673 \\
\hline C & -0.750076 & 1.251714 & 1.622058 & C & -2.076124 & -1.537746 & -1.849891 \\
\hline O & -0.244146 & 0.165974 & 1.276424 & C & -5.526022 & -1.549297 & -0.453514 \\
\hline O & -0.849363 & 1.558733 & 2.886257 & C & -3.348274 & -1.943797 & -1.552722 \\
\hline C & -0.280025 & 0.665613 & 3.857966 & C & -5.920382 & 0.534049 & 0.698522 \\
\hline $\mathrm{H}$ & 0.776056 & 0.498795 & 3.634728 & $\mathrm{H}$ & -4.341320 & 1.966928 & 0.705558 \\
\hline $\mathrm{H}$ & -0.825387 & -0.279403 & 3.856309 & $\mathrm{H}$ & -1.387774 & -2.173781 & -2.396647 \\
\hline $\mathrm{H}$ & -0.399738 & 1.171287 & 4.813317 & C & -6.357538 & -0.743912 & 0.279806 \\
\hline $\mathrm{Cu}$ & 0.890170 & -0.270610 & -0.245291 & $\mathrm{H}$ & -5.858174 & -2.529518 & -0.783619 \\
\hline N & 2.541804 & 0.259134 & -1.091241 & $\mathrm{H}$ & -3.697300 & -2.917847 & -1.879388 \\
\hline N & 1.731463 & -1.747877 & 0.640513 & $\mathrm{H}$ & -6.588138 & 1.164017 & 1.277089 \\
\hline C & 3.630187 & -0.436294 & -1.118073 & $\mathrm{H}$ & -7.355850 & -1.080871 & 0.538330 \\
\hline O & 4.726355 & 0.198479 & -1.456460 & C & -3.338074 & 2.772704 & -2.448191 \\
\hline C & 2.846700 & -2.304853 & 0.311161 & $\mathrm{H}$ & -2.987953 & 1.920678 & -3.038543 \\
\hline O & 3.185371 & -3.383566 & 0.979648 & $\mathrm{H}$ & -3.387188 & 3.646693 & -3.101565 \\
\hline C & 4.373760 & 1.575031 & -1.752395 & $\mathrm{H}$ & -4.359415 & 2.537356 & -2.128664 \\
\hline $\mathrm{H}$ & 4.539440 & 1.727791 & -2.819576 & $\mathrm{O}$ & -0.339513 & 0.117306 & -1.717415 \\
\hline $\mathrm{H}$ & 5.035941 & 2.214814 & -1.170166 & $C$ & -0.133451 & 0.761161 & -2.989612 \\
\hline C & 2.884648 & 1.676138 & -1.335006 & $\mathrm{H}$ & -0.790197 & 0.297818 & -3.727782 \\
\hline C & 1.065161 & -2.588764 & 1.665081 & $\mathrm{H}$ & 0.907150 & 0.593365 & -3.267374 \\
\hline C & 2.191047 & -3.586867 & 2.021678 & $\mathrm{H}$ & -0.352494 & 1.827746 & -2.896496 \\
\hline $\mathrm{H}$ & 2.680513 & -3.372975 & 2.972955 & $\mathrm{C}$ & 2.639148 & 2.536714 & -0.111719 \\
\hline $\mathrm{H}$ & 1.882444 & -4.630523 & 1.982752 & C & 2.343760 & 3.889451 & -0.286258 \\
\hline C & 3.761937 & -1.914045 & -0.828950 & C & 2.801908 & 2.033306 & 1.182657 \\
\hline C & 5.220187 & -2.280103 & -0.503252 & C & 2.238216 & 4.734380 & 0.813777 \\
\hline $\mathrm{H}$ & 5.294964 & -3.352529 & -0.319153 & $\mathrm{H}$ & 2.211559 & 4.291974 & -1.287484 \\
\hline $\mathrm{H}$ & 5.583463 & -1.749122 & 0.380063 & C & 2.671967 & 2.875494 & 2.284530 \\
\hline $\mathrm{H}$ & 5.862797 & -2.025756 & -1.347302 & $\mathrm{H}$ & 3.061513 & 0.988755 & 1.341396 \\
\hline C & 3.279811 & -2.689214 & -2.087130 & C & 2.402013 & 4.229372 & 2.101314 \\
\hline $\mathrm{H}$ & 3.374405 & -3.763359 & -1.909020 & $\mathrm{H}$ & 2.029651 & 5.788775 & 0.664575 \\
\hline $\mathrm{H}$ & 3.911025 & -2.428069 & -2.940483 & $\mathrm{H}$ & 2.817328 & 2.481752 & 3.285837 \\
\hline $\mathrm{H}$ & 2.238105 & -2.456423 & -2.327921 & $\mathrm{H}$ & 2.328275 & 4.890314 & 2.958929 \\
\hline C & -2.456900 & 3.040236 & -1.253672 & $\mathrm{H}$ & 2.283821 & 2.055569 & -2.165541 \\
\hline C & -2.012895 & 1.994486 & -0.426854 & $\mathrm{H}$ & 0.813350 & -1.956849 & 2.518493 \\
\hline C & -2.465469 & 0.605854 & -0.733016 & $\mathrm{C}$ & -0.190439 & -3.230714 & 1.116928 \\
\hline
\end{tabular}




\begin{tabular}{|c|c|c|c|c|c|c|c|}
\hline C & -0.110441 & -4.143144 & 0.059654 & C & -3.456668 & -1.884546 & -2.377488 \\
\hline C & -1.428106 & -2.970036 & 1.702118 & $\mathrm{H}$ & -3.615514 & -1.439052 & -3.360687 \\
\hline C & -1.247352 & -4.812796 & -0.377045 & $\mathrm{H}$ & -3.805529 & -2.916421 & -2.372139 \\
\hline $\mathrm{H}$ & 0.849667 & -4.364797 & -0.403894 & C & -4.131543 & 0.389219 & 0.401008 \\
\hline C & -2.568260 & -3.637893 & 1.264740 & C & -5.445846 & 0.982651 & -0.138793 \\
\hline $\mathrm{H}$ & -1.500700 & -2.258597 & 2.519760 & $\mathrm{H}$ & -6.109009 & 0.176694 & -0.455055 \\
\hline C & -2.475251 & -4.569075 & 0.235590 & $\mathrm{H}$ & -5.269279 & 1.643069 & -0.991847 \\
\hline $\mathrm{H}$ & -1.172924 & -5.539914 & -1.179341 & $\mathrm{H}$ & -5.942196 & 1.556019 & 0.645110 \\
\hline $\mathrm{H}$ & -3.525588 & -3.436600 & 1.734147 & $\mathrm{C}$ & -4.415760 & -0.538776 & 1.615156 \\
\hline \multirow[t]{2}{*}{$\mathrm{H}$} & -3.358889 & -5.109529 & -0.089026 & $\mathrm{H}$ & -5.099565 & -1.335157 & 1.310776 \\
\hline & & & & $\mathrm{H}$ & -4.893850 & 0.037684 & 2.411314 \\
\hline \multicolumn{2}{|c|}{ P2-D } & & & $\mathrm{H}$ & -3.491972 & -0.984793 & 1.996806 \\
\hline C & 4.359390 & 2.509236 & 0.869063 & C & 3.928434 & 1.216862 & 1.182029 \\
\hline C & 3.903901 & 3.175159 & -0.261639 & C & 2.986542 & 0.596865 & 0.347808 \\
\hline C & 2.500356 & 1.290816 & -0.775552 & $\mathrm{C}$ & 2.604070 & -0.817869 & 0.622858 \\
\hline C & 2.982128 & 2.562722 & -1.095625 & C & 1.466949 & -1.133050 & 1.385502 \\
\hline $\mathrm{H}$ & 4.272264 & 4.168241 & -0.495699 & C & 3.442998 & -1.844490 & 0.188350 \\
\hline $\mathrm{H}$ & 2.616690 & 3.070035 & -1.979653 & C & 1.167415 & -2.493225 & 1.694697 \\
\hline $\mathrm{H}$ & 5.086818 & 2.991280 & 1.515947 & C & 0.577003 & -0.108301 & 1.870124 \\
\hline C & 1.480494 & 0.702877 & -1.660755 & C & 3.154944 & -3.198112 & 0.501796 \\
\hline O & 0.513384 & 0.002779 & -1.304305 & C & 0.049289 & -2.793725 & 2.496725 \\
\hline $\mathrm{O}$ & 1.649417 & 0.996342 & -2.924609 & C & 2.044831 & -3.507095 & 1.236883 \\
\hline C & 0.650957 & 0.572492 & -3.863976 & $\mathrm{C}$ & -0.467693 & -0.465423 & 2.739189 \\
\hline $\mathrm{H}$ & -0.330539 & 0.944768 & -3.561061 & $\mathrm{H}$ & 0.945956 & 0.918501 & 1.881328 \\
\hline $\mathrm{H}$ & 0.641232 & -0.517270 & -3.928728 & $\mathrm{H}$ & 3.816979 & -3.989177 & 0.171405 \\
\hline $\mathrm{H}$ & 0.951938 & 1.004993 & -4.815545 & C & -0.763523 & -1.801020 & 3.018323 \\
\hline $\mathrm{Cu}$ & -0.745591 & 0.112292 & 0.210660 & $\mathrm{H}$ & -0.157468 & -3.834866 & 2.726774 \\
\hline N & -1.926139 & 1.456861 & 0.939576 & $\mathrm{H}$ & 1.828816 & -4.541204 & 1.484035 \\
\hline N & -2.197888 & -0.655321 & -0.819828 & $\mathrm{H}$ & -1.063586 & 0.316850 & 3.199759 \\
\hline C & -3.213318 & 1.487942 & 0.878218 & $\mathrm{H}$ & -1.589904 & -2.054496 & 3.672880 \\
\hline O & -3.797894 & 2.576131 & 1.327029 & $\mathrm{C}$ & 4.503285 & 0.492217 & 2.371879 \\
\hline C & -3.459992 & -0.458800 & -0.654603 & $\mathrm{H}$ & 3.722005 & 0.045515 & 2.994959 \\
\hline O & -4.272399 & -1.139220 & -1.435898 & $\mathrm{H}$ & 5.091120 & 1.168397 & 2.996580 \\
\hline C & -2.765493 & 3.469624 & 1.817857 & $\mathrm{H}$ & 5.164640 & -0.317763 & 2.045175 \\
\hline $\mathrm{H}$ & -2.911567 & 3.573364 & 2.893672 & $\mathrm{O}$ & 4.514316 & -1.464341 & -0.513680 \\
\hline $\mathrm{H}$ & -2.901396 & 4.430831 & 1.323166 & C & 5.470882 & -2.433532 & -0.934640 \\
\hline C & -1.441714 & 2.759527 & 1.433033 & $\mathrm{H}$ & 5.904120 & -2.955977 & -0.075996 \\
\hline C & -2.015756 & -1.709983 & -1.842864 & $\mathrm{H}$ & 6.247824 & -1.868799 & -1.4469 \\
\hline
\end{tabular}




\begin{tabular}{lrrr} 
H & 5.023392 & -3.151598 & -1.629421 \\
C & -0.633109 & 3.495867 & 0.385035 \\
C & 0.360431 & 4.386104 & 0.793002 \\
C & -0.942740 & 3.388325 & -0.974134 \\
C & 1.012974 & 5.185088 & -0.140706 \\
H & 0.612425 & 4.474856 & 1.846958 \\
C & -0.276458 & 4.175667 & -1.909218 \\
H & -1.726156 & 2.711248 & -1.307661 \\
C & 0.691407 & 5.086010 & -1.491622 \\
H & 1.769637 & 5.889202 & 0.189368 \\
H & -0.533761 & 4.100447 & -2.961236 \\
H & 1.191994 & 5.718421 & -2.217863 \\
H & -0.824637 & 2.597288 & 2.322865 \\
H & -1.350439 & -1.325362 & -2.617552 \\
C & -1.422186 & -2.964112 & -1.241449 \\
C & -2.098180 & -3.644681 & -0.224195 \\
C & -0.235491 & -3.491760 & -1.746340 \\
C & -1.609161 & -4.853314 & 0.257480 \\
H & -3.029665 & -3.248402 & 0.176026 \\
C & 0.252525 & -4.705117 & -1.268354 \\
H & 0.298443 & -2.966017 & -2.533487 \\
C & -0.437285 & -5.390541 & -0.273364 \\
H & -2.154437 & -5.389594 & 1.027888 \\
H & 1.164848 & -5.120591 & -1.683758 \\
H & -0.070671 & -6.348725 & 0.081656 \\
& & & \\
\hline
\end{tabular}

$\begin{array}{rrrr}\text { O } & 0.569867 & 1.380408 & -0.701245 \\ \text { O } & -1.232749 & 2.646362 & -0.980243 \\ \text { C } & -0.611689 & 3.230670 & -2.134870 \\ \text { H } & -0.388416 & 2.461140 & -2.876678 \\ \text { H } & -1.343009 & 3.936236 & -2.523077 \\ \text { H } & 0.302875 & 3.746456 & -1.839438 \\ \text { Cu } & 1.769383 & 0.084583 & 0.135338\end{array}$

$\begin{array}{llll}N & 3.378462 & 0.843496 & -0.715757\end{array}$

$\begin{array}{llll}N & 2.745580 & -1.580664 & 0.518827\end{array}$

$\begin{array}{llll}\text { C } & 4.562508 & 0.337790 & -0.775470\end{array}$

$\begin{array}{llll}\mathrm{O} & 5.523358 & 1.141089 & -1.182505\end{array}$

$\begin{array}{llll}\text { C } & 3.963795 & -1.885872 & 0.235952\end{array}$

$\begin{array}{llll}\text { O } & 4.358778 & -3.096198 & 0.582822\end{array}$

$\begin{array}{llll}\text { C } & 4.944443 & 2.442505 & -1.449570\end{array}$

$\begin{array}{llll}H & 5.465659 & 3.167528 & -0.824055\end{array}$

$\begin{array}{llll}H & 5.112834 & 2.663192 & -2.503822\end{array}$

$\begin{array}{llll}\text { C } & 3.448899 & 2.273070 & -1.079192\end{array}$

$\begin{array}{llll}\text { C } & 2.077617 & -2.773924 & 1.098462\end{array}$

$\begin{array}{llll}\text { C } & 3.279212 & -3.716452 & 1.328118\end{array}$

$\begin{array}{llll}H & 3.136535 & -4.720359 & 0.930436\end{array}$

$\begin{array}{llll}H & 3.596243 & -3.765216 & 2.371218\end{array}$

$\begin{array}{llll}\text { C } & 5.014705 & -1.068518 & -0.471472\end{array}$

$\begin{array}{llll}\text { C } & 5.331398 & -1.765259 & -1.822033\end{array}$

$\begin{array}{llll}H & 5.705090 & -2.773795 & -1.633363\end{array}$

$\begin{array}{llll}H & 4.440182 & -1.826827 & -2.454529\end{array}$

$\begin{array}{llll}H & 6.103188 & -1.201441 & -2.350602\end{array}$

TS1-A

$\begin{array}{llll}\text { C } & 6.282909 & -1.013476 & 0.413503\end{array}$

$\begin{array}{llll}\text { C } & -2.688793 & 0.006277 & 2.839827\end{array}$

$\begin{array}{llll}H & 6.630997 & -2.027937 & 0.614235\end{array}$

$\begin{array}{llll}\text { C } & -3.216836 & 1.183769 & 2.267748\end{array}$

$\begin{array}{llll}\text { C } & -1.246487 & 1.251834 & 0.863690\end{array}$

$\begin{array}{llll}\text { C } & -2.518489 & 1.762457 & 1.249178\end{array}$

$\begin{array}{llll}H & -4.197150 & 1.527244 & 2.571735\end{array}$

$\begin{array}{llll}H & -2.910848 & 2.631335 & 0.733522\end{array}$

$\begin{array}{llll}H & -3.025089 & -0.347774 & 3.808018\end{array}$

\begin{tabular}{llll}
\hline & -1.368743 & -0.279973 & 2.666880
\end{tabular}

$\begin{array}{llll}\text { C } & -0.674611 & 0.211397 & 1.618104\end{array}$

$\begin{array}{llll}\mathrm{O} & 0.448357 & -0.329817 & 1.478534\end{array}$

$\begin{array}{llll}\text { C } & -0.558291 & 1.748134 & -0.297140\end{array}$

$\begin{array}{llll}H & 7.071816 & -0.467931 & -0.106191\end{array}$

$\begin{array}{llll}H & 6.082231 & -0.515547 & 1.367148\end{array}$

$\begin{array}{llll}H & 1.615212 & -2.482993 & 2.042190\end{array}$

$\begin{array}{llll}H & 2.810852 & 2.432240 & -1.952716\end{array}$

$\begin{array}{llll}\text { C } & 1.022557 & -3.314501 & 0.156012\end{array}$

$\begin{array}{llll}\text { C } & -0.319799 & -3.324711 & 0.532211\end{array}$

$\begin{array}{llll}\text { C } & 1.388167 & -3.837850 & -1.089738\end{array}$

$\begin{array}{llll}\text { C } & -1.283290 & -3.871565 & -0.312199\end{array}$

$\begin{array}{llll}H & -0.612058 & -2.902881 & 1.489586\end{array}$

$\begin{array}{llll}\text { C } & 0.427098 & -4.384569 & -1.933022\end{array}$ 


\begin{tabular}{|c|c|c|c|c|c|c|c|}
\hline $\mathrm{H}$ & 2.432605 & -3.844925 & -1.396611 & O & -2.010157 & -0.916860 & -1.141047 \\
\hline C & -0.911569 & -4.407966 & -1.540775 & $C$ & -1.102699 & -1.012462 & -2.232176 \\
\hline $\mathrm{H}$ & -2.325738 & -3.872678 & -0.010340 & $\mathrm{H}$ & -0.781681 & -0.021056 & -2.568558 \\
\hline $\mathrm{H}$ & 0.722240 & -4.805752 & -2.888759 & $\mathrm{H}$ & -0.246069 & -1.566083 & -1.847243 \\
\hline $\mathrm{H}$ & -1.659778 & -4.848135 & -2.192307 & $\mathrm{H}$ & -1.549301 & -1.567233 & -3.062297 \\
\hline C & 2.976619 & 3.168465 & 0.047563 & & & & \\
\hline C & 2.444825 & 4.422389 & -0.257746 & \multicolumn{2}{|c|}{ TS1-B } & & \\
\hline C & 3.084960 & 2.781150 & 1.384674 & $C$ & -3.900469 & -0.999753 & -2.352864 \\
\hline C & 2.009847 & 5.270614 & 0.756053 & C & -1.652620 & -1.252964 & -1.496829 \\
\hline $\mathrm{H}$ & 2.384129 & 4.746887 & -1.294706 & C & -2.924014 & -1.796818 & -1.836773 \\
\hline C & 2.639686 & 3.625563 & 2.400157 & $\mathrm{H}$ & -4.888501 & -1.364296 & -2.600565 \\
\hline $\mathrm{H}$ & 3.532593 & 1.824892 & 1.648092 & $\mathrm{H}$ & -3.090455 & -2.858334 & -1.693305 \\
\hline C & 2.097904 & 4.869073 & 2.087543 & $\mathrm{O}$ & -2.337766 & 0.811611 & -2.437261 \\
\hline $\mathrm{H}$ & 1.606153 & 6.246853 & 0.507313 & C & -1.375223 & 0.084502 & -1.819967 \\
\hline $\mathrm{H}$ & 2.730612 & 3.316103 & 3.436495 & $\mathrm{O}$ & -0.308340 & 0.721655 & -1.651771 \\
\hline $\mathrm{H}$ & 1.759178 & 5.529884 & 2.878642 & C & -0.659417 & -2.035258 & -0.808182 \\
\hline C & -3.475304 & -1.729169 & 1.956906 & $\mathrm{O}$ & 0.438523 & -1.611224 & -0.386230 \\
\hline C & -3.788235 & -1.308651 & 0.833955 & $\mathrm{O}$ & -0.995888 & -3.290484 & -0.619330 \\
\hline C & -4.150344 & -0.565956 & -0.280886 & C & -0.054311 & -4.147242 & 0.045878 \\
\hline C & -5.470943 & 0.020601 & -0.374615 & $\mathrm{H}$ & 0.171850 & -3.748575 & 1.037676 \\
\hline C & -3.223352 & -0.390297 & -1.330953 & $\mathrm{H}$ & -0.553366 & -5.111111 & 0.121285 \\
\hline C & -5.805040 & 0.755388 & -1.542226 & $\mathrm{H}$ & 0.860489 & -4.228609 & -0.542442 \\
\hline C & -6.435335 & -0.107199 & 0.645871 & $\mathrm{Cu}$ & 1.162784 & 0.203257 & -0.498405 \\
\hline C & -3.585074 & 0.330065 & -2.486890 & $\mathrm{~N}$ & 2.778231 & -0.348758 & 0.511329 \\
\hline C & -7.087721 & 1.345516 & -1.658809 & $\mathrm{~N}$ & 1.890825 & 2.041397 & -0.655516 \\
\hline C & -4.841908 & 0.876851 & -2.576200 & C & 3.813412 & 0.360443 & 0.810360 \\
\hline C & -7.675058 & 0.477070 & 0.507590 & $\mathrm{O}$ & 4.726335 & -0.223784 & 1.561621 \\
\hline $\mathrm{H}$ & -6.200387 & -0.680148 & 1.536977 & C & 3.064837 & 2.466950 & -0.339117 \\
\hline $\mathrm{H}$ & -2.886246 & 0.450321 & -3.305490 & $\mathrm{O}$ & 3.347529 & 3.713225 & -0.667183 \\
\hline C & -8.008399 & 1.210804 & -0.649665 & $C$ & 4.195130 & -1.505707 & 1.971705 \\
\hline $\mathrm{H}$ & -7.331927 & 1.902233 & -2.558938 & $\mathrm{H}$ & 4.987292 & -2.244906 & 1.865112 \\
\hline $\mathrm{H}$ & -5.114537 & 1.426923 & -3.473058 & $\mathrm{H}$ & 3.900979 & -1.410435 & 3.019970 \\
\hline $\mathrm{H}$ & -8.409987 & 0.366064 & 1.298536 & C & 3.004840 & -1.719929 & 1.014039 \\
\hline $\mathrm{H}$ & -8.991410 & 1.659917 & -0.740000 & C & 1.122378 & 3.179158 & -1.225707 \\
\hline C & -3.309737 & -2.815462 & 2.943641 & C & 2.235666 & 4.225844 & -1.443601 \\
\hline $\mathrm{H}$ & -2.310766 & -2.786111 & 3.391121 & $\mathrm{H}$ & 1.992391 & 5.215239 & -1.059024 \\
\hline $\mathrm{H}$ & -4.044899 & -2.718674 & 3.749245 & $\mathrm{H}$ & 2.569937 & 4.292894 & -2.480714 \\
\hline $\mathrm{H}$ & -3.450882 & -3.790347 & 2.469753 & C & 4.176434 & 1.759123 & 0.388047 \\
\hline
\end{tabular}




\begin{tabular}{|c|c|c|c|c|c|c|c|}
\hline C & 4.536618 & 2.590796 & 1.643928 & C & -1.544642 & 0.725452 & 1.485035 \\
\hline $\mathrm{H}$ & 4.838497 & 3.595808 & 1.344814 & C & -4.228994 & -2.563513 & 1.803282 \\
\hline $\mathrm{H}$ & 3.684522 & 2.666733 & 2.327337 & C & -0.807391 & -1.565107 & 2.917450 \\
\hline $\mathrm{H}$ & 5.366403 & 2.117441 & 2.171118 & C & -2.992338 & -2.597721 & 2.380740 \\
\hline C & 5.401821 & 1.656888 & -0.558775 & C & -0.319662 & 0.652186 & 2.117114 \\
\hline $\mathrm{H}$ & 5.707781 & 2.658930 & -0.865722 & $\mathrm{H}$ & -1.823167 & 1.628783 & 0.950170 \\
\hline $\mathrm{H}$ & 6.232587 & 1.182053 & -0.033155 & $\mathrm{H}$ & -4.898744 & -3.406757 & 1.916444 \\
\hline $\mathrm{H}$ & 5.166176 & 1.068692 & -1.451086 & C & 0.057940 & -0.499031 & 2.836565 \\
\hline $\mathrm{H}$ & 0.682297 & 2.865522 & -2.171768 & $\mathrm{H}$ & -0.542986 & -2.450425 & 3.489858 \\
\hline $\mathrm{H}$ & 2.112944 & -2.039778 & 1.558530 & $\mathrm{H}$ & -2.697720 & -3.478627 & 2.945022 \\
\hline C & 0.032552 & 3.630082 & -0.276774 & $\mathrm{H}$ & 0.341543 & 1.513169 & 2.083078 \\
\hline C & -1.282348 & 3.754868 & -0.722858 & $\mathrm{H}$ & 1.014260 & -0.527340 & 3.349858 \\
\hline C & 0.343535 & 3.983517 & 1.039719 & C & -5.133946 & 2.614937 & -1.428766 \\
\hline C & -2.270524 & 4.233068 & 0.135795 & $\mathrm{H}$ & -4.451441 & 3.201185 & -2.053264 \\
\hline $\mathrm{H}$ & -1.531773 & 3.480555 & -1.744093 & $\mathrm{H}$ & -5.986266 & 2.315847 & -2.047867 \\
\hline C & -0.646025 & 4.441035 & 1.902899 & $\mathrm{H}$ & -5.508162 & 3.248545 & -0.621020 \\
\hline $\mathrm{H}$ & 1.369756 & 3.916189 & 1.396747 & $\mathrm{O}$ & -5.816927 & -1.306600 & 0.482874 \\
\hline C & -1.957816 & 4.568617 & 1.449639 & C & -6.786595 & -2.348145 & 0.616711 \\
\hline H & -3.286247 & 4.357089 & -0.224138 & $\mathrm{H}$ & -7.042377 & -2.507375 & 1.667915 \\
\hline $\mathrm{H}$ & -0.393035 & 4.714049 & 2.922262 & $\mathrm{H}$ & -7.663552 & -1.993821 & 0.078218 \\
\hline $\mathrm{H}$ & -2.728553 & 4.941786 & 2.116054 & $\mathrm{H}$ & -6.425495 & -3.276963 & 0.164323 \\
\hline C & 3.304359 & -2.712747 & -0.094238 & C & -3.628945 & 0.386135 & -2.469421 \\
\hline C & 3.416508 & -4.061977 & 0.253505 & $\mathrm{H}$ & -4.204474 & 1.002582 & -3.152022 \\
\hline C & 3.493027 & -2.335533 & -1.421472 & & & & \\
\hline C & 3.703498 & -5.019669 & -0.713058 & \multicolumn{2}{|c|}{ TS1-C } & & \\
\hline $\mathrm{H}$ & 3.276506 & -4.369806 & 1.288296 & C & 3.201962 & 2.043519 & 1.210282 \\
\hline C & 3.771955 & -3.295383 & -2.393394 & C & 2.748496 & 3.314505 & 1.650393 \\
\hline $\mathrm{H}$ & 3.428011 & -1.290108 & -1.710740 & C & 0.496322 & 2.430430 & 1.579860 \\
\hline C & 3.875716 & -4.637466 & -2.042940 & C & 1.406590 & 3.490721 & 1.827069 \\
\hline $\mathrm{H}$ & 3.794664 & -6.063326 & -0.429567 & $\mathrm{H}$ & 3.466971 & 4.107156 & 1.813154 \\
\hline $\mathrm{H}$ & 3.917458 & -2.990378 & -3.424867 & $\mathrm{H}$ & 1.016137 & 4.443034 & 2.168630 \\
\hline $\mathrm{H}$ & 4.099878 & -5.382686 & -2.798945 & $\mathrm{H}$ & 4.222998 & 1.727277 & 1.381941 \\
\hline C & -4.455865 & 1.422178 & -0.880455 & $\mathrm{O}$ & 2.357427 & 0.984174 & 1.320211 \\
\hline C & -4.129510 & 0.715049 & 0.085588 & C & 1.015910 & 1.149696 & 1.291167 \\
\hline C & -3.746001 & -0.325997 & 0.922649 & $\mathrm{O}$ & 0.394856 & 0.090028 & 1.056278 \\
\hline C & -2.452610 & -0.353133 & 1.565794 & C & -0.925062 & 2.586852 & 1.778021 \\
\hline C & -4.633232 & -1.417354 & 1.065502 & $\mathrm{O}$ & -1.792158 & 1.722554 & 1.512661 \\
\hline C & -2.073023 & -1.509974 & 2.288499 & 0 & -1.280689 & 3.732785 & 2.304772 \\
\hline
\end{tabular}




\begin{tabular}{|c|c|c|c|c|c|c|c|}
\hline C & -2.668951 & 3.935277 & 2.621241 & $\mathrm{H}$ & -1.565075 & 5.883374 & -1.002181 \\
\hline $\mathrm{H}$ & -3.276181 & 3.838673 & 1.720769 & $\mathrm{H}$ & 0.804204 & 2.693363 & -2.630535 \\
\hline $\mathrm{H}$ & -2.723559 & 4.946574 & 3.017660 & $\mathrm{H}$ & 0.552418 & 5.087458 & -2.019313 \\
\hline $\mathrm{H}$ & -2.988181 & 3.209001 & 3.370467 & C & -0.423061 & -2.628919 & 2.297714 \\
\hline $\mathrm{Cu}$ & -1.489181 & 0.039728 & 0.589509 & C & 0.780412 & -2.411357 & 2.965979 \\
\hline$N$ & -3.000311 & 0.290093 & -0.664742 & C & -1.610266 & -2.719370 & 3.029506 \\
\hline N & -1.450288 & -1.869376 & 0.184954 & C & 0.800263 & -2.293311 & 4.352851 \\
\hline C & -3.564984 & -0.597177 & -1.408347 & $\mathrm{H}$ & 1.703071 & -2.325694 & 2.398211 \\
\hline O & -4.396373 & -0.148790 & -2.327917 & $C$ & -1.592651 & -2.592951 & 4.415000 \\
\hline C & -2.247747 & -2.559133 & -0.549001 & $\mathrm{H}$ & -2.555703 & -2.898151 & 2.520147 \\
\hline O & -2.016053 & -3.856566 & -0.606592 & C & -0.385360 & -2.381132 & 5.078125 \\
\hline C & -4.362652 & 1.300768 & -2.294543 & $\mathrm{H}$ & 1.741804 & -2.133688 & 4.868617 \\
\hline $\mathrm{H}$ & -5.389853 & 1.652369 & -2.208575 & $\mathrm{H}$ & -2.517228 & -2.670376 & 4.978025 \\
\hline $\mathrm{H}$ & -3.926889 & 1.637954 & -3.236881 & $\mathrm{H}$ & -0.369152 & -2.291492 & 6.159472 \\
\hline C & -3.480077 & 1.629329 & -1.065256 & C & 3.463357 & 2.269266 & -0.835698 \\
\hline $\mathrm{H}$ & -4.085849 & 2.021554 & -0.242717 & C & 3.590483 & 1.072567 & -1.141737 \\
\hline C & -0.443334 & -2.766726 & 0.793336 & C & 3.500013 & 3.688930 & -1.237756 \\
\hline $\mathrm{H}$ & 0.531554 & -2.493328 & 0.380010 & $\mathrm{H}$ & 2.629433 & 4.219480 & -0.838973 \\
\hline C & -0.901520 & -4.150584 & 0.276688 & $\mathrm{H}$ & 4.400255 & 4.172761 & -0.844966 \\
\hline $\mathrm{H}$ & -0.145575 & -4.664846 & -0.315168 & $\mathrm{H}$ & 3.511416 & 3.778729 & -2.326973 \\
\hline $\mathrm{H}$ & -1.275908 & -4.803406 & 1.066711 & C & 4.954268 & -0.893774 & -0.886953 \\
\hline C & -3.456678 & -2.100610 & -1.328024 & C & 3.713951 & -0.309338 & -1.226218 \\
\hline C & -3.409240 & -2.699206 & -2.750717 & C & 4.085487 & -3.079273 & -1.407810 \\
\hline $\mathrm{H}$ & -3.354675 & -3.787054 & -2.687601 & C & 2.617302 & -1.140231 & -1.664126 \\
\hline $\mathrm{H}$ & -2.543267 & -2.335926 & -3.312561 & C & 5.124522 & -2.296641 & -0.982412 \\
\hline $\mathrm{H}$ & -4.312702 & -2.425271 & -3.297282 & C & 2.816598 & -2.539500 & -1.761475 \\
\hline C & -4.717995 & -2.611187 & -0.576993 & C & 1.362793 & -0.607893 & -2.032635 \\
\hline $\mathrm{H}$ & -4.691966 & -3.701803 & -0.523537 & $\mathrm{H}$ & 6.072074 & -2.754546 & -0.727731 \\
\hline $\mathrm{H}$ & -5.615342 & -2.310794 & -1.123069 & $\mathrm{H}$ & 1.944913 & -4.436484 & -2.324450 \\
\hline $\mathrm{H}$ & -4.765918 & -2.204210 & 0.437694 & $\mathrm{H}$ & 4.233165 & -4.152917 & -1.487895 \\
\hline C & -2.344946 & 2.591614 & -1.344217 & C & 0.366392 & -1.432290 & -2.500975 \\
\hline C & -2.482754 & 3.940555 & -1.017218 & $\mathrm{H}$ & 1.203405 & 0.462328 & -1.953483 \\
\hline C & -1.156325 & 2.152165 & -1.930473 & $\mathrm{H}$ & -0.582870 & -1.003354 & -2.812020 \\
\hline C & -1.442237 & 4.834944 & -1.253775 & C & 0.562891 & -2.824811 & -2.606874 \\
\hline $\mathrm{H}$ & -3.412662 & 4.300801 & -0.583855 & $\mathrm{H}$ & -0.226131 & -3.460580 & -2.996527 \\
\hline C & -0.111714 & 3.043394 & -2.162840 & C & 1.769305 & -3.367866 & -2.233830 \\
\hline $\mathrm{H}$ & -1.047708 & 1.109599 & -2.217884 & O & 5.903764 & -0.064521 & -0.4718 \\
\hline C & -0.252073 & 4.386198 & -1.821176 & $\mathrm{C}$ & 7.214343 & -0.561885 & -0.1894 \\
\hline
\end{tabular}




\begin{tabular}{|c|c|c|c|c|c|c|c|}
\hline $\mathrm{H}$ & 7.192159 & -1.257890 & 0.654449 & $\mathrm{H}$ & 5.088326 & -0.308539 & 0.635959 \\
\hline $\mathrm{H}$ & 7.803913 & 0.314577 & 0.073027 & C & 2.411432 & -2.749983 & -1.429875 \\
\hline \multirow[t]{2}{*}{$\mathrm{H}$} & 7.644575 & -1.042093 & -1.072756 & C & 2.603392 & -2.584176 & -2.954472 \\
\hline & & & & $\mathrm{H}$ & 3.631140 & -2.281268 & -3.163558 \\
\hline \multicolumn{2}{|c|}{ TS1-D } & & & $\mathrm{H}$ & 1.920245 & -1.828963 & -3.353616 \\
\hline C & -2.102903 & 3.487765 & 1.231202 & $\mathrm{H}$ & 2.408862 & -3.532721 & -3.457353 \\
\hline C & -3.277554 & 2.923272 & 1.785936 & C & 3.323535 & -3.888745 & -0.896891 \\
\hline C & -2.120613 & 0.798212 & 1.883065 & $\mathrm{H}$ & 4.365896 & -3.654244 & -1.121177 \\
\hline C & -3.265720 & 1.597060 & 2.118656 & $\mathrm{H}$ & 3.062389 & -4.828346 & -1.388121 \\
\hline $\mathrm{H}$ & -4.143014 & 3.552829 & 1.946551 & $\mathrm{H}$ & 3.212438 & -4.011463 & 0.184993 \\
\hline $\mathrm{H}$ & -4.135353 & 1.138257 & 2.576048 & C & -2.368388 & -2.734678 & -0.379620 \\
\hline $\mathrm{H}$ & -1.882199 & 4.542473 & 1.359383 & C & -3.531934 & -3.336942 & 0.102826 \\
\hline O & -0.953238 & 2.769350 & 1.277821 & $\mathrm{C}$ & -2.436642 & -1.440486 & -0.896202 \\
\hline C & -0.960021 & 1.420480 & 1.377966 & C & -4.741335 & -2.647959 & 0.094888 \\
\hline O & 0.123328 & 0.896670 & 1.043418 & $\mathrm{H}$ & -3.494082 & -4.350512 & 0.496396 \\
\hline C & -2.058679 & -0.594607 & 2.272796 & C & -3.640557 & -0.738192 & -0.876481 \\
\hline O & -1.119154 & -1.377590 & 2.002689 & $\mathrm{H}$ & -1.545406 & -0.961519 & -1.299742 \\
\hline O & -3.073941 & -1.002207 & 2.988650 & C & -4.793879 & -1.340020 & -0.380760 \\
\hline C & -3.053850 & -2.347855 & 3.501909 & $\mathrm{H}$ & -5.638745 & -3.129711 & 0.469452 \\
\hline $\mathrm{H}$ & -2.982815 & -3.057300 & 2.677744 & $\mathrm{H}$ & -3.666148 & 0.280700 & -1.248562 \\
\hline $\mathrm{H}$ & -3.997809 & -2.460347 & 4.030107 & $\mathrm{H}$ & -5.732734 & -0.795761 & -0.372131 \\
\hline $\mathrm{H}$ & -2.211783 & -2.467564 & 4.185466 & C & 2.989023 & 0.457085 & 2.054187 \\
\hline $\mathrm{Cu}$ & 0.347838 & -1.015119 & 0.782220 & $\mathrm{C}$ & 2.732169 & 1.672975 & 2.685525 \\
\hline N & 0.146552 & -2.679746 & -0.290331 & C & 3.243656 & -0.680573 & 2.824997 \\
\hline N & 2.139296 & -0.742049 & 0.067301 & C & 2.738734 & 1.754894 & 4.074930 \\
\hline C & 0.983084 & -3.151535 & -1.150487 & $\mathrm{H}$ & 2.518865 & 2.553755 & 2.085218 \\
\hline O & 0.567767 & -4.175531 & -1.872938 & C & 3.240607 & -0.601229 & 4.214524 \\
\hline C & 2.819875 & -1.474946 & -0.737863 & $\mathrm{H}$ & 3.456563 & -1.633420 & 2.343041 \\
\hline O & 4.061216 & -1.091684 & -0.969904 & $\mathrm{C}$ & 2.989678 & 0.618344 & 4.840281 \\
\hline C & -0.825525 & -4.406175 & -1.555287 & $\mathrm{H}$ & 2.549438 & 2.706335 & 4.561681 \\
\hline $\mathrm{H}$ & -0.954304 & -5.471682 & -1.371718 & $\mathrm{H}$ & 3.446109 & -1.486146 & 4.808311 \\
\hline $\mathrm{H}$ & -1.415168 & -4.099633 & -2.422305 & $\mathrm{H}$ & 2.997453 & 0.683506 & 5.923475 \\
\hline C & -1.074501 & -3.514915 & -0.318469 & $\mathrm{C}$ & -2.478382 & 3.871393 & -0.737050 \\
\hline $\mathrm{H}$ & -1.071327 & -4.114714 & 0.598148 & C & -1.624658 & 3.225147 & -1.364295 \\
\hline C & 2.984175 & 0.376345 & 0.546446 & C & -3.577542 & 4.864416 & -0.777484 \\
\hline $\mathrm{H}$ & 2.575956 & 1.301466 & 0.132876 & $\mathrm{H}$ & -3.392485 & 5.691129 & -0.083494 \\
\hline C & 4.347920 & 0.030842 & -0.090868 & $\mathrm{H}$ & -3.682425 & 5.276545 & -1.783260 \\
\hline $\mathrm{H}$ & 4.757228 & 0.833351 & -0.701769 & $\mathrm{H}$ & -4.522790 & 4.389545 & -0.495709 \\
\hline
\end{tabular}




\begin{tabular}{|c|c|c|c|c|c|c|c|}
\hline C & -0.941149 & 1.398892 & -2.782899 & $\mathrm{H}$ & 3.920449 & 0.424728 & -0.466006 \\
\hline C & -0.596841 & 2.465333 & -1.927299 & $\mathrm{H}$ & 4.896630 & 0.105055 & -1.935649 \\
\hline C & 1.383577 & 1.021681 & -3.289604 & $\mathrm{Cu}$ & -0.538056 & 0.545031 & -0.681023 \\
\hline C & 0.791568 & 2.822869 & -1.745923 & $\mathrm{~N}$ & -2.433430 & 1.134257 & -0.750440 \\
\hline C & 0.068468 & 0.667897 & -3.452091 & $\mathrm{~N}$ & -0.073123 & 2.146733 & 0.380862 \\
\hline C & 1.784069 & 2.091128 & -2.449324 & C & -2.881442 & 2.315895 & -0.494620 \\
\hline C & 1.204701 & 3.882230 & -0.907851 & $\mathrm{O}$ & -4.076110 & 2.609201 & -0.971539 \\
\hline $\mathrm{H}$ & -0.194599 & -0.134236 & -4.131154 & C & -0.862559 & 3.087485 & 0.780037 \\
\hline $\mathrm{H}$ & 3.890889 & 1.907061 & -2.903081 & O & -0.352802 & 3.951243 & 1.636462 \\
\hline $\mathrm{H}$ & 2.150557 & 0.486547 & -3.844128 & C & -4.473556 & 1.507148 & -1.832182 \\
\hline C & 2.537336 & 4.205855 & -0.793035 & $\mathrm{H}$ & -5.528418 & 1.308603 & -1.651781 \\
\hline $\mathrm{H}$ & 0.460214 & 4.453288 & -0.365198 & $\mathrm{H}$ & -4.318282 & 1.833727 & -2.862884 \\
\hline $\mathrm{H}$ & 2.834727 & 5.034371 & -0.158018 & $\mathrm{C}$ & -3.534847 & 0.366322 & -1.405107 \\
\hline C & 3.523159 & 3.493157 & -1.510882 & C & 1.280386 & 2.449418 & 0.894082 \\
\hline $\mathrm{H}$ & 4.565701 & 3.782485 & -1.428641 & $\mathrm{C}$ & 0.956202 & 3.459020 & 2.010395 \\
\hline C & 3.149313 & 2.454145 & -2.326241 & $\mathrm{H}$ & 1.633569 & 4.309919 & 2.053499 \\
\hline O & -2.243398 & 1.162429 & -2.949754 & $\mathrm{H}$ & 0.854967 & 2.997264 & 2.996661 \\
\hline C & -2.676831 & 0.264407 & -3.970181 & C & -2.271397 & 3.399643 & 0.352945 \\
\hline $\mathrm{H}$ & -2.370985 & -0.762713 & -3.746294 & C & -2.225540 & 4.725099 & -0.450051 \\
\hline $\mathrm{H}$ & -2.295540 & 0.577057 & -4.946546 & $\mathrm{H}$ & -1.825594 & 5.522879 & 0.178710 \\
\hline \multirow[t]{2}{*}{$\mathrm{H}$} & -3.763982 & 0.322781 & -3.964505 & $\mathrm{H}$ & -1.596848 & 4.629570 & -1.340618 \\
\hline & & & & $\mathrm{H}$ & -3.236195 & 4.995740 & -0.760818 \\
\hline \multicolumn{2}{|c|}{ TS2-A } & & & $\mathrm{C}$ & -3.168564 & 3.561278 & 1.606064 \\
\hline C & -0.004270 & -4.384000 & -1.616490 & $\mathrm{H}$ & -2.765652 & 4.346560 & 2.248015 \\
\hline C & 1.192401 & -4.351331 & -2.510789 & $\mathrm{H}$ & -4.177169 & 3.844388 & 1.298748 \\
\hline C & 1.154757 & -2.056756 & -1.859651 & $\mathrm{H}$ & -3.222871 & 2.628613 & 2.177085 \\
\hline C & 1.785507 & -3.153575 & -2.582197 & $\mathrm{H}$ & 1.720051 & 1.536410 & 1.299934 \\
\hline $\mathrm{H}$ & 1.533287 & -5.242126 & -3.022563 & $\mathrm{H}$ & -3.131776 & -0.163549 & -2.267085 \\
\hline $\mathrm{H}$ & 2.690111 & -2.966833 & -3.149262 & $\mathrm{C}$ & 2.199609 & 3.024380 & -0.166955 \\
\hline $\mathrm{H}$ & -0.636122 & -5.259776 & -1.755939 & C & 3.546520 & 3.192415 & 0.165556 \\
\hline O & -0.863720 & -3.261564 & -1.877065 & $\mathrm{C}$ & 1.754789 & 3.418223 & -1.426213 \\
\hline C & -0.264446 & -2.086312 & -1.734432 & C & 4.436240 & 3.745820 & -0.748323 \\
\hline O & -1.029842 & -1.107313 & -1.533861 & $\mathrm{H}$ & 3.906089 & 2.882387 & 1.145232 \\
\hline C & 1.850191 & -0.843423 & -1.547379 & C & 2.647005 & 3.966644 & -2.347154 \\
\hline O & 1.356160 & 0.118044 & -0.903188 & $\mathrm{H}$ & 0.711744 & 3.295678 & -1.703083 \\
\hline O & 3.105247 & -0.816322 & -1.918752 & C & 3.987205 & 4.132017 & -2.010957 \\
\hline C & 3.902206 & 0.323335 & -1.552586 & $\mathrm{H}$ & 5.478798 & 3.877613 & -0.477114 \\
\hline $\mathrm{H}$ & 3.498743 & 1.227411 & -2.010175 & $\mathrm{H}$ & 2.291035 & 4.271483 & -3.326131 \\
\hline
\end{tabular}




\begin{tabular}{|c|c|c|c|c|c|c|c|}
\hline $\mathrm{H}$ & 4.678714 & 4.565797 & -2.725670 & $\mathrm{H}$ & -1.999890 & -1.484927 & 3.521611 \\
\hline C & -4.163134 & -0.617603 & -0.438296 & & & & \\
\hline C & -4.019352 & -1.989726 & -0.639471 & \multicolumn{4}{|c|}{ TS2-B } \\
\hline C & -4.938397 & -0.161400 & 0.634301 & $C$ & 3.761844 & -1.935245 & -1.811813 \\
\hline C & -4.657038 & -2.894710 & 0.207804 & $C$ & 4.419986 & -0.743298 & -2.430132 \\
\hline $\mathrm{H}$ & -3.414786 & -2.353780 & -1.463640 & $C$ & 2.378503 & 0.288845 & -1.742452 \\
\hline C & -5.574281 & -1.063231 & 1.480598 & $C$ & 3.706948 & 0.384257 & -2.338089 \\
\hline $\mathrm{H}$ & -5.076776 & 0.905259 & 0.800415 & $\mathrm{H}$ & 5.396997 & -0.822136 & -2.889074 \\
\hline C & -5.439055 & -2.434968 & 1.264234 & $\mathrm{H}$ & 4.059502 & 1.343713 & -2.698558 \\
\hline $\mathrm{H}$ & -4.556532 & -3.960718 & 0.030031 & $\mathrm{H}$ & 4.196644 & -2.884608 & -2.121205 \\
\hline $\mathrm{H}$ & -6.190518 & -0.698458 & 2.296192 & $\mathrm{O}$ & 2.385459 & -2.014913 & -2.207749 \\
\hline $\mathrm{H}$ & -5.950954 & -3.139940 & 1.911461 & $\mathrm{C}$ & 1.673016 & -0.933434 & -1.905247 \\
\hline C & 0.444093 & -4.315462 & -0.129512 & $\mathrm{O}$ & 0.432511 & -1.119495 & -1.812639 \\
\hline C & 0.884293 & -3.214316 & 0.394640 & $C$ & 1.682420 & 1.413206 & -1.202761 \\
\hline C & 1.249757 & -2.234720 & 1.285160 & $\mathrm{O}$ & 0.534247 & 1.385795 & -0.694529 \\
\hline C & 2.640468 & -1.862326 & 1.470807 & $\mathrm{O}$ & 2.382156 & 2.528528 & -1.170659 \\
\hline C & 0.230285 & -1.560471 & 2.014525 & $\mathrm{C}$ & 1.724449 & 3.718274 & -0.702540 \\
\hline C & 2.957846 & -0.867965 & 2.429589 & $\mathrm{H}$ & 0.866296 & 3.947752 & -1.335630 \\
\hline C & 3.680748 & -2.461996 & 0.739681 & $\mathrm{H}$ & 1.399069 & 3.584439 & 0.331542 \\
\hline C & 0.579313 & -0.582542 & 2.974202 & $\mathrm{H}$ & 2.475165 & 4.502933 & -0.774361 \\
\hline C & 4.306797 & -0.493599 & 2.634612 & $\mathrm{Cu}$ & -0.797914 & -0.061273 & -0.773191 \\
\hline C & 1.902018 & -0.272276 & 3.172556 & $\mathrm{~N}$ & -2.131666 & -1.527205 & -0.941816 \\
\hline C & 4.989435 & -2.077818 & 0.954627 & $\mathrm{~N}$ & -2.106242 & 1.034419 & 0.226421 \\
\hline $\mathrm{H}$ & 3.450340 & -3.228403 & 0.005876 & C & -3.382383 & -1.492479 & -0.639180 \\
\hline $\mathrm{H}$ & -0.184875 & -0.096326 & 3.567544 & $\mathrm{O}$ & -4.111693 & -2.524000 & -1.024601 \\
\hline C & 5.309514 & -1.088666 & 1.904646 & $C$ & -3.300905 & 0.705175 & 0.581025 \\
\hline $\mathrm{H}$ & 4.541002 & 0.256231 & 3.385255 & $\mathrm{O}$ & -3.916563 & 1.529717 & 1.406406 \\
\hline $\mathrm{H}$ & 2.159353 & 0.465420 & 3.929447 & $C$ & -3.265438 & -3.366329 & -1.850068 \\
\hline $\mathrm{H}$ & 5.784832 & -2.551346 & 0.388019 & $\mathrm{H}$ & -3.442504 & -4.400658 & -1.559598 \\
\hline $\mathrm{H}$ & 6.345061 & -0.809911 & 2.066721 & $\mathrm{H}$ & -3.566438 & -3.207028 & -2.887574 \\
\hline C & 0.375816 & -5.607979 & 0.649003 & C & -1.843407 & -2.861542 & -1.544985 \\
\hline $\mathrm{H}$ & -0.665603 & -5.939173 & 0.716676 & $C$ & -1.805586 & 2.362797 & 0.797765 \\
\hline $\mathrm{H}$ & 0.945509 & -6.379633 & 0.119235 & C & -2.968603 & 2.548799 & 1.795779 \\
\hline $\mathrm{H}$ & 0.782895 & -5.502154 & 1.655417 & $\mathrm{H}$ & -3.466501 & 3.514213 & 1.718190 \\
\hline $\mathrm{O}$ & -1.014526 & -1.902081 & 1.732671 & $\mathrm{H}$ & -2.686944 & 2.345040 & 2.831211 \\
\hline C & -2.103927 & -1.313551 & 2.446564 & $C$ & -4.145956 & -0.464612 & 0.151378 \\
\hline $\mathrm{H}$ & -2.166111 & -0.242549 & 2.226729 & $C$ & -5.301453 & 0.091182 & -0.721072 \\
\hline $\mathrm{H}$ & -2.993589 & -1.814162 & 2.073182 & $\mathrm{H}$ & -5.890269 & 0.804257 & -0.140715 \\
\hline
\end{tabular}




\begin{tabular}{|c|c|c|c|c|c|c|c|}
\hline $\mathrm{H}$ & -4.917107 & 0.594233 & -1.613717 & C & -0.221742 & 0.723374 & 3.573933 \\
\hline $\mathrm{H}$ & -5.950427 & -0.730218 & -1.030586 & C & 1.887807 & 1.932727 & 3.140355 \\
\hline C & -4.712343 & -1.169813 & 1.408975 & C & -0.660624 & -1.338173 & 2.417257 \\
\hline $\mathrm{H}$ & -5.297703 & -0.460757 & 1.996466 & $\mathrm{H}$ & 0.844512 & -2.067619 & 1.081765 \\
\hline $\mathrm{H}$ & -5.360604 & -1.994118 & 1.106777 & $\mathrm{H}$ & 3.707030 & 2.952084 & 2.712256 \\
\hline $\mathrm{H}$ & -3.905955 & -1.566317 & 2.034786 & C & -1.057585 & -0.340867 & 3.327441 \\
\hline $\mathrm{H}$ & -0.850094 & 2.299226 & 1.324297 & $\mathrm{H}$ & -0.498048 & 1.489606 & 4.293700 \\
\hline $\mathrm{H}$ & -1.267230 & -2.716652 & -2.457831 & $\mathrm{H}$ & 1.576188 & 2.687397 & 3.858241 \\
\hline C & -1.731764 & 3.461292 & -0.245511 & $\mathrm{H}$ & -1.302894 & -2.191920 & 2.232780 \\
\hline C & -1.382708 & 4.744335 & 0.185666 & $\mathrm{H}$ & -2.004493 & -0.427811 & 3.850937 \\
\hline C & -1.999520 & 3.247331 & -1.594942 & C & 4.698015 & -2.859486 & 0.439242 \\
\hline C & -1.294122 & 5.795532 & -0.720164 & $\mathrm{H}$ & 4.322432 & -3.869702 & 0.246774 \\
\hline $\mathrm{H}$ & -1.175360 & 4.926418 & 1.238821 & $\mathrm{H}$ & 5.710530 & -2.787650 & 0.027011 \\
\hline C & -1.902169 & 4.297891 & -2.506753 & $\mathrm{H}$ & 4.744697 & -2.696132 & 1.516684 \\
\hline $\mathrm{H}$ & -2.287767 & 2.260986 & -1.947183 & $\mathrm{O}$ & 4.628586 & 1.185204 & 0.881492 \\
\hline C & -1.547832 & 5.571818 & -2.073106 & $C$ & 5.488065 & 2.322565 & 1.008651 \\
\hline $\mathrm{H}$ & -1.029572 & 6.788954 & -0.372235 & $\mathrm{H}$ & 5.871668 & 2.404690 & 2.029008 \\
\hline $\mathrm{H}$ & -2.113744 & 4.120712 & -3.556501 & $\mathrm{H}$ & 6.309486 & 2.137185 & 0.319862 \\
\hline $\mathrm{H}$ & -1.480386 & 6.389973 & -2.782546 & $\mathrm{H}$ & 4.956388 & 3.232722 & 0.717063 \\
\hline C & -1.064719 & -3.725448 & -0.573644 & & & & \\
\hline C & 0.243024 & -4.110072 & -0.865726 & \multicolumn{2}{|c|}{ TS2-C } & & \\
\hline C & -1.652984 & -4.161164 & 0.618294 & C & 2.198671 & -3.544017 & -1.643207 \\
\hline C & 0.961342 & -4.897750 & 0.031984 & C & 2.248059 & -4.179616 & -0.290348 \\
\hline $\mathrm{H}$ & 0.702150 & -3.792976 & -1.795340 & C & 1.237897 & -2.098145 & 0.277560 \\
\hline C & -0.936285 & -4.946049 & 1.515680 & C & 1.790520 & -3.388746 & 0.685021 \\
\hline $\mathrm{H}$ & -2.684879 & -3.900201 & 0.848891 & $\mathrm{H}$ & 2.647383 & -5.175705 & -0.149754 \\
\hline C & 0.378153 & -5.310012 & 1.226592 & $\mathrm{H}$ & 1.789162 & -3.660955 & 1.733831 \\
\hline $\mathrm{H}$ & 1.971725 & -5.209707 & -0.215319 & $\mathrm{H}$ & 2.348665 & -4.237498 & -2.468644 \\
\hline $\mathrm{H}$ & -1.405889 & -5.286372 & 2.433179 & $\mathrm{O}$ & 0.893728 & -2.968597 & -1.877613 \\
\hline $\mathrm{H}$ & 0.933868 & -5.931819 & 1.921017 & C & 0.540119 & -2.068145 & -0.978029 \\
\hline C & 3.812062 & -1.850183 & -0.254599 & $\mathrm{O}$ & -0.378174 & -1.282566 & -1.318648 \\
\hline C & 3.168374 & -0.945693 & 0.412094 & C & 1.027507 & -1.035407 & 1.224770 \\
\hline C & 2.707490 & -0.047541 & 1.336249 & $\mathrm{O}$ & 0.379106 & 0.018438 & 1.004840 \\
\hline C & 1.420080 & -0.183215 & 1.996432 & $\mathrm{O}$ & 1.647486 & -1.199076 & 2.368431 \\
\hline C & 3.512576 & 1.109262 & 1.573070 & C & 1.615852 & -0.122289 & 3.317269 \\
\hline C & 1.022703 & 0.824593 & 2.909167 & $\mathrm{H}$ & 0.585869 & 0.096204 & 3.606089 \\
\hline C & 0.553720 & -1.270038 & 1.761718 & $\mathrm{H}$ & 2.090708 & 0.761595 & 2.885578 \\
\hline C & 3.088840 & 2.088793 & 2.499913 & $\mathrm{H}$ & 2.186935 & -0.480579 & 4.170685 \\
\hline
\end{tabular}




\begin{tabular}{|c|c|c|c|c|c|c|c|}
\hline $\mathrm{Cu}$ & -1.219186 & -0.017084 & -0.147089 & $\mathrm{H}$ & 4.785226 & 3.264582 & 2.208621 \\
\hline N & -3.023640 & -0.656674 & -0.638426 & $\mathrm{H}$ & 3.536289 & 3.792739 & 0.156326 \\
\hline$N$ & -1.998453 & 1.597919 & 0.660030 & $\mathrm{H}$ & 5.594759 & -0.833187 & 3.194908 \\
\hline C & -4.166157 & -0.160959 & -0.313755 & $\mathrm{H}$ & 5.773567 & 1.585008 & 3.733966 \\
\hline $\mathrm{O}$ & -5.216127 & -0.934264 & -0.498433 & $C$ & 4.434255 & -2.621674 & -2.609957 \\
\hline C & -3.246497 & 1.913169 & 0.767750 & $\mathrm{H}$ & 4.106574 & -2.741951 & -3.647983 \\
\hline O & -3.506718 & 3.038382 & 1.402757 & $\mathrm{H}$ & 4.949322 & -3.540261 & -2.307224 \\
\hline C & -4.754470 & -2.179911 & -1.084734 & $\mathrm{H}$ & 5.139168 & -1.791004 & -2.553066 \\
\hline $\mathrm{H}$ & -5.150251 & -2.224881 & -2.100210 & $\mathrm{O}$ & 2.062968 & 0.555458 & -2.641628 \\
\hline $\mathrm{H}$ & -5.158389 & -2.993976 & -0.483742 & C & 1.548193 & 1.474952 & -3.603161 \\
\hline C & -3.211130 & -2.069095 & -1.031691 & $\mathrm{H}$ & 0.772708 & 2.104294 & -3.157022 \\
\hline C & -1.191845 & 2.711391 & 1.197306 & $\mathrm{H}$ & 1.121537 & 0.857152 & -4.391279 \\
\hline C & -2.261648 & 3.525576 & 1.957978 & $\mathrm{H}$ & 2.350403 & 2.096146 & -4.012371 \\
\hline $\mathrm{H}$ & -2.285224 & 3.317847 & 3.030186 & C & -2.537466 & -2.996611 & -0.038656 \\
\hline $\mathrm{H}$ & -2.213216 & 4.598145 & 1.778007 & C & -1.930674 & -4.168454 & -0.492451 \\
\hline C & -4.472019 & 1.207121 & 0.239429 & $C$ & -2.519117 & -2.703339 & 1.328004 \\
\hline C & -5.509282 & 1.076616 & 1.379022 & C & -1.321167 & -5.038474 & 0.406397 \\
\hline $\mathrm{H}$ & -5.760431 & 2.067023 & 1.761174 & $\mathrm{H}$ & -1.927523 & -4.399604 & -1.554316 \\
\hline $\mathrm{H}$ & -5.123039 & 0.469086 & 2.203314 & C & -1.899132 & -3.569305 & 2.226600 \\
\hline $\mathrm{H}$ & -6.416931 & 0.607100 & 0.997668 & $\mathrm{H}$ & -3.003634 & -1.805130 & 1.705499 \\
\hline C & -5.051426 & 2.069085 & -0.915605 & $C$ & -1.301723 & -4.739788 & 1.766784 \\
\hline $\mathrm{H}$ & -5.314655 & 3.059681 & -0.538751 & $\mathrm{H}$ & -0.858117 & -5.949865 & 0.042626 \\
\hline $\mathrm{H}$ & -5.954792 & 1.591873 & -1.302512 & $\mathrm{H}$ & -1.901676 & -3.339238 & 3.287559 \\
\hline $\mathrm{H}$ & -4.331151 & 2.176926 & -1.732295 & $\mathrm{H}$ & -0.831285 & -5.422191 & 2.467413 \\
\hline C & 3.233366 & -2.400320 & -1.724527 & $\mathrm{H}$ & -2.771619 & -2.217564 & -2.019902 \\
\hline C & 3.070864 & -1.306711 & -1.039559 & $\mathrm{H}$ & -0.439900 & 2.305962 & 1.877693 \\
\hline C & 3.244590 & 0.021253 & -0.705914 & C & -0.519695 & 3.524506 & 0.104955 \\
\hline C & 3.923125 & 0.414068 & 0.511705 & $C$ & -0.935279 & 3.480986 & -1.225277 \\
\hline C & 2.686283 & 1.004763 & -1.562236 & $C$ & 0.466460 & 4.441285 & 0.480512 \\
\hline C & 4.027240 & 1.793331 & 0.815196 & $C$ & -0.381150 & 4.350832 & -2.165377 \\
\hline C & 4.497491 & -0.525133 & 1.390006 & $\mathrm{H}$ & -1.711382 & 2.786664 & -1.536046 \\
\hline C & 2.806622 & 2.372895 & -1.235438 & $C$ & 1.010395 & 5.316721 & -0.452892 \\
\hline C & 4.698310 & 2.203603 & 1.991720 & $\mathrm{H}$ & 0.795594 & 4.487134 & 1.516490 \\
\hline C & 3.454676 & 2.736081 & -0.083859 & $C$ & 0.587111 & 5.273823 & -1.781387 \\
\hline C & 5.147271 & -0.101286 & 2.529888 & $\mathrm{H}$ & -0.728279 & 4.323505 & -3.194014 \\
\hline $\mathrm{H}$ & 4.424833 & -1.584609 & 1.163225 & $\mathrm{H}$ & 1.756041 & 6.042639 & -0.143221 \\
\hline $\mathrm{H}$ & 2.374917 & 3.135371 & -1.871656 & $\mathrm{H}$ & 0.998888 & 5.967266 & -2.507545 \\
\hline C & 5.249310 & 1.270165 & 2.838085 & & & & \\
\hline
\end{tabular}


TS2-D

\begin{tabular}{|c|c|c|c|}
\hline C & -1.806309 & 3.717372 & -1.282462 \\
\hline C & -1.716537 & 4.271342 & 0.103313 \\
\hline C & -0.950657 & 2.048326 & 0.516703 \\
\hline C & -1.334567 & 3.369633 & 1.012457 \\
\hline $\mathrm{H}$ & -1.982072 & 5.298286 & 0.317971 \\
\hline $\mathrm{H}$ & -1.266047 & 3.577388 & 2.073705 \\
\hline $\mathrm{H}$ & -1.897342 & 4.472009 & -2.061650 \\
\hline O & -0.606348 & 2.987434 & -1.613404 \\
\hline C & -0.316909 & 2.010142 & -0.770655 \\
\hline O & 0.483331 & 1.145029 & -1.203365 \\
\hline C & -0.723261 & 0.955081 & 1.432776 \\
\hline O & -0.021952 & -0.059190 & 1.216391 \\
\hline O & -1.359071 & 1.067932 & 2.577291 \\
\hline C & -1.195180 & 0.009963 & 3.533738 \\
\hline $\mathrm{H}$ & -0.146414 & -0.076793 & 3.825700 \\
\hline $\mathrm{H}$ & -1.543361 & -0.933430 & 3.107919 \\
\hline $\mathrm{H}$ & -1.801789 & 0.303531 & 4.388222 \\
\hline $\mathrm{Cu}$ & 1.419201 & -0.133665 & -0.11207 \\
\hline$N$ & 3.128970 & 0.337894 & -0.979974 \\
\hline N & 2.270247 & -1.736739 & 0.671919 \\
\hline C & 4.238053 & -0.312430 & -0.971725 \\
\hline O & 5.316258 & 0.355520 & -1.321986 \\
\hline C & 3.409230 & -2.256197 & 0.356277 \\
\hline O & 3.722544 & -3.388433 & 0.948643 \\
\hline C & 4.920263 & 1.724754 & -1.606544 \\
\hline $\mathrm{H}$ & 5.173642 & 1.925168 & -2.647467 \\
\hline $\mathrm{H}$ & 5.496775 & 2.372893 & -0.945942 \\
\hline C & 3.395804 & 1.748542 & -1.324230 \\
\hline C & 1.597288 & -2.618038 & 1.647001 \\
\hline C & 2.644550 & -3.744059 & 1.848034 \\
\hline $\mathrm{H}$ & 3.061952 & -3.784328 & 2.854349 \\
\hline $\mathrm{H}$ & 2.280187 & -4.727838 & 1.551324 \\
\hline C & 4.425061 & -1.775968 & -0.657266 \\
\hline C & 5.851682 & -2.036225 & -0.134751 \\
\hline $\mathrm{H}$ & 5.987653 & -3.102485 & 0.049645 \\
\hline $\mathrm{H}$ & 6.046195 & -1.492692 & 0.793947 \\
\hline & 6.579436 & -1.714729 & -0.881049 \\
\hline
\end{tabular}

$\begin{array}{llll}\text { C } & 4.181418 & -2.571195 & -1.970302\end{array}$

$\begin{array}{llll}H & 4.317420 & -3.638893 & -1.781767\end{array}$

$\begin{array}{llll}H & 4.909516 & -2.258013 & -2.723083\end{array}$

$\begin{array}{llll}\text { H } & 3.173642 & -2.397653 & -2.359338\end{array}$

$\begin{array}{llll}\text { C } & -2.988501 & 2.733308 & -1.359836\end{array}$

$\begin{array}{llll}\text { C } & -2.968337 & 1.592170 & -0.735417\end{array}$

$\begin{array}{llll}\text { C } & -3.466719 & 0.336466 & -0.448666\end{array}$

$\begin{array}{llll}\text { C } & -3.404563 & -0.739249 & -1.418961\end{array}$

$\begin{array}{llll}\text { C } & -4.179952 & 0.165178 & 0.765607\end{array}$

C $\quad-4.167605 \quad-1.907226 \quad-1.173066$

$\begin{array}{llll}\text { C } & -2.658298 & -0.650870 & -2.609908\end{array}$

$\begin{array}{llll}\text { C } & -4.883169 & -1.034333 & 1.008483\end{array}$

$\begin{array}{llll}\text { C } & -4.207165 & -2.936868 & -2.142643\end{array}$

$\begin{array}{llll}\text { C } & -4.881115 & -2.017802 & 0.051337\end{array}$

$\begin{array}{llll}\text { C } & -2.701582 & -1.674764 & -3.532701\end{array}$

$\begin{array}{llll}H & -2.056254 & 0.231523 & -2.803953\end{array}$

$\begin{array}{llll}H & -5.449030 & -1.171444 & 1.921455\end{array}$

$\begin{array}{llll}\text { C } & -3.489017 & -2.822009 & -3.308857\end{array}$

$\begin{array}{llll}\text { H } & -4.819565 & -3.814823 & -1.955835\end{array}$

$\begin{array}{llll}\text { H } & -5.452012 & -2.924850 & 0.233309\end{array}$

$\begin{array}{llll}H & -2.131904 & -1.589413 & -4.452805\end{array}$

H $\quad-3.529188 \quad-3.605883 \quad-4.057661$

$\begin{array}{llll}\text { C } & -4.199065 & 3.182809 & -2.141829\end{array}$

$\begin{array}{llll}\text { H } & -3.925822 & 3.336299 & -3.191185\end{array}$

$\begin{array}{llll}H & -4.557500 & 4.137547 & -1.740550\end{array}$

$\begin{array}{llll}H & -5.009617 & 2.454529 & -2.088970\end{array}$

$\begin{array}{llll}\text { O } & -4.157317 & 1.197115 & 1.595280\end{array}$

$\begin{array}{llll}\text { C } & -4.887872 & 1.147453 & 2.819098\end{array}$

$\begin{array}{llll}H & -5.958002 & 1.024912 & 2.629095\end{array}$

$\begin{array}{llll}H & -4.707545 & 2.106302 & 3.301369\end{array}$

$\begin{array}{llll}H & -4.520536 & 0.336961 & 3.456698\end{array}$

$\begin{array}{llll}\text { C } & 2.959719 & 2.665892 & -0.199330\end{array}$

$\begin{array}{llll}\text { C } & 2.353140 & 3.887171 & -0.493154\end{array}$

$\begin{array}{llll}\text { C } & 3.145922 & 2.298873 & 1.137271\end{array}$

$\begin{array}{llll}\text { C } & 1.938049 & 4.730921 & 0.533087\end{array}$

$\begin{array}{llll}H & 2.193695 & 4.176137 & -1.528378\end{array}$

$\begin{array}{llll}\text { C } & 2.720525 & 3.138873 & 2.164169\end{array}$

$\begin{array}{llll}H & 3.638862 & 1.360977 & 1.385926\end{array}$ 


\begin{tabular}{|c|c|c|c|c|c|c|c|}
\hline C & 2.115717 & 4.356642 & 1.862722 & $\mathrm{~N}$ & -1.024922 & 1.963405 & 0.510654 \\
\hline $\mathrm{H}$ & 1.471933 & 5.680881 & 0.292977 & C & -3.763722 & 1.011851 & -0.032353 \\
\hline $\mathrm{H}$ & 2.880885 & 2.851175 & 3.198651 & O & -5.036667 & 0.810318 & -0.315397 \\
\hline $\mathrm{H}$ & 1.795914 & 5.017723 & 2.661856 & $C$ & -2.059675 & 2.503150 & 1.060323 \\
\hline $\mathrm{H}$ & 2.831284 & 1.993810 & -2.226643 & $\mathrm{O}$ & -1.827467 & 3.522656 & 1.864025 \\
\hline $\mathrm{H}$ & 1.449322 & -2.051125 & 2.570298 & C & -5.102562 & -0.291396 & -1.258085 \\
\hline C & 0.262006 & -3.129953 & 1.157047 & $\mathrm{H}$ & -5.917975 & -0.943501 & -0.949476 \\
\hline C & 0.017455 & -3.343671 & -0.199421 & $\mathrm{H}$ & -5.309302 & 0.137866 & -2.240655 \\
\hline C & -0.714649 & -3.471485 & 2.092975 & C & -3.709498 & -0.939276 & -1.154081 \\
\hline C & -1.193175 & -3.889947 & -0.615194 & $\mathrm{C}$ & 0.151567 & 2.798146 & 0.840313 \\
\hline $\mathrm{H}$ & 0.775137 & -3.098505 & -0.940148 & $\mathrm{C}$ & -0.392199 & 3.631531 & 2.016889 \\
\hline C & -1.920050 & -4.030413 & 1.679446 & $\mathrm{H}$ & -0.130779 & 4.687305 & 1.972978 \\
\hline $\mathrm{H}$ & -0.524505 & -3.320251 & 3.153517 & $\mathrm{H}$ & -0.138990 & 3.213323 & 2.994629 \\
\hline C & -2.158129 & -4.241318 & 0.323942 & $\mathrm{C}$ & -3.518194 & 2.170634 & 0.897698 \\
\hline $\mathrm{H}$ & -1.380080 & -4.048221 & -1.671995 & C & -4.235336 & 3.432583 & 0.357573 \\
\hline $\mathrm{H}$ & -2.665781 & -4.312839 & 2.415968 & $\mathrm{H}$ & -4.103489 & 4.258933 & 1.057970 \\
\hline \multirow[t]{2}{*}{$\mathrm{H}$} & -3.090966 & -4.690246 & -0.001233 & $\mathrm{H}$ & -3.836004 & 3.730500 & -0.616801 \\
\hline & & & & $\mathrm{H}$ & -5.301881 & 3.228061 & 0.251767 \\
\hline \multicolumn{2}{|c|}{ TS3-A } & & & $C$ & -4.095110 & 1.772291 & 2.281845 \\
\hline C & 1.977127 & -4.097428 & -1.538893 & $\mathrm{H}$ & -3.950007 & 2.591944 & 2.988257 \\
\hline C & 2.394191 & -3.508746 & -2.780894 & $\mathrm{H}$ & -5.165700 & 1.578084 & 2.188742 \\
\hline C & 1.685452 & -1.528266 & -1.630413 & $\mathrm{H}$ & -3.603168 & 0.874649 & 2.670381 \\
\hline C & 2.278662 & -2.169928 & -2.831584 & $\mathrm{H}$ & 0.967460 & 2.150796 & 1.170185 \\
\hline $\mathrm{H}$ & 2.652186 & -4.127163 & -3.632176 & $\mathrm{H}$ & -3.289921 & -1.145640 & -2.138509 \\
\hline $\mathrm{H}$ & 2.442695 & -1.588178 & -3.730915 & C & 0.612333 & 3.651221 & -0.327473 \\
\hline $\mathrm{H}$ & 1.736737 & -5.157200 & -1.514816 & C & 1.820341 & 4.340861 & -0.193425 \\
\hline O & 0.020609 & -3.340410 & -1.653089 & $\mathrm{C}$ & -0.131233 & 3.801742 & -1.495874 \\
\hline C & 0.109908 & -2.116693 & -1.669815 & C & 2.280055 & 5.166415 & -1.214270 \\
\hline O & -0.805214 & -1.265452 & -1.742951 & $\mathrm{H}$ & 2.408218 & 4.233833 & 0.716452 \\
\hline C & 1.756964 & -0.018018 & -1.642978 & $\mathrm{C}$ & 0.331631 & 4.622945 & -2.523430 \\
\hline O & 0.937754 & 0.729264 & -1.109519 & $\mathrm{H}$ & -1.075268 & 3.277814 & -1.616432 \\
\hline O & 2.830226 & 0.423355 & -2.225096 & C & 1.536800 & 5.305843 & -2.385851 \\
\hline C & 3.074610 & 1.853255 & -2.211431 & $\mathrm{H}$ & 3.215256 & 5.704136 & -1.094773 \\
\hline $\mathrm{H}$ & 3.948796 & 1.992101 & -2.842244 & $\mathrm{H}$ & -0.256829 & 4.736611 & -3.428470 \\
\hline $\mathrm{H}$ & 2.207300 & 2.386155 & -2.603097 & $\mathrm{H}$ & 1.890855 & 5.952579 & -3.181937 \\
\hline $\mathrm{H}$ & 3.275532 & 2.163129 & -1.186126 & C & -3.668493 & -2.187193 & -0.299071 \\
\hline $\mathrm{Cu}$ & -0.951439 & 0.295472 & -0.542691 & C & -3.089822 & -3.356600 & -0.788363 \\
\hline$N$ & -2.927096 & 0.157831 & -0.513833 & C & -4.221361 & -2.181924 & 0.9870 \\
\hline
\end{tabular}




\begin{tabular}{|c|c|c|c|c|c|c|c|}
\hline C & -3.051034 & -4.504829 & 0.000369 & C & -1.527752 & 4.662792 & -1.967580 \\
\hline $\mathrm{H}$ & -2.662749 & -3.372454 & -1.785777 & C & -1.449147 & 2.374094 & -1.266650 \\
\hline C & -4.185587 & -3.327680 & 1.773605 & C & -2.021607 & 3.426257 & -2.143718 \\
\hline $\mathrm{H}$ & -4.697049 & -1.284861 & 1.378669 & $\mathrm{H}$ & -1.734047 & 5.498224 & -2.625612 \\
\hline C & -3.595109 & -4.491997 & 1.281087 & $\mathrm{H}$ & -2.678786 & 3.153883 & -2.959948 \\
\hline $\mathrm{H}$ & -2.604083 & -5.411781 & -0.393744 & $\mathrm{H}$ & 0.117344 & 5.627767 & -0.909398 \\
\hline $\mathrm{H}$ & -4.626968 & -3.318146 & 2.765058 & $\mathrm{O}$ & 0.747358 & 3.387272 & -1.738316 \\
\hline $\mathrm{H}$ & -3.575452 & -5.389402 & 1.891134 & $C$ & 0.161471 & 2.306435 & -1.709325 \\
\hline C & 2.293895 & -3.439612 & -0.290019 & $\mathrm{O}$ & 0.647060 & 1.172125 & -1.938116 \\
\hline C & 2.175848 & -2.090247 & -0.320906 & C & -2.091789 & 1.015609 & -1.419274 \\
\hline C & 2.349137 & -1.207945 & 0.860931 & $\mathrm{O}$ & -1.572976 & -0.030558 & -1.018787 \\
\hline C & 3.656179 & -0.790806 & 1.263381 & $\mathrm{O}$ & -3.249800 & 1.032793 & -1.999417 \\
\hline C & 1.250333 & -0.775893 & 1.565472 & C & -3.942327 & -0.229295 & -2.171633 \\
\hline C & 3.783732 & 0.036663 & 2.416961 & $\mathrm{H}$ & -3.315129 & -0.922703 & -2.732482 \\
\hline C & 4.826852 & -1.163215 & 0.552987 & $\mathrm{H}$ & -4.172231 & -0.649387 & -1.192716 \\
\hline C & 1.371939 & 0.035330 & 2.712190 & $\mathrm{H}$ & -4.846190 & 0.021942 & -2.720491 \\
\hline C & 5.076324 & 0.452146 & 2.829585 & $\mathrm{Cu}$ & 0.376243 & -0.429459 & -0.828456 \\
\hline C & 2.618380 & 0.426030 & 3.128292 & $\mathrm{~N}$ & 2.226340 & -1.036977 & -1.113400 \\
\hline C & 6.061385 & -0.739748 & 0.976604 & $\mathrm{~N}$ & -0.023080 & -2.002380 & 0.262448 \\
\hline $\mathrm{H}$ & 4.742698 & -1.788925 & -0.330719 & C & 2.701672 & -2.200815 & -0.826005 \\
\hline $\mathrm{H}$ & 0.474154 & 0.330603 & 3.246972 & $\mathrm{O}$ & 3.855913 & -2.512994 & -1.384810 \\
\hline C & 6.190424 & 0.072367 & 2.126953 & C & 0.799905 & -2.919418 & 0.645658 \\
\hline $\mathrm{H}$ & 5.169536 & 1.073249 & 3.715819 & O & 0.352423 & -3.757399 & 1.557396 \\
\hline $\mathrm{H}$ & 2.728703 & 1.042466 & 4.016032 & C & 4.170897 & -1.452223 & -2.328708 \\
\hline $\mathrm{H}$ & 6.950382 & -1.032923 & 0.427593 & $\mathrm{H}$ & 5.240512 & -1.259938 & -2.269695 \\
\hline $\mathrm{H}$ & 7.175341 & 0.390821 & 2.451459 & $\mathrm{H}$ & 3.904010 & -1.817437 & -3.323092 \\
\hline C & 2.612327 & -4.265390 & 0.922444 & C & 3.290366 & -0.289726 & -1.853444 \\
\hline $\mathrm{H}$ & 1.811376 & -4.988986 & 1.113067 & C & -1.332732 & -2.262750 & 0.894865 \\
\hline $\mathrm{H}$ & 3.538113 & -4.831636 & 0.778266 & C & -0.942887 & -3.275840 & 1.990062 \\
\hline $\mathrm{H}$ & 2.734576 & -3.636761 & 1.806553 & $\mathrm{H}$ & -1.610287 & -4.133001 & 2.060302 \\
\hline O & -0.011446 & -1.104690 & 1.101310 & $\mathrm{H}$ & -0.801458 & -2.812105 & 2.968835 \\
\hline C & -0.632826 & -2.208271 & 1.781882 & C & 2.188564 & -3.222478 & 0.152504 \\
\hline $\mathrm{H}$ & -0.738424 & -1.984970 & 2.847405 & C & 2.160429 & -4.622626 & -0.507697 \\
\hline $\mathrm{H}$ & -1.610140 & -2.342251 & 1.324006 & $\mathrm{H}$ & 1.842323 & -5.368194 & 0.223234 \\
\hline \multirow[t]{2}{*}{$\mathrm{H}$} & -0.030063 & -3.111273 & 1.647787 & $\mathrm{H}$ & 1.473185 & -4.648130 & -1.359054 \\
\hline & & & & $\mathrm{H}$ & 3.161448 & -4.879479 & -0.858443 \\
\hline \multicolumn{2}{|c|}{ TS3-B } & & & C & 3.165066 & -3.209243 & 1.357618 \\
\hline$C$ & -0.550268 & 4.769998 & -0.918634 & $\mathrm{H}$ & 2.830566 & -3.930017 & 2.106102 \\
\hline
\end{tabular}




\begin{tabular}{|c|c|c|c|c|c|c|c|}
\hline $\mathrm{H}$ & 4.164348 & -3.492532 & 1.020845 & $\mathrm{H}$ & -4.018599 & 0.522468 & 3.116019 \\
\hline $\mathrm{H}$ & 3.207666 & -2.214730 & 1.811973 & $C$ & 1.763882 & -0.120334 & 3.258102 \\
\hline $\mathrm{H}$ & -1.701788 & -1.338572 & 1.340149 & $\mathrm{H}$ & 0.265546 & -0.949311 & 4.539358 \\
\hline $\mathrm{H}$ & 2.837885 & 0.243283 & -2.688932 & $\mathrm{H}$ & -2.146621 & -0.492412 & 4.317413 \\
\hline C & -2.369206 & -2.800386 & -0.071321 & $\mathrm{H}$ & 3.046580 & 0.924003 & 1.855639 \\
\hline C & -3.702821 & -2.803934 & 0.346879 & $\mathrm{H}$ & 2.586545 & -0.608916 & 3.770450 \\
\hline C & -2.043148 & -3.332269 & -1.316383 & $C$ & -0.285371 & 4.661857 & 1.611254 \\
\hline C & -4.697158 & -3.335026 & -0.468799 & $\mathrm{H}$ & 0.810815 & 4.686245 & 1.643403 \\
\hline $\mathrm{H}$ & -3.964078 & -2.393057 & 1.320492 & $\mathrm{H}$ & -0.645364 & 5.689707 & 1.713249 \\
\hline C & -3.039955 & -3.854120 & -2.139984 & $\mathrm{H}$ & -0.638593 & 4.086842 & 2.468860 \\
\hline $\mathrm{H}$ & -1.010752 & -3.341220 & -1.654916 & $\mathrm{O}$ & -3.718237 & 2.110363 & 0.916525 \\
\hline C & -4.367043 & -3.856842 & -1.719174 & $C$ & -5.083243 & 1.914624 & 1.267890 \\
\hline $\mathrm{H}$ & -5.728165 & -3.345657 & -0.129359 & $\mathrm{H}$ & -5.293166 & 2.312964 & 2.265350 \\
\hline $\mathrm{H}$ & -2.776533 & -4.270695 & -3.107215 & $\mathrm{H}$ & -5.658643 & 2.469920 & 0.528651 \\
\hline $\mathrm{H}$ & -5.139577 & -4.275549 & -2.355822 & $\mathrm{H}$ & -5.354556 & 0.853764 & 1.222248 \\
\hline C & 3.979213 & 0.681250 & -0.914926 & & & & \\
\hline C & 3.684954 & 2.042481 & -0.979886 & \multicolumn{2}{|c|}{ TS3-C } & & \\
\hline C & 4.904305 & 0.228035 & 0.032841 & C & -2.412569 & 3.711995 & -1.196465 \\
\hline C & 4.292943 & 2.937634 & -0.101744 & $C$ & -1.704579 & 4.077028 & 0.003272 \\
\hline $\mathrm{H}$ & 2.987298 & 2.410934 & -1.724607 & C & -1.405276 & 1.703273 & 0.087550 \\
\hline C & 5.522304 & 1.123123 & 0.900057 & $C$ & -1.209212 & 3.046683 & 0.707715 \\
\hline $\mathrm{H}$ & 5.161653 & -0.826733 & 0.096198 & $\mathrm{H}$ & -1.494850 & 5.115071 & 0.229881 \\
\hline C & 5.212038 & 2.481276 & 0.838874 & $\mathrm{H}$ & -0.544358 & 3.167564 & 1.556343 \\
\hline $\mathrm{H}$ & 4.064712 & 3.996665 & -0.170802 & $\mathrm{H}$ & -2.551182 & 4.462484 & -1.970527 \\
\hline $\mathrm{H}$ & 6.251921 & 0.762934 & 1.618287 & $\mathrm{O}$ & -0.736110 & 2.702230 & -2.040041 \\
\hline $\mathrm{H}$ & 5.698663 & 3.180446 & 1.511128 & $C$ & -0.417812 & 1.834349 & -1.235724 \\
\hline C & -0.750790 & 4.047884 & 0.322899 & $\mathrm{O}$ & 0.597290 & 1.094843 & -1.305209 \\
\hline C & -1.259845 & 2.800710 & 0.179333 & $C$ & -1.115131 & 0.572610 & 1.055189 \\
\hline C & -1.450904 & 1.838861 & 1.287584 & $\mathrm{O}$ & -0.241737 & -0.296002 & 0.963825 \\
\hline C & -0.350975 & 1.218214 & 1.947914 & O & -1.948189 & 0.609486 & 2.050948 \\
\hline C & -2.751612 & 1.545661 & 1.668864 & $C$ & -1.997883 & -0.509990 & 2.959763 \\
\hline C & -0.609838 & 0.344645 & 3.043677 & $\mathrm{H}$ & -1.043529 & -0.614421 & 3.477914 \\
\hline C & 0.998793 & 1.405435 & 1.538392 & $\mathrm{H}$ & -2.238991 & -1.410283 & 2.391602 \\
\hline C & -3.004191 & 0.704097 & 2.781387 & $\mathrm{H}$ & -2.798831 & -0.269238 & 3.654112 \\
\hline C & 0.473447 & -0.308242 & 3.686150 & $\mathrm{Cu}$ & 1.427318 & -0.139506 & -0.116381 \\
\hline C & -1.952118 & 0.138491 & 3.454086 & $\mathrm{~N}$ & 3.196083 & 0.606027 & -0.566912 \\
\hline C & 2.025197 & 0.749364 & 2.175839 & $N$ & 2.273409 & -1.670157 & 0.755972 \\
\hline $\mathrm{H}$ & 1.241433 & 2.105203 & 0.743427 & C & 4.362825 & 0.143098 & -0.279957 \\
\hline
\end{tabular}




\begin{tabular}{|c|c|c|c|c|c|c|c|}
\hline 0 & 5.386304 & 0.922633 & -0.557807 & C & -4.498309 & 2.538289 & -2.064612 \\
\hline c & 3.531397 & -1.946173 & 0.846137 & $\mathrm{H}$ & -4.242353 & 2.789486 & -3.099914 \\
\hline $\mathrm{O}$ & 3.836490 & -3.070621 & 1.459884 & $\mathrm{H}$ & -5.228685 & 3.273884 & -1.712747 \\
\hline C & 4.874000 & 2.121690 & -1.196519 & $\mathrm{H}$ & -4.970628 & 1.554734 & -2.052701 \\
\hline $\mathrm{H}$ & 5.223670 & 2.110984 & -2.229689 & O & -1.730748 & -0.491487 & -1.972894 \\
\hline $\mathrm{H}$ & 5.287581 & 2.978041 & -0.664955 & C & -1.042132 & -1.467141 & -2.738057 \\
\hline C & 3.335687 & 1.988570 & -1.066734 & $\mathrm{H}$ & -0.703720 & -2.296543 & -2.108016 \\
\hline C & 1.497169 & -2.802377 & 1.313220 & $\mathrm{H}$ & -0.183161 & -0.947166 & -3.163545 \\
\hline C & 2.610891 & -3.654097 & 1.967137 & $\mathrm{H}$ & -1.669810 & -1.848939 & -3.549950 \\
\hline $\mathrm{H}$ & 2.641530 & -3.572259 & 3.054904 & C & 2.706590 & 2.993543 & -0.121216 \\
\hline $\mathrm{H}$ & 2.593712 & -4.700799 & 1.666303 & C & 2.156796 & 4.166911 & -0.640924 \\
\hline C & 4.726414 & -1.190566 & 0.318539 & C & 2.730213 & 2.805611 & 1.263548 \\
\hline C & 5.728813 & -0.968385 & 1.476579 & C & 1.666128 & 5.151564 & 0.212199 \\
\hline $\mathrm{H}$ & 6.027288 & -1.932054 & 1.892188 & $\mathrm{H}$ & 2.120775 & 4.317411 & -1.716545 \\
\hline $\mathrm{H}$ & 5.289081 & -0.361893 & 2.274500 & C & 2.224407 & 3.784694 & 2.117427 \\
\hline $\mathrm{H}$ & 6.616865 & -0.458625 & 1.100130 & $\mathrm{H}$ & 3.169090 & 1.905395 & 1.688303 \\
\hline C & 5.378108 & -2.050610 & -0.797990 & C & 1.699960 & 4.964506 & 1.592823 \\
\hline $\mathrm{H}$ & 5.684399 & -3.014766 & -0.387079 & $\mathrm{H}$ & 1.268329 & 6.072210 & -0.203561 \\
\hline $\mathrm{H}$ & 6.263470 & -1.537329 & -1.179534 & $\mathrm{H}$ & 2.267722 & 3.638730 & 3.192444 \\
\hline $\mathrm{H}$ & 4.683179 & -2.218318 & -1.626911 & $\mathrm{H}$ & 1.333684 & 5.739971 & 2.258358 \\
\hline C & -3.264896 & 2.551648 & -1.210777 & $\mathrm{H}$ & 2.849991 & 2.056161 & -2.041797 \\
\hline C & -2.772852 & 1.494580 & -0.518943 & $\mathrm{H}$ & 0.806484 & -2.413861 & 2.064210 \\
\hline C & -3.387188 & 0.147696 & -0.486982 & C & 0.724117 & -3.535525 & 0.238737 \\
\hline C & -4.533533 & -0.117930 & 0.321060 & C & 1.357263 & -3.943983 & -0.940069 \\
\hline C & -2.825706 & -0.852665 & -1.261386 & C & -0.602728 & -3.899381 & 0.462805 \\
\hline C & -5.095164 & -1.425532 & 0.297149 & C & 0.675414 & -4.718744 & -1.872473 \\
\hline C & -5.121259 & 0.860455 & 1.165632 & $\mathrm{H}$ & 2.395623 & -3.678344 & -1.126587 \\
\hline C & -3.396167 & -2.145878 & -1.294244 & C & -1.282140 & -4.686773 & -0.464078 \\
\hline C & -6.230039 & -1.710339 & 1.100683 & $\mathrm{H}$ & -1.102889 & -3.590876 & 1.377211 \\
\hline C & -4.503718 & -2.411834 & -0.531961 & C & -0.644903 & -5.098399 & -1.631147 \\
\hline C & -6.216233 & 0.551653 & 1.933351 & $\mathrm{H}$ & 1.177431 & -5.041395 & -2.778876 \\
\hline $\mathrm{H}$ & -4.697534 & 1.859510 & 1.204151 & $\mathrm{H}$ & -2.307764 & -4.982327 & -0.268378 \\
\hline H & -2.970392 & -2.917129 & -1.923634 & $\mathrm{H}$ & -1.169909 & -5.721023 & -2.348481 \\
\hline C & -6.781396 & -0.744357 & 1.901425 & & & & \\
\hline $\mathrm{H}$ & -6.657746 & -2.708443 & 1.067196 & \multicolumn{4}{|c|}{ TS3-D } \\
\hline $\mathrm{H}$ & -4.950978 & -3.401642 & -0.564798 & C & 2.193778 & 3.891056 & 0.835243 \\
\hline $\mathrm{H}$ & -6.658450 & 1.310973 & 2.570718 & C & 1.443795 & 4.191338 & -0.353242 \\
\hline $\mathrm{H}$ & -7.651403 & -0.967613 & 2.509637 & C & 1.265244 & 1.800185 & -0.388962 \\
\hline
\end{tabular}




\begin{tabular}{|c|c|c|c|c|c|c|c|}
\hline C & 0.984364 & 3.120426 & -1.020906 & $C$ & 3.093947 & 2.766366 & 0.863948 \\
\hline $\mathrm{H}$ & 1.180002 & 5.212102 & -0.600868 & C & 2.658873 & 1.669672 & 0.195061 \\
\hline $\mathrm{H}$ & 0.298777 & 3.189514 & -1.858387 & $C$ & 3.381921 & 0.372852 & 0.202366 \\
\hline $\mathrm{H}$ & 2.324997 & 4.671427 & 1.580752 & C & 3.330207 & -0.502344 & 1.331765 \\
\hline $\mathrm{O}$ & 0.605885 & 2.825914 & 1.747045 & C & 4.251516 & 0.108620 & -0.848362 \\
\hline C & 0.342816 & 1.894939 & 0.992053 & C & 4.150068 & -1.665175 & 1.336269 \\
\hline O & -0.529315 & 1.013931 & 1.189358 & C & 2.529562 & -0.243522 & 2.476684 \\
\hline C & 0.924084 & 0.657249 & -1.331547 & $C$ & 5.033781 & -1.072878 & -0.851708 \\
\hline O & 0.082411 & -0.231124 & -1.171667 & C & 4.142922 & -2.525330 & 2.464431 \\
\hline $\mathrm{O}$ & 1.632059 & 0.729327 & -2.415349 & C & 4.977519 & -1.926413 & 0.216775 \\
\hline C & 1.546709 & -0.355376 & -3.361695 & $C$ & 2.542014 & -1.096177 & 3.552675 \\
\hline $\mathrm{H}$ & 0.528655 & -0.435941 & -3.746253 & $\mathrm{H}$ & 1.933648 & 0.661155 & 2.531553 \\
\hline $\mathrm{H}$ & 1.844360 & -1.281623 & -2.866507 & $\mathrm{H}$ & 5.702110 & -1.287483 & -1.676731 \\
\hline $\mathrm{H}$ & 2.241047 & -0.088175 & -4.154493 & $C$ & 3.354024 & -2.253184 & 3.552005 \\
\hline $\mathrm{Cu}$ & -1.443324 & -0.256503 & 0.088717 & $\mathrm{H}$ & 4.789117 & -3.399290 & 2.458063 \\
\hline$N$ & -3.167156 & 0.336197 & 0.829356 & $\mathrm{H}$ & 5.599819 & -2.817210 & 0.221815 \\
\hline N & -2.304435 & -1.850103 & -0.667727 & $\mathrm{H}$ & 1.937554 & -0.867055 & 4.424919 \\
\hline C & -4.310909 & -0.252158 & 0.785537 & $\mathrm{H}$ & 3.365520 & -2.907264 & 4.417585 \\
\hline O & -5.347924 & 0.452522 & 1.183702 & $C$ & 4.327451 & 2.830871 & 1.716427 \\
\hline C & -3.493962 & -2.292297 & -0.426665 & $\mathrm{H}$ & 4.050400 & 2.814131 & 2.777310 \\
\hline $\mathrm{O}$ & -3.792215 & -3.470054 & -0.931305 & $\mathrm{H}$ & 4.890763 & 3.749716 & 1.530152 \\
\hline C & -4.871695 & 1.763021 & 1.589744 & $\mathrm{H}$ & 4.980170 & 1.978466 & 1.522734 \\
\hline $\mathrm{H}$ & -5.093292 & 1.872826 & 2.651491 & $\mathrm{O}$ & 4.321610 & 1.045813 & -1.817307 \\
\hline $\mathrm{H}$ & -5.422238 & 2.503685 & 1.009701 & $C$ & 5.262708 & 0.888701 & -2.868272 \\
\hline C & -3.353211 & 1.730430 & 1.275236 & $\mathrm{H}$ & 6.285381 & 0.831669 & -2.481064 \\
\hline C & -1.590177 & -2.837325 & -1.507531 & $\mathrm{H}$ & 5.161452 & 1.778441 & -3.488905 \\
\hline C & -2.606231 & -4.002944 & -1.571024 & $\mathrm{H}$ & 5.046475 & -0.001770 & -3.470464 \\
\hline $\mathrm{H}$ & -2.882457 & -4.298923 & -2.582109 & C & -2.913737 & 2.721659 & 0.215610 \\
\hline $\mathrm{H}$ & -2.288049 & -4.873964 & -0.995484 & C & -2.393811 & 3.956666 & 0.607260 \\
\hline C & -4.609118 & -1.671755 & 0.380921 & $C$ & -3.069438 & 2.446856 & -1.146313 \\
\hline C & -5.912909 & -1.711803 & -0.449202 & C & -2.058868 & 4.913060 & -0.347332 \\
\hline $\mathrm{H}$ & -6.149556 & -2.744020 & -0.710846 & $\mathrm{H}$ & -2.256333 & 4.175688 & 1.662629 \\
\hline $\mathrm{H}$ & -5.819799 & -1.127805 & -1.369747 & C & -2.717385 & 3.398107 & -2.102585 \\
\hline $\mathrm{H}$ & -6.734391 & -1.302679 & 0.140520 & $\mathrm{H}$ & -3.493481 & 1.498641 & -1.470189 \\
\hline C & -4.775415 & -2.503709 & 1.682282 & C & -2.219774 & 4.637161 & -1.703809 \\
\hline $\mathrm{H}$ & -5.028194 & -3.534891 & 1.426604 & $\mathrm{H}$ & -1.682352 & 5.880165 & -0.028756 \\
\hline $\mathrm{H}$ & -5.589055 & -2.083935 & 2.278429 & $\mathrm{H}$ & -2.861592 & 3.182807 & -3.156936 \\
\hline $\mathrm{H}$ & -3.858331 & -2.496736 & 2.279420 & $\mathrm{H}$ & -1.974315 & 5.389467 & -2.446973 \\
\hline
\end{tabular}




$\begin{array}{lrrr}\text { H } & -2.760332 & 1.883847 & 2.179357 \\ \text { H } & -1.447966 & -2.388543 & -2.495893 \\ \mathrm{C} & -0.248653 & -3.240325 & -0.944129 \\ \mathrm{C} & -0.022079 & -3.281018 & 0.432404 \\ \mathrm{C} & 0.762857 & -3.634426 & -1.819887 \\ \mathrm{C} & 1.210096 & -3.698451 & 0.926852 \\ \mathrm{H} & -0.810210 & -3.002864 & 1.129698 \\ \mathrm{C} & 1.988793 & -4.069841 & -1.325303 \\ \mathrm{H} & 0.587187 & -3.617667 & -2.893564 \\ \mathrm{C} & 2.211983 & -4.100160 & 0.048510 \\ \mathrm{H} & 1.389584 & -3.711580 & 1.996548 \\ \mathrm{H} & 2.765685 & -4.392009 & -2.011420 \\ \mathrm{H} & 3.164726 & -4.442802 & 0.436776\end{array}$




\section{Gram-scale synthesis of 3w}

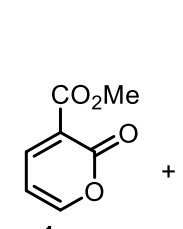

1a<smiles>COc1ccc2ccccc2c1C#CBr</smiles>

2I

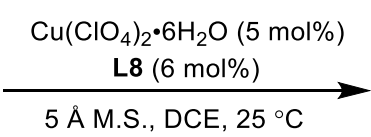

5 Å M.S., DCE, $25^{\circ} \mathrm{C}$

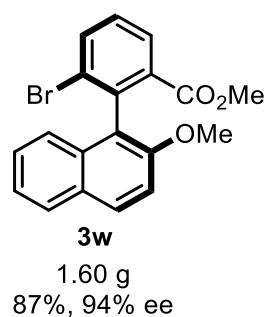

A mixture of $\mathrm{Cu}\left(\mathrm{ClO}_{4}\right)_{2} \bullet 6 \mathrm{H}_{2} \mathrm{O}(92.6 \mathrm{mg}, 0.25 \mathrm{mmol}), 5 \AA$ M.S. (1.25 g) and $\mathbf{L 8}$ (227.5 mg, $0.30 \mathrm{mmol})$ in DCE $(25.0 \mathrm{~mL})$ was stirred at $25^{\circ} \mathrm{C}$ for $2 \mathrm{~h}$. Then, 2-pyrone $1 \mathrm{a}(0.77 \mathrm{~g}, 5.0 \mathrm{mmol})$ was added. After stirred at $25^{\circ} \mathrm{C}$ for $20 \mathrm{~min}$, alkyne $2 \mathrm{l}(1.95 \mathrm{~g}$, $7.5 \mathrm{mmol}$ ) was added in one portion. After the reaction mixture was stirred at this temperature for $72 \mathrm{~h}$, the crude mixture was filtrated through celite and washed with dichloromethane. The filtrate was concentrated under reduced pressure. The residue was purified by flash column chromatography (silica gel, ethyl acetate/petroleum ether $1: 20)$ to afford $3 \mathbf{w}(1.60 \mathrm{~g}, 87 \%, 94 \%$ ee $)$. 


\section{Synthetic transformations}

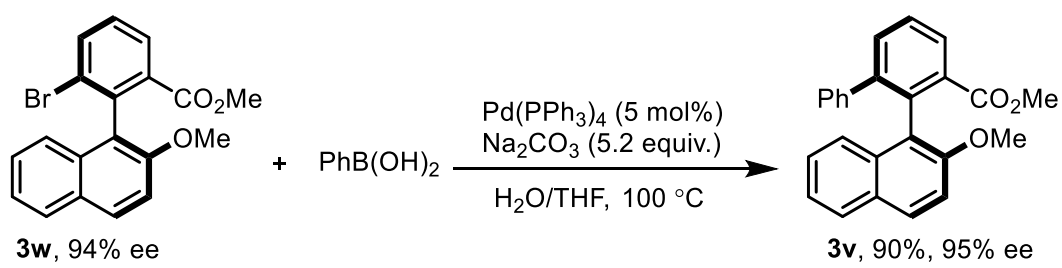

To a stirred solution of $\mathbf{3 w}(37.0 \mathrm{mg}, 0.1 \mathrm{mmol}), \mathrm{Pd}\left(\mathrm{PPh}_{3}\right)_{4}(5.8 \mathrm{mg}, 0.005 \mathrm{mmol})$ and $\mathrm{PhB}(\mathrm{OH})_{2}(24.4 \mathrm{mg}, 0.2 \mathrm{mmol})$ in THF $(1.0 \mathrm{~mL})$ was added $\mathrm{Na}_{2} \mathrm{CO}_{3}$ (aq., $2.0 \mathrm{M}$, $0.26 \mathrm{~mL}$ ). The mixture was stirred at $100{ }^{\circ} \mathrm{C}$ for $24 \mathrm{~h}$. Then, the mixture was quenched with saturated $\mathrm{NH}_{4} \mathrm{Cl}$ aqueous $(3.0 \mathrm{~mL})$. The aqueous layer was extracted with ethyl acetate $(3.0 \mathrm{~mL} \times 3)$. The combined organic layers were washed with brine $(3.0 \mathrm{~mL})$, dried over $\mathrm{Na}_{2} \mathrm{SO}_{4}$, and concentrated under reduced pressure. The residue was purified by flash column chromatography (silica gel, ethyl acetate/petroleum ether 1:20) to afford $3 \mathbf{v}$ as colorless oil $(33.1 \mathrm{mg}, 90 \%, 95 \%$ ee $)$

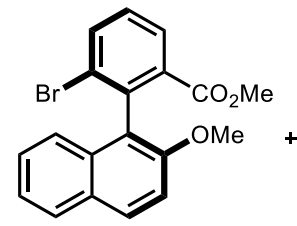

3 w, $94 \%$ ee

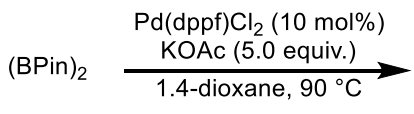

1.4-dioxane, $90^{\circ} \mathrm{C}$

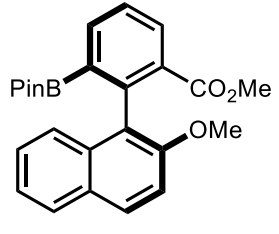

$4,43 \%, 95 \%$ ee

To a stirred solution of $\mathbf{3 w}(37.0 \mathrm{mg}, 0.1 \mathrm{mmol}), \mathrm{Pd}(\mathrm{dppf}) \mathrm{Cl}_{2}(7.3 \mathrm{mg}, 0.01 \mathrm{mmol})$ and (BPin) 2 (102 mg, $0.4 \mathrm{mmol})$ in 1,4-dioxane $(0.5 \mathrm{~mL})$ was added KOAc $(49.1 \mathrm{mg}$, $0.5 \mathrm{mmol}$ ). The mixture was stirred at $90{ }^{\circ} \mathrm{C}$ for $24 \mathrm{~h}$. Then, the mixture was quenched with $\mathrm{H}_{2} \mathrm{O}(3.0 \mathrm{~mL})$. The aqueous layer was extracted with ethyl acetate $(3.0 \mathrm{~mL} \times 3)$. The combined organic layers were washed with brine $(3.0 \mathrm{~mL})$, dried over $\mathrm{Na}_{2} \mathrm{SO}_{4}$, and concentrated under reduced pressure. The residue was purified by flash column chromatography (silica gel, $100 \mathrm{~mL}$ dichloromethane/petroleum ether 2:3, $200 \mathrm{~mL}$ ethyl acetate/petroleum ether 1:20) to afford 4 as colorless oil (18.0 mg, 43\%, 95\% ee) 
<smiles>COc1cccc(PC)c1-c1c(OC)ccc2ccccc12</smiles>

The enantiomeric excess was determined by CHIRALCEL OD-H column, hexanes $/ i-\mathrm{PrOH}, 97: 3 \mathrm{v} / \mathrm{v}, v=0.3 \mathrm{~mL} / \mathrm{min}, \lambda=254 \mathrm{~nm}$, $25^{\circ} \mathrm{C}, \mathrm{t}_{\mathrm{R}}($ major $)=24.8 \mathrm{~min}, \mathrm{t}_{\mathrm{R}}($ minor $)=23.0 \mathrm{~min} ;[\alpha]_{\mathrm{D}}{ }^{24}=+117.26$ $\left(c=1.0, \mathrm{CHCl}_{3}\right)$.

4: ${ }^{1} \mathrm{H}$ NMR (400 MHz, $\left.\mathrm{CHCl}_{3}\right) \delta 8.09(\mathrm{dd}, J=7.6,1.2 \mathrm{~Hz}, 1 \mathrm{H}), 7.93(\mathrm{dd}, J=7.2,1.2$ $\mathrm{Hz}, 1 \mathrm{H}), 7.84(\mathrm{~d}, J=9.2 \mathrm{~Hz}, 1 \mathrm{H}), 7.78-7.74(\mathrm{~m}, 1 \mathrm{H}), 7.48$ (t, $J=7.6 \mathrm{~Hz}, 1 \mathrm{H}), 7.30$ (d, $J=9.2 \mathrm{~Hz}, 1 \mathrm{H}), 7.27-7.21(\mathrm{~m}, 2 \mathrm{H}), 7.15-7.11(\mathrm{~m}, 1 \mathrm{H}), 3.79(\mathrm{~s}, 3 \mathrm{H}), 3.45(\mathrm{~s}, 3 \mathrm{H})$, 0.85 (s, 6H), 0.67 (s, 6H); ${ }^{13} \mathrm{C}$ NMR (101 MHz, $\left.\mathrm{CHCl}_{3}\right) \delta 168.0,153.8,142.6,137.8$, 134.3, 132.1, 131.8, 129.1, 128.6, 127.7, 126.8, 126.1, 125.6, 125.0, 123.1, 113.7, 83.4, 57.0, 51.8, 24.5, 24.1; FT-IR (neat): $v_{\max }=2977,2945,2839,1731,1593,1510,1461$, 1357, 1313, 1287, 1271, 1252, 1202, 1138, 1108, 1080, 1064, 852, 805, 747, 682, 667 $\mathrm{cm}^{-1}$; HRMS (ESI): exact mass calculated for: $\mathrm{C}_{25} \mathrm{H}_{27} \mathrm{BO}_{5} \mathrm{Na}[\mathrm{M}+\mathrm{Na}]^{+}$: 441.1844 , found 441.8706 .

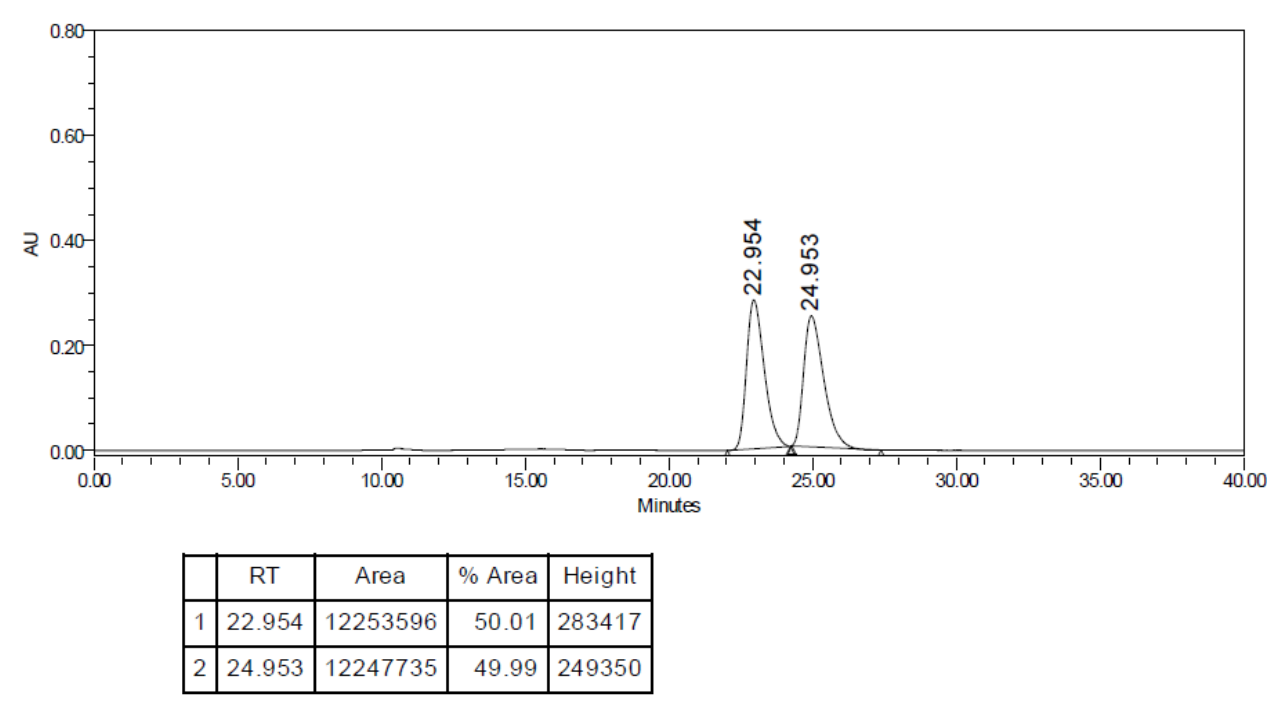




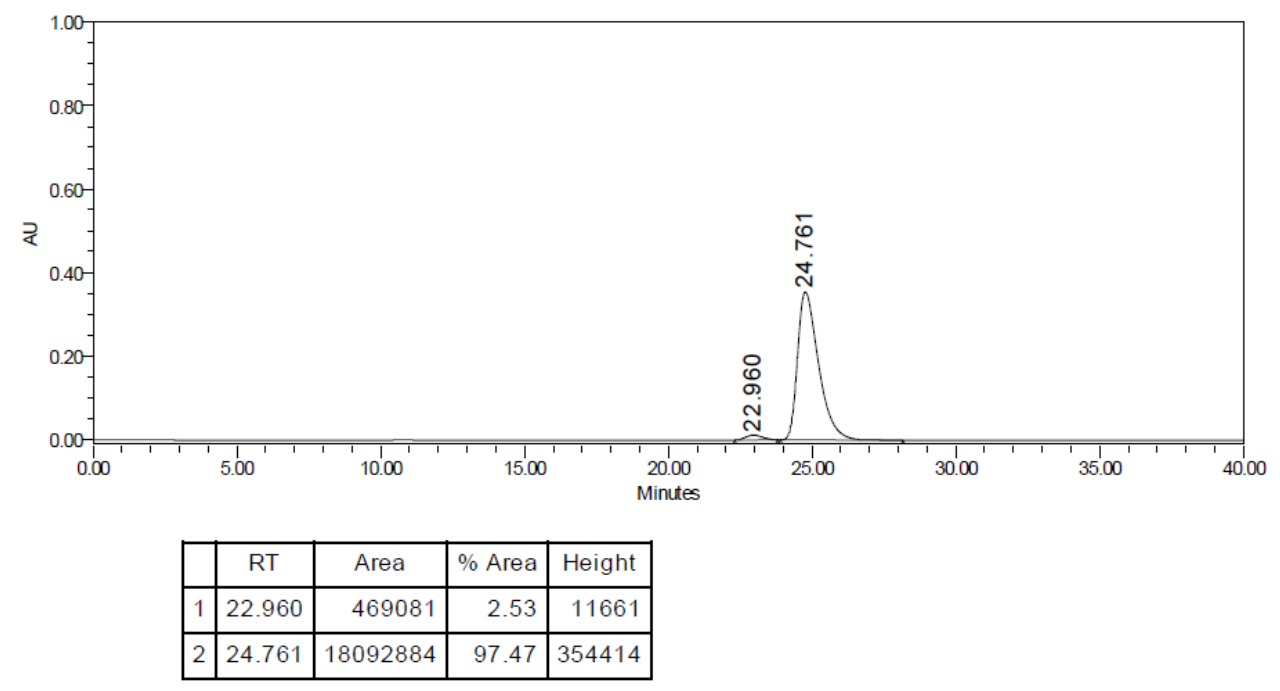

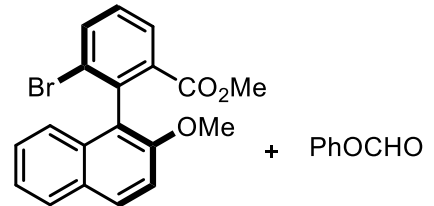

$3 w, 94 \%$ ee

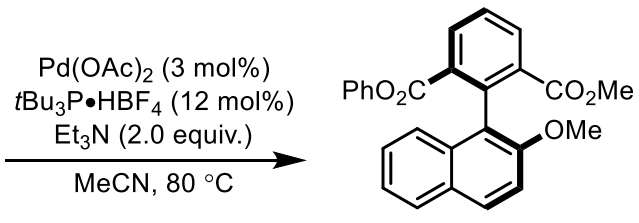

$\mathbf{5}, 83 \%, 95 \%$ ee

To a stirred solution of $\mathbf{3 w}(37.0 \mathrm{mg}, 0.1 \mathrm{mmol}), \mathrm{Pd}(\mathrm{OAc})_{2}(0.7 \mathrm{mg}, 0.003 \mathrm{mmol})$ $t \mathrm{Bu}_{3} \mathrm{P} \cdot \mathrm{HBF}_{4}(3.5 \mathrm{mg}, 0.012 \mathrm{mmol})$ and phenyl formate $(24.4 \mathrm{mg}, 0.2 \mathrm{mmol})$ in $\mathrm{MeCN}$ $(0.16 \mathrm{~mL})$ was added triethylamine $(28 \mu \mathrm{L})$. The mixture was stirred at $80{ }^{\circ} \mathrm{C}$ for $33 \mathrm{~h}$. Then, the mixture was quenched with $\mathrm{H}_{2} \mathrm{O}(3.0 \mathrm{~mL})$. The aqueous layer was extracted with ethyl acetate $(3.0 \mathrm{~mL} \times 3)$. The combined organic layers were washed with brine (3.0 mL), dried over $\mathrm{Na}_{2} \mathrm{SO}_{4}$, and concentrated under reduced pressure. The residue was purified by flash column chromatography (silica gel, ethyl acetate/petroleum ether $1: 20)$ to afford $\mathbf{5}$ as colorless oil $(34.3 \mathrm{mg}, 83 \%, 95 \%$ ee $)$<smiles></smiles>

The enantiomeric excess was determined by CHIRALPAK AD-H column, hexanes $/ i-\mathrm{PrOH}, 90: 10 \mathrm{v} / \mathrm{v}, v=1.0 \mathrm{~mL} / \mathrm{min}, \lambda=254 \mathrm{~nm}$, $25^{\circ} \mathrm{C}, \mathrm{t}_{\mathrm{R}}($ major $)=15.3 \mathrm{~min}, \mathrm{t}_{\mathrm{R}}($ minor $)=17.6 \mathrm{~min} ;[\alpha]_{\mathrm{D}}{ }^{20}=+63.54$ $\left(c=1.0, \mathrm{CHCl}_{3}\right)$.

5: ${ }^{1} \mathrm{H}$ NMR (400 MHz, $\left.\mathrm{CHCl}_{3}\right) \delta 8.23(\mathrm{dd}, J=7.6,1.2 \mathrm{~Hz}, 1 \mathrm{H}), 8.17(\mathrm{dd}, J=7.6,1.2$ Hz, 1H), $7.86(\mathrm{~d}, J=9.2 \mathrm{~Hz}, 1 \mathrm{H}), 7.81-7.78(\mathrm{~m}, 1 \mathrm{H}), 7.63(\mathrm{t}, J=7.6 \mathrm{~Hz}, 1 \mathrm{H}), 7.33-$ $7.27(\mathrm{~m}, 3 \mathrm{H}), 7.25-7.23(\mathrm{~m}, 1 \mathrm{H}), 7.15-7.10(\mathrm{~m}, 2 \mathrm{H}), 7.04(\mathrm{tt}, J=7.2,1.2 \mathrm{~Hz}, 1 \mathrm{H})$, $6.39-6.36(\mathrm{~m}, 2 \mathrm{H}), 3.80(\mathrm{~s}, 3 \mathrm{H}), 3.39(\mathrm{~s}, 3 \mathrm{H}) ;{ }^{13} \mathrm{C} \mathrm{NMR}\left(101 \mathrm{MHz}, \mathrm{CHCl}_{3}\right) \delta 67.4$, 
$166.1,153.6,150.5,137.4,134.0,133.8,133.5,133.4,129.6,129.2,128.9,128.2,127.8$, $126.8,125.7,124.2,123.5,122.2,121.1,113.3,56.7,52.1$; FT-IR (neat): $v_{\max }=3064$, 2950, 2839, 1731, 1622, 1593, 1509, 1488, 1456, 1432, 1303, 1271, 1253, 1188, 1161, 1143, 1127, 1098, 1082, 1064, 1021, 807, $748 \mathrm{~cm}^{-1}$; HRMS (EI): exact mass calculated for: $\mathrm{C}_{26} \mathrm{H}_{20} \mathrm{O}_{5}[\mathrm{M}]^{+}:$412.1305, found 412.1308 .
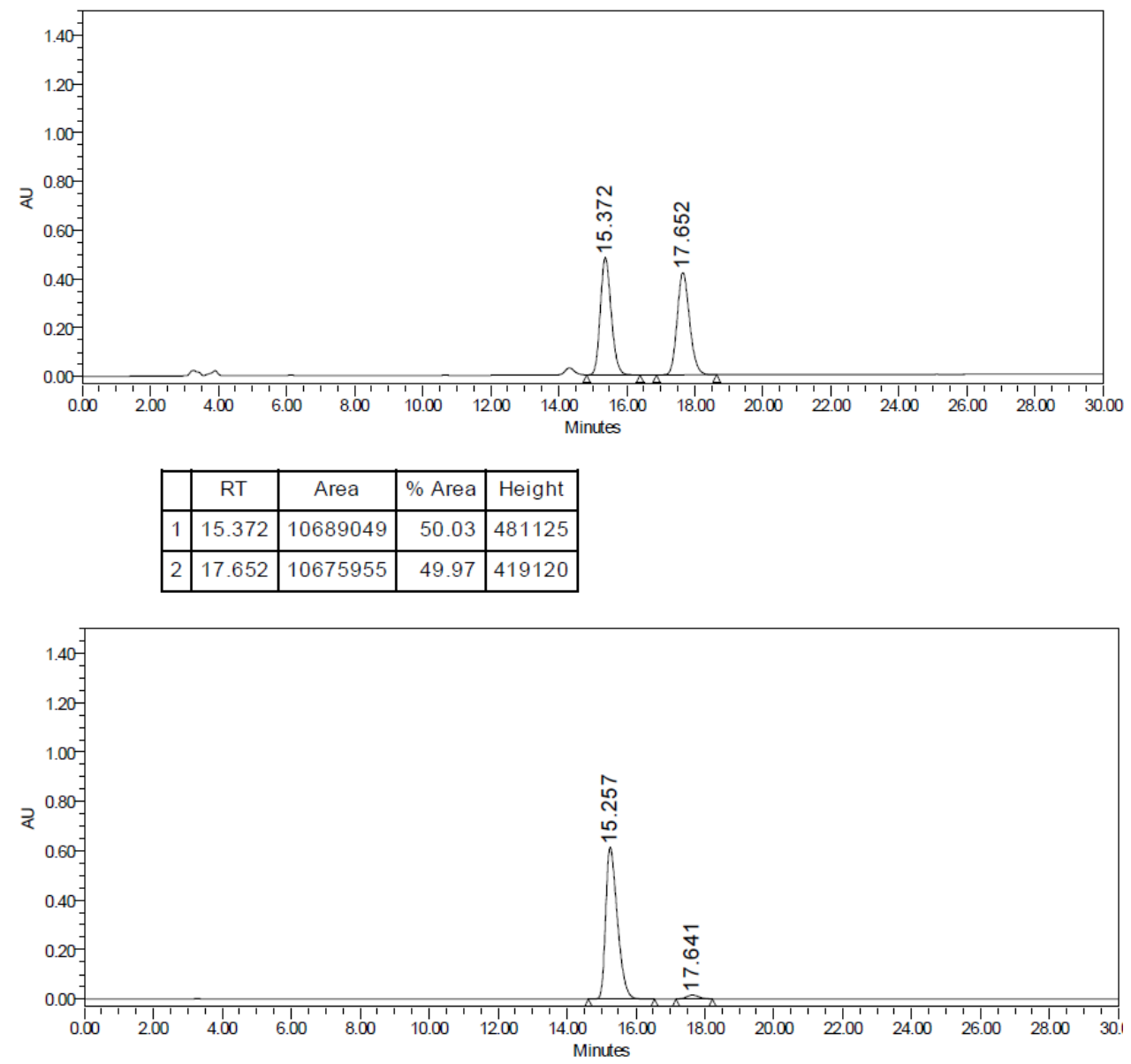

\begin{tabular}{|l|c|r|r|r|}
\hline & RT & \multicolumn{1}{|c|}{ Area } & \% Area & Height \\
\hline 1 & 15.257 & 14409762 & 97.30 & 614316 \\
\hline 2 & 17.641 & 399645 & 2.70 & 16026 \\
\hline
\end{tabular}

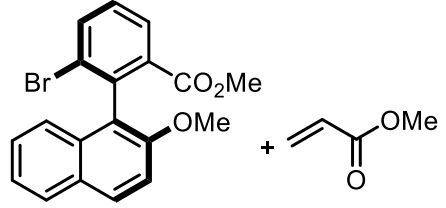

$3 w, 94 \%$ ee

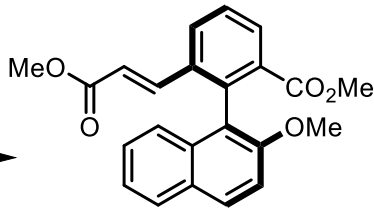

6, $97 \%, 95 \%$ ee

To a stirred solution of $\mathbf{3 w}(37.0 \mathrm{mg}, 0.1 \mathrm{mmol}), \mathrm{Pd}\left(t \mathrm{Bu}_{3} \mathrm{P}\right)_{2}(5.1 \mathrm{mg}, 0.01 \mathrm{mmol})$, and methyl acrylate $(17.2 \mathrm{mg}, 0.2 \mathrm{mmol})$ in THF $(1.0 \mathrm{~mL})$ was added $N, N$ - 
dicyclohexylmethylamine $(78.1 \mathrm{mg}, 0.4 \mathrm{mmol})$. The mixture was stirred at $100{ }^{\circ} \mathrm{C}$ for $13 \mathrm{~h}$. Then, the mixture was quenched with saturated $\mathrm{NH}_{4} \mathrm{Cl}$ aqueous $(3.0 \mathrm{~mL})$. The aqueous layer was extracted with ethyl acetate $(3.0 \mathrm{~mL} \times 3)$. The combined organic layers were washed with brine $(3.0 \mathrm{~mL})$, dried over $\mathrm{Na}_{2} \mathrm{SO}_{4}$, and concentrated under reduced pressure. The residue was purified by flash column chromatography (silica gel, ethyl acetate/petroleum ether 1:20) to afford 6 as colorless oil (36.3 mg, 97\%, 95\% ee)<smiles>COC(=O)/C=C/c1cccc(OC)c1-c1c(OC)ccc2ccccc12</smiles>

The enantiomeric excess was determined by CHIRALCEL OD-H column, hexanes $/ i-\mathrm{PrOH}, 90: 10 \mathrm{v} / \mathrm{v}, \mathrm{v}=1.0 \mathrm{~mL} / \mathrm{min}, \lambda$ $=254 \mathrm{~nm}, 25^{\circ} \mathrm{C}, \mathrm{t}_{\mathrm{R}}($ major $)=13.4 \mathrm{~min}, \mathrm{t}_{\mathrm{R}}($ minor $)=38.9 \mathrm{~min}$; $[\alpha]_{\mathrm{D}}^{23}=+30.74\left(c=1.0, \mathrm{CHCl}_{3}\right)$.

6: ${ }^{1} \mathrm{H}$ NMR (400 MHz, $\left.\mathrm{CHCl}_{3}\right) \delta 8.04(\mathrm{dd}, J=8.0,1.2 \mathrm{~Hz}, 1 \mathrm{H}), 7.95$ - $7.92(\mathrm{~m}, 2 \mathrm{H})$, $7.82(\mathrm{dd}, J=7.2,1.6 \mathrm{~Hz}, 1 \mathrm{H}), 7.54$ (t, $J=7.6 \mathrm{~Hz}, 1 \mathrm{H}), 7.37$ (d, $J=9.2 \mathrm{~Hz}, 1 \mathrm{H}), 7.32$ $7.24(\mathrm{~m}, 2 \mathrm{H}), 7.22(\mathrm{~d}, J=16.0 \mathrm{~Hz}, 1 \mathrm{H}), 7.01(\mathrm{~d}, J=8.0 \mathrm{~Hz}, 1 \mathrm{H}), 6.32(\mathrm{~d}, J=16.0 \mathrm{~Hz}$, 1H), $3.80(\mathrm{~s}, 3 \mathrm{H}), 3.60$ (s, 3H), $3.36(\mathrm{~s}, 3 \mathrm{H}) ;{ }^{13} \mathrm{C} \mathrm{NMR}\left(101 \mathrm{MHz}, \mathrm{CHCl}_{3}\right) \delta 167.4$, 167.0, 154.0, 142.9, 138.0, 135.8, 133.4, 133.3, 131.8, 130.2, 129.8, 129.0, 128.2, 128.0, 126.8, 124.3, 123.6, 120.8, 119.7, 113.3, 56.7, 52.0, 51.6; FT-IR (neat): $v_{\max }=3060$, $3011,2050,2839,1715,1634,1593,1509,1451,1433,1314,1270,1254,1191,1169$, 1133, 1086, 1063, 1020, 986, 807, 763, 749, 700, $642 \mathrm{~cm}^{-1}$; HRMS (EI): exact mass calculated for: $\mathrm{C}_{23} \mathrm{H}_{20} \mathrm{O}_{5}[\mathrm{M}]^{+}: 376.1305$, found 376.1309 .

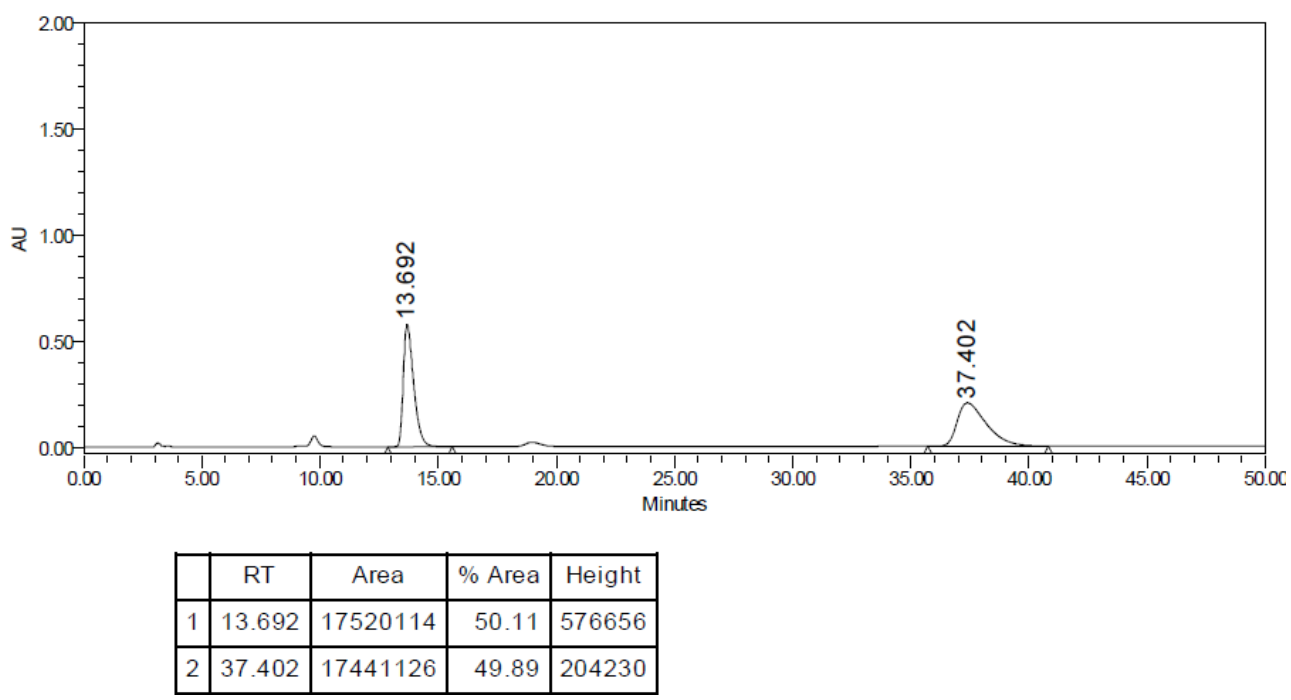



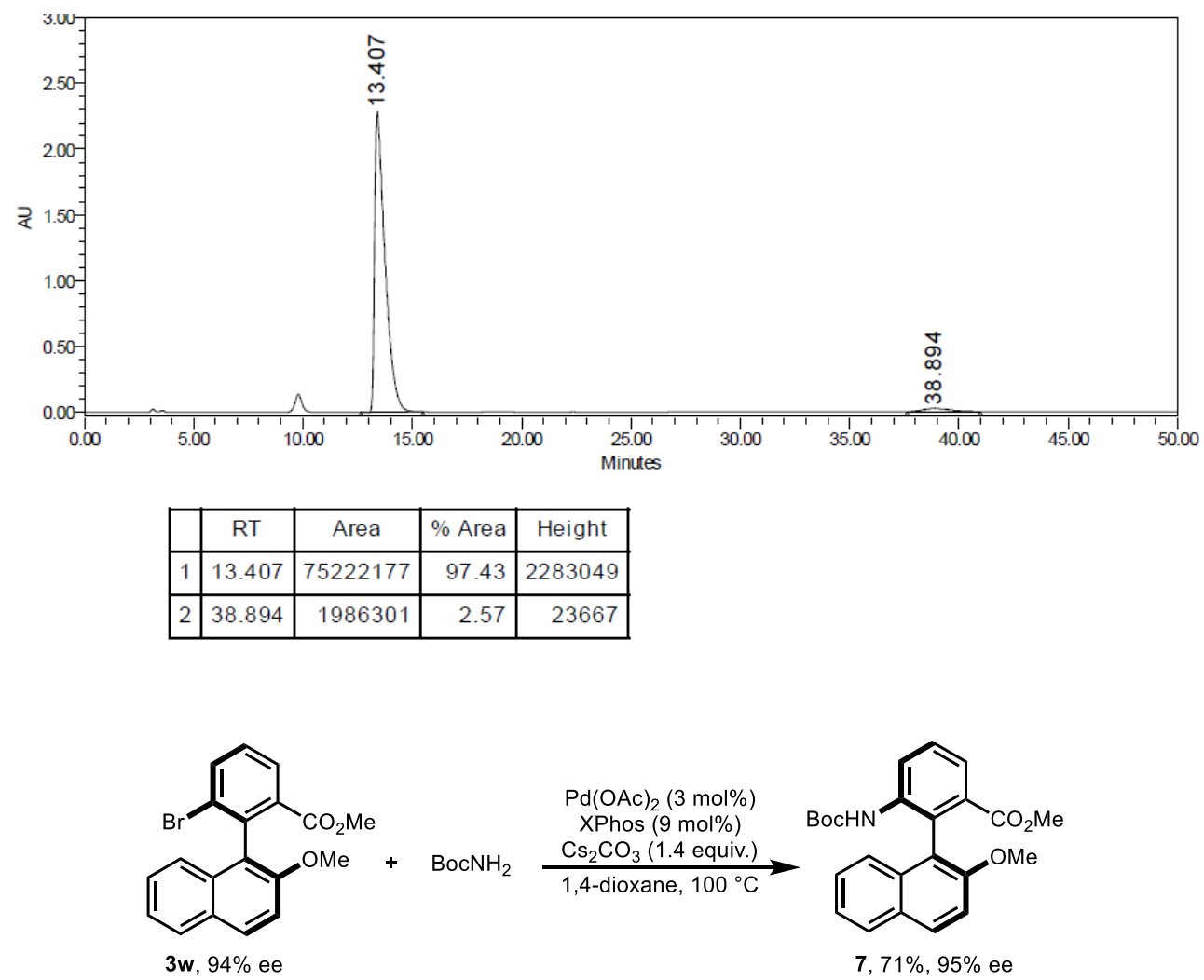

To a stirred solution of $\mathbf{3 w}(37.0 \mathrm{mg}, 0.1 \mathrm{mmol}), \mathrm{Pd}(\mathrm{OAc})_{2}(0.7 \mathrm{mg}, 0.003 \mathrm{mmol})$ and XPhos $(4.3 \mathrm{mg}, 0.009 \mathrm{mmol})$ in 1,4-dioxane $(1.0 \mathrm{~mL})$ was added $\mathrm{Cs}_{2} \mathrm{CO}_{3}(45.6 \mathrm{mg}$, $0.14 \mathrm{mmol}$ ). The mixture was stirred at $100{ }^{\circ} \mathrm{C}$ for $20 \mathrm{~h}$. Then, the mixture was quenched with saturated $\mathrm{NH}_{4} \mathrm{Cl}$ aqueous $(3.0 \mathrm{~mL})$. The aqueous layer was extracted with ethyl acetate $(3.0 \mathrm{~mL} \times 3)$. The combined organic layers were washed with brine $(3.0 \mathrm{~mL})$, dried over $\mathrm{Na}_{2} \mathrm{SO}_{4}$, and concentrated under reduced pressure. The residue was purified by flash column chromatography (silica gel, ethyl acetate/petroleum ether 1:20) to afford 7 as colorless oil (28.9 $\mathrm{mg}, 71 \%, 95 \%$ ee).

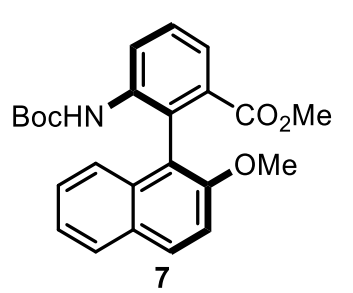

The enantiomeric excess was determined by CHIRALPAK IG column, hexanes $/ i$-PrOH, $90: 10 \mathrm{v} / \mathrm{v}, v=1.0 \mathrm{~mL} / \mathrm{min}, \lambda=254 \mathrm{~nm}$, $25^{\circ} \mathrm{C}, \mathrm{t}_{\mathrm{R}}($ major $)=7.9 \mathrm{~min}, \mathrm{t}_{\mathrm{R}}($ minor $)=7.4 \mathrm{~min} ;[\alpha]_{\mathrm{D}}{ }^{21}=+10.96$ $\left(c=1.0, \mathrm{CHCl}_{3}\right)$.

7: ${ }^{1} \mathrm{H}$ NMR (400 MHz, $\left.\mathrm{CHCl}_{3}\right) \delta 8.38(\mathrm{~d}, J=8.4 \mathrm{~Hz}, 1 \mathrm{H}), 7.97(\mathrm{~d}, J=8.8 \mathrm{~Hz}, 1 \mathrm{H}), 7.85$ $(\mathrm{dd}, J=6.8,2.0 \mathrm{~Hz}, 1 \mathrm{H}), 7.73(\mathrm{dd}, J=7.6,1.2 \mathrm{~Hz}, 1 \mathrm{H}), 7.50(\mathrm{t}, J=8.0 \mathrm{~Hz}, 1 \mathrm{H}), 7.40$ (d, $J=8.8 \mathrm{~Hz}, 1 \mathrm{H}), 7.37-7.30(\mathrm{~m}, 2 \mathrm{H}), 7.18-7.15(\mathrm{~m}, 1 \mathrm{H}), 6.11$ (br, 1H), 3.84 (s, $3 \mathrm{H}), 3.37$ (s, 3H), 1.34 (s, 9H); ${ }^{13} \mathrm{C}$ NMR (101 MHz, $\left.\mathrm{CHCl}_{3}\right) \delta 167.4,154.3,153.0$, 
$137.8,133.3,132.4,130.6,129.2,128.4,128.2,127.2,126.1,124.8,124.1,124.0,123.3$, 118.3, 113.5, 80.6, 56.7, 51.8, 28.3; FT-IR (neat): $v_{\max }=2964,2934,1725,1508,1466$, 1426, 1367, 1304, 1257, 1225, 1141, 1085, 1063, 1020, 869, 807, $751 \mathrm{~cm}^{-1}$; HRMS (EI): exact mass calculated for: $\mathrm{C}_{24} \mathrm{H}_{25} \mathrm{NO}_{5}[\mathrm{M}]^{+}$: 407.1727, found 407.1724.
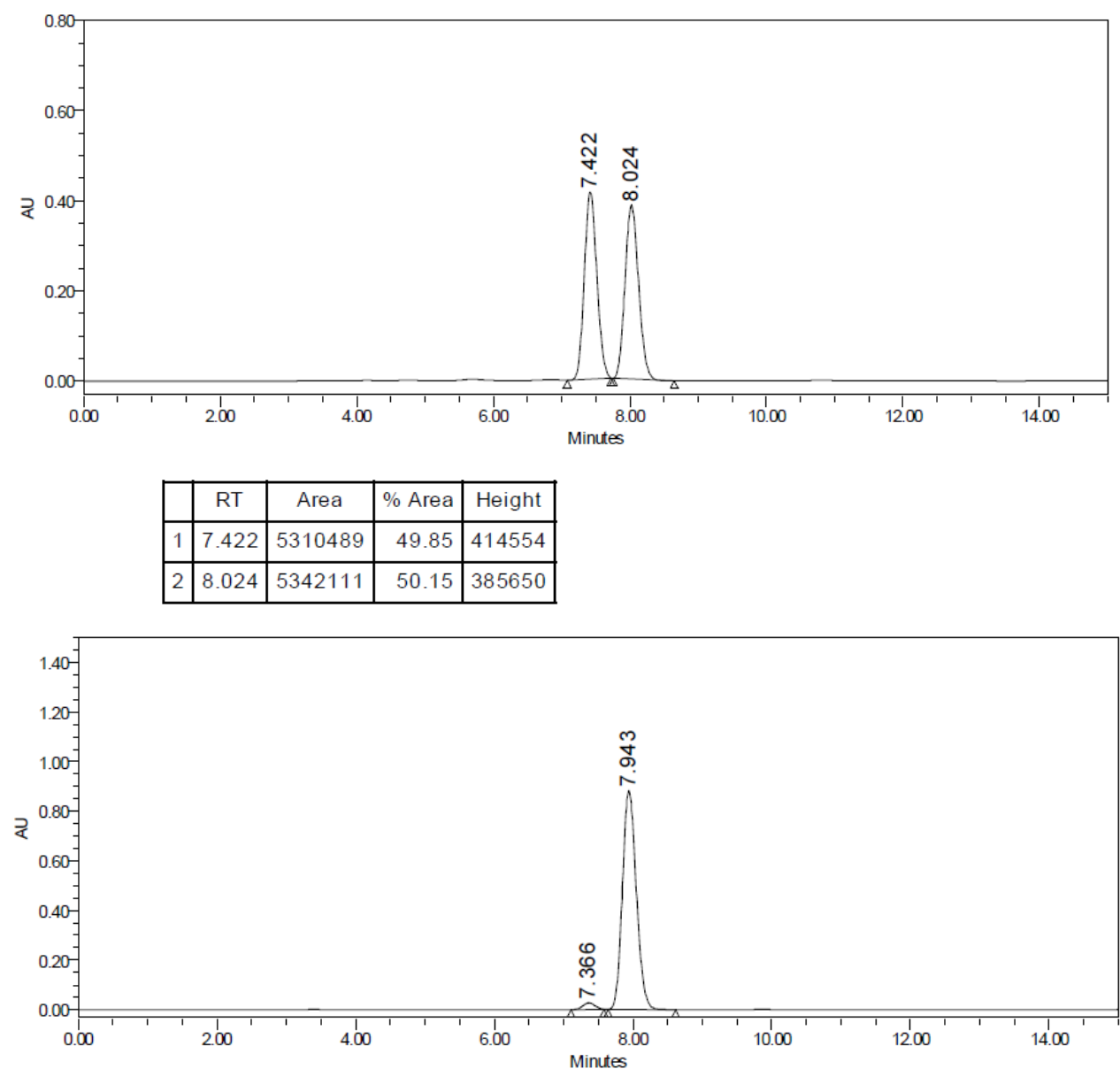

\begin{tabular}{|c|c|r|r|r|}
\hline & RT & Area & \% Area & Height \\
\hline 1 & 7.366 & 323556 & 2.56 & 26809 \\
\hline 2 & 7.943 & 12311689 & 97.44 & 882021 \\
\hline
\end{tabular}

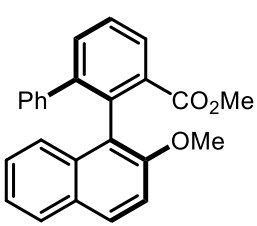

$3 v, 90 \%, 95 \%$ ee $\frac{\text { f) DIBAL-H, DCM, } 0{ }^{\circ} \mathrm{C}}{\text { then,DMP, DCM, } 25^{\circ} \mathrm{C}}$

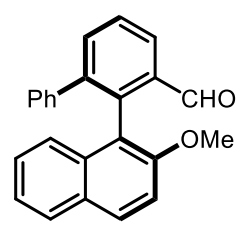

$8,86 \%, 95 \%$ ee

To a stirred solution of $3 \mathrm{v}(51.4 \mathrm{mg}, 0.14 \mathrm{mmol})$ in DCM $(0.46 \mathrm{~mL})$ was added DIBAL-H (1.0 M in THF, $0.42 \mathrm{~mL})$ dropwise at $0{ }^{\circ} \mathrm{C}$. The mixture was stirred at $0{ }^{\circ} \mathrm{C}$ for $5 \mathrm{~h}$. Then, the mixture was quenched with saturated potassium sodium tartrate 
aqueous $(2.0 \mathrm{~mL})$. The aqueous layer was extracted with DCM $(4.0 \mathrm{~mL} \times 3)$. The combined organic layers were washed with brine $(4.0 \mathrm{~mL})$, dried over $\mathrm{Na}_{2} \mathrm{SO}_{4}$, and concentrated under reduced pressure. The residue was used in the next step without purification.

To a solution of above residue in DCM $(1.4 \mathrm{~mL})$ was added Dess-Martin periodinane (149 $\mathrm{mg}, 0.35 \mathrm{mmol}$ ) at $25^{\circ} \mathrm{C}$. After stirred for $1 \mathrm{~h}$, the mixture was quenched with saturated $\mathrm{Na}_{2} \mathrm{~S}_{2} \mathrm{O}_{3}$ aqueous $(2.5 \mathrm{~mL})$. The aqueous layer was extracted with DCM (3.0 $\mathrm{mL} \times 3)$. The combined organic layers were washed with brine $(3.0 \mathrm{~mL})$, dried over $\mathrm{Na}_{2} \mathrm{SO}_{4}$, and concentrated under reduced pressure. The residue was purified by flash column chromatography (silica gel, ethyl acetate/petroleum ether 1:20) to afford $\mathbf{8}$ as colorless oil (40.7 mg, 86\%, 95\% ee).

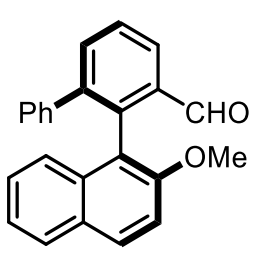

8

The enantiomeric excess was determined by CHIRALPAK AD-H column, hexanes/i-PrOH, 90:10 v/v, $v=1.0 \mathrm{~mL} / \mathrm{min}, \lambda=254 \mathrm{~nm}, 25$ ${ }^{\circ} \mathrm{C}, \mathrm{t}_{\mathrm{R}}($ major $)=6.0 \mathrm{~min}, \mathrm{t}_{\mathrm{R}}($ minor $)=5.4 \mathrm{~min} ;[\alpha]_{\mathrm{D}}^{22}=+53.92(c=1.0$, $\mathrm{CHCl}_{3}$ ).

8: ${ }^{1} \mathrm{H}$ NMR (400 MHz, $\left.\mathrm{CHCl}_{3}\right) \delta 9.49(\mathrm{~s}, 1 \mathrm{H}), 8.13(\mathrm{dd}, \mathrm{J}=7.6,1.2 \mathrm{~Hz}, 1 \mathrm{H}), 7.82(\mathrm{~d}, \mathrm{~J}$ $=8.8 \mathrm{~Hz}, 1 \mathrm{H}), 7.74(\mathrm{dd}, \mathrm{J}=8.0,1.2 \mathrm{~Hz}, 3 \mathrm{H}), 7.64(\mathrm{t}, \mathrm{J}=7.6 \mathrm{~Hz}, 1 \mathrm{H}), 7.32-7.27(\mathrm{~m}$, 2H), $7.21-7.18(\mathrm{~m}, 1 \mathrm{H}), 7.16(\mathrm{~d}, \mathrm{~J}=8.8 \mathrm{~Hz}, 1 \mathrm{H}), 7.03-6.98(\mathrm{~m}, 5 \mathrm{H}), 3.66(\mathrm{~s}, 3 \mathrm{H})$; ${ }^{13} \mathrm{C} \mathrm{NMR}\left(101 \mathrm{MHz}, \mathrm{CHCl}_{3}\right) \delta 193.2,154.6,144.3,140.5,139.0,135.7,135.3,134.4$, $130.4,128.6,128.4,128.3,128.2,127.4,127.2,126.9,126.2,124.7,123.7,118.4,112.3$, 56.1; FT-IR (neat): $v_{\max }=2840,1686,1591,1508,1461,1367,1334,1271,1253,1236$, 1147, 1123, 1061, 1022, 805, 746, 699, 638, $582 \mathrm{~cm}^{-1}$; HRMS (EI): exact mass calculated for: $\mathrm{C}_{24} \mathrm{H}_{18} \mathrm{O}_{2}[\mathrm{M}]^{+}: 338.1301$, found 338.1306 . 


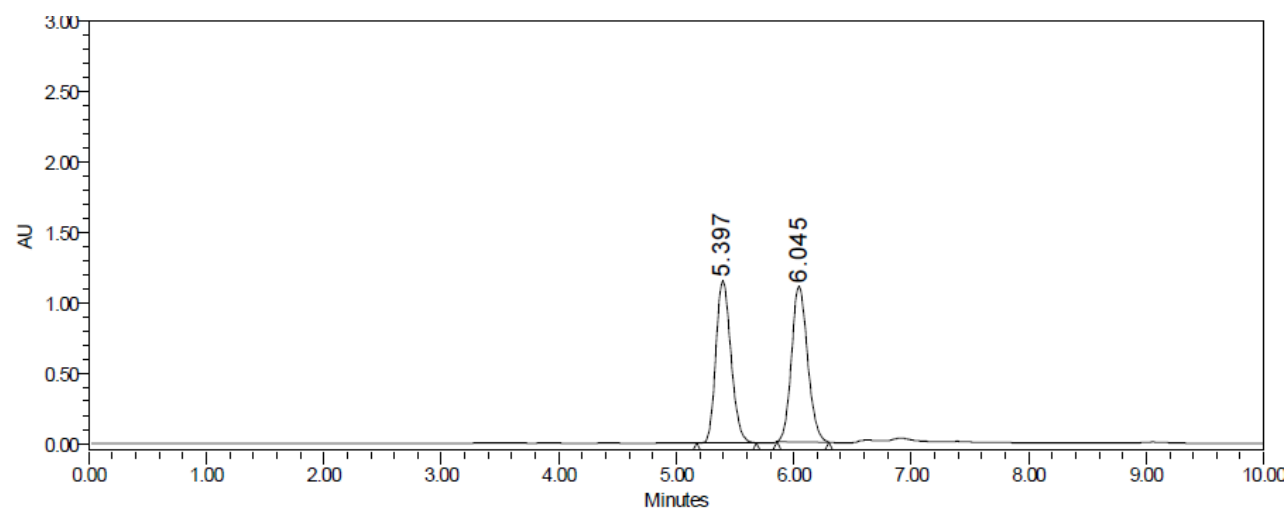

\begin{tabular}{|c|c|c|r|c|}
\hline & RT & Area & \% Area & Height \\
\hline 1 & 5.397 & 10292284 & 49.48 & 1153155 \\
\hline 2 & 6.045 & 10508439 & 50.52 & 1105949 \\
\hline
\end{tabular}

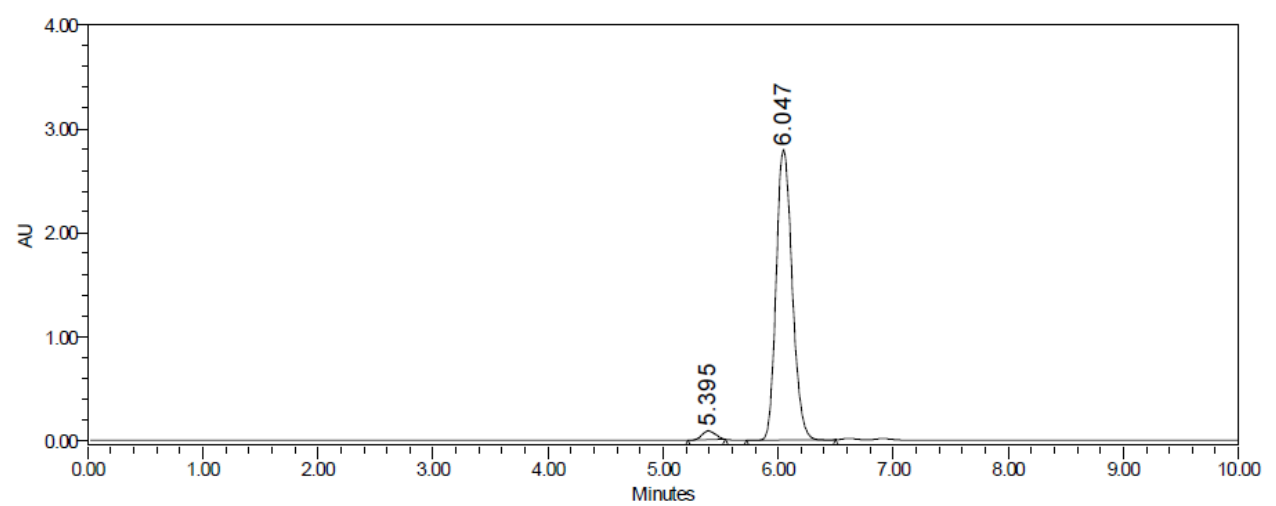

\begin{tabular}{|c|c|r|r|r|}
\hline & RT & \multicolumn{1}{|c|}{ Area } & $\%$ Area & Height \\
\hline 1 & 5.395 & 698192 & 2.51 & 85628 \\
\hline 2 & 6.047 & 27071560 & 97.49 & 2799316 \\
\hline
\end{tabular}

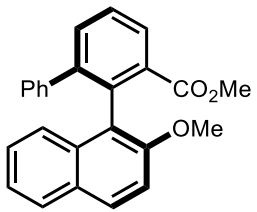

$3 \mathbf{v}, 90 \%, 95 \%$ ee

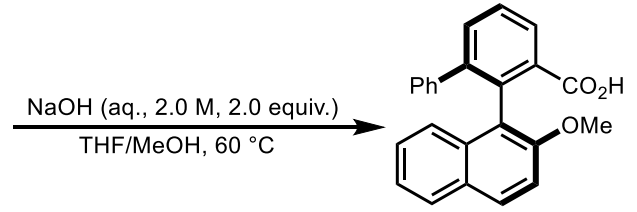

9, $91 \%, 96 \%$ ee

To a stirred solution of $3 \mathbf{v}(73.6 \mathrm{mg}, 0.2 \mathrm{mmol})$ in THF $(0.2 \mathrm{~mL})$ and $\mathrm{MeOH}(0.2 \mathrm{~mL})$ was added fresh prepared $\mathrm{NaOH}$ (aq., $2.0 \mathrm{M}, 0.2 \mathrm{~mL}$ ). The reaction was stirred at $60^{\circ} \mathrm{C}$ for $24 \mathrm{~h}$. Then, the solvents were removed under reduced pressure. The residue was dissolved with ethyl acetate $(6.0 \mathrm{~mL})$ and acidified with $\mathrm{HCl}$ (aq., 1.0 M, 6.0 mL). The aqueous layer was extracted with ethyl acetate $(6.0 \mathrm{~mL} \times 2)$. The combined organic layers were washed with brine $(6.0 \mathrm{~mL})$, dried over $\mathrm{Na}_{2} \mathrm{SO}_{4}$, and concentrated under reduced pressure. The residue was purified by flash column chromatography (silica gel, ethyl acetate/petroleum ether 1:5) to afford 9 as white solid (64.8 $\mathrm{mg}, 91 \%, 96 \%$ ee). 


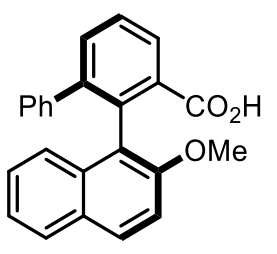

9

The enantiomeric excess was determined by CHIRALPAK AD-H column, hexanes $/ i-\mathrm{PrOH}, 90: 10 \mathrm{v} / \mathrm{v}, v=1.0 \mathrm{~mL} / \mathrm{min}, \lambda=254 \mathrm{~nm}, 25$ ${ }^{\circ} \mathrm{C}, \mathrm{t}_{\mathrm{R}}($ major $)=7.9 \mathrm{~min}, \mathrm{t}_{\mathrm{R}}($ minor $)=11.7$ min; m.p. $182.4-184.3{ }^{\circ} \mathrm{C}$; $[\alpha]_{\mathrm{D}}^{25}=+38.84\left(c=1.0, \mathrm{CHCl}_{3}\right)$.

9: ${ }^{1} \mathrm{H}$ NMR (400 MHz, $\left.\mathrm{CHCl}_{3}\right) \delta 8.01(\mathrm{dd}, \mathrm{J}=8.0,1.6 \mathrm{~Hz}, 1 \mathrm{H}), 7.67-7.65(\mathrm{~m}, 2 \mathrm{H})$, $7.60(\mathrm{dd}, \mathrm{J}=7.6,1.2 \mathrm{~Hz}, 1 \mathrm{H}), 7.51(\mathrm{t}, \mathrm{J}=7.6 \mathrm{~Hz}, 1 \mathrm{H}), 7.22-7.17(\mathrm{~m}, 2 \mathrm{H}), 7.14-7.12$ $(\mathrm{m}, 1 \mathrm{H}), 6.97(\mathrm{~d}, \mathrm{~J}=9.2 \mathrm{~Hz}, 1 \mathrm{H}), 6.95-6.88(\mathrm{~m}, 5 \mathrm{H}), 3.48(\mathrm{~s}, 3 \mathrm{H}) ;{ }^{13} \mathrm{C}$ NMR $(101 \mathrm{MHz}$, $\left.\mathrm{CHCl}_{3}\right) \delta 172.4,153.8,144.4,141.0,136.1,134.4,133.5,131.7,129.9,129.3,128.6$, 128.6, 127.9, 127.7, 127.1, 126.6, 126.3, 124.4, 123.2, 122.3, 112.7, 56.1; FT-IR (neat): $v_{\max }=3560,2918,2853,1683,1623,1594,1510,1460,1415,1306,1269,1253,1148$, 1127, 1090, 904, 788, 746, 698, $642 \mathrm{~cm}^{-1}$; HRMS (EI): exact mass calculated for: $\mathrm{C}_{24} \mathrm{H}_{18} \mathrm{O}_{3}[\mathrm{M}]^{+}: 354.1250$, found 354.1250.

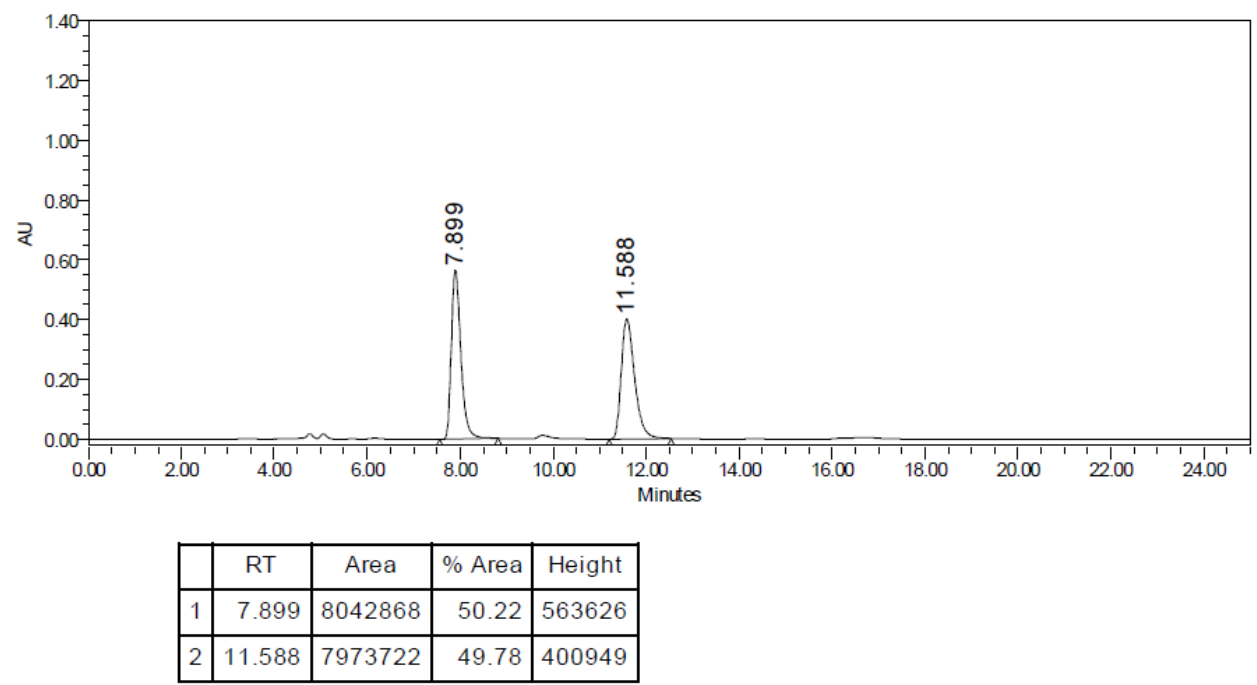




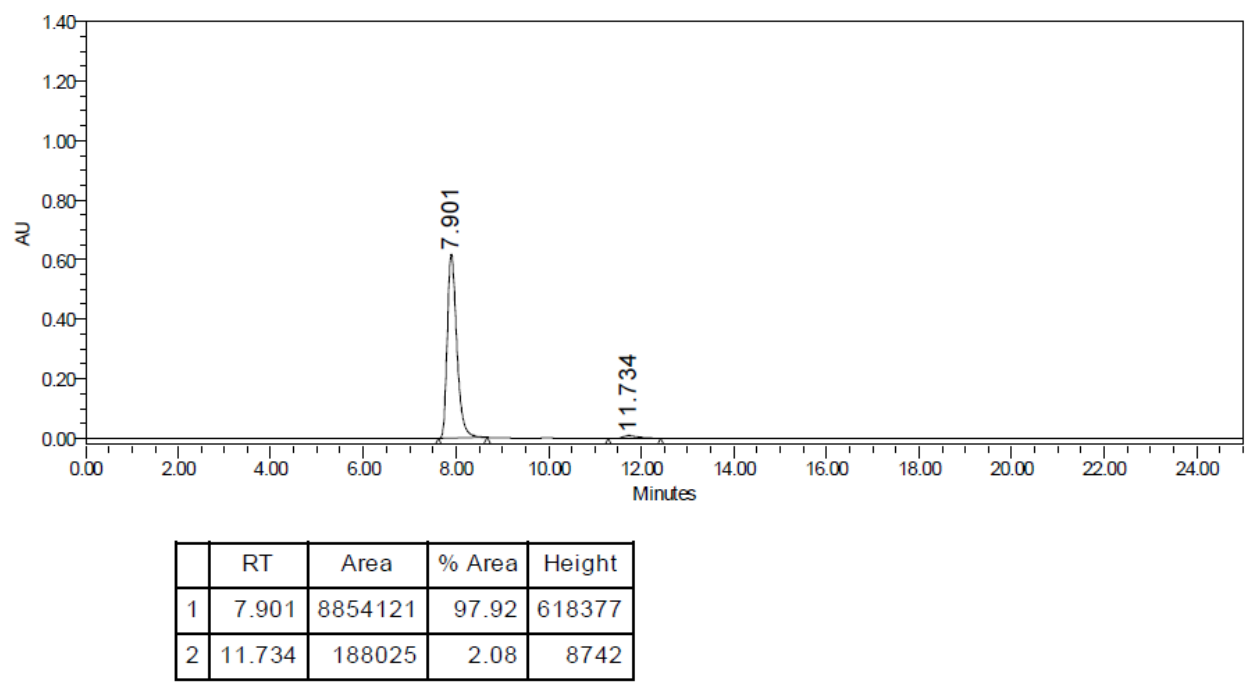

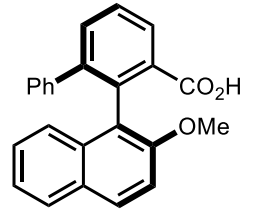

9, $96 \%$ ee

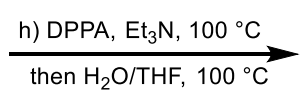

then $\mathrm{H}_{2} \mathrm{O} / \mathrm{THF}, 100^{\circ} \mathrm{C}$

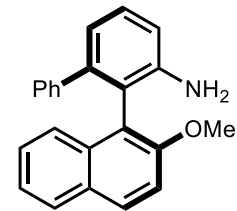

$10,75 \%, 96 \%$ ee

To a stirred solution of $9(35.4 \mathrm{mg}, 0.1 \mathrm{mmol})$ and Et3N (50.6 mg, $0.5 \mathrm{mmol})$ in toluene $(1.0 \mathrm{~mL})$ was added diphenylphosphoryl azide $(55.0 \mathrm{mg}, 0.2 \mathrm{mmol})$. After stirring at $25^{\circ} \mathrm{C}$ for 30 minutes, the reaction was heated to $100{ }^{\circ} \mathrm{C}$ for another $12 \mathrm{~h}$. The solvent was removed under reduced pressure and replaced with $\mathrm{H}_{2} \mathrm{O}(0.5 \mathrm{~mL})$ and THF $(0.5 \mathrm{~mL})$. Then, the reaction was stirred at $100^{\circ} \mathrm{C}$ for $24 \mathrm{~h}$. THF was removed under reduced pressure, the residue was quenched with saturated $\mathrm{K}_{2} \mathrm{CO}_{3}(3.0 \mathrm{~mL})$. The aqueous layer was extracted with ethyl acetate $(5.0 \mathrm{~mL} \times 3)$. The combined organic layers were washed with brine $(5.0 \mathrm{~mL})$, dried over $\mathrm{Na}_{2} \mathrm{SO}_{4}$, and concentrated under reduced pressure. The residue was purified by flash column chromatography (silica gel, ethyl acetate/petroleum ether 1:5) to afford $\mathbf{1 0}$ as colorless oil (24.4 mg, 75\%, 96\% ee).<smiles>COc1ccc2ccccc2c1-c1c(N)cccc1-c1ccccc1</smiles>

10

The enantiomeric excess was determined by CHIRALPAK AD-H column, hexanes $/ i-\mathrm{PrOH}, 90: 10 \mathrm{v} / \mathrm{v}, v=1.0 \mathrm{~mL} / \mathrm{min}, \lambda=254 \mathrm{~nm}, 25$ ${ }^{\circ} \mathrm{C}, \mathrm{t}_{\mathrm{R}}($ major $)=13.5 \mathrm{~min}, \mathrm{t}_{\mathrm{R}}($ minor $)=7.8 \mathrm{~min} ;[\alpha]_{\mathrm{D}}{ }^{22}=-17.22(c=$ $\left.1.0, \mathrm{CHCl}_{3}\right)$.

10: ${ }^{1} \mathrm{H}$ NMR (400 MHz, $\left.\mathrm{CHCl}_{3}\right) \delta 7.78-7.74(\mathrm{~m}, 2 \mathrm{H}), 7.51(\mathrm{~d}, \mathrm{~J}=8.4 \mathrm{~Hz}, 1 \mathrm{H}), 7.37$ $(\mathrm{td}, \mathrm{J}=6.4,1.8 \mathrm{~Hz}, 1 \mathrm{H}), 7.34-7.29(\mathrm{~m}, 2 \mathrm{H}), 7.13(\mathrm{~d}, \mathrm{~J}=8.8 \mathrm{~Hz}, 1 \mathrm{H}), 6.99-6.94(\mathrm{~m}$, 
$5 \mathrm{H}), 6.91(\mathrm{~d}, \mathrm{~J}=8.0 \mathrm{~Hz}, 1 \mathrm{H}), 6.86(\mathrm{~d}, \mathrm{~J}=8.0 \mathrm{~Hz}, 1 \mathrm{H}), 3.62(\mathrm{~s}, 3 \mathrm{H}), 3.43(\mathrm{br}, 1 \mathrm{H}) ;{ }^{13} \mathrm{C}$ NMR $\left(101 \mathrm{MHz}, \mathrm{CHCl}_{3}\right) \delta 154.7,144.9,144.1,142.3,133.4,129.8,129.0,128.7,128.6$, 128.2, 127.1, 127.0, 126.2, 125.0, 123.7, 120.3, 120.2, 119.9, 114.5, 113.5, 56.3; FT-IR (neat): $v_{\max }=3375,3056,2929,2837,1609,1592,1507,1459,1265,1251,1065,1020$, 907, 809, 747, 700, $661 \mathrm{~cm}^{-1}$; HRMS (EI): exact mass calculated for: $\mathrm{C}_{23} \mathrm{H}_{19} \mathrm{NO}[\mathrm{M}]^{+}$: 325.1461 , found 325.1463 .
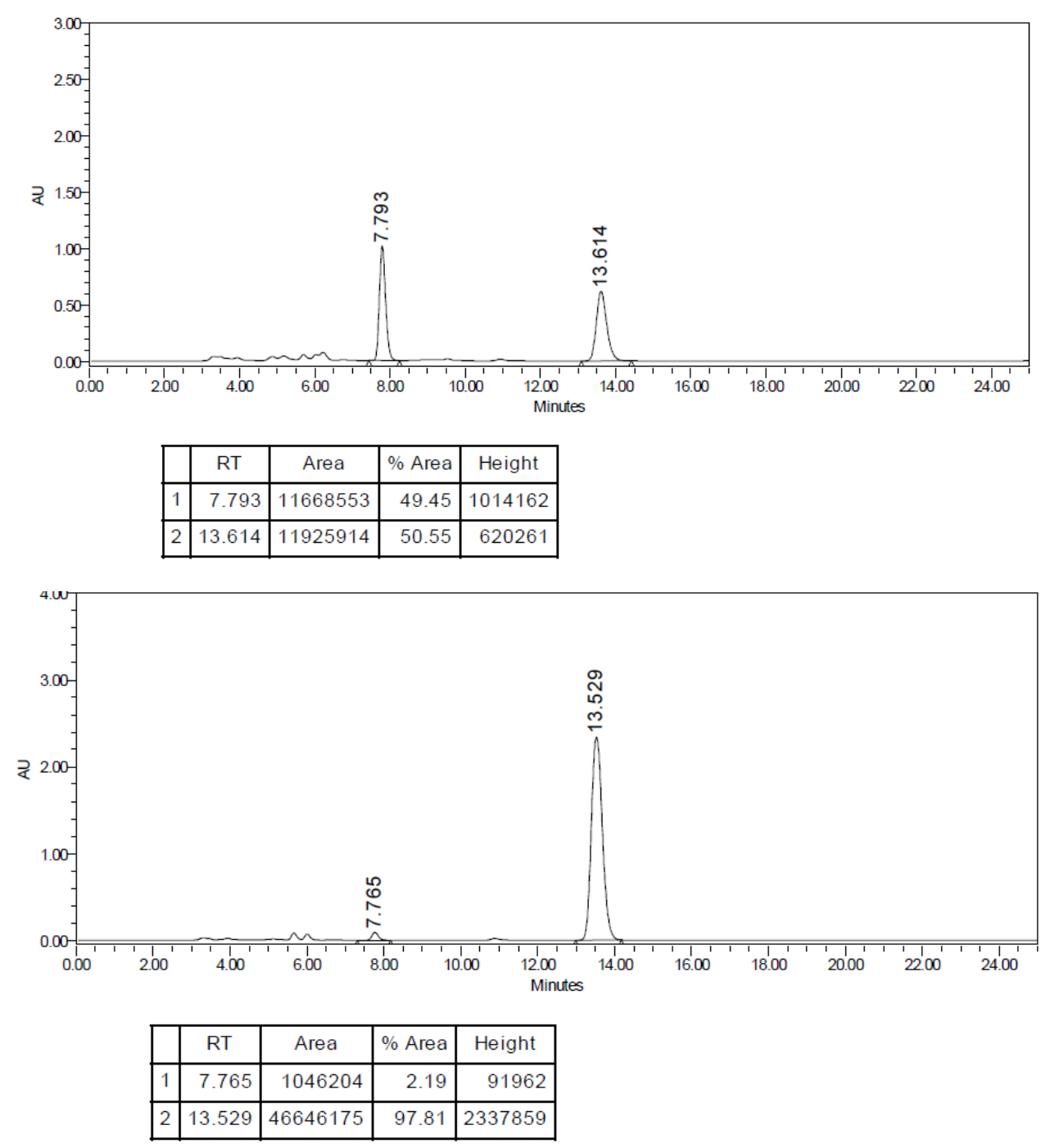


\section{X-ray Crystallographic Data of 3w (CCDC 2054016)}

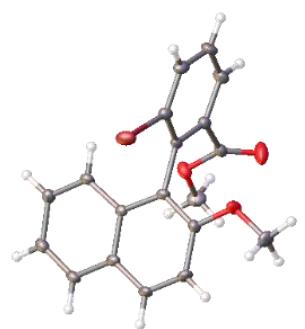

Table 1 Crystal data and structure refinement for $\mathbf{3 w}$.

Compound

Empirical formula

Formula weight

Temperature/K

Crystal system

Space group

$\mathrm{a} / \AA$

$\mathrm{b} / \AA$

$\mathrm{c} / \AA$

$\alpha /{ }^{\circ}$

$\beta /{ }^{\circ}$

$\gamma /{ }^{\circ}$

Volume $/ \AA^{3}$

$\mathrm{Z}$

$\rho_{\text {calc } g / \mathrm{cm}^{3}}$

$\mu / \mathrm{mm}^{-1}$

$\mathrm{F}(000)$

Crystal size $/ \mathrm{mm}^{3}$

Radiation 3w

$\mathrm{C}_{19} \mathrm{H}_{15} \mathrm{BrO}_{3}$

371.22

100.00(10)

monoclinic

$\mathrm{P} 2{ }_{1}$

10.37730(10)

12.87060(10)

12.07660(10)

90

98.1650(10)

90

1596.62(2)

4

1.544

3.615

752.0

$0.42 \times 0.38 \times 0.36$

$\mathrm{CuK \alpha}(\lambda=1.54184)$

$2 \Theta$ range for data collection/ ${ }^{\circ} 7.396$ to 149.104

Index ranges

Reflections collected

$-12 \leq \mathrm{h} \leq 12,-16 \leq \mathrm{k} \leq 16,-15 \leq 1 \leq 15$

40187

Independent reflections

Data/restraints/parameters

Goodness-of-fit on $\mathrm{F}^{2}$

$6445\left[\mathrm{R}_{\text {int }}=0.0515, \mathrm{R}_{\text {sigma }}=0.0240\right]$

$6445 / 1 / 420$

1.052

Final $R$ indexes $[\mathrm{I}>=2 \sigma(\mathrm{I})] \quad \mathrm{R}_{1}=0.0248, \mathrm{wR}_{2}=0.0649$

Final $\mathrm{R}$ indexes [all data] $\quad \mathrm{R}_{1}=0.0251, \mathrm{wR}_{2}=0.0649$

Largest diff. peak/hole / e $\AA^{-3} 0.49 /-0.59$

Flack parameter

Table 2 Fractional Atomic Coordinates $\left(\times 10^{4}\right)$ and Equivalent Isotropic Displacement Parameters $\left(\AA^{2} \times 10^{3}\right)$ for exp_1491. Ueq is defined as $1 / 3$ of of the trace of the orthogonalised UIJ tensor.
Atom
$x$ 


\begin{tabular}{|c|c|c|c|c|}
\hline $\mathrm{Br} 1$ & $3344.5(3)$ & $590.7(3)$ & $5959.0(2)$ & $21.66(10)$ \\
\hline $\mathrm{Br} 2$ & $2247.8(3)$ & $5645.3(3)$ & $-1017.6(2)$ & $25.03(10)$ \\
\hline $\mathrm{O} 3$ & $5202(2)$ & $3362.5(19)$ & 2013.2(19) & 22.1(5) \\
\hline $\mathrm{O} 2$ & $3543(2)$ & $3873.3(18)$ & 2883(2) & $22.9(5)$ \\
\hline $\mathrm{O} 4$ & $3537(2)$ & 8051.3(19) & $2874(2)$ & $23.0(5)$ \\
\hline $\mathrm{O} 1$ & $2355(2)$ & 1249.2(19) & $2475(2)$ & $22.9(5)$ \\
\hline O6 & $2073(2)$ & $8616(2)$ & $-614(2)$ & $24.4(5)$ \\
\hline O5 & $4674(3)$ & $9277(2)$ & $2124(2)$ & $35.7(6)$ \\
\hline C11 & $3964(3)$ & $1983(2)$ & $4259(3)$ & $14.0(6)$ \\
\hline C18 & $4429(3)$ & $3288(2)$ & $2812(3)$ & $15.8(6)$ \\
\hline $\mathrm{C} 25$ & 1299(3) & $6855(2)$ & $1564(2)$ & $14.0(6)$ \\
\hline $\mathrm{C} 27$ & $1222(3)$ & $5576(3)$ & $3028(2)$ & $18.4(5)$ \\
\hline $\mathrm{C} 23$ & $-549(3)$ & $8024(2)$ & $1039(3)$ & $17.8(6)$ \\
\hline C12 & $4466(3)$ & $1237(2)$ & $5039(3)$ & $17.7(6)$ \\
\hline $\mathrm{C} 1$ & $2034(3)$ & 2941(2) & $4839(3)$ & $16.2(6)$ \\
\hline $\mathrm{C} 28$ & $10(3)$ & $5975(2)$ & $3233(3)$ & $18.5(6)$ \\
\hline C33 & $4885(3)$ & $6222(3)$ & $-409(3)$ & $20.7(7)$ \\
\hline C13 & $5754(3)$ & $923(2)$ & $5196(3)$ & $21.3(7)$ \\
\hline C31 & $3337(3)$ & $7103(2)$ & $627(3)$ & $14.5(6)$ \\
\hline C9 & $1755(3)$ & $1864(2)$ & $3175(3)$ & $17.9(6)$ \\
\hline $\mathrm{C} 21$ & $1360(3)$ & $8165(2)$ & 123(3) & $16.9(6)$ \\
\hline $\mathrm{C} 10$ & $2559(3)$ & $2263(2)$ & $4089(3)$ & $15.8(6)$ \\
\hline C32 & $3629(3)$ & $6404(3)$ & $-180(3)$ & $17.7(6)$ \\
\hline C6 & $686(3)$ & $3194(3)$ & $4649(3)$ & $19.9(6)$ \\
\hline C29 & $-574(3)$ & $6768(2)$ & $2592(3)$ & $18.1(6)$ \\
\hline C37 & $4220(3)$ & $8415(2)$ & $2103(3)$ & $17.8(6)$ \\
\hline C15 & $6149(3)$ & 2094(3) & $3736(3)$ & $19.5(6)$ \\
\hline $\mathrm{C} 24$ & $42(3)$ & $7214(2)$ & $1730(3)$ & $16.0(6)$ \\
\hline $\mathrm{C} 22$ & $75(3)$ & $8483(3)$ & $238(3)$ & $19.2(6)$ \\
\hline $\mathrm{C} 14$ & $6599(3)$ & $1355(3)$ & $4533(3)$ & $21.9(7)$ \\
\hline $\mathrm{C} 5$ & $185(3)$ & $3907(3)$ & $5374(3)$ & $27.6(8)$ \\
\hline $\mathrm{C} 26$ & $1854(3)$ & $6009(2)$ & $2216(3)$ & $16.2(6)$ \\
\hline $\mathrm{C} 20$ & 1964(3) & $7369(2)$ & $765(3)$ & $14.5(6)$ \\
\hline C34 & $5904(3)$ & $6753(3)$ & 204(3) & $24.5(7)$ \\
\hline $\mathrm{C} 8$ & 419(3) & $2107(3)$ & 2991(3) & $23.0(7)$ \\
\hline C38 & $3298(4)$ & $8777(3)$ & $3739(3)$ & $25.4(7)$ \\
\hline C35 & $5662(3)$ & $7450(3)$ & $1030(3)$ & $22.8(7)$ \\
\hline $\mathrm{C} 3$ & $2312(4)$ & 4099(3) & $6442(3)$ & $24.0(7)$ \\
\hline $\mathrm{C} 2$ & $2821(3)$ & $3407(3)$ & $5763(3)$ & $19.1(6)$ \\
\hline C19 & $4902(3)$ & $4213(3)$ & $1244(3)$ & $24.3(7)$ \\
\hline $\mathrm{C} 7$ & $-100(3)$ & $2752(3)$ & $3709(3)$ & $24.8(7)$ \\
\hline $\mathrm{C} 4$ & $967(4)$ & $4352(3)$ & $6248(3)$ & $28.0(8)$ \\
\hline $\mathrm{C} 30$ & $1468(3)$ & $9408(3)$ & $-1343(3)$ & $23.5(7)$ \\
\hline $\mathrm{C} 16$ & $4858(3)$ & $2423(2)$ & $3611(3)$ & $15.5(6)$ \\
\hline $\mathrm{C} 17$ & $1562(3)$ & $749(3)$ & $1567(3)$ & $27.4(7)$ \\
\hline
\end{tabular}


Table 3 Anisotropic Displacement Parameters $\left(\AA^{2} \times 10^{3}\right)$ for exp_1491. The Anisotropic displacement factor exponent takes the form: $2 \pi^{2}\left[h^{2} a^{* 2} U_{11}+2 h k a * b * U_{12}+\ldots\right]$.

\begin{tabular}{|c|c|c|c|c|c|c|}
\hline Atom & $\mathbf{U}_{11}$ & $\mathbf{U}_{22}$ & $\mathbf{U}_{33}$ & $\mathbf{U}_{23}$ & $\mathbf{U}_{13}$ & $\mathbf{U}_{12}$ \\
\hline $\mathrm{Br} 1$ & $18.71(15)$ & $23.68(17)$ & $23.21(16)$ & $5.00(16)$ & $5.09(11)$ & $1.96(14)$ \\
\hline $\mathrm{Br} 2$ & $18.33(16)$ & $29.56(18)$ & $25.37(17)$ & $-9.39(17)$ & $-3.16(11)$ & $3.52(16)$ \\
\hline $\mathrm{O} 3$ & $21.2(11)$ & $25.7(12)$ & 21.1(12) & $3.8(10)$ & $8.7(9)$ & 7.1(9) \\
\hline $\mathrm{O} 2$ & $17.7(11)$ & $21.2(11)$ & $31.4(13)$ & $5.1(10)$ & $9.1(10)$ & $7.1(9)$ \\
\hline $\mathrm{O} 4$ & $23.5(12)$ & $23.2(12)$ & $23.8(12)$ & $-3.5(10)$ & $8.7(9)$ & $-6.4(9)$ \\
\hline $\mathrm{O} 1$ & $16.3(11)$ & $25.6(12)$ & $24.6(12)$ & $-8.9(10)$ & $-4.1(9)$ & $-1.5(9)$ \\
\hline O6 & $16.0(11)$ & $28.4(12)$ & $29.2(13)$ & $15.6(10)$ & $4.7(10)$ & $4.9(9)$ \\
\hline O5 & $46.5(17)$ & 21.1(13) & $43.3(16)$ & $-2.8(12)$ & $20.1(13)$ & $-11.0(12)$ \\
\hline $\mathrm{C} 11$ & $10.4(13)$ & $13.4(13)$ & $17.8(14)$ & $-3.0(11)$ & $0.3(10)$ & $-1.2(11)$ \\
\hline C18 & $12.4(13)$ & $16.9(14)$ & $18.3(14)$ & $-3.3(12)$ & $2.3(11)$ & $-0.7(11)$ \\
\hline $\mathrm{C} 25$ & $11.6(13)$ & $12.7(13)$ & $17.3(14)$ & $-2.4(11)$ & $0.9(10)$ & $-0.6(11)$ \\
\hline $\mathrm{C} 27$ & $14.6(12)$ & $17.9(13)$ & $21.9(13)$ & $3.7(15)$ & $-0.1(10)$ & $-0.6(14)$ \\
\hline $\mathrm{C} 23$ & $8.7(13)$ & $16.9(14)$ & $27.4(16)$ & $-0.6(12)$ & $0.8(11)$ & $1.4(11)$ \\
\hline C12 & $16.2(14)$ & $17.7(15)$ & $18.9(15)$ & $-3.3(12)$ & $1.7(11)$ & $0.2(11)$ \\
\hline $\mathrm{C} 1$ & $14.1(13)$ & $15.8(14)$ & $19.5(15)$ & $3.8(12)$ & $5.6(11)$ & $1.7(11)$ \\
\hline $\mathrm{C} 28$ & $15.0(14)$ & $20.6(14)$ & $20.6(15)$ & $-1.9(12)$ & $5.1(11)$ & $-4.0(11)$ \\
\hline C33 & $20.5(15)$ & $22.1(16)$ & $21.0(16)$ & $2.6(13)$ & $8.1(12)$ & $5.9(12)$ \\
\hline C13 & $17.4(14)$ & $20.1(15)$ & $25.3(16)$ & $2.3(12)$ & $-0.2(12)$ & $3.4(11)$ \\
\hline C31 & $11.2(13)$ & $16.5(14)$ & $16.3(14)$ & $5.7(11)$ & $3.0(10)$ & $1.7(11)$ \\
\hline C9 & $13.8(14)$ & $17.8(15)$ & $22.0(15)$ & $0.3(12)$ & $1.9(12)$ & $-1.2(11)$ \\
\hline $\mathrm{C} 21$ & $13.5(13)$ & $17.3(14)$ & $20.1(15)$ & $3.8(12)$ & $3.2(11)$ & $-1.1(11)$ \\
\hline C10 & $9.3(13)$ & $16.6(14)$ & $21.4(15)$ & $1.6(12)$ & $2.3(11)$ & $0.1(11)$ \\
\hline C32 & $14.2(14)$ & $19.5(15)$ & $19.5(15)$ & $3.5(12)$ & $2.4(11)$ & $2.7(11)$ \\
\hline C6 & $14.0(14)$ & $19.3(15)$ & $28.0(17)$ & $9.8(13)$ & $8.6(12)$ & $1.4(12)$ \\
\hline C29 & $11.8(13)$ & $20.1(15)$ & $23.3(15)$ & $-2.2(13)$ & $5.3(11)$ & $-0.7(11)$ \\
\hline $\mathrm{C} 37$ & $11.7(13)$ & 19.1(15) & $22.4(15)$ & $3.5(12)$ & $1.3(11)$ & $-1.3(11)$ \\
\hline C15 & $11.1(14)$ & $21.9(16)$ & $25.4(16)$ & $-2.4(13)$ & $1.9(12)$ & $0.1(12)$ \\
\hline $\mathrm{C} 24$ & $10.6(13)$ & $15.5(14)$ & $21.3(15)$ & $-2.9(12)$ & $0.2(11)$ & $0.0(11)$ \\
\hline $\mathrm{C} 22$ & $13.1(14)$ & $17.8(14)$ & 25.1(16) & $2.6(12)$ & $-2.5(12)$ & $4.5(12)$ \\
\hline C14 & $10.7(13)$ & $25.0(16)$ & $29.4(17)$ & $-0.4(14)$ & $0.4(12)$ & $4.3(12)$ \\
\hline $\mathrm{C} 5$ & $20.9(17)$ & $27.3(18)$ & $38(2)$ & $12.0(15)$ & $15.5(15)$ & $9.6(13)$ \\
\hline $\mathrm{C} 26$ & $9.7(13)$ & $18.6(14)$ & $20.2(15)$ & $-0.5(12)$ & $1.6(11)$ & $0.2(10)$ \\
\hline $\mathrm{C} 20$ & $9.3(13)$ & $16.2(14)$ & $17.4(14)$ & $-0.9(12)$ & $-0.1(10)$ & $0.5(11)$ \\
\hline C34 & $12.3(14)$ & $28.8(18)$ & $34.0(18)$ & $2.4(15)$ & $9.1(13)$ & $2.2(13)$ \\
\hline $\mathrm{C} 8$ & $14.6(14)$ & $25.5(17)$ & $26.7(17)$ & $2.7(14)$ & $-5.0(12)$ & $-3.9(13)$ \\
\hline C38 & $26.7(18)$ & $27.5(17)$ & $22.8(16)$ & $-6.7(14)$ & $6.4(14)$ & $3.7(14)$ \\
\hline C35 & $12.6(14)$ & 26.1(17) & $29.7(17)$ & $0.2(14)$ & $2.6(12)$ & $-2.6(13)$ \\
\hline $\mathrm{C} 3$ & $29.5(18)$ & $19.7(16)$ & $24.1(16)$ & $-0.6(13)$ & $8.3(14)$ & $2.9(13)$ \\
\hline
\end{tabular}




$\begin{array}{lrrrrrr}\text { C2 } & 17.9(15) & 18.7(15) & 21.9(16) & 2.3(13) & 6.9(12) & 0.3(12) \\ \text { C19 } & 23.8(16) & 28.6(18) & 21.1(16) & 6.0(14) & 4.8(13) & 4.7(14) \\ \text { C7 } & 9.4(14) & 29.4(18) & 36(2) & 9.9(15) & 3.3(13) & 1.9(12) \\ \text { C4 } & 34(2) & 22.6(17) & 31.7(19) & 5.1(14) & 18.5(16) & 7.7(15) \\ \text { C30 } & 24.0(16) & 23.7(17) & 21.8(17) & 10.1(14) & -0.3(13) & 4.2(13) \\ \text { C16 } & 12.5(14) & 14.4(14) & 19.1(15) & -3.5(12) & 1.2(11) & 1.1(11) \\ \text { C17 } & 24.8(15) & 37(2) & 19.0(14) & -4.8(16) & -2.3(12) & -8.3(16) \\ \text { C36 } & 13.6(14) & 17.6(15) & 19.2(14) & 3.7(12) & 3.0(11) & 0.8(11)\end{array}$

Table 4 Bond Lengths for exp_1491.

Atom Atom Length/Å Atom Atom Length/Å

$\begin{array}{llllll}\mathrm{B} 1 & \mathrm{C} 12 & 1.910(3) & \mathrm{C} 1 & \mathrm{C} 6 & 1.422(4) \\ \mathrm{B} 22 & \mathrm{C} 32 & 1.902(3) & \mathrm{C} 1 & \mathrm{C} 2 & 1.419(5) \\ \mathrm{O} 3 & \mathrm{C} 18 & 1.343(4) & \mathrm{C} 28 & \mathrm{C} 29 & 1.370(5) \\ \mathrm{O} 3 & \mathrm{C} 19 & 1.441(4) & \mathrm{C} 33 & \mathrm{C} 32 & 1.391(4) \\ \mathrm{O} 2 & \mathrm{C} 18 & 1.202(4) & \mathrm{C} 33 & \mathrm{C} 34 & 1.382(5) \\ \mathrm{O} 4 & \mathrm{C} 37 & 1.332(4) & \mathrm{C} 13 & \mathrm{C} 14 & 1.384(5) \\ \mathrm{O} 4 & \mathrm{C} 38 & 1.449(4) & \mathrm{C} 31 & \mathrm{C} 32 & 1.391(4) \\ \mathrm{O} 1 & \mathrm{C} 9 & 1.370(4) & \mathrm{C} 31 & \mathrm{C} 20 & 1.497(4) \\ \mathrm{O} 1 & \mathrm{C} 17 & 1.427(4) & \mathrm{C} 31 & \mathrm{C} 36 & 1.395(4) \\ \mathrm{O} 6 & \mathrm{C} 21 & 1.365(4) & \mathrm{C} 9 & \mathrm{C} 10 & 1.384(4) \\ \mathrm{O} 6 & \mathrm{C} 30 & 1.432(4) & \mathrm{C} 9 & \mathrm{C} 8 & 1.408(4) \\ \mathrm{O} 5 & \mathrm{C} 37 & 1.205(4) & \mathrm{C} 21 & \mathrm{C} 22 & 1.421(4) \\ \mathrm{C} 11 & \mathrm{C} 12 & 1.394(4) & \mathrm{C} 21 & \mathrm{C} 20 & 1.381(4) \\ \mathrm{C} 11 & \mathrm{C} 10 & 1.487(4) & \mathrm{C} 6 & \mathrm{C} 5 & 1.416(5) \\ \mathrm{C} 11 & \mathrm{C} 16 & 1.414(4) & \mathrm{C} 6 & \mathrm{C} 7 & 1.420(5) \\ \mathrm{C} 18 & \mathrm{C} 16 & 1.498(4) & \mathrm{C} 29 & \mathrm{C} 24 & 1.418(4) \\ \mathrm{C} 25 & \mathrm{C} 24 & 1.425(4) & \mathrm{C} 37 & \mathrm{C} 36 & 1.494(5) \\ \mathrm{C} 25 & \mathrm{C} 26 & 1.417(4) & \mathrm{C} 15 & \mathrm{C} 14 & 1.386(5) \\ \mathrm{C} 25 & \mathrm{C} 20 & 1.426(4) & \mathrm{C} 15 & \mathrm{C} 16 & 1.393(4) \\ \mathrm{C} 27 & \mathrm{C} 28 & 1.413(4) & \mathrm{C} 5 & \mathrm{C} 4 & 1.363(6) \\ \mathrm{C} 27 & \mathrm{C} 26 & 1.373(4) & \mathrm{C} 34 & \mathrm{C} 35 & 1.390(5) \\ \mathrm{C} 23 & \mathrm{C} 24 & 1.419(4) & \mathrm{C} 8 & \mathrm{C} 7 & 1.366(5) \\ \mathrm{C} 23 & \mathrm{C} 22 & 1.372(5) & \mathrm{C} 35 & \mathrm{C} 36 & 1.393(4) \\ \mathrm{C} 12 & \mathrm{C} 13 & 1.384(4) & \mathrm{C} 3 & \mathrm{C} 2 & 1.367(5) \\ \mathrm{C} 1 & \mathrm{C} 10 & 1.422(4) & \mathrm{C} 3 & \mathrm{C} 4 & 1.420(5)\end{array}$

Table 5 Bond Angles for exp_1491.

\begin{tabular}{lllrlllr} 
Atom Atom Atom & \multicolumn{1}{c}{ Angle $^{\circ}$} & \multicolumn{3}{c}{ Atom Atom Atom } & Angle $^{\circ}$ \\
C18 & O3 & C19 & $114.9(2)$ & C9 & C10 & C1 & $119.5(3)$ \\
C37 & O4 & C38 & $116.1(3)$ & C33 & C32 & Br2 & $117.7(2)$ \\
C9 & O1 & C17 & $118.1(3)$ & C33 & C32 & C31 & $123.5(3)$
\end{tabular}




$\begin{array}{lll}\text { C21 } & \text { O6 } & \text { C30 } \\ \text { C12 } & \text { C11 } & \text { C10 } \\ \text { C12 } & \text { C11 } & \text { C16 } \\ \text { C16 } & \text { C11 } & \text { C10 } \\ \text { O3 } & \text { C18 } & \text { C16 } \\ \text { O2 } & \text { C18 } & \text { O3 } \\ \text { O2 } & \text { C18 } & \text { C16 } \\ \text { C24 } & \text { C25 } & \text { C20 } \\ \text { C26 } & \text { C25 } & \text { C24 } \\ \text { C26 } & \text { C25 } & \text { C20 } \\ \text { C26 } & \text { C27 } & \text { C28 } \\ \text { C22 } & \text { C23 } & \text { C24 } \\ \text { C11 } & \text { C12 } & \text { Br1 } \\ \text { C13 } & \text { C12 } & \text { Br1 } \\ \text { C13 } & \text { C12 } & \text { C11 } \\ \text { C10 } & \text { C1 } & \text { C6 } \\ \text { C2 } & \text { C1 } & \text { C10 } \\ \text { C2 } & \text { C1 } & \text { C6 } \\ \text { C29 } & \text { C28 } & \text { C27 } \\ \text { C34 } & \text { C33 } & \text { C32 } \\ \text { C12 } & \text { C13 } & \text { C14 } \\ \text { C32 } & \text { C31 } & \text { C20 } \\ \text { C32 } & \text { C31 } & \text { C36 } \\ \text { C36 } & \text { C31 } & \text { C20 } \\ \text { O1 } & \text { C9 } & \text { C10 } \\ \text { O1 } & \text { C9 } & \text { C8 } \\ \text { C10 } & \text { C9 } & \text { C8 } \\ \text { O6 } & \text { C21 } & \text { C22 } \\ \text { O6 } & \text { C21 } & \text { C20 } \\ \text { C20 } & \text { C21 } & \text { C22 } \\ \text { C1 } & \text { C10 } & \text { C11 } \\ \text { C9 } & \text { C10 } & \text { C11 }\end{array}$

$\begin{array}{lllll}118.2(3) & \mathrm{C} 31 & \mathrm{C} 32 & \mathrm{~B} 2 & 118.8(2) \\ 121.6(3) & \mathrm{C} 5 & \mathrm{C} 6 & \mathrm{C} 1 & 119.2(3) \\ 116.1(3) & \mathrm{C} 5 & \mathrm{C} 6 & \mathrm{C} 7 & 122.3(3) \\ 122.2(3) & \mathrm{C} 7 & \mathrm{C} 6 & \mathrm{C} 1 & 118.5(3) \\ 111.3(2) & \mathrm{C} 28 & \mathrm{C} 29 & \mathrm{C} 24 & 120.5(3) \\ 123.2(3) & \mathrm{O} 4 & \mathrm{C} 37 & \mathrm{C} 36 & 112.2(3) \\ 125.5(3) & \mathrm{O} 5 & \mathrm{C} 37 & \mathrm{O} 4 & 123.6(3) \\ 119.1(3) & \mathrm{O} 5 & \mathrm{C} 37 & \mathrm{C} 36 & 124.1(3) \\ 118.6(3) & \mathrm{C} 14 & \mathrm{C} 15 & \mathrm{C} 16 & 120.6(3) \\ 122.3(3) & \mathrm{C} 23 & \mathrm{C} 24 & \mathrm{C} 25 & 118.9(3) \\ 120.3(3) & \mathrm{C} 29 & \mathrm{C} 24 & \mathrm{C} 25 & 119.3(3) \\ 121.6(3) & \mathrm{C} 29 & \mathrm{C} 24 & \mathrm{C} 23 & 121.8(3) \\ 119.6(2) & \mathrm{C} 23 & \mathrm{C} 22 & \mathrm{C} 21 & 119.2(3) \\ 116.8(2) & \mathrm{C} 13 & \mathrm{C} 14 & \mathrm{C} 15 & 119.9(3) \\ 123.6(3) & \mathrm{C} 4 & \mathrm{C} 5 & \mathrm{C} 6 & 121.4(3) \\ 119.7(3) & \mathrm{C} 27 & \mathrm{C} 26 & \mathrm{C} 25 & 120.8(3) \\ 122.0(3) & \mathrm{C} 25 & \mathrm{C} 20 & \mathrm{C} 31 & 121.9(3) \\ 118.2(3) & \mathrm{C} 21 & \mathrm{C} 20 & \mathrm{C} 25 & 120.0(3) \\ 120.4(3) & \mathrm{C} 21 & \mathrm{C} 20 & \mathrm{C} 31 & 118.0(3) \\ 118.5(3) & \mathrm{C} 33 & \mathrm{C} 34 & \mathrm{C} 35 & 120.0(3) \\ 118.8(3) & \mathrm{C} 7 & \mathrm{C} 8 & \mathrm{C} 9 & 120.2(3) \\ 122.1(3) & \mathrm{C} 34 & \mathrm{C} 35 & \mathrm{C} 36 & 120.0(3) \\ 116.3(3) & \mathrm{C} 2 & \mathrm{C} 3 & \mathrm{C} 4 & 120.3(3) \\ 121.4(3) & \mathrm{C} 3 & \mathrm{C} 2 & \mathrm{C} 1 & 121.2(3) \\ 115.5(3) & \mathrm{C} 8 & \mathrm{C} 7 & \mathrm{C} 6 & 121.2(3) \\ 123.7(3) & \mathrm{C} 5 & \mathrm{C} 4 & \mathrm{C} 3 & 119.6(3) \\ 120.8(3) & \mathrm{C} 11 & \mathrm{C} 16 & \mathrm{C} 18 & 120.9(3) \\ 123.0(3) & \mathrm{C} 15 & \mathrm{C} 16 & \mathrm{C} 11 & 122.1(3) \\ 115.9(3) & \mathrm{C} 15 & \mathrm{C} 16 & \mathrm{C} 18 & 121.6(3) \\ 121.1(3) & \mathrm{C} 31 & \mathrm{C} 36 & \mathrm{C} 37 & 116.2(3) \\ 121.2(3) & \mathrm{C} 35 & \mathrm{C} 36 & \mathrm{C} 31 & \\ 119.3(3) & \mathrm{C} 35 & \mathrm{C} 36 & \mathrm{C} 37 & \end{array}$

Table 6 Hydrogen Atom Coordinates $\left(\AA \times 10^{4}\right)$ and Isotropic Displacement Parameters $\left(\AA^{2} \times 10^{3}\right)$ for exp_1491.

\begin{tabular}{lrrrr} 
Atom & \multicolumn{1}{c}{$\boldsymbol{x}$} & $\boldsymbol{y}$ & $\boldsymbol{z}$ & $\boldsymbol{U}(\mathbf{e q})$ \\
H27 & 1594.19 & 5016.68 & 3444.68 & 22 \\
H23 & -1378.31 & 8248.72 & 1131.62 & 21 \\
H28 & -393.42 & 5696.85 & 3806.16 & 22 \\
H33 & 5036.59 & 5753.35 & -962.16 & 25 \\
H13 & 6048.11 & 430.61 & 5736.49 & 26 \\
H29 & -1380.7 & 7015.78 & 2723.14 & 22 \\
H15 & 6713.25 & 2371.4 & 3280.78 & 23
\end{tabular}




$\begin{array}{lrrrr}\text { H22 } & -338.49 & 8996.81 & -224.09 & 23 \\ \text { H14 } & 7466.51 & 1150.25 & 4622.77 & 26 \\ \text { H5 } & -695.04 & 4074.65 & 5250.33 & 33 \\ \text { H26 } & 2656.01 & 5745.29 & 2092.96 & 19 \\ \text { H34 } & 6752.26 & 6645.03 & 62.88 & 29 \\ \text { H8 } & -112.27 & 1825.98 & 2378.42 & 28 \\ \text { H38A } & 2893.09 & 9391.91 & 3399.83 & 38 \\ \text { H38B } & 4108.64 & 8958.34 & 4182.66 & 38 \\ \text { H38C } & 2733.7 & 8461.45 & 4207.04 & 38 \\ \text { H35 } & 6348.96 & 7801.89 & 1446.39 & 27 \\ \text { H3 } & 2848.96 & 4406.15 & 7033.95 & 29 \\ \text { H2 } & 3699.72 & 3237.01 & 5910.63 & 36 \\ \text { H19A } & 5475.28 & 4194.21 & 686.8 & 36 \\ \text { H19B } & 5012.9 & 4858.87 & 1644.58 & 36 \\ \text { H19C } & 4016.75 & 4153.87 & 888.48 & 30 \\ \text { H7 } & -984.27 & 2905.62 & 3579.03 & 34 \\ \text { H4 } & 621.64 & 4819.44 & 6714.06 & 35 \\ \text { H30A } & 696.42 & 9134.51 & -1777.15 & 35 \\ \text { H30B } & 2062.15 & 9636.08 & -1833.82 & 35 \\ \text { H30C } & 1240.55 & 9985.41 & -905.44 & 41 \\ \text { H17A } & 1132.76 & 1264.92 & 1071.5 & 41 \\ \text { H17B } & 922.89 & 325.13 & 1852.15 & 1167.07 \\ \text { H17C } & 2097.8 & 321.55 & & \end{array}$




\section{References}

1. Liang, X.-W.; Zhao, Y.; Si, X.-G.; Xu, M.-M.; Tan, J.-H.; Zhang, Z.-M.; Zheng. C.G.; Zheng, C.; Cai, Q. Enantioselective Synthesis of Arene cis-Dihydrodiols from 2-Pyrones. Angew. Chem. Int. Ed. 2019, 58, 14562-14567.

2. Zhang, Y.; Hsung, R. P.; Tracey, M. R.; Kurtz, K. C. M.; Vera, E. L. Copper SulfatePentahydrate-1,10-Phenanthroline Catalyzed Amidations of Alkynyl Bromides. Synthesis of Heteroaromatic Amine Substituted Ynamides. Org. Lett. 2004, 6, $1151-1154$.

3. (a) Nishida, G.; Noguchi, K.; Hirano, M.; Tanaka, K. Asymmetric Assembly of Aromatic Rings To Produce Tetra-ortho-Substituted Axially Chiral Biaryl Phosphorus Compounds. Angew. Chem. Int. Ed. 2007, 46, 3951-3954. (b) Zheng, S.-C.; Wang, Q.; Zhu, J. Catalytic Atropenantioselective Heteroannulation between Isocyanoacetates and Alkynyl Ketones: Synthesis of Enantioenriched Axially Chiral 3-Arylpyrroles. Angew. Chem. Int. Ed. 2019, 58, 1494-1498.

4. Aikawa, K.; Hioki, Y.; Shimizu, N.; Mikami, K. Catalytic Asymmetric Synthesis of Stable Oxetenes via Lewis Acid-Promoted [2 + 2] Cycloaddition. J. Am. Chem. Soc. 2011, 133, 20092-20095.

5. Jiang, Q.; Li, H.; Zhang, X.; Xu, B.; Su, W. Pd-Catalyzed Decarboxylative Sonogashira Reaction via Decarboxylative Bromination. Org. Lett. 2018, 20, $2424-2427$.

6. Zhao, D.; Gao, C.; Su, X.; He, Y.; You, J.; Xue, Y. Copper-catalyzed decarboxylative cross-coupling of alkynyl carboxylic acids with aryl halides. Chem. Commun., 2010, 46, 9049-9051.

7. Frisch, M. J.; Trucks, G. W.; Schlegel, H. B.; Scuseria, G. E.; Robb, M. A.; Cheeseman, J. R.; Scalmani, G.; Barone, V.; Petersson, G. A.; Nakatsuji, H.; Li, X.; Caricato, M.; Marenich, A. V.; Bloino, J.; Janesko, B. G.; Gomperts, R.; Mennucci, B.; Hratchian, H. P.; Ortiz, J. V.; Izmaylov, A. F.; Sonnenberg, J. L.; WilliamsYoung, D.; Ding, F.; Lipparini, F.; Egidi, F.; Goings, J.; Peng, B.; Petrone, A.; Henderson, T.; Ranasinghe, D.; Zakrzewski, V. G.; Gao, J.; Rega, N.; Zheng, G.; Liang, W.; Hada, M.; Ehara, M.; Toyota, K.; Fukuda, R.; Hasegawa, J.; Ishida, M.; Nakajima, T.; Honda, Y.; Kitao, O.; Nakai, H.; Vreven, T.; Throssell, K.; Montgomery, J. A., Jr.; Peralta, J. E.; Ogliaro, F.; Bearpark, M. J.; Heyd, J. J.; Brothers, E. N.; Kudin, K. N.; Staroverov, V. N.; Keith, T. A.; Kobayashi, R.; 
Normand, J.; Raghavachari, K.; Rendell, A. P.; Burant, J. C.; Iyengar, S. S.; Tomasi, J.; Cossi, M.; Millam, J. M.; Klene, M.; Adamo, C.; Cammi, R.; Ochterski, J. W.; Martin, R. L.; Morokuma, K.; Farkas, O.; Foresman, J. B.; Fox, D. J. Gaussian, Inc., Wallingford CT, 2016.

8. Chai, J.-D.; Head-Gordon, M. Long-Range Corrected Hybrid Density Functionals with Damped Atom-Atom Dispersion Corrections. Phys. Chem. Chem. Phys. 2008, 10, 6615-6620.

9. Hay, P. J.; Wadt, W. R. Ab initio Effective Core Potentials for Molecular Calculations. Potentials for $\mathrm{K}$ to $\mathrm{Au}$ Including the Outermost Core Orbitals. $J$. Chem. Phys. 1985, 82, 299-310.

10. Neese, F.; Wennmohs, F.; Becker, U.; Riplinger, C. The ORCA quantum chemistry program package. J. Chem. Phys. 2020, 152, 224108.

11. Mardirossian, N.; Head-Gordon, M. $\omega \mathrm{B} 97 \mathrm{M}-\mathrm{V}$ : A combinatorially optimized, range-separated hybrid, meta-GGA density functional with VV10 nonlocal correlation. J. Chem. Phys. 2016, 144, 214110.

12. Weigend, F.; Ahlrichs, R. Balanced basis sets of split valence, triple zeta valence and quadruple zeta valence quality for $\mathrm{H}$ to $\mathrm{Rn}$ : Design and assessment of accuracy. Phys. Chem. Chem. Phys. 2005, 7, 3297-3305.

13. Marenich, A. V.; Cramer, C. J.; Truhlar, D. G. Universal Solvation Model Based on Solute Electron Density and on a Continuum Model of the Solvent Defined by the Bulk Dielectric Constant and Atomic Surface Tensions. J. Phys. Chem. B 2009, 113, 6378-6396.

14. Legault, C. Y. CYLview, 1.0b; Université de Sherbrooke, 2009 (http://www.cylview.org). 


\section{Copies of NMR Spectra}

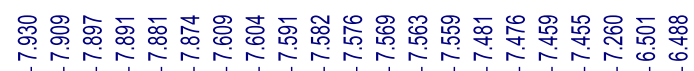

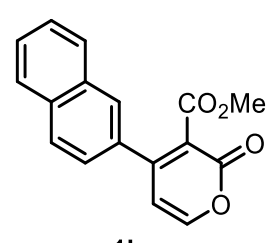

$1 \mathrm{~h}$

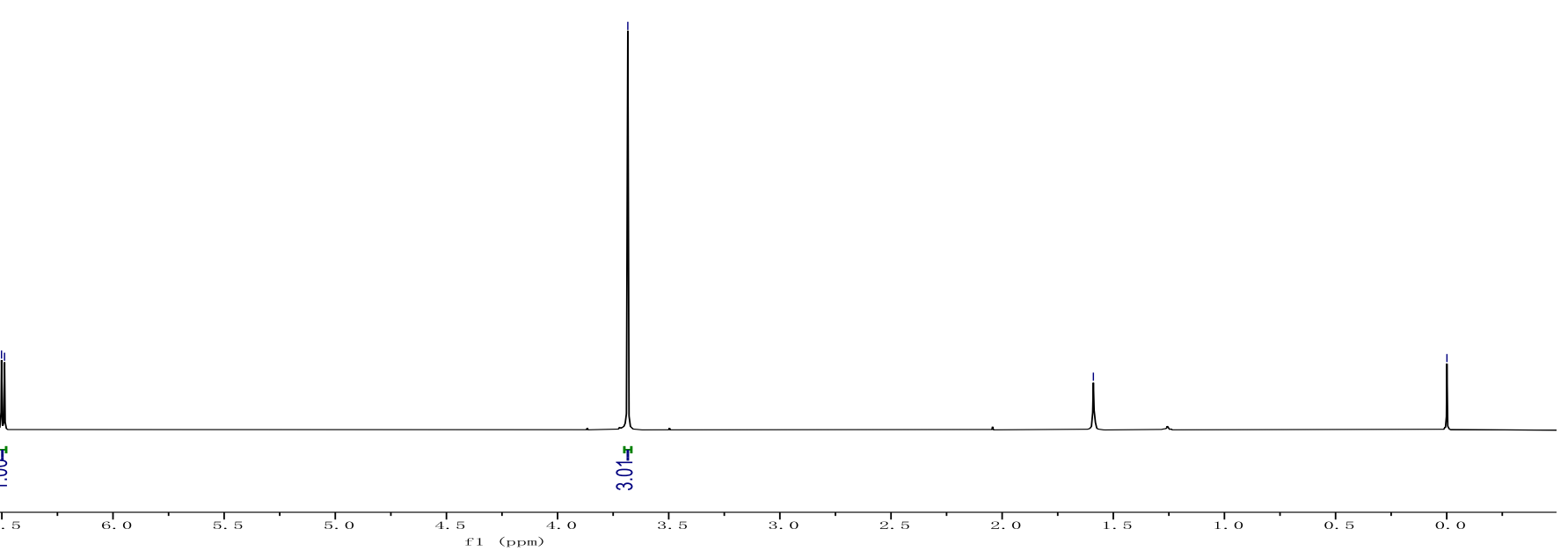




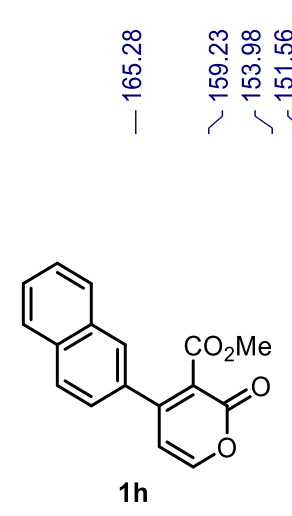

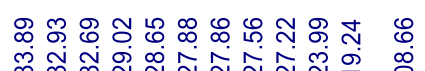

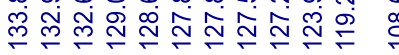

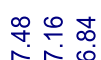

송

空

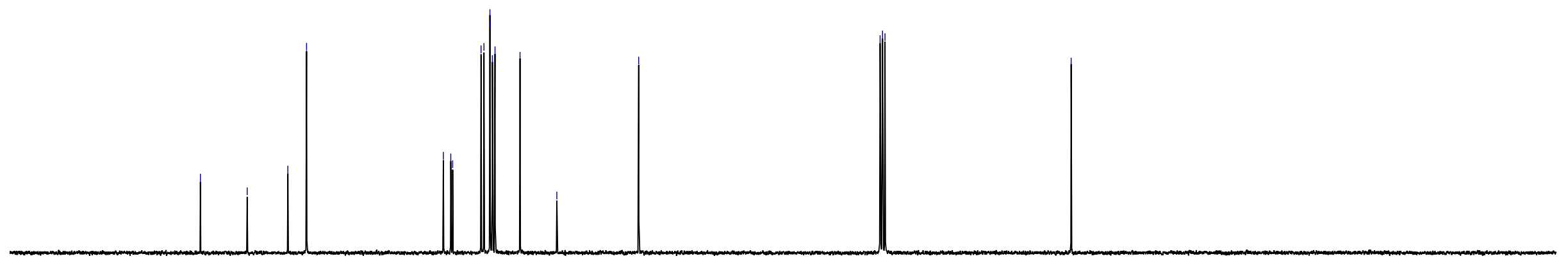




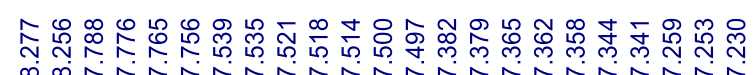

$\stackrel{\sim}{\sim}$

$\stackrel{\text { Ñ }}{\text { I }}$

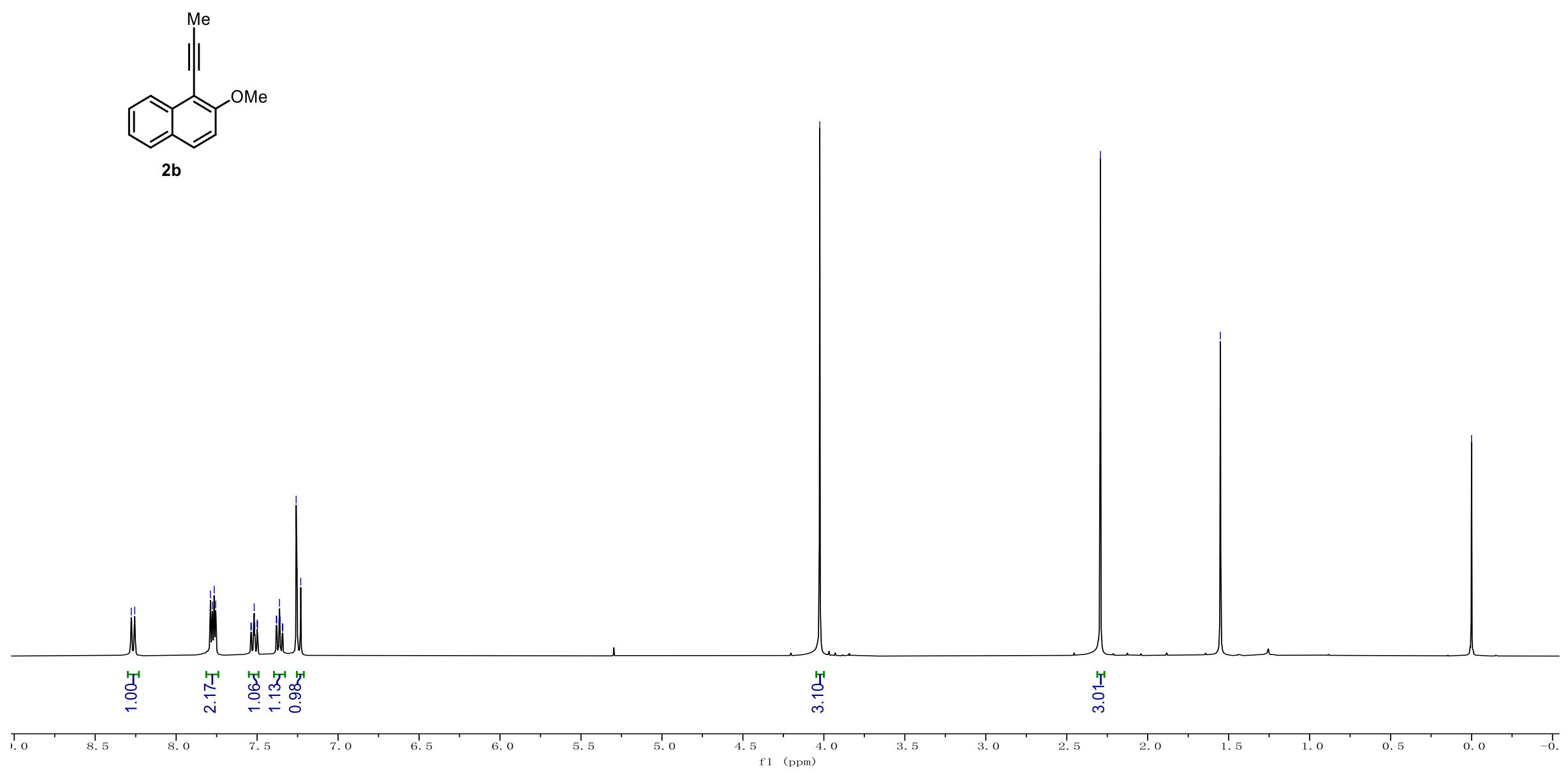



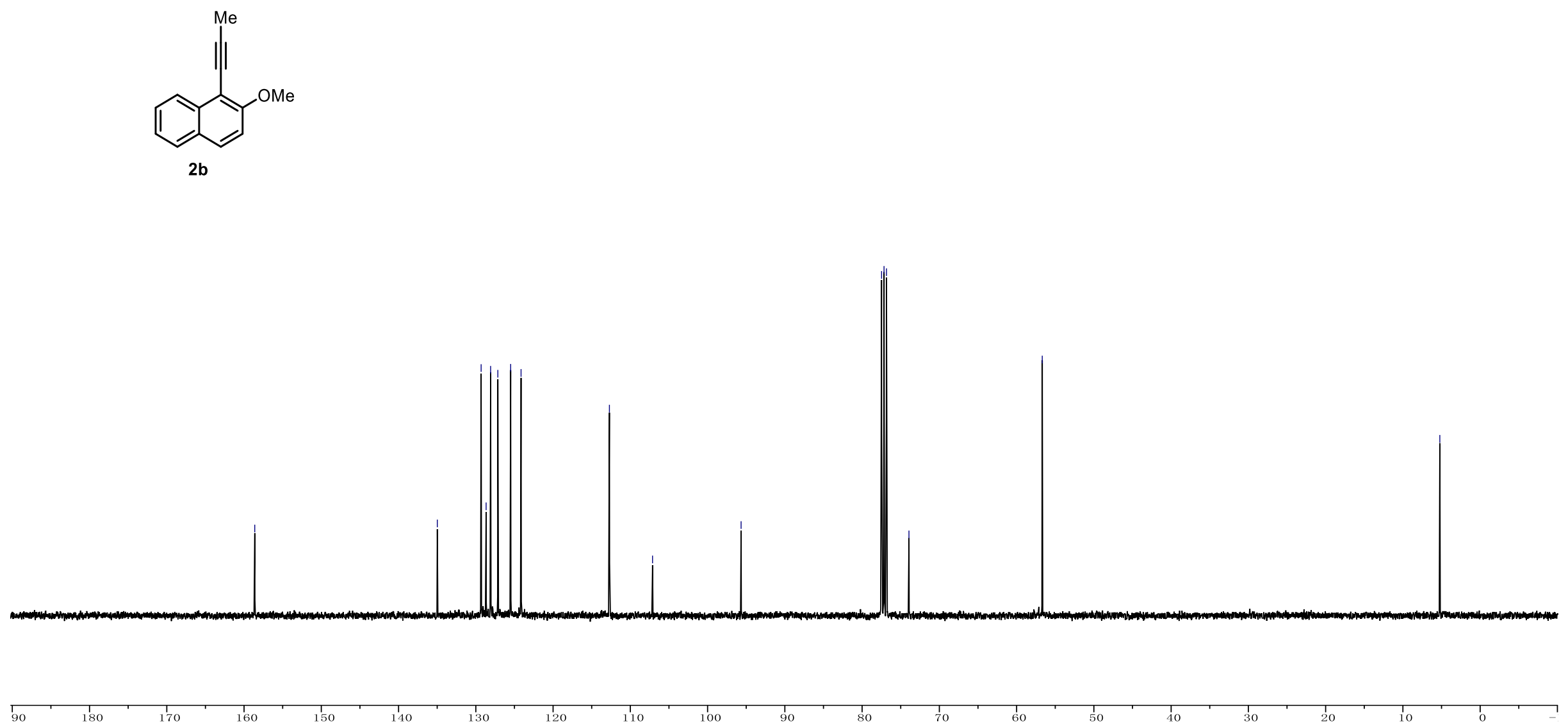

120
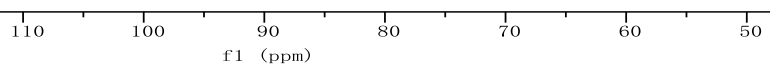

1,40

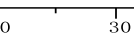

$10 \quad{ }_{20}^{1}$ 


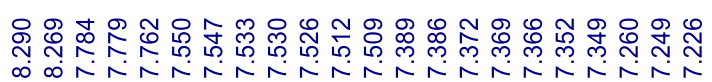

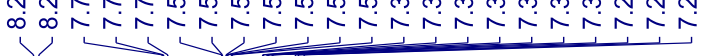

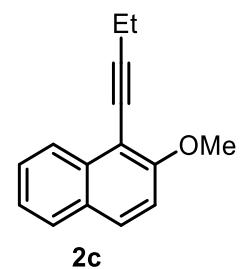

2c

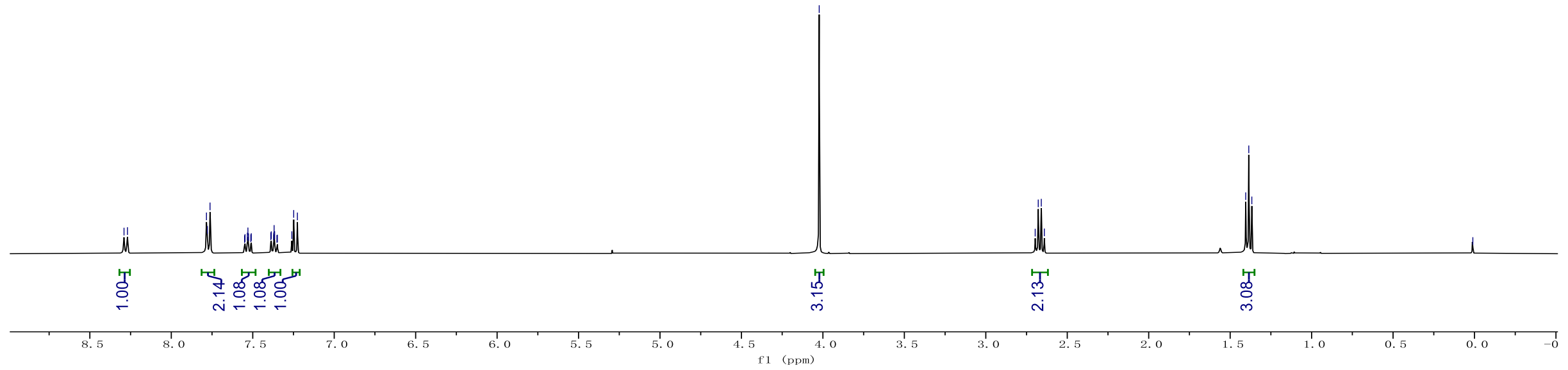



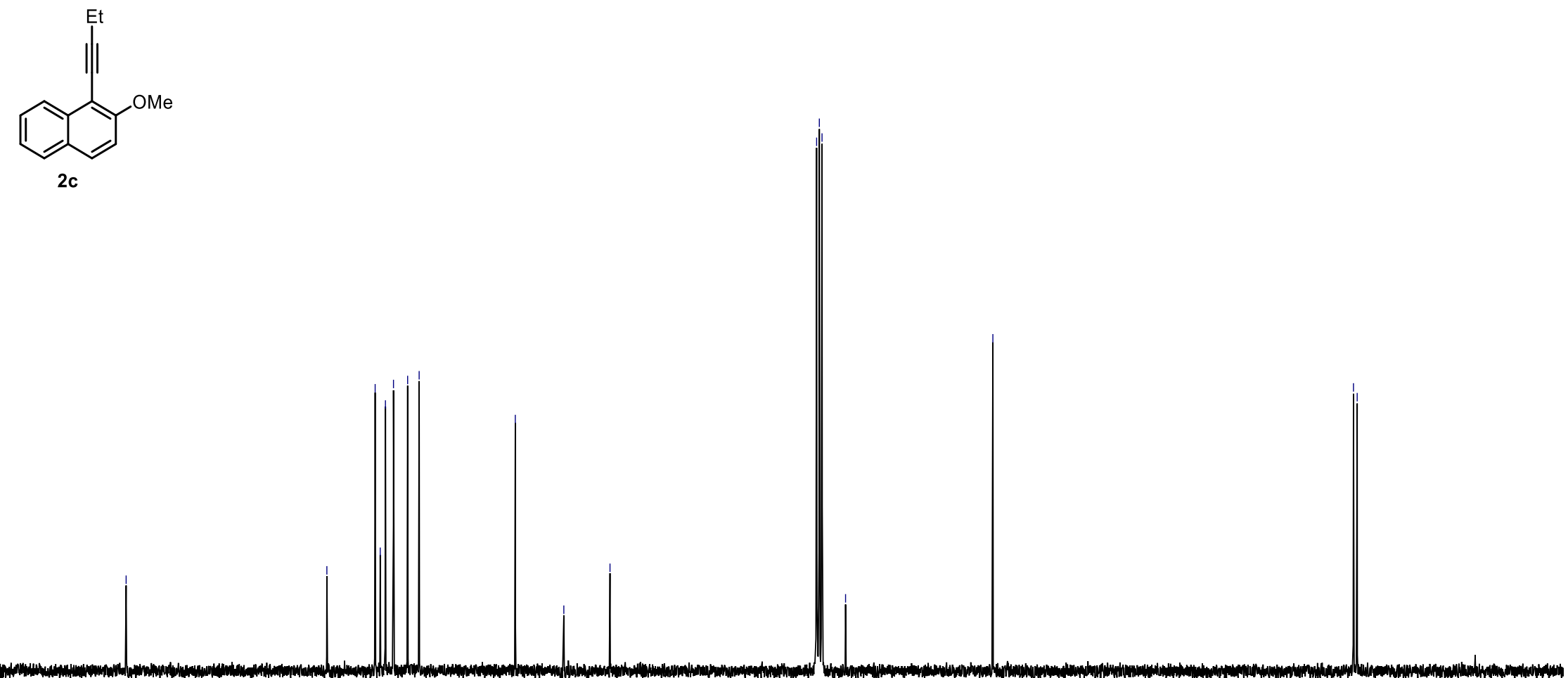

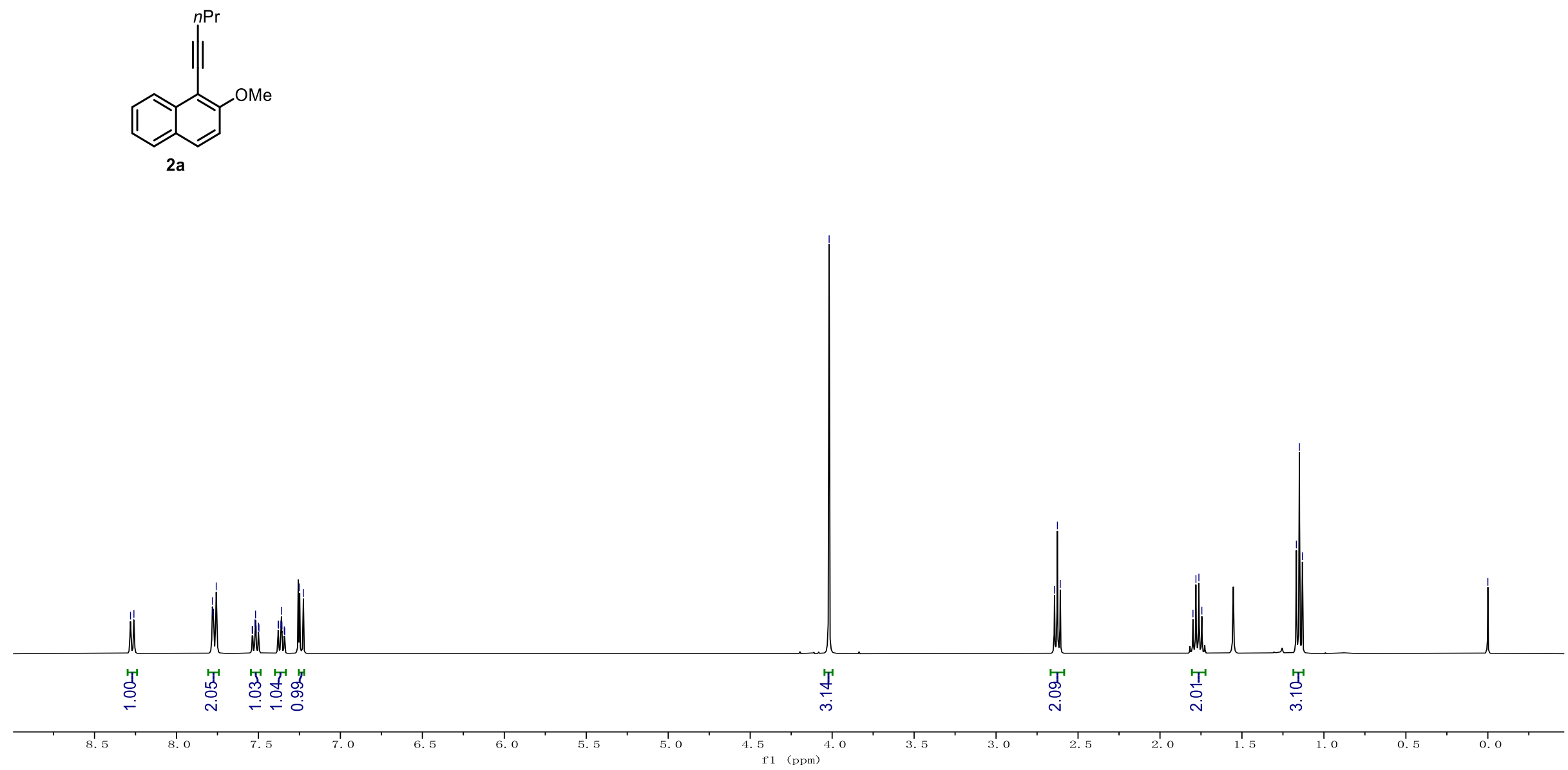
ஜ
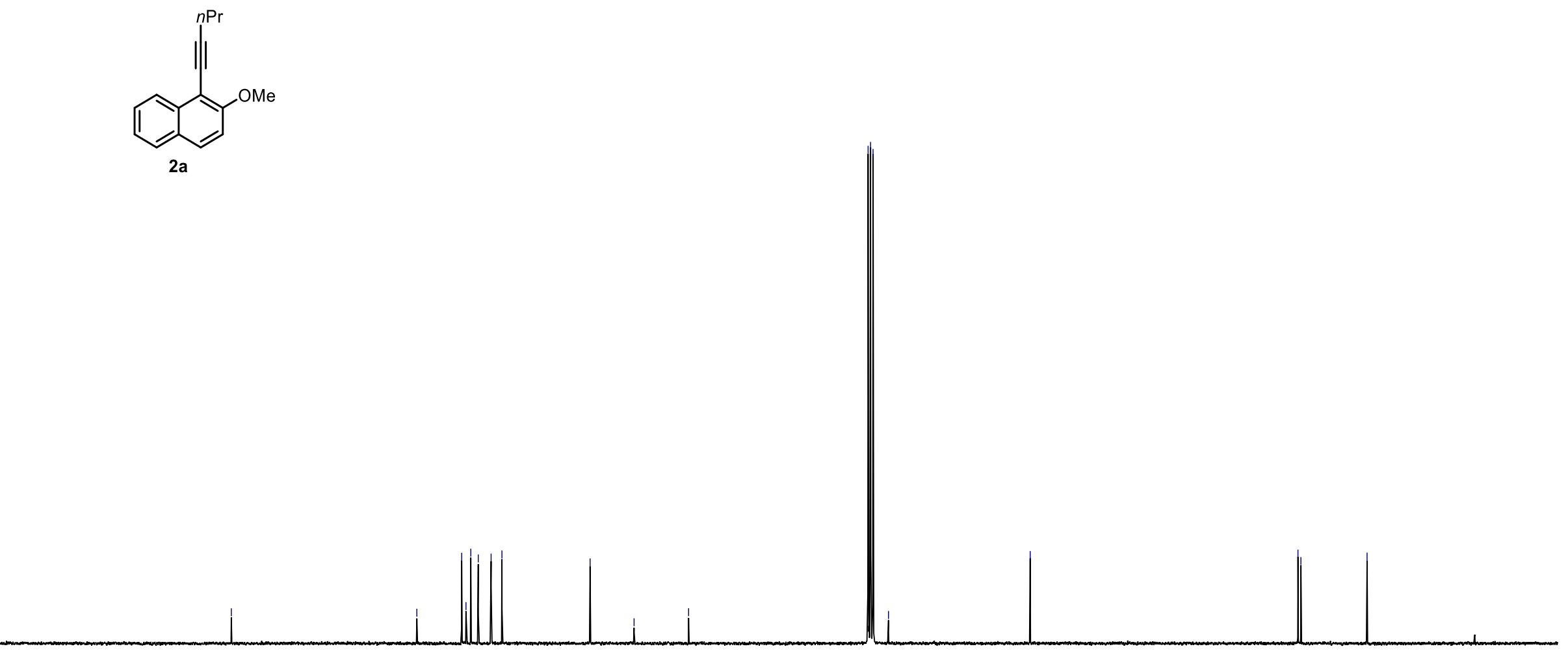


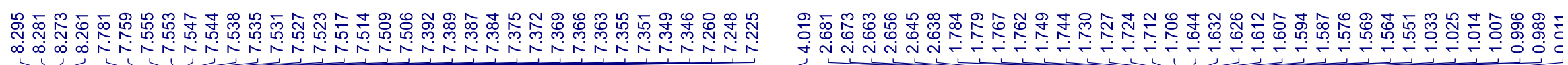
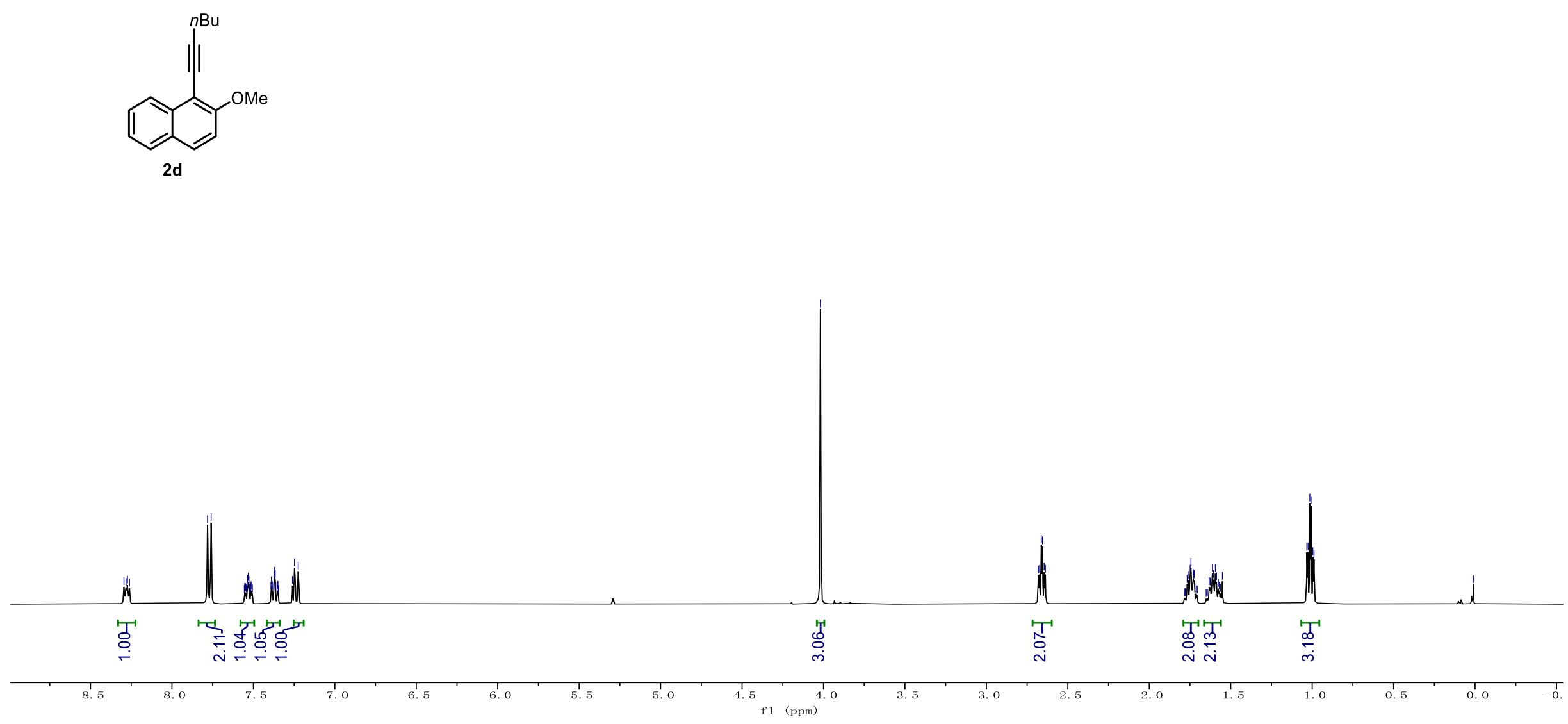


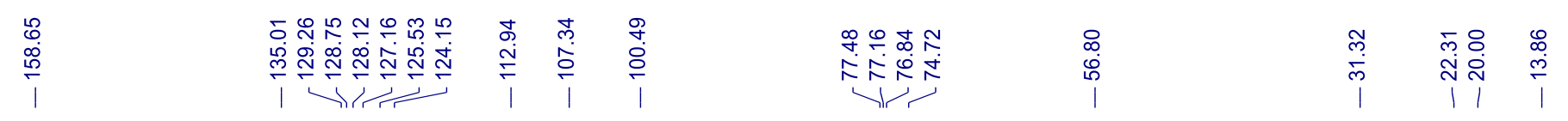
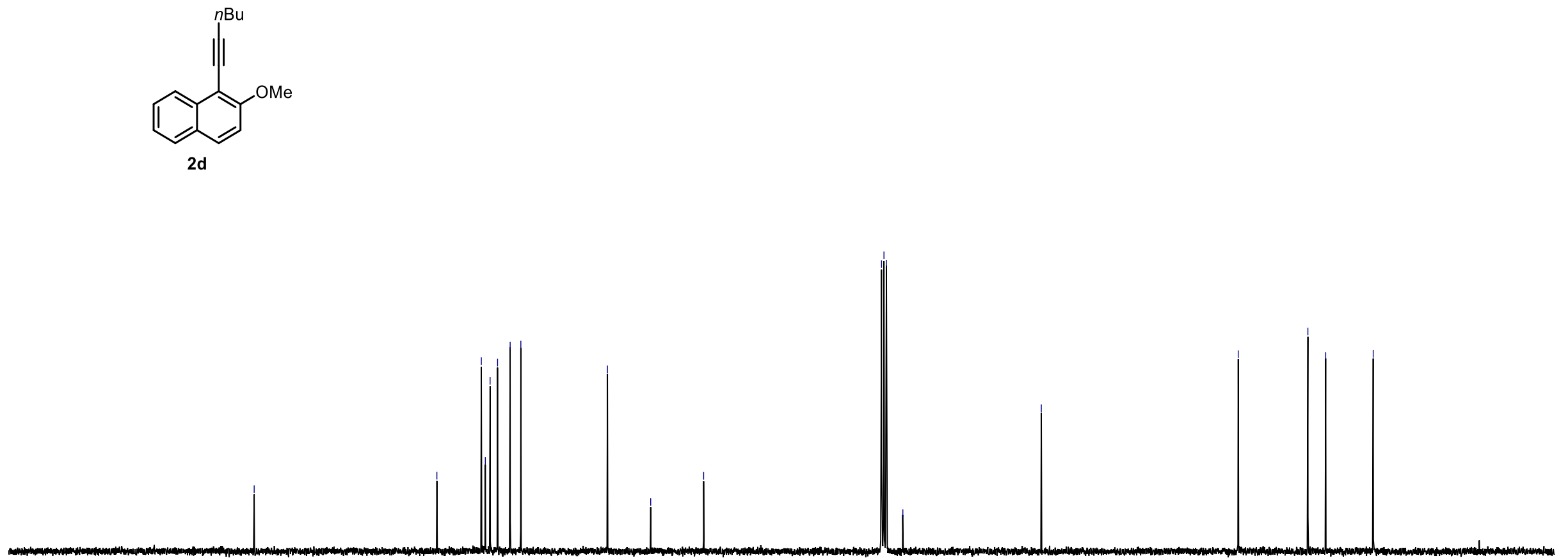

90

150

$140 \quad 130$

${ }_{120}^{1}$

90 

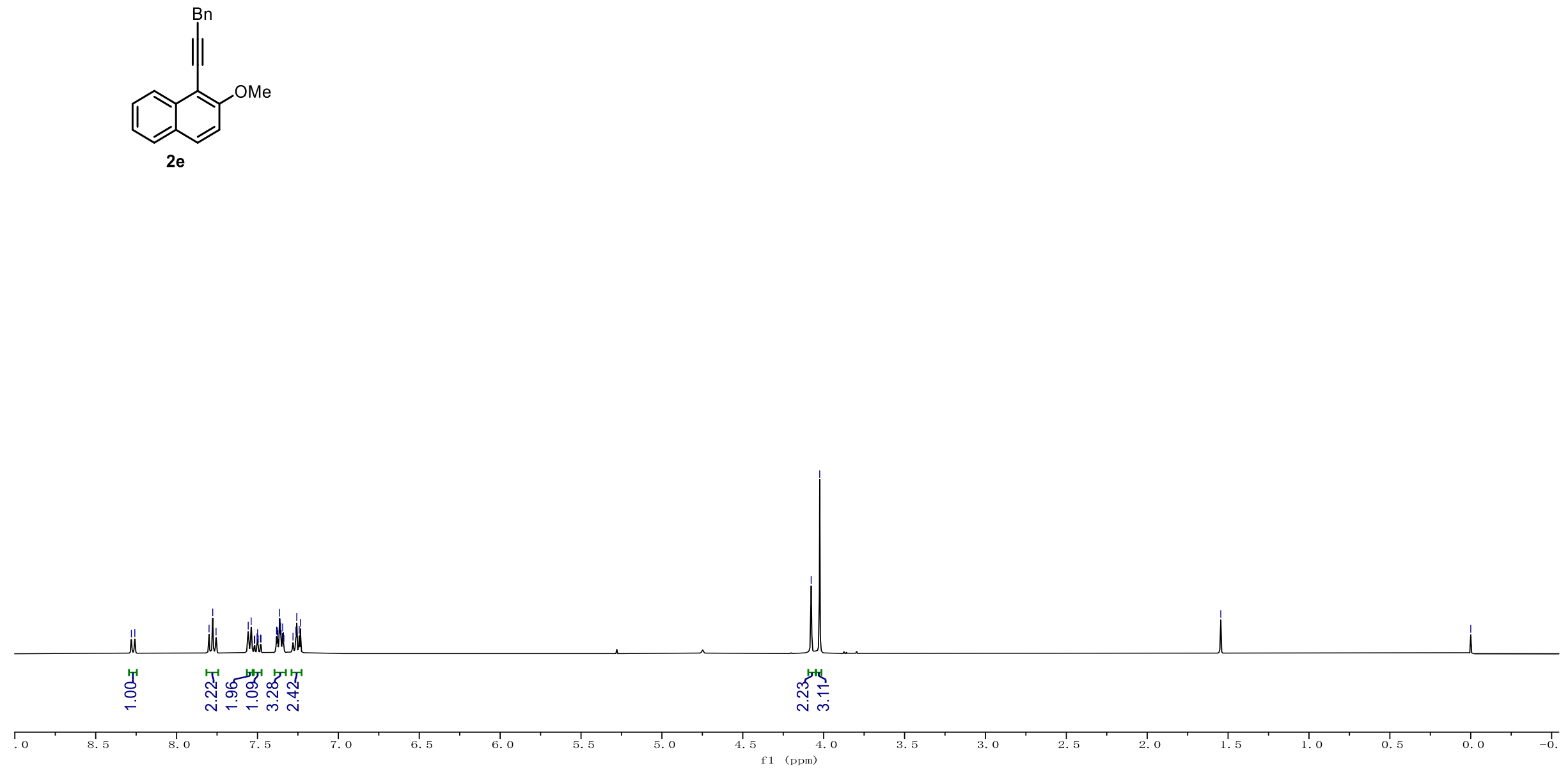


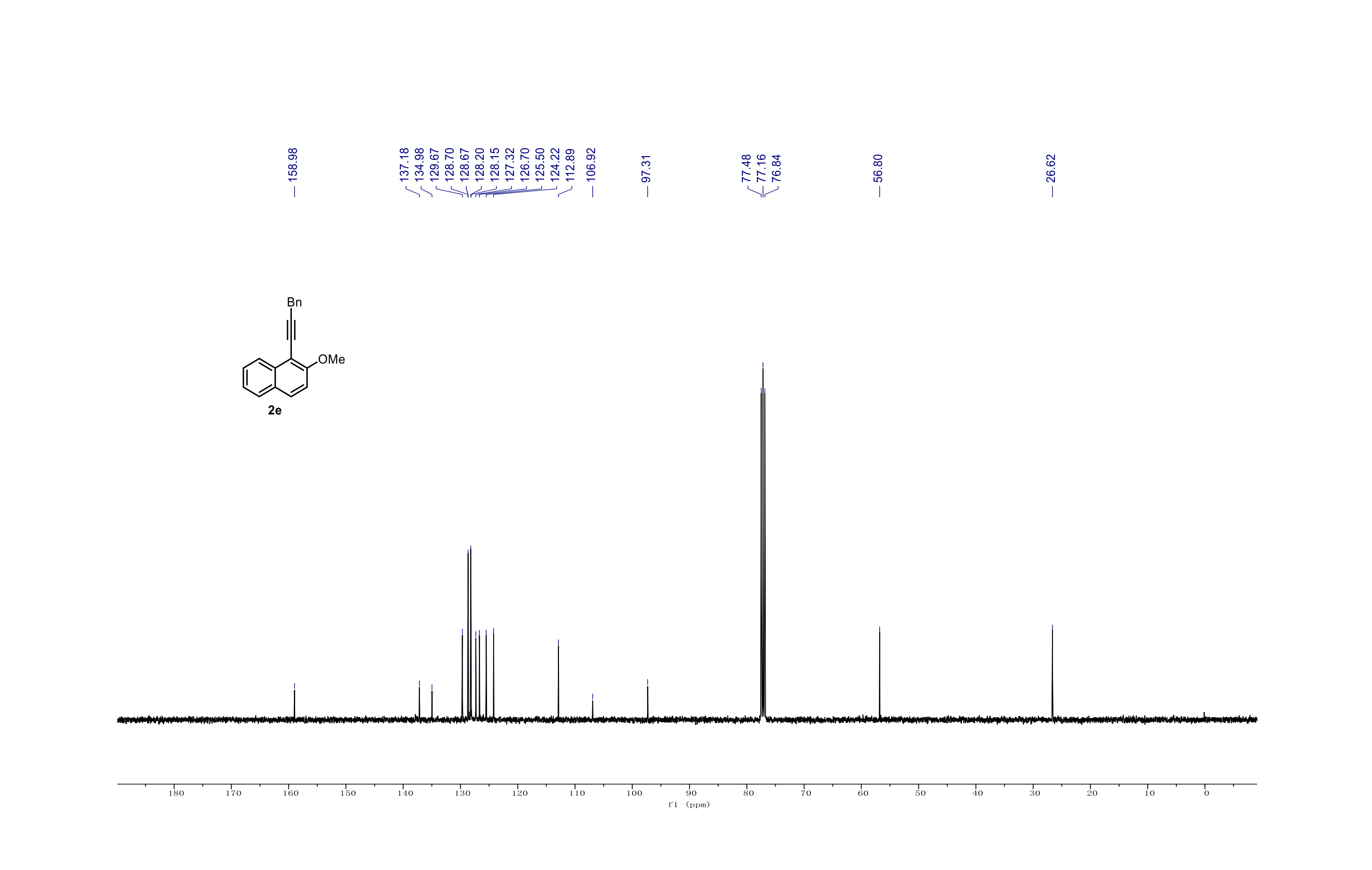




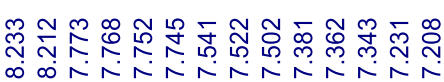

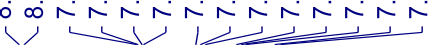

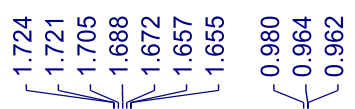

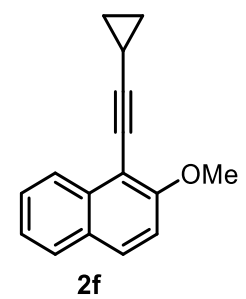

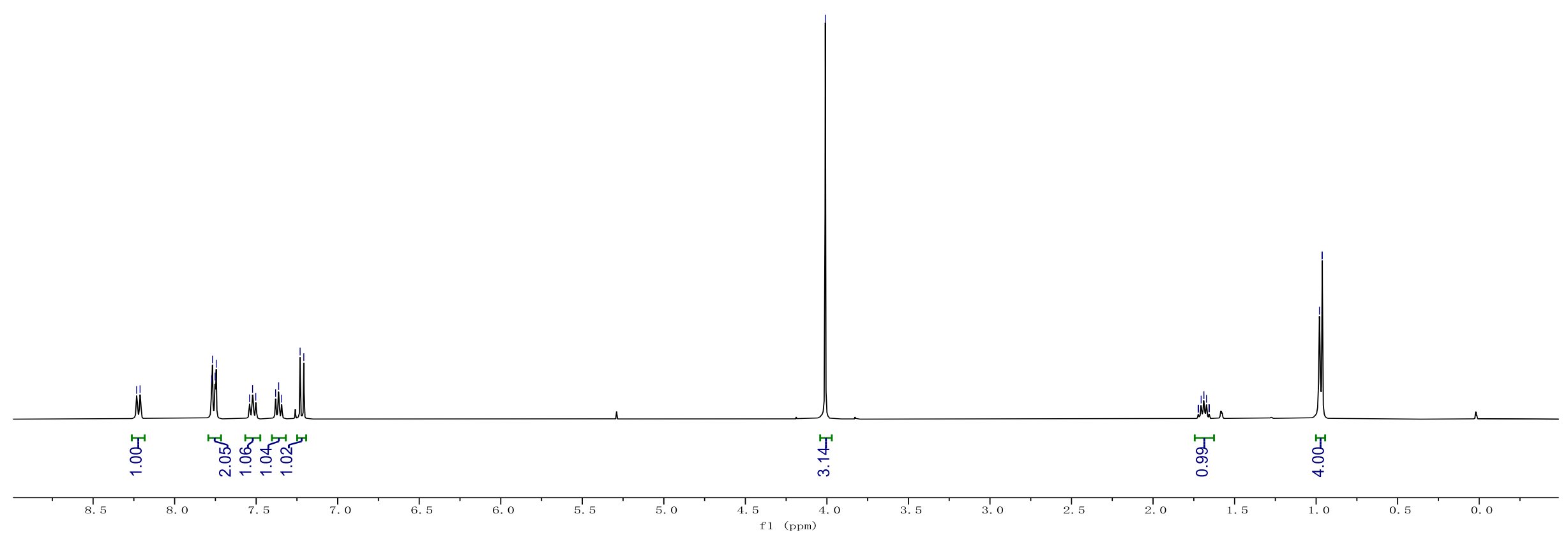



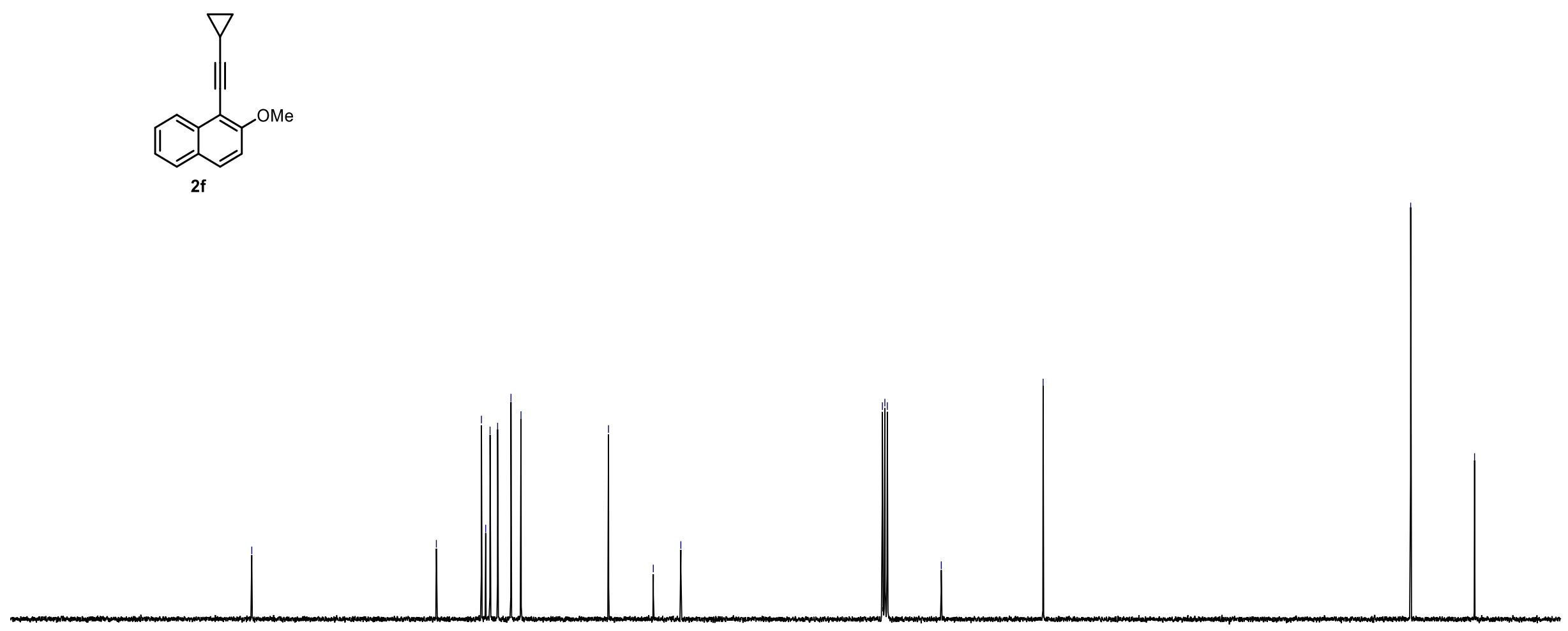

90 


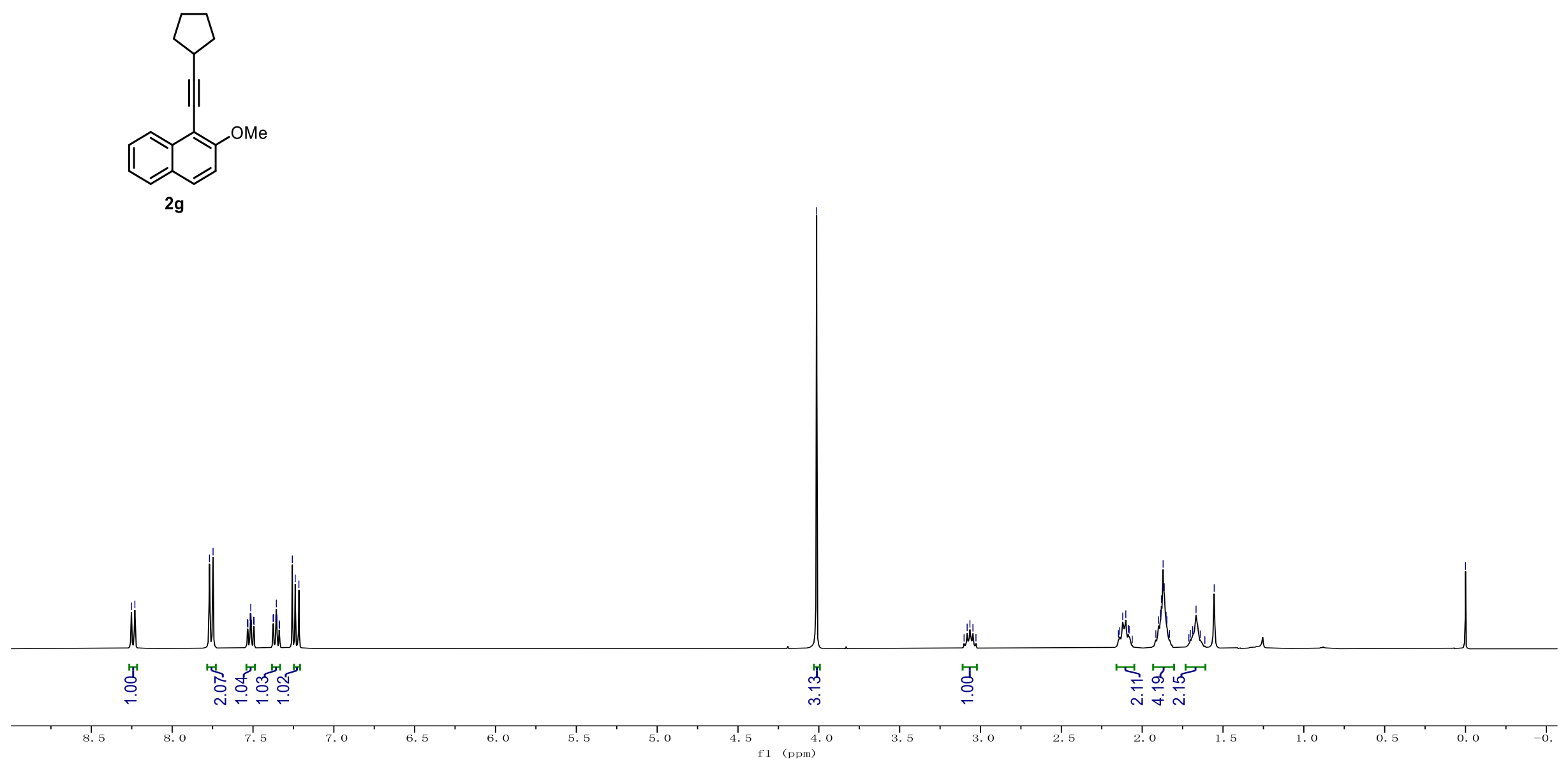




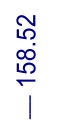

|

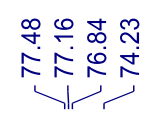

\[ 0 \]
0
0
0
0

承苍

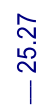

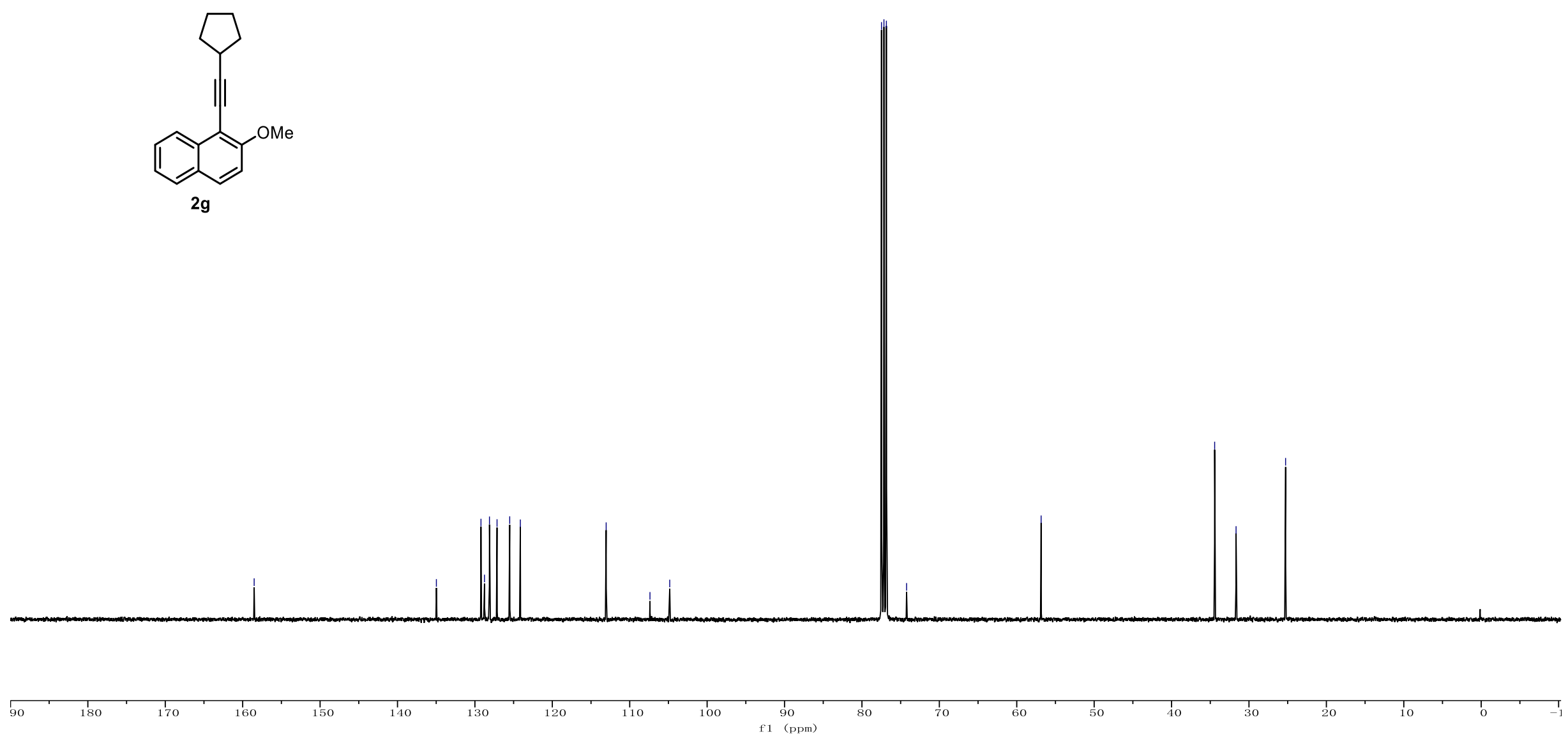

150 

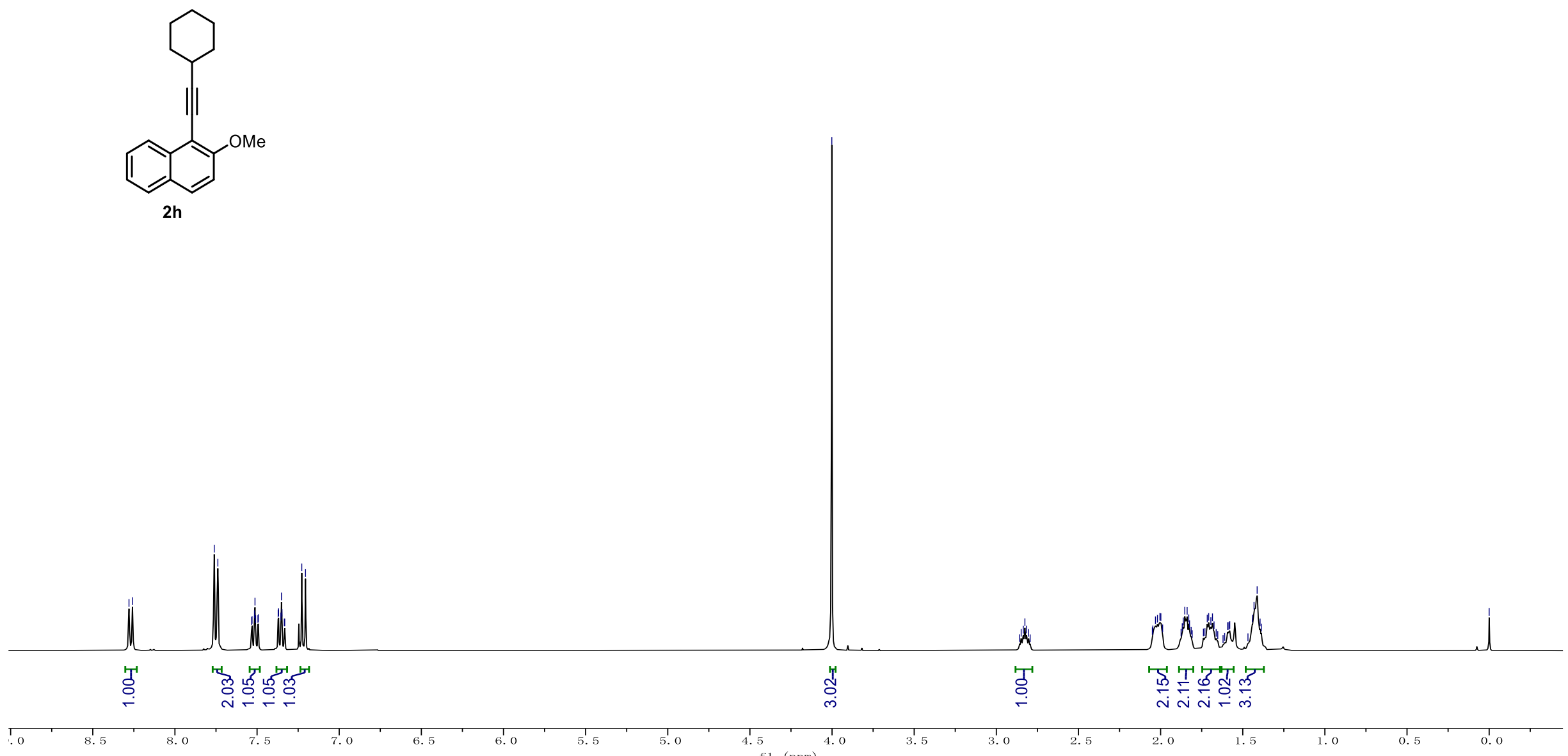


\begin{tabular}{|c|c|c|c|c|c|}
\hline $\begin{array}{l}8 \\
0 \\
\infty \\
\infty \\
\infty \\
1 \\
1\end{array}$ & 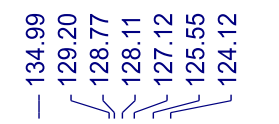 & 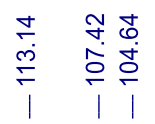 & 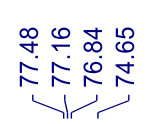 & $\begin{array}{l}\infty \\
\infty \\
0 \\
0 \\
0 \\
1\end{array}$ & 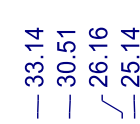 \\
\hline
\end{tabular}
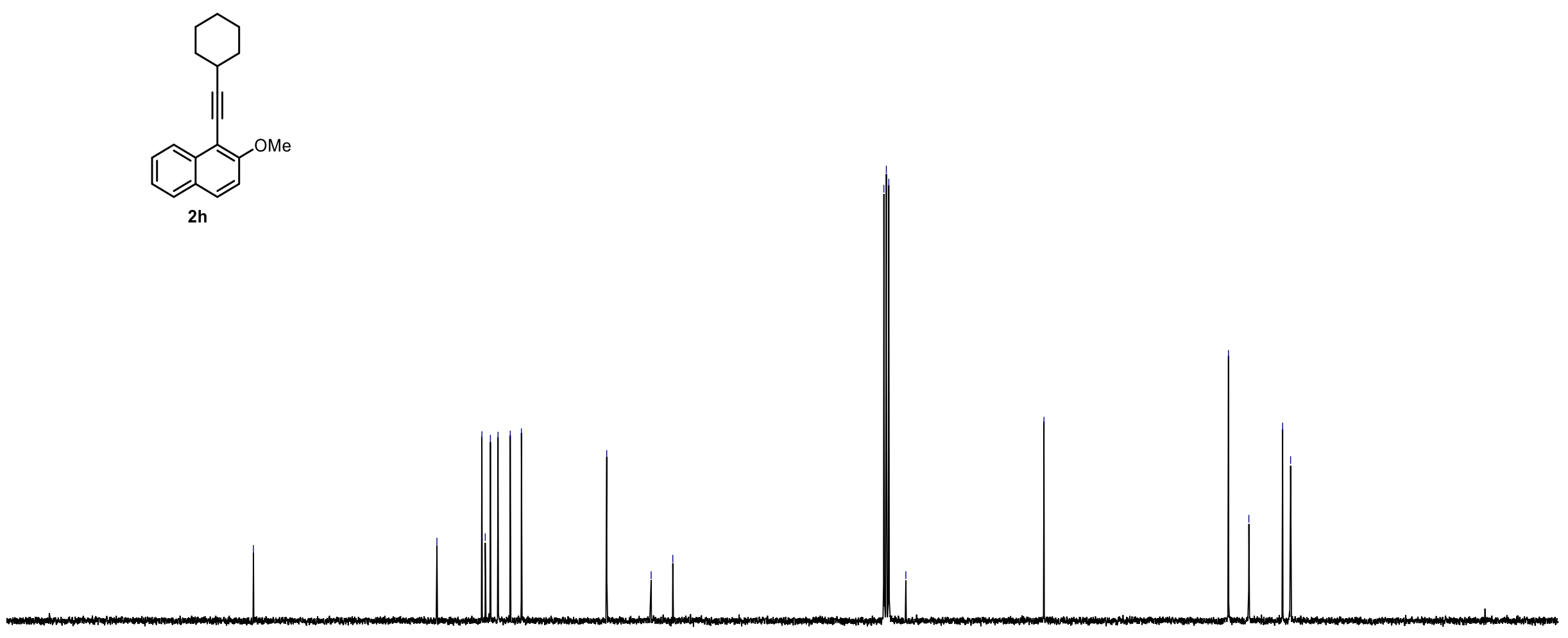


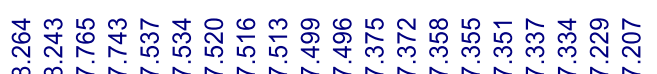

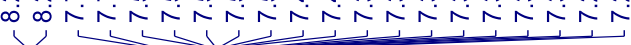

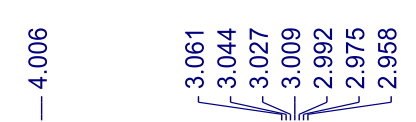

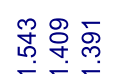

it

8
$\vdots$
0
1
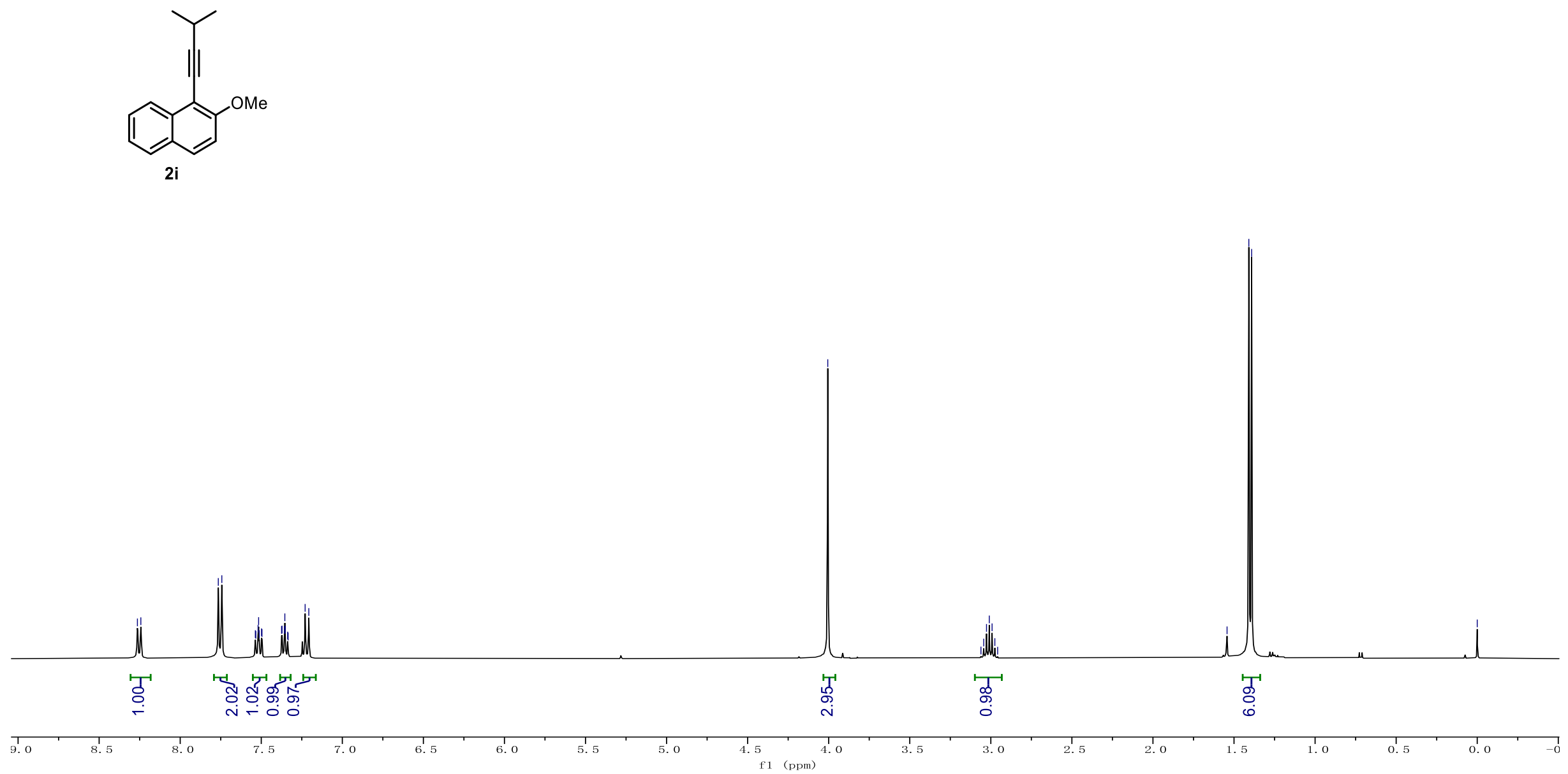

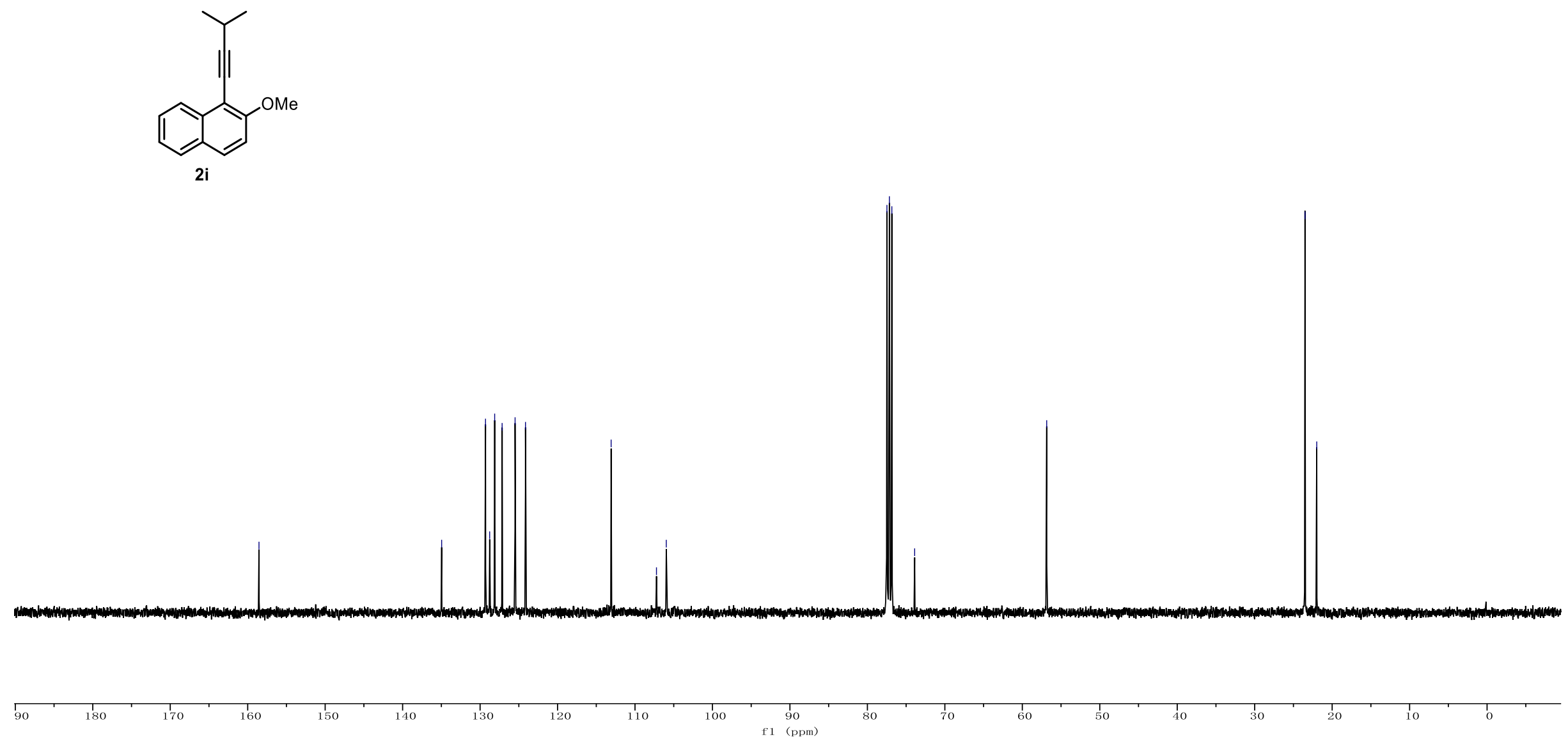


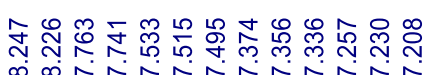

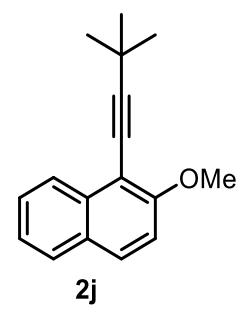

$\sum_{42}^{2}$

为

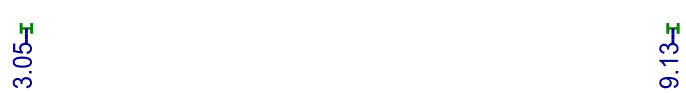

\begin{tabular}{llll}
\hline 1.0 & 8.5 & 1.0 & 1.5
\end{tabular} 


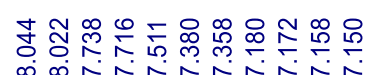

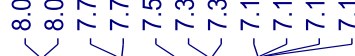

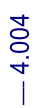
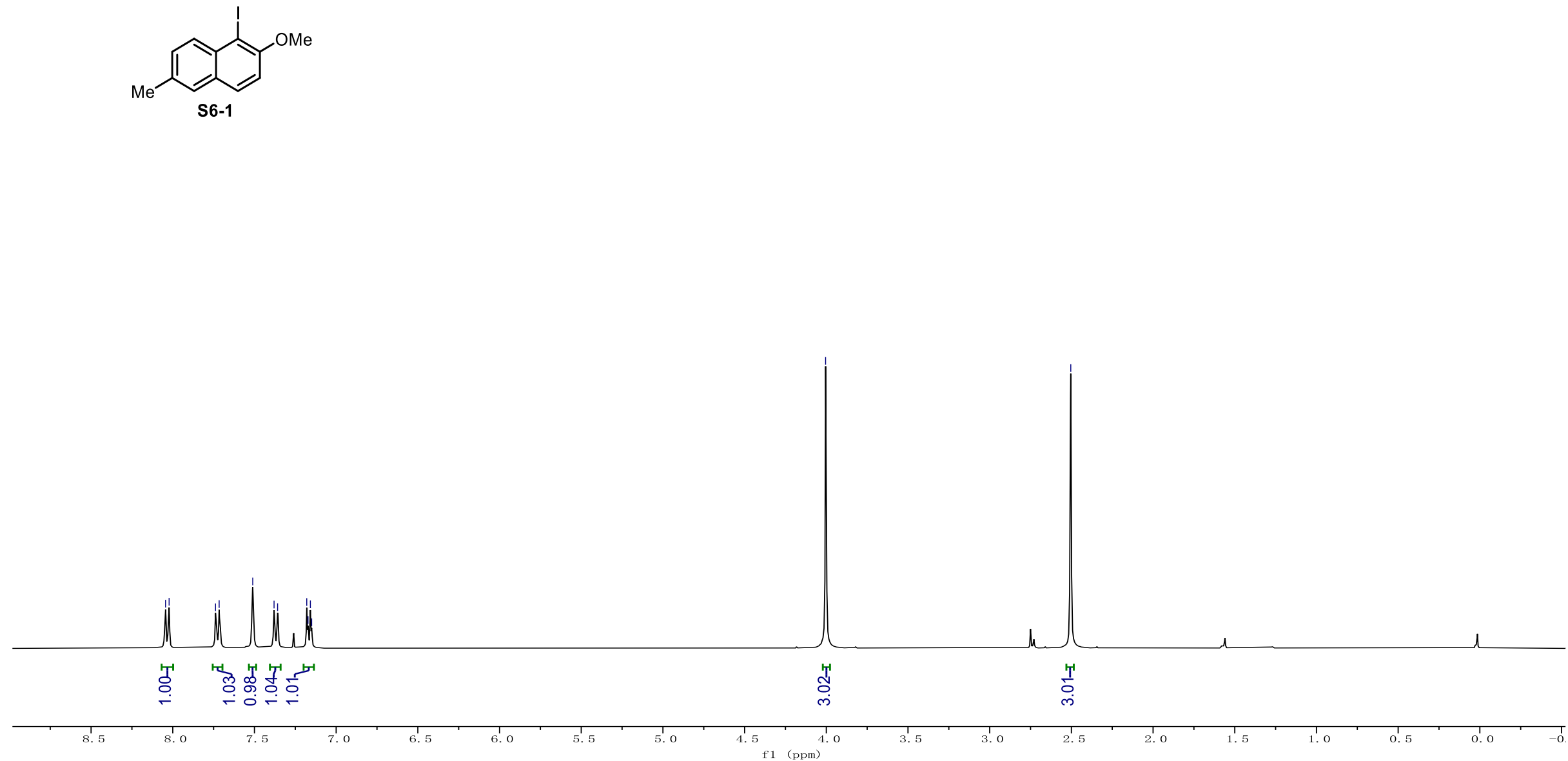

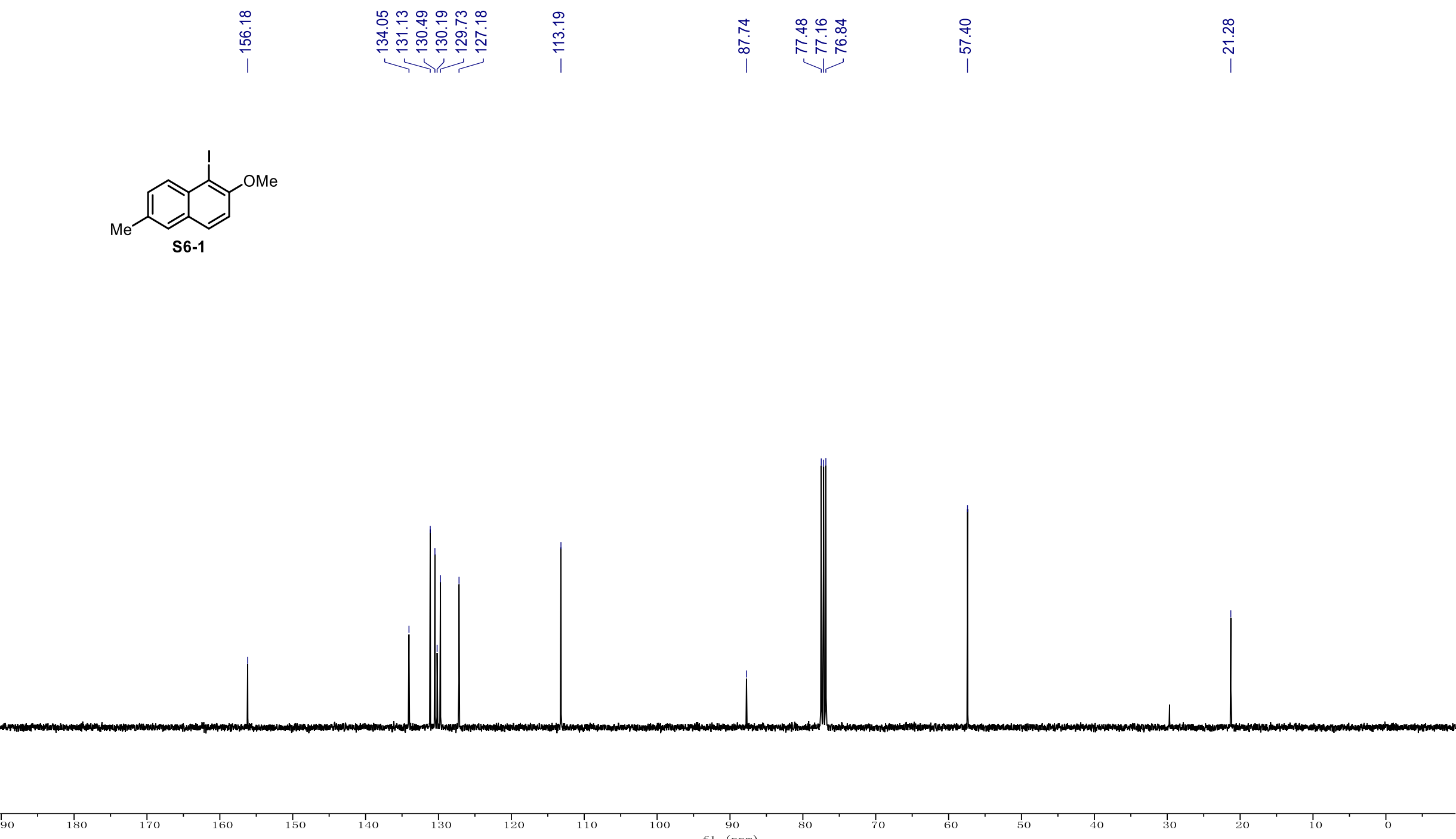
象早

욝
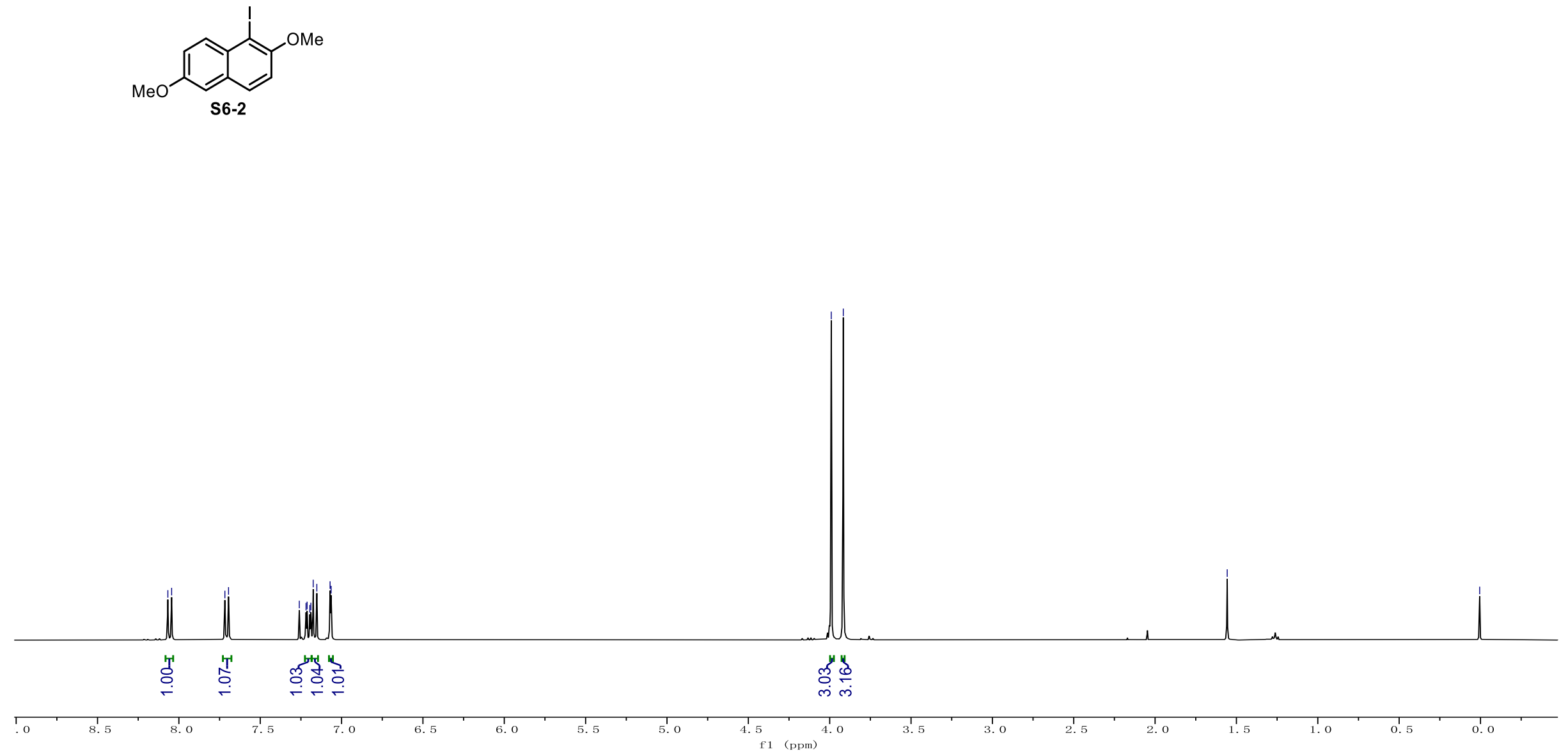


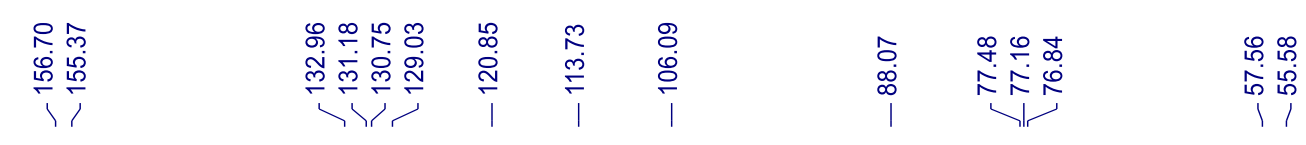
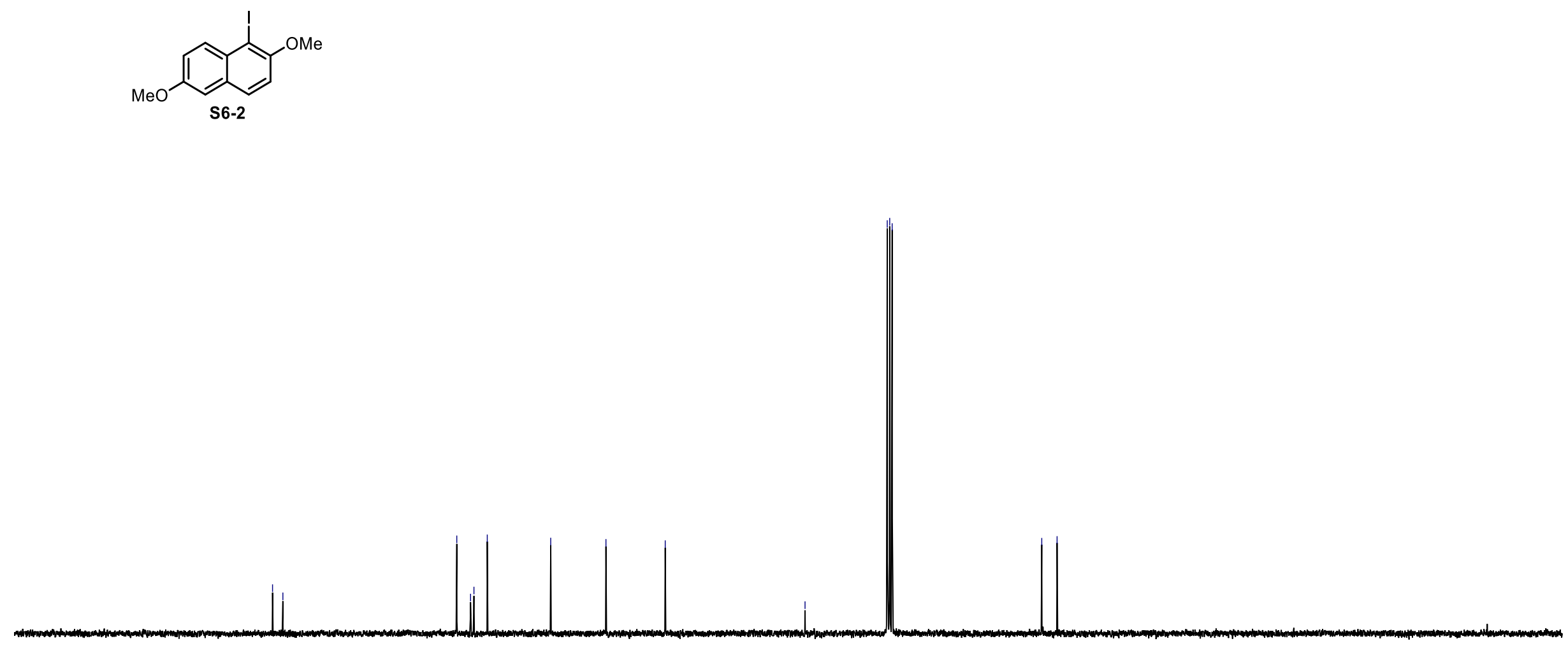

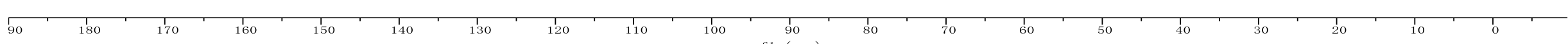




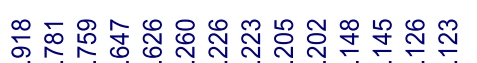

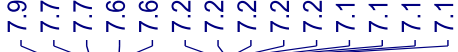

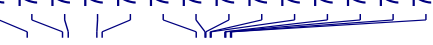

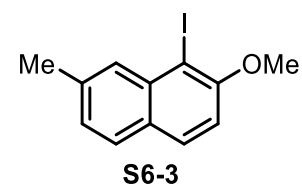

Will ili

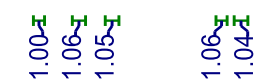

8.5

8. 0 , $7.5,7$

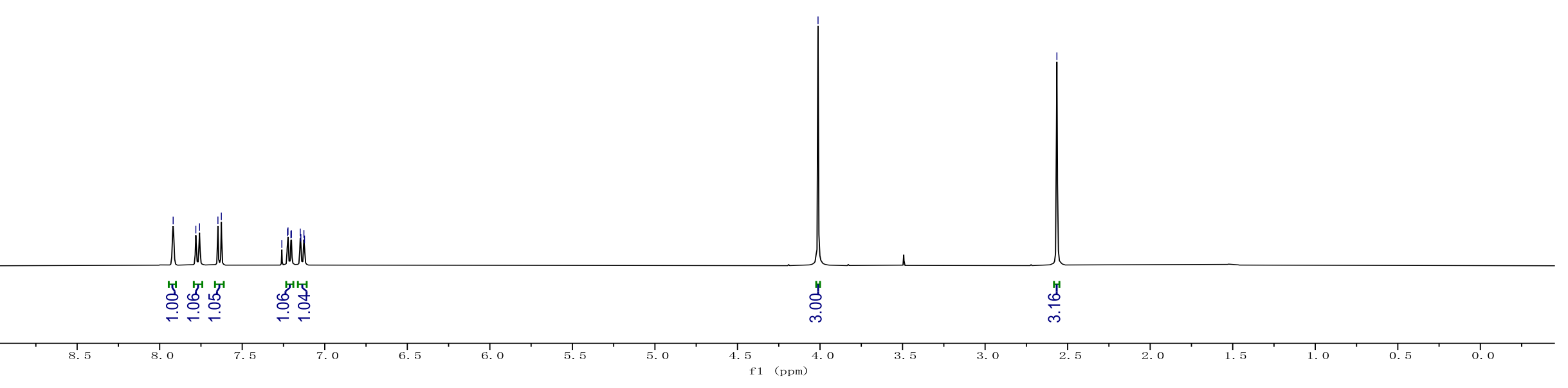




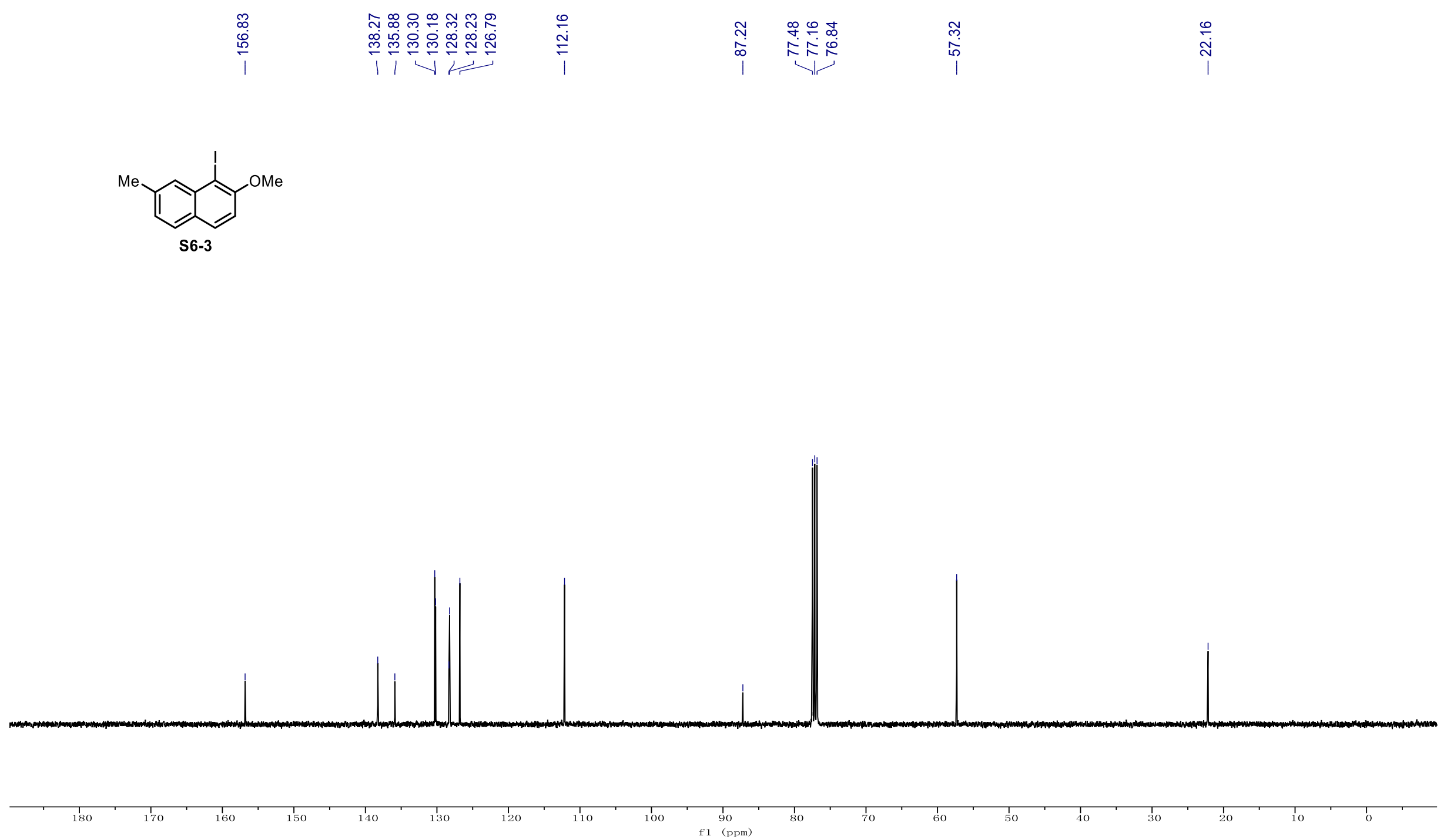




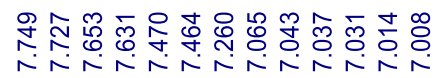
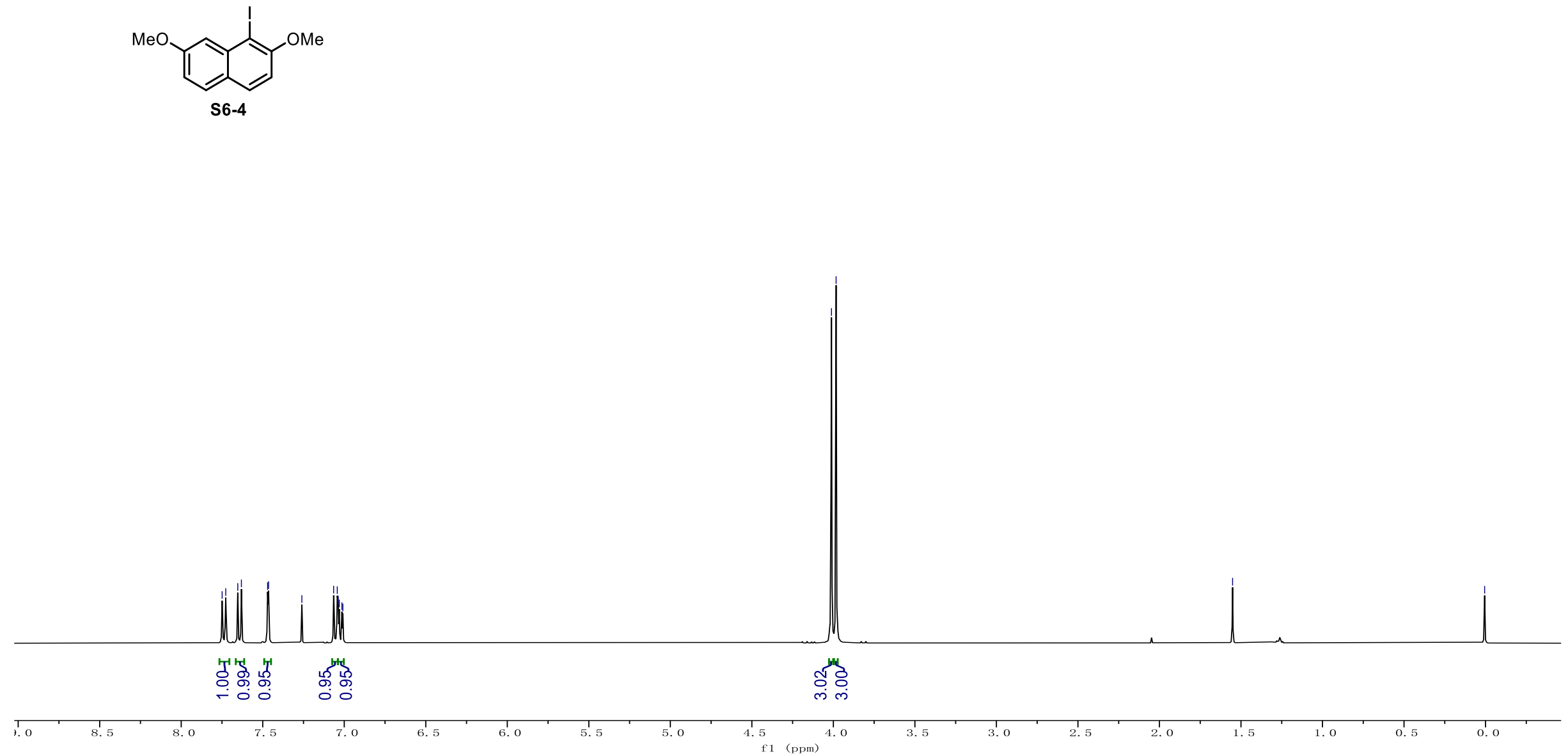


官官
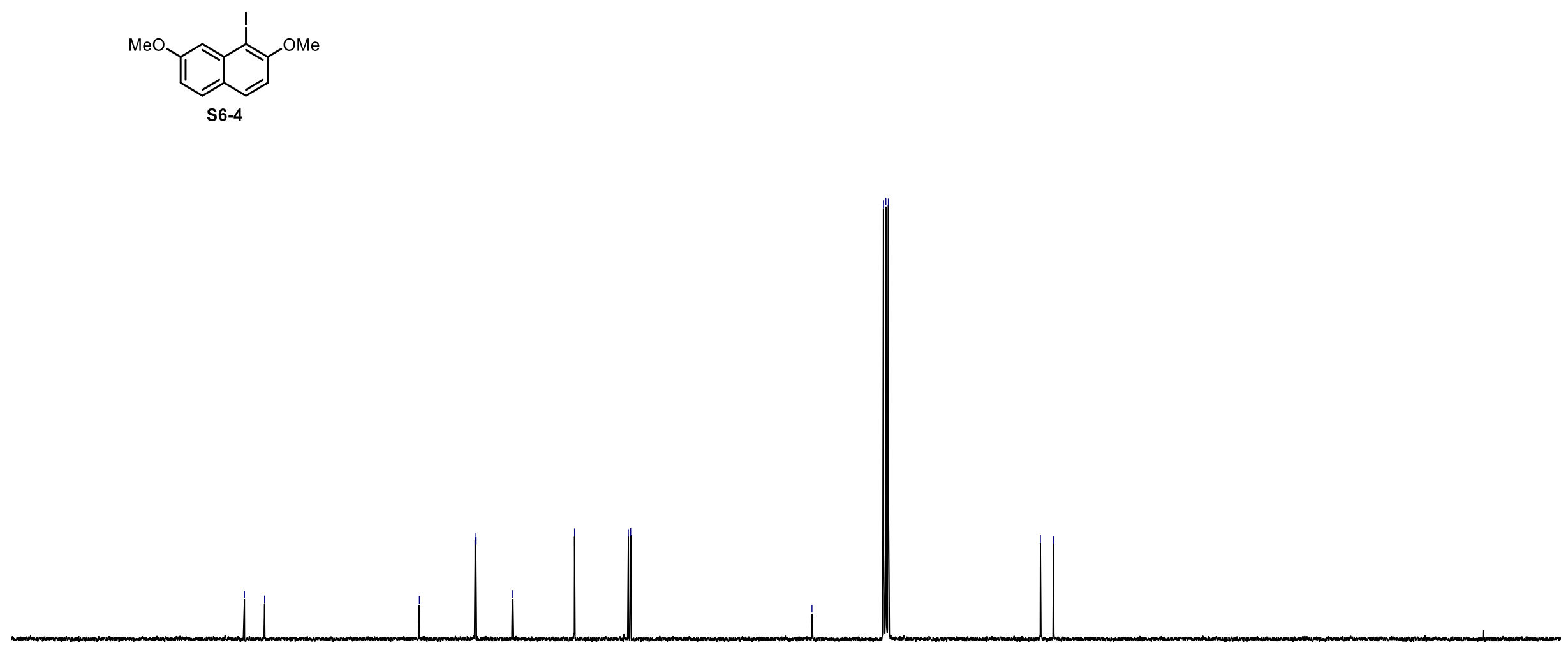

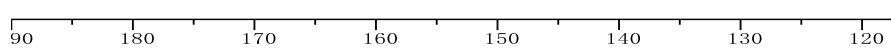



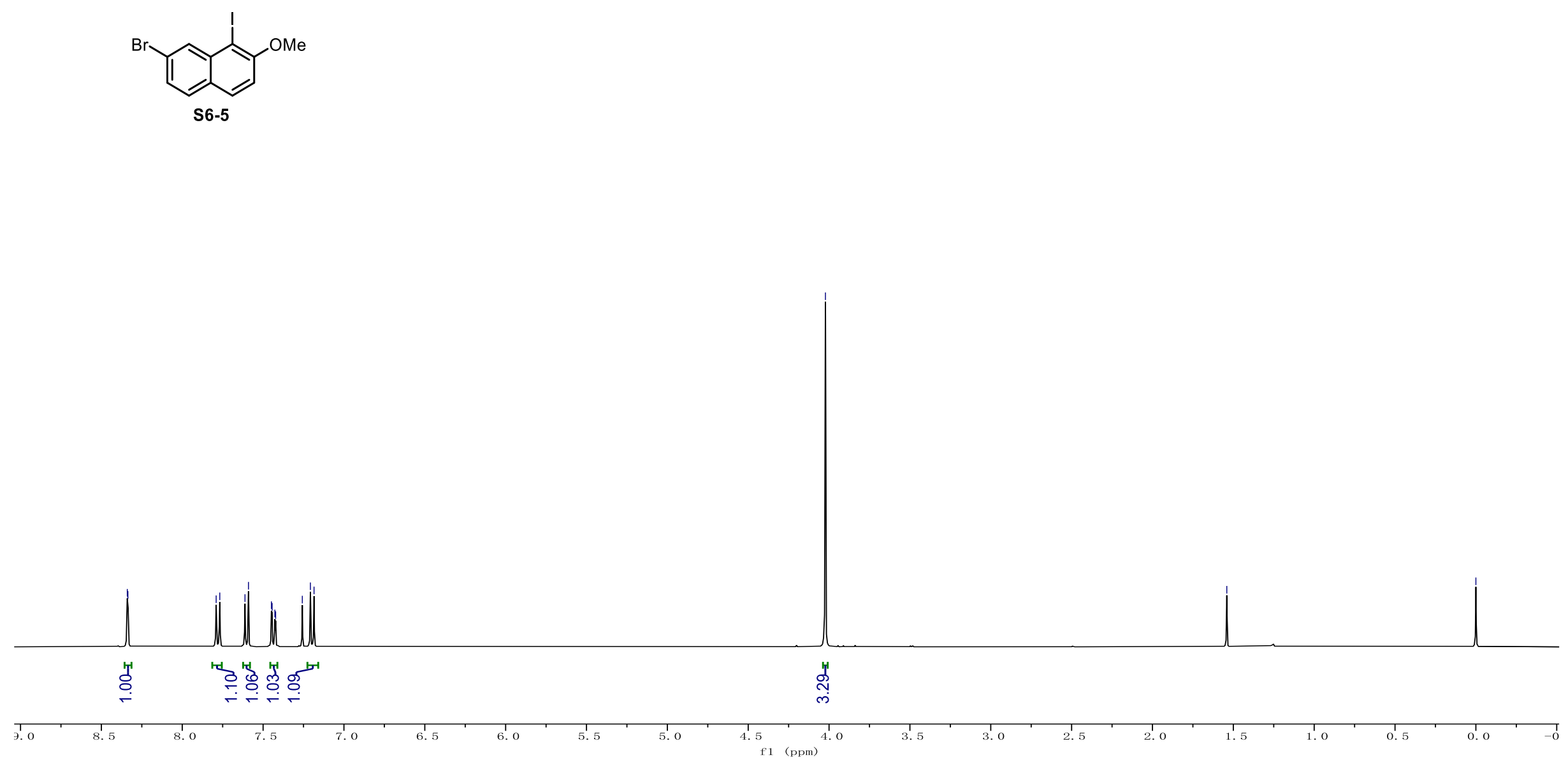
$\frac{1}{\frac{10}{20}}$

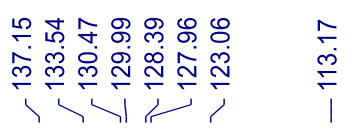

ñ
0
0

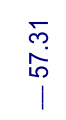
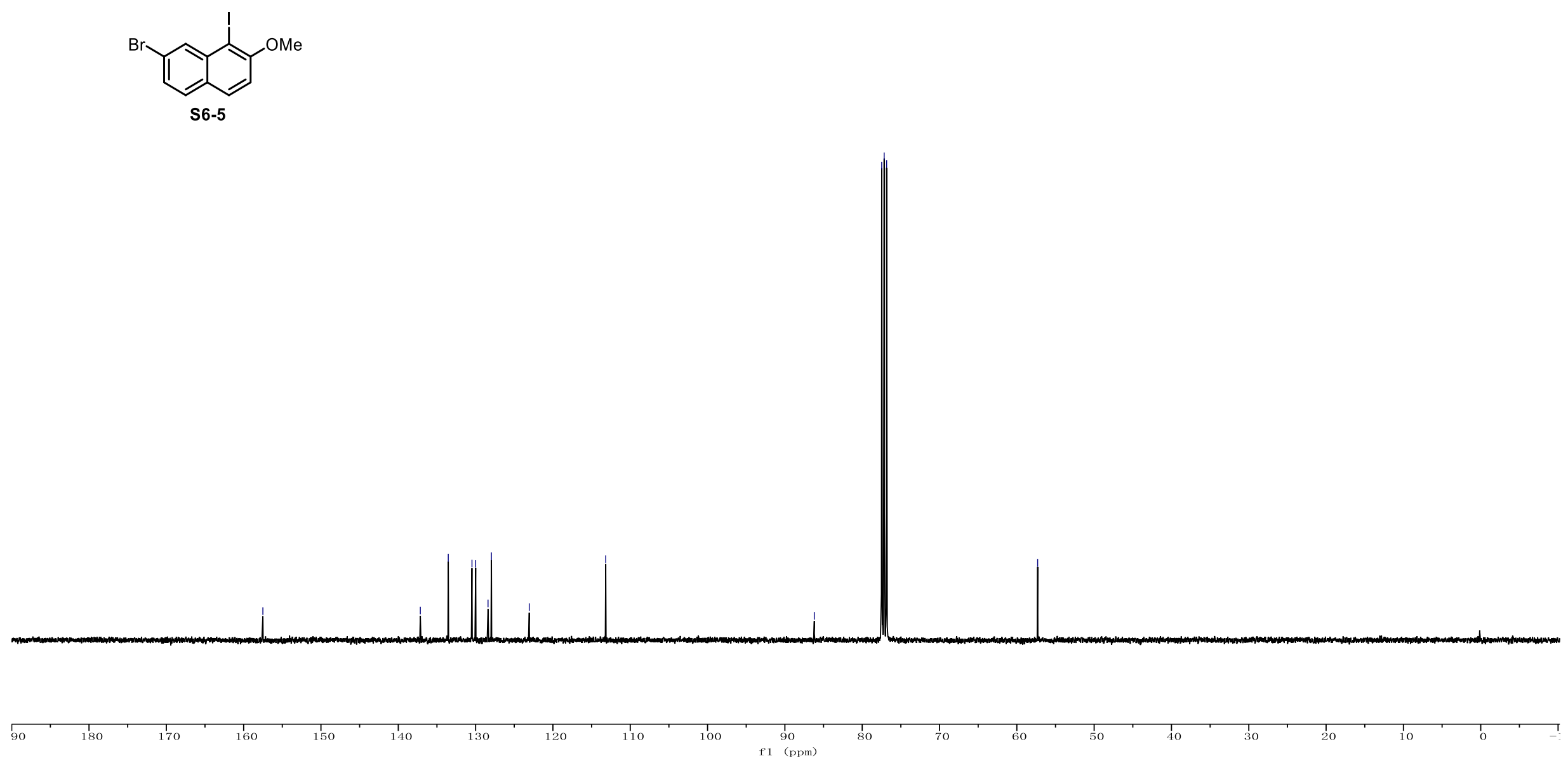

165 


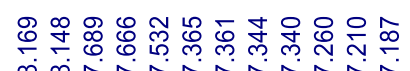

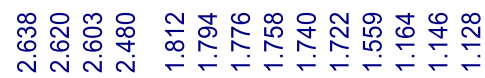

$\sqrt{n \pi r}$

N N N
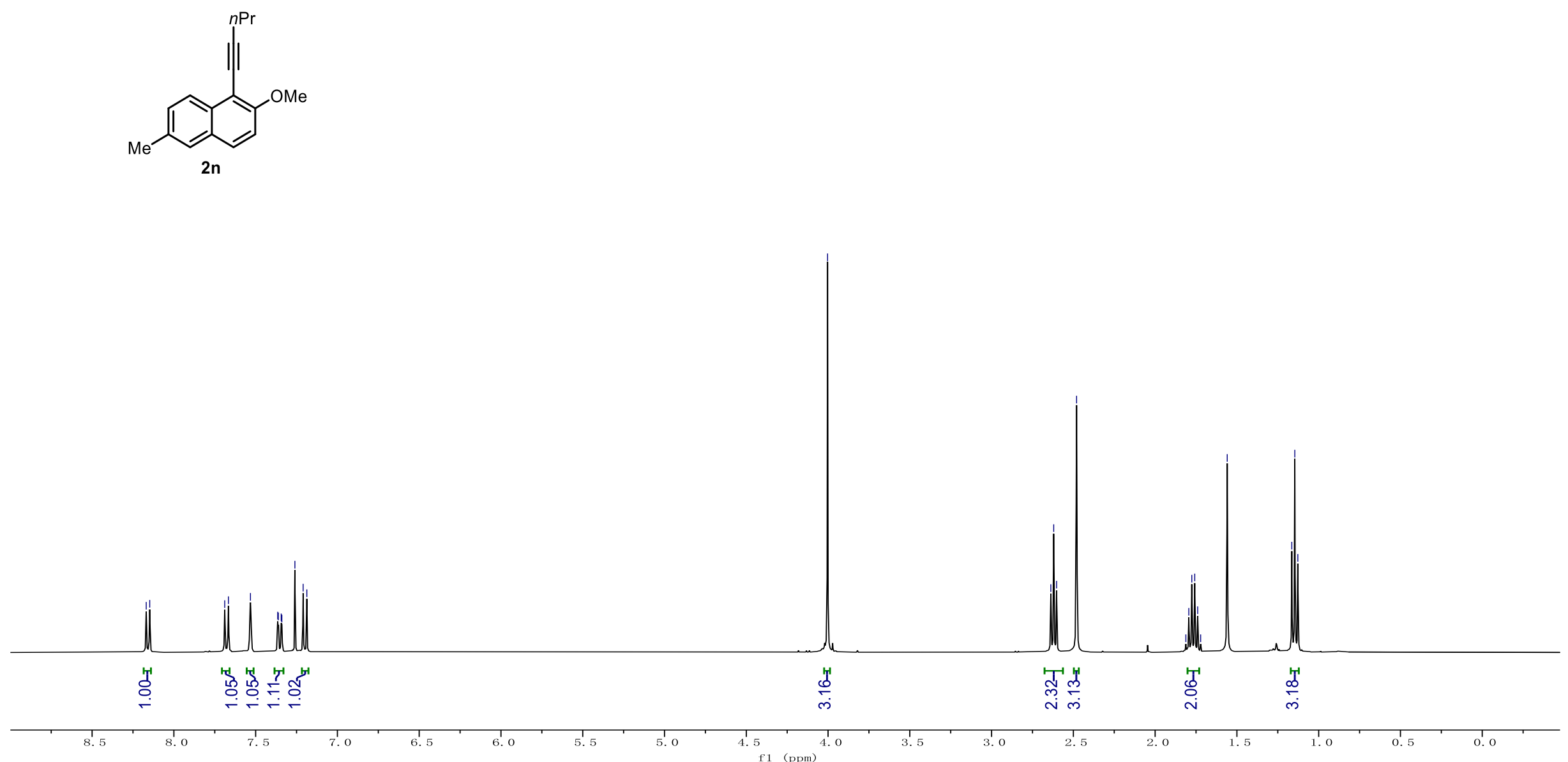


\begin{tabular}{|c|c|c|c|c|c|}
\hline$\underbrace{m}$ & 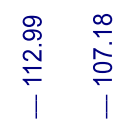 & $\frac{\hat{0}}{\grave{O}}$ & 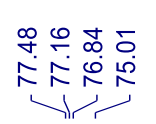 & $\begin{array}{ll}\infty \\
0 \\
0 \\
0 \\
1\end{array}$ & 茴 \\
\hline
\end{tabular}
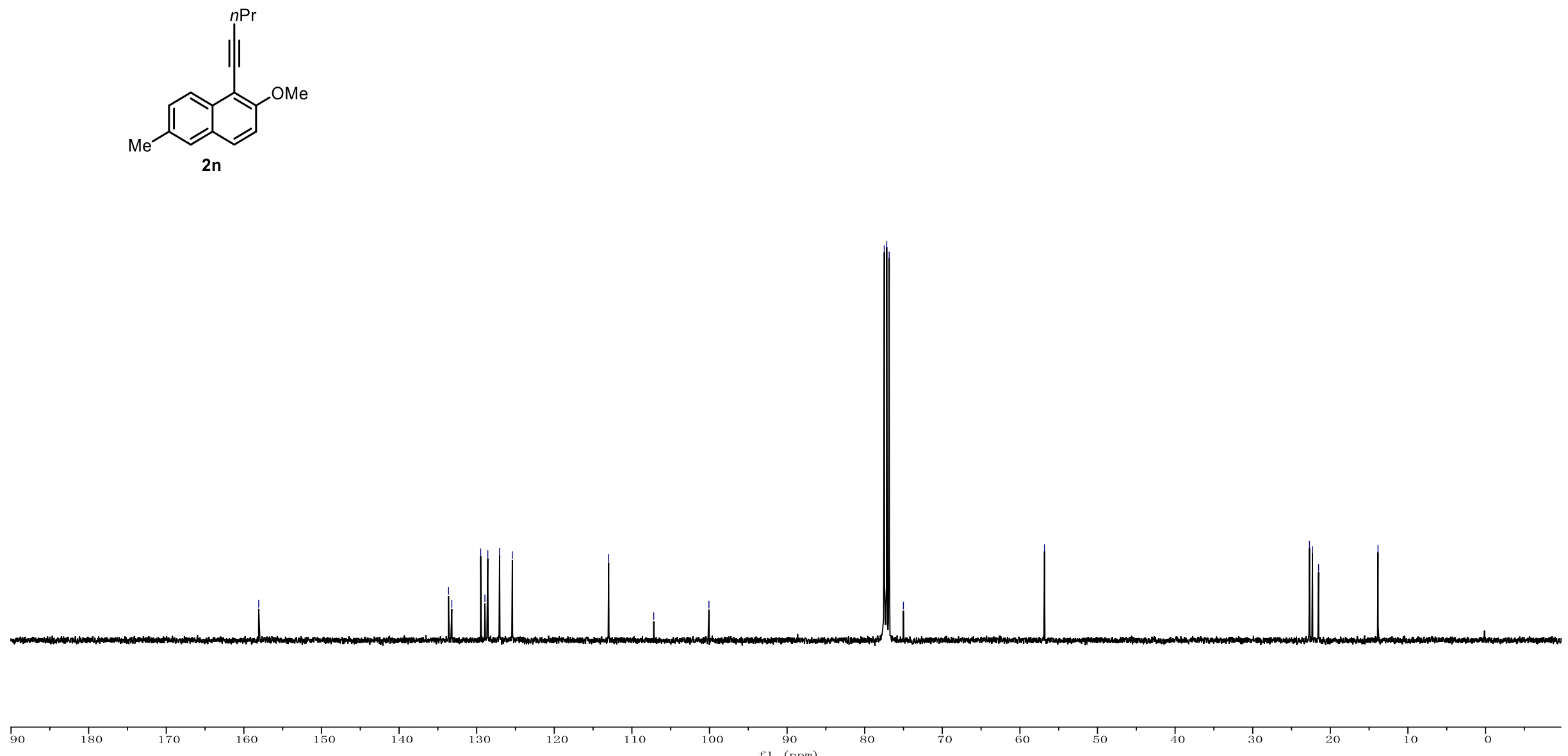


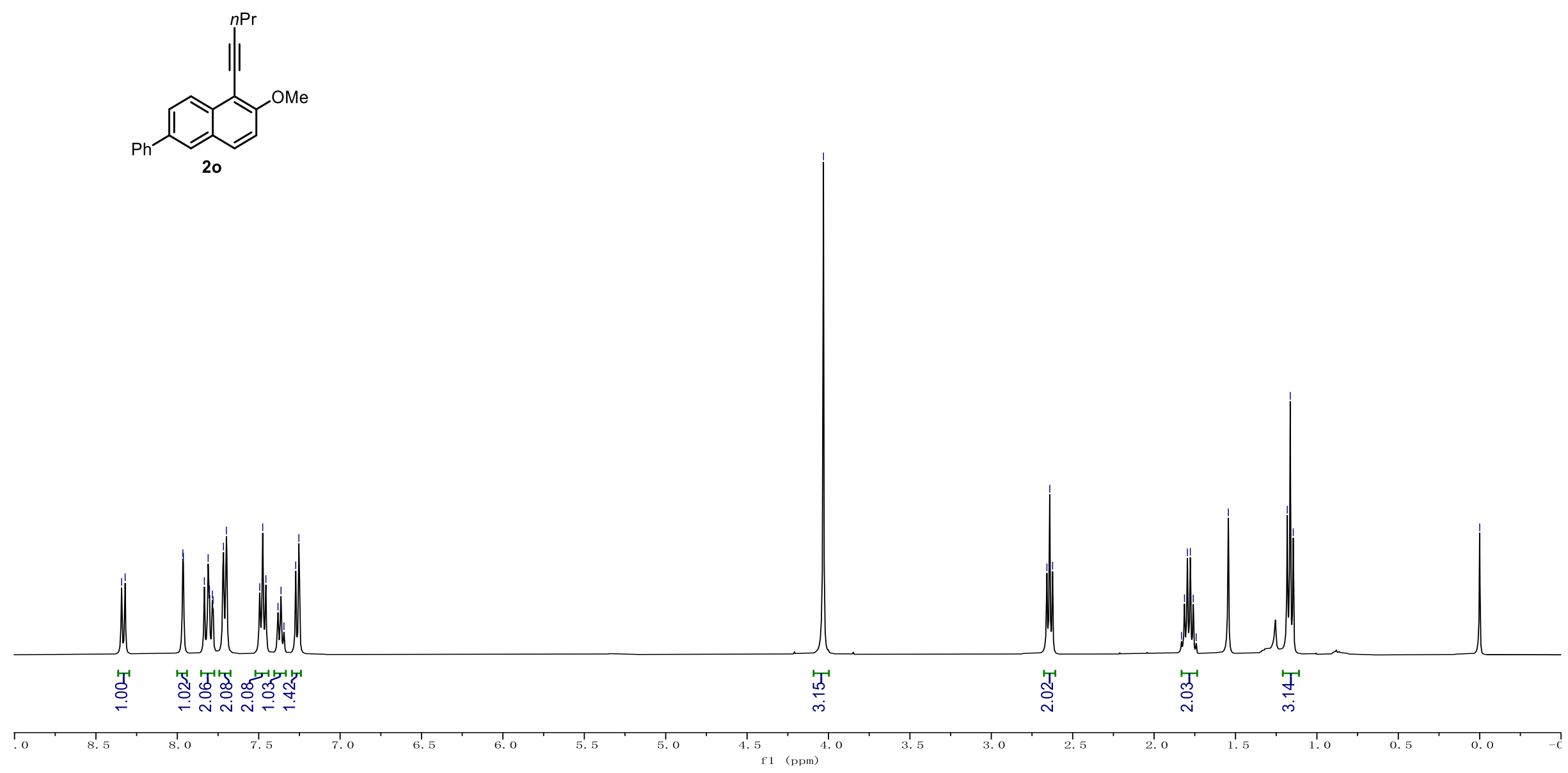




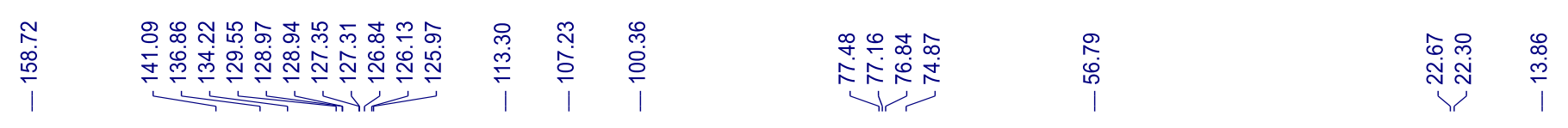
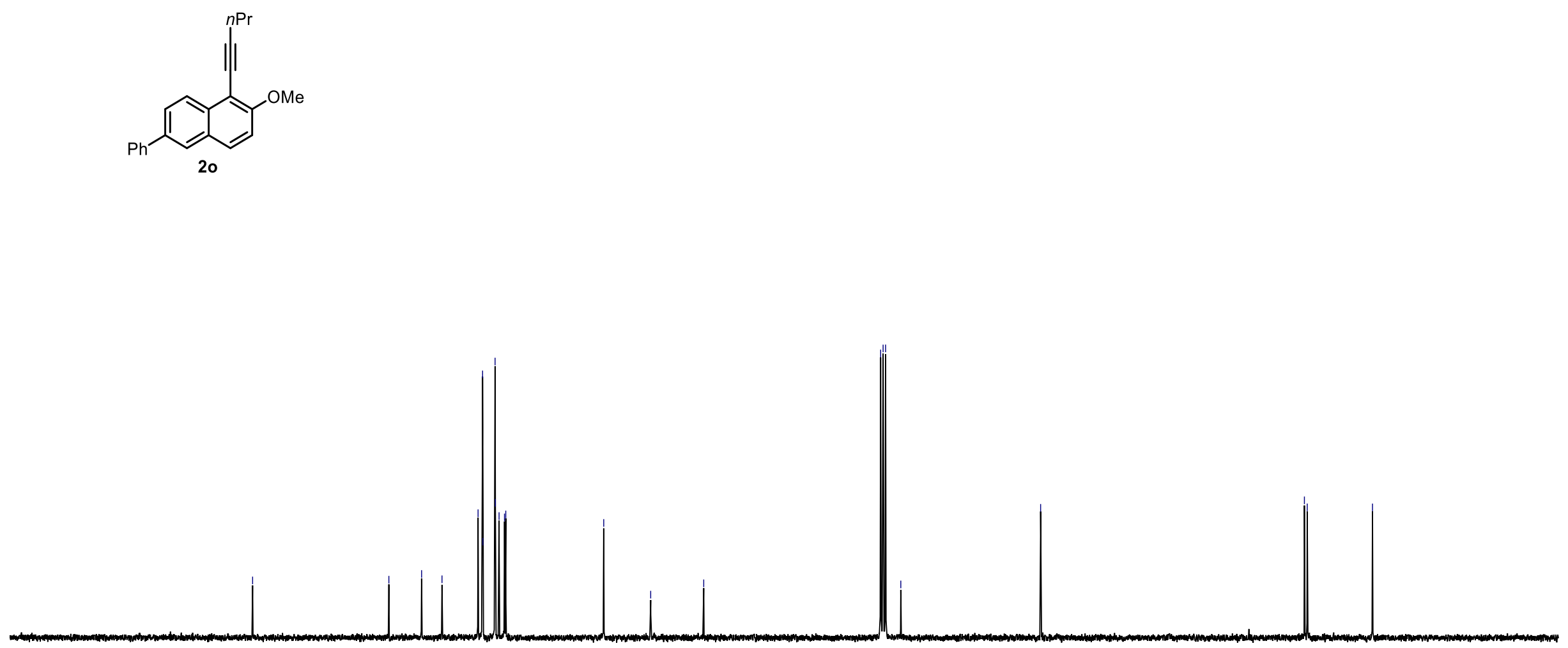


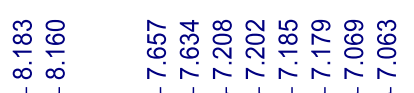

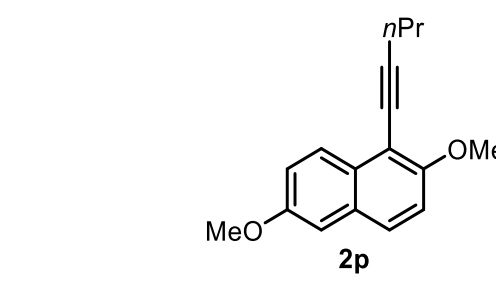

$2 \mathrm{p}$

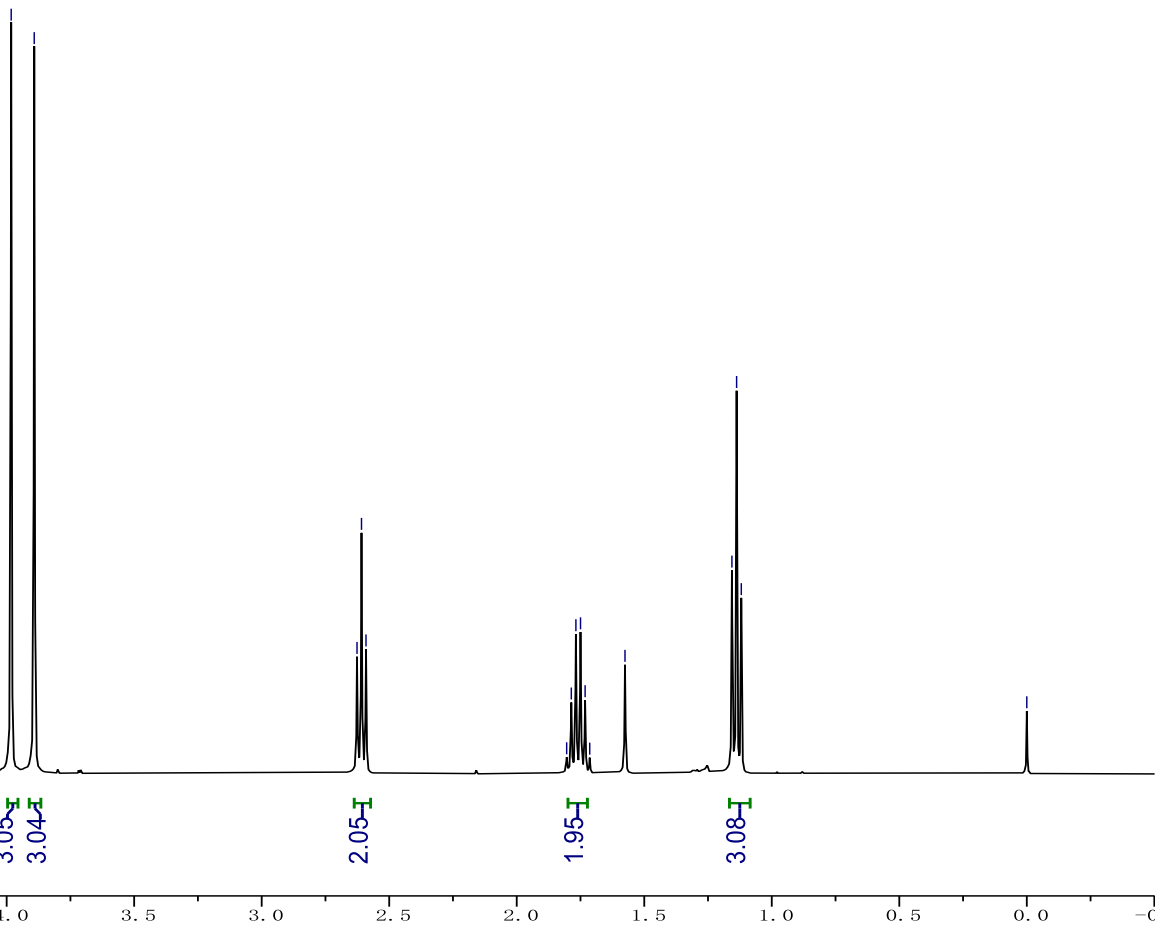




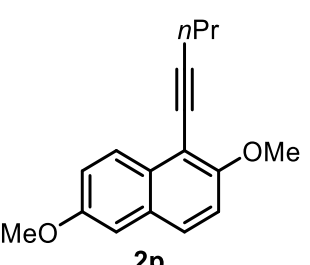

$2 p$
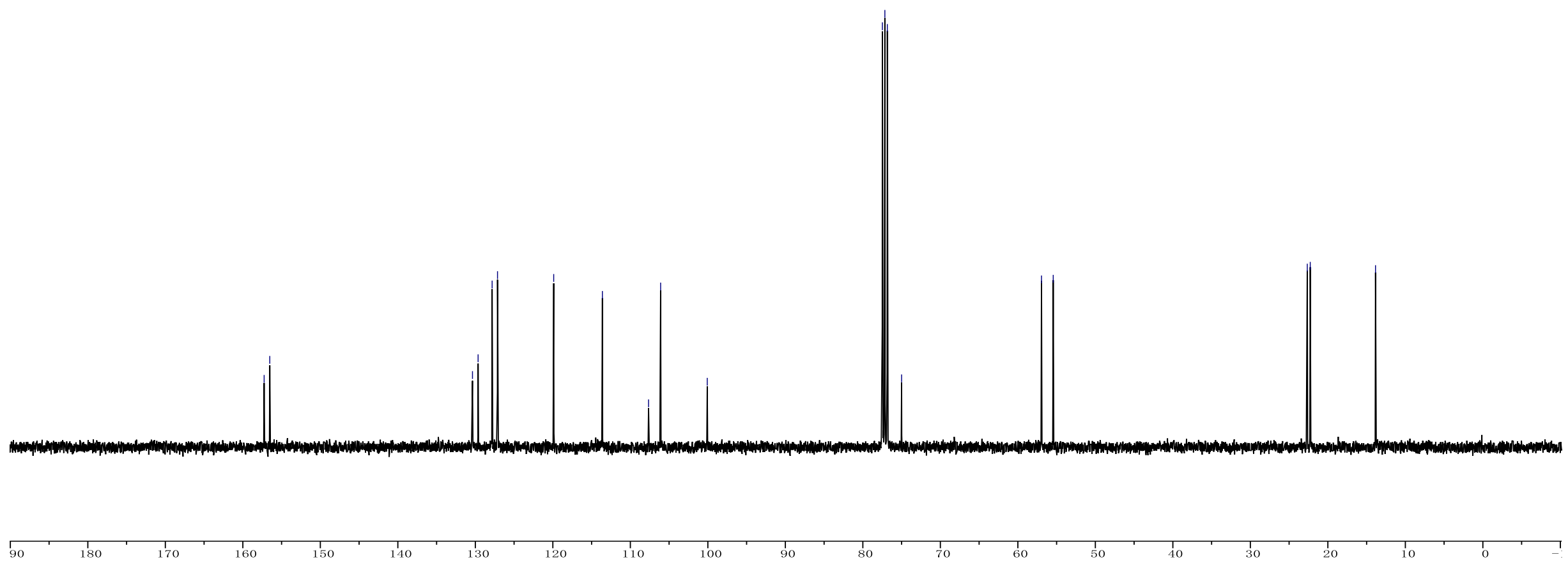
160 ${ }_{110}^{1} \cdot 100$ 90 


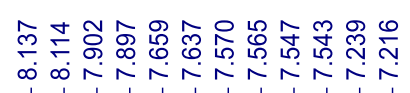

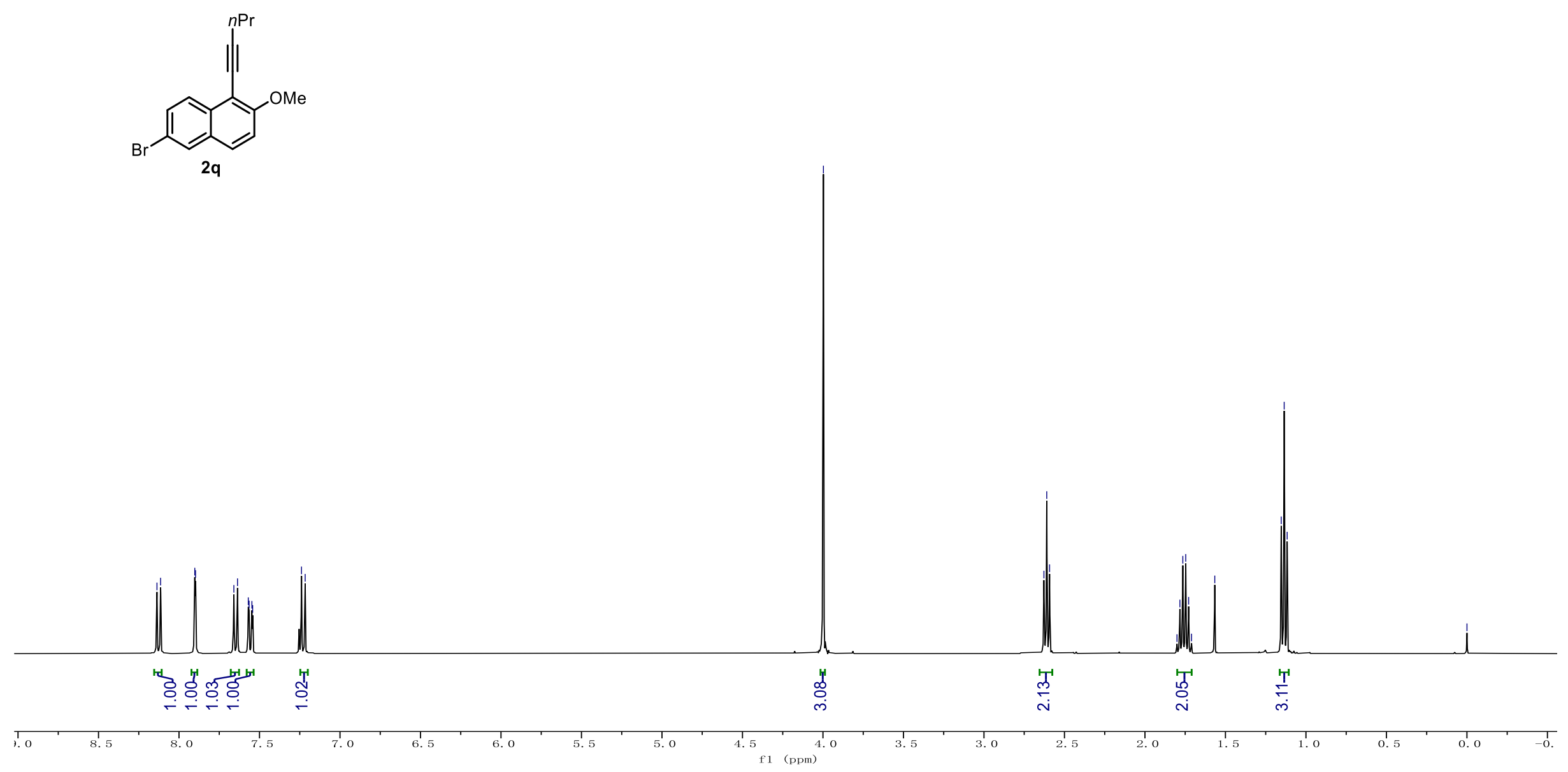



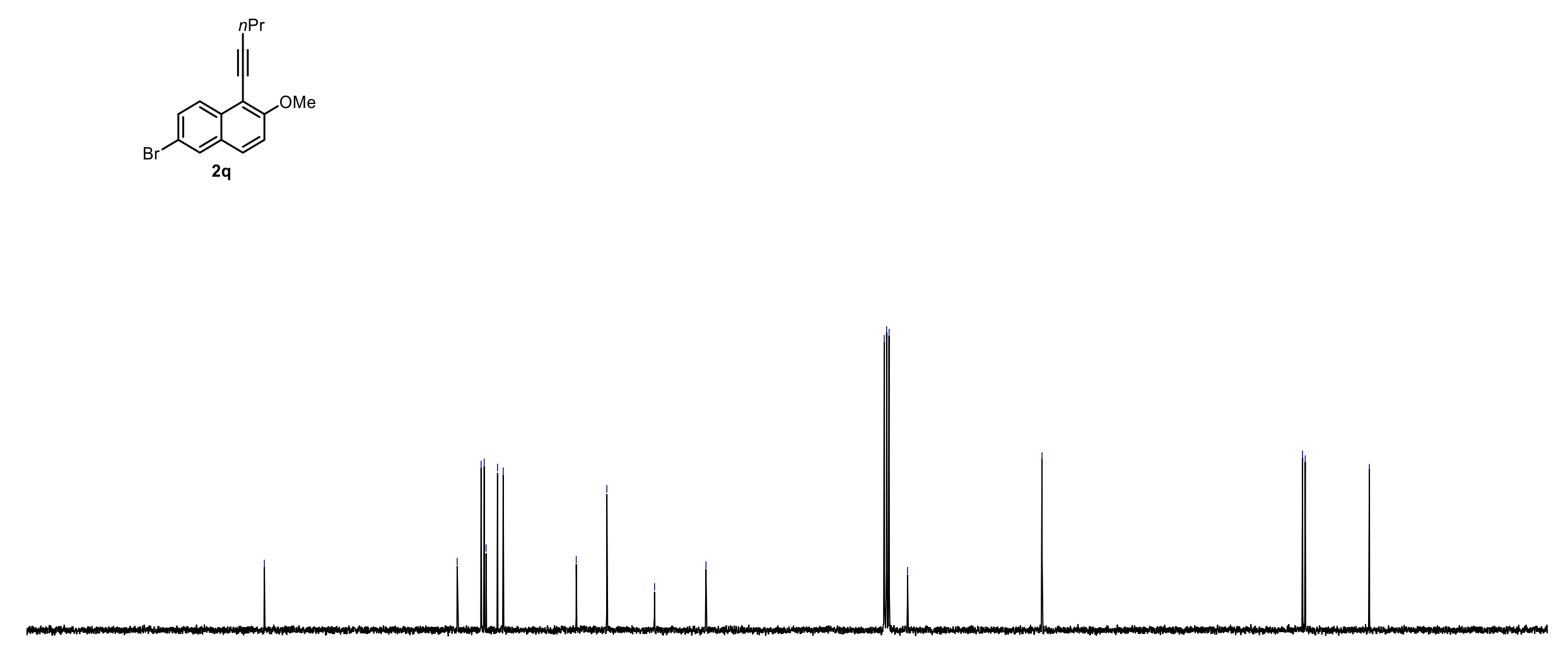

173 


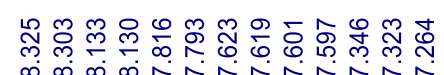
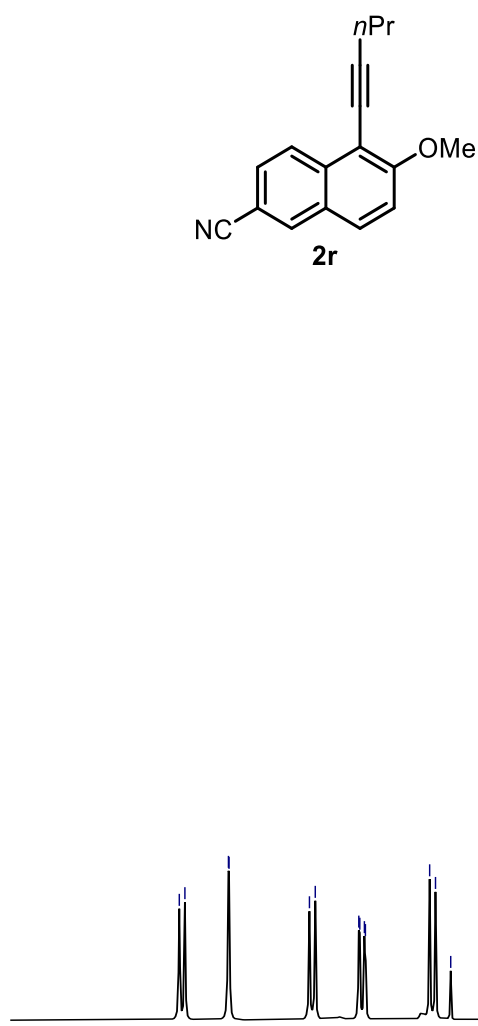

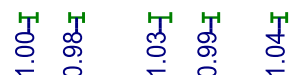
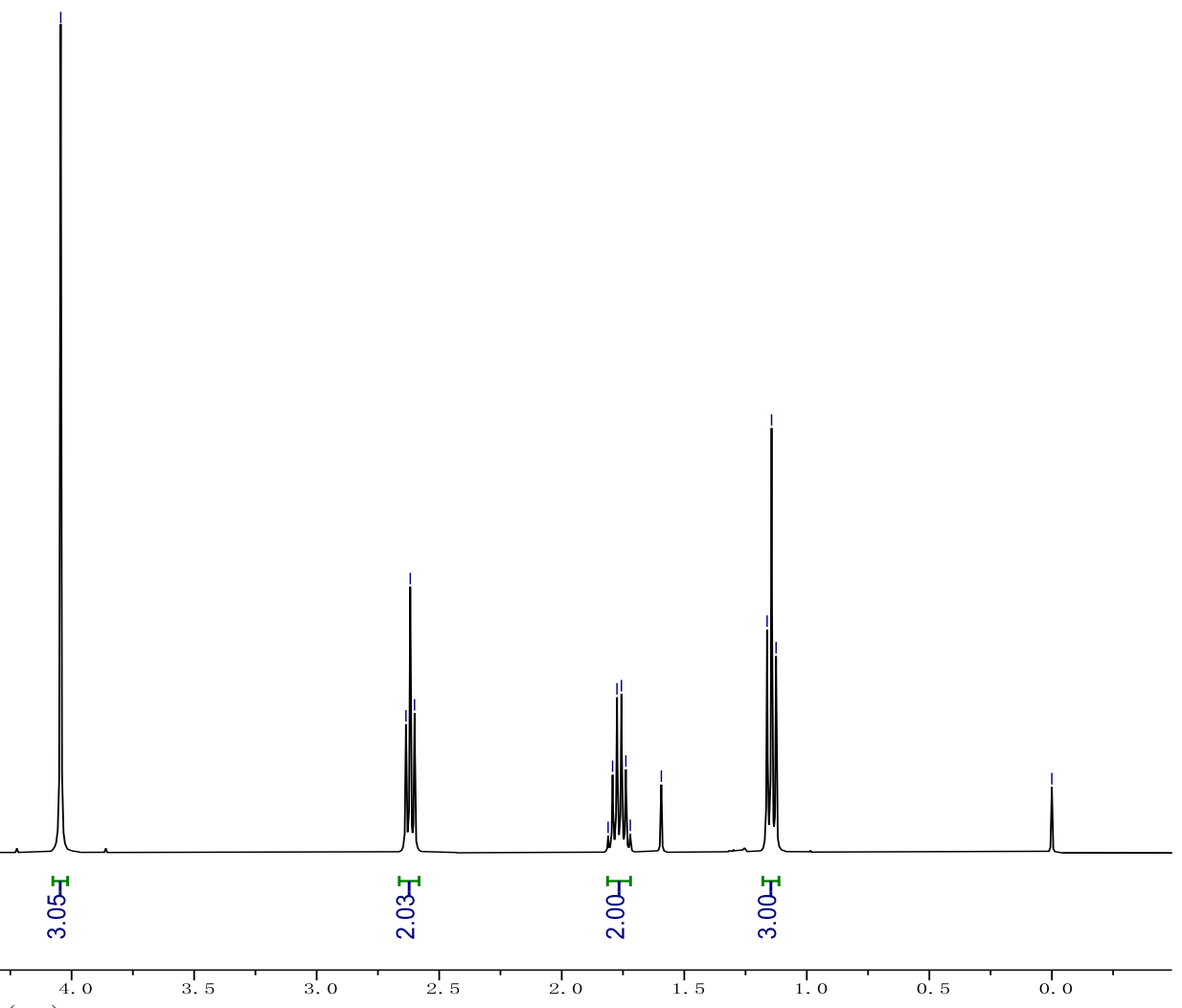


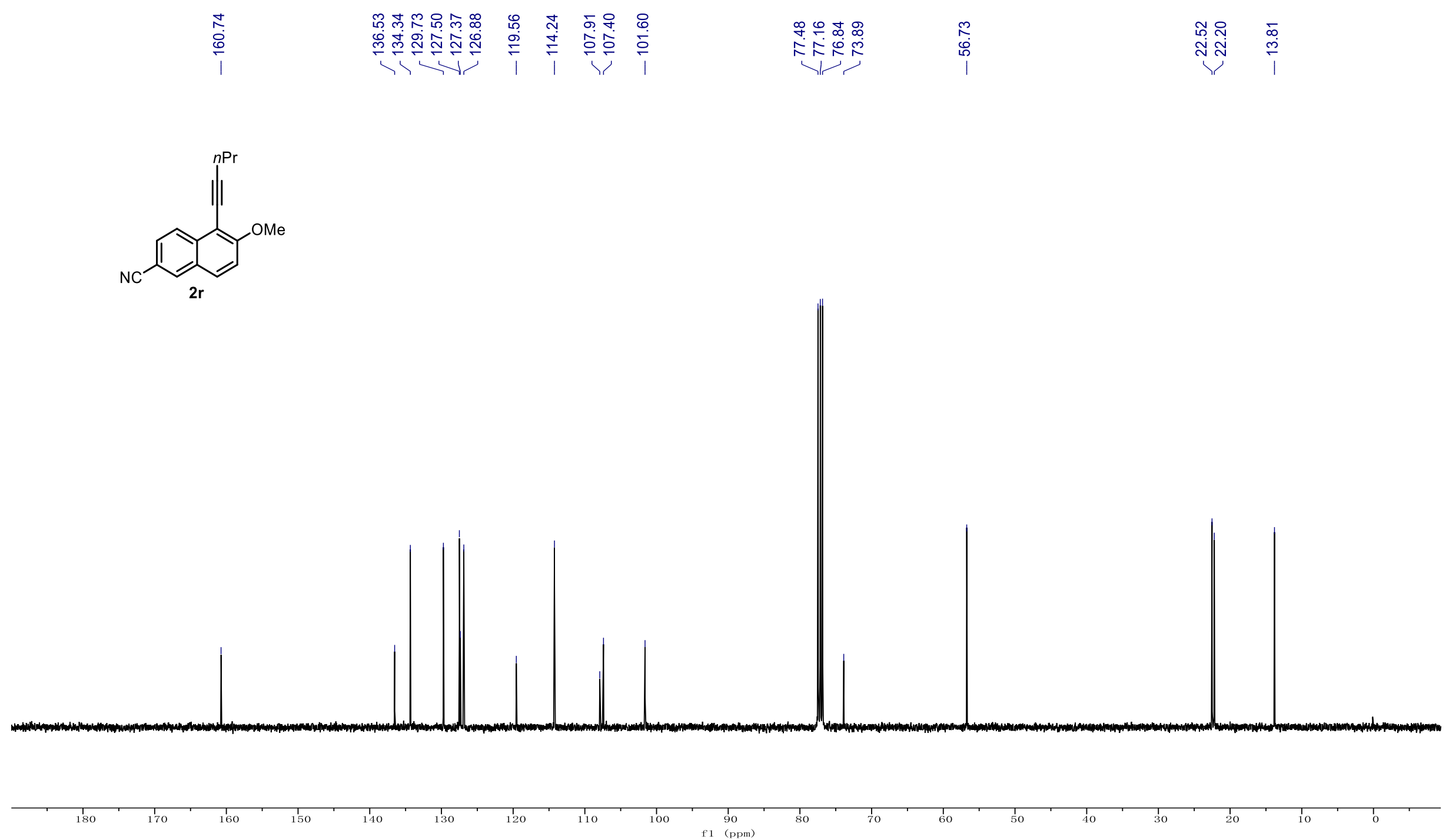




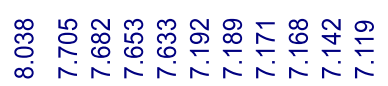

管
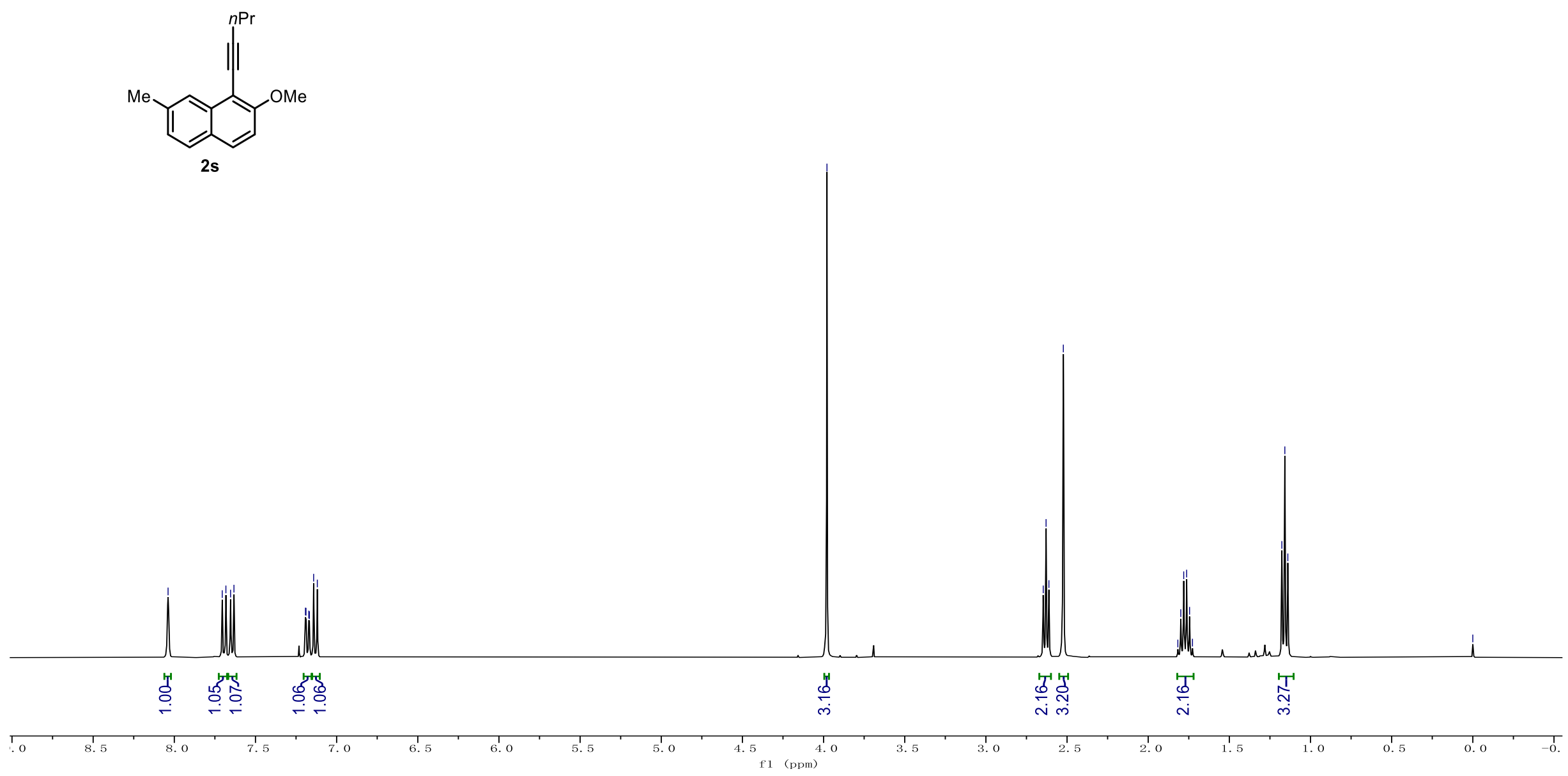


\begin{tabular}{|c|c|c|c|c|c|c|}
\hline 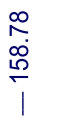 & 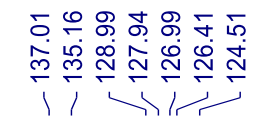 & $\stackrel{\substack{\infty \\
\stackrel{\infty}{\risingdotseq}}}{\uparrow}$ & 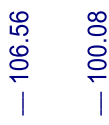 & 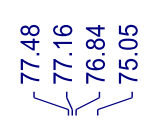 & $\begin{array}{c}R \\
0 \\
0 \\
0 \\
1\end{array}$ & 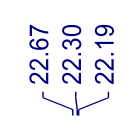 \\
\hline
\end{tabular}
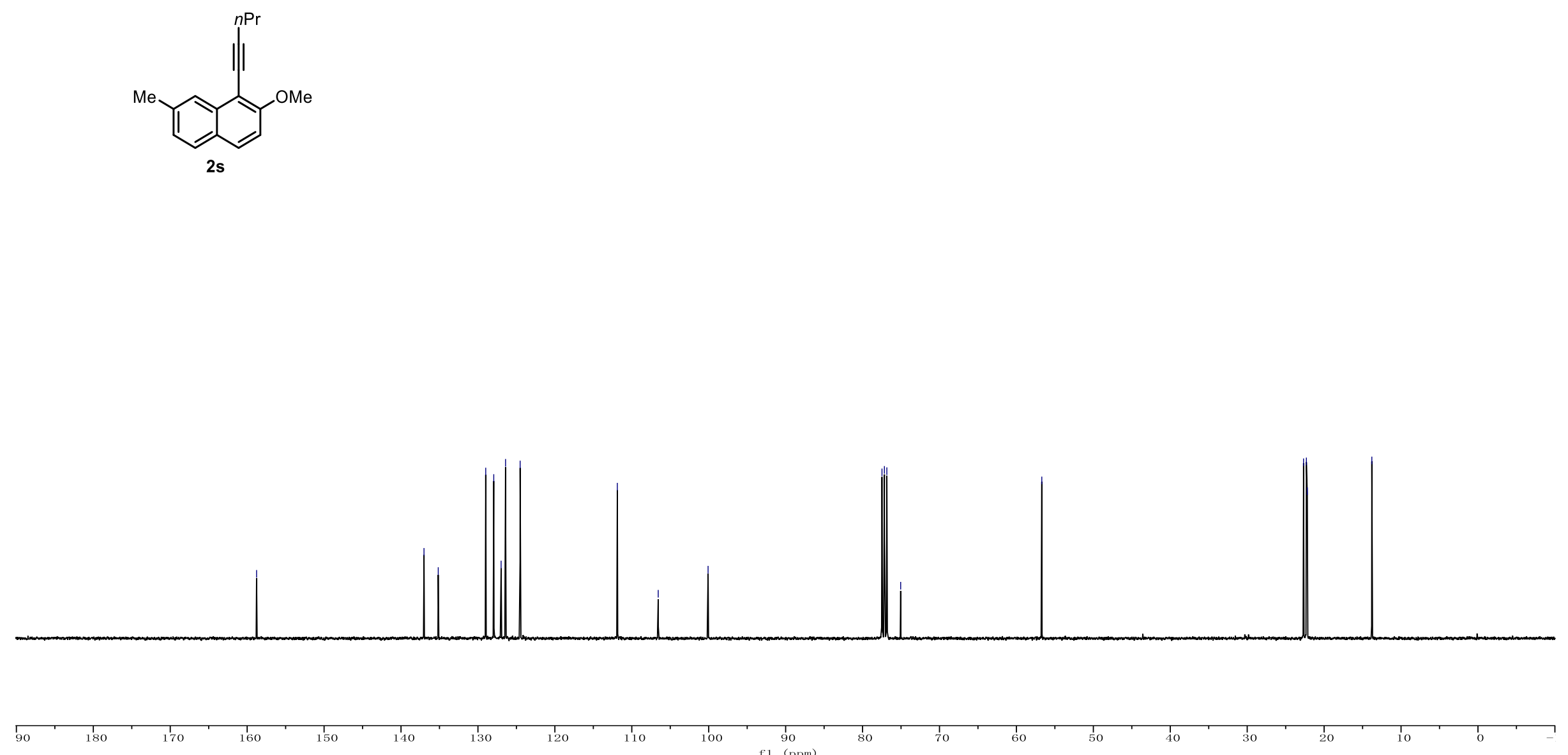

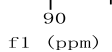




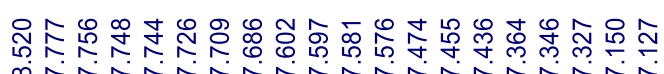

里

$\underbrace{-10}$

o<smiles>CC=Cc1cc(P)ccc1OC</smiles>

$2 t$

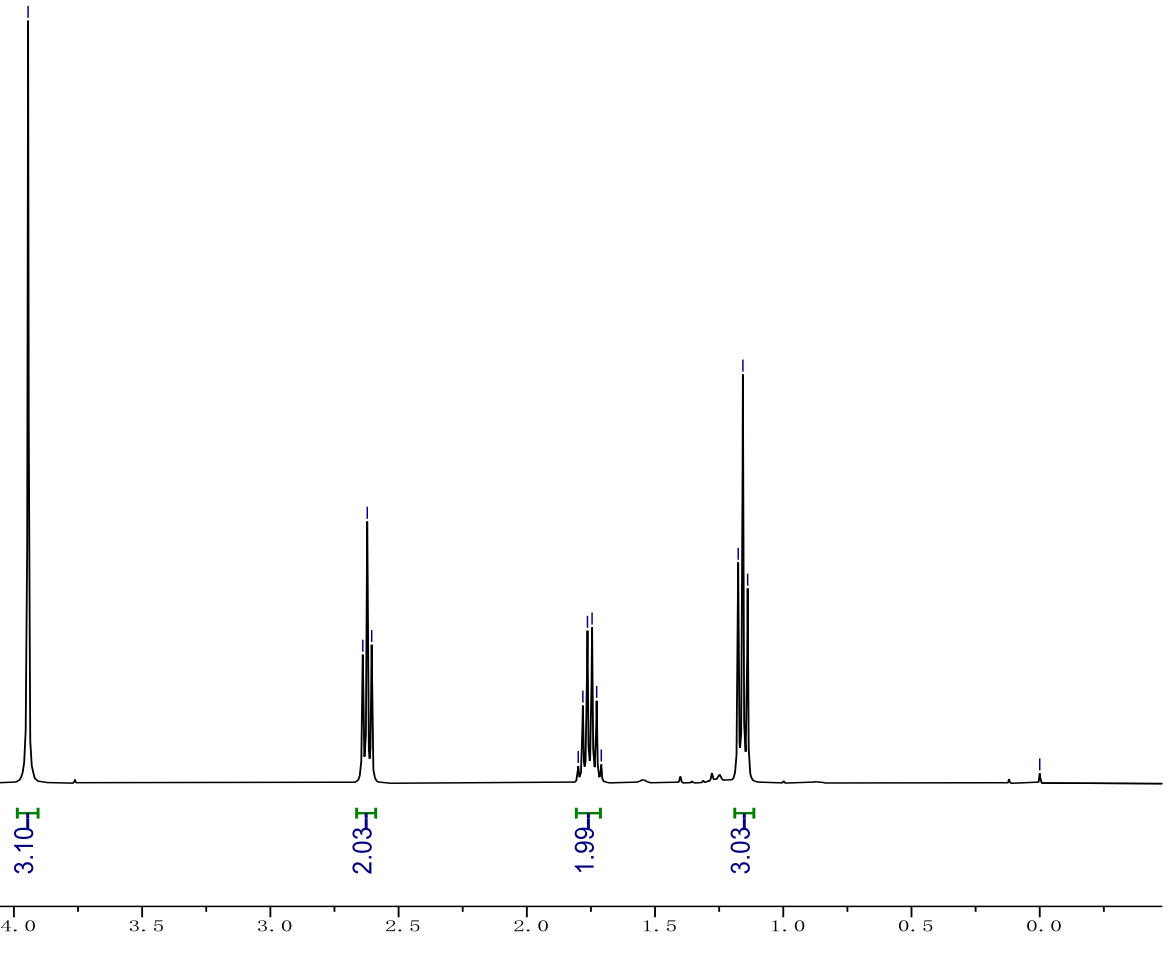




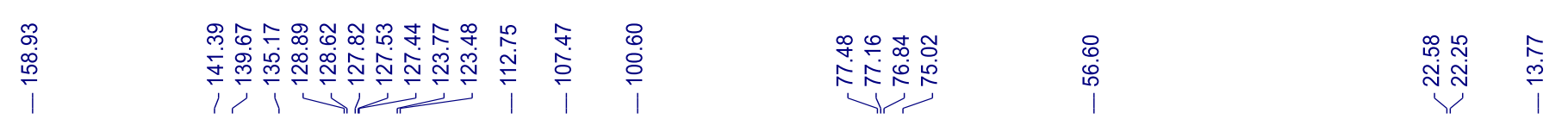
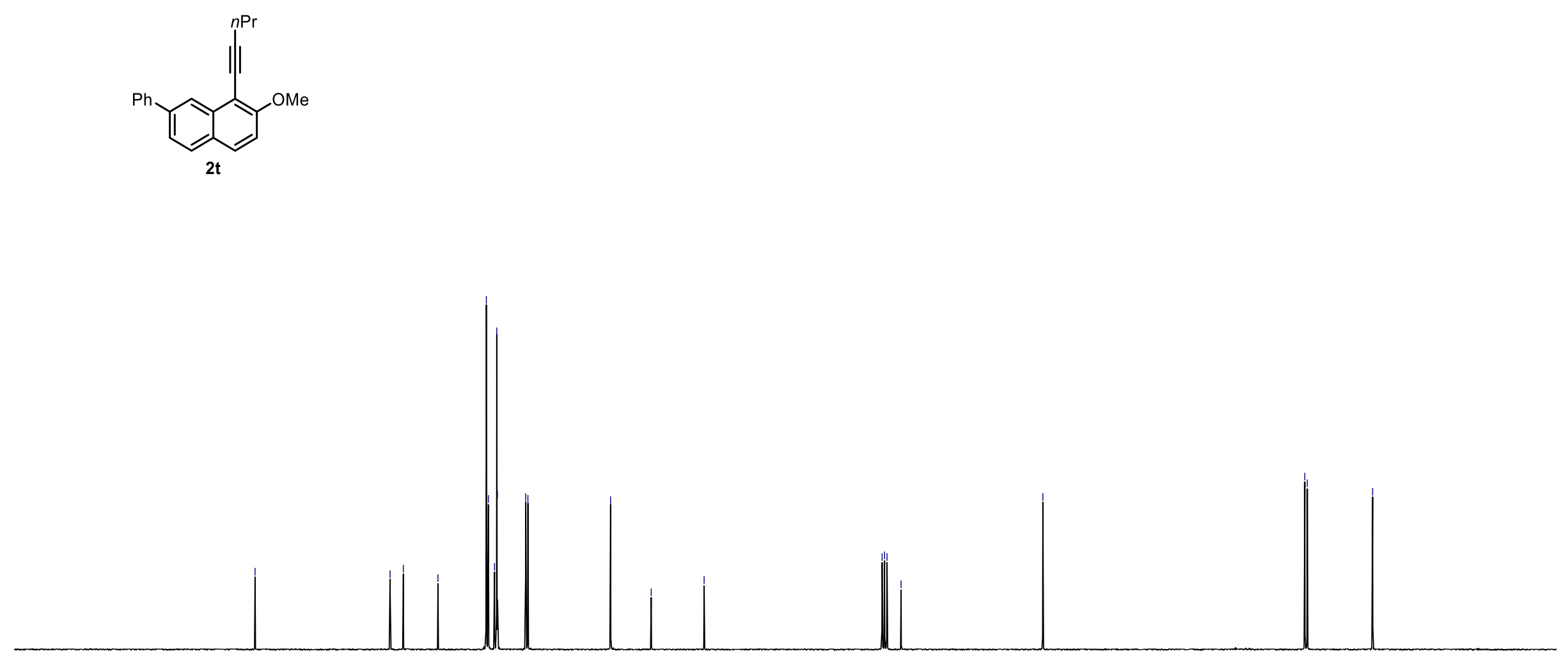

90

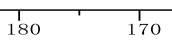

$170 \quad \quad 160$

$\stackrel{90}{\mathrm{f} 1} \stackrel{(\mathrm{ppm})}{ }$ 


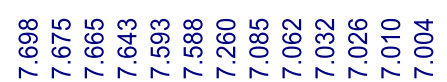

亭管

谓

总

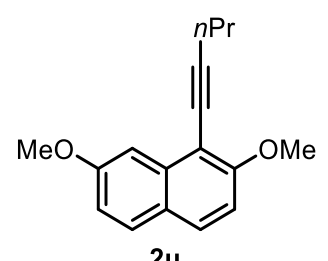

$2 \mathrm{u}$

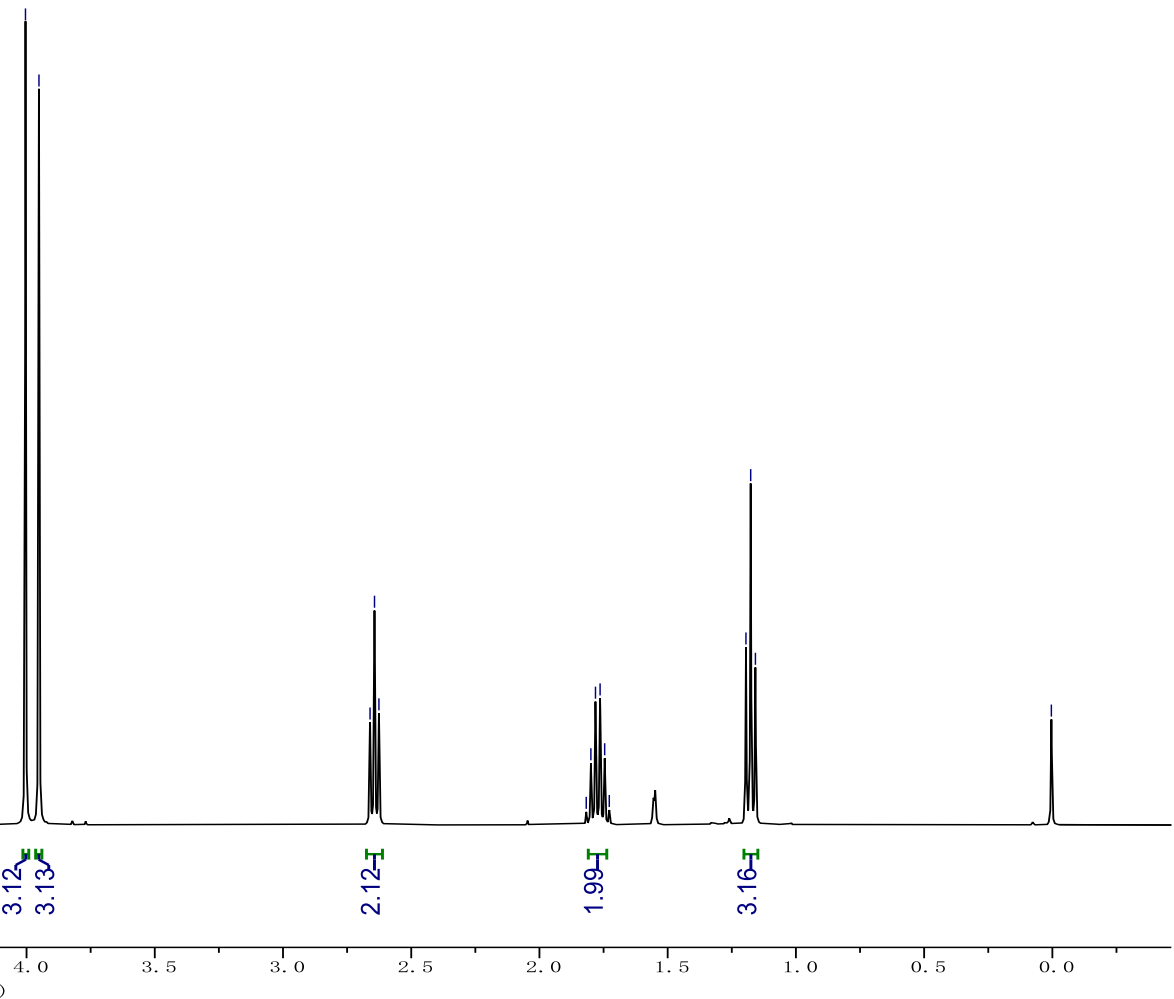


空点

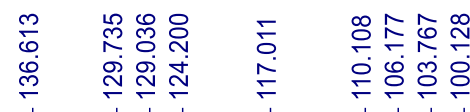

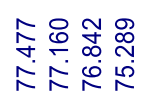

我恋

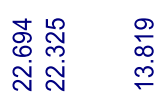<smiles>COc1ccc2ccc(OC)c(C#CC(C)C)c2c1</smiles>
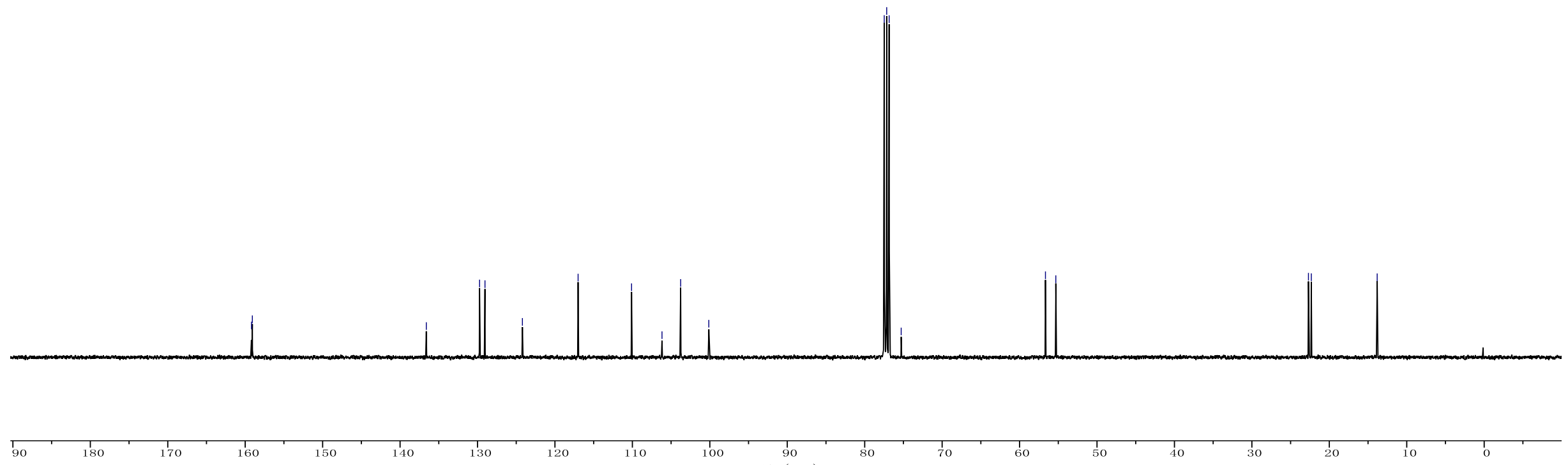
$\stackrel{90}{90}$ $60 \quad \frac{1}{60}$ 


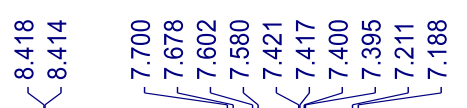

চ্.্ণে

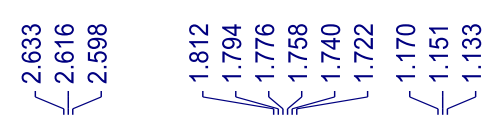
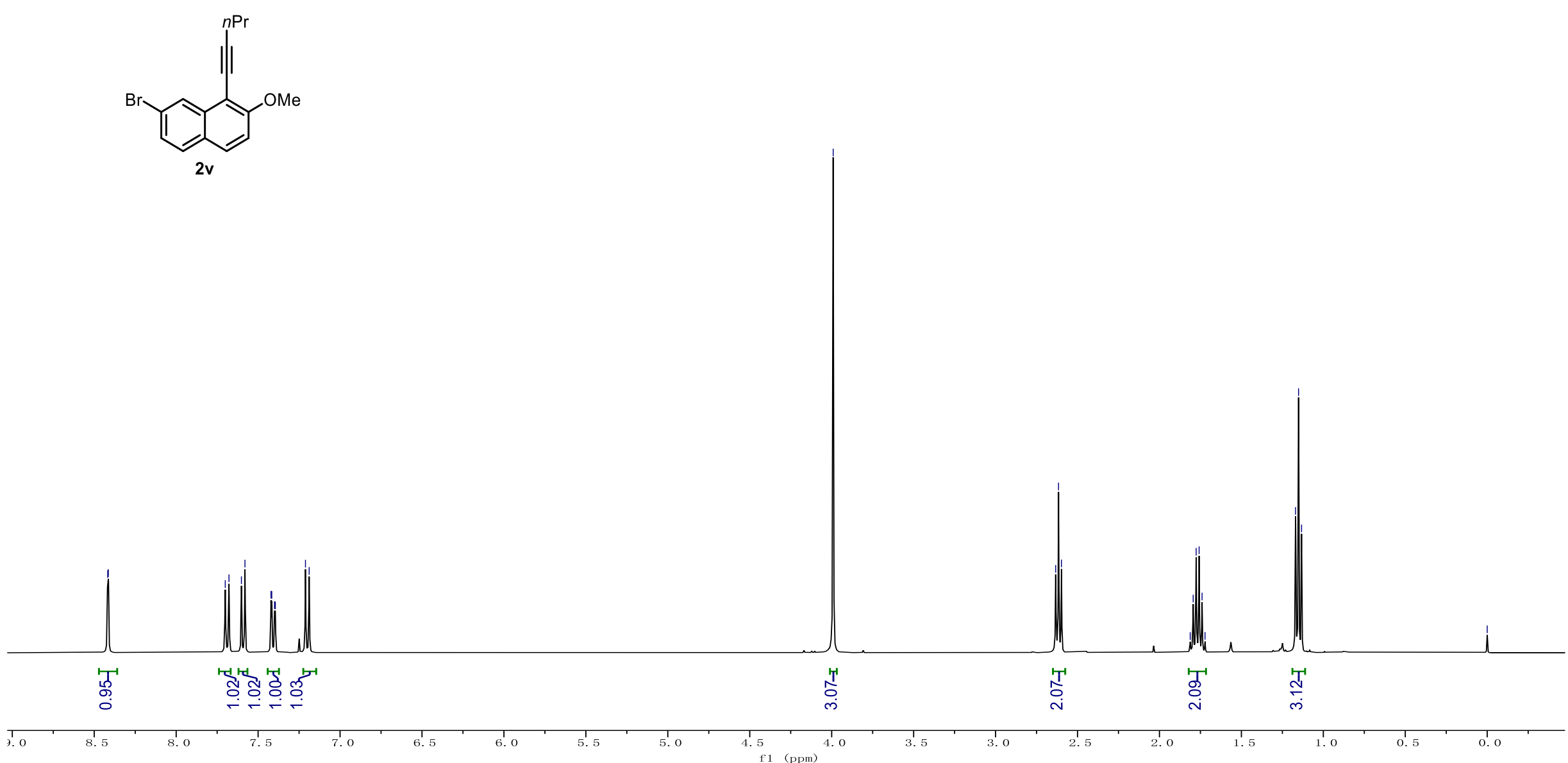

꼬ำ
|

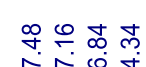

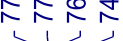
0
0
0
0

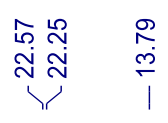
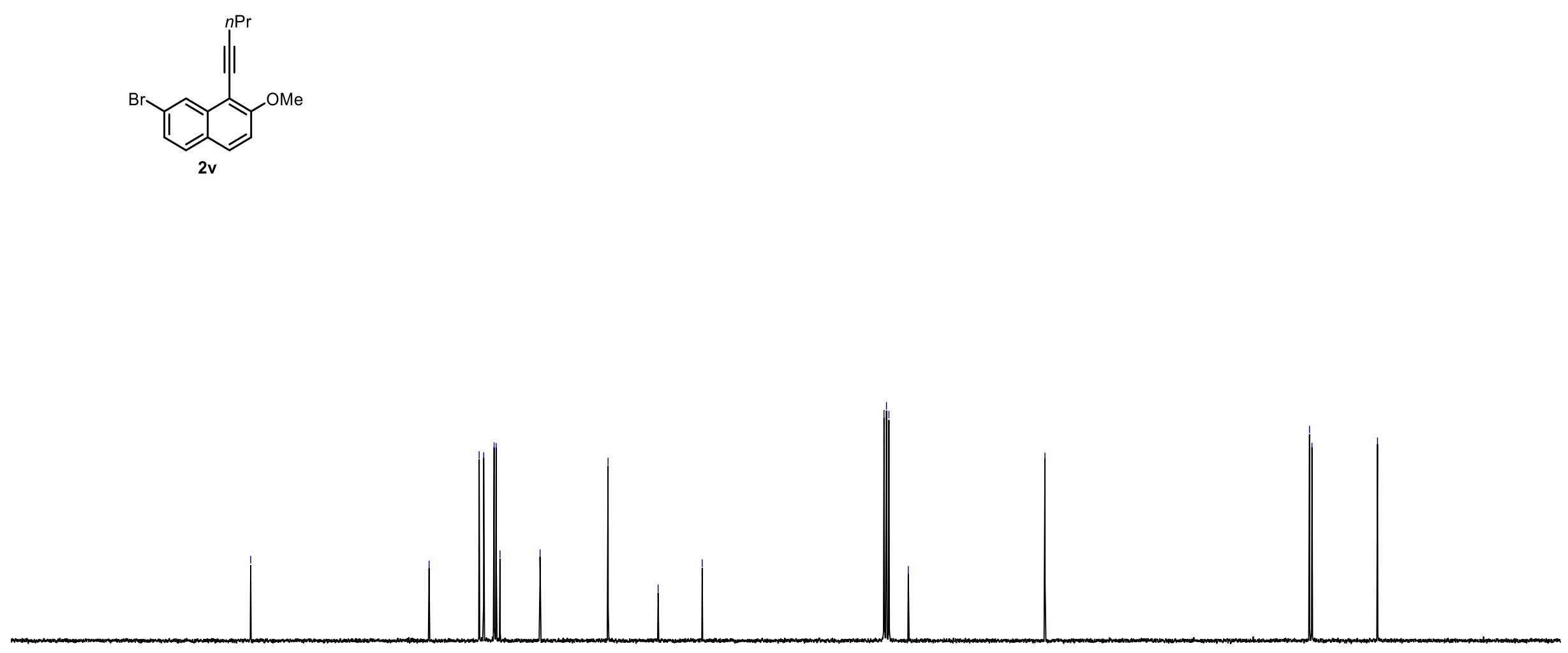

90
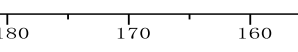

150

140 90
f1 $(\mathrm{ppm})$ 


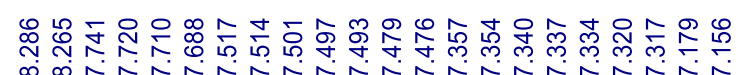

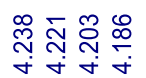

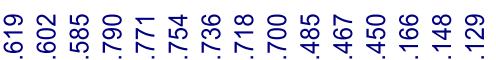

ت
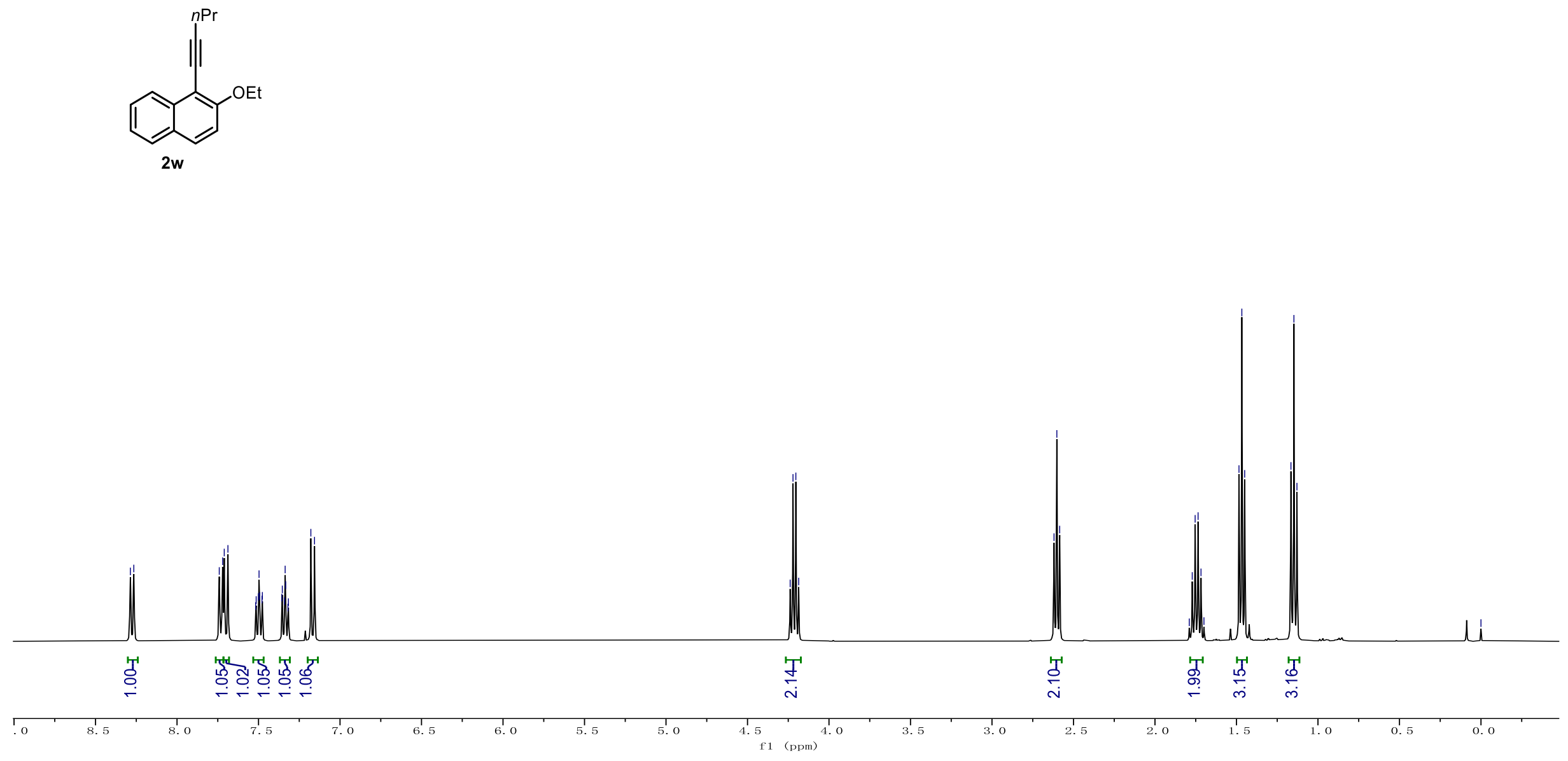
$\underset{\substack{n \\ \infty \\ \infty}}{\stackrel{\infty}{\infty}}$

|

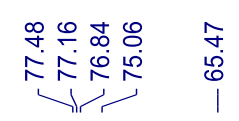

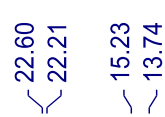
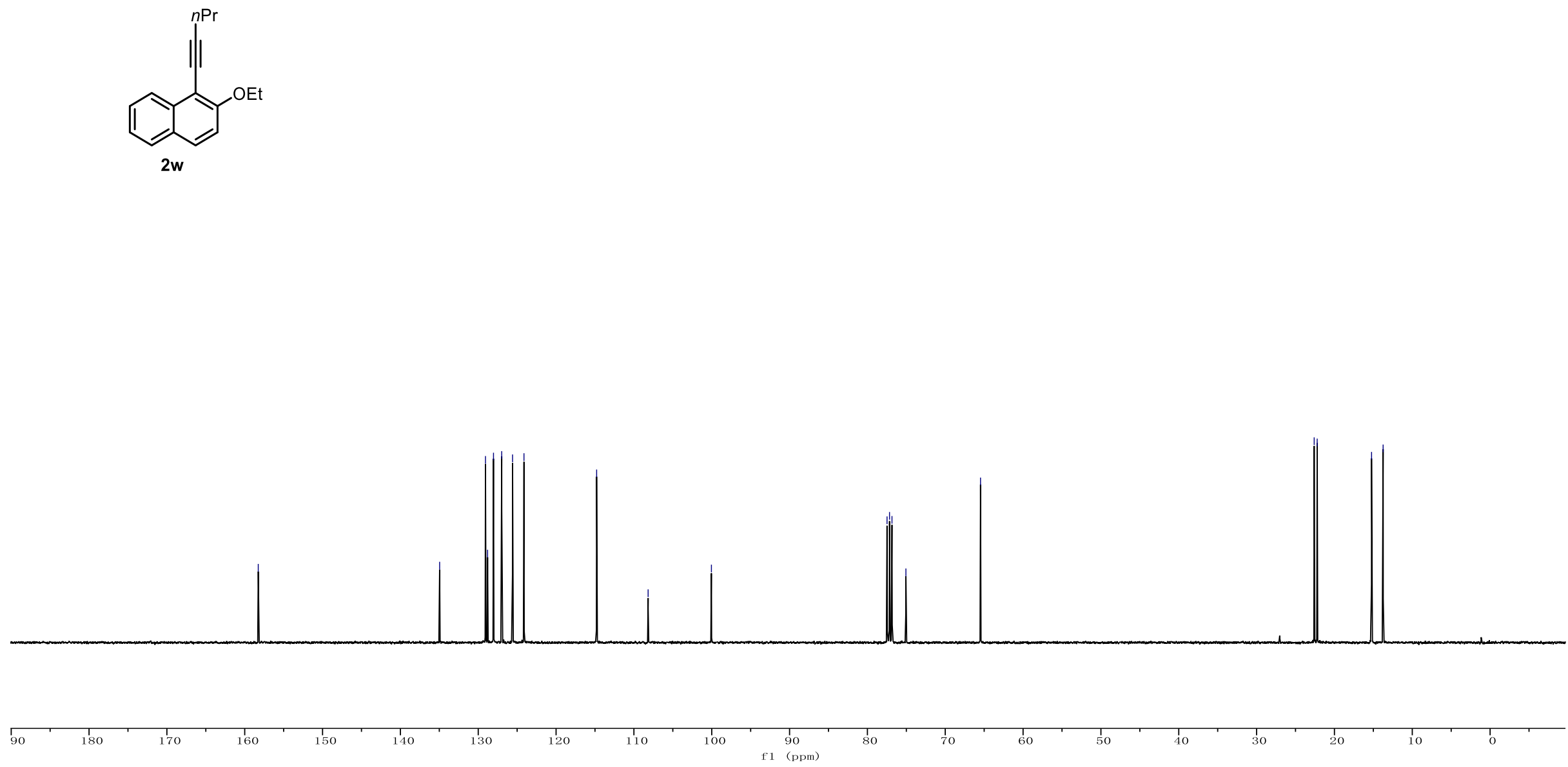

185 


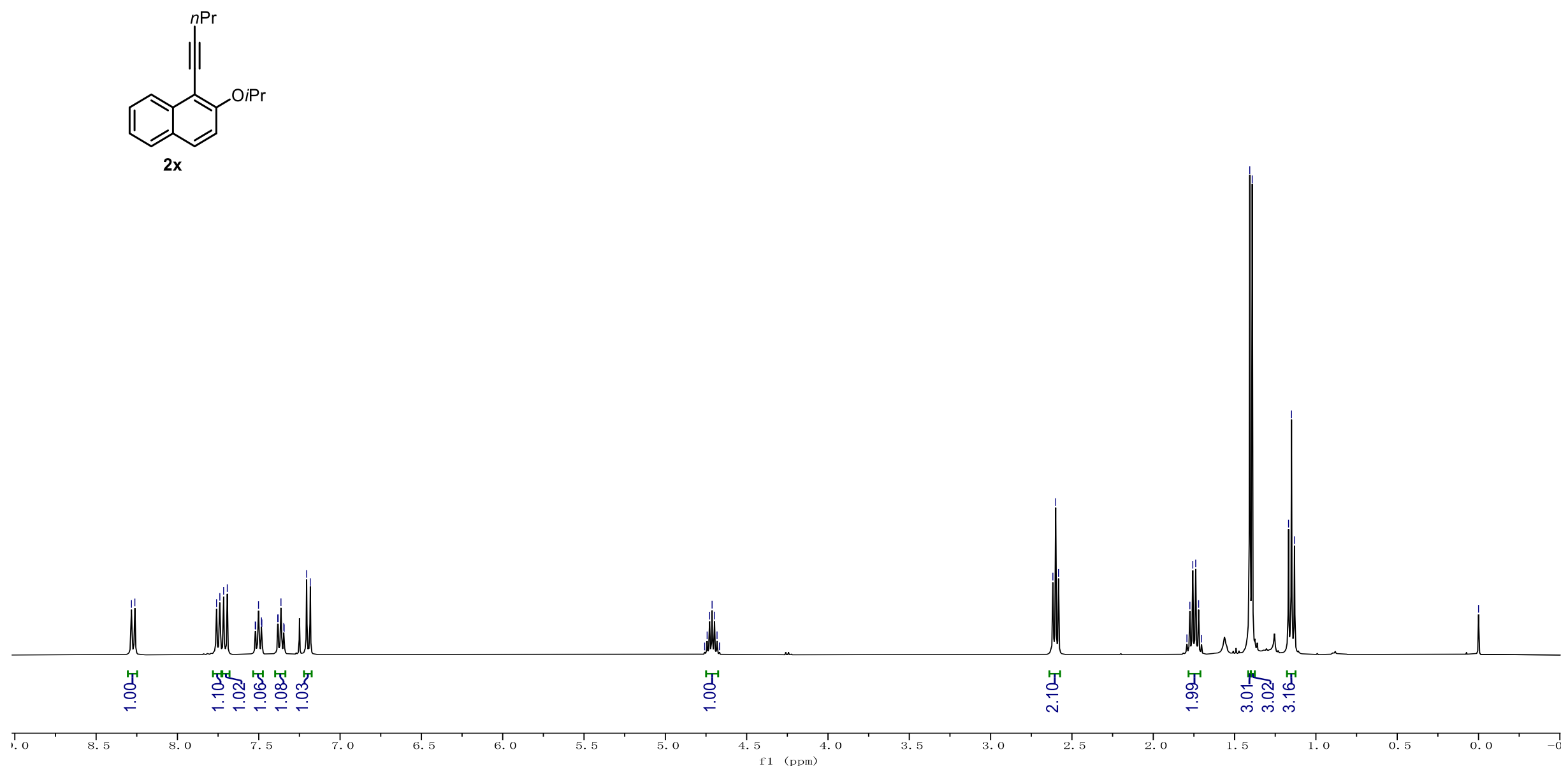

$2 \mathrm{x}$ 


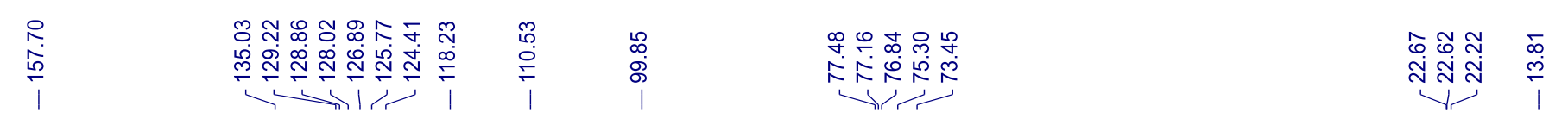
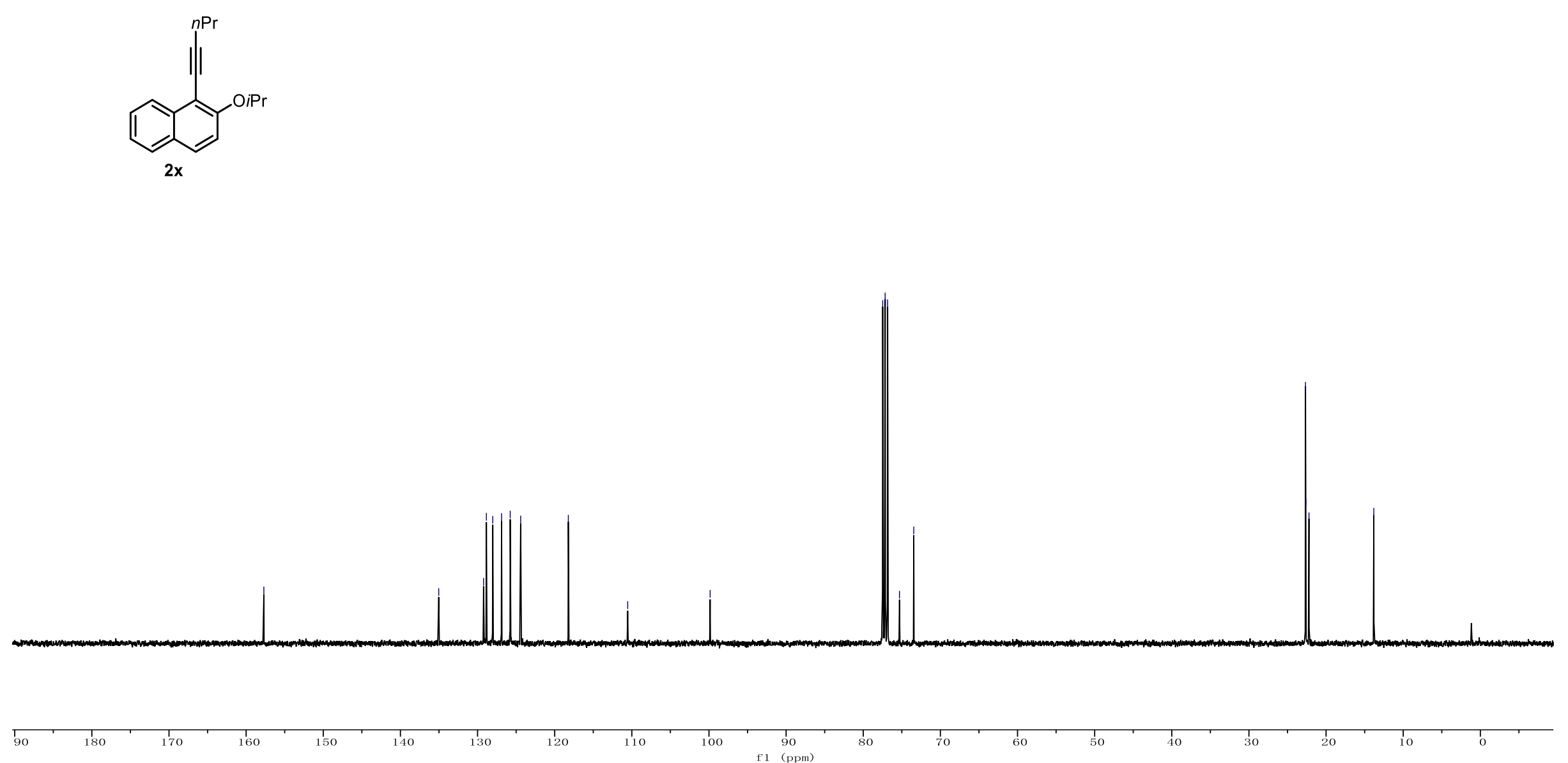


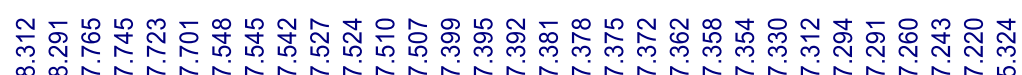

o

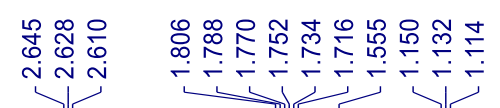
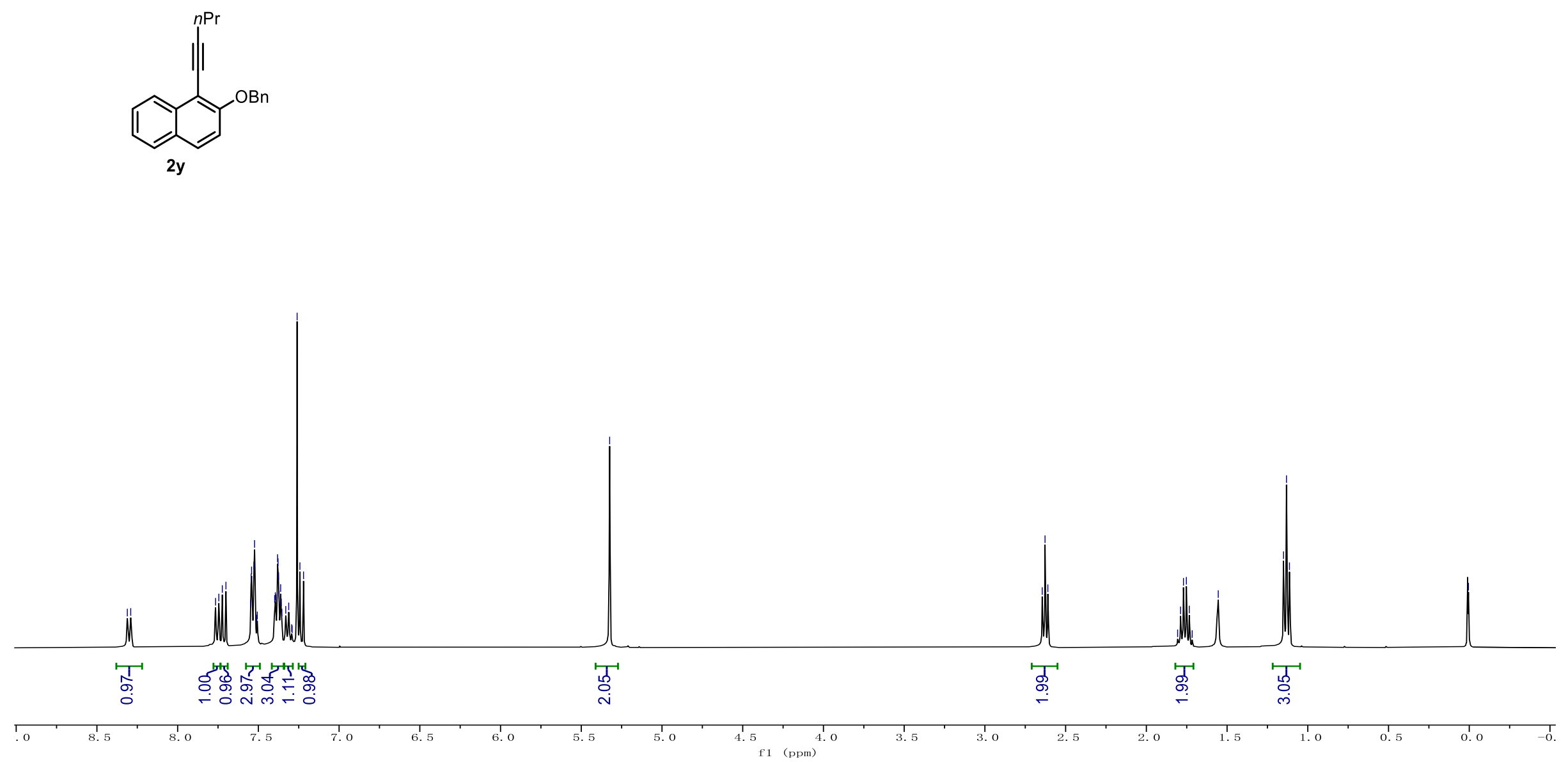


\begin{tabular}{|c|c|c|c|}
\hline 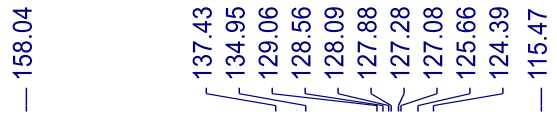 & 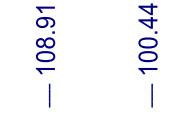 & 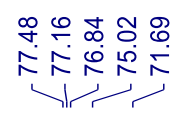 & స్ \\
\hline
\end{tabular}
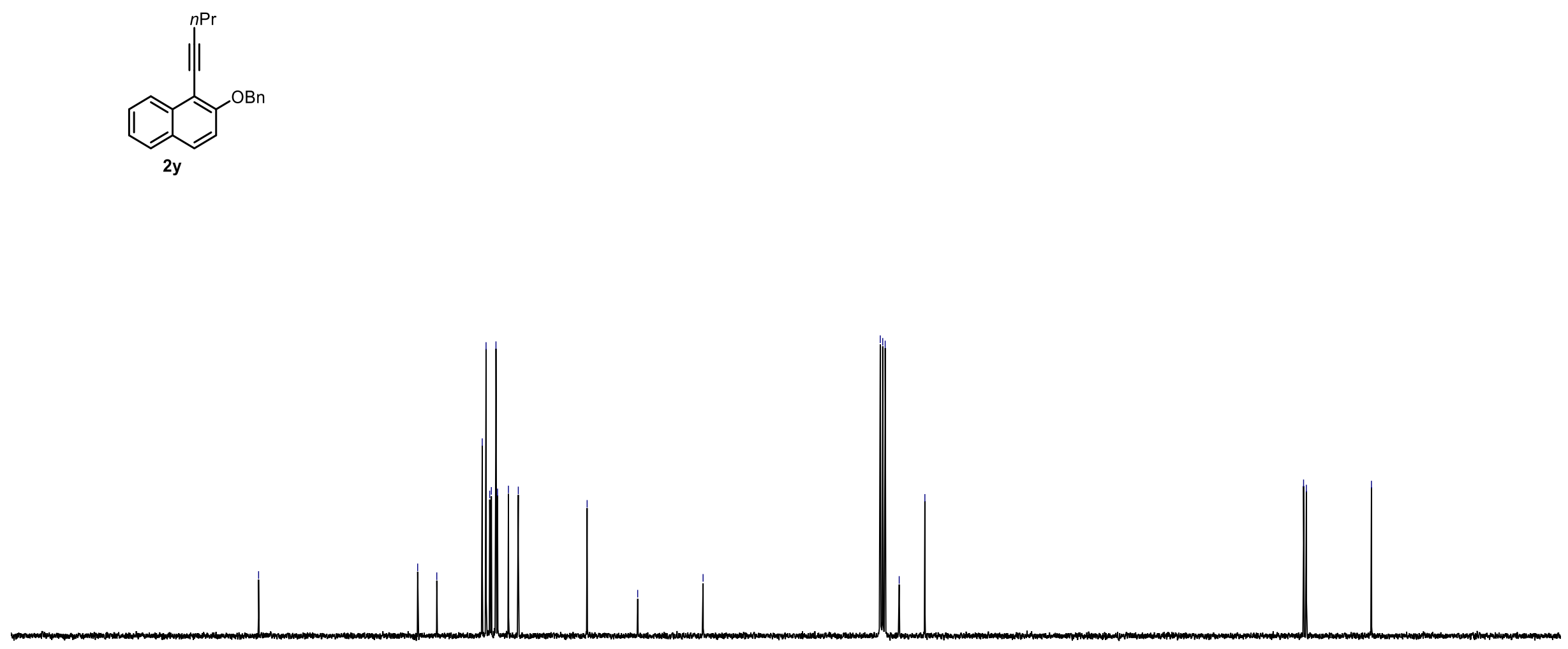

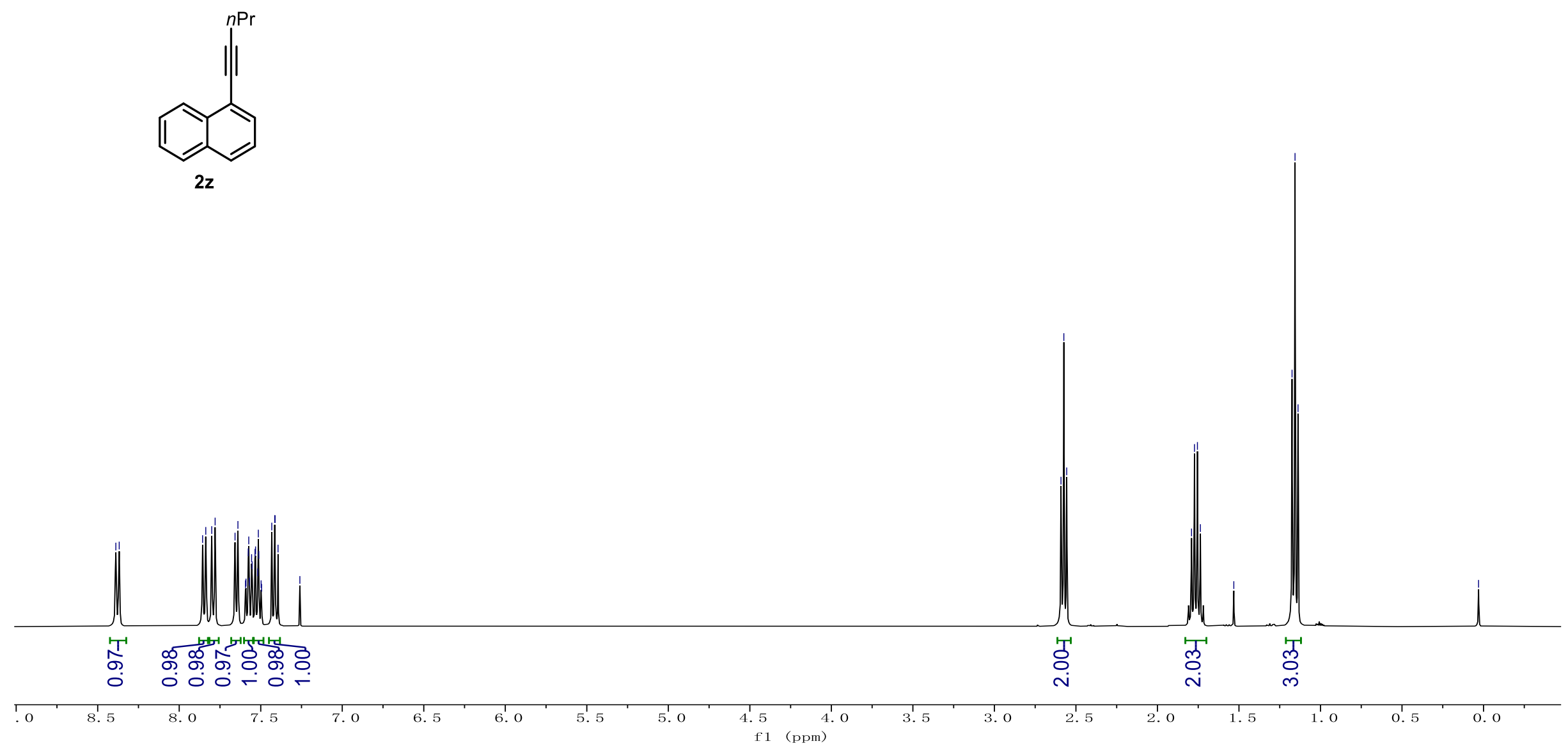

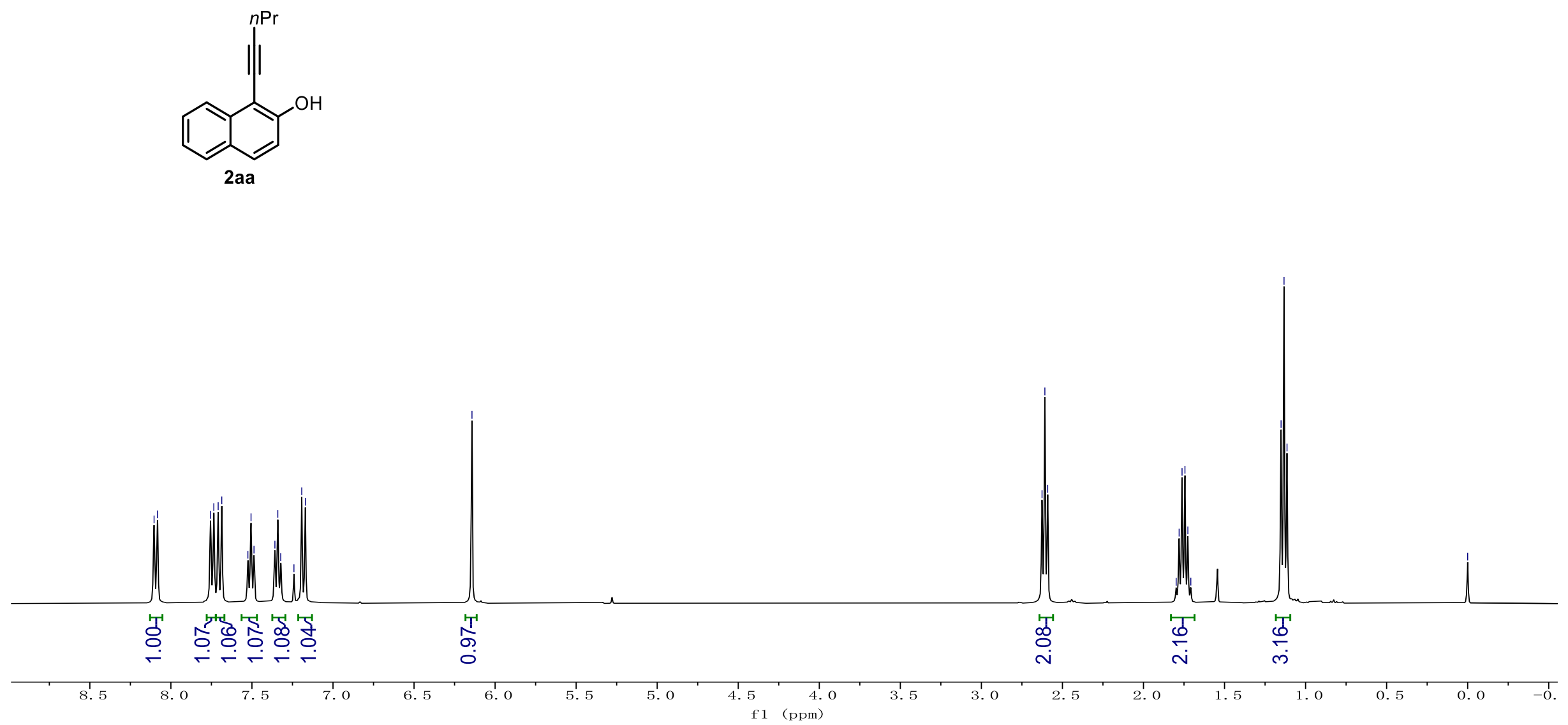


\begin{tabular}{|c|c|c|c|}
\hline 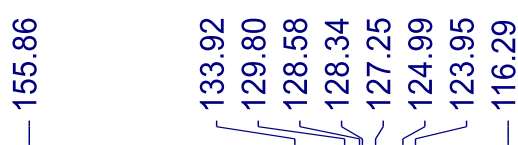 & 范 & 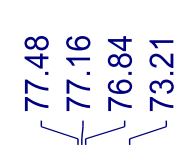 & îñ \\
\hline
\end{tabular}

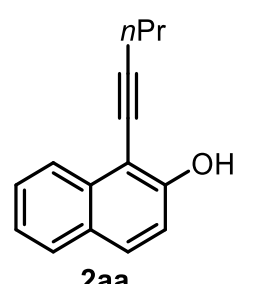

2aa

1.

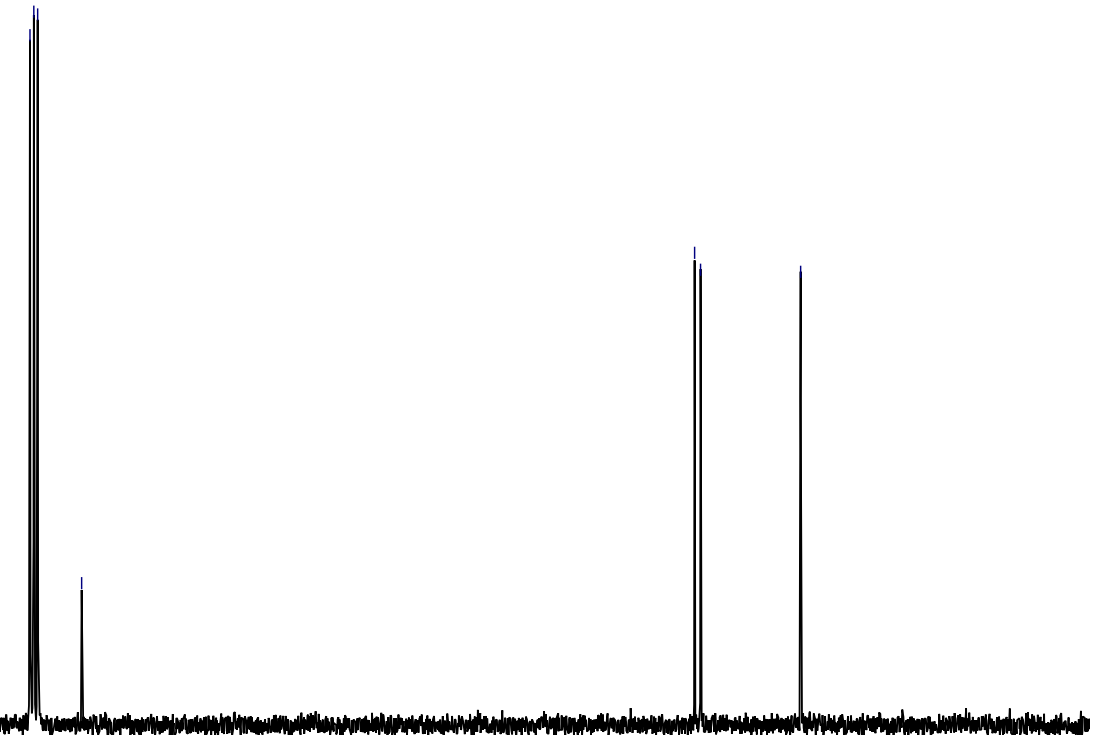

90
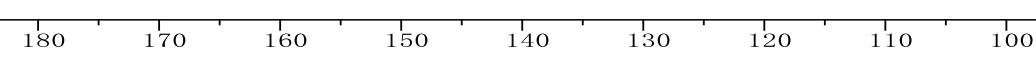

f1 $\stackrel{9}{90}(\mathrm{ppm})$ 


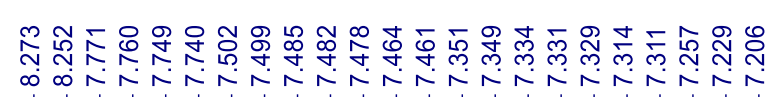
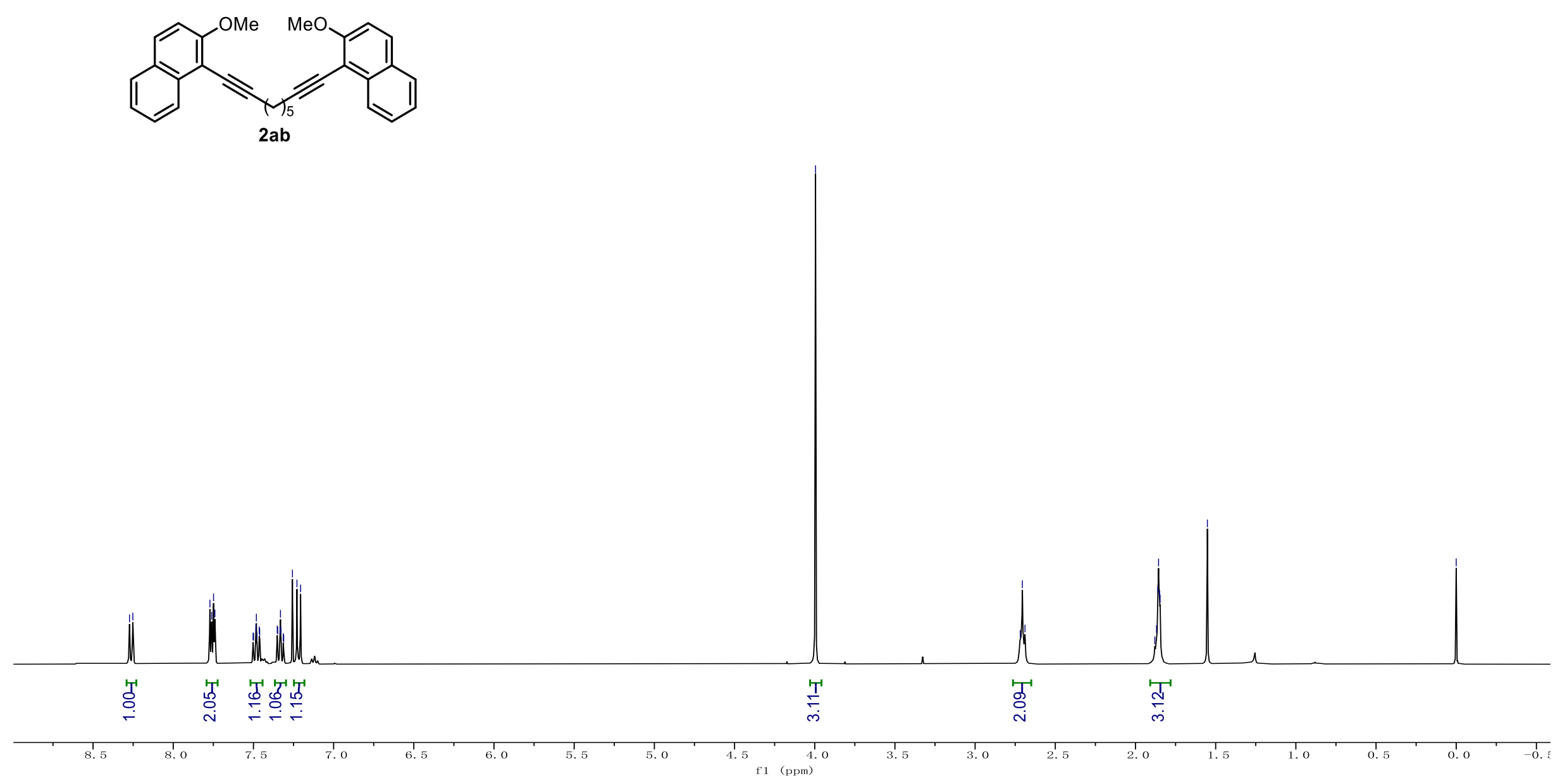
$\infty$
0
$\infty$
$\stackrel{\infty}{\circ}$
$\stackrel{1}{1}$

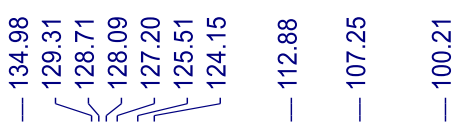

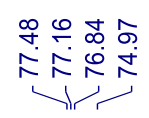

$\infty$
2
0
1
1

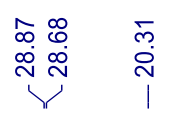
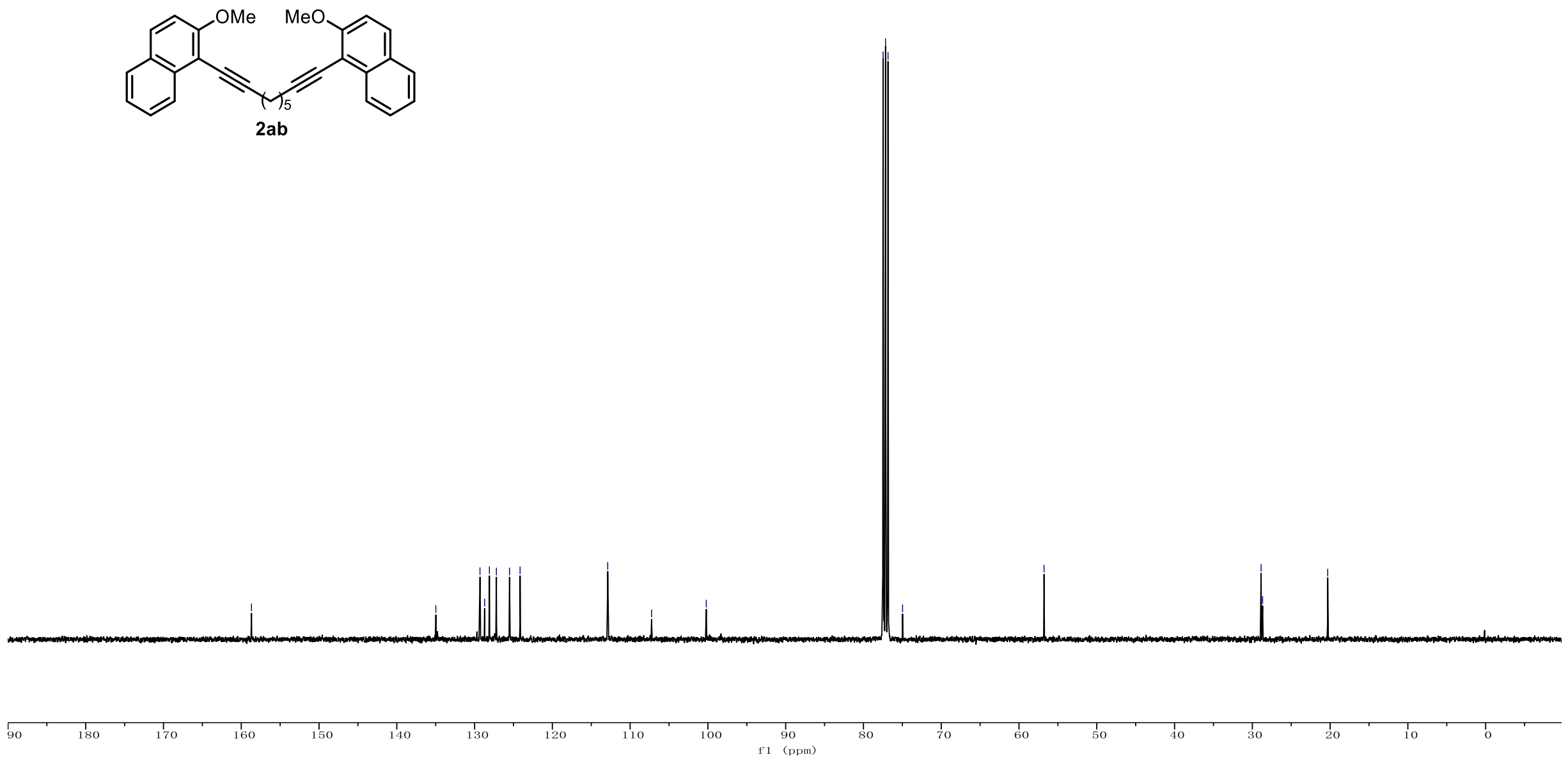

194 

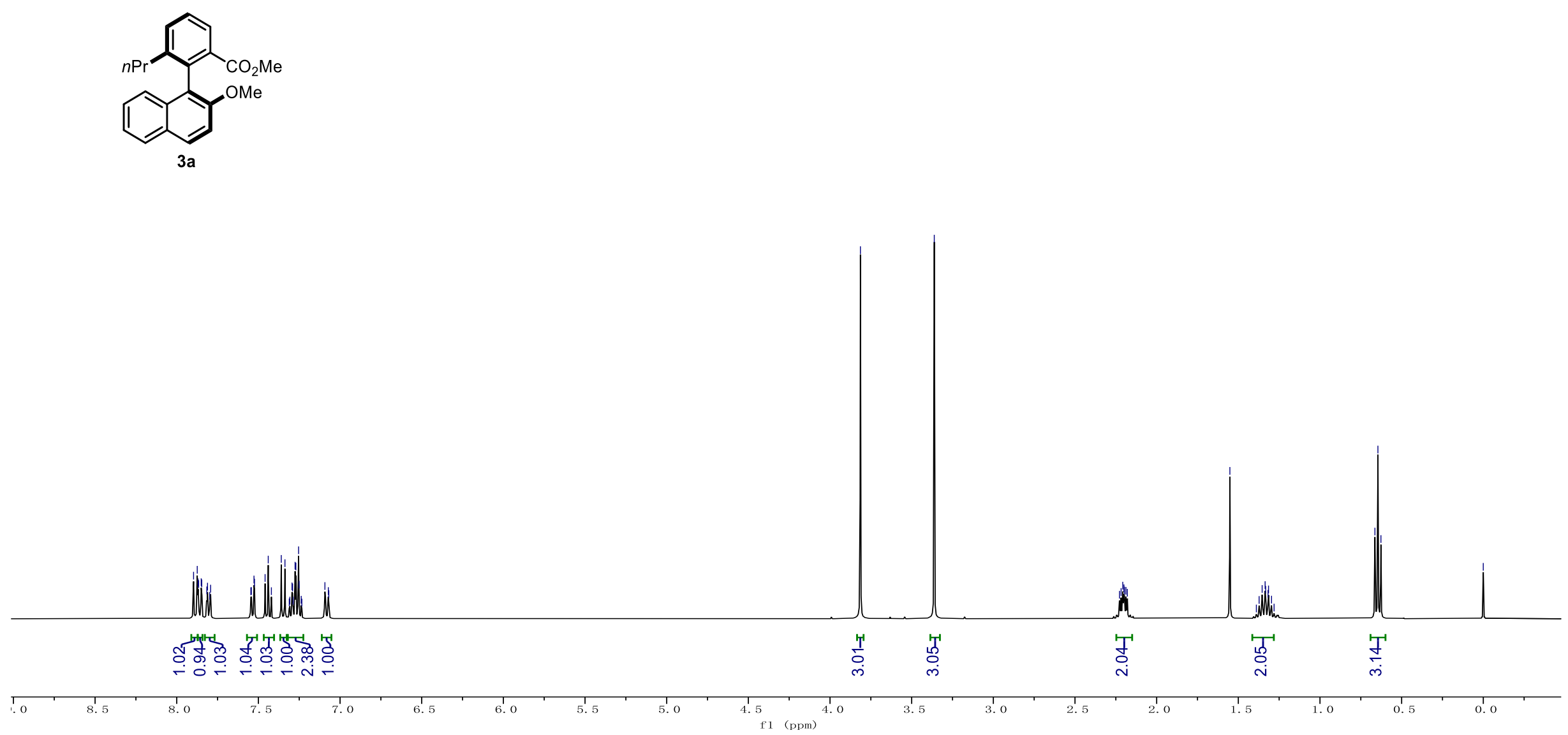
<smiles>COc1cccc2c1C(=O)c1cccc3cccc(c13)O2</smiles> 


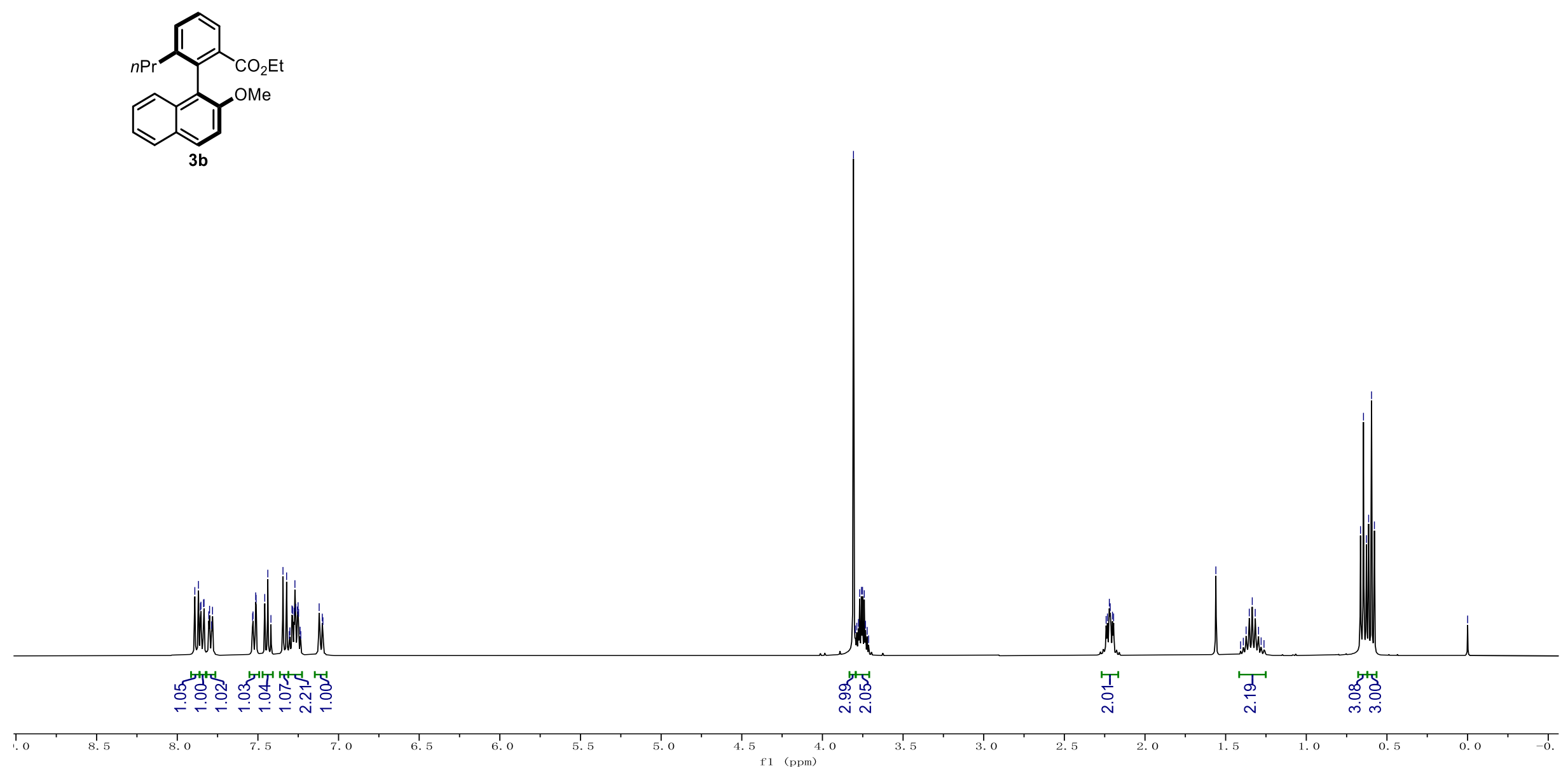




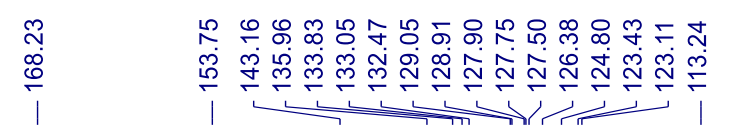

$\sqrt{1}$

$\begin{array}{ll}1 & \\ m & 0 \\ 0 & 0 \\ 0 & 0 \\ 1 & 1 \\ 0 & 0\end{array}$

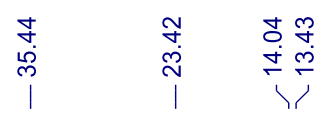
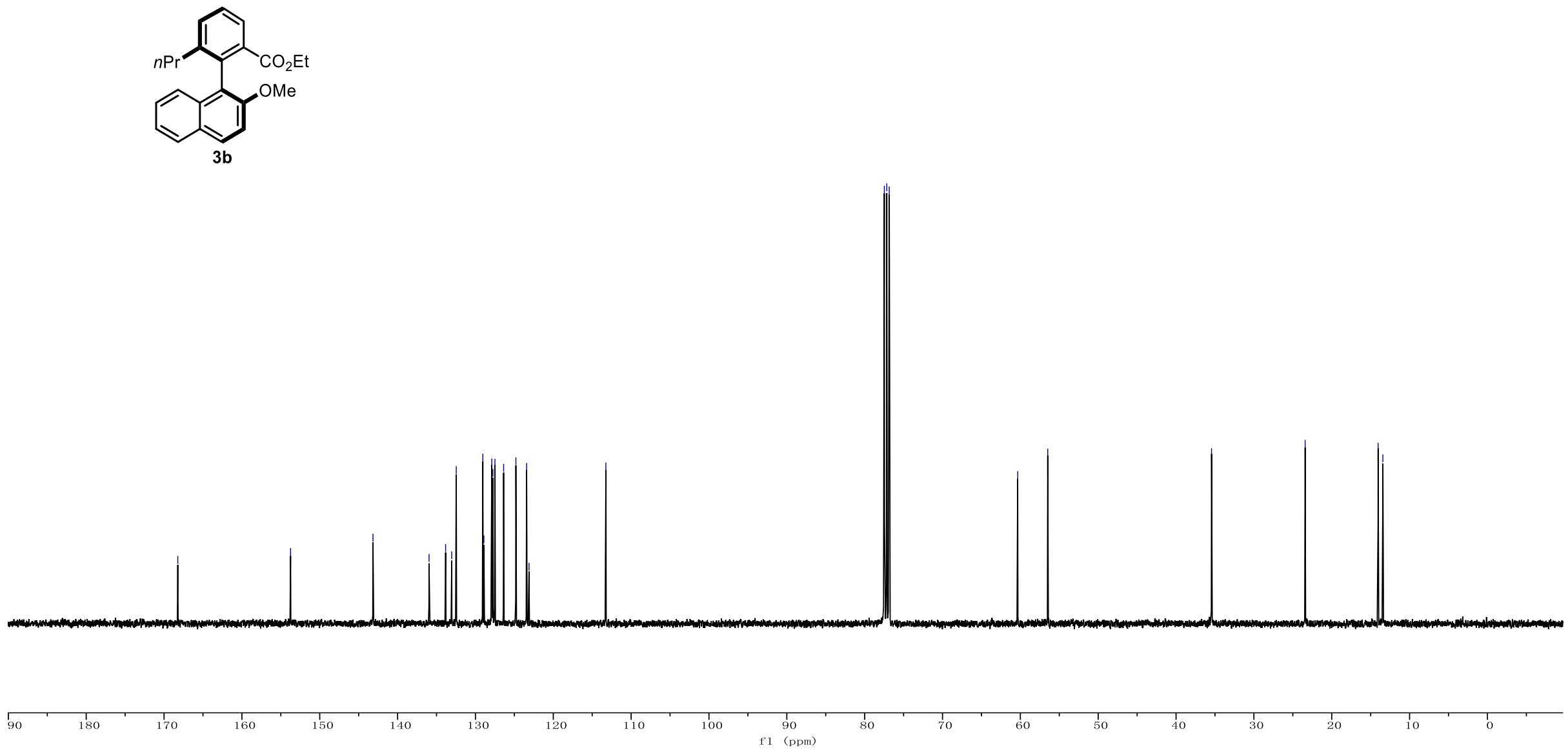

198 


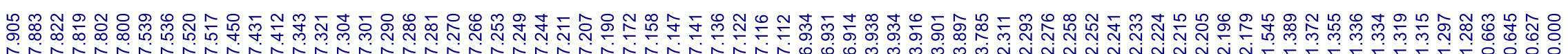<smiles>O=C(Oc1ccccc1)c1cccc2ccccc12</smiles>

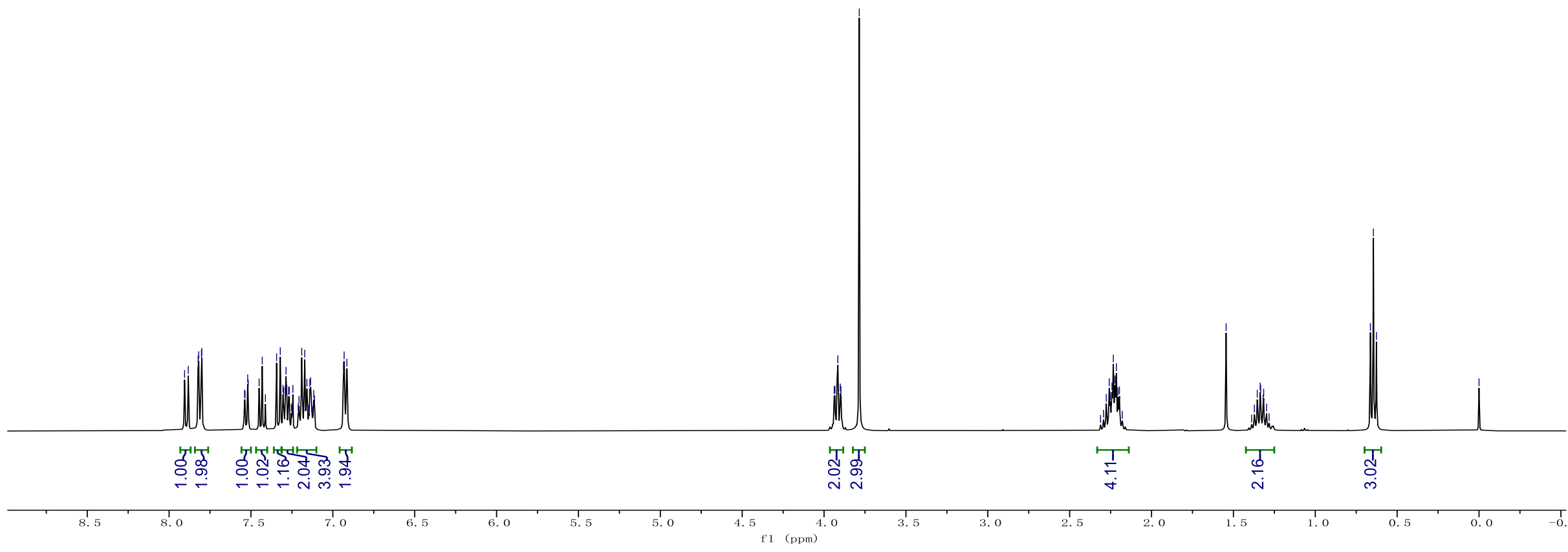



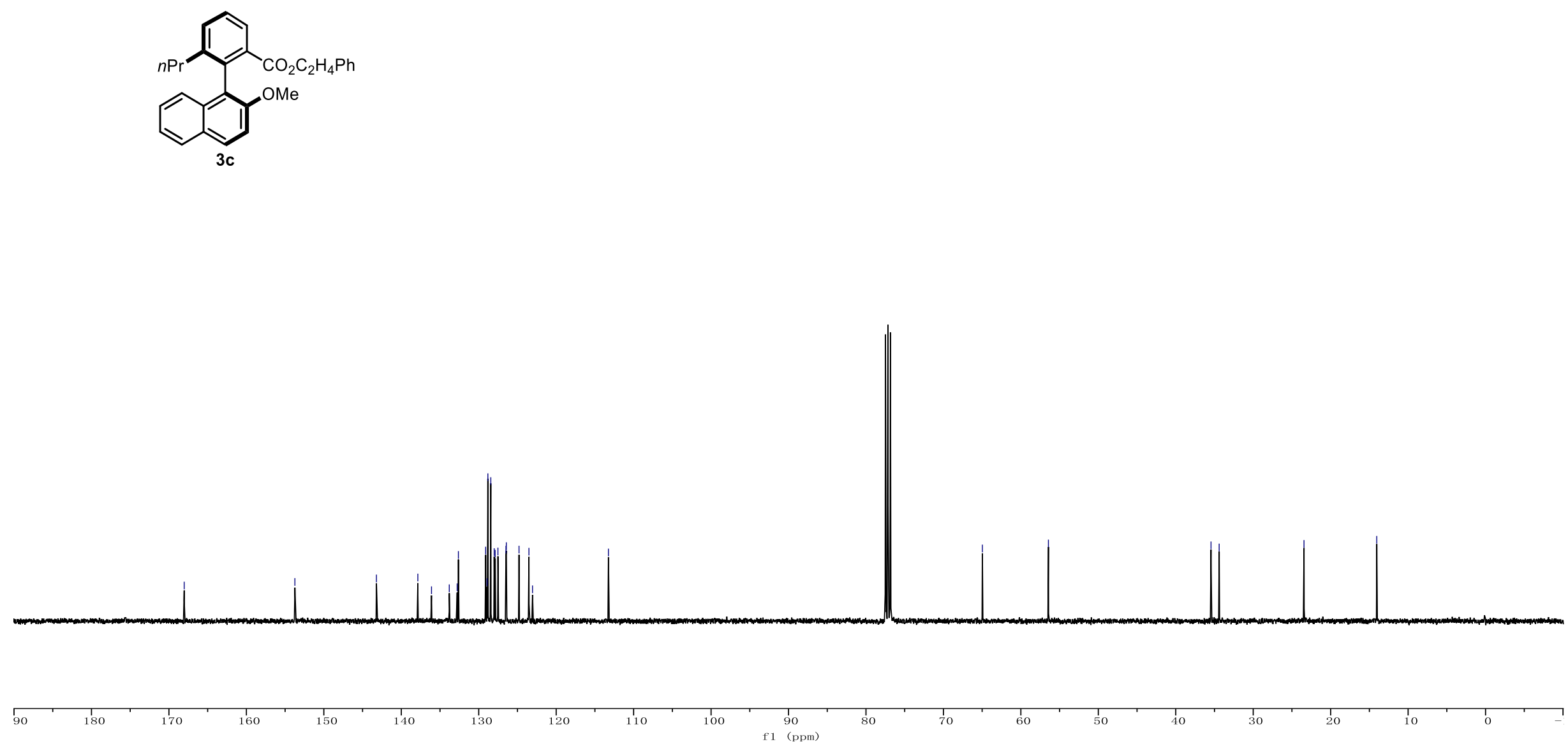

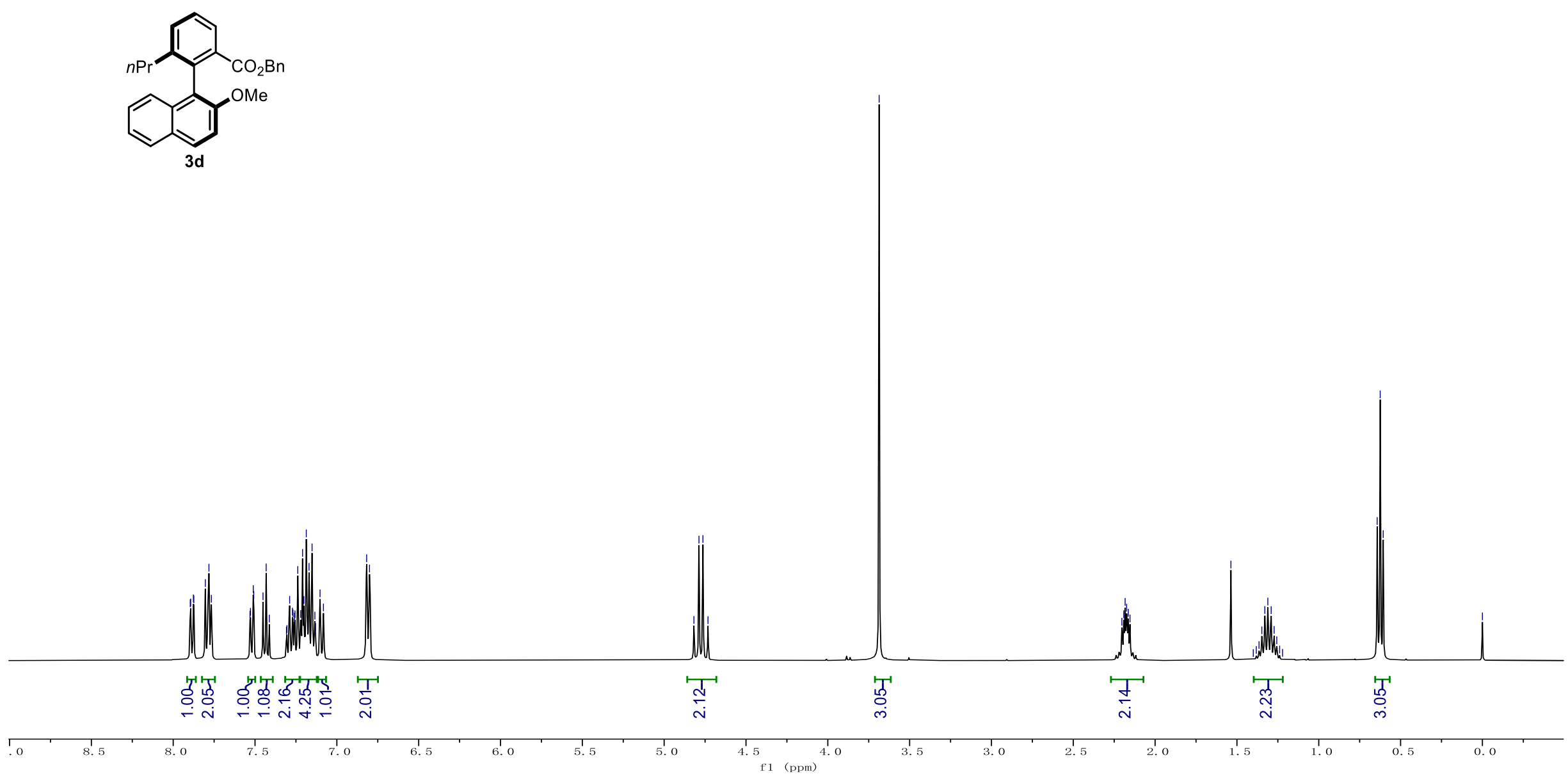


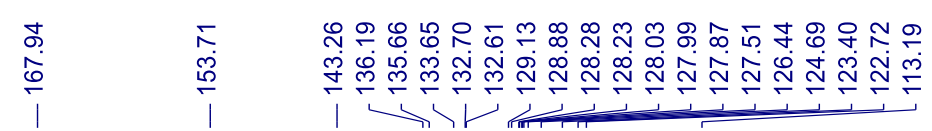

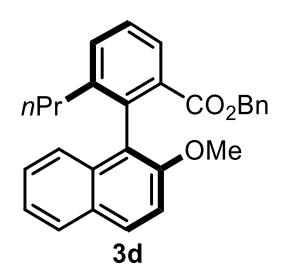

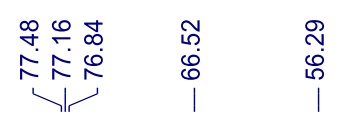

正

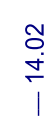
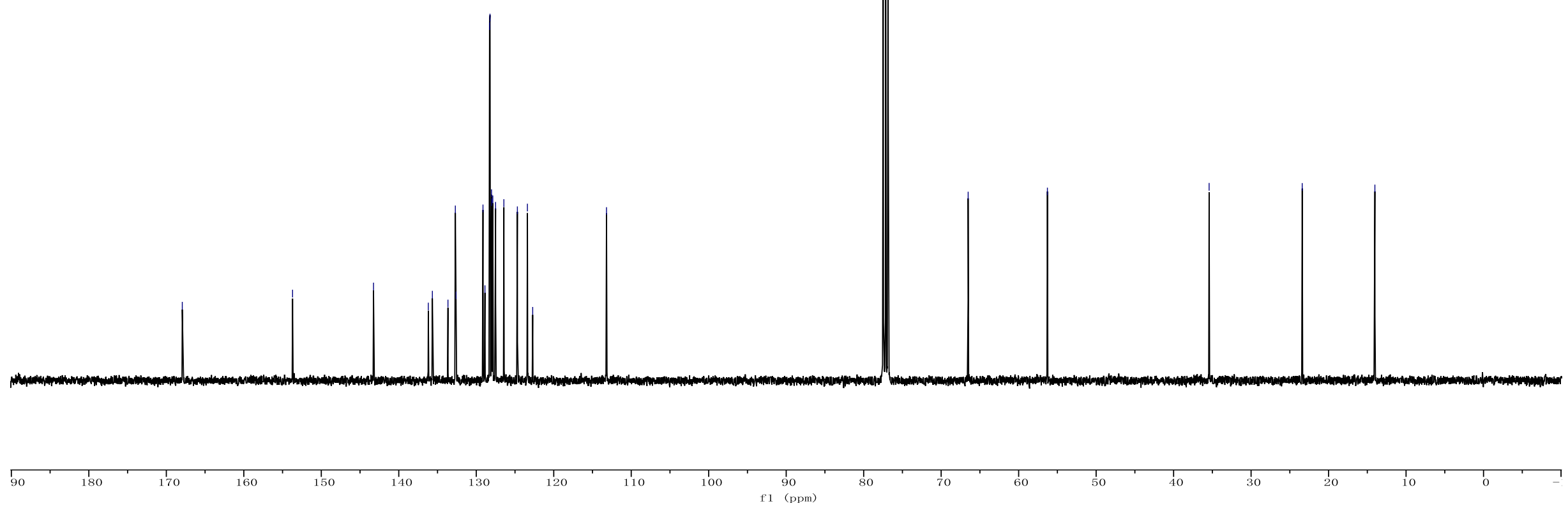

202 

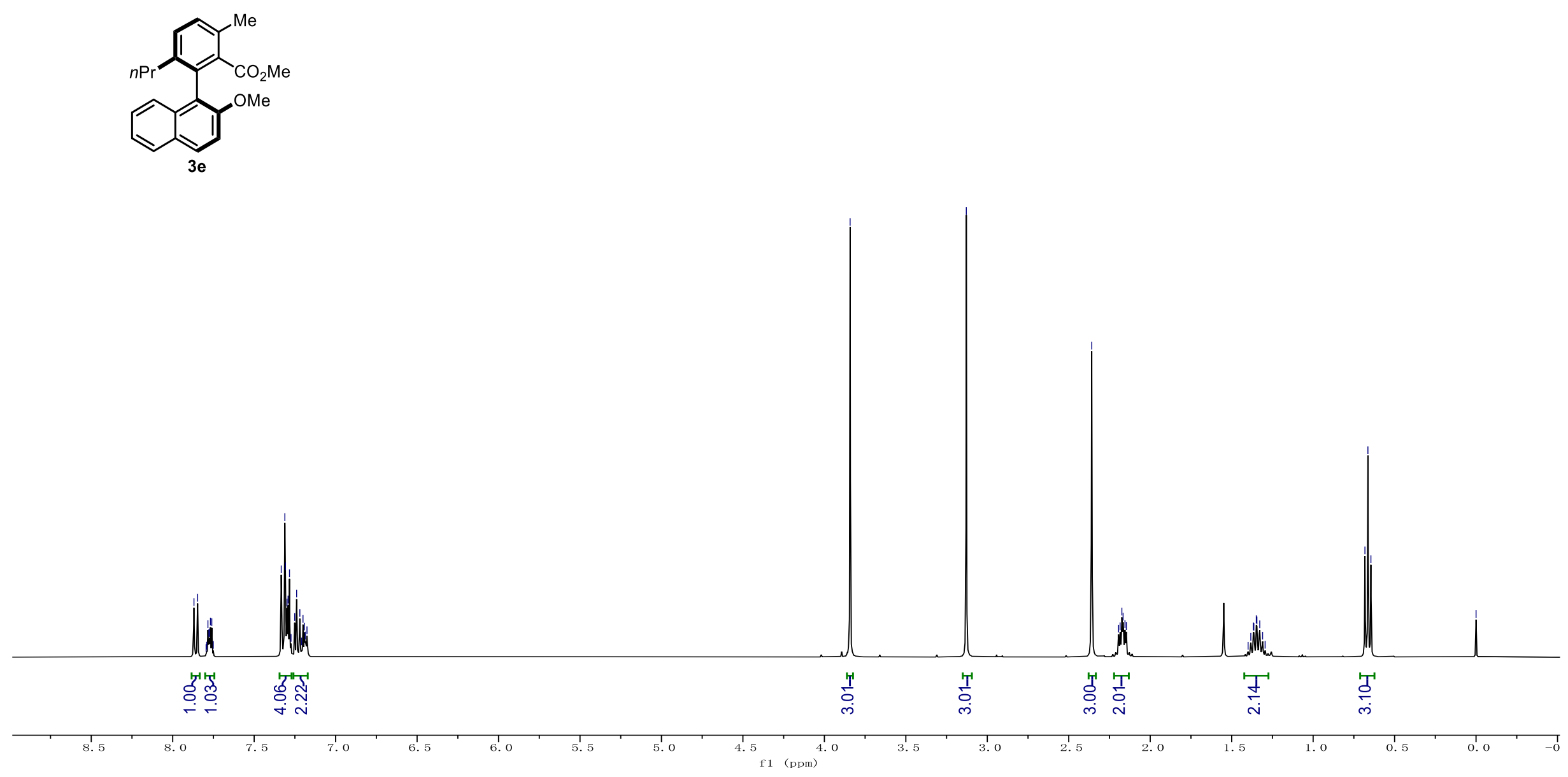


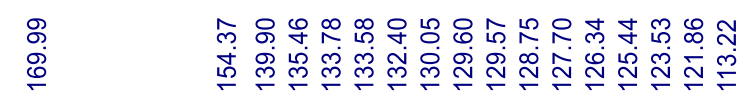

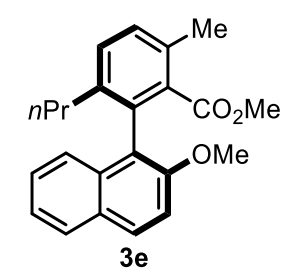



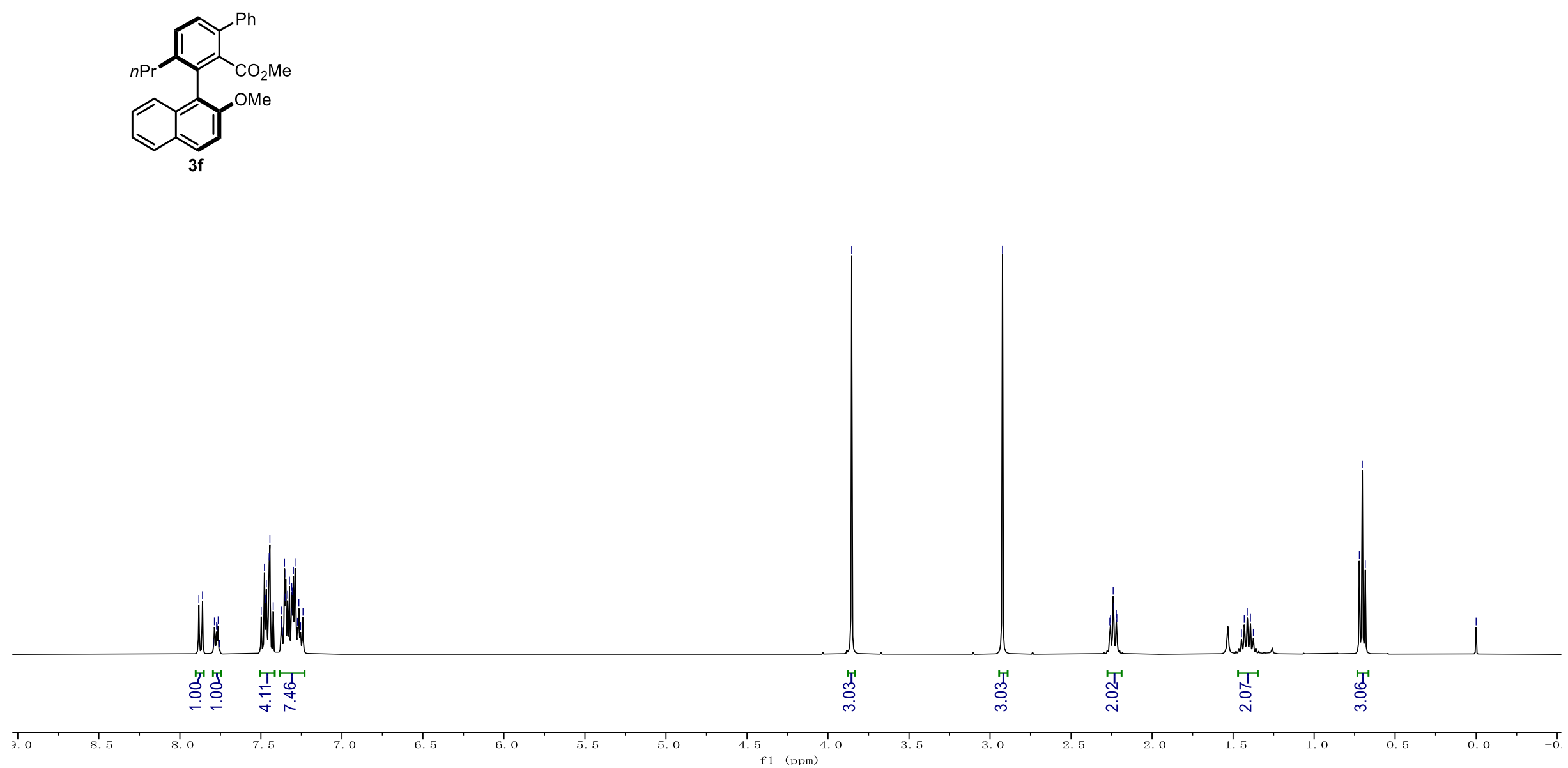
車

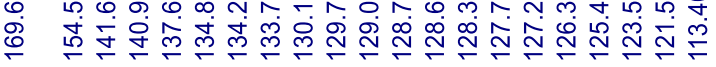

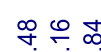

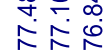

$\underset{\substack{0 \\ i}}{i}$

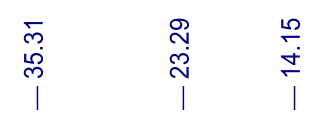
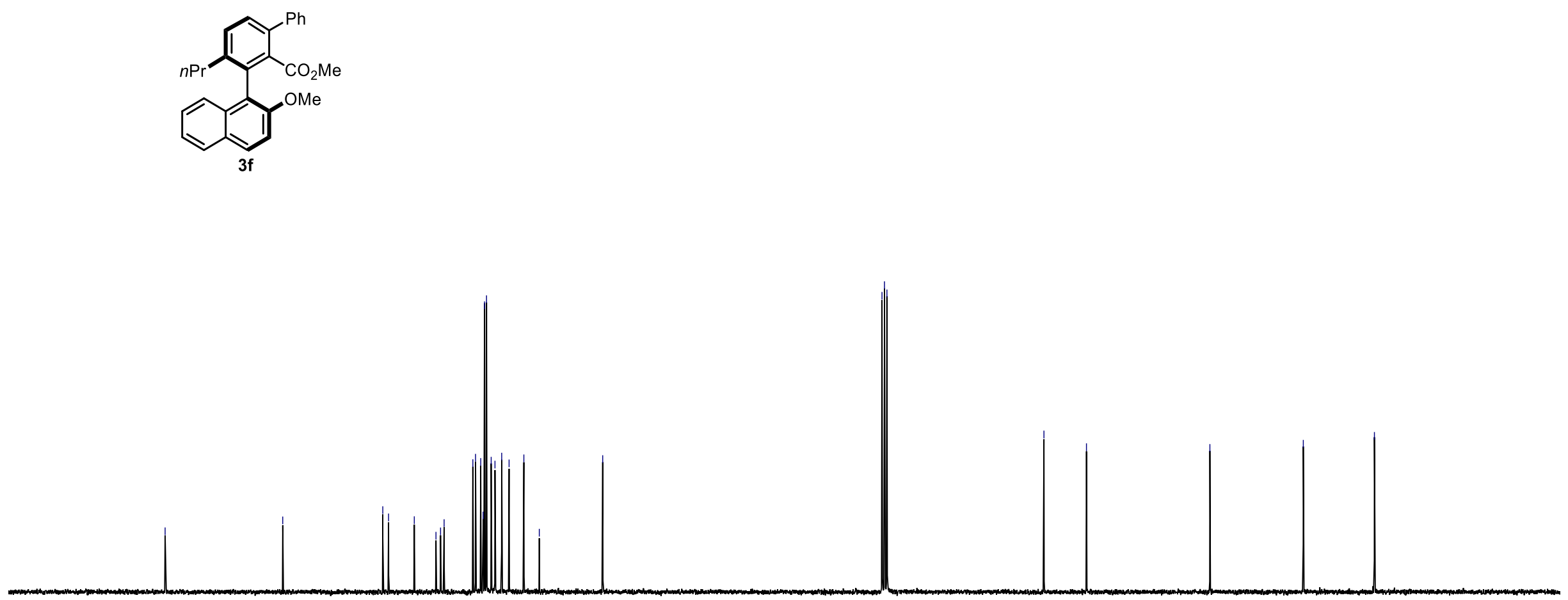

180

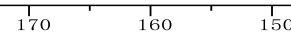

$140 \quad \frac{1}{130}$

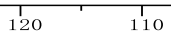

90

180 $\quad 70$

$\frac{1}{60}, \quad 10$

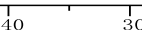



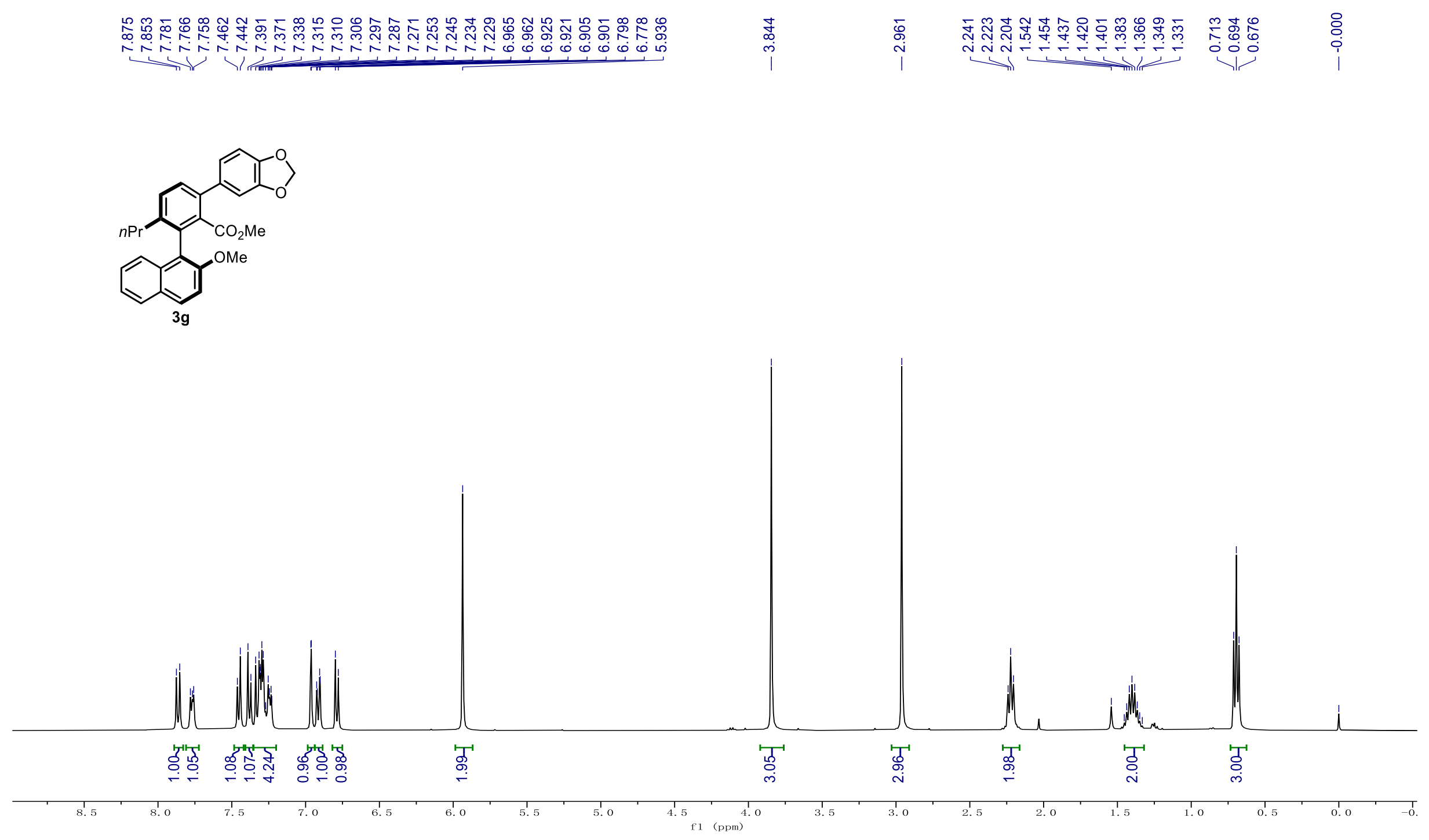

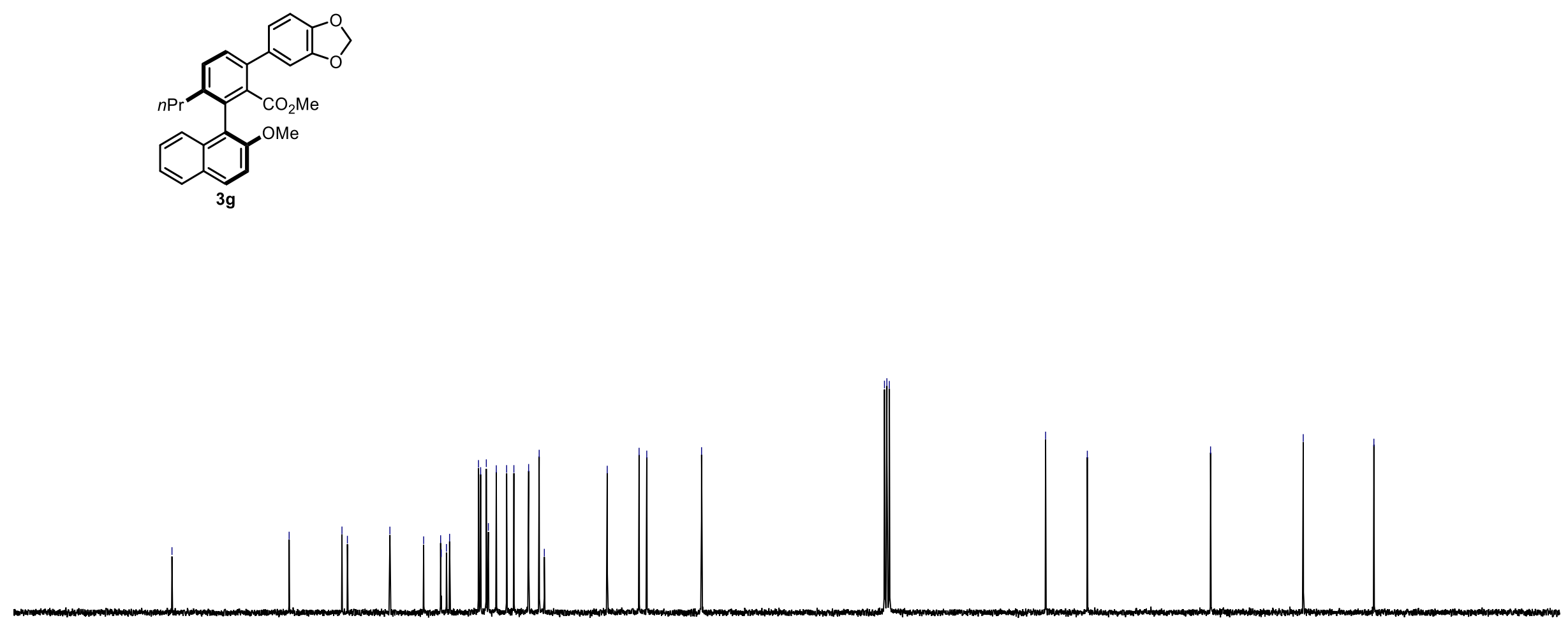

90 180 $170+160$ $150+140$
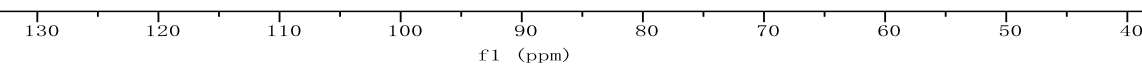

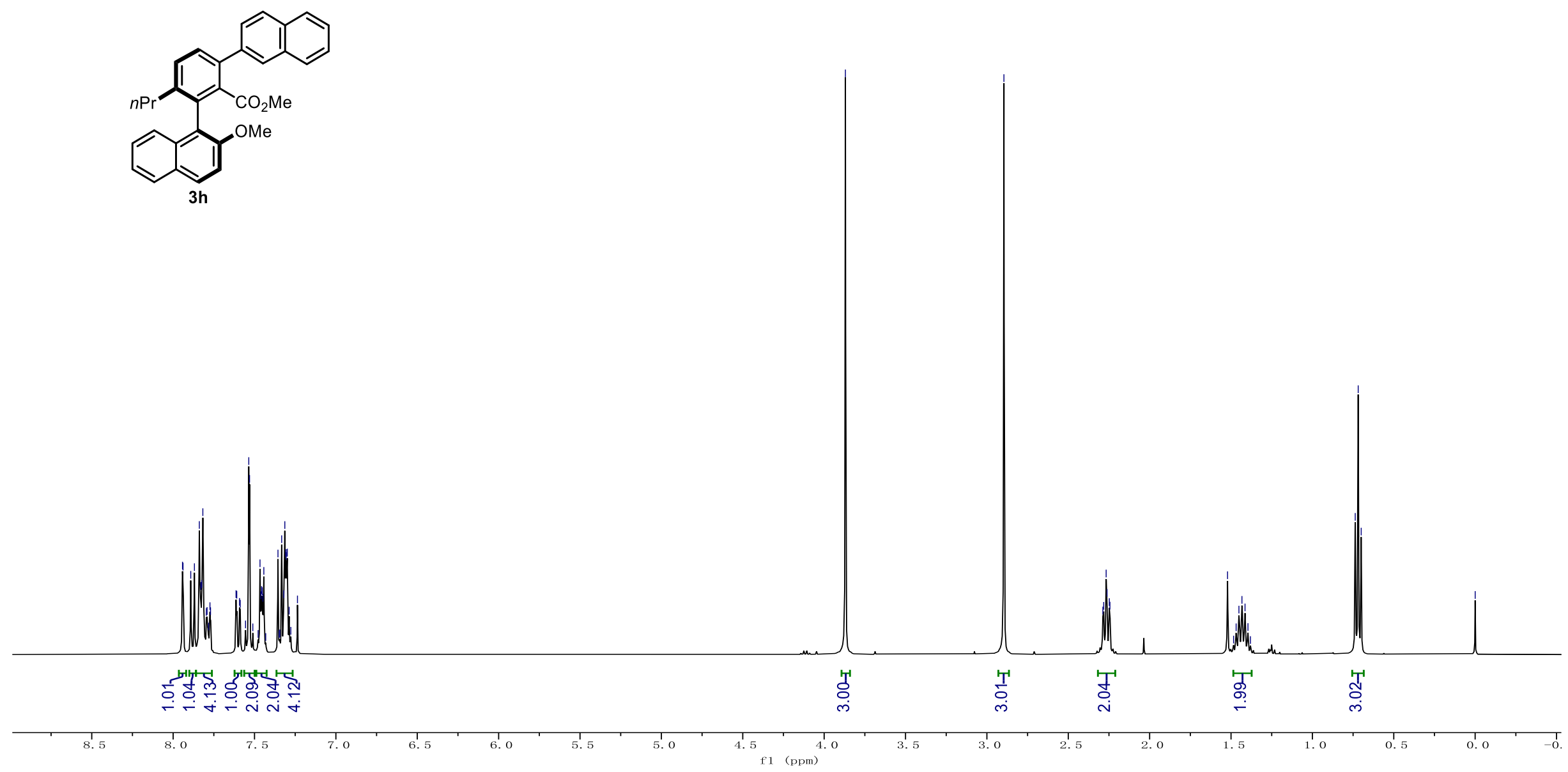


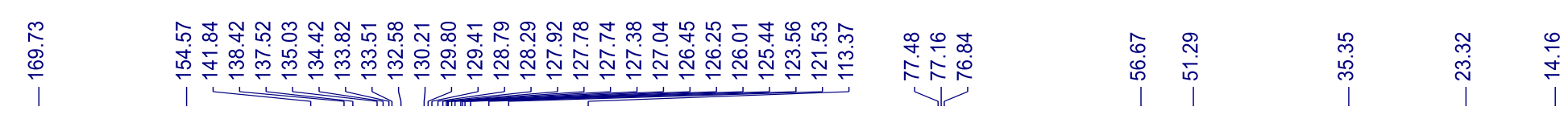
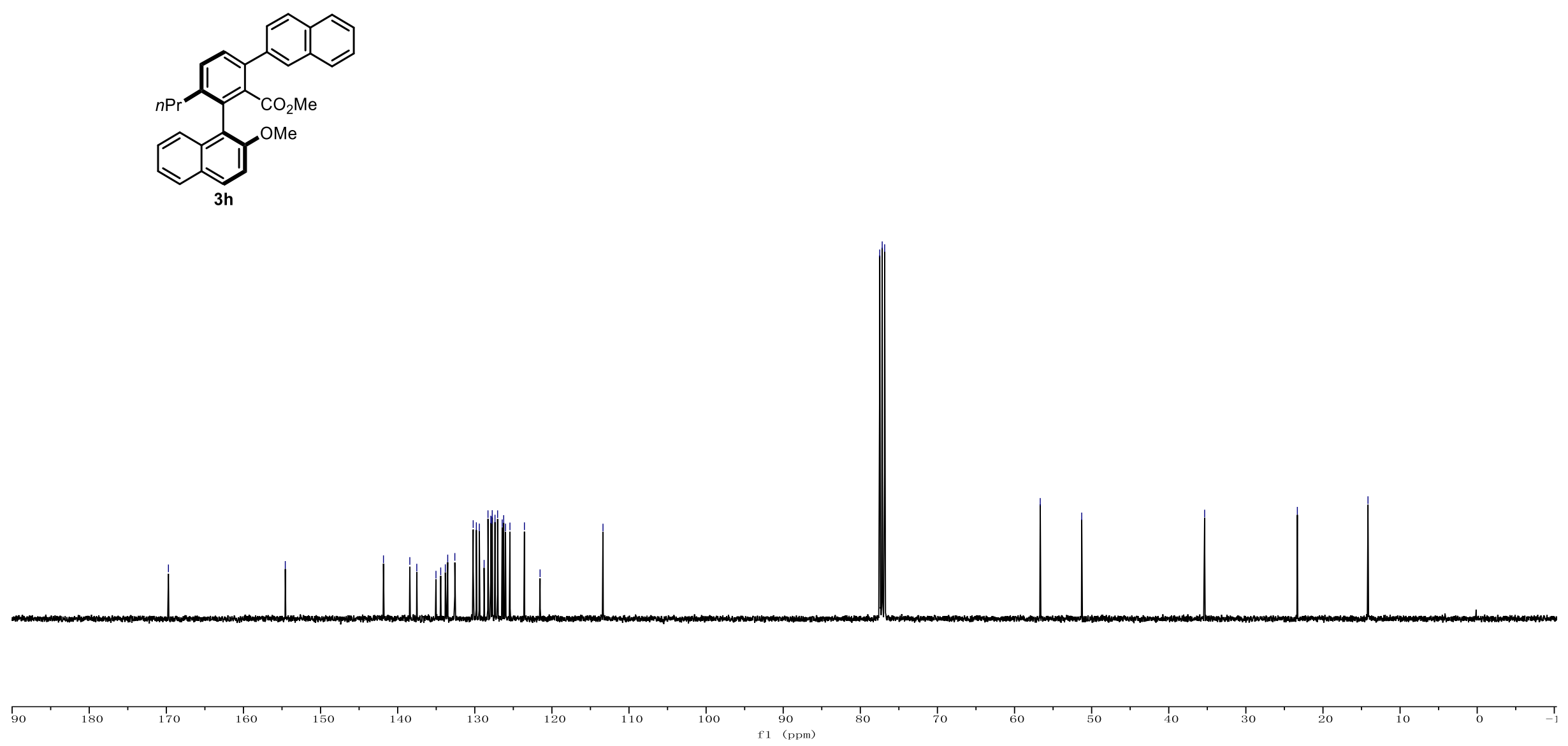


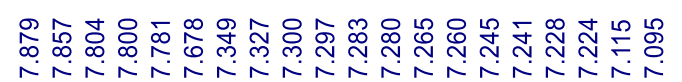
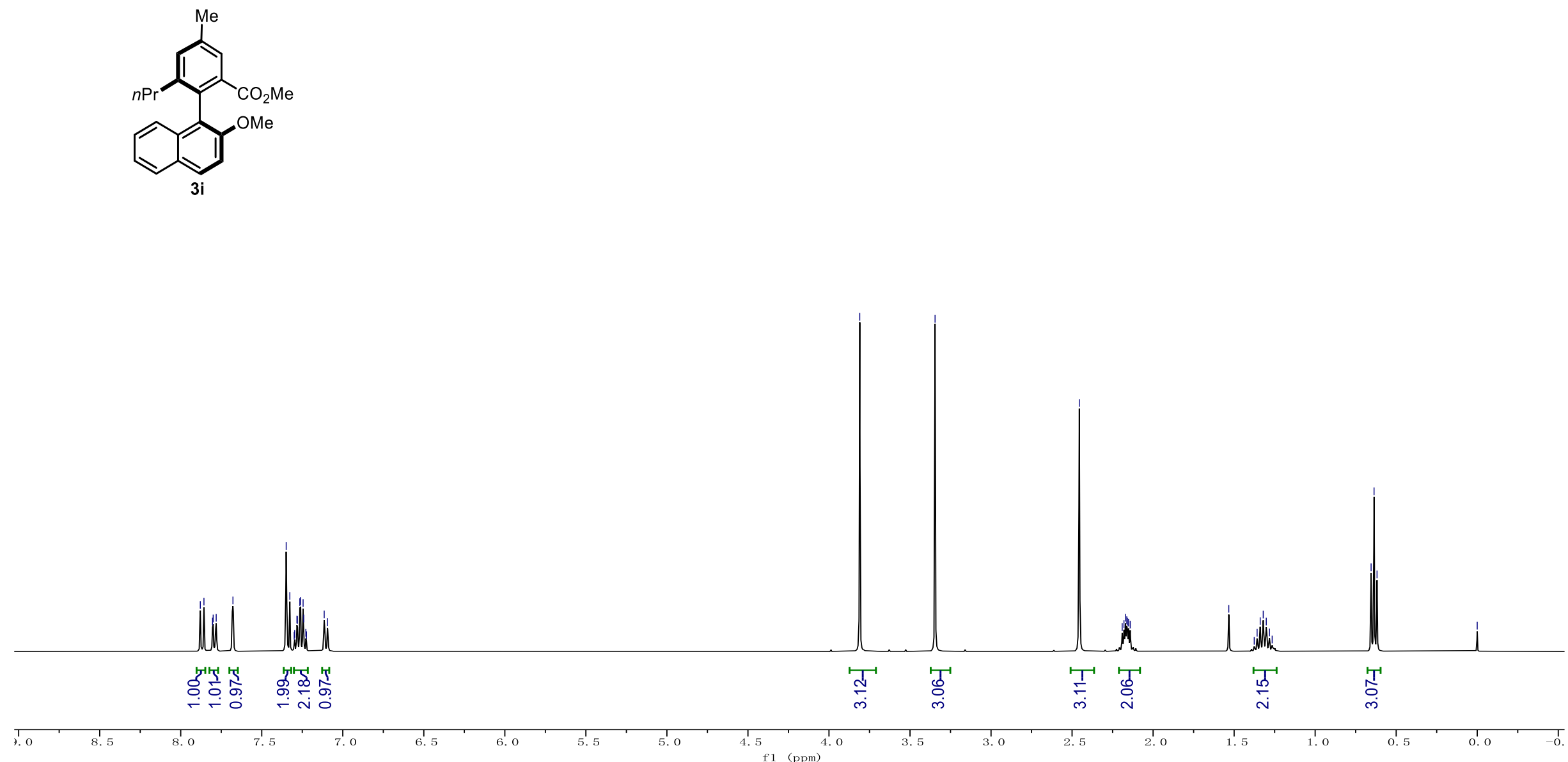


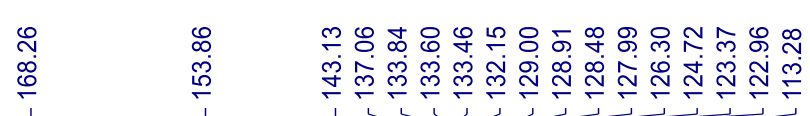

号음

송

in

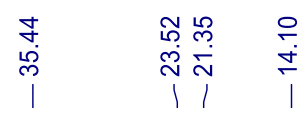
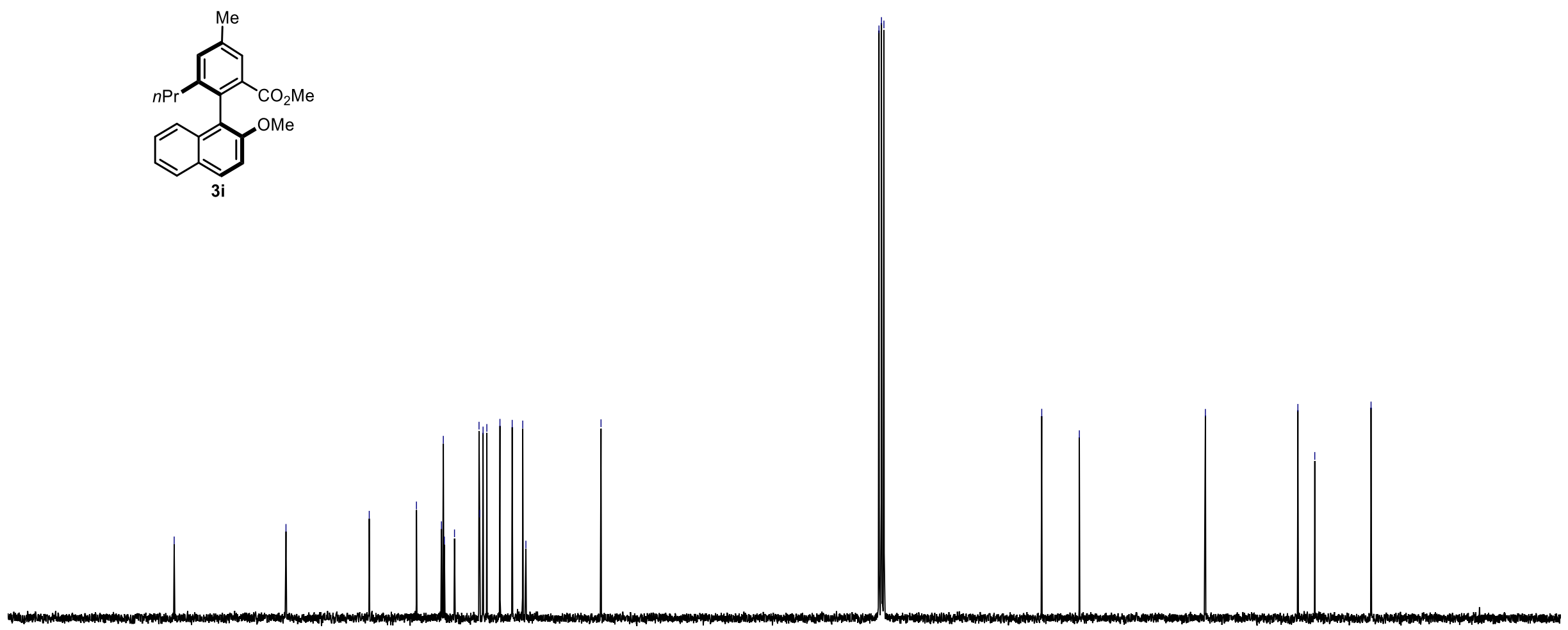

180
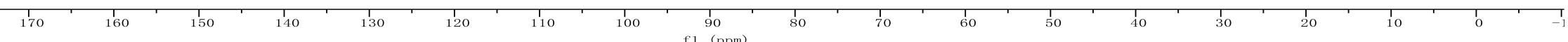

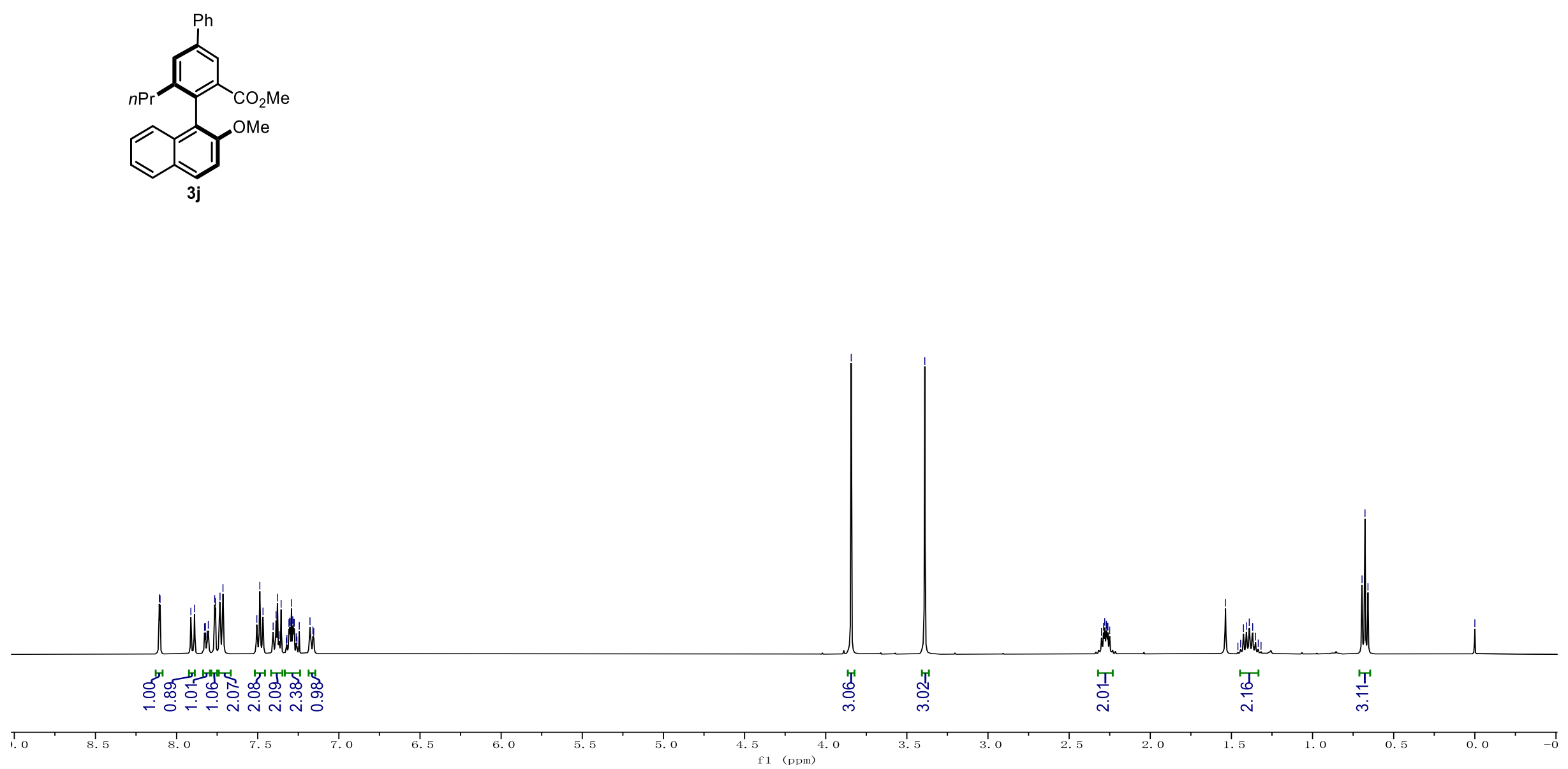
|

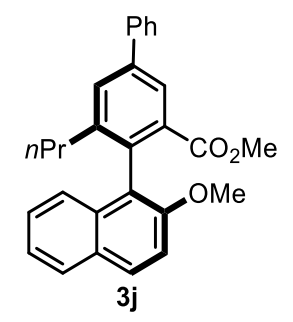

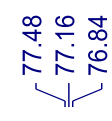

L
0
0
0

产

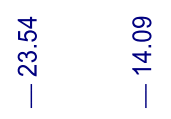
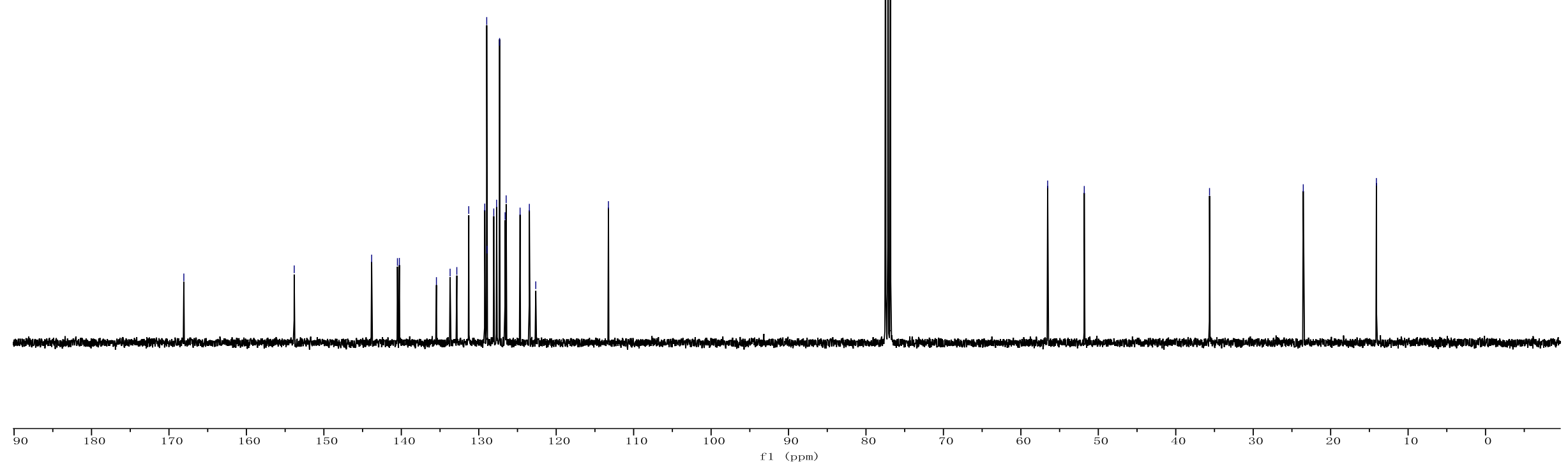

214 


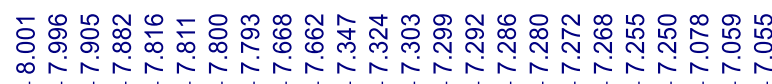
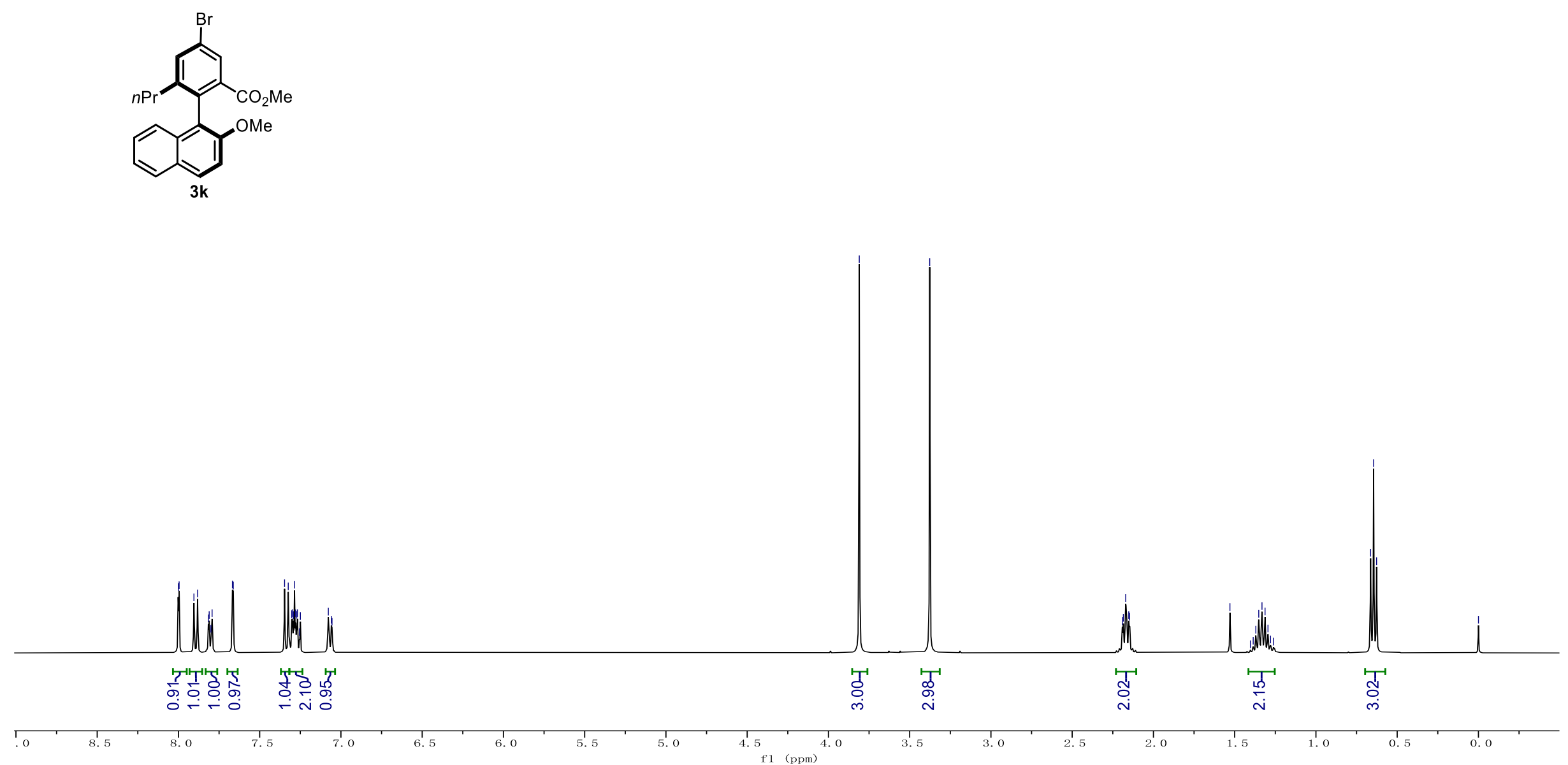


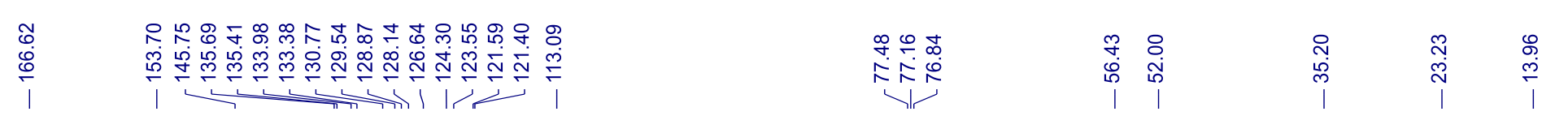
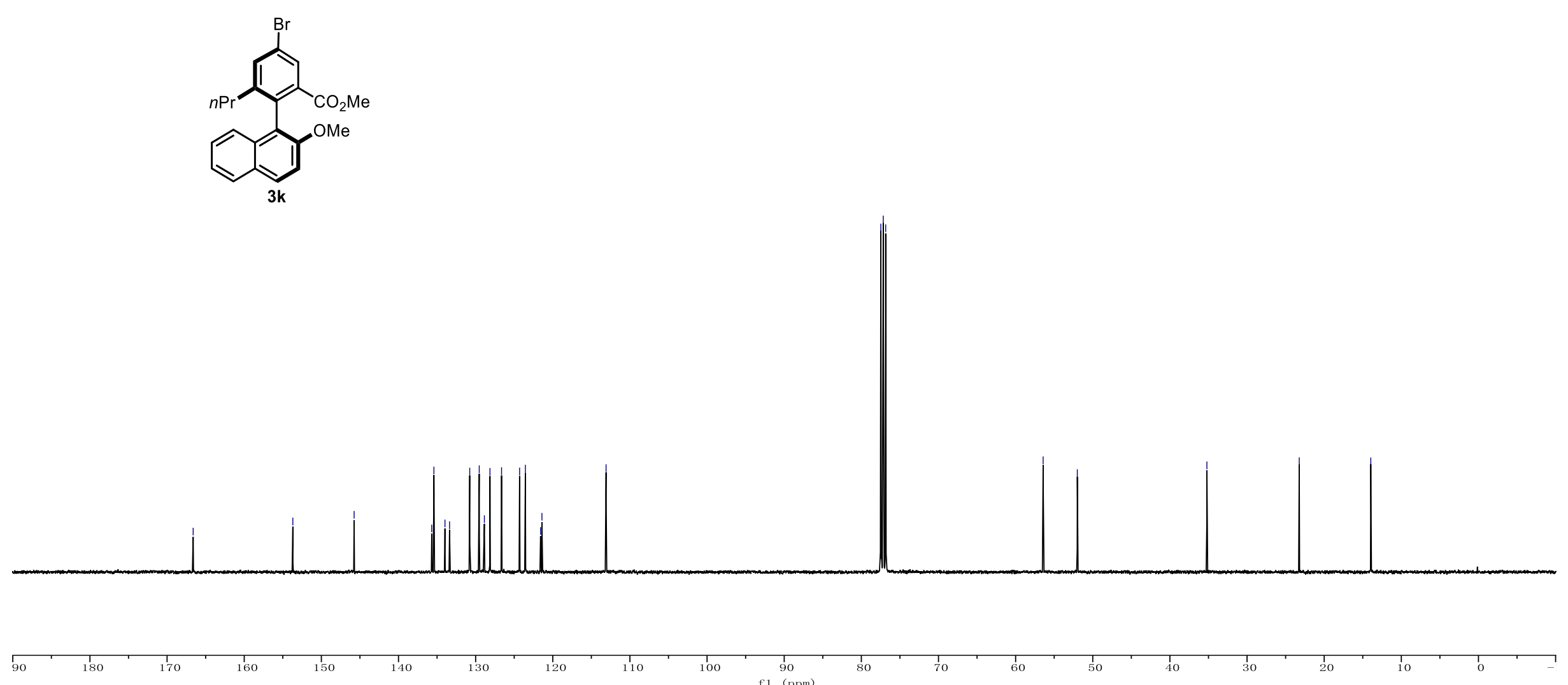


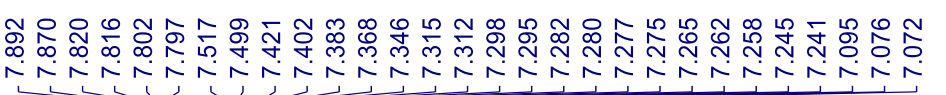

总

:
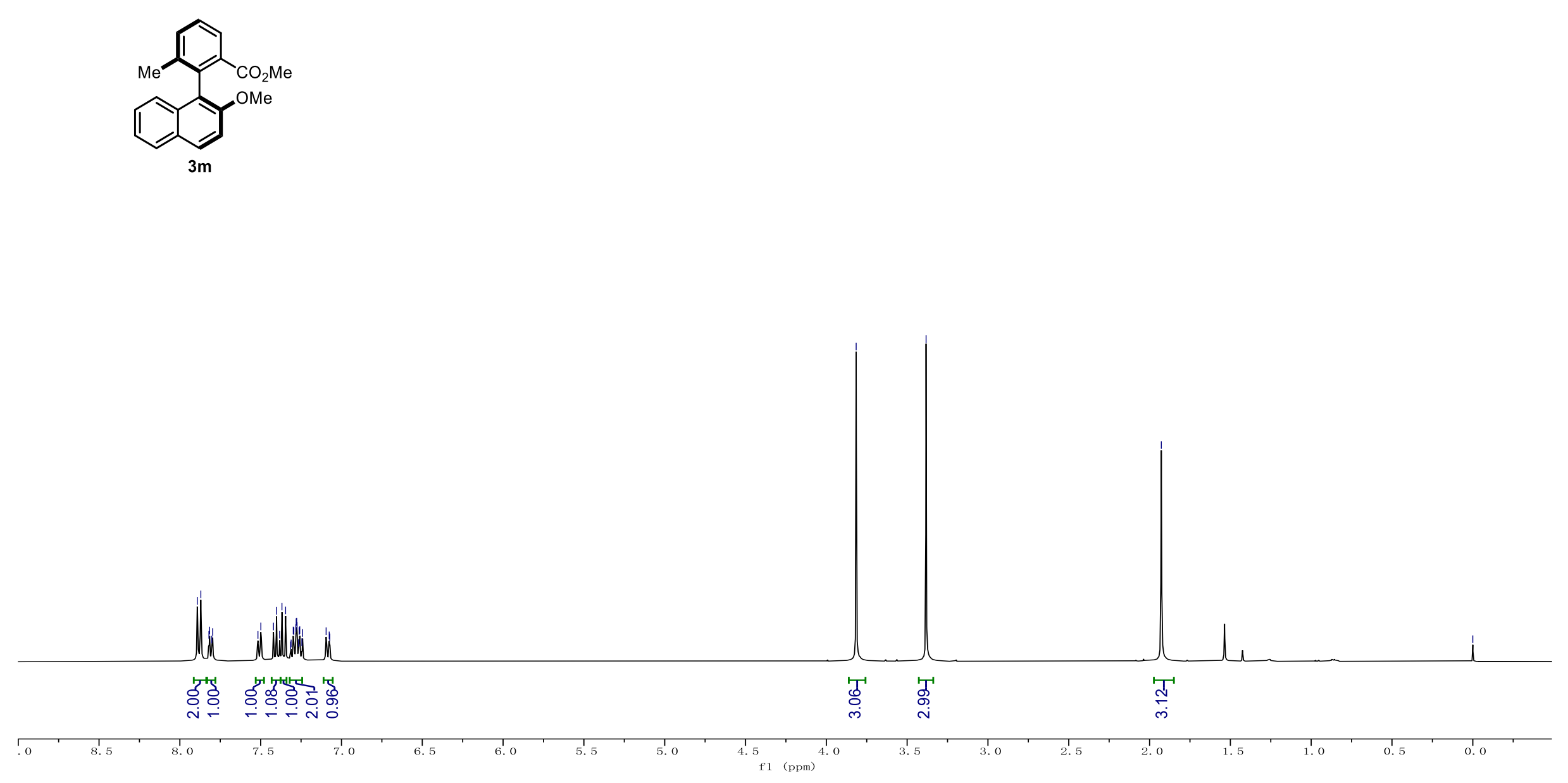

217 

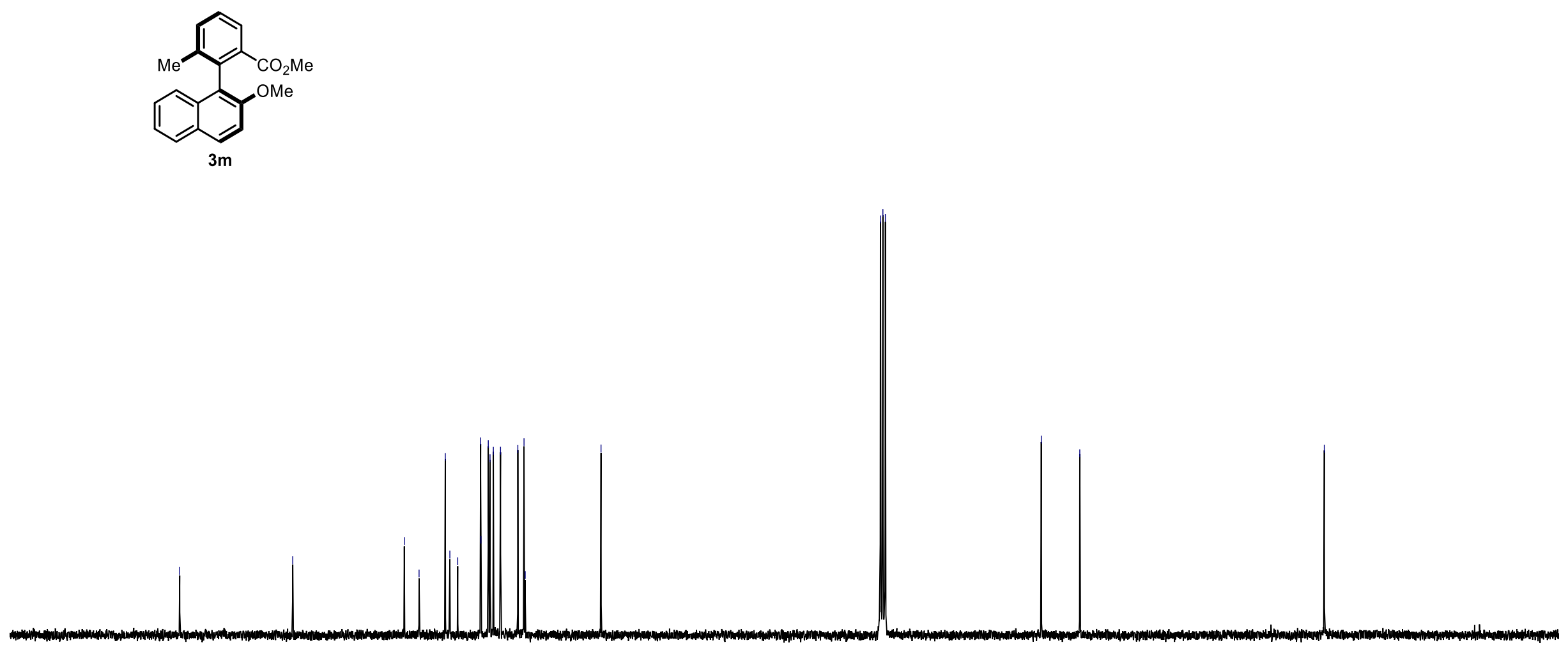

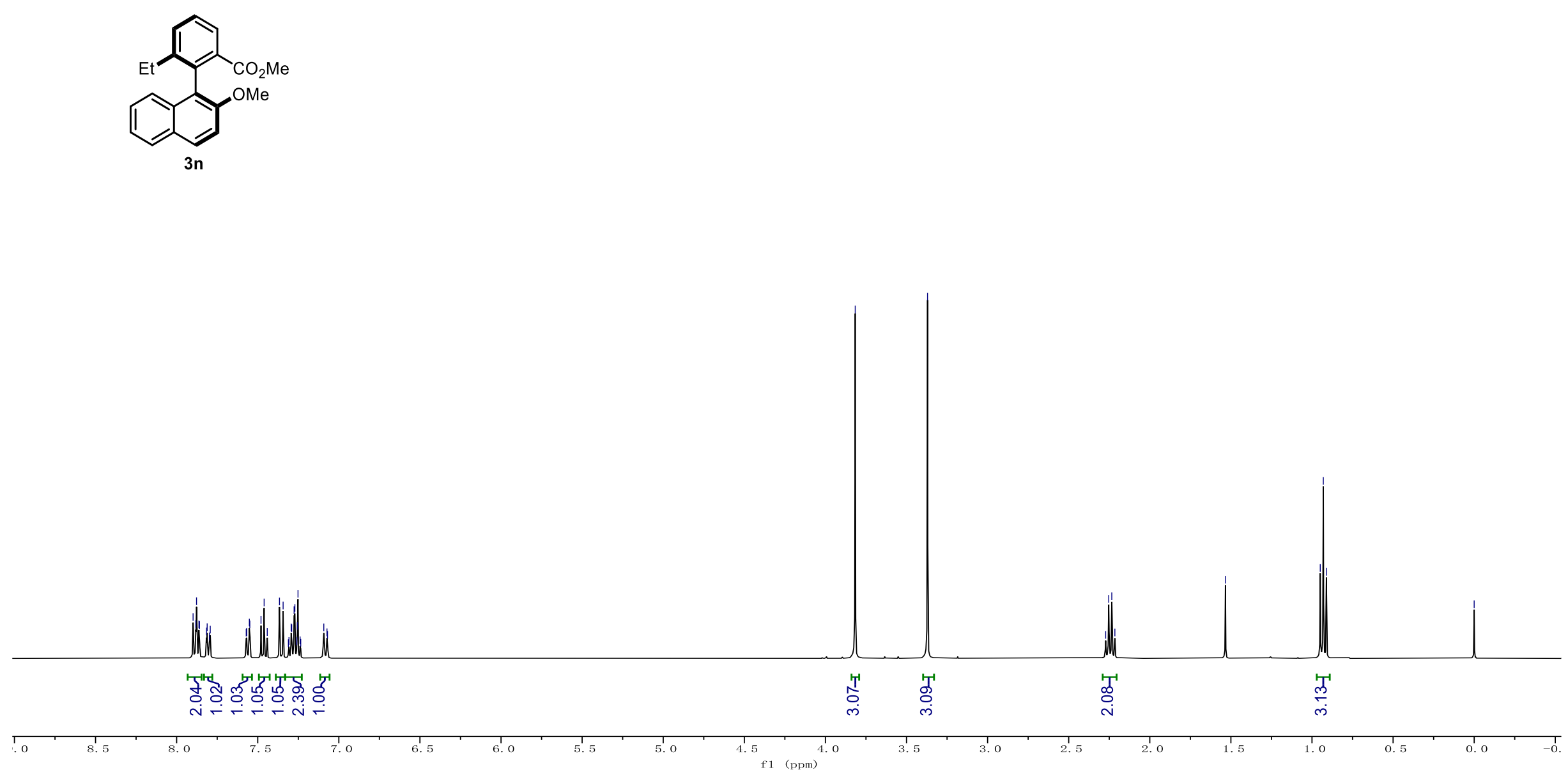


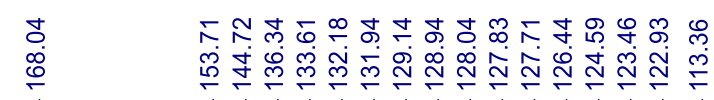

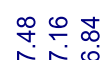

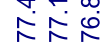

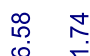

1

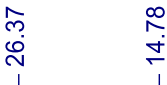
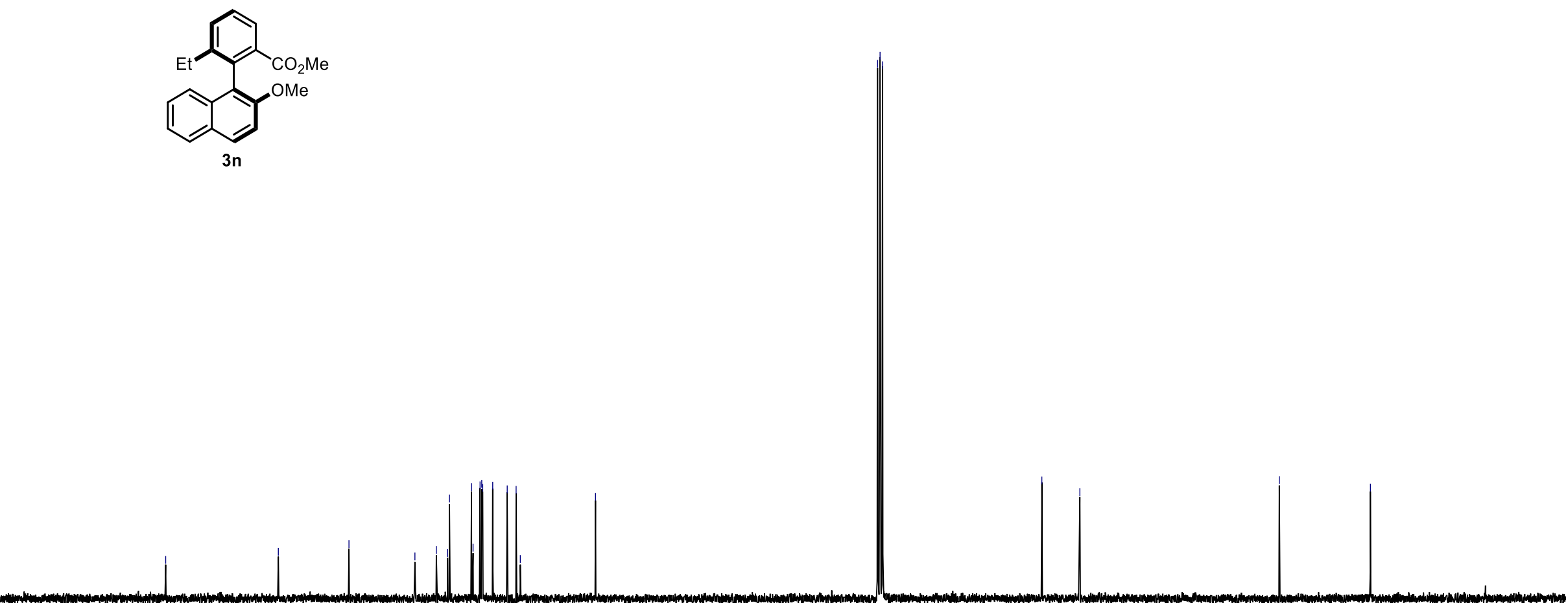


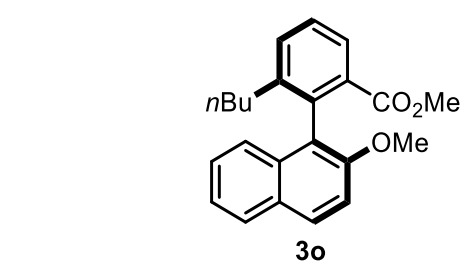

30

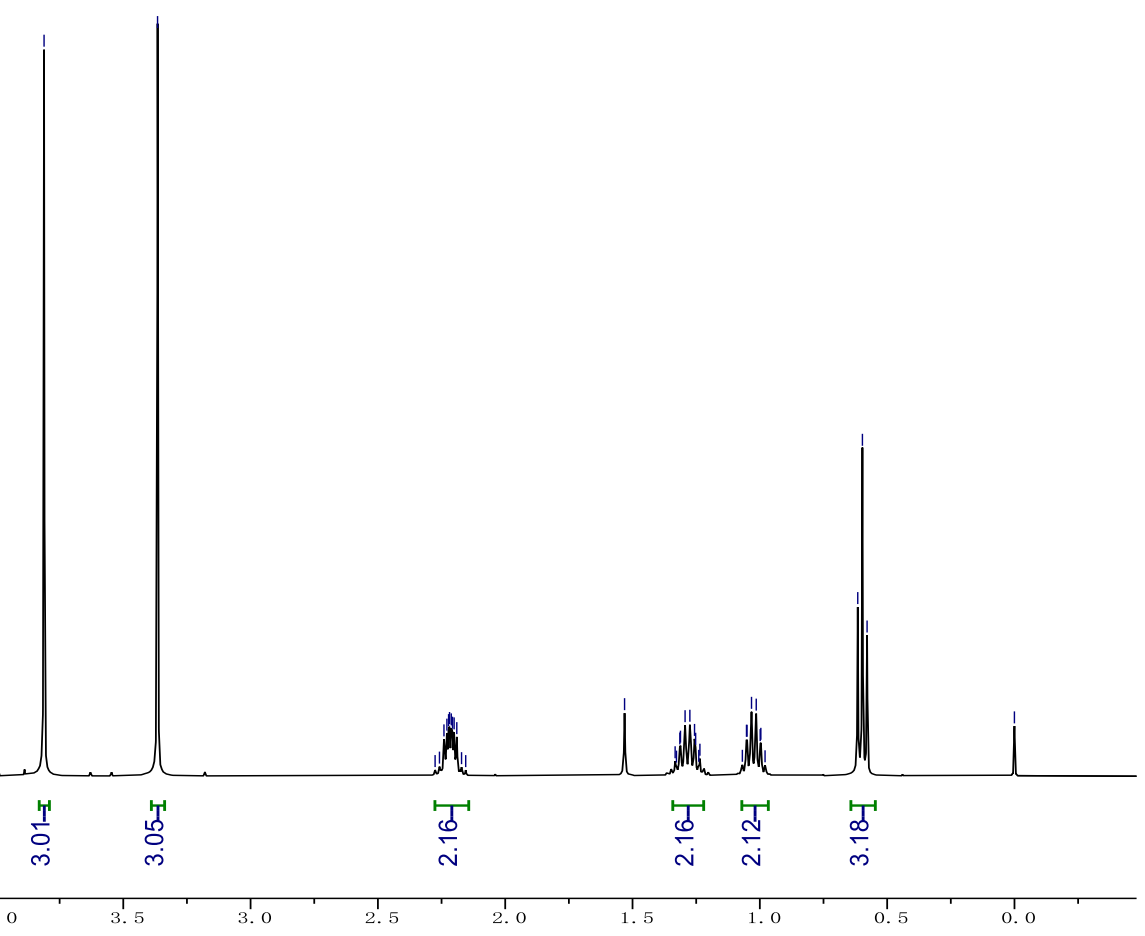




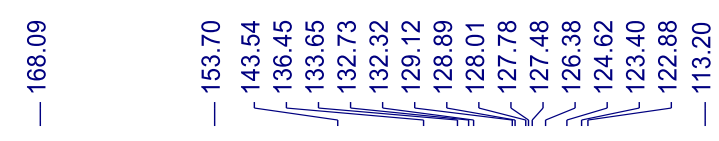

采ำ

每

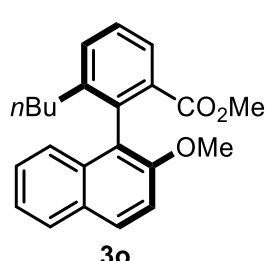

30
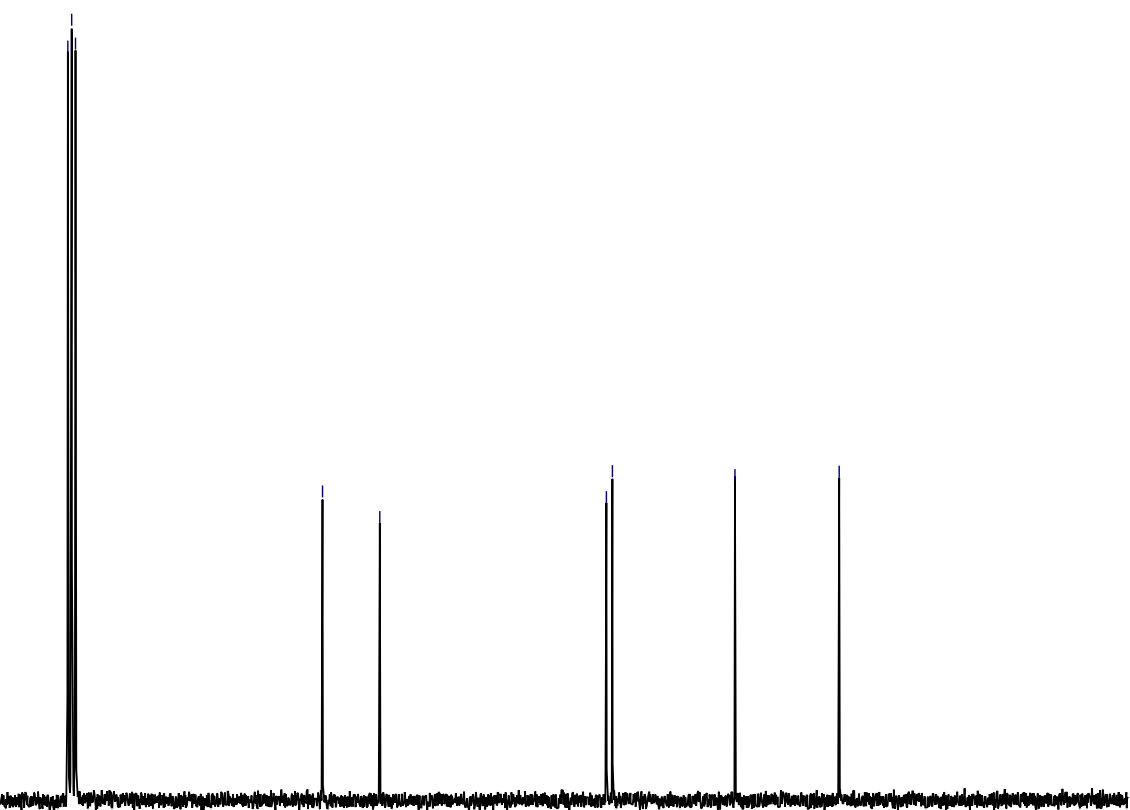

90 160 150
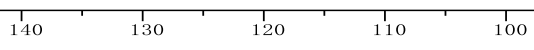

$\stackrel{1}{90}$
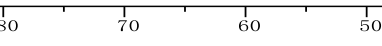

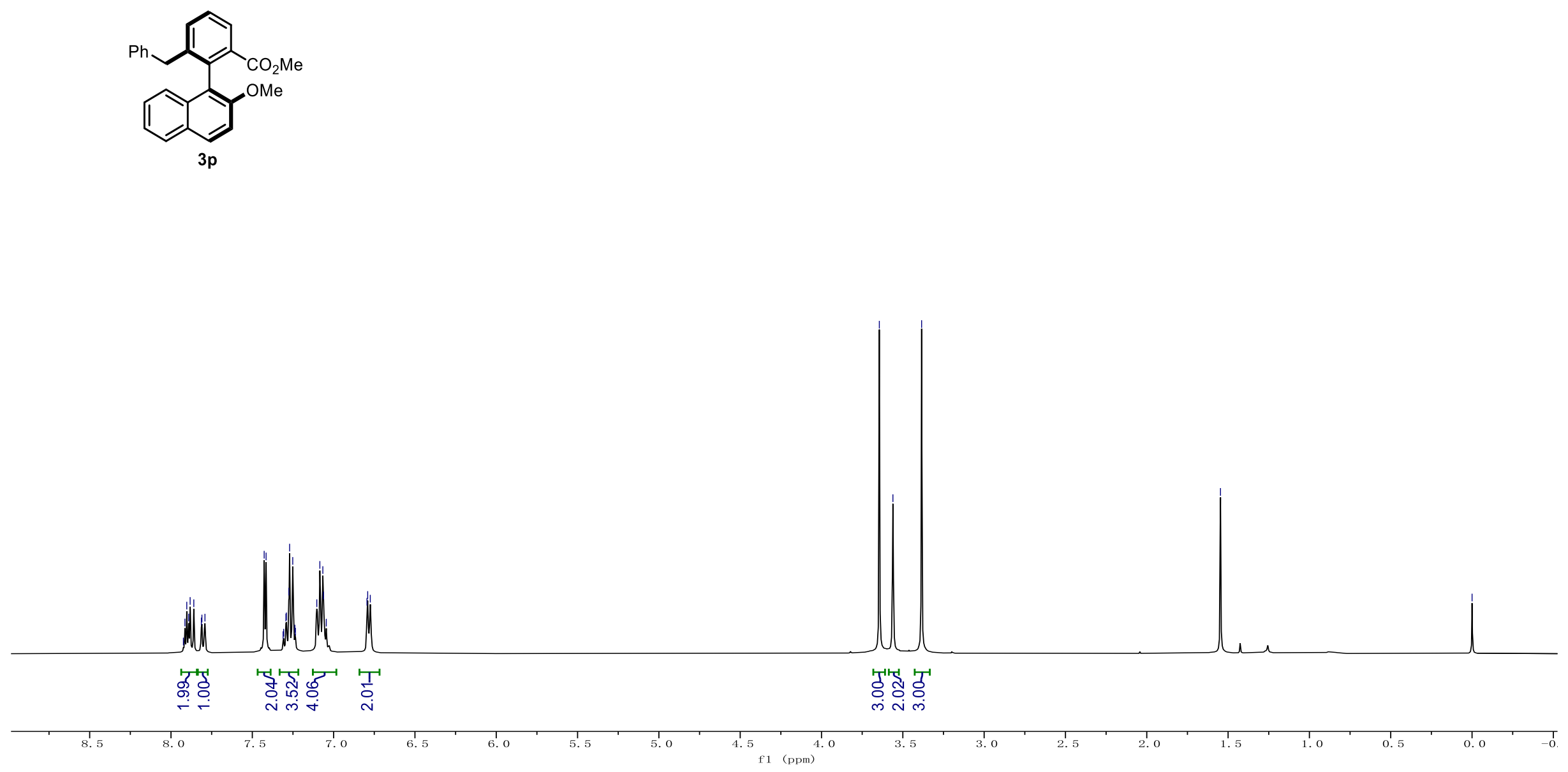
$\underbrace{1}$

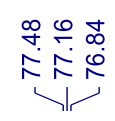

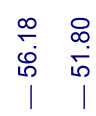

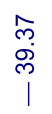
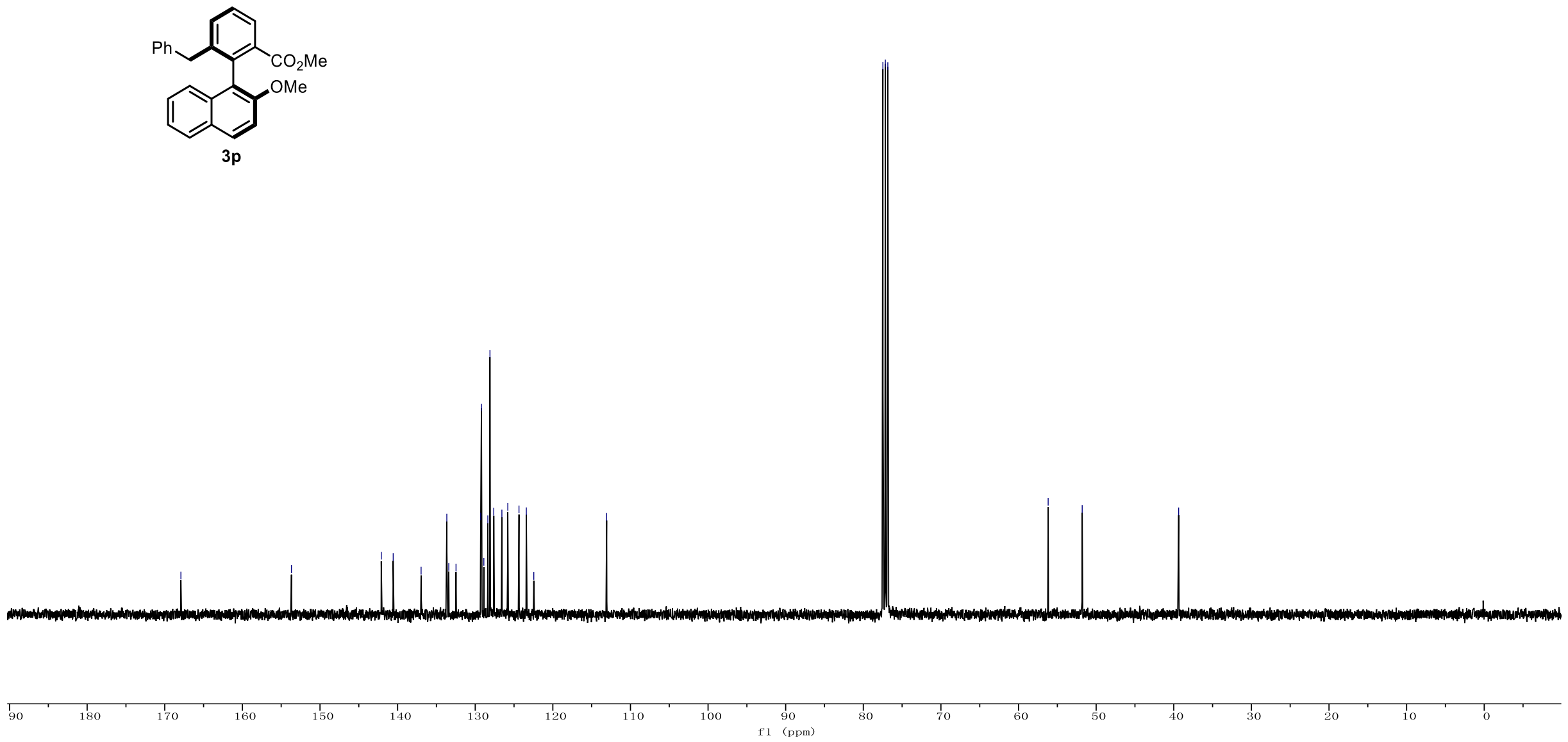

224 

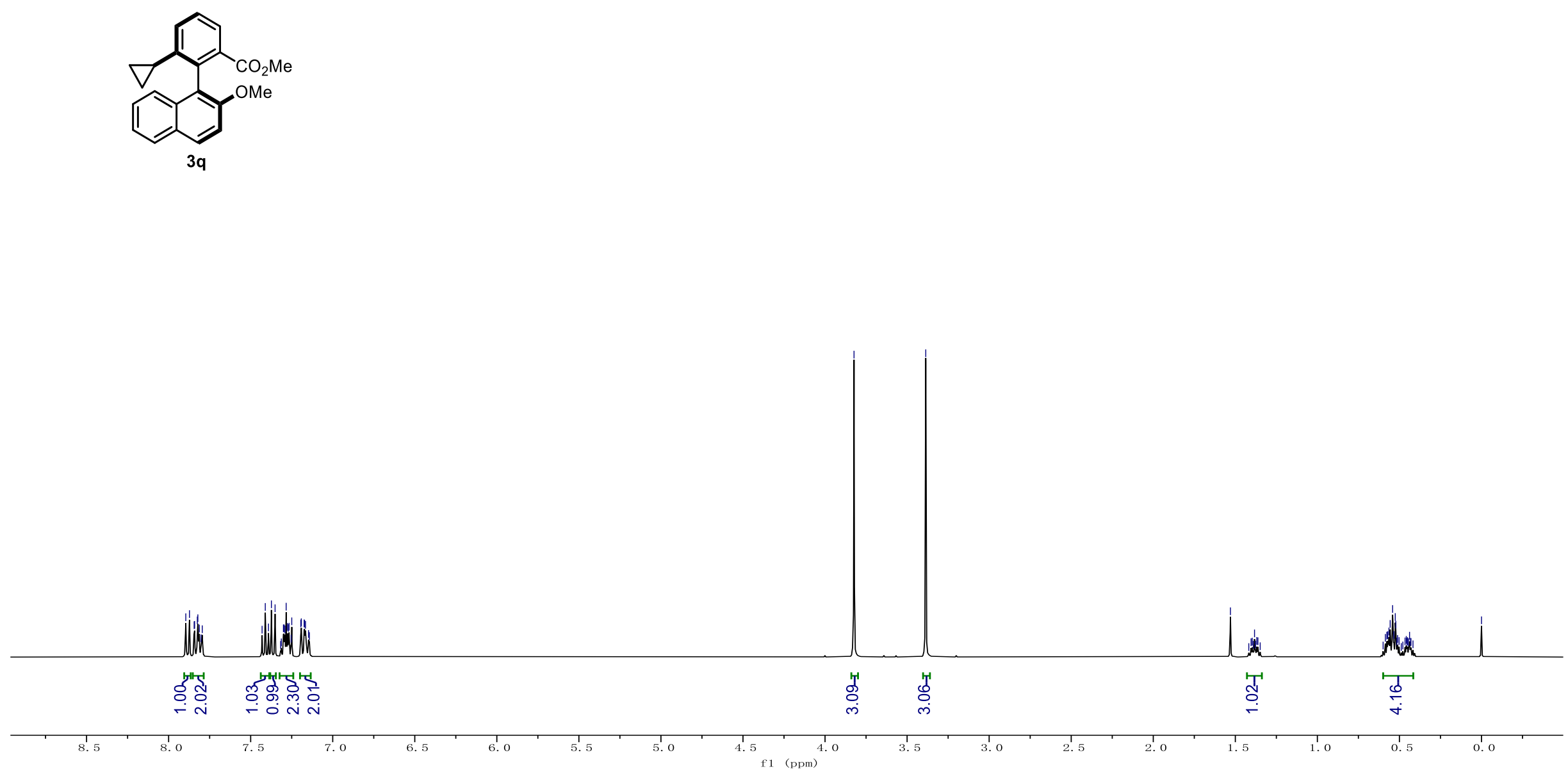


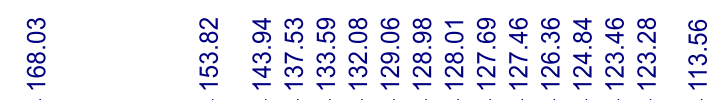

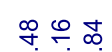

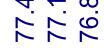

$\underset{0}{1} \stackrel{0}{0}$

竞芦柋
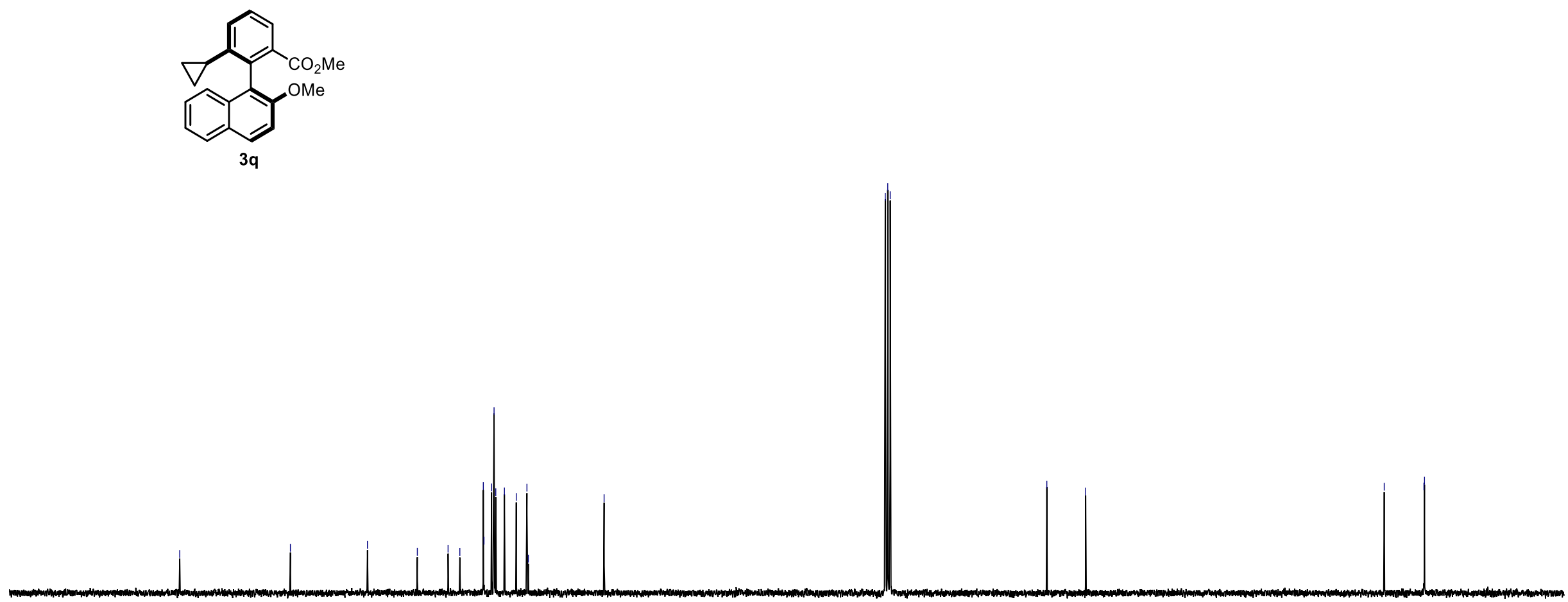


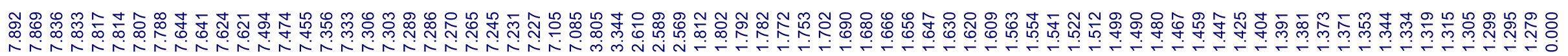

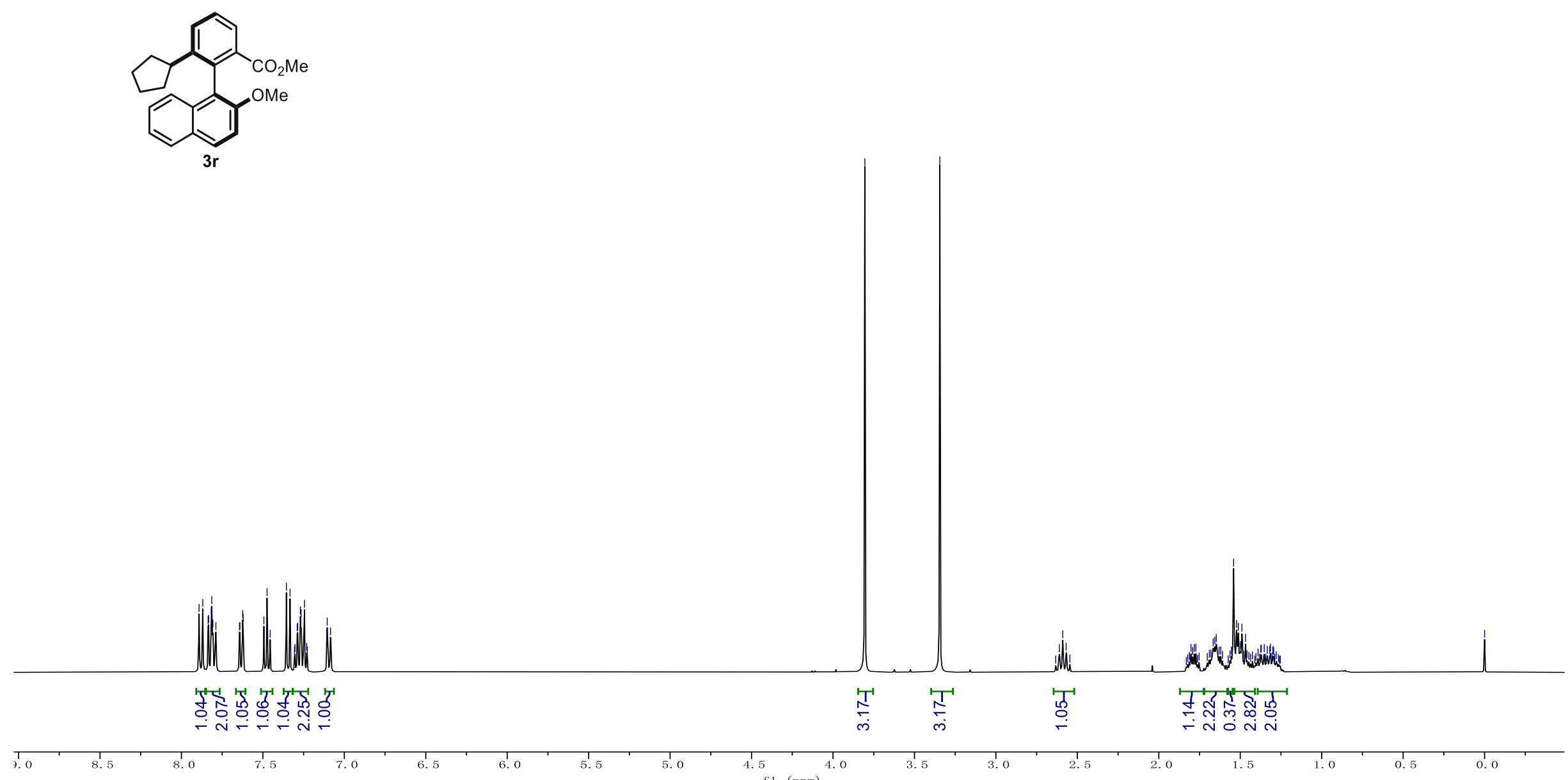




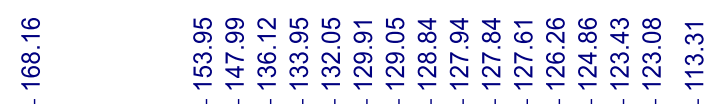

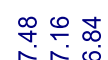

定通

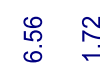

is in

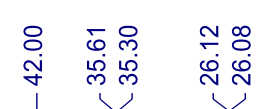
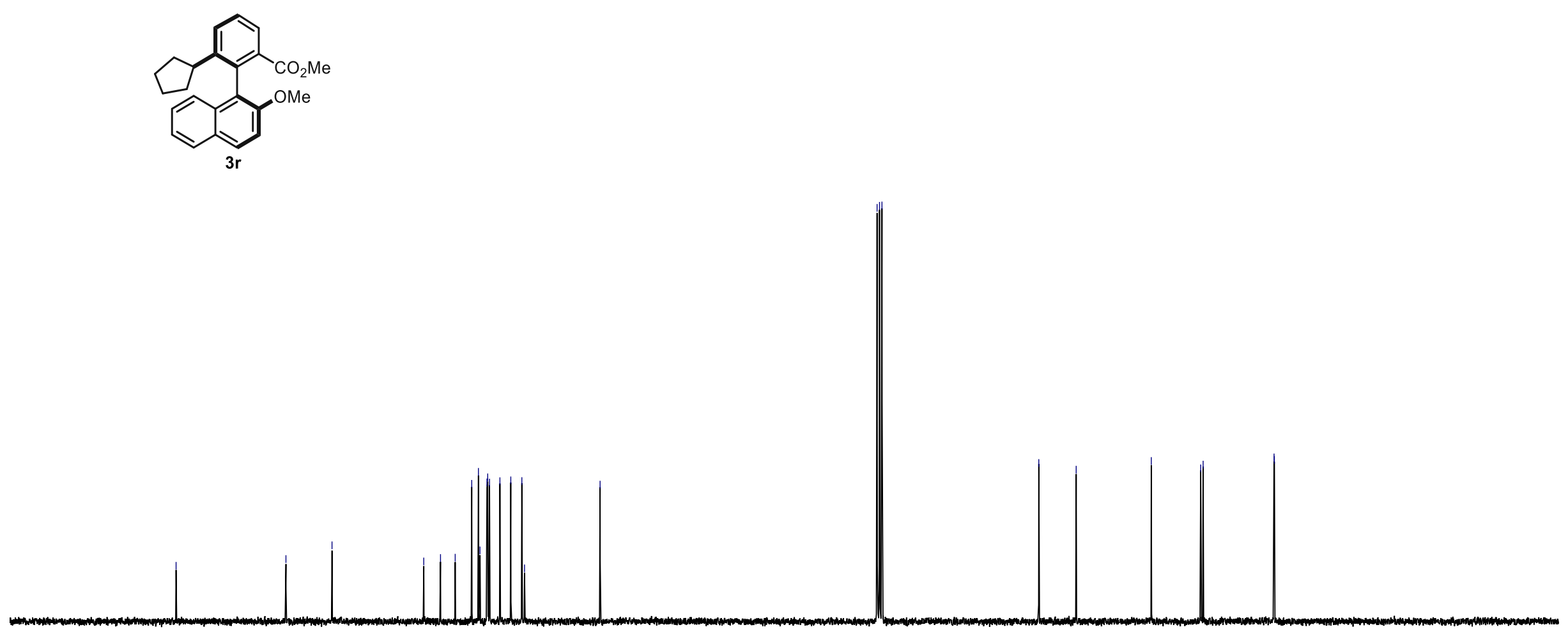

180

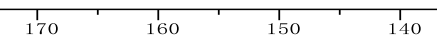

130

90

80

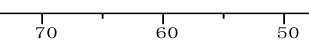

40

30 


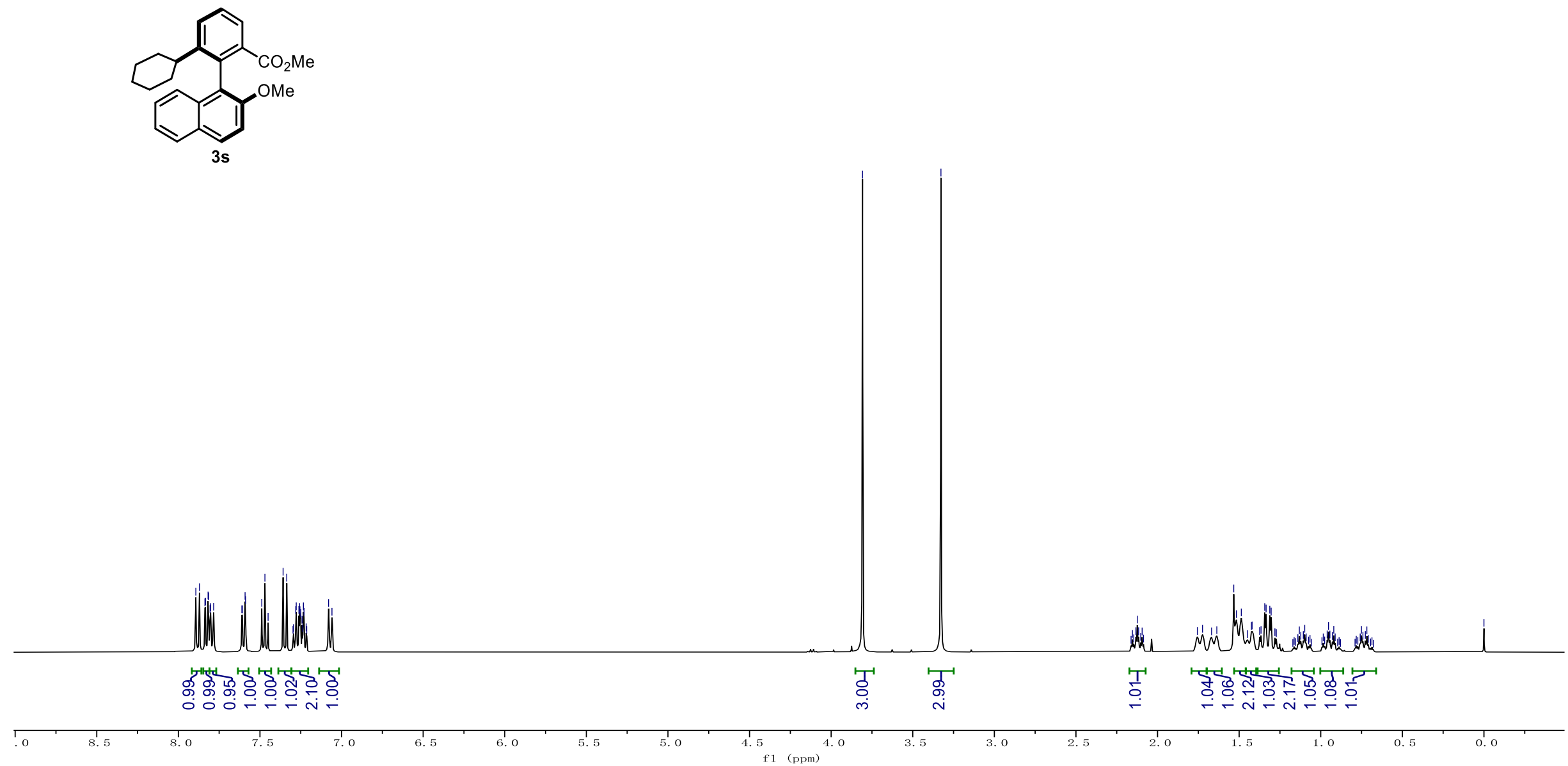



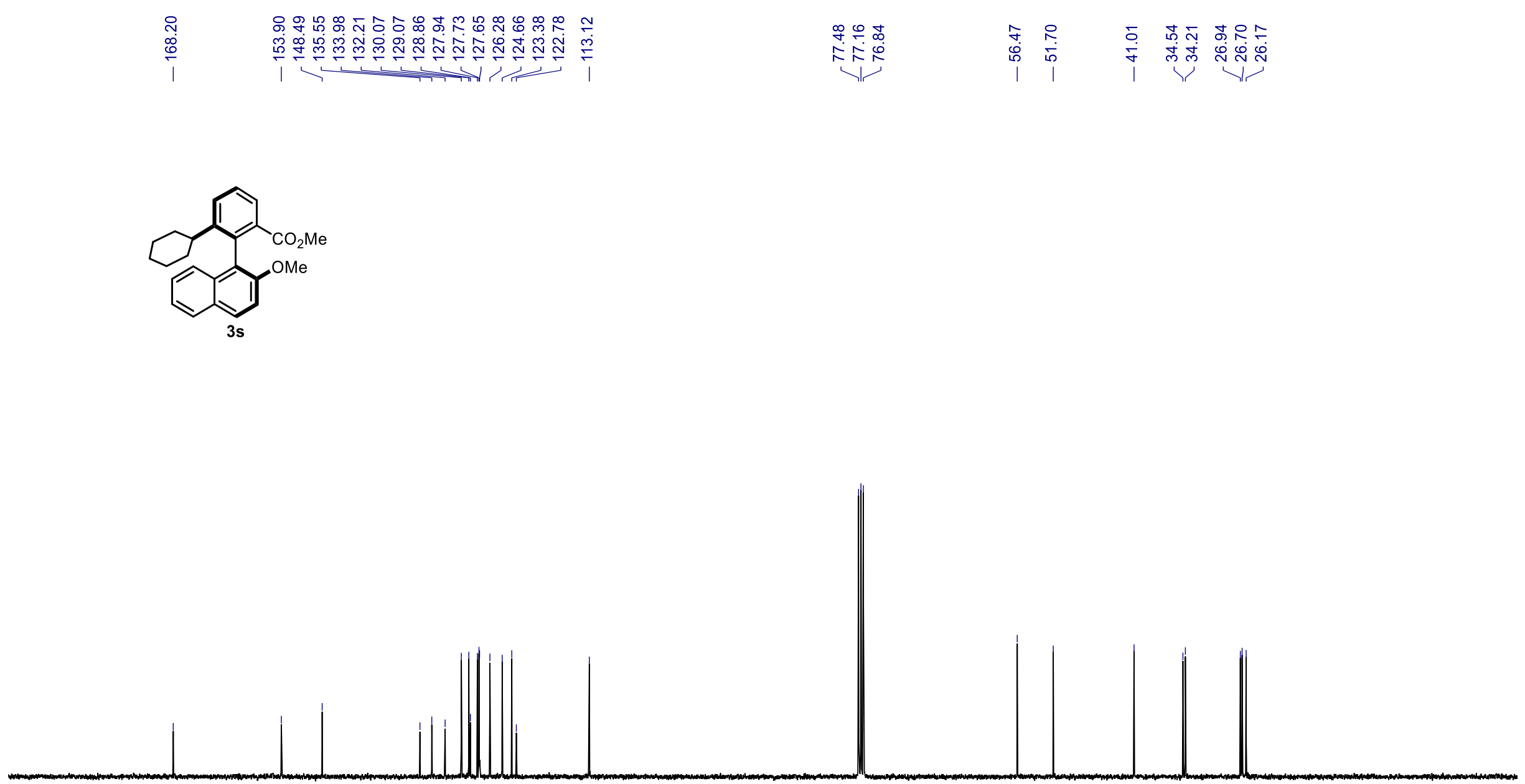

90
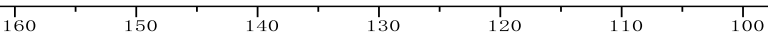

90

$\frac{1}{60}+50$

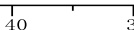



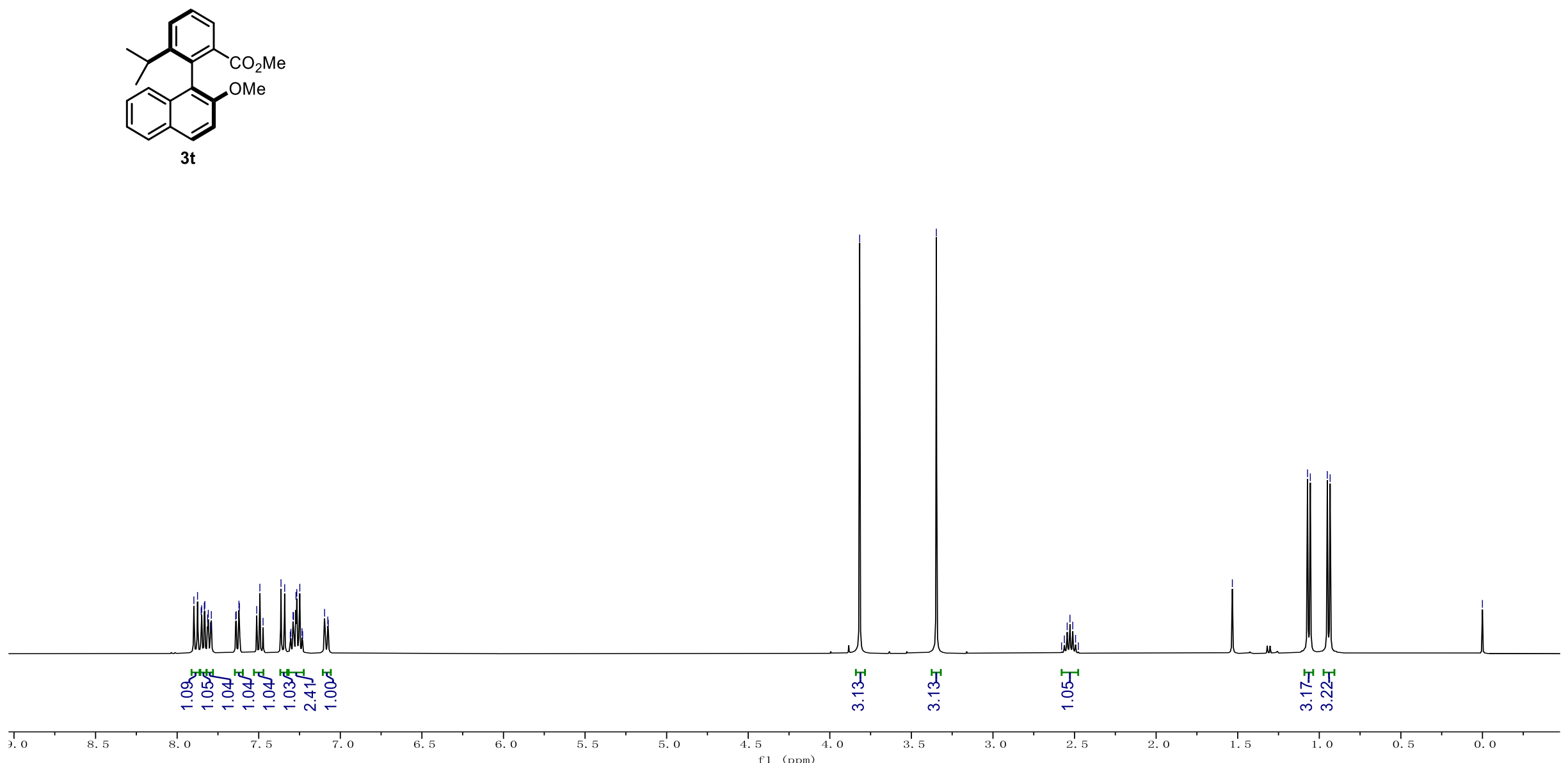

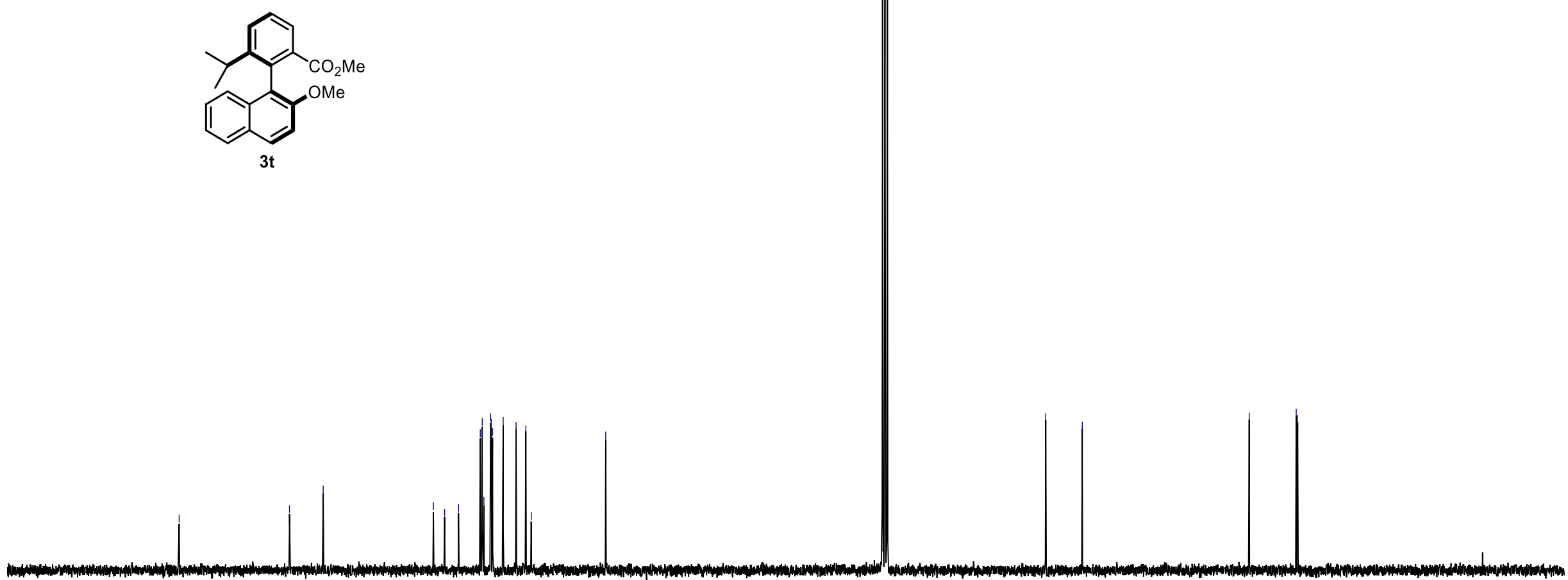
90 180 170 $160 \quad 150$
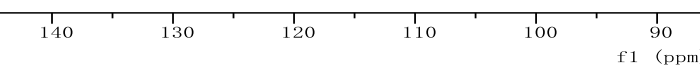

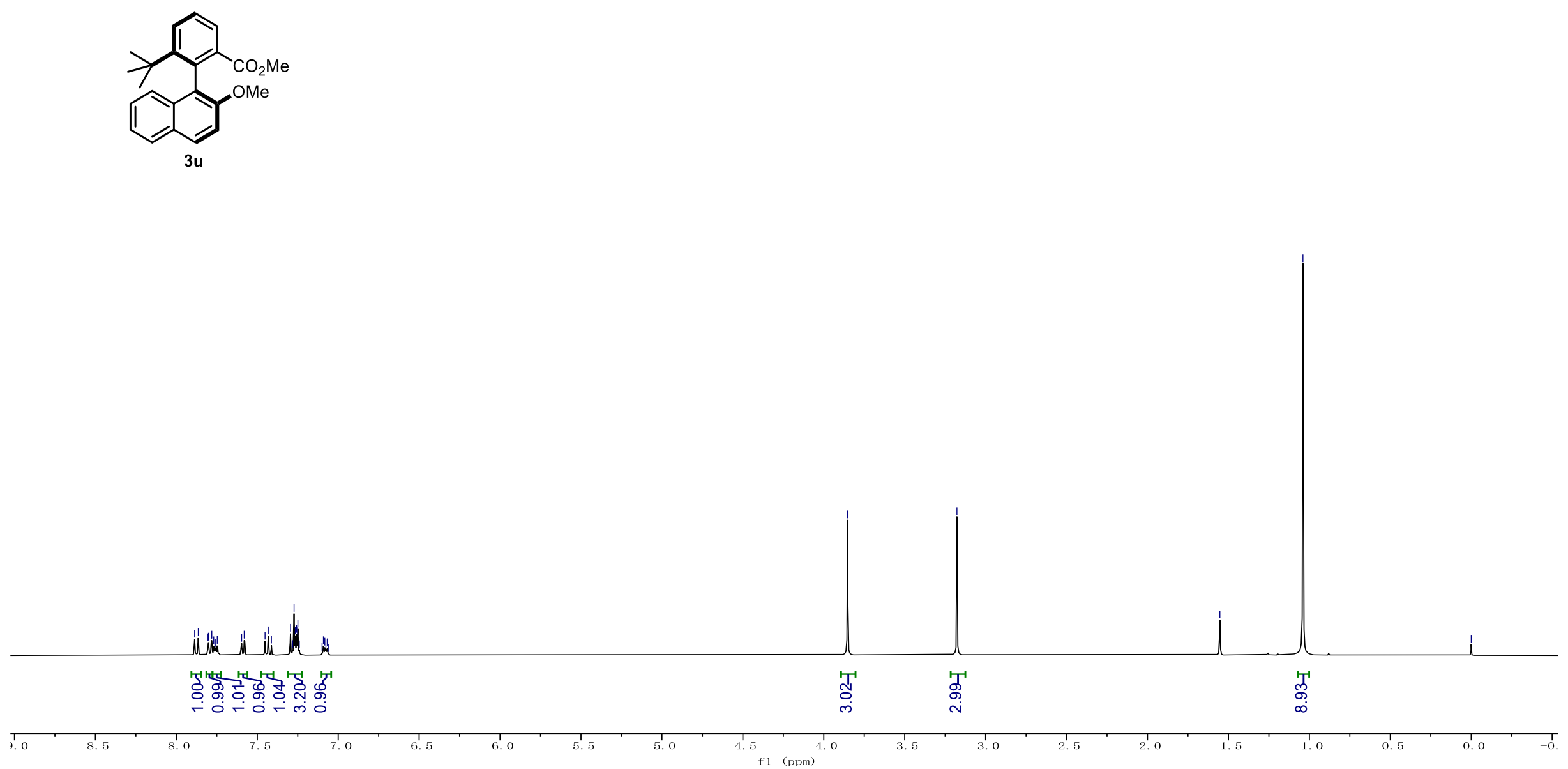


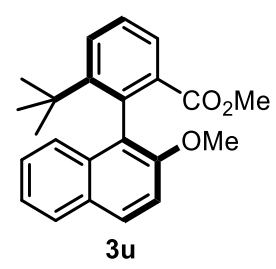


<smiles>COc1cccc(C)c1-c1c(OC)cccc1OC</smiles>

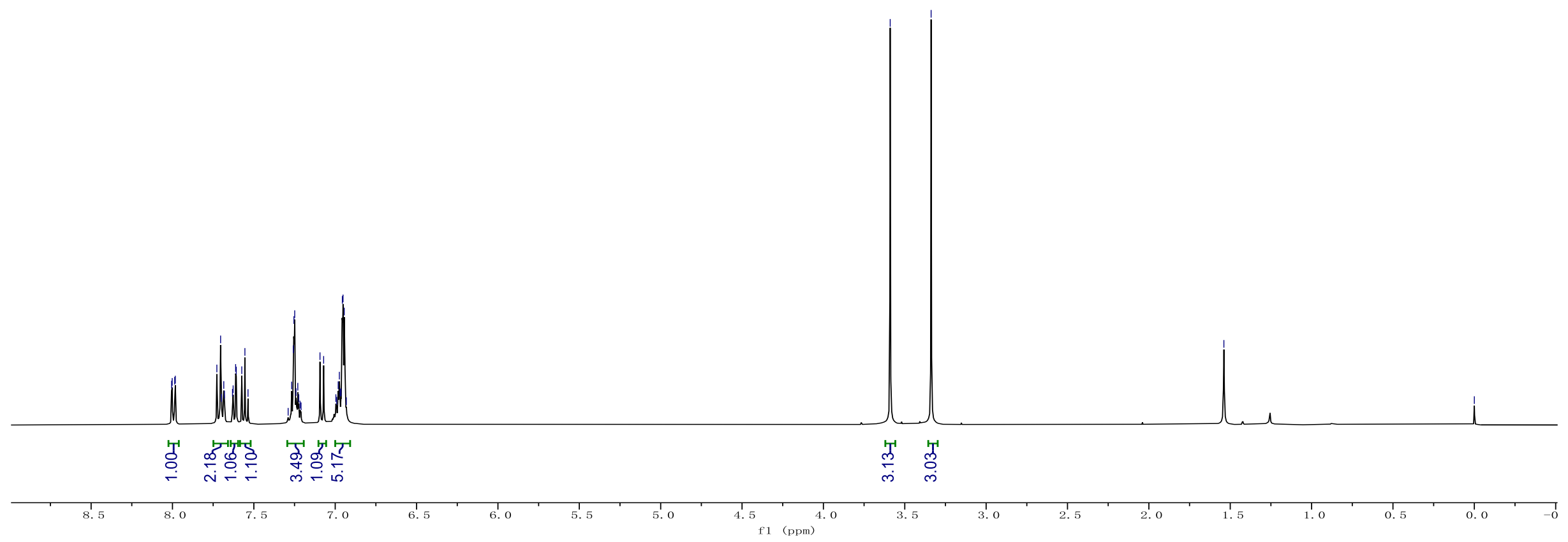




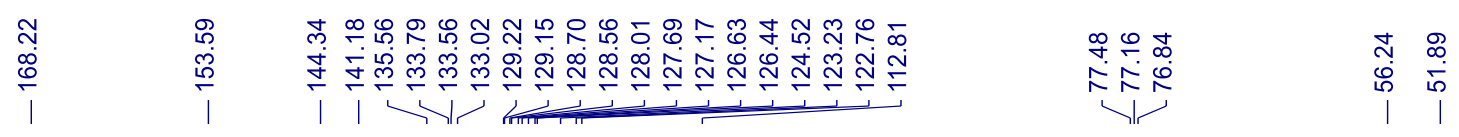
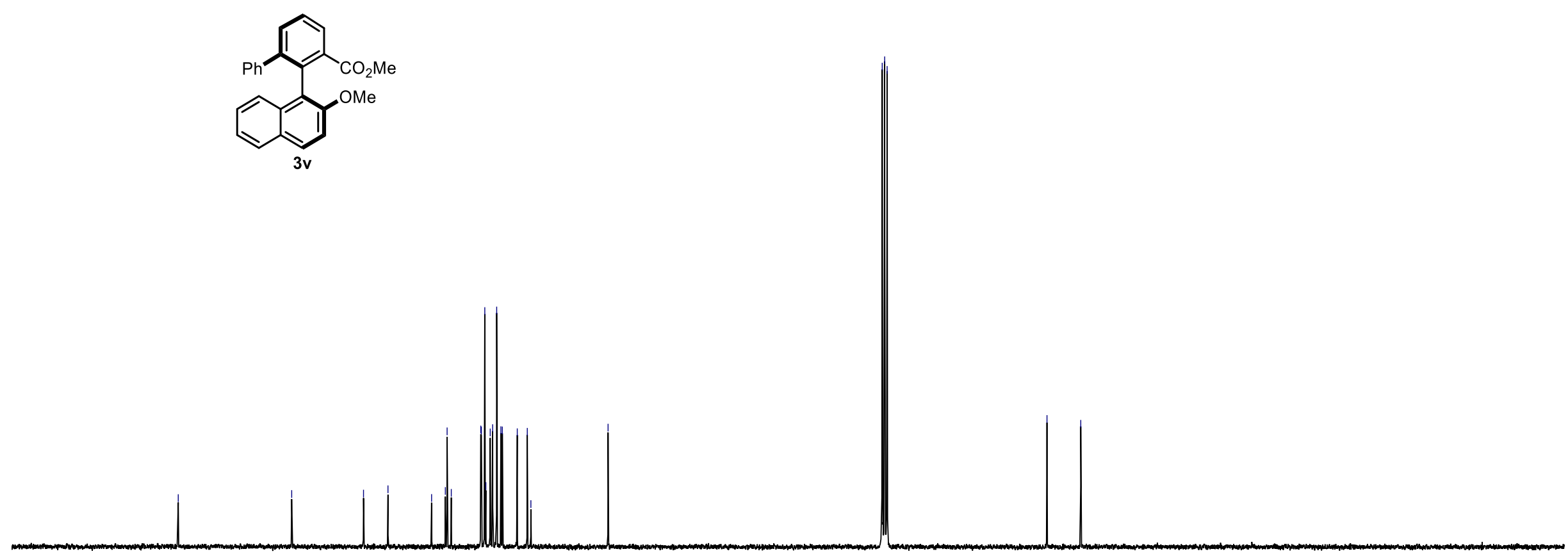


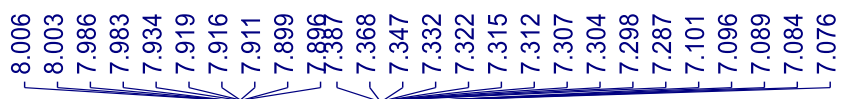

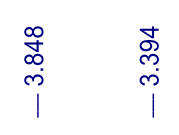

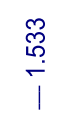

$\stackrel{8}{\circ}$
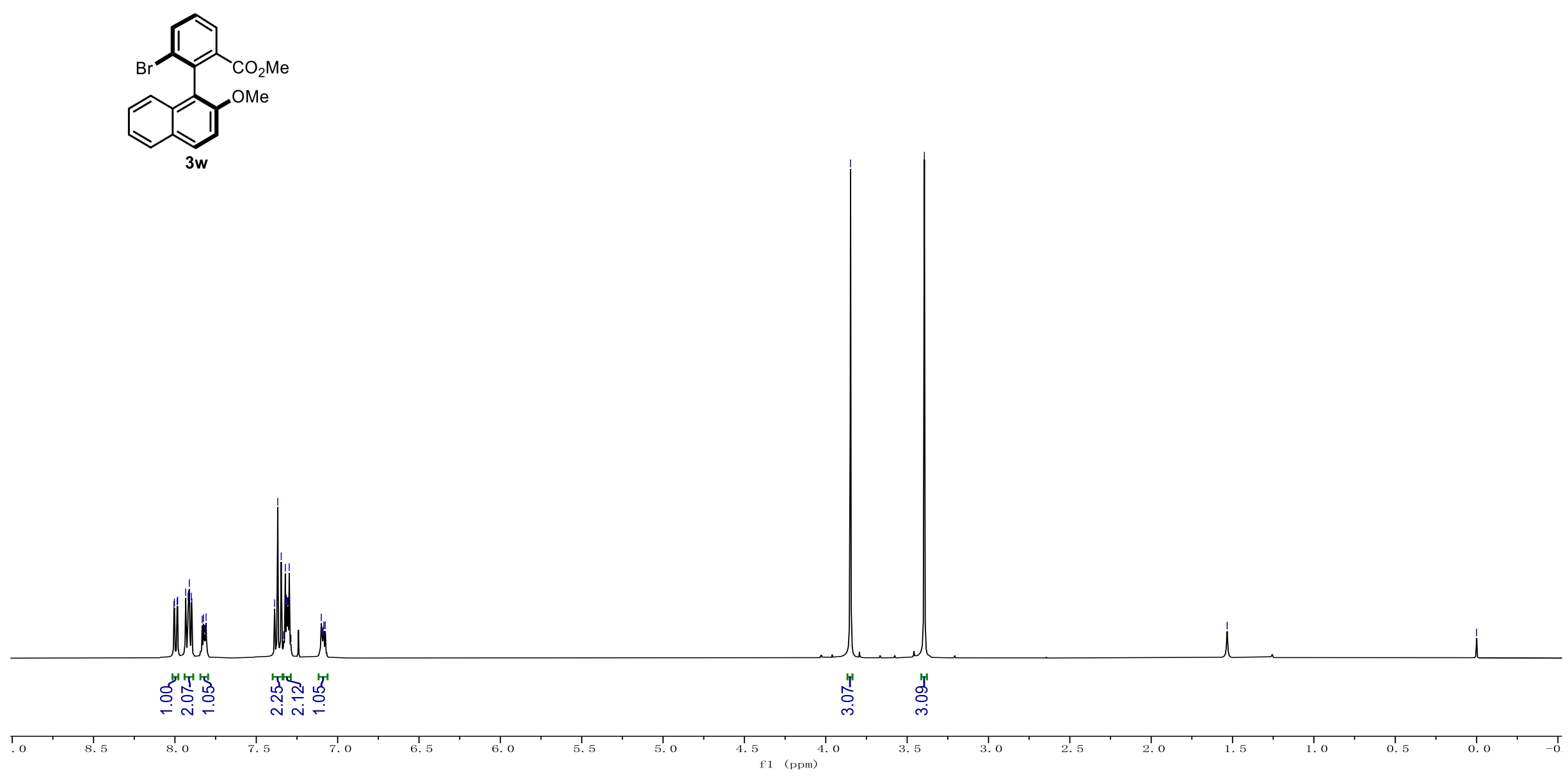

237 


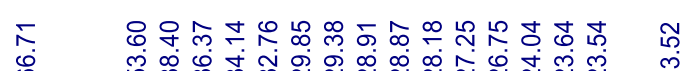

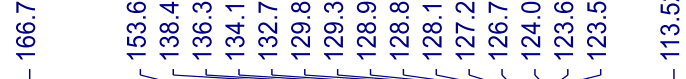

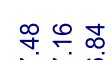

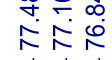

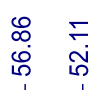

3w

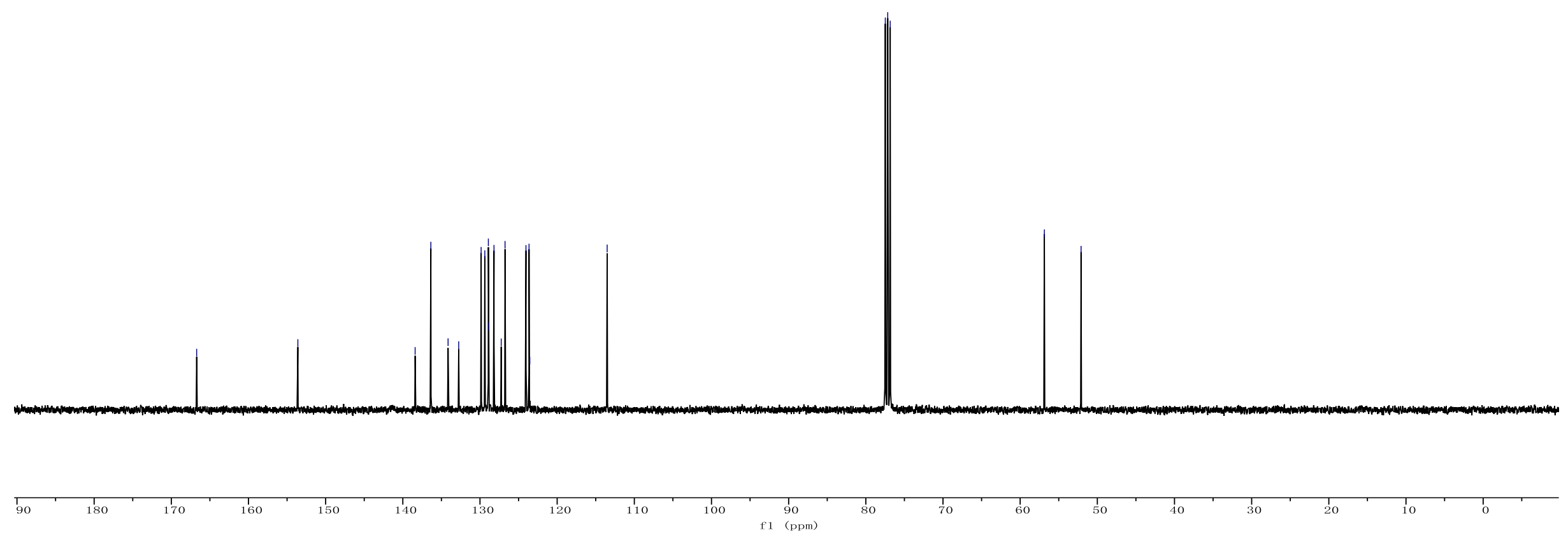



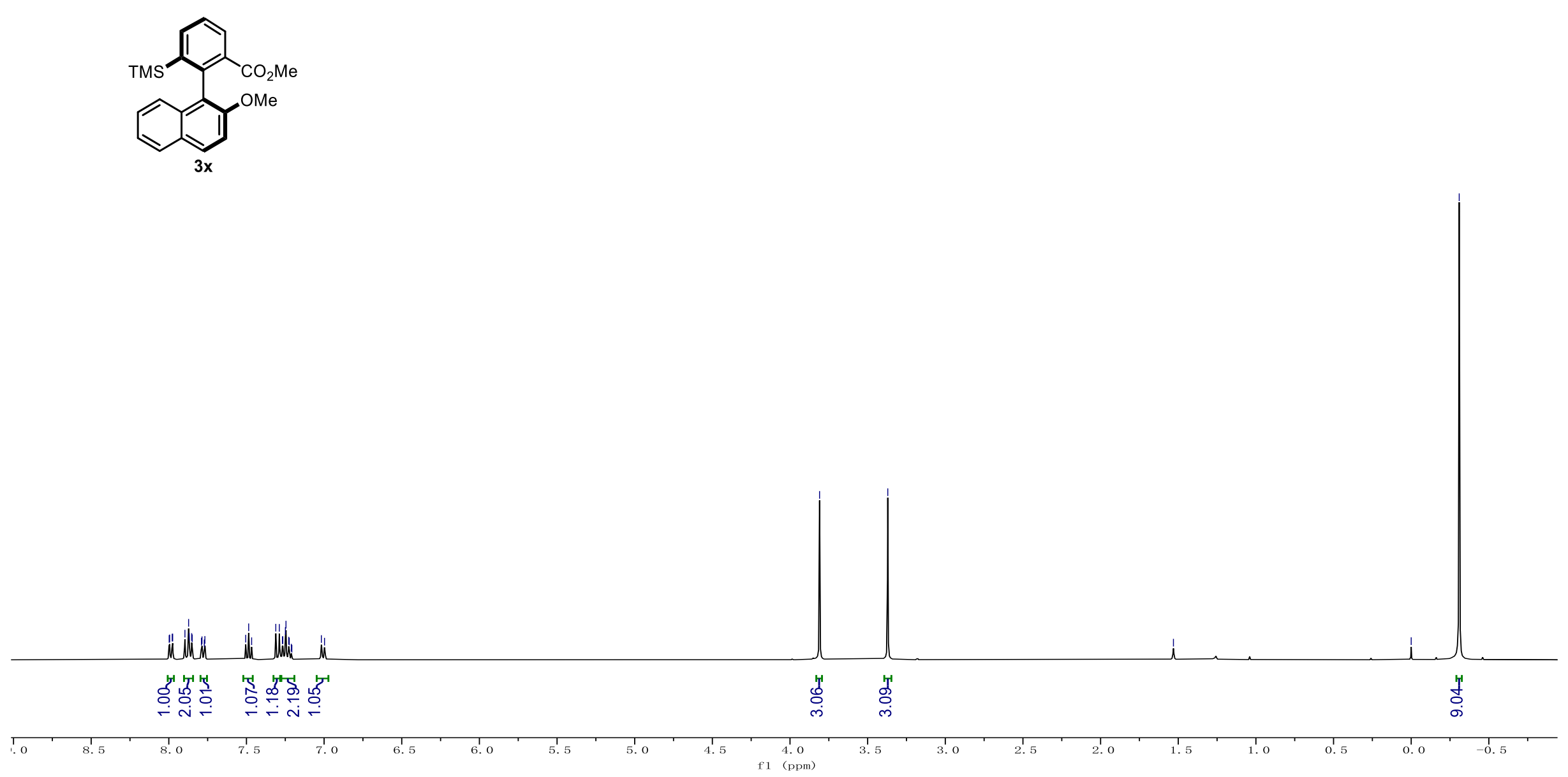

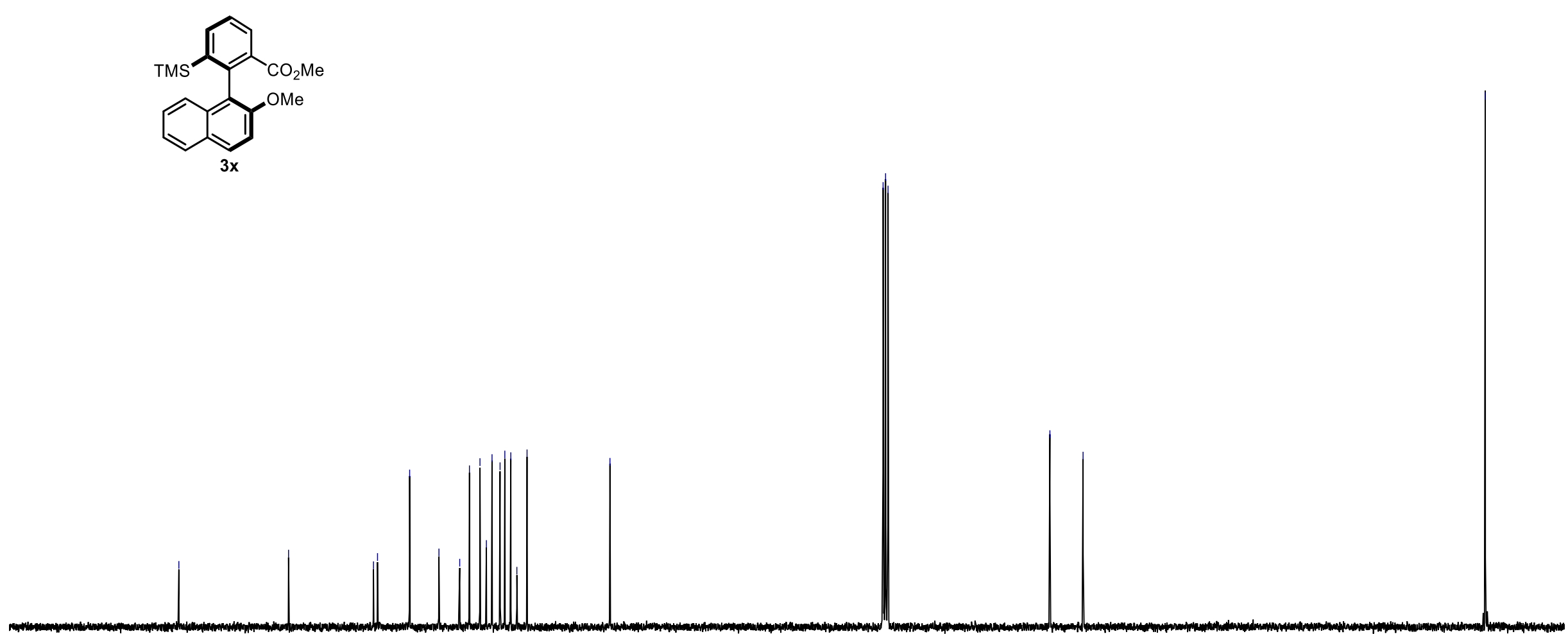


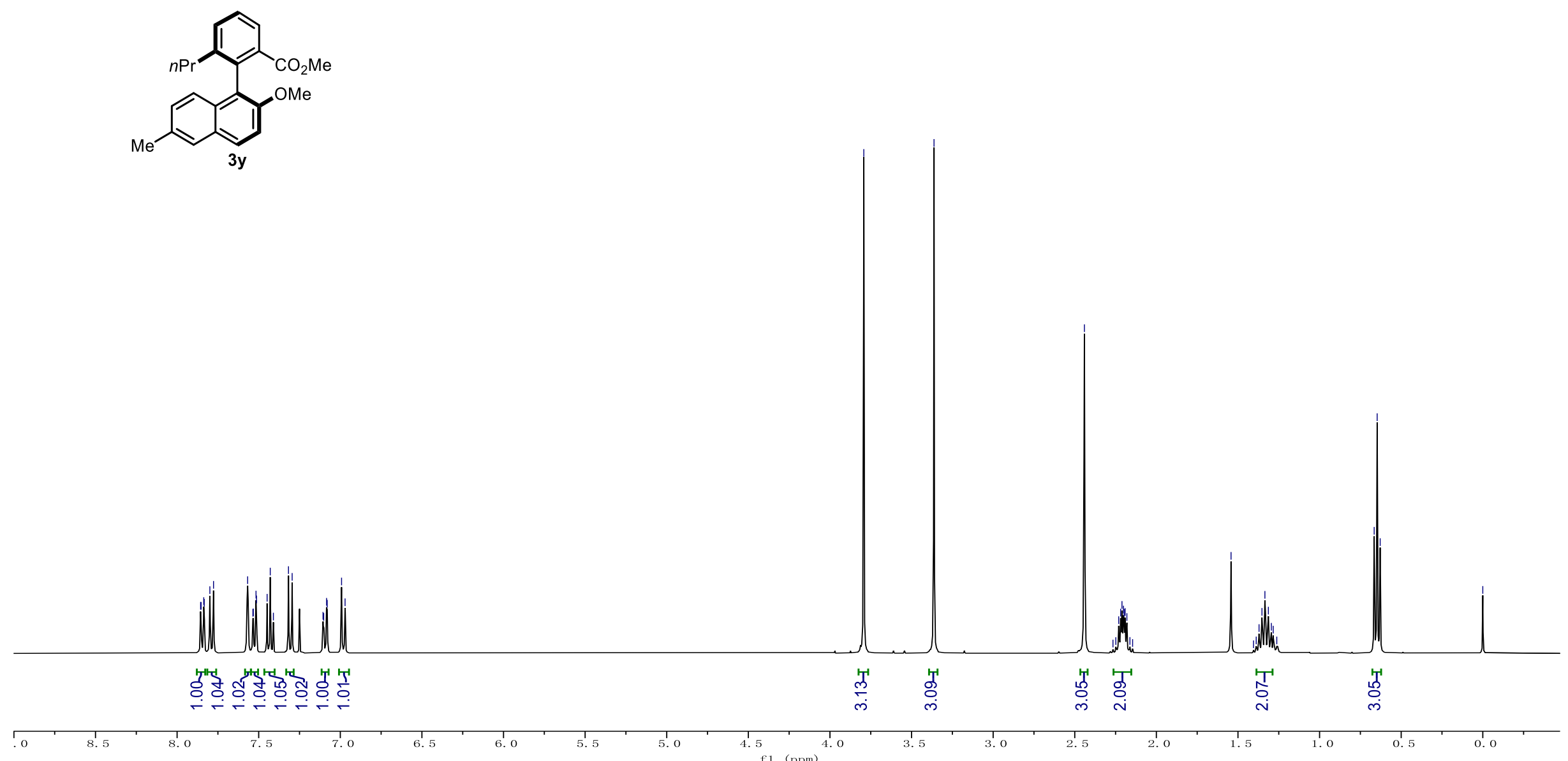


<smiles>COc1ccc2cc(C)ccc2c1-c1c(C(C)=O)cccc1C(C)C</smiles>
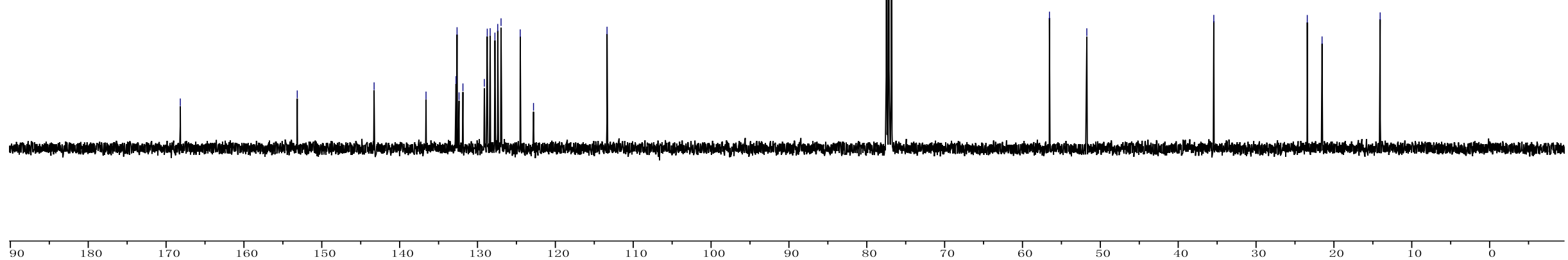
1
$170 \quad 160$ 150 140 130 ${ }_{100}^{100} \quad 90$ 

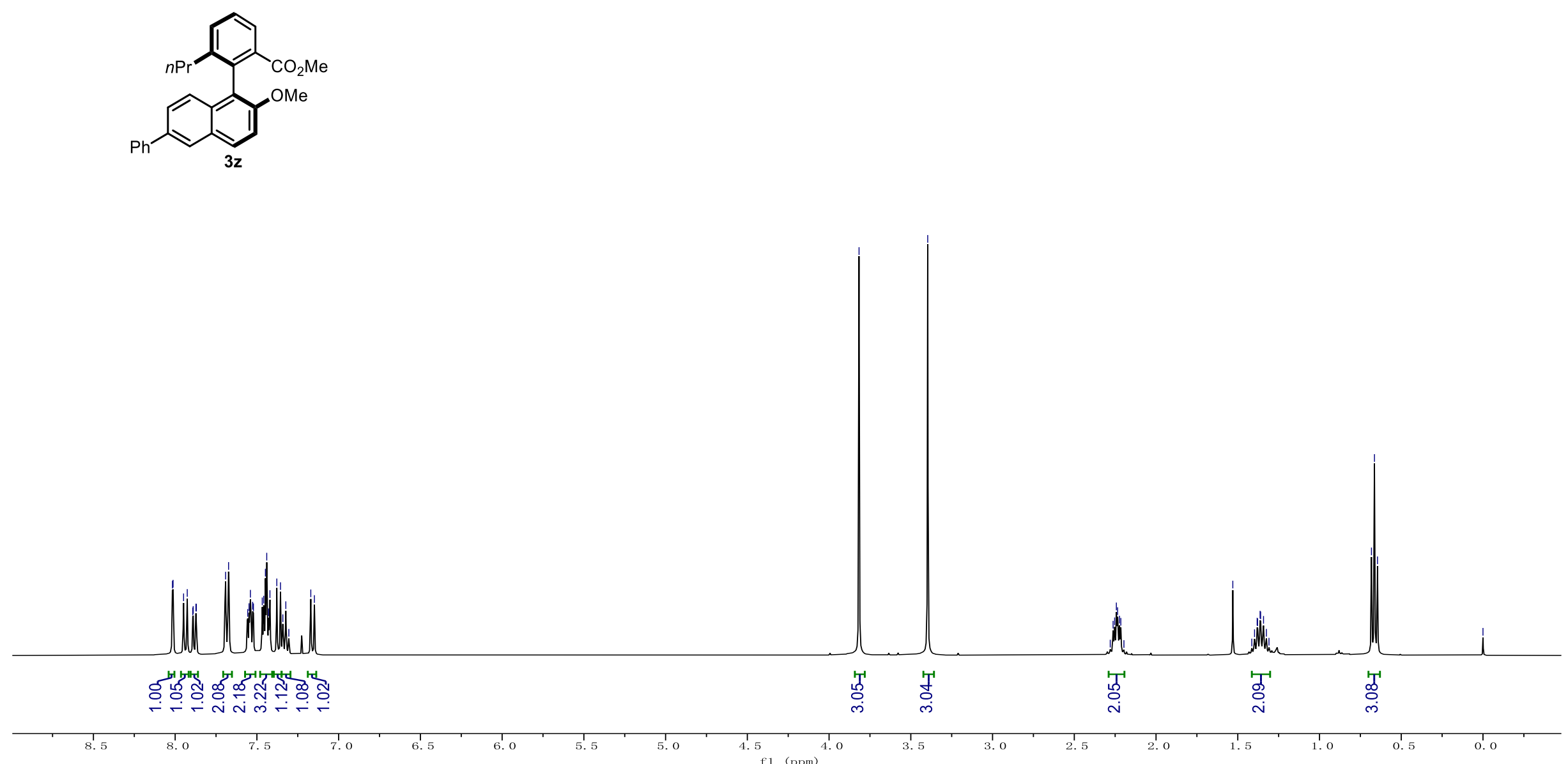


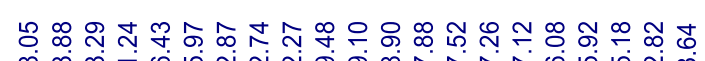

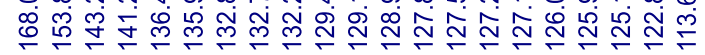

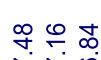

人命

$\underset{\substack{10 \\ 0}}{\substack{0 \\ 0}}$

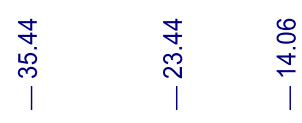<smiles>COc1cccc(OC)c1-c1c(C)ccc2cc(I)ccc12</smiles>

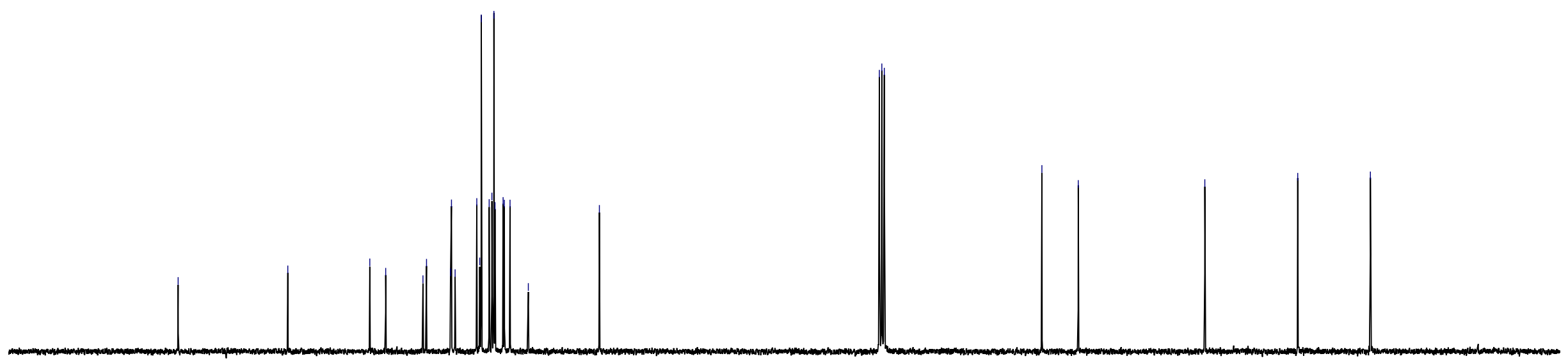




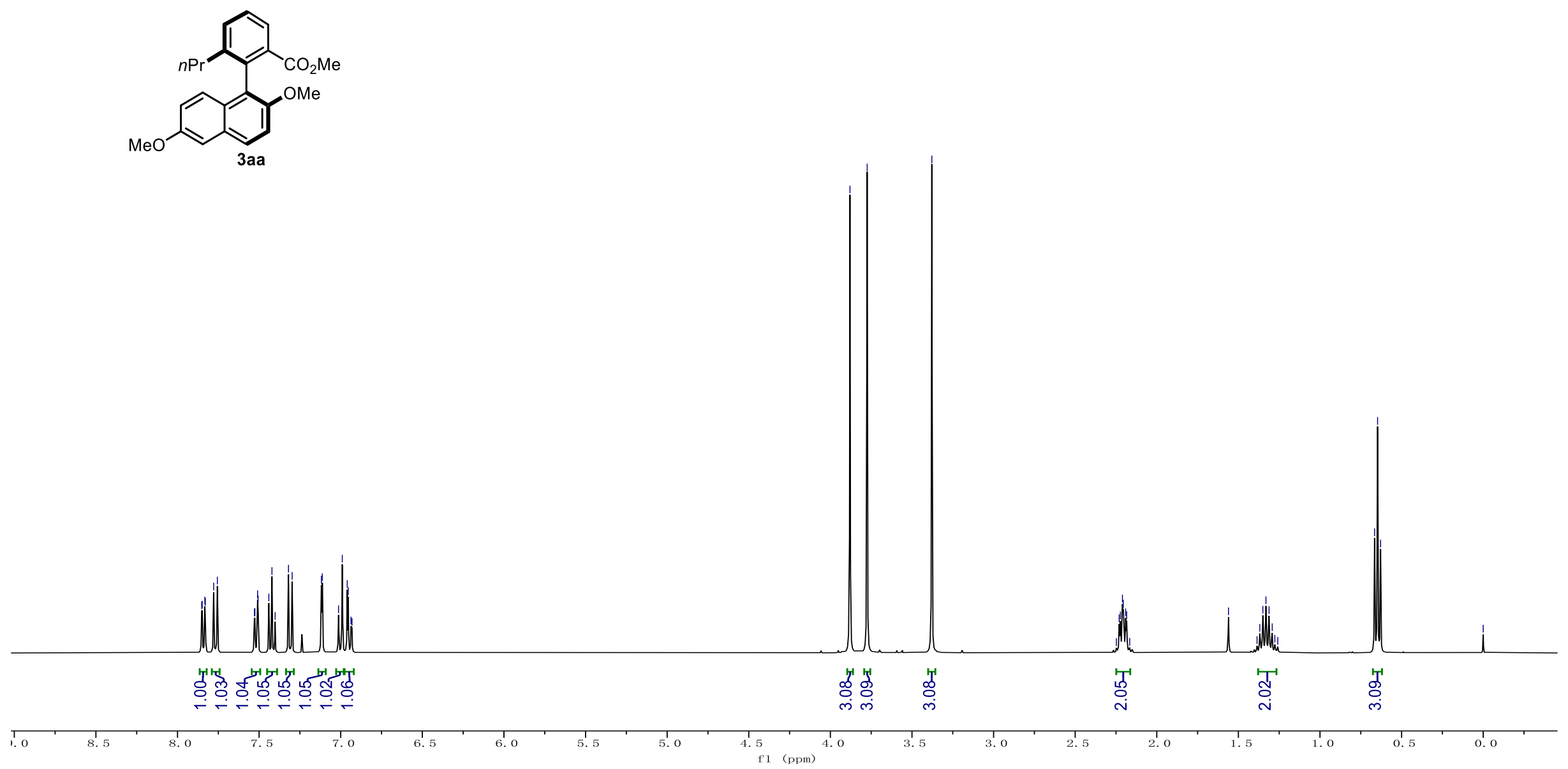




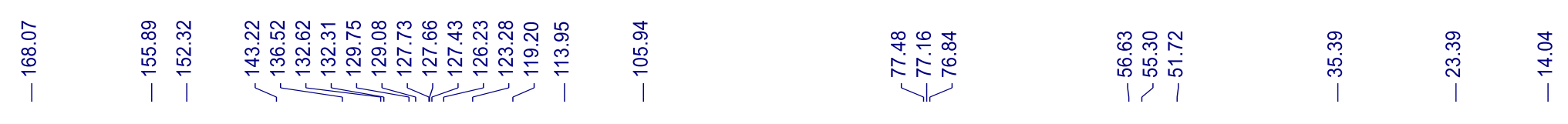
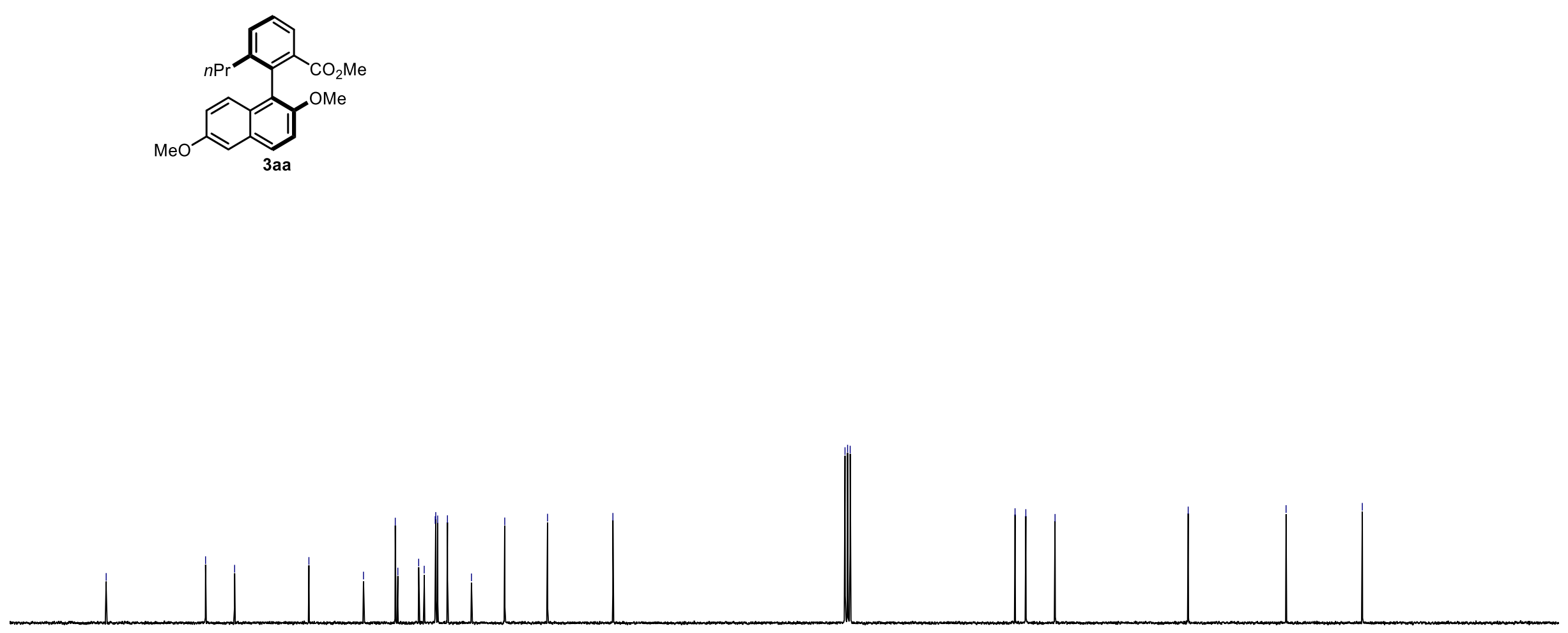


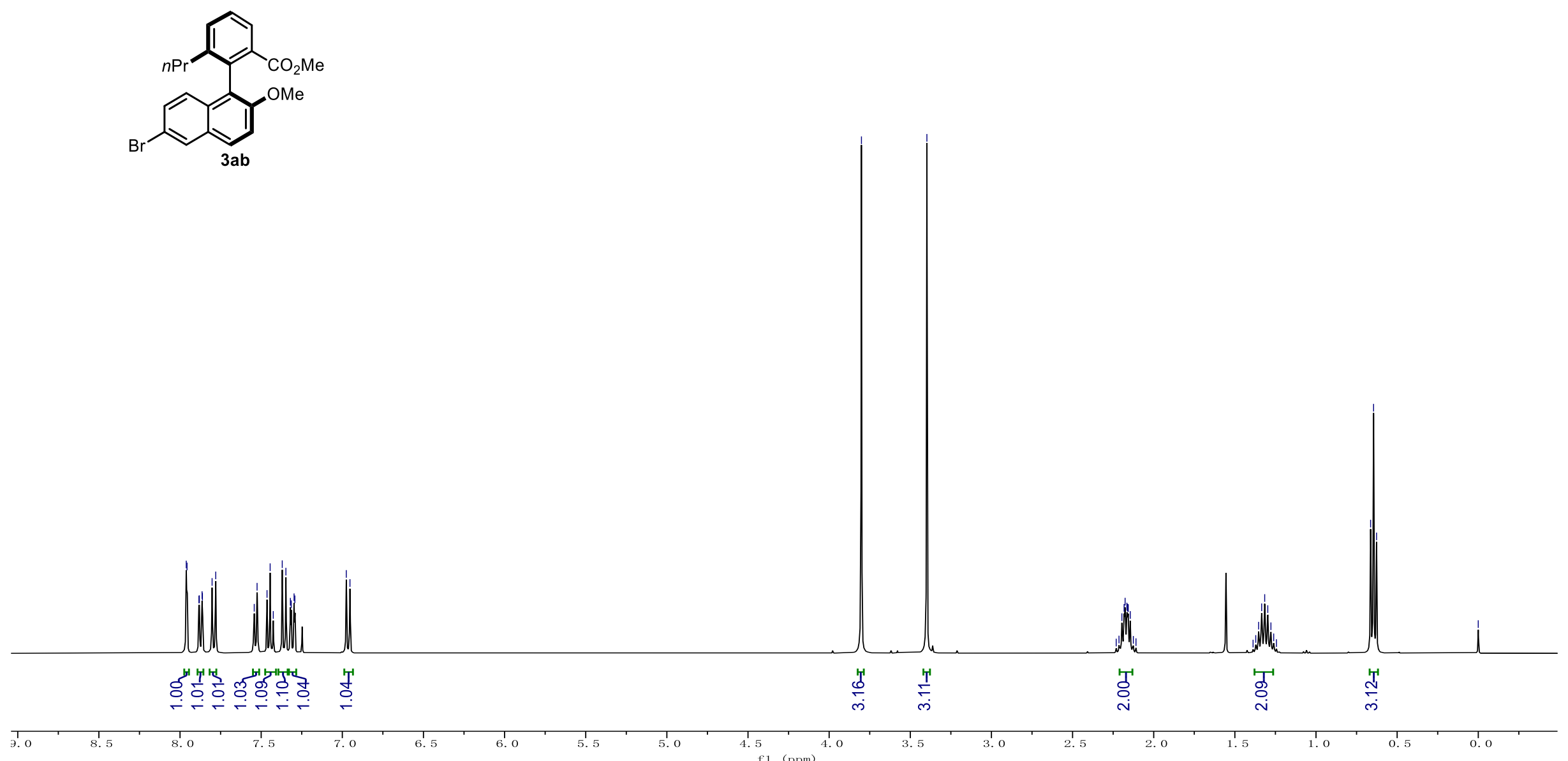




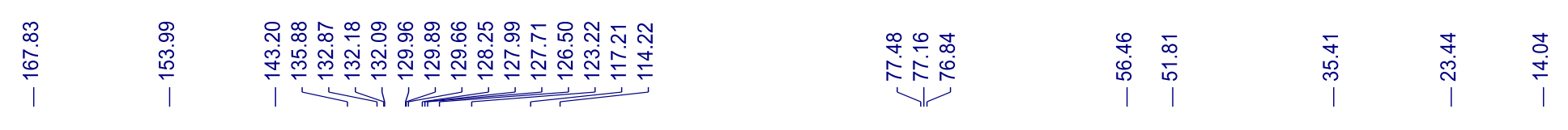
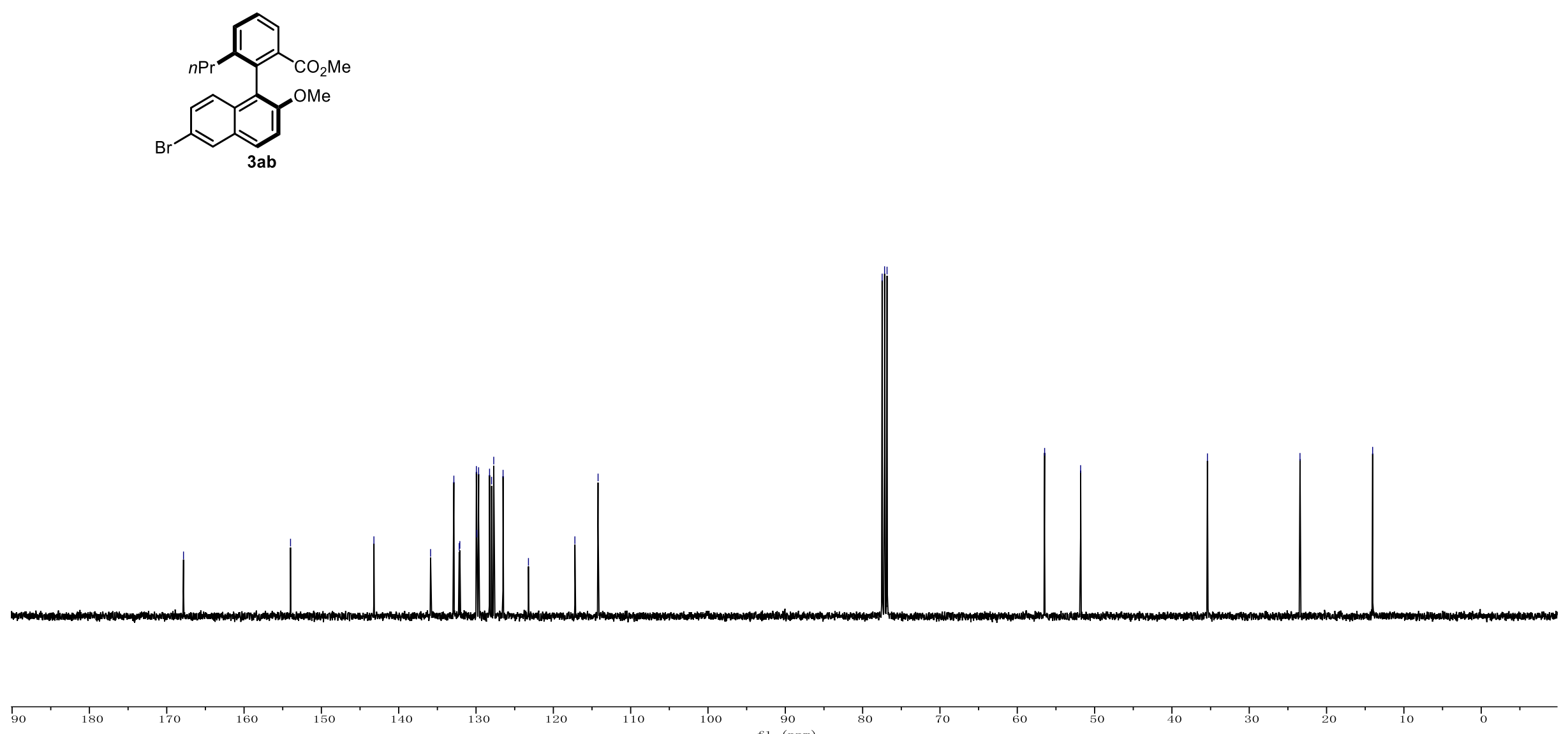


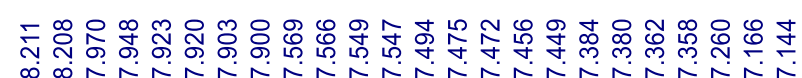

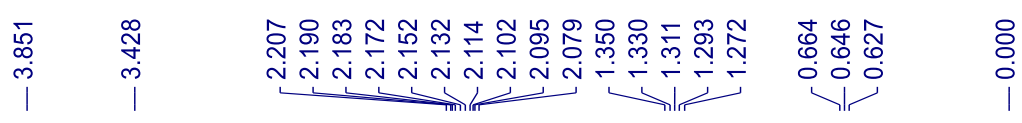
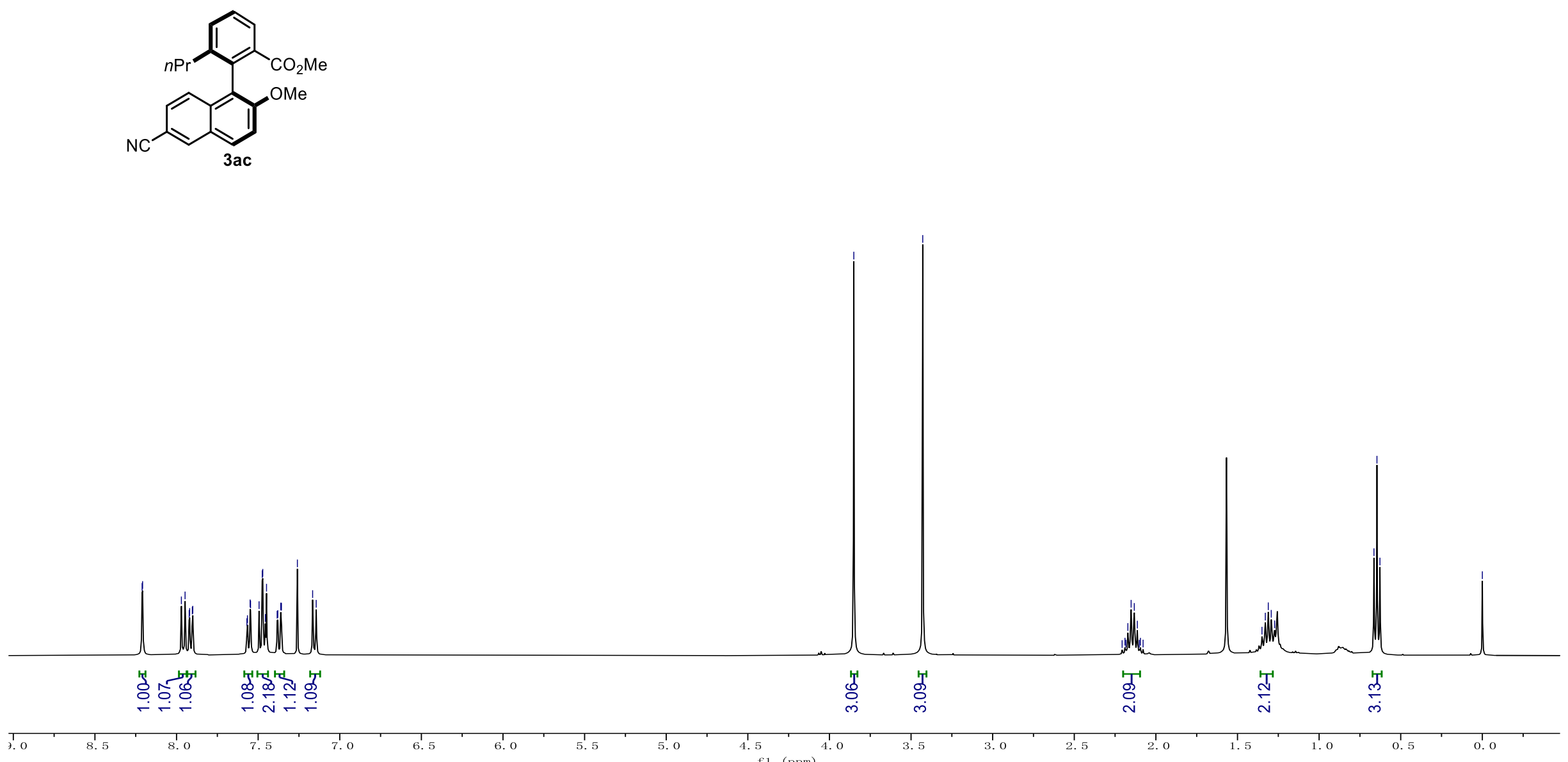

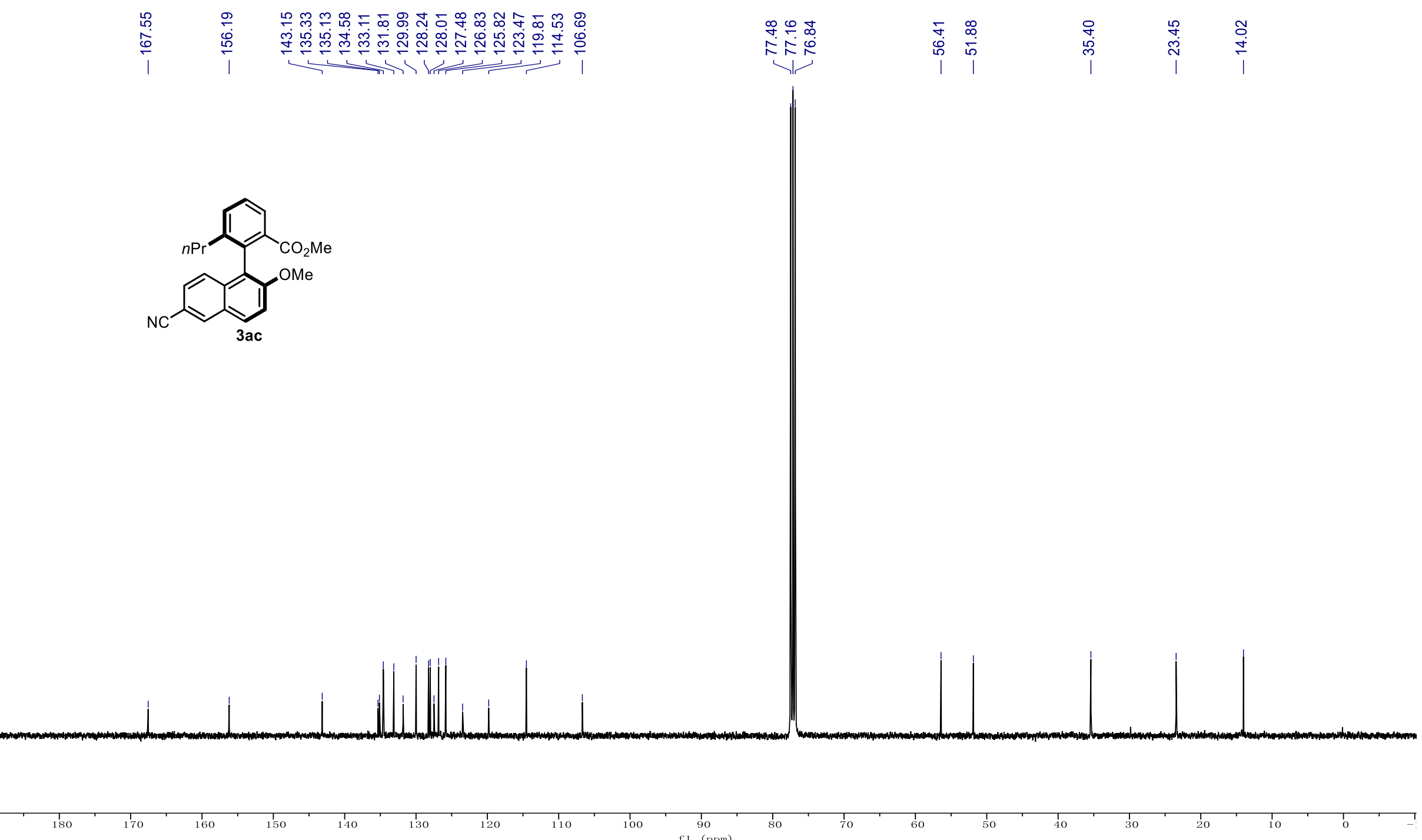


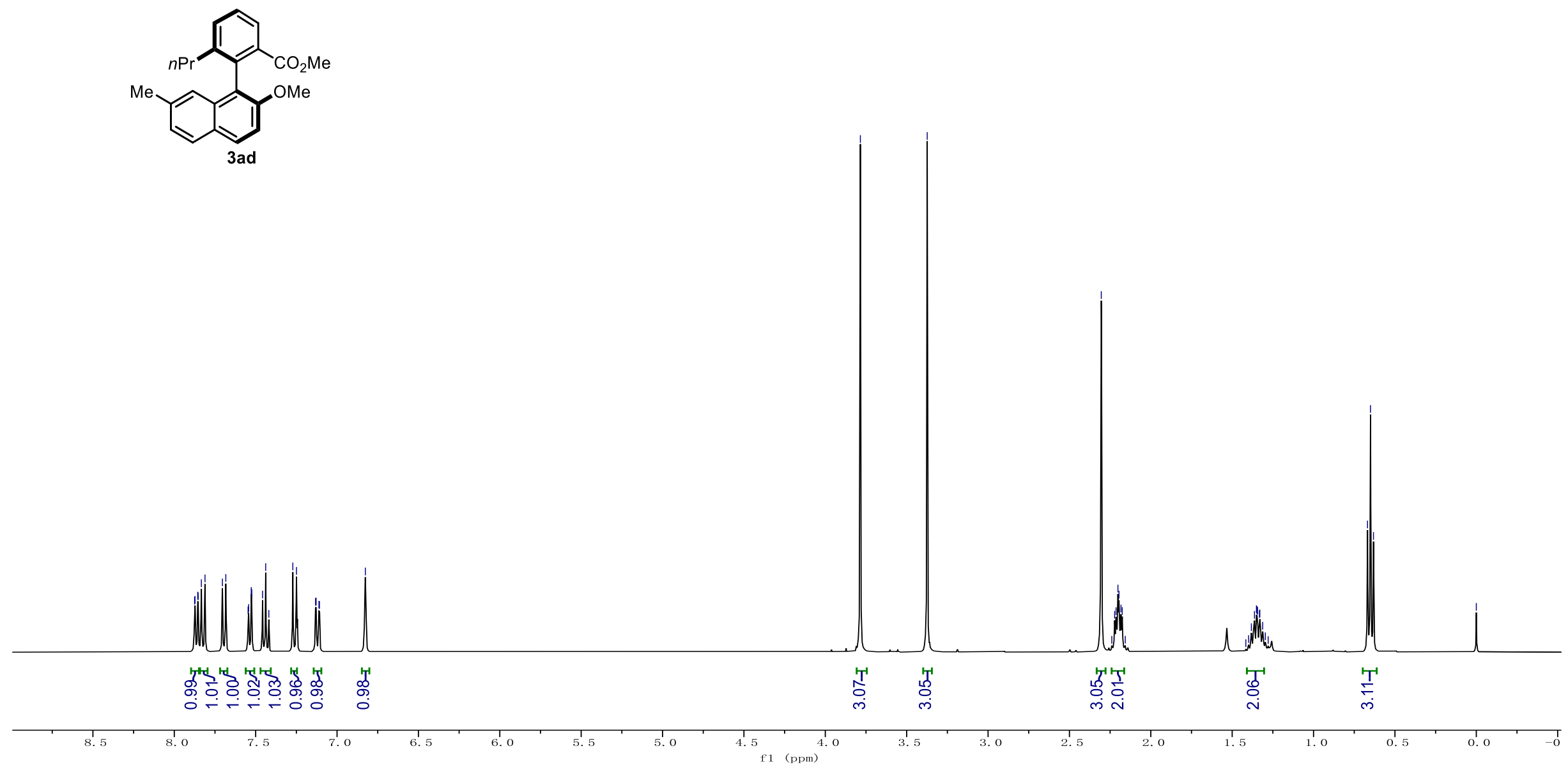




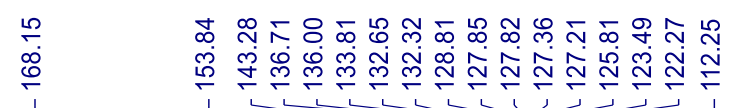

守

송

$\underset{\substack{0 \\ 0}}{5}$

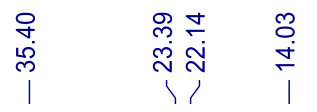
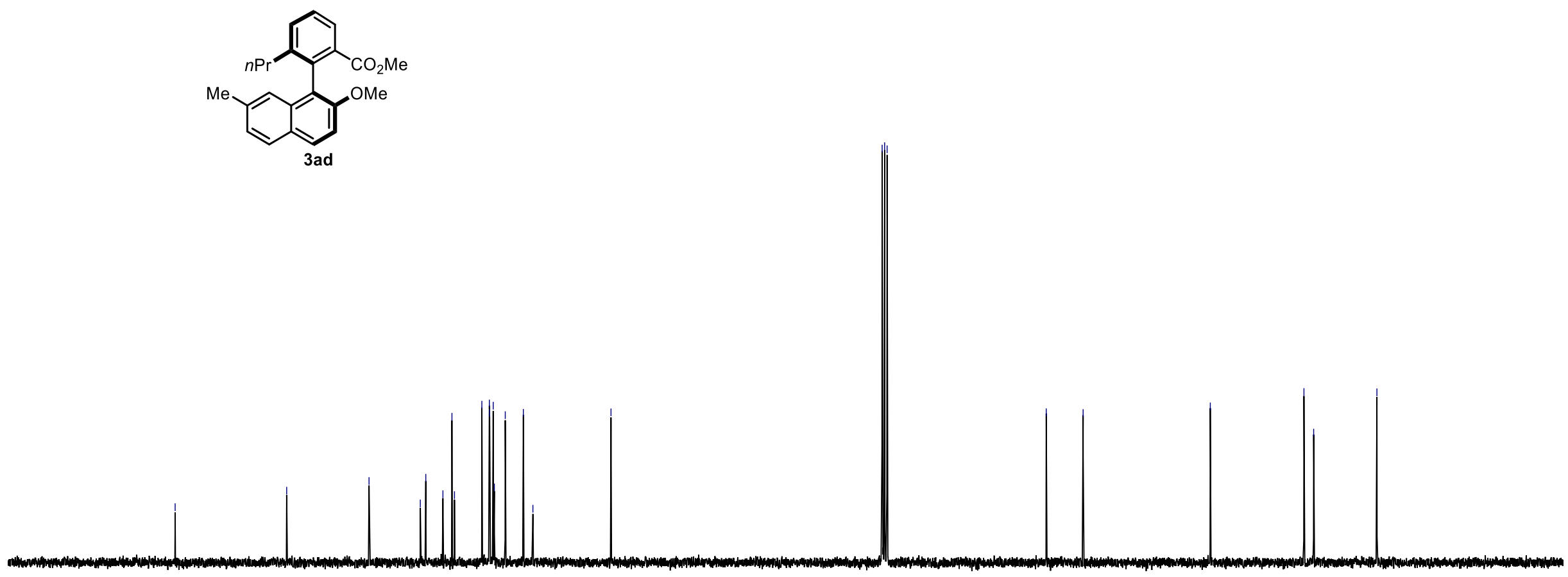

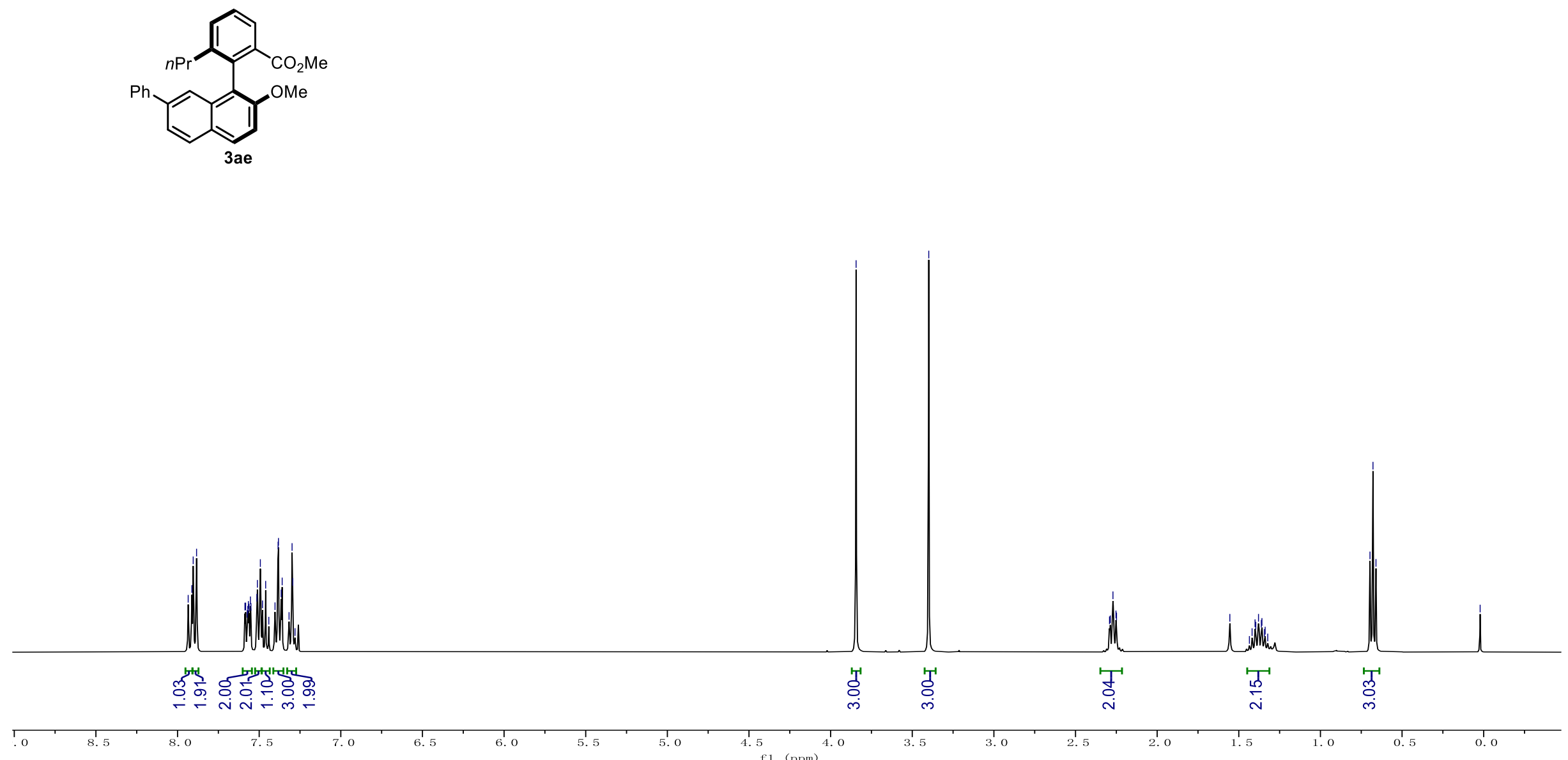
車

它

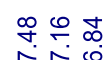

人下゚

in

过

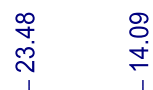
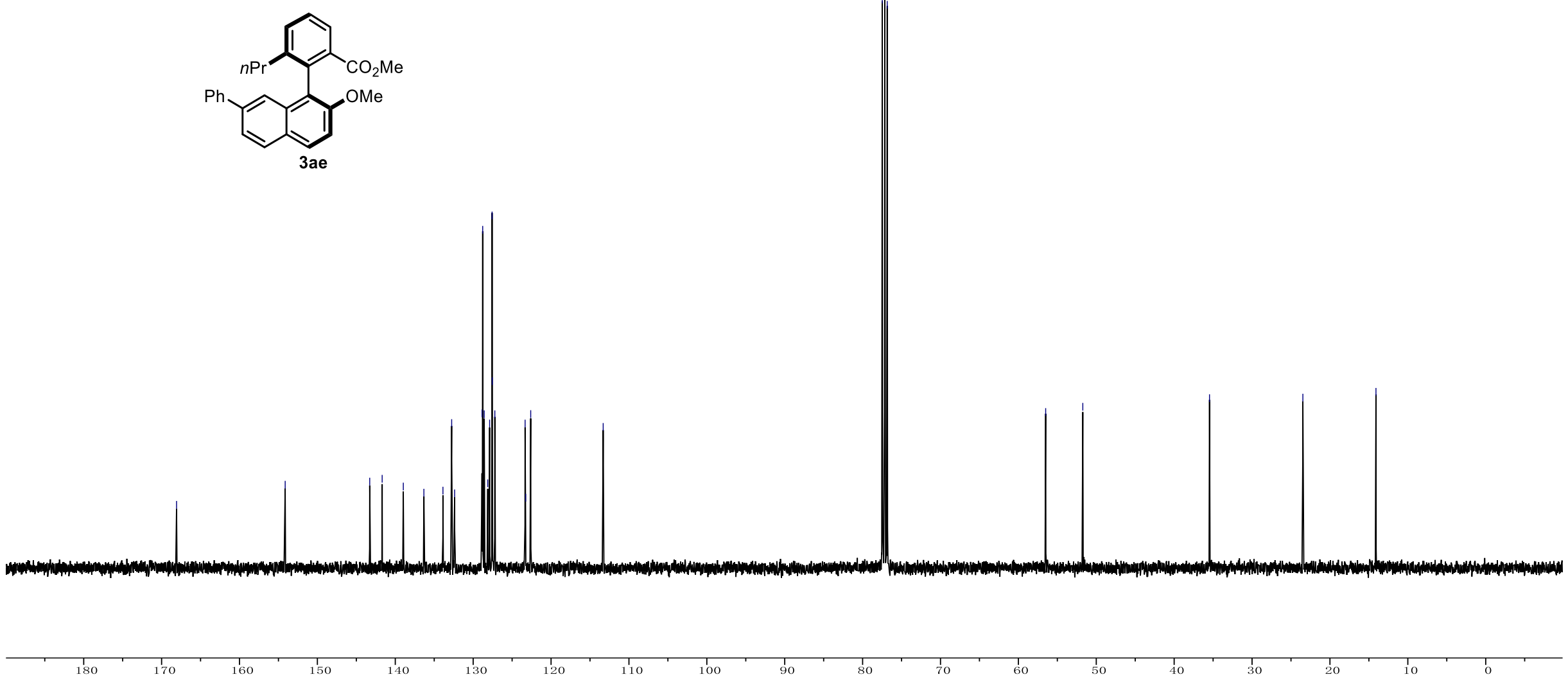

140
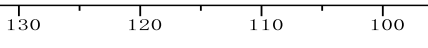

$\stackrel{1}{90}$

80

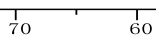

$50+40$

$40 \quad 30$ 


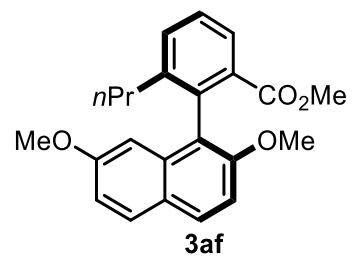

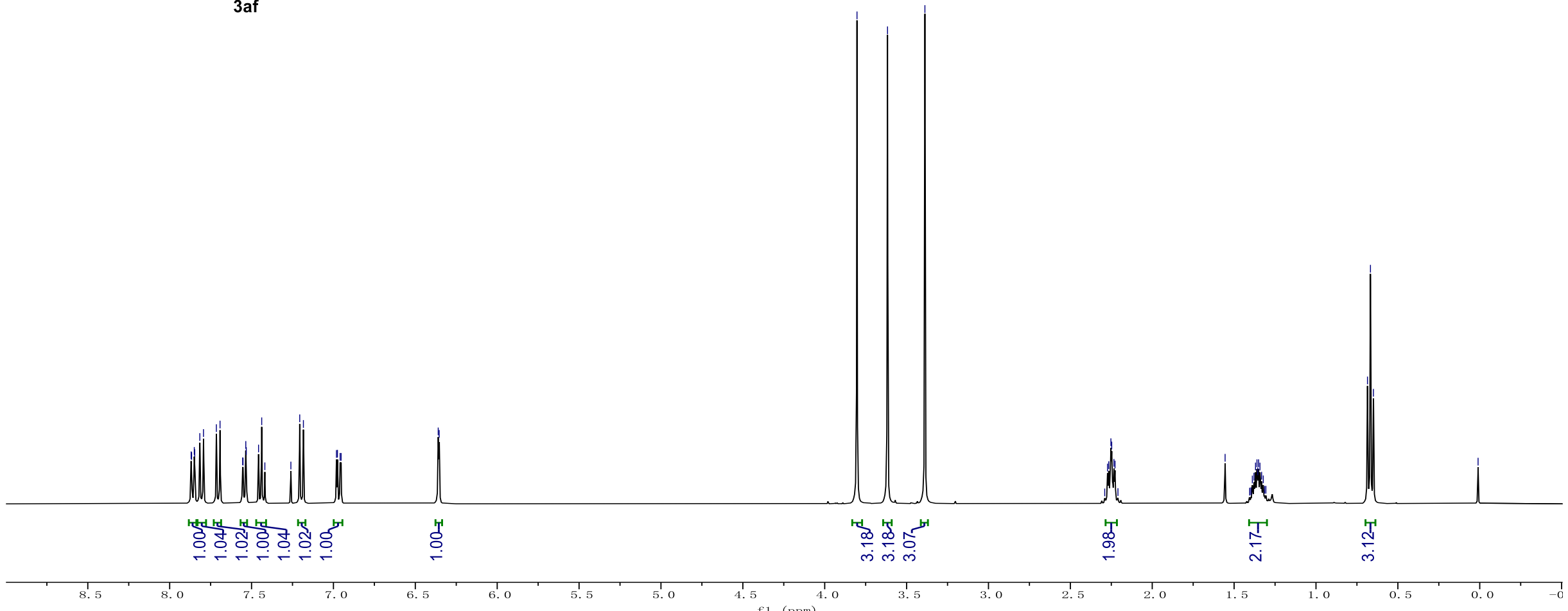




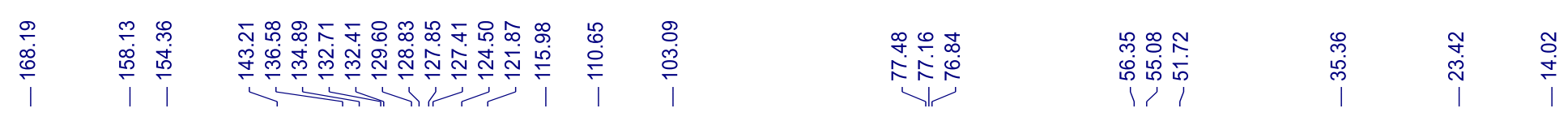
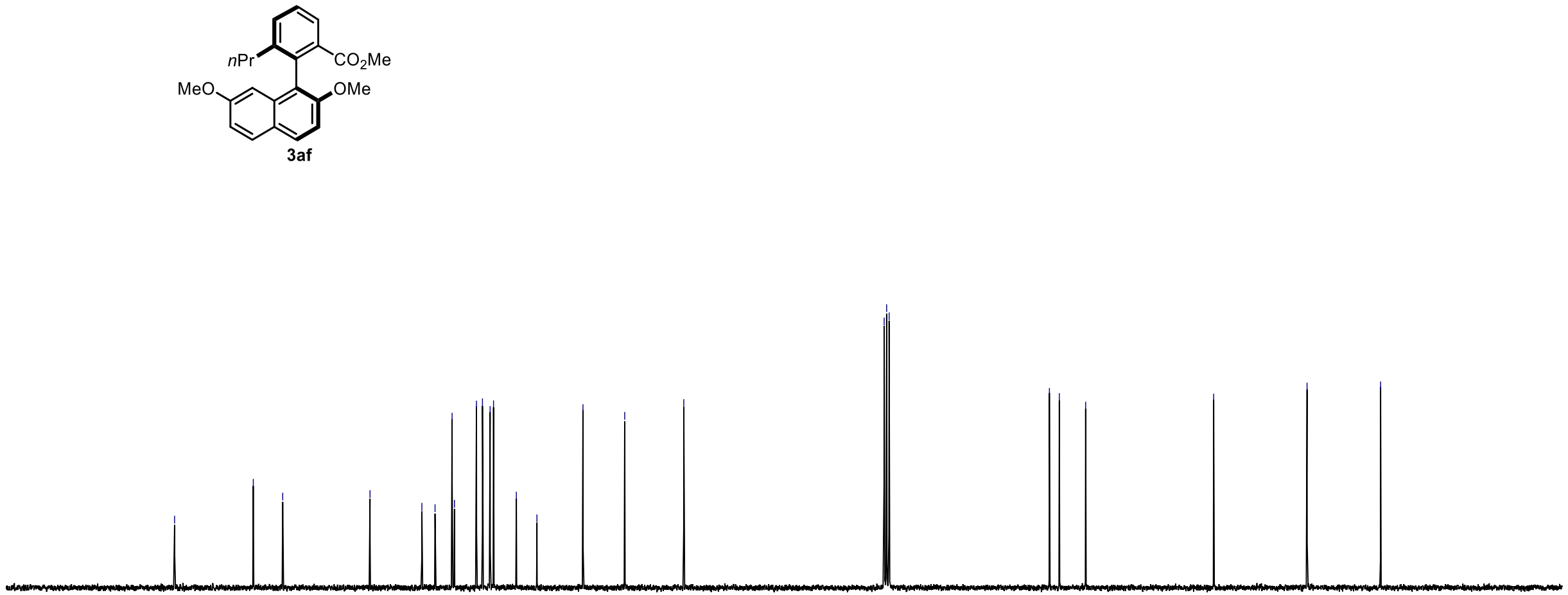


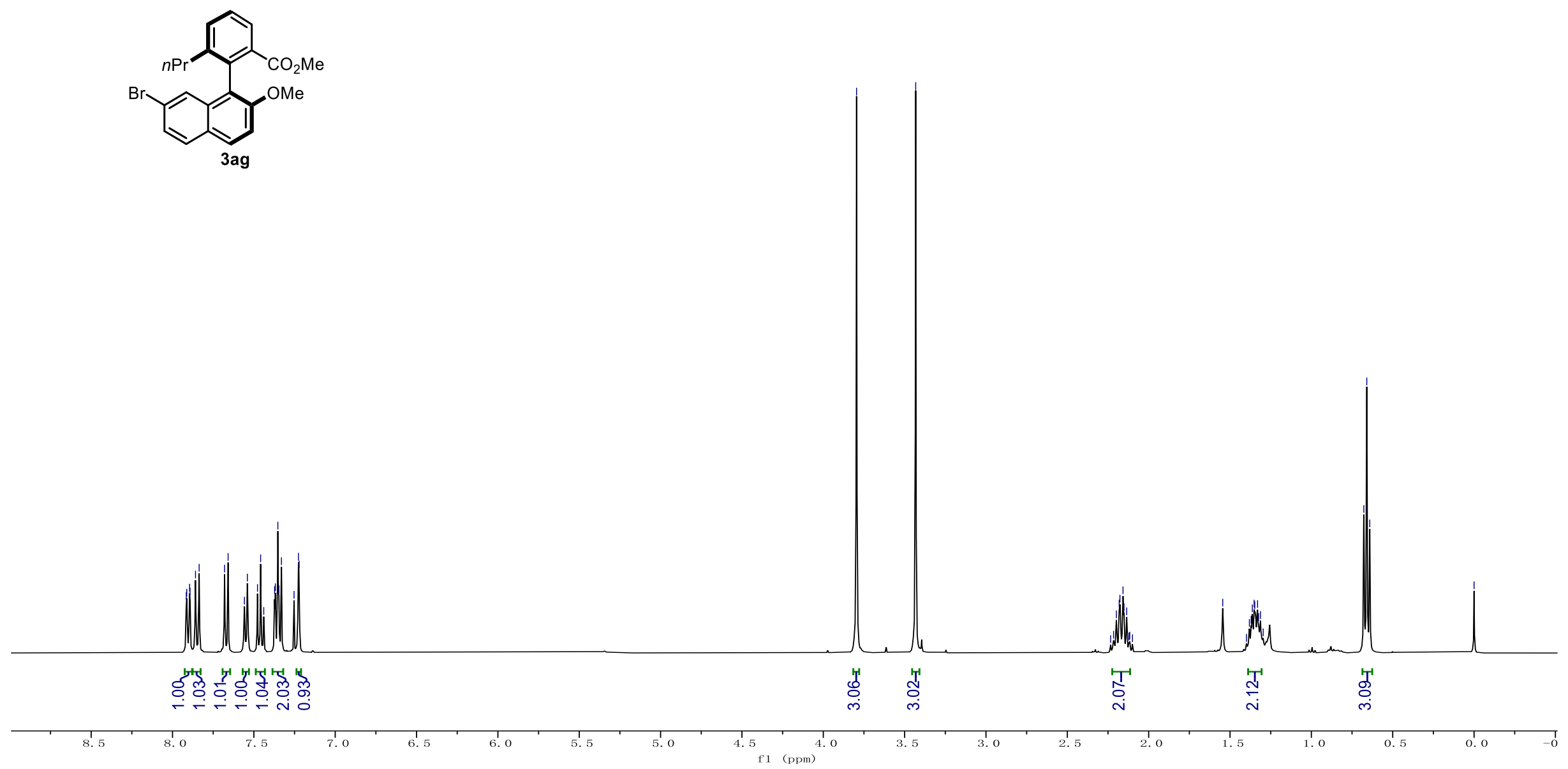




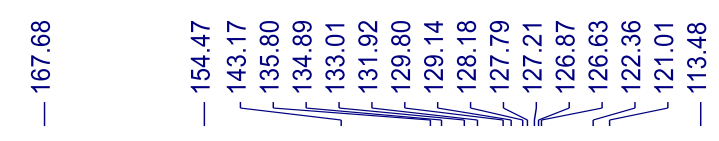

尔电哭

企是

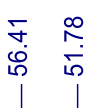

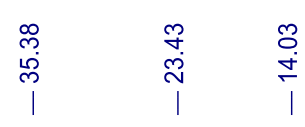
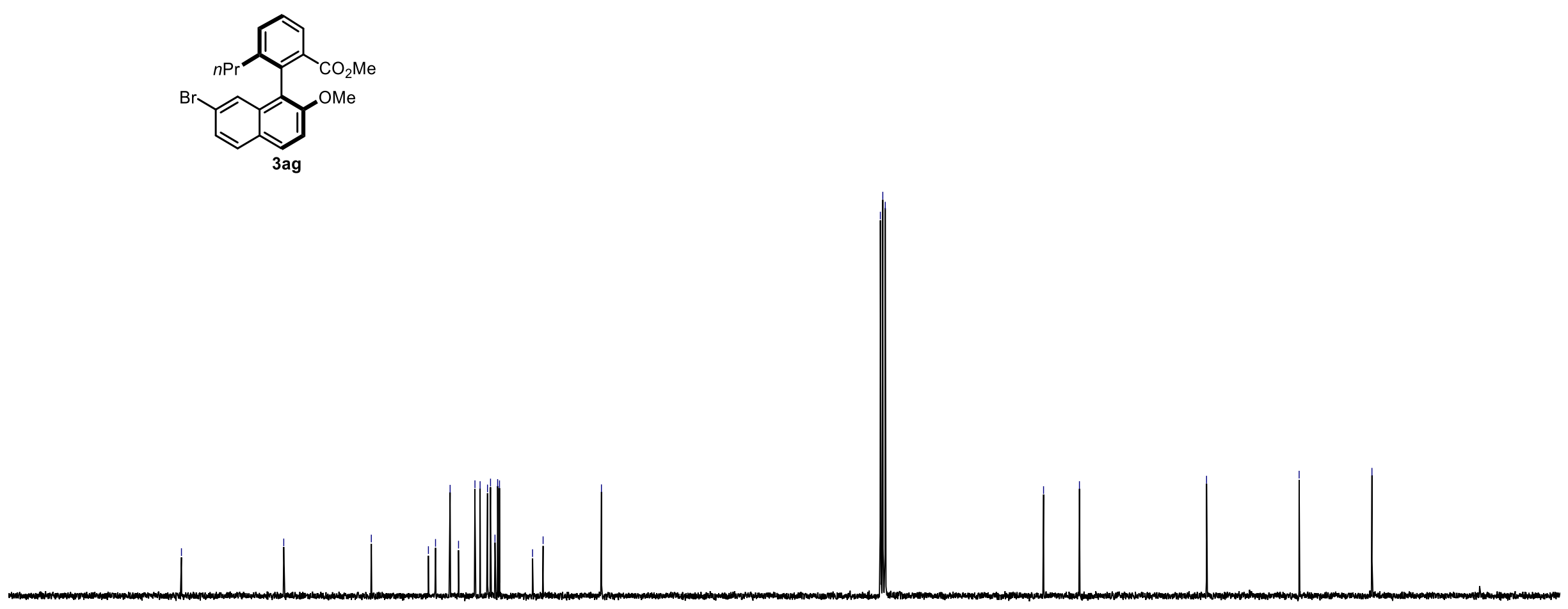

90
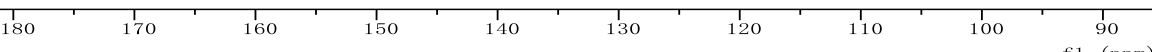

8
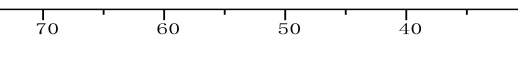
<smiles>COc1cccc(C)c1Cc1cccc2ccccc12</smiles>

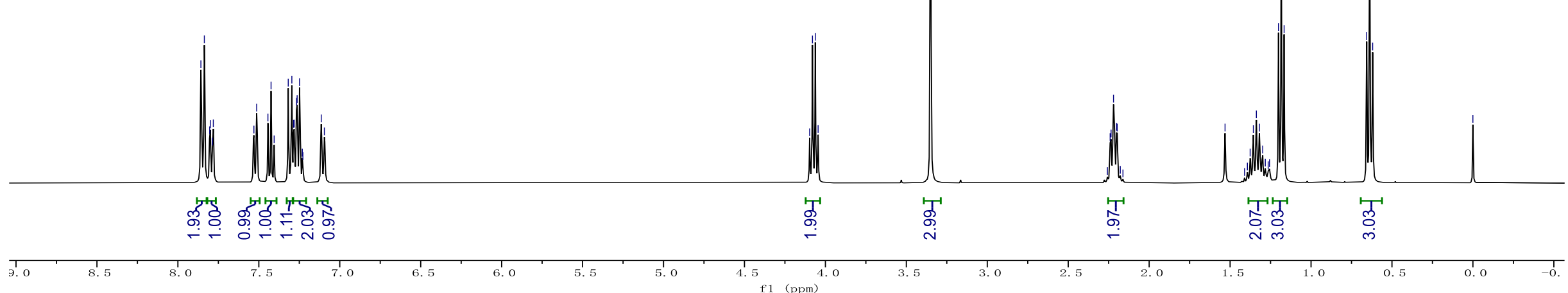



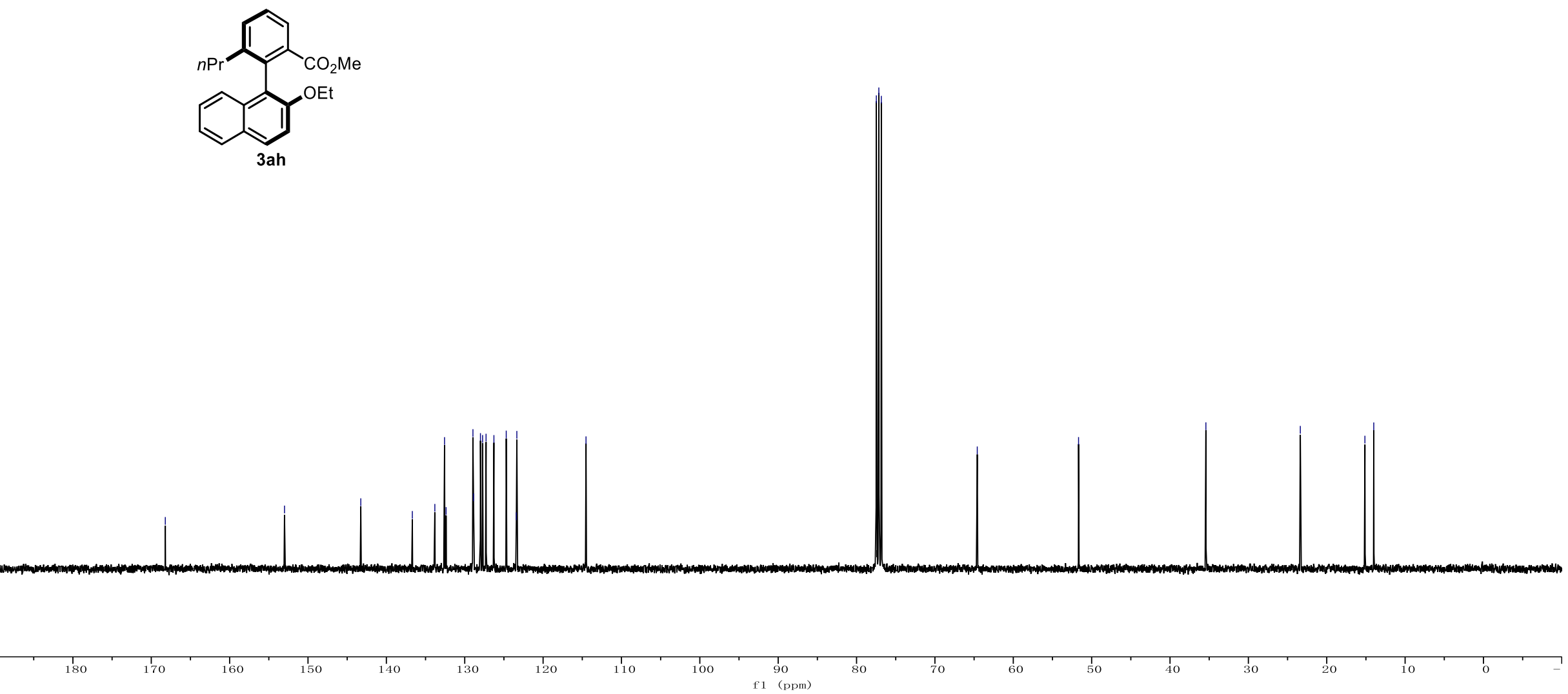
<smiles>[GeH3]</smiles>

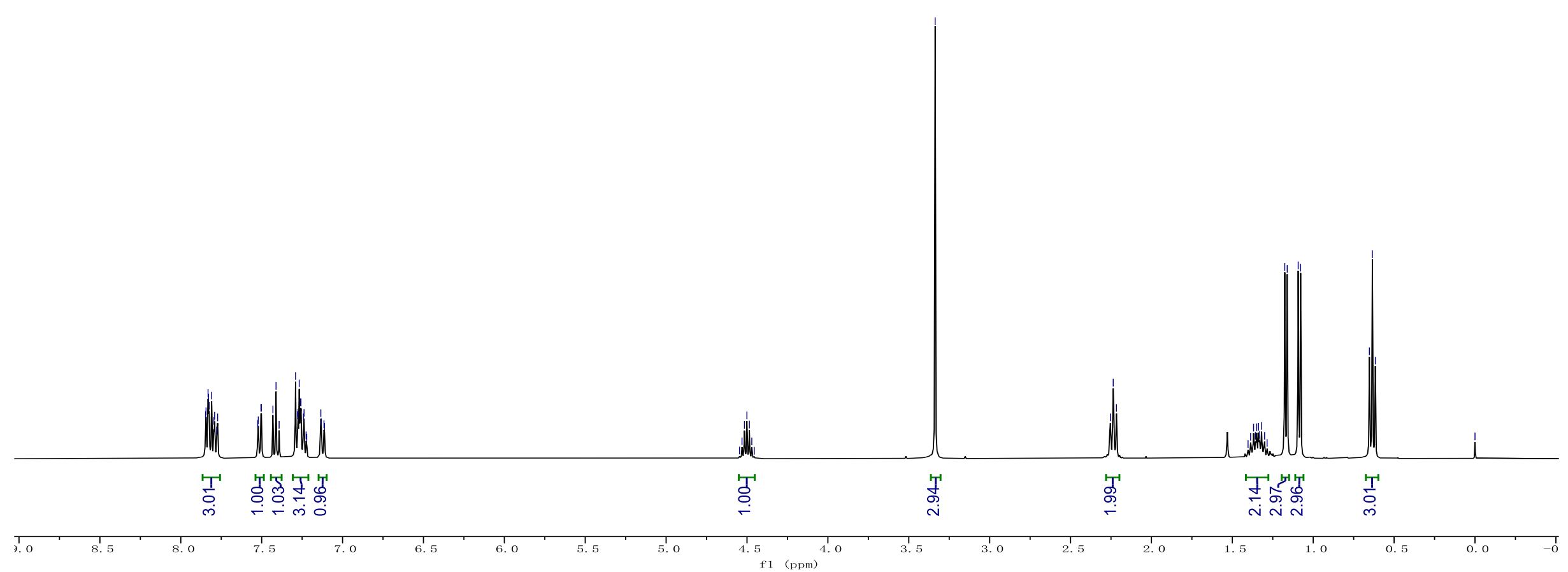




\begin{tabular}{|c|c|c|c|c|c|}
\hline 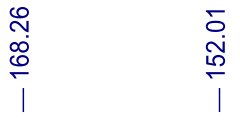 & $\underbrace{O}$ & 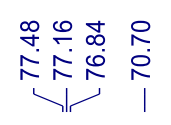 & 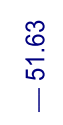 & 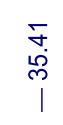 & 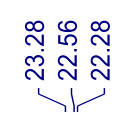 \\
\hline
\end{tabular}

3ai
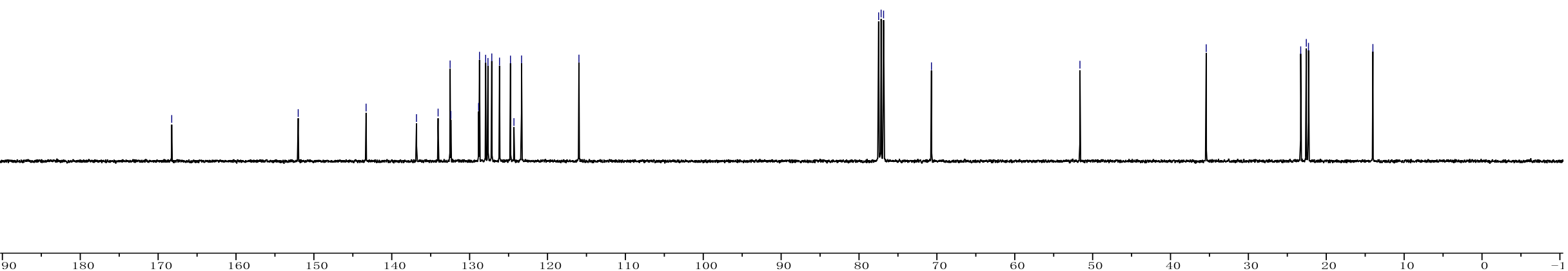

$\stackrel{1}{90}$

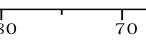

$60 \quad 50$

4

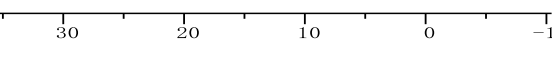



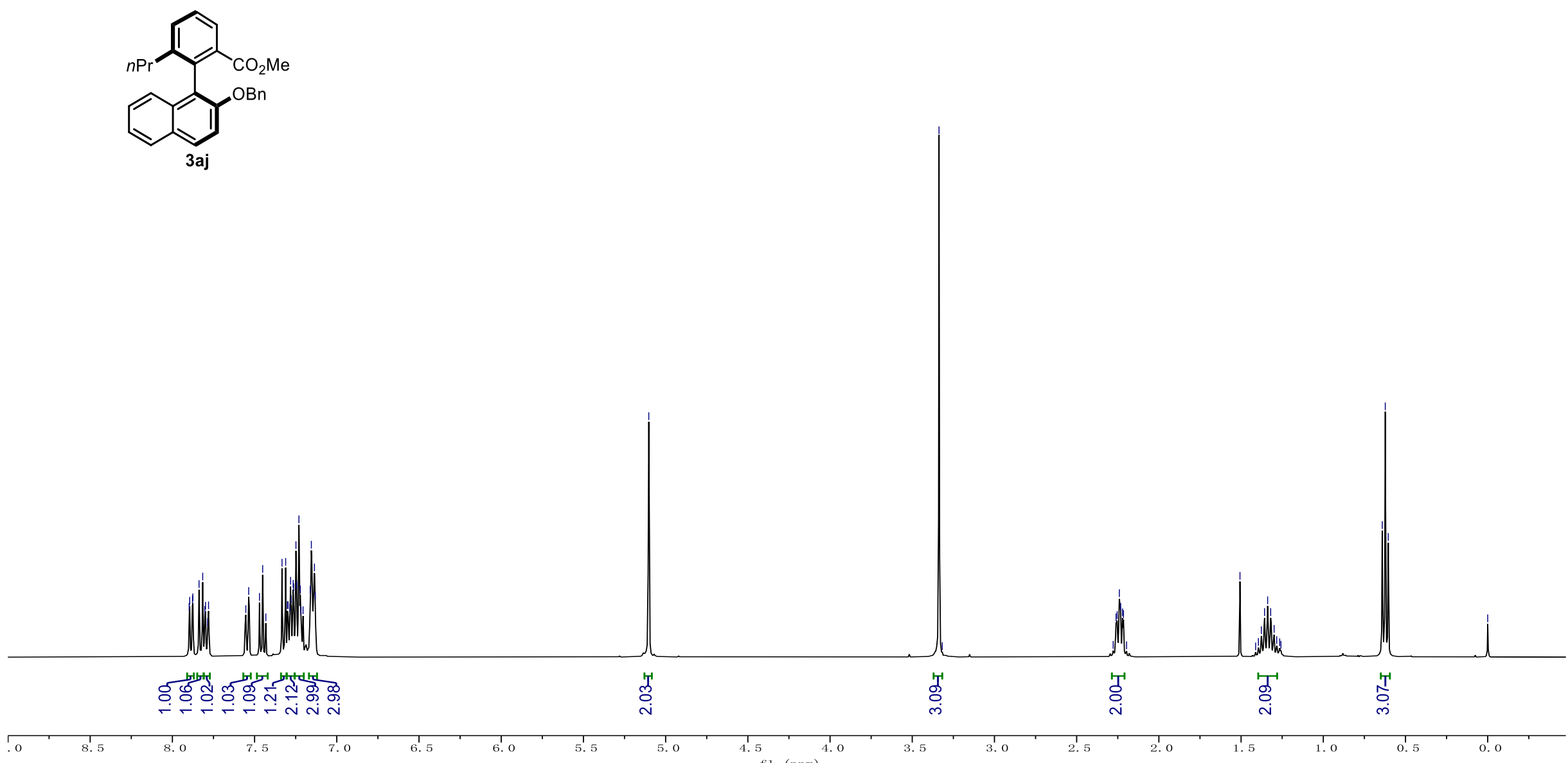


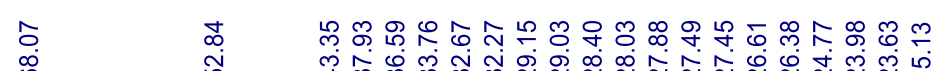

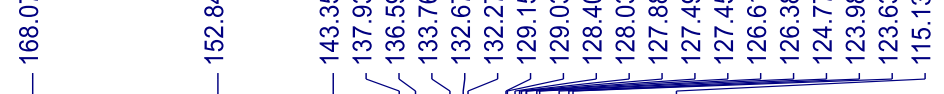

守

N余

$\frac{i n}{\stackrel{1}{5}}$

$\begin{array}{lll}0 & \hat{n} & + \\ i & \stackrel{+}{j} & + \\ 1 & i & 1\end{array}$

son

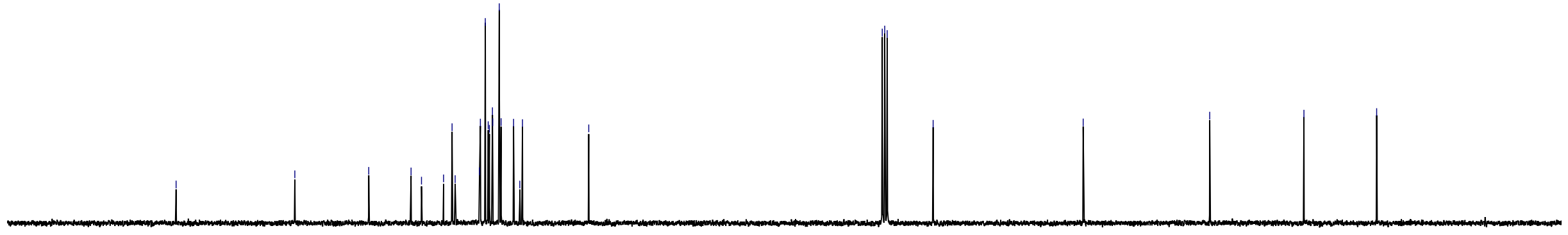




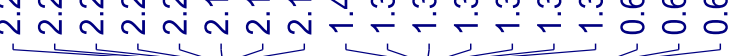
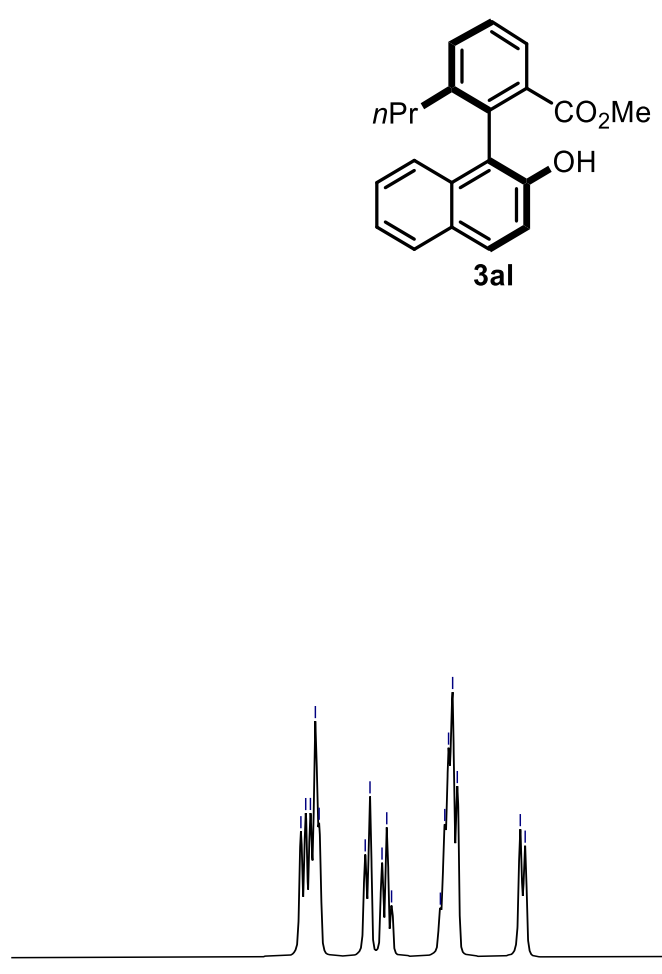

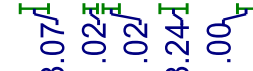

焉

$\frac{\pi}{\pi}$
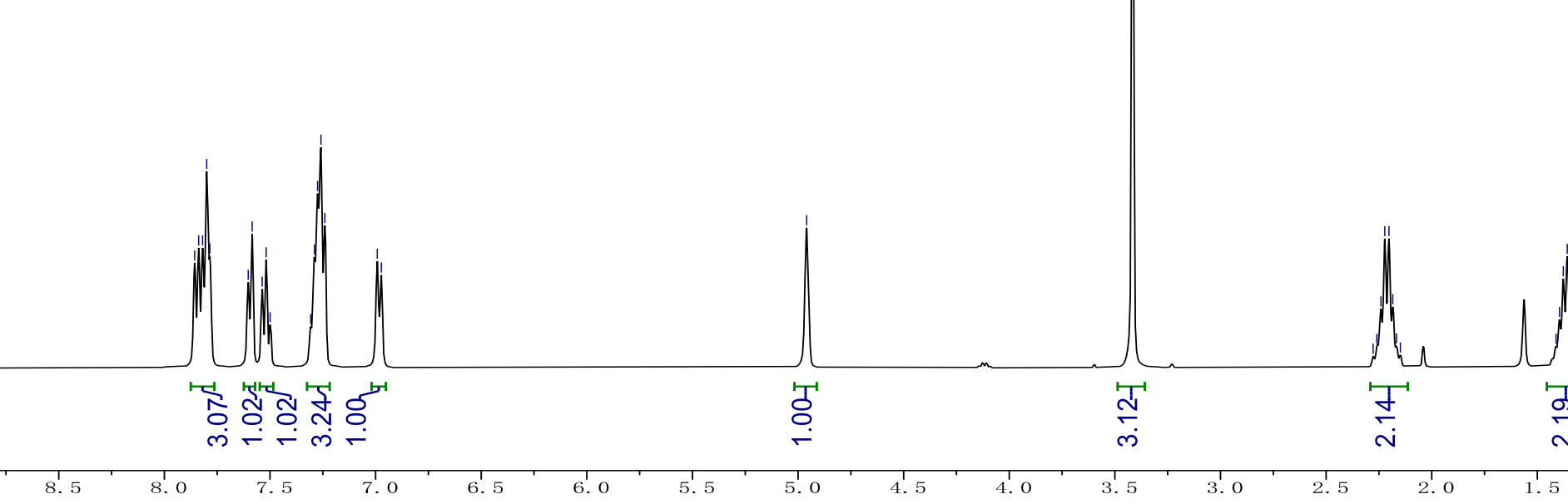

6. 0

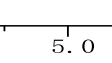

4. 5 ( 1 (ppm) 


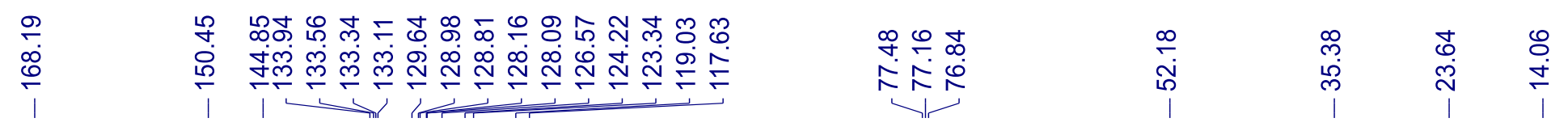
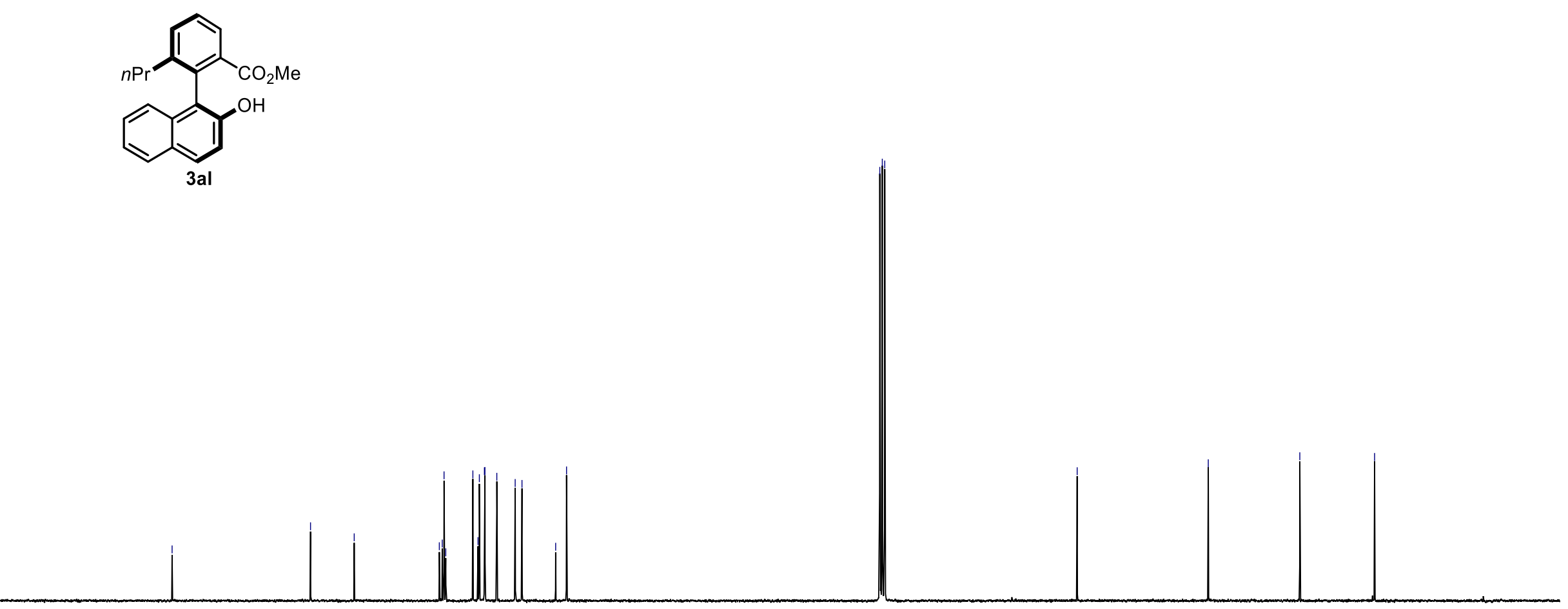

90
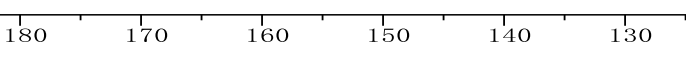

f1 $\stackrel{1}{90}(\mathrm{ppm})$ 

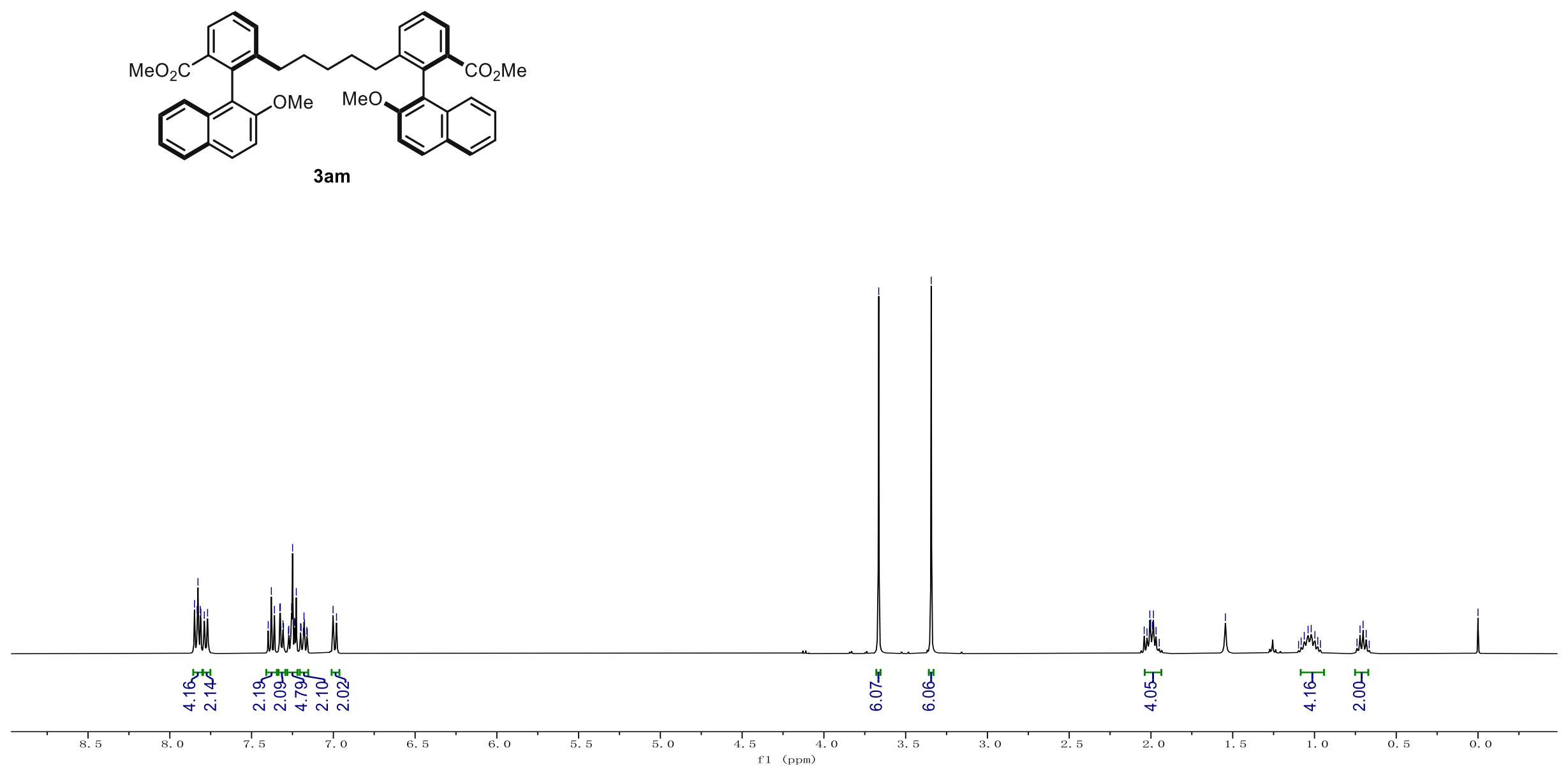


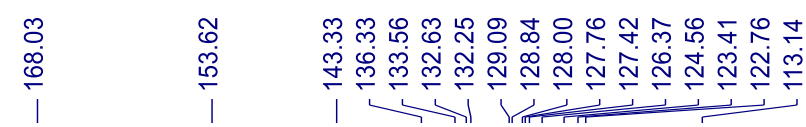
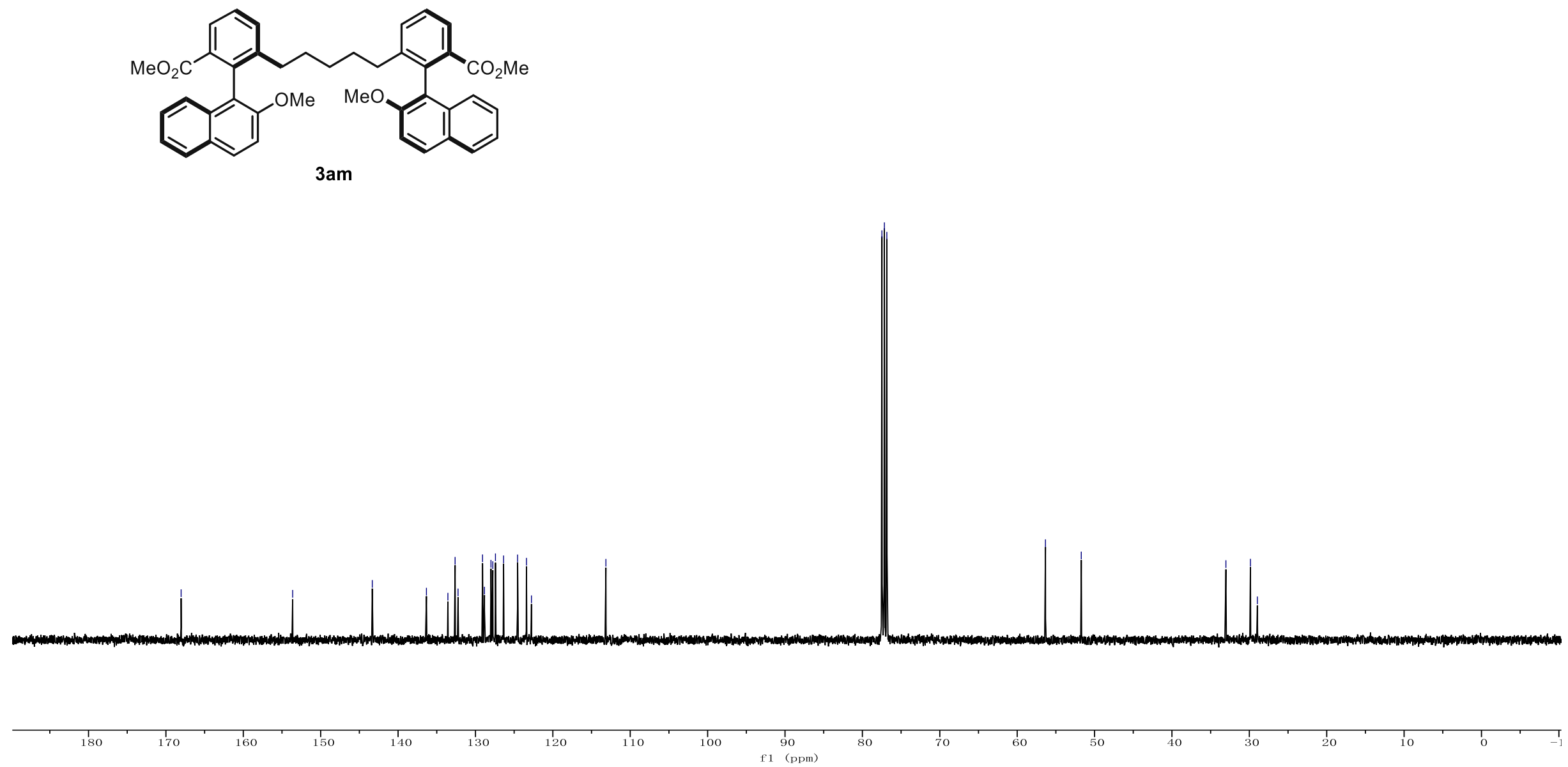


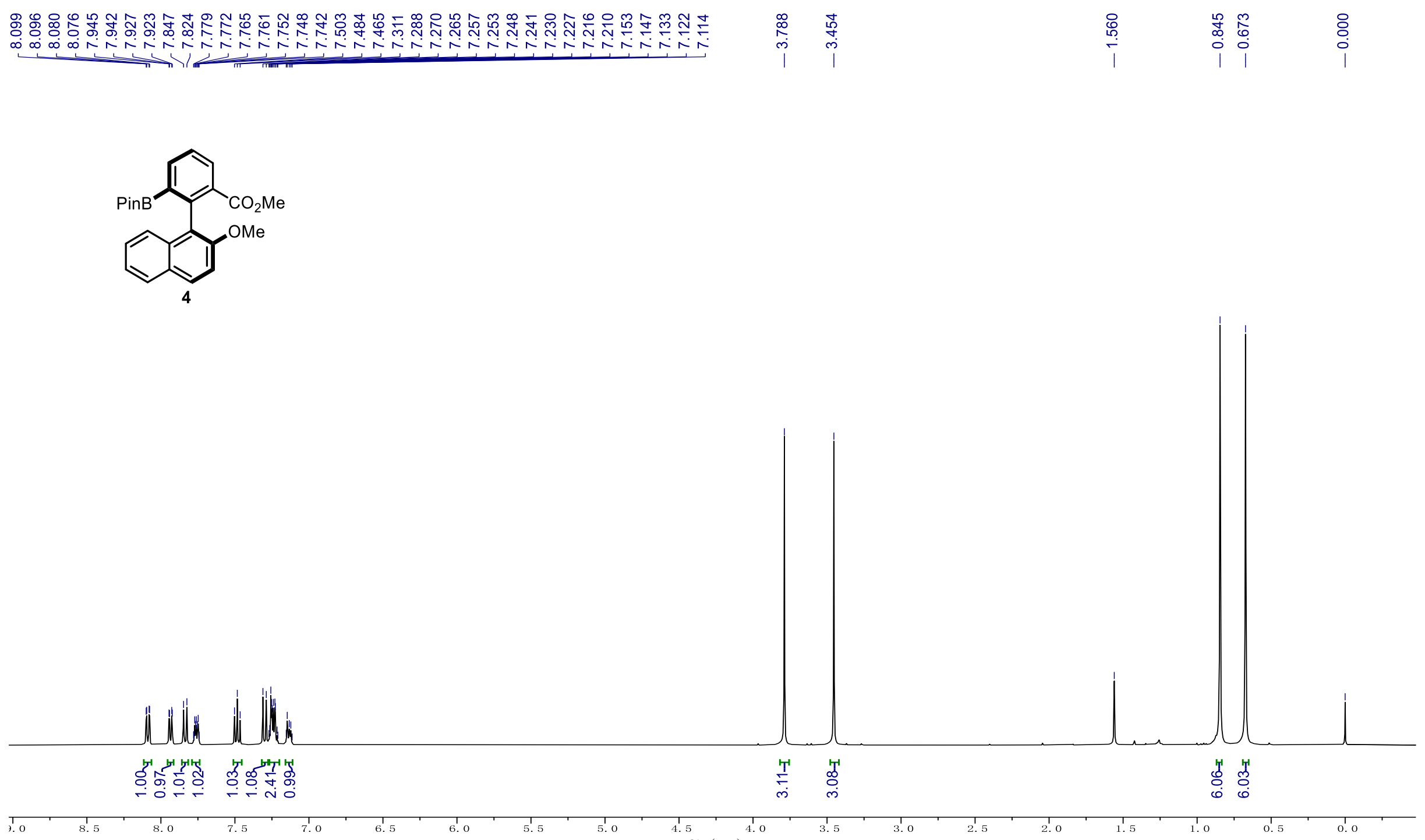

whe llik 
<smiles>COc1cccc(P)c1-c1c(OC)ccc2cccc(O)c12</smiles>

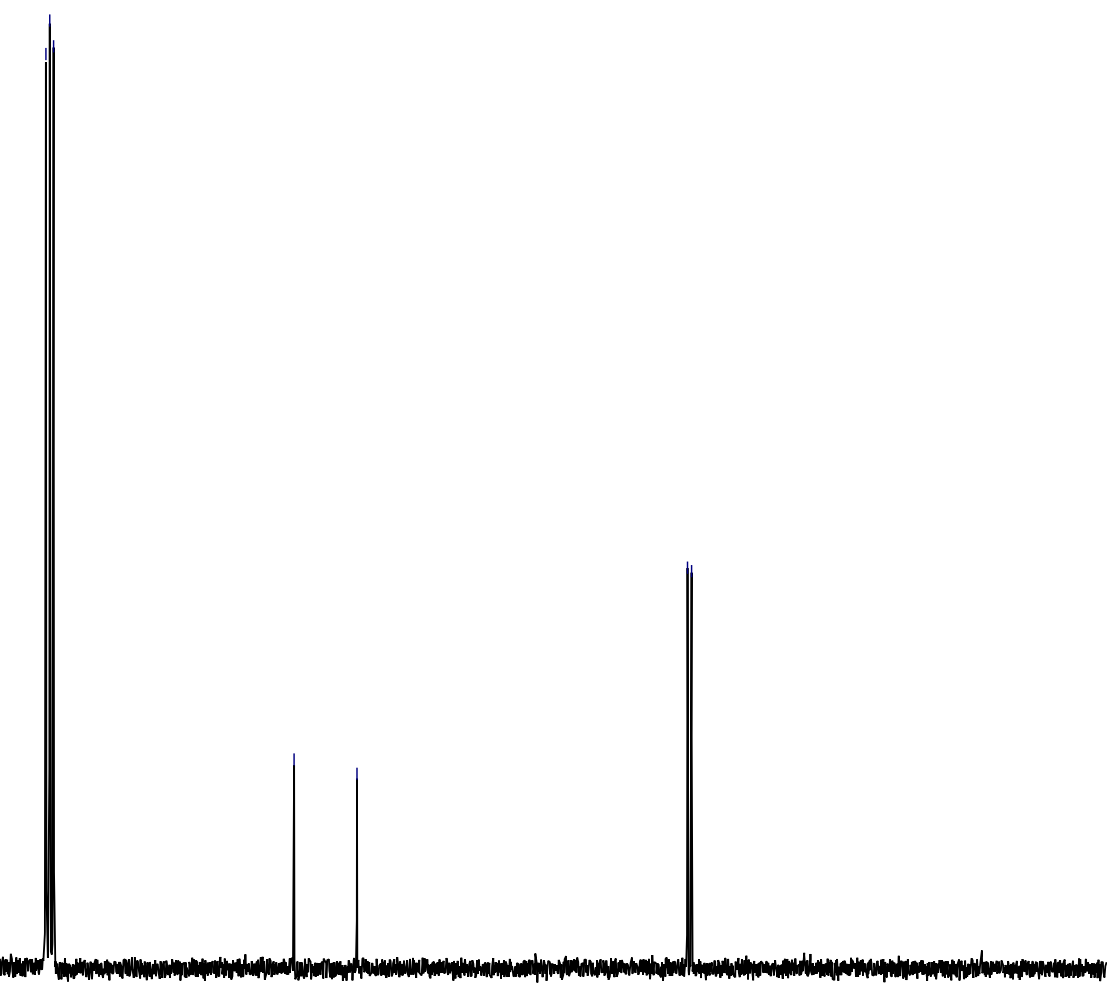



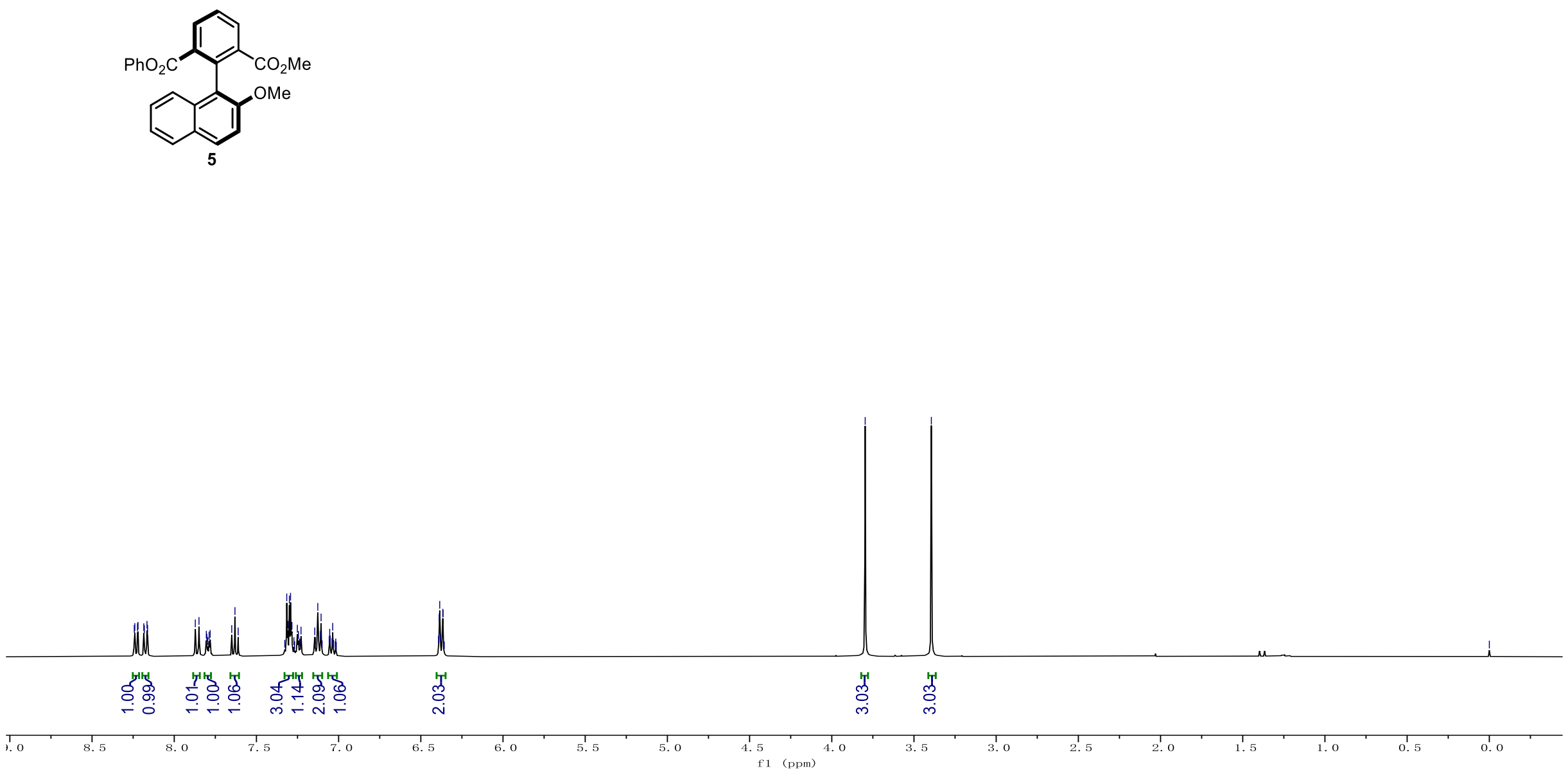


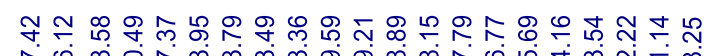

它它

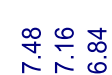

兵㲾

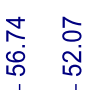

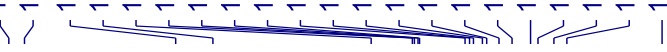
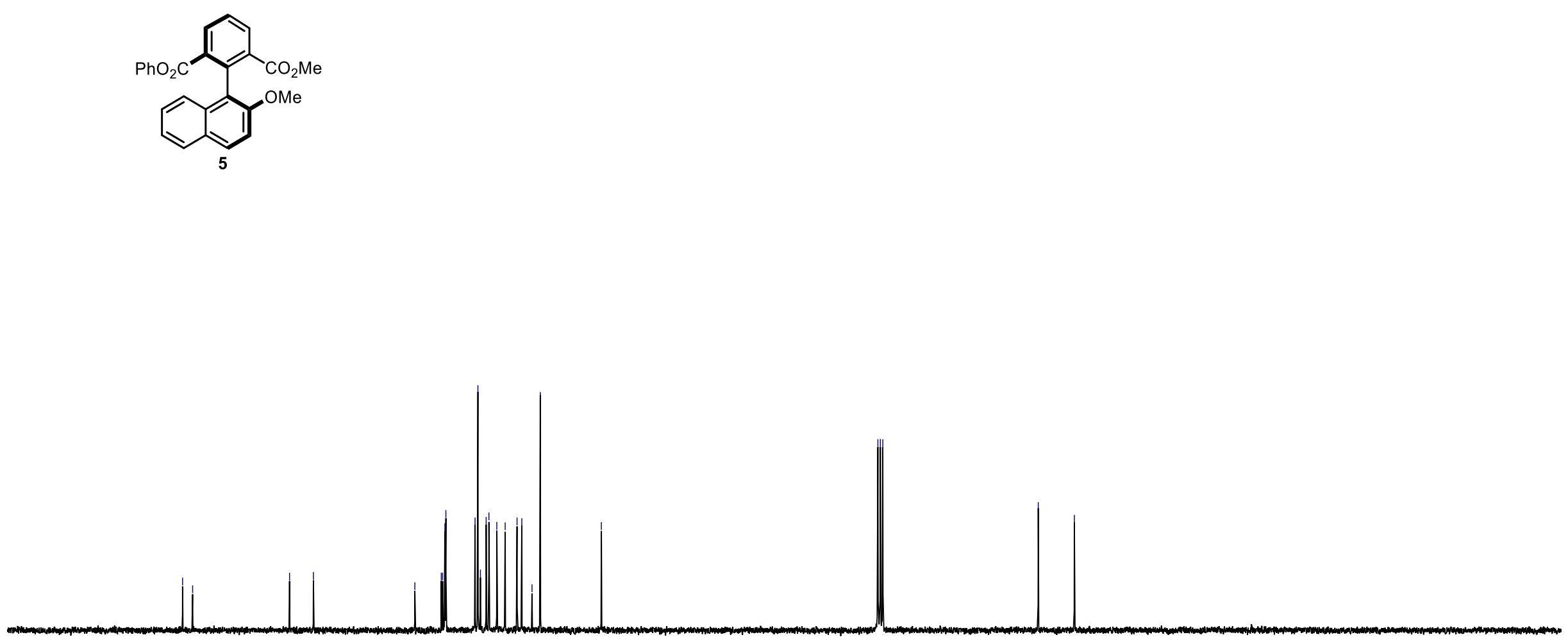

90
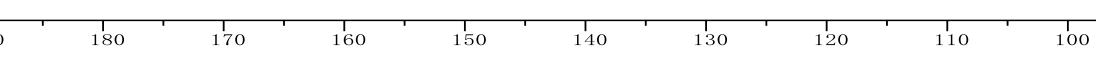

100

$\stackrel{90}{90}(\mathrm{ppm})$

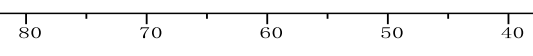

30 


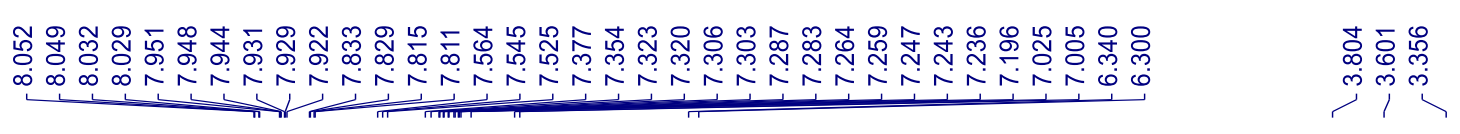

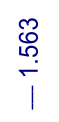

o̊
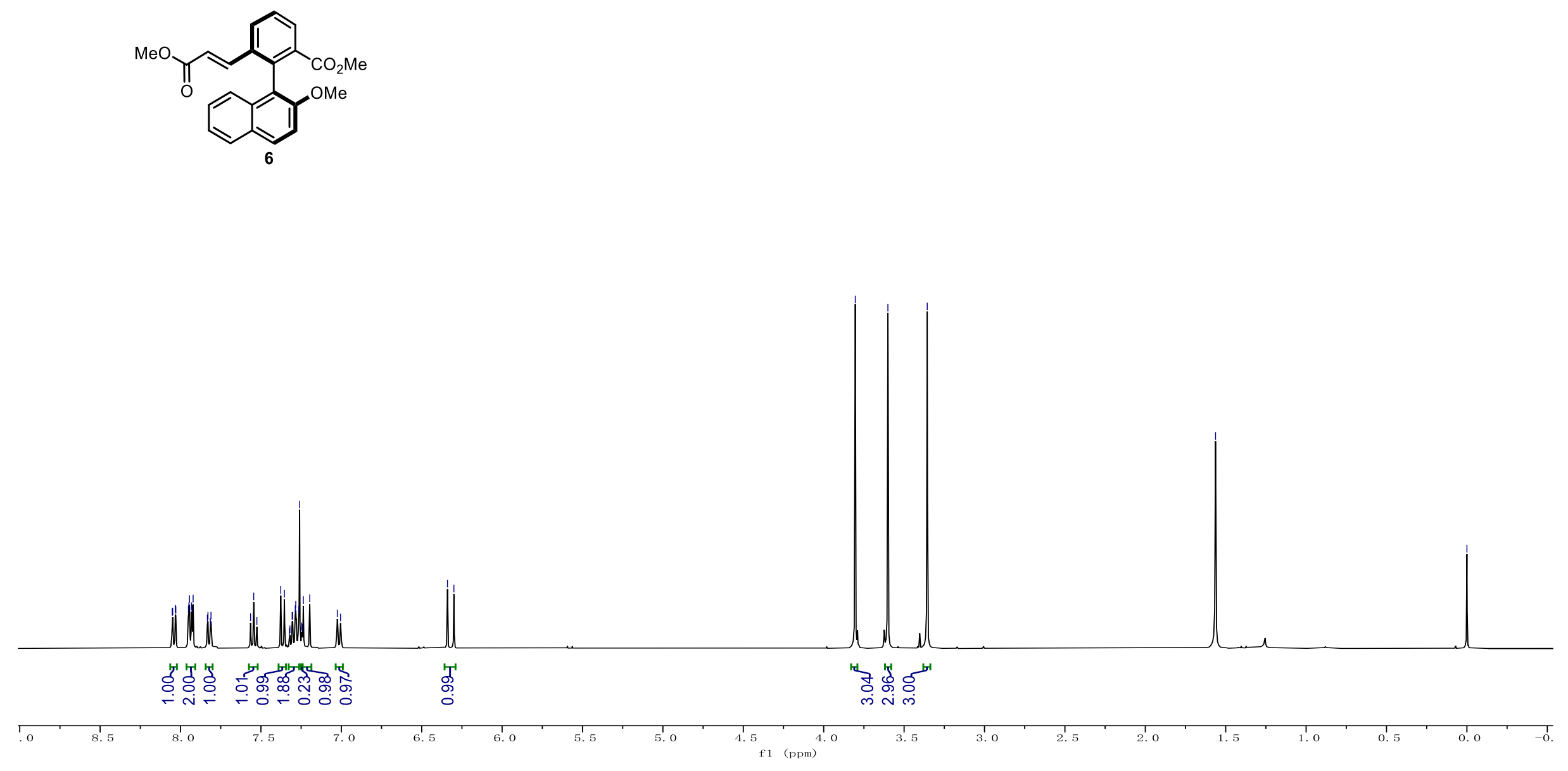

273 


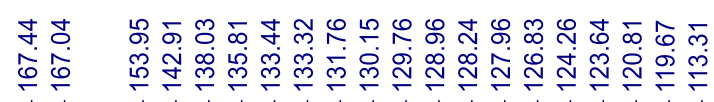
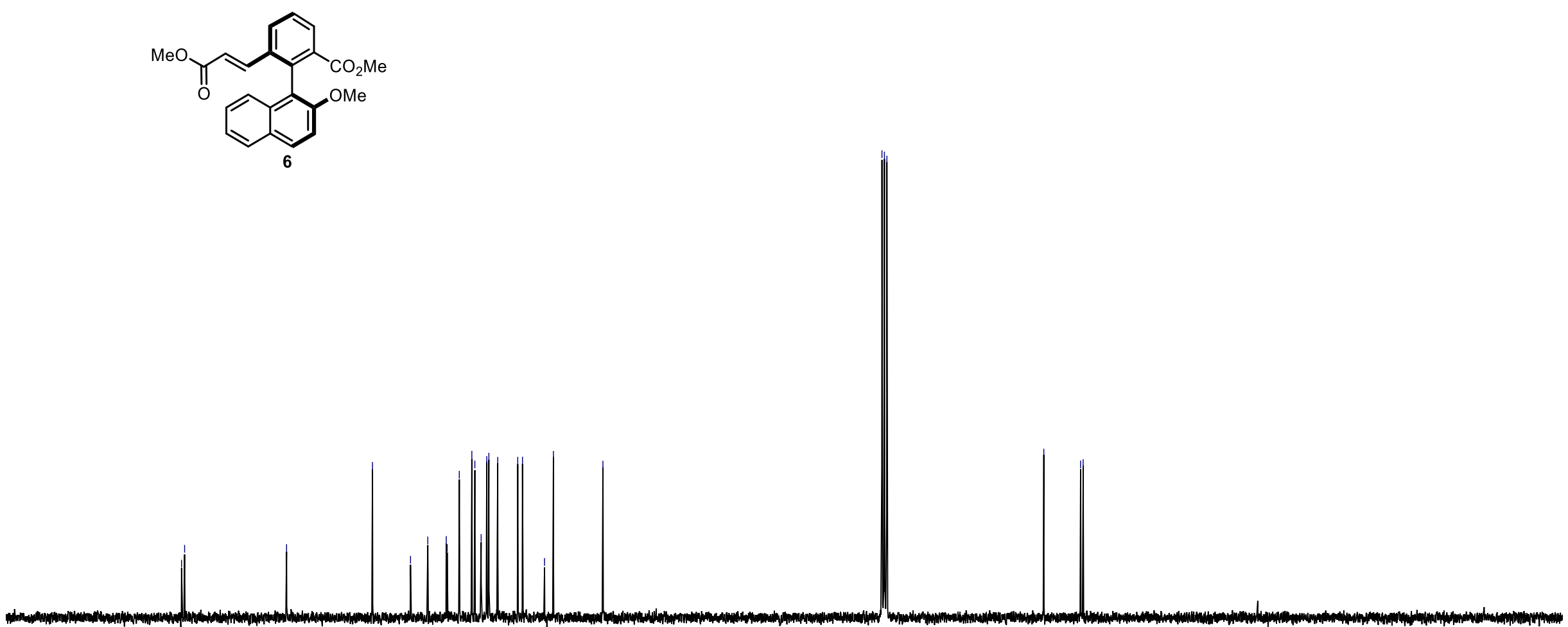


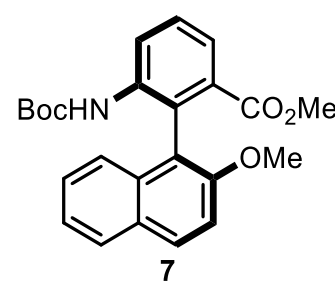




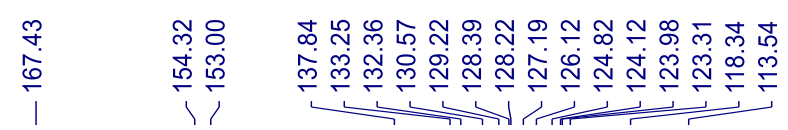

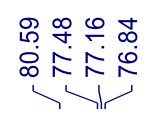

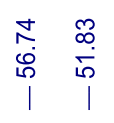

$\stackrel{\substack{N \\ \infty}}{1}$
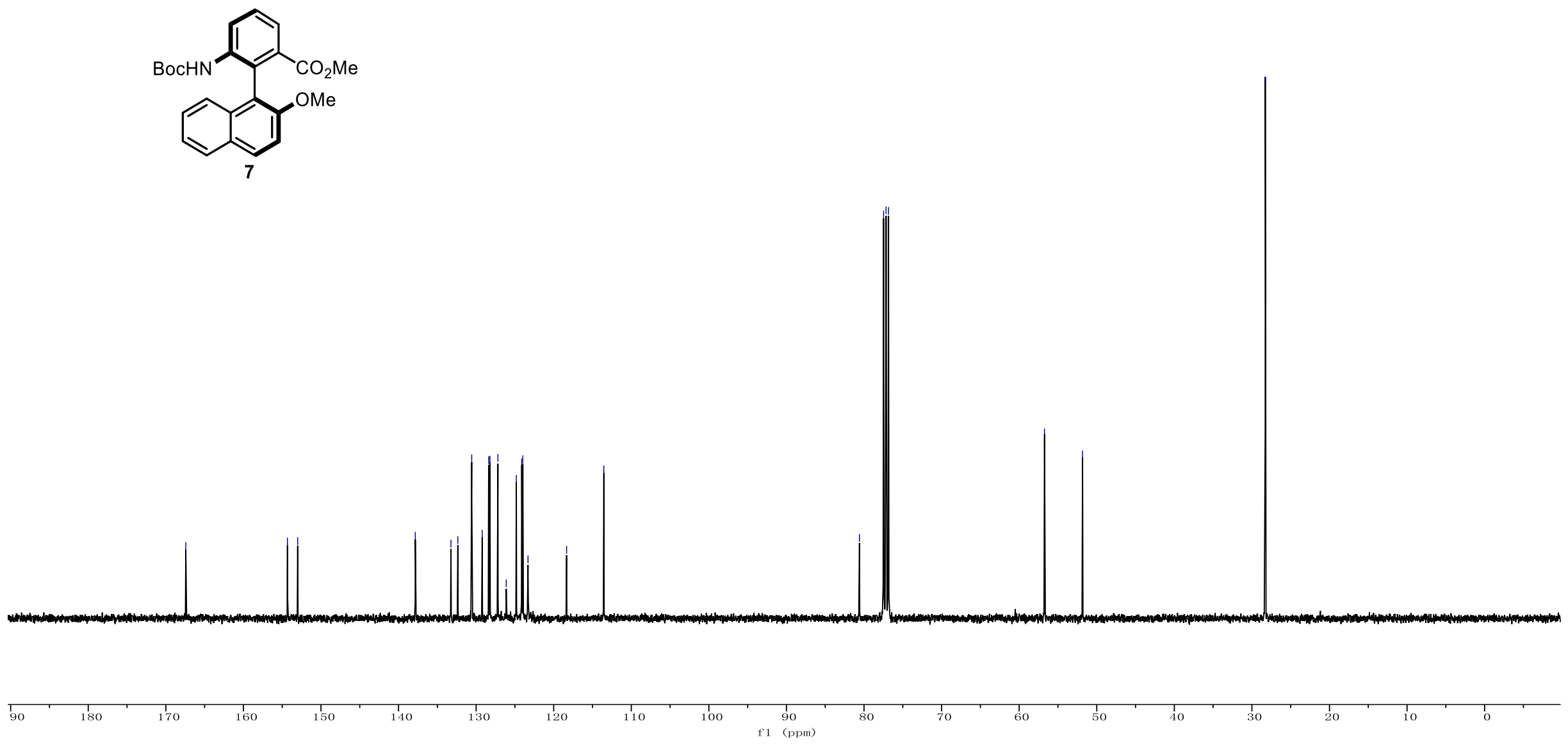

276 

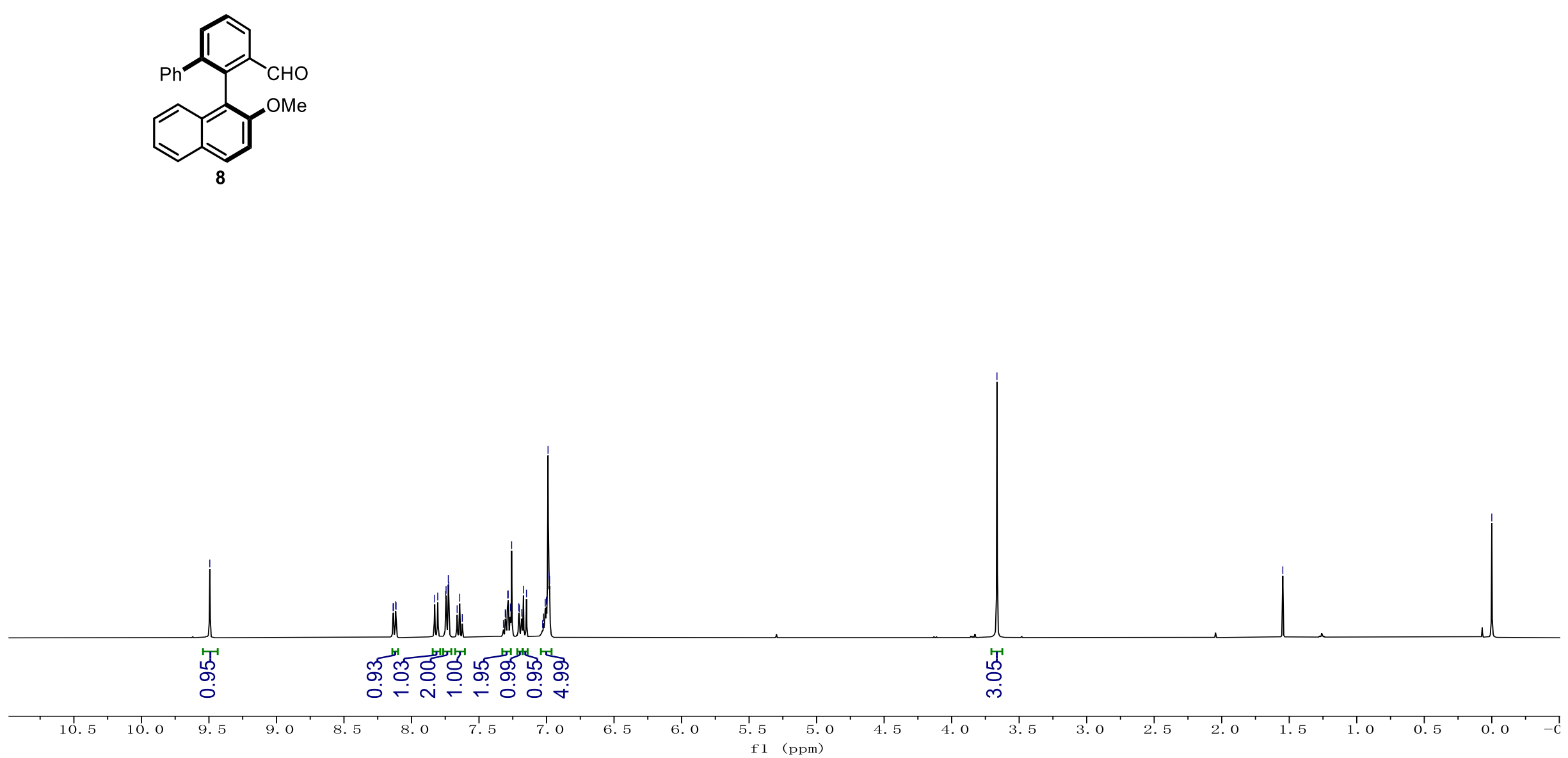

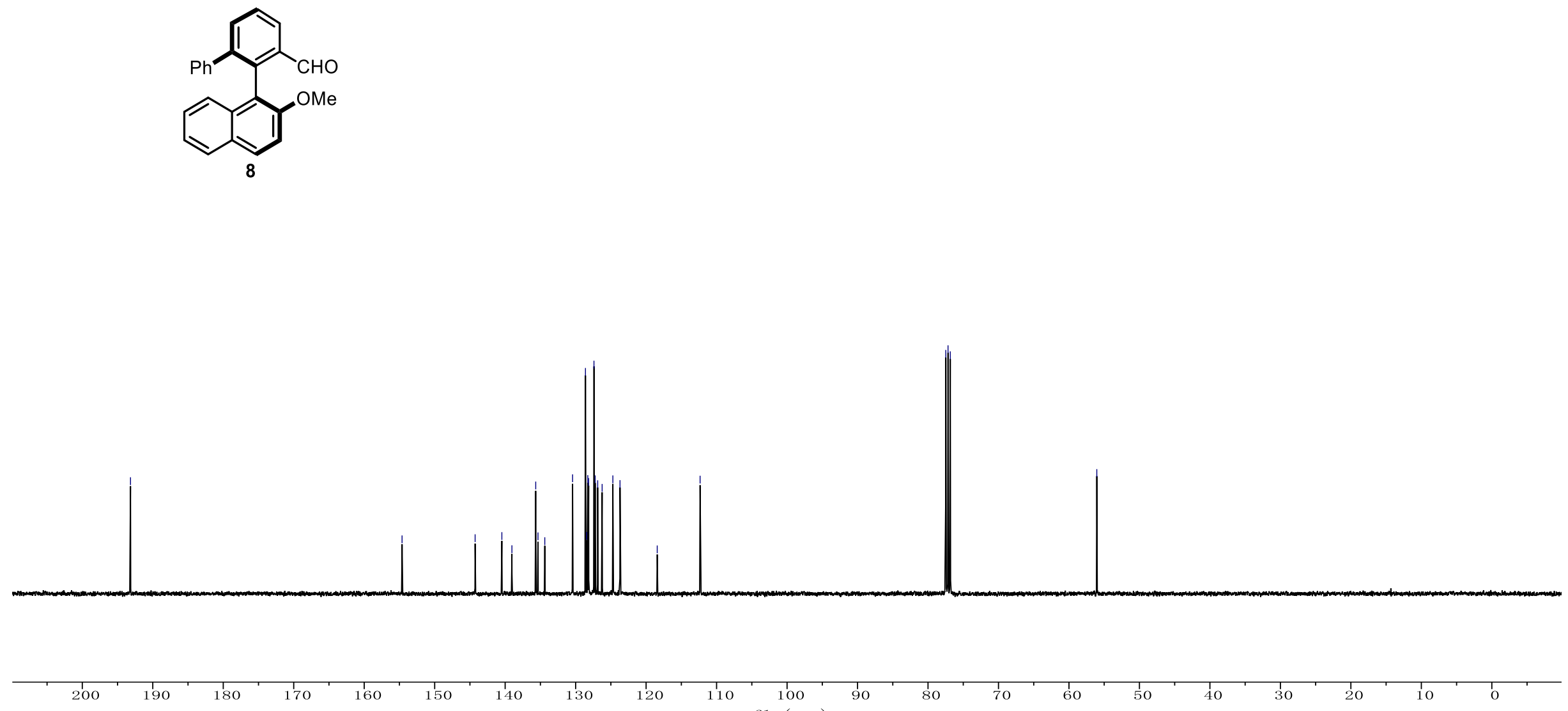

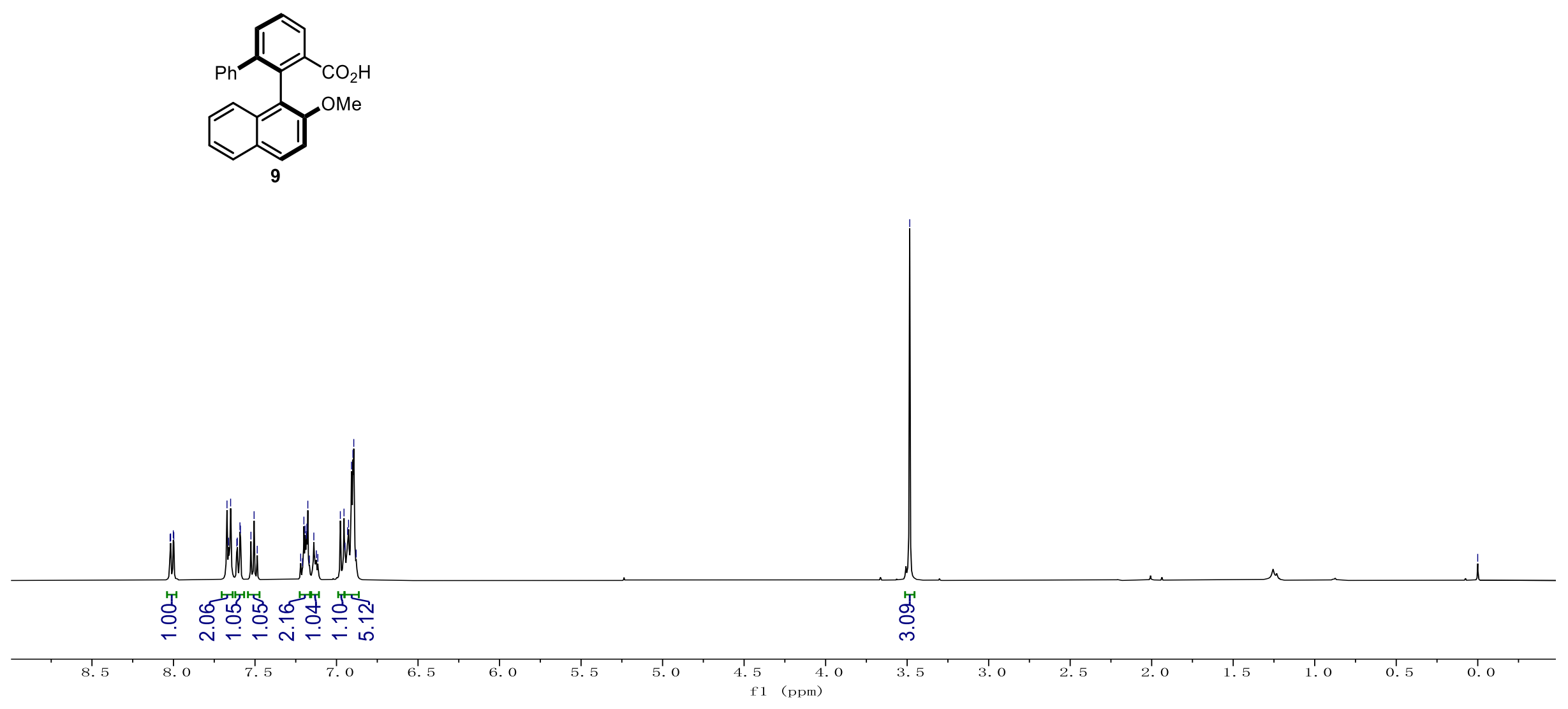


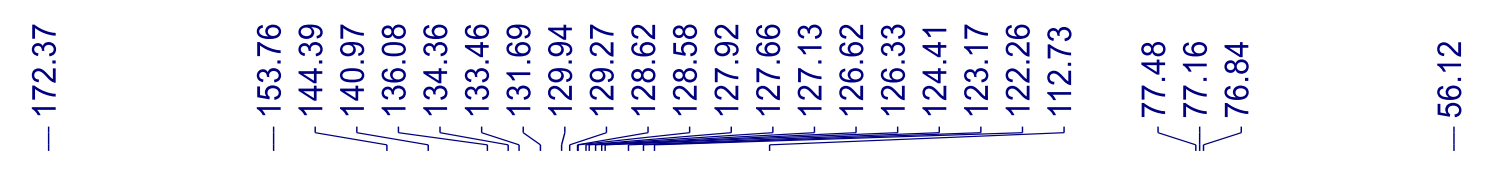
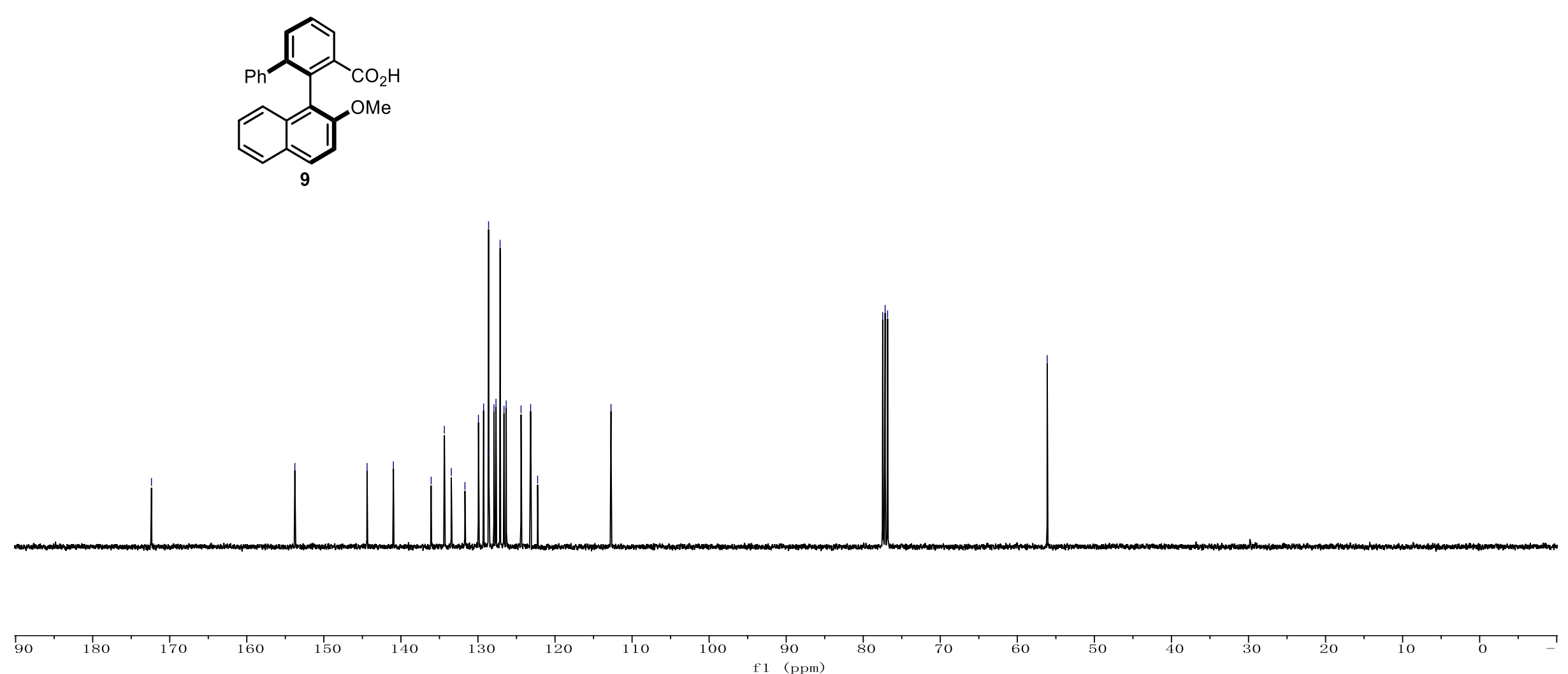

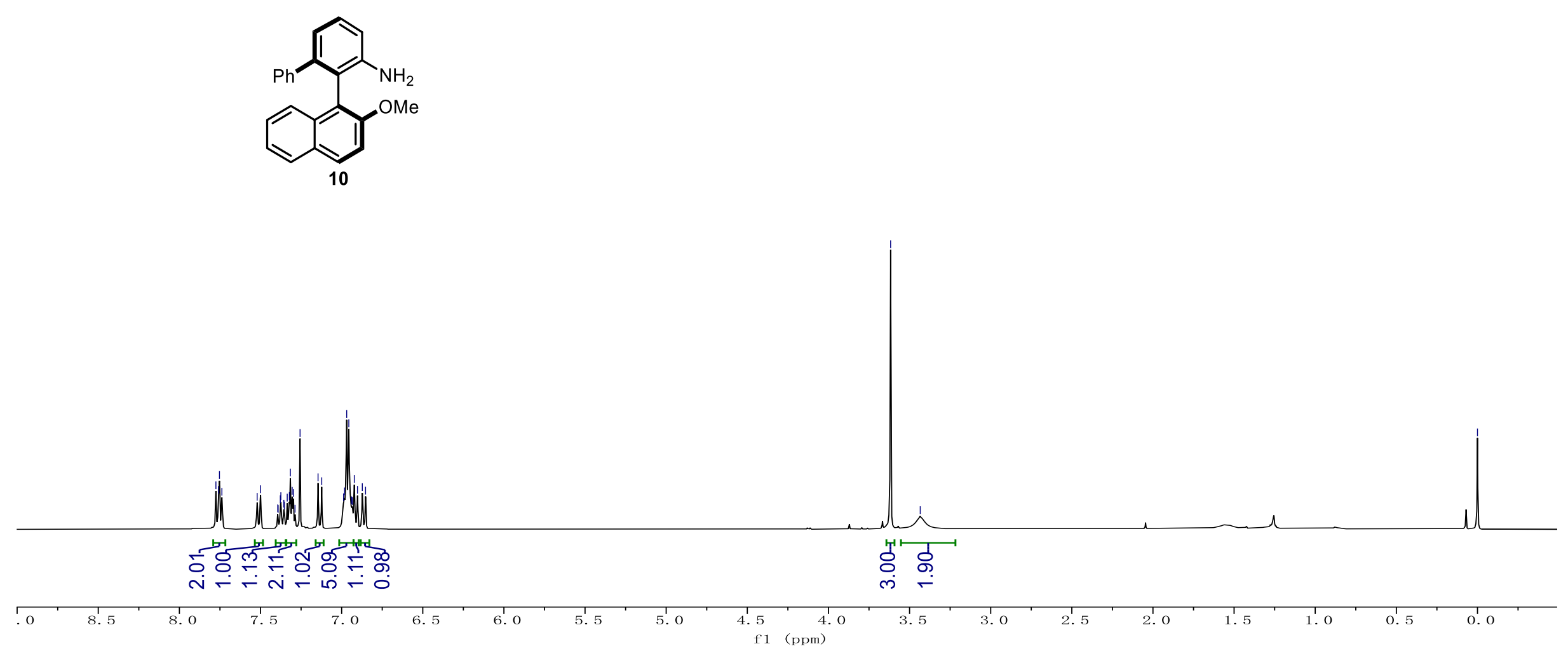


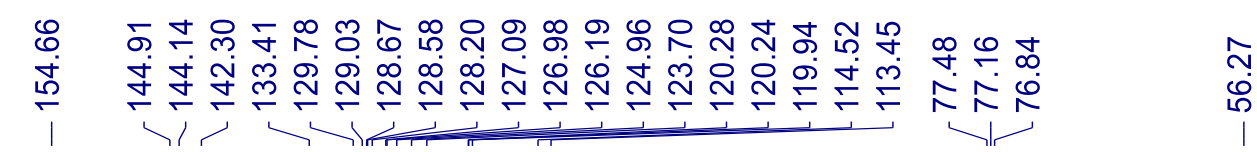
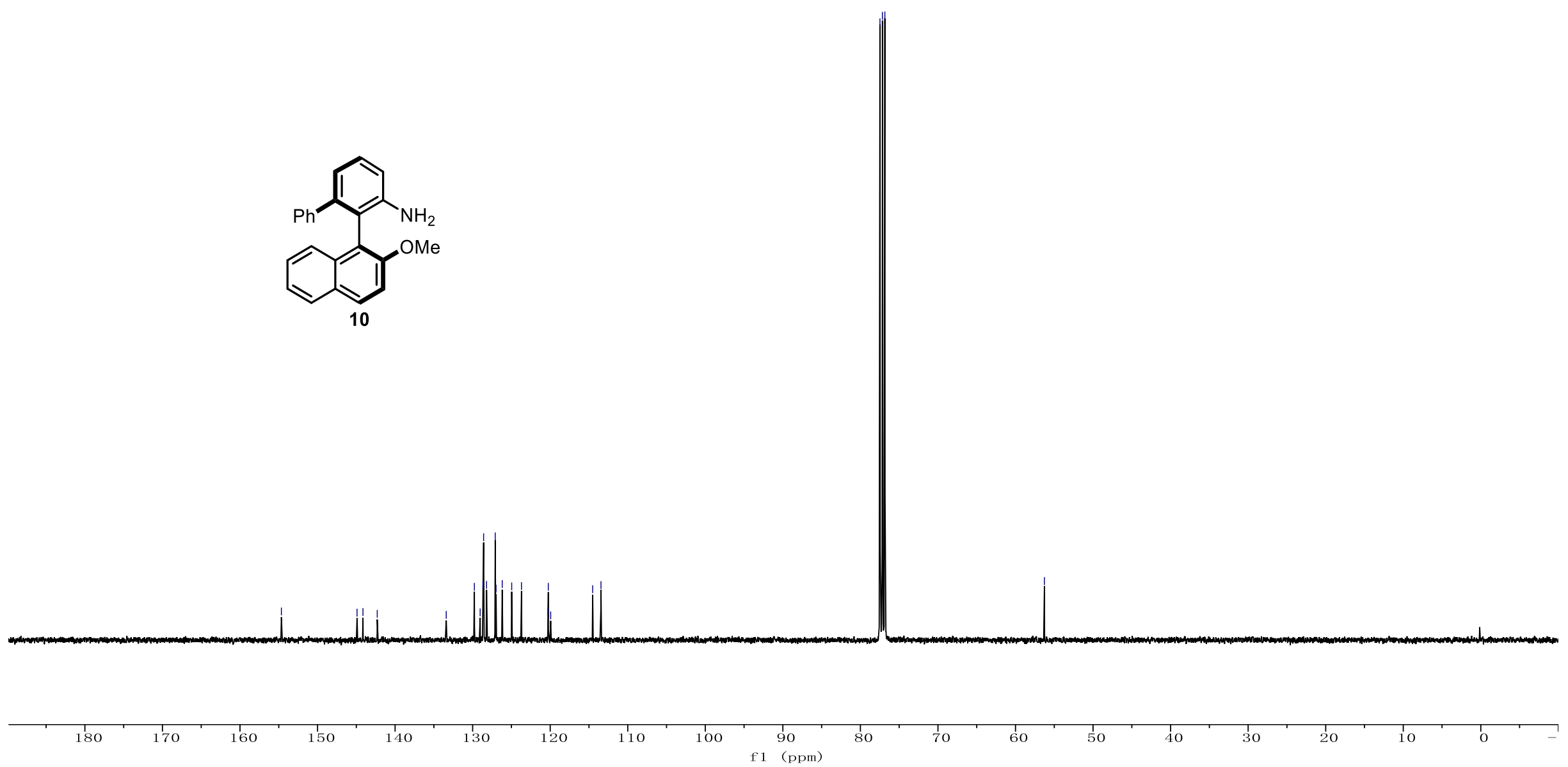